\title{
Tubular Piles - Buckling Design in a Complex Situation
}

\author{
Zur Erlangung des akademischen Grades eines \\ DOKTOR-INGENIEURS \\ von der Fakultät für \\ Bauingenieur-, Geo- und Umweltwissenschaften \\ der Universität Fridericiana zu Karlsruhe (TH) \\ genehmigte \\ DISSERTATION \\ von
}

Dipl.-Ing. Anton Hübner

aus München

Tag der mündlichen

Prüfung:

02.05.2007

Hauptreferent:

Univ.-Prof. Dr.-Ing. habil. Helmut Saal

Korreferent:

Prof. Dr. Ir. Alain Holeyman

Karlsruhe 2007 


\section{Kurzfassung (Abstract in German)}

Die analytische und experimentelle Forschung zur Stabilität von Schalentragwerken befasst sich hinsichtlich Beanspruchung und Beanspruchbarkeit größtenteils mit den elementaren Grundfällen. Die auf diesem Stand der Technik basierenden Normen bieten für viele reale Bauwerke keine Lösungsansätze oder führen durch grobe Abschätzungen zu einer deutlichen Überbemessung der Konstruktion. Beispiele hierfür sind kombinierte Spundwände, wie sie im Hafenbau eingesetzt werden. Bei diesen Systemen werden längs- oder spiralgeschweißte Rohre mit Längen um $30 \mathrm{~m}$ als pfahlartige, vorwiegend horizontal belastete Primärelemente verwendet. Aufgrund der großen Tiefgänge der neuen Generation von Schiffen sind kombinierte Spundwände bis $40 \mathrm{~m}$ Höhe in Planung. Für diese Bauwerke, deren Tragsicherheit bisher auf der Basis der Biegespannungen (d.h. Fließen durch die axialen Manteldruckspannungen) nachgewiesen wurde, wird mit Einführung des Eurocodes ein Beulsicherheitsnachweis erforderlich, obwohl bis jetzt kein Fall bekannt ist, bei dem eine solche Konstruktion beulte. Da das Verhältnis von Radius zu Wanddicke bei den Rohren kleiner als 50 ist, wurde diese Versagensform bisher kaum in Erwägung gezogen. Der zusätzlich geforderte Beulsicherheitsnachweis führt mit den verfügbaren Regeln zu $10 \%$ - 20 \% größeren Wanddicken der Rohrpfähle.

Da die Lastabtragungsmechanismen einer kombinierten Spundwand, wie in der Arbeit gezeigt, denen eines horizontal belasteten Einzelpfahls ähneln, wird das System aus Gründen der besseren Betrachtungsweise und Effizienz dementsprechend vereinfacht. Die Belastungen und die Interaktionen zwischen umgebendem Boden und Bauwerk führen zu komplizierten Randbedingungen und nicht-linearen Spannungsverteilungen, für die es derzeit keine Bemessungsregeln für den Beulsicherheitsnachweis gibt. Diese „realen Randbedingungen“ umfassen den Einfluss des Bodens, der Lastabtragung und der Imperfektionssensibilität auf die Beulsicherheit sowie veränderliche Druckverteilungen in Längs- und Umfangsrichtung. In der Arbeit wird auf die Problematik und die Unzulänglichkeiten beim Beulsicherheitsnachweis solcher Strukturen hingewiesen. In diesem Zusammenhang werden einzelne Aspekte des Beulversagens erörtert und anhand numerischer Berechnungen und experimenteller Untersuchungen diskutiert:

- Randbedingungen und Belastungen: In Vergleichsberechnungen wird dargelegt, dass die Beullast der Rohrpfähle maßgebend durch die Außendruckverteilung bestimmt wird. In diesem Zusammenhang werden Ansätze für die Ermittlung der ideellen Beullasten entwickelt, um den veränderlichen Außendruck in Längs- und Umfangsrichtung zu berücksichtigen. Als weiterer Aspekt wird der Reduktionsfaktor für die theoretische Beullast unter Außendruck zur Berücksichtigung von Imperfektionen diskutiert, der nach gängigen Bemessungsregeln unabhängig von der Geometrie ist. Basierend auf numerischen und experimentellen Untersuchungen für lange Kreiszylinderschalen wird ein neuer Bemes- 
sungsvorschlag zur Ermittlung des Reduktionsfaktors gemacht. Die Berücksichtigung von größeren Reduktionsfaktoren für längere Kreiszylinder führt zu einem signifikanten Anstieg des kritischen Bemessungsbeuldrucks.

- Bettung: Im ersten Schritt werden unterschiedliche Boden- und BodenBauwerk-Interaktionsmodelle analysiert und anhand numerischer Berechungen diskutiert. Zum Vergleich werden verschiedene Belastungsversuche an Modellrohrpfählen durchgeführt. Für die weiteren Untersuchungen werden Bodemodelle gewählt, die für den weiteren Verlauf effizient sind, aber ebenso alle erforderlichen Parameter berücksichtigen. Es wird gezeigt, dass für die Steigerung der Beullast eines Zylinders unter Axiallast im Gegensatz zu Zylindern unter Außendruck sehr große Bettungen erforderlich sind. Die Dickwandigkeit der untersuchten Zylinder bestärkt dieses Verhalten. Zur Bestimmung der horizontalen Bettungssteifigkeit von Sand bei kleinen Dehnungen wurden Versuche durchgeführt (Bender Tests). Basierend auf weiteren Versuchsergebnissen aus Modellversuchen an Rohrpfählen und numerischen Berechnungen werden Vorschläge zur Berücksichtigung des unterstützenden Effekts des Bodens auf die Beullast aufgeführt. Hierbei wird die Bettung des Zylinders in radialer, tangentialer und axialer Richtung berücksichtigt.

- Geometrische Imperfektionen: Der Einfluss geometrischer Imperfektionen auf eine Kreiszylinderschale, die im Boden eingebettet ist, kann von dem einer ungebetteten Schale abweichen. Daher werden die radialen Formabweichungen und die Aufstandsimperfektionen betrachtet, um den Einfluss der Imperfektionen auf den Abminderungsfaktor im Beulsicherheitsnachweis festzulegen. Numerische Untersuchungen zeigen, dass die geometrischen Imperfektionen bei einer Druckbeanspruchung in Meridianrichtung der Rohrpfähle von kombinierten Spundwänden von geringer Bedeutung sind.

Die aktuellen Bemessungsregelwerke zum Schalenbeulen bieten für viele reale Bauwerke keine Lösungsansätze oder führen durch grobe Abschätzungen zu einer deutlichen Überbemessung der Konstruktion. Daher wurden für den Fall von kombinierten Spundwänden detaillierte Untersuchungen durchgeführt. Aus den interpretierten Ergebnissen der vorhergehenden Teilgebiete werden schließlich Bemessungsvorschläge entwickelt, um die bestehenden Beulsicherheitsnachweise um problemspezifischere Ansätze zu erweitern. 


\section{Abstract}

Shells have been thoroughly studied over the last century resulting in a sophisticated compendium of standards on shell structures: in the first place the European Standard on Shell Design, providing the designer and the analyst with a comprehensive and very general tool to assess all types of shell structures. The design rules have been kept so general that they may result in extremely conservative constructions, since several special influences are not taken into account. An example is a large thin-walled tube used in harbour constructions. The latter tubes are commonly employed as parts of combiwalls or as piles. Large diameter steel pile pipes are manufactured by longitudinally or spirally welding a hot-rolled steel strip of thickness $10 \mathrm{~mm}$ to $24 \mathrm{~mm}$. The usual length of the piles ranges from $20 \mathrm{~m}$ to $30 \mathrm{~m}$ with a diameter of $900 \mathrm{~mm}-2400 \mathrm{~mm}$. Increasing drafts of ships even demand for new harbour constructions with longer tubes measuring up to $40 \mathrm{~m}$. The assessment of these tubes is mainly based on stress design regarding the stresses from bending moments of the pile. Due to experimental experience with cylinders of a slenderness radius over thickness of about 50 the problem of shell buckling has been disregarded so far. The design procedure will change with the introduction of the Eurocodes, as for most harbour constructions a buckling assessment will become necessary resulting in a $10 \%-20 \%$ larger wall thickness. For a design according to the common shell buckling rules several extremely conservative assumptions have to be made. Although, many years of practice revealed no bad experience with respect to buckling. The reason for the positive difference in the carrying behaviour between tubular piles and the laboratory shells arises from the complexity of the problem far beyond the basic rules of the codes.

Since the load transfer mechanisms of a combiwall are similar to those of a horizontally loaded pile as shown in the work, the system is simplified due to efficiency, accordingly. This work describes the design procedure for tubular piles and points out the conservative assumptions in the assessment of the investigated problem. In the next step these conservative assumptions are discussed in order to provide suggestions and comments for enhancing design rules for tubular piles regarding shell buckling. The discussion is based on "real boundary conditions" governing the carrying behaviour of the investigated structure with respect to shell buckling: Influence of the soil, the load transfer mechanisms, and the imperfection sensitivity on the buckling behaviour, and the varying pressure distributions in longitudinal and circumferential direction. The following aspects of the buckling failure are discussed based on numerical and experimental investigations:

- Boundary conditions and loading: Comparing analyses reveal the significant influence of the external pressure distribution on the carrying capacity of tubular piles. In this context approaches for evaluating the ideal buckling loads are developed for considering the influence of a pressure distribution varying in cir- 
circumferential and longitudinal direction. Further, the reduction factor for the theoretical buckling pressure taking imperfections into account is investigated in detail. As in current codes the latter factor is independent of the geometry of the shell, this aspect is enhanced by introducing a new design concept for obtaining a reduction factor depending on the length. The proposal is based on numerical and experimental evidence. The consideration of a larger reduction factor for long cylinders, significantly increases the critical buckling load for externally pressurized cylinders.

- Foundation: In a first step different soil and soil-structure interaction models are analysed and discussed based on numerical calculations. For comparison different loading experiments are executed with a reduced scale test set-up for tubular piles. In the following investigations, soil models are chosen which are efficient in use but also take all necessary parameters into account. It is shown that for an increase of the buckling load of a cylinder subjected to axial load, a far larger stiffness of the surrounding material is necessary in comparison to an externally pressurized cylinder. The rather large wall thicknesses of the cylinders emphasize this behaviour. In order to obtain the horizontal modulus of subgrade reaction for sand subjected to small strains laboratory experiments are performed (Bender Tests). Based on additional test results from the reduced scale tests and numerical analyses different proposals are given for considering the supporting effect of the soil. In this context the supporting effect in radial, circumferential, and axial direction is discussed separately.

- Geometrical imperfections: The aspect of geometrical imperfections is investigated with the aim to determine the influence of radial shape deformations and uneven supports on the buckling load of cylinders. For this study the special problem of combiwalls is set aside and the problem is studied for the general case of a loaded cylindrical shell. The intention is to obtain a better founded knowledge of the buckling load reductions arising from uneven supports, as it is assumed that this aspect does not play a decisive role for combiwalls due to the surrounding soil. Numerical investigations show that geometrical imperfections do not influence the carrying capacity of tubular piles in combiwalls subjected to axial loads considerably.

Current design rules for shell buckling provide no solutions for many real constructions or result in a significant underestimation of the carrying capacity by proposing rough approximations. Thus, for the case of combiwalls improved assessment rules based on the different aspects influencing the carrying behaviour in a different way are derived and given as design proposals for improving and extending the existing design codes on shell buckling. 


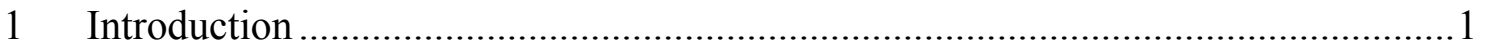

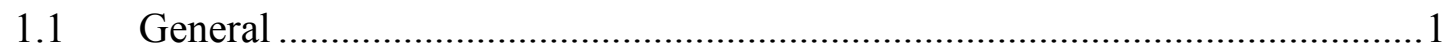

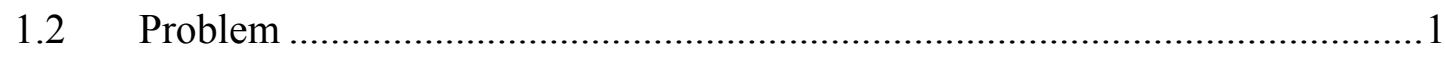

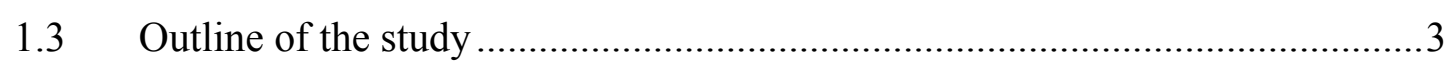

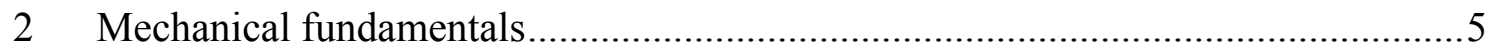

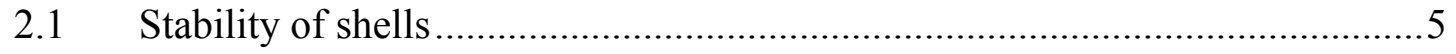

2.2 Fundamentals of continuum mechanics .......................................................

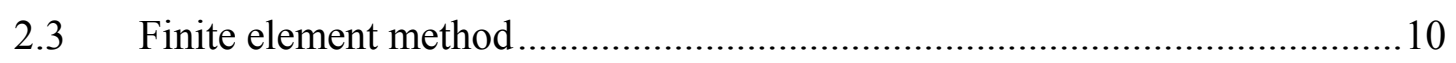

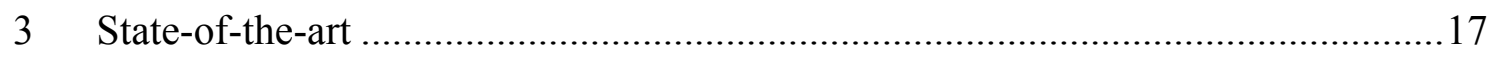

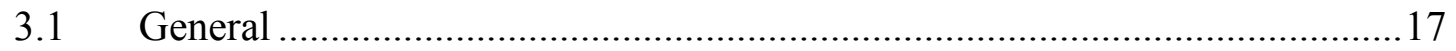

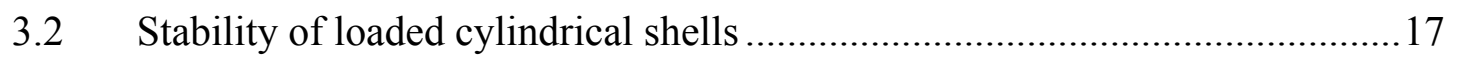

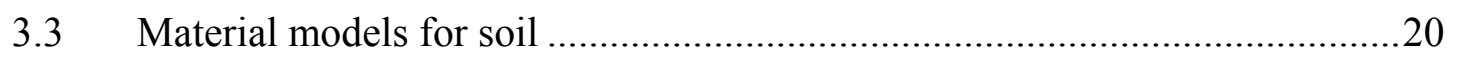

3.4 Approaches for modelling the soil-structure interaction (SSI) ......................23

4 Common practical design of combiwalls ..........................................................2

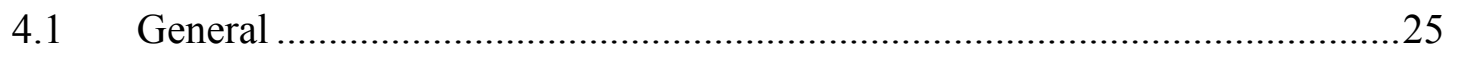

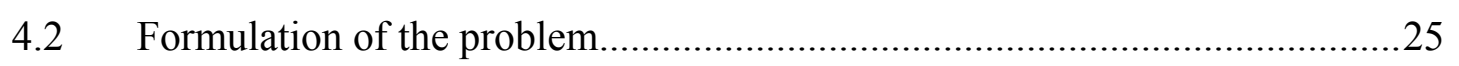

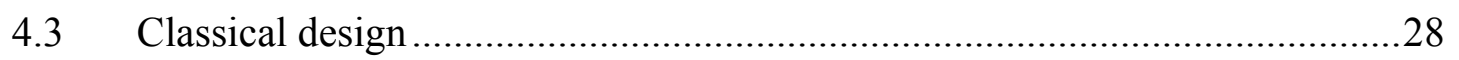

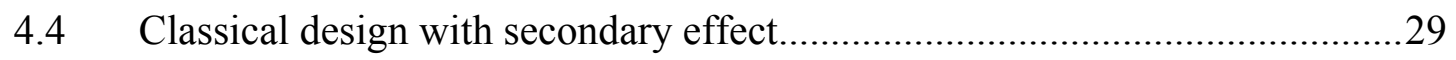

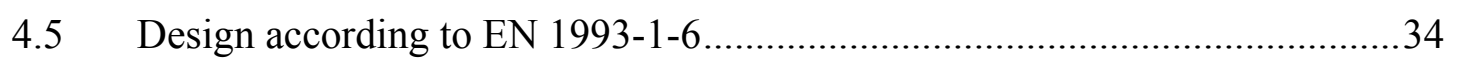

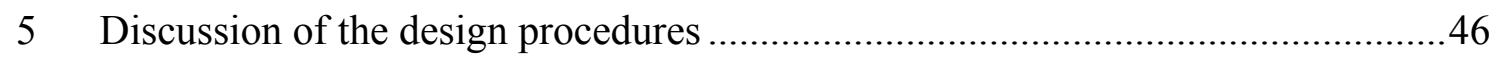

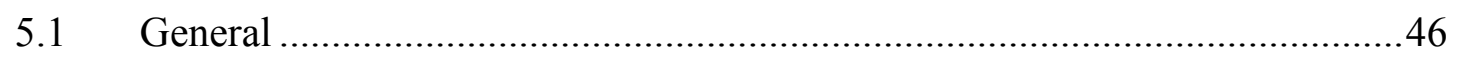

5.2 Comparison between combiwall and single pile.............................................46

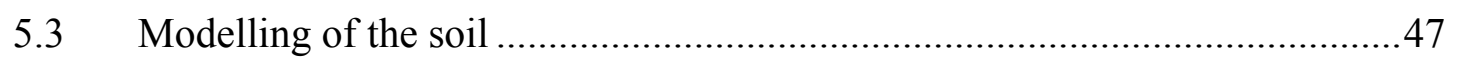

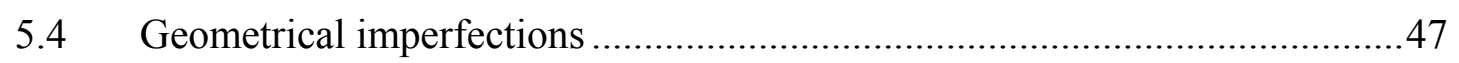

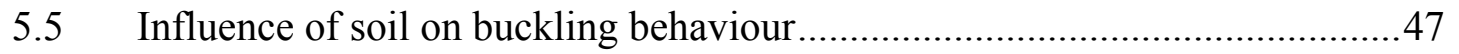

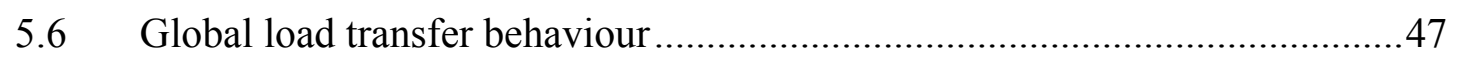

5.7 Boundary conditions and loads - external pressure ........................................4

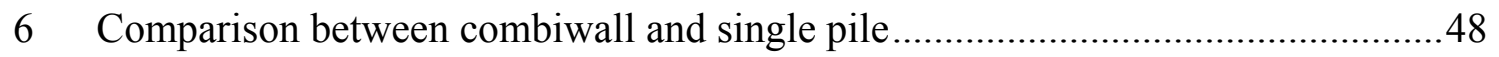

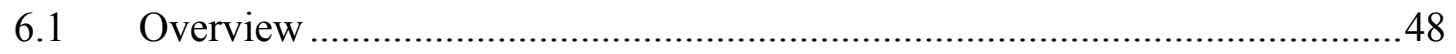

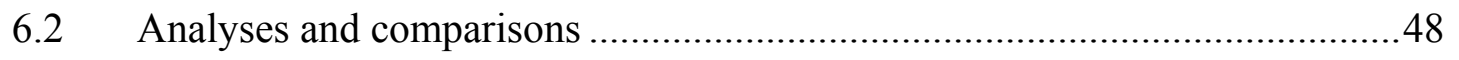

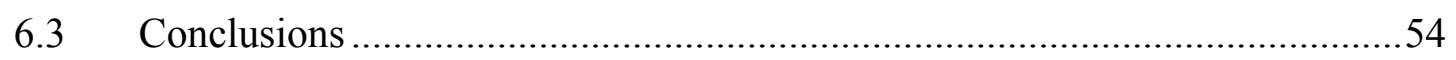


7 Investigation of soil and soil-structure interaction ..........................................56

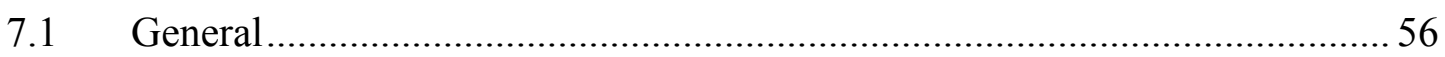

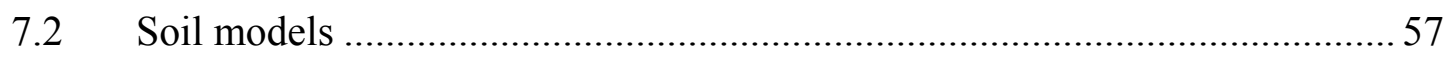

7.3 Comparison of different soil models for a loaded block ............................... 74

7.4 Comparison of different soil models for a strip footing ............................... 76

7.5 Comparison of different soil models for a laterally loaded pile ..................... 81

7.6 Discussion of the soil-structure interaction models ..................................... 103

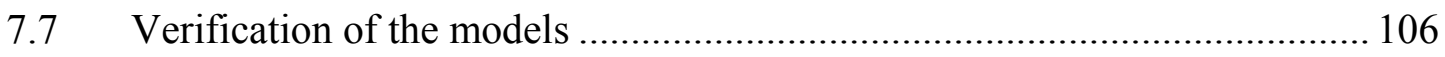

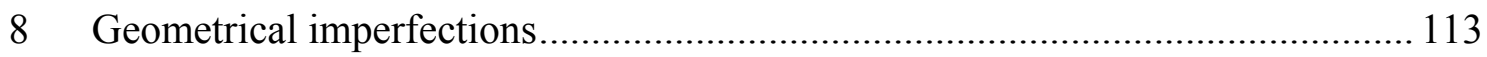

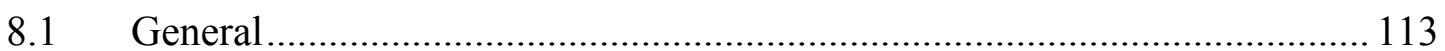

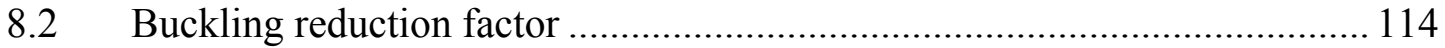

8.3 Geometrically imperfect boundary conditions ....................................... 116

8.4 Bending moment and geometrically imperfect boundary conditions ............ 122

8.5 Summary and conclusions ...................................................................... 127

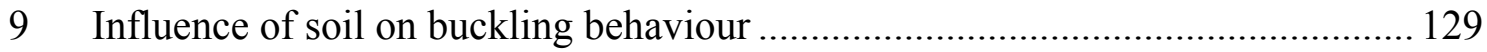

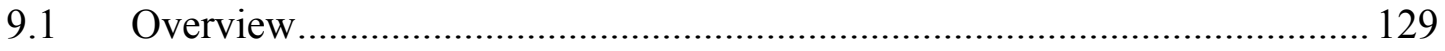

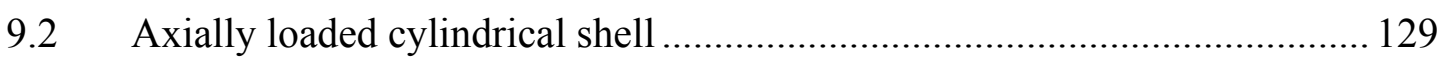

9.3 Cylindrical shell subjected to external pressure ......................................... 135

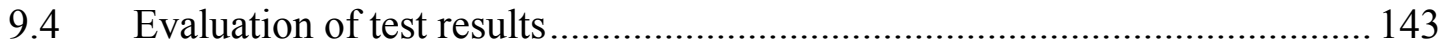

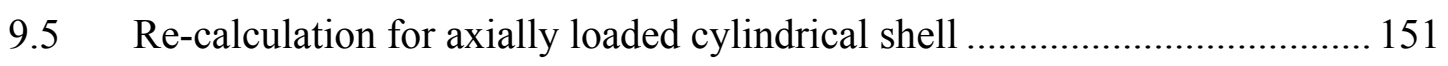

9.6 Back-calculation for cylindrical shell subjected to external pressure............ 156

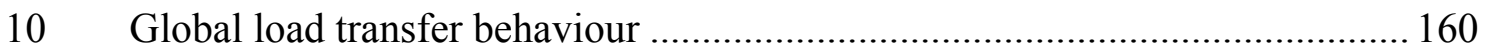

10.1 Load transfer mechanisms of the bending moment over the pile length ....... 160

10.2 Load transfer in the area of the maximum bending moment ....................... 167

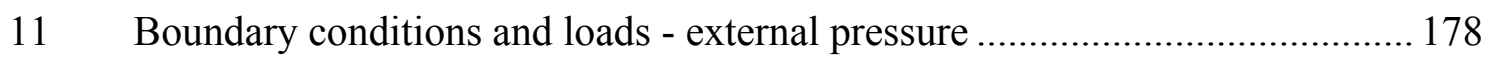

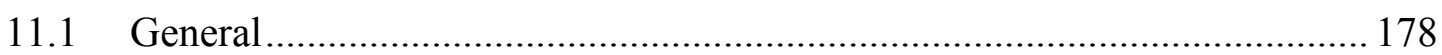

11.2 Investigation of the shell's resistance for external pressure ....................... 179

11.3 Investigation of the loading for external pressure .................................... 182

11.4 Summary and comparison of previous analyses...................................... 183

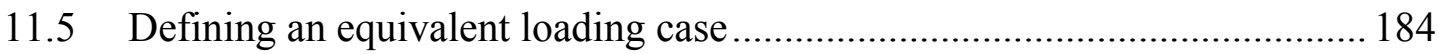

11.6 Evaluation of the elastic buckling reduction factor ................................... 197 


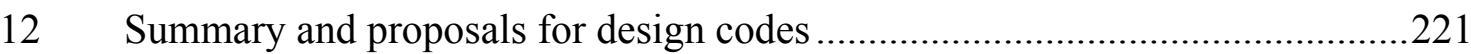

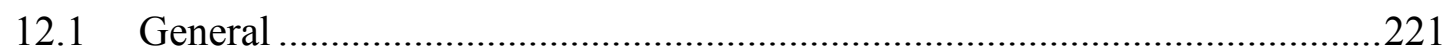

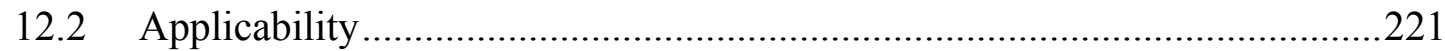

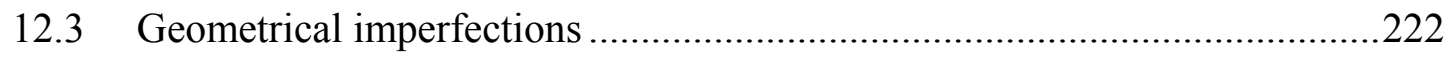

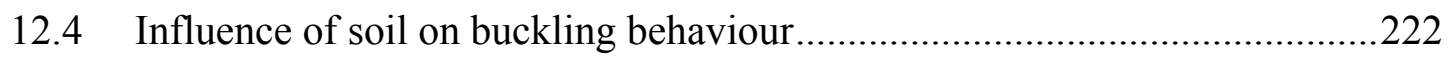

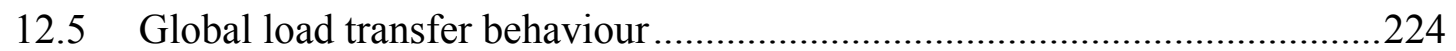

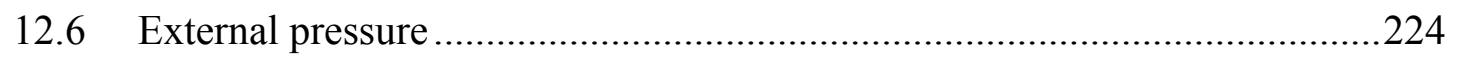

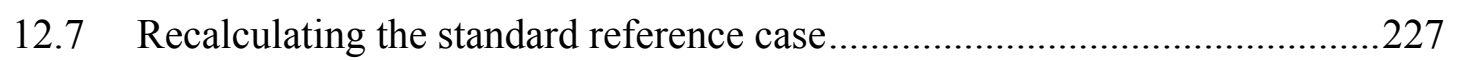

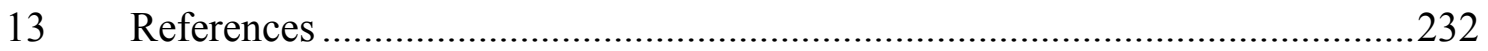

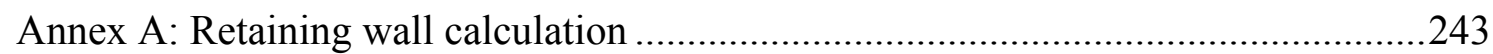

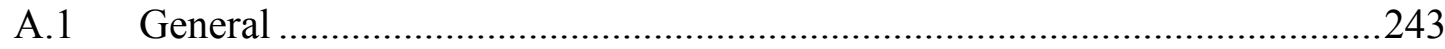

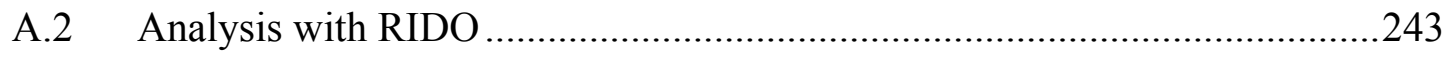

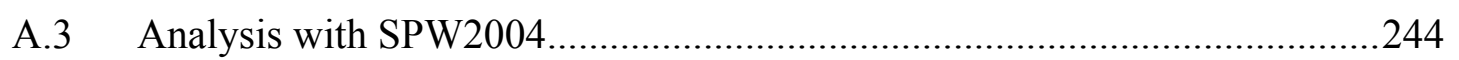

A.4 Comparison RIDO - SPW2004 _............................................................24

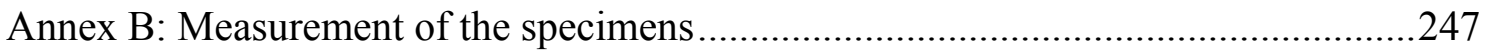

B.1 Preliminary investigation of specimens made of non-alloyed steel ..............247

B.2 Investigation of test tube for the reduced scale tests .................................252

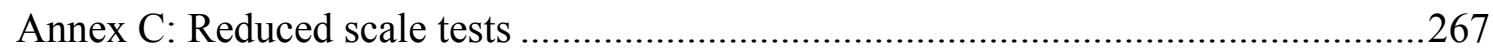

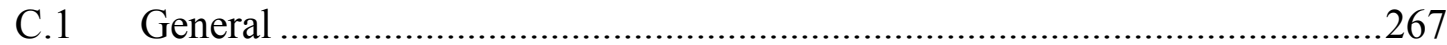

C.2 Test and measuring program for the laboratory tests ................................267

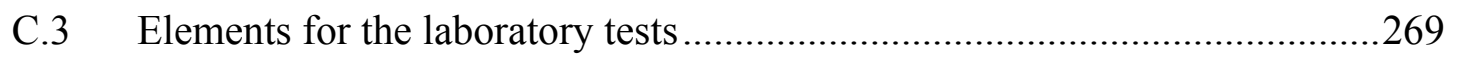

C.4 Set-up and execution of laboratory tests ...............................................2 271

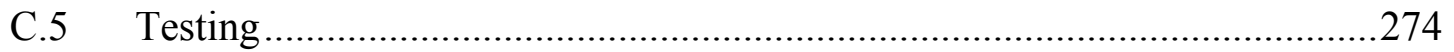

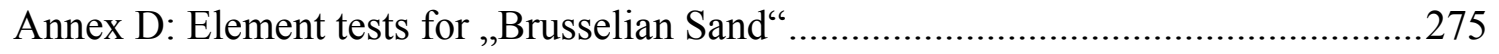

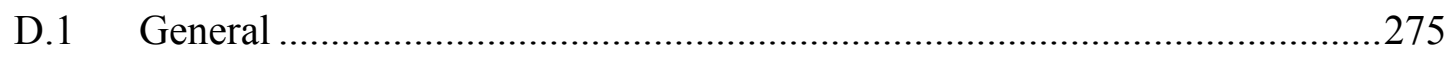

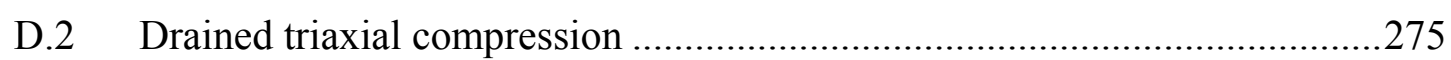

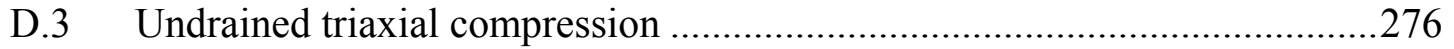

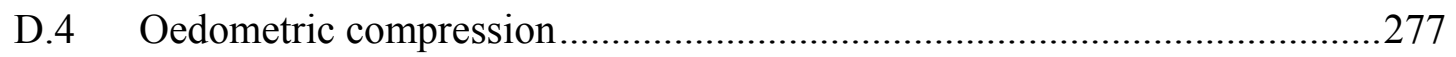

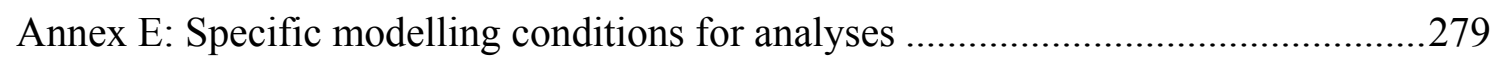

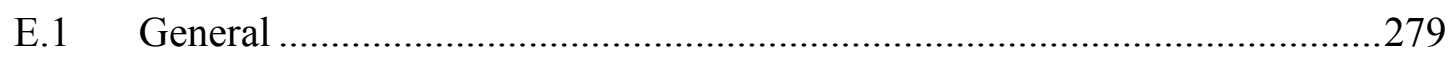

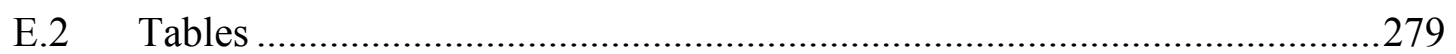




\section{Notation list}
length $=l$
force $=f$
time $=t$
angle $=a$
mass $=m$

\section{Greek letters}

\begin{tabular}{|c|c|c|}
\hline$\Gamma_{u}, \Gamma_{\sigma}$ & {$[-]$} & surface definition \\
\hline$\Delta$ & {$[l]$} & radial dilation \\
\hline$\Delta l$ & {$[-]$} & arc length \\
\hline$\Delta w_{k}, \Delta w$ & {$[l]$} & imperfection amplitude \\
\hline$\stackrel{\circ}{\sigma}$ & {$\left[f / l^{2}\right]$} & Jaumann stress tensor \\
\hline$\alpha_{\text {column }}$ & {$[-]$} & linear elastic reduction factor for column \\
\hline$\alpha_{G}, \beta_{G}$ & {$[a]$} & geometrical coefficients \\
\hline$\alpha_{x}, \alpha_{\varphi}$ & {$[-]$} & linear buckling reduction factor \\
\hline$\alpha, \beta$ & {$[-]$} & exponents (hypoplasticity) \\
\hline$\alpha, \theta$ & {$[a]$} & angle \\
\hline$\alpha_{\varphi}^{*}$ & {$[-]$} & adopted buckling reduction factor for external pressure \\
\hline$\beta$ & {$[-]$} & plastic range factor from EN 1993-1-6 \\
\hline$\beta_{g}, \beta_{s}$ & {$[-]$} & reduction factor from EN 1993-5 \\
\hline$\beta_{x, \text { sand }}, \beta_{\varphi, \text { sand }}$ & {$[-]$} & factor for increasing buckling stress due to sand \\
\hline$\chi_{x}, \chi_{\varphi}$ & {$[-]$} & buckling reduction factor \\
\hline$\delta$ & {$[a]$} & angle of interface friction \\
\hline$\varepsilon$ & {$[-]$} & slenderness from EN 1993-1-1 \\
\hline$\varepsilon$ & {$[-]$} & strain tensor \\
\hline$\varepsilon, \Delta \varepsilon$ & {$[-]$} & general imperfection amplitude \\
\hline$\varepsilon_{c, m}$ & {$[-]$} & circumferential strain in centre of tube wall \\
\hline$\varepsilon_{c, o}$ & {$[-]$} & circumferential strain on outside of the tube \\
\hline$\varepsilon_{l, m}$ & {$[-]$} & longitudinal strain in centre of tube wall \\
\hline$\varepsilon_{l, o}$ & {$[-]$} & longitudinal strain on outside of the tube \\
\hline$\gamma_{M}$ & {$[-]$} & safety factor for resistance \\
\hline$\gamma, \gamma^{\prime}, \gamma_{\min ,}, \gamma_{\max }, \gamma_{s}$ & {$\left[f / l^{3}\right]$} & density for different states \\
\hline$\eta$ & {$[-]$} & trial function \\
\hline$\eta$ & {$[-]$} & interaction factor for buckling \\
\hline$\eta_{L}$ & {$[-]$} & normalized length \\
\hline$\varphi$ & {$[a]$} & effective angle of internal friction \\
\hline$\varphi_{c}$ & {$[a]$} & critical friction angle \\
\hline$\varphi_{\text {load }}$ & {$[a]$} & load angle \\
\hline
\end{tabular}




\begin{tabular}{|c|c|c|}
\hline$\varphi_{\text {rot }}$ & {$[a]$} & rotation of pile \\
\hline$\kappa$ & {$[-]$} & buckling reduction factor \\
\hline$\kappa_{c}$ & {$[-]$} & curvature \\
\hline$\kappa_{e l}$ & {$[-]$} & elastic curvature \\
\hline$\kappa_{p l}$ & {$[-]$} & plastic (residual) curvature \\
\hline$\lambda$ & {$[-]$} & dimensionless load parameter \\
\hline $\bar{\lambda}$ & {$[-]$} & relative slenderness of the shell \\
\hline$\lambda_{0}$ & {$[-]$} & squash limit relative slenderness \\
\hline$\lambda_{c l}$ & {$[-]$} & axial buckling half-wave length \\
\hline$\lambda_{E V, i}$ & {$[-]$} & eigenvalue \\
\hline$\overline{\lambda_{p}}$ & {$[-]$} & plastic limit relative slenderness \\
\hline$\overline{\lambda_{x}}, \overline{\lambda_{\varphi}}, \overline{\lambda_{\tau}}$ & {$[-]$} & relative slenderness of a shell \\
\hline$\mu$ & {$[-]$} & Poisson ratio \\
\hline$\mu_{f r i c,} \mu_{l i m}$ & {$[-]$} & friction coefficients \\
\hline$\mu_{\varepsilon}$ & {$[-]$} & strain ratio from EN 1993-5 \\
\hline$v$ & {$[a]$} & dilation angle \\
\hline$\theta_{L}$ & {$[a]$} & Lode angle \\
\hline$\rho$ & {$\left[m / l^{3}\right]$} & density \\
\hline$\sigma$ & {$\left[f \mid l^{2}\right]$} & stress tensor \\
\hline$\sigma_{1}, \sigma_{2}, \sigma_{3}$ & {$\left[f / l^{2}\right]$} & soil stresses; 3 refers to vertical direction \\
\hline$\sigma_{c l}$ & {$\left[f / l^{2}\right]$} & classical buckling stress (ideal buckling stress) \\
\hline$\sigma_{k}$ & {$\left[f / l^{2}\right]$} & reference stress \\
\hline$\sigma_{m}$ & {$\left[f / l^{2}\right]$} & mean pressure stress \\
\hline$\sigma_{n}$ & {$\left[f / l^{2}\right]$} & normal stresses \\
\hline$\sigma_{r}$ & {$\left[f / l^{2}\right]$} & radial stress \\
\hline$\sigma_{r e f}$ & {$\left[f / l^{2}\right]$} & reference stress \\
\hline$\sigma_{v}$ & {$\left[f / l^{2}\right]$} & effective vertical stress \\
\hline$\sigma_{v}^{*}$ & {$\left[f / l^{2}\right]$} & fixed vertical confinement vertical stress \\
\hline$\sigma_{x}, \sigma_{\varphi}$ & {$\left[f / l^{2}\right]$} & stresses \\
\hline$\sigma_{x, R c}, \sigma_{\varphi, R c}, \sigma_{\tau, R c}$ & {$\left[f / l^{2}\right]$} & critical buckling stresses \\
\hline$\sigma_{x, R d}, \sigma_{\varphi, R d}$ & {$\left[f / l^{2}\right]$} & design stress resistance \\
\hline$\tau$ & {$\left[f / l^{2}\right]$} & shear stresses \\
\hline$\tau_{R d}$ & {$\left[f / l^{2}\right]$} & design shear stress resistance \\
\hline$\tau_{y}$ & {$\left[f / l^{2}\right]$} & shear stress in direction $y$ \\
\hline$\omega$ & {$[-]$} & dimensionless length parameter \\
\hline$\omega_{G}$ & {$[-]$} & empirical power index \\
\hline$\zeta$ & {$[-]$} & constant \\
\hline$\zeta_{L}$ & {$[-]$} & normalized length \\
\hline
\end{tabular}




\section{$\underline{\text { Latin letters }}$}

\begin{tabular}{|c|c|c|}
\hline$A$ & {$\left[l^{2}\right]$} & area of cross-section \\
\hline$a$ & {$[l]$} & radius considering ovalisation effect \\
\hline$A, A_{R}$ & {$[-]$} & empirical correction factor \\
\hline$a_{f}$ & {$[-]$} & load fraction ratio \\
\hline$B$ & {$[l]$} & system width \\
\hline$b$ & {$[-]$} & load factor \\
\hline$b, b^{*}$ & {$\left[f / l^{3}\right]$} & mass forces \\
\hline$b_{b}, h_{b}$ & {$[l]$} & geometry of bulge \\
\hline$b_{B A}$ & {$[-]$} & constant \\
\hline$b_{c}$ & {$[-]$} & constant \\
\hline$B_{P}$ & {$[-]$} & variable \\
\hline$c$ & {$\left[f / l^{2}\right]$} & cohesion \\
\hline$C$ & {$[-]$} & coefficient \\
\hline$c_{1}$ & {$[-]$} & factor from EN 1993-5 \\
\hline$C_{1}, C_{2}, C_{3}$ & {$[-]$} & coefficients \\
\hline$C_{c}$ & {$\left[f / l^{2}\right]$} & compression index \\
\hline$C_{L}$ & {$[-]$} & equivalent pressure correction factor \\
\hline$C_{u}$ & {$[-]$} & coefficient of uniformity \\
\hline$C_{x}, C_{\varphi}, C_{\tau}$ & {$[-]$} & coefficient in buckling strength assessment \\
\hline$d$ & {$[l]$} & global node displacements \\
\hline $\boldsymbol{D}$ & {$[-]$} & tensor of the deformation rate $\boldsymbol{D}$ \\
\hline$D, d$ & {$[l]$} & diameter (middle surface) \\
\hline$d_{50}$ & {$[l]$} & mean diameter of the grain \\
\hline$D_{i}$ & {$[l]$} & inner diameter \\
\hline$D_{o}$ & {$[l]$} & outer diameter \\
\hline$D_{p l}$ & {$\left[f l^{2}\right]$} & flexural plate stiffness \\
\hline$D_{r}$ & {$[-]$} & relative density \\
\hline $\boldsymbol{e}$ & {$[l]$} & unit vector \\
\hline $\boldsymbol{E}$ & {$[-]$} & Green-Lagrangian strain tensor \\
\hline E & {$\left[f / l^{2}\right]$} & Young’s modulus \\
\hline$e$ & {$[l]$} & ovalisation \\
\hline$e, e_{0, i n i}$ & {$[-]$} & void ratio, initial void ratio \\
\hline$E^{50}$ & {$\left[f / l^{2}\right]$} & $\begin{array}{l}\text { Young's modulus of soil at } 50 \% \text { of failure load from } \\
\text { triaxial tests }\end{array}$ \\
\hline$e_{c 0}$ & {$[-]$} & critical void ratio for a stress-free state \\
\hline$e_{d 0}$ & {$[-]$} & minimum void ratio for a stress-free state \\
\hline$e_{i 0}$ & {$[-]$} & maximum void ratio for a stress-free state \\
\hline$e_{\max }$ & {$[-]$} & maximum void ratio \\
\hline$e_{\min }$ & {$[-]$} & minimum void ratio \\
\hline$E_{\text {oed }}$ & {$\left[f / l^{2}\right]$} & oedometer modulus \\
\hline$e_{p h}$ & {$[f / l]$} & passive earth resistance \\
\hline
\end{tabular}




\begin{tabular}{|c|c|c|}
\hline$E_{p} I_{p}$ & {$\left[f l^{2}\right]$} & flexural stiffness of the pile \\
\hline$E_{\text {ref }}$ & {$\left[f / l^{2}\right]$} & reference Young's modulus \\
\hline$E_{S}$ & {$\left[f / l^{2}\right]$} & soil modulus (1D model) \\
\hline $\boldsymbol{F}$ & {$[-]$} & deformation gradient \\
\hline$F$ & {$[f]$} & force or load \\
\hline$f$ & {$[-]$} & dimensionless parameter \\
\hline$f_{b}, f_{e}, f_{d}$ & {$[-]$} & scalar functions \\
\hline Fload & {$[f]$} & energetically equivalent global node vector \\
\hline$f_{s f}$ & {$\left[f / l^{2}\right]$} & skin friction \\
\hline$F_{s f}$ & [-] & scalar stress function \\
\hline$F_{\text {sheet }}$ & {$[f / l]$} & force transferred from sheeting to pile \\
\hline$f_{y}, f_{y, k}$ & {$\left[f / l^{2}\right]$} & yield stress (characteristic value) \\
\hline$g$ & {$[l]$} & gap \\
\hline$G$ & {$\left[f / l^{2}\right]$} & shear modulus \\
\hline$g_{\text {int }}$ & {$[-]$} & interaction factor \\
\hline$g_{s l}$ & {$\left[f / l^{2}\right]$} & stiffness of shear layer \\
\hline$H_{\text {combi, }} H_{\text {pile }}$ & {$[f l l]$} & shear force per unit thickness \\
\hline$h_{s}$ & {$\left[f / l^{2}\right]$} & granular stiffness \\
\hline${ }_{i} C,{ }_{0} C$ & {$[-]$} & geometrical position of a body \\
\hline$I_{y}, I_{z}$ & {$\left[l^{4}\right]$} & second order moment of inertia \\
\hline $\boldsymbol{K}$ & {$[f / l]$} & global stiffness matrix \\
\hline$k$ & {$\left[f / l^{2}\right]$} & modulus of subgrade reaction (two-dimensional case) \\
\hline K & {$[-]$} & dimensionless coefficients of lateral earth pressure \\
\hline $\boldsymbol{K}_{0}$ & {$[f / l]$} & material and initial stiffness matrix \\
\hline$K_{0}$ & {$[-]$} & earth pressure coefficient at rest \\
\hline$K_{a}$ & {$[-]$} & active earth pressure coefficient \\
\hline$k_{i}$ & {$\left[f / l^{2}\right]$} & initial stiffness \\
\hline$k_{n}$ & {$\left[f / l^{3}\right]$} & normal coefficient of subgrade reaction \\
\hline$k_{S}$ & {$\left[f / l^{3}\right]$} & coefficient of subgrade reaction \\
\hline$k_{s t}$ & {$[f l l]$} & spring stiffness \\
\hline$k_{t, c}$ & {$\left[f / l^{3}\right]$} & circumferential coefficient of subgrade reaction \\
\hline$k_{t, m}$ & {$\left[f / l^{3}\right]$} & meridional coefficient of subgrade reaction \\
\hline $\boldsymbol{K}_{\Delta}$ & {$[f l l]$} & differential stiffness matrix \\
\hline$l$ & {$[l]$} & length \\
\hline$L, L_{0}$ & {$[l]$} & elastic length \\
\hline$L_{e m b}$ & {$[l]$} & embedded length of the pile \\
\hline$l_{g}$ & {$[l]$} & gauge length \\
\hline$m$ & {$[f l / l]$} & moments transferred from sheetings to piles in combiwalls \\
\hline$m_{\text {eff }}$ & {$[f l / l]$} & local design plastic moment of resistance \\
\hline$m_{h}$ & {$\left[f / l^{3}\right]$} & $\begin{array}{l}\text { ratio of coefficient of subgrade reaction and depth below } \\
\text { ground surface }\end{array}$ \\
\hline$m_{p l, R d}$ & {$[f l / l]$} & design plastic moment of resistance for the shell \\
\hline
\end{tabular}




\begin{tabular}{|c|c|c|}
\hline$M_{\text {red,Rd }}$ & {$[f l]$} & reduced moment of resistance from EN 1993-5 \\
\hline$M_{S d}$ & {$[f l]$} & resultant design moment \\
\hline$M_{y}, M_{z}$ & {$[f l]$} & bending moment \\
\hline$n$ & {$[-]$} & normal unit vector \\
\hline$N$ & {$[f]$} & normal force \\
\hline$n$ & {$[-]$} & exponent (hypoplasticity) \\
\hline$n_{h}$ & {$\left[f / l^{3}\right]$} & constant of lateral subgrade reaction \\
\hline$N_{p l, R d}$ & {$[f]$} & design plastic resistance to normal force \\
\hline$N_{\text {red,Rd }}$ & {$[f]$} & reduced plastic resistance to normal force from EN 1993-5 \\
\hline$N_{S d}$ & {$[f]$} & resultant design normal force \\
\hline$P$ & {$[f / l]$} & shear force per unit thickness \\
\hline$p$ & {$[f / l]$} & lateral soil reaction or resistance \\
\hline$p^{*}$ & {$\left[f / l^{2}\right]$} & equivalent constant pressure \\
\hline$P_{0}$ & {$[f]$} & initial load \\
\hline Pcrit, prit $_{1}$ & {$[f],\left[f / l^{2}\right]$} & critical buckling load, pressure \\
\hline$p_{\text {crit }, 0.5}$ & {$\left[f / l^{2}\right]$} & critical buckling stress; half pressure \\
\hline$p_{\text {criti.1.0 }}$ & {$\left[f / l^{2}\right]$} & critical buckling stress; full pressure \\
\hline$p_{E}$ & {$\left[f / l^{2}\right]$} & earth pressure \\
\hline$P_{i}, P_{0}$ & {$[-]$} & name of position \\
\hline$p_{\text {int }}$ & {$\left[f / l^{2}\right]$} & internal pressure \\
\hline$p_{m}$ & {$\left[f / l^{2}\right]$} & averaged total pressure \\
\hline$p_{\text {ref }}$ & {$\left[f / l^{2}\right]$} & reference pressure \\
\hline$p_{s d}$ & {$[f / l]$} & soil resistance close to surface \\
\hline$p_{s t}$ & {$[f / l]$} & soil resistance close in deeper regions \\
\hline$p_{W}$ & {$\left[f / l^{2}\right]$} & water pressure \\
\hline$Q$ & {$[-]$} & fabrication quality class \\
\hline$q$ & {$\left[f / l^{2}\right]$} & pressure (normal to surface) \\
\hline$q_{c}$ & {$\left[f / l^{2}\right]$} & cone resistance \\
\hline$Q_{l}$ & {$[f]$} & load increment \\
\hline$R, r$ & {$[l]$} & radius (middle surface) \\
\hline$R_{\text {eff }}$ & {$[l]$} & effective pile radius \\
\hline$r_{\text {out }}$ & [1] & outer boundary radius \\
\hline$S_{y}$ & {$\left[l^{3}\right]$} & first order moment of inertia \\
\hline$t$ & {$[l]$} & wall thickness \\
\hline$t$ & {$[t]$} & time (section 2) \\
\hline$t^{*}$ & {$\left[f / l^{2}\right]$} & surface loads \\
\hline$t_{a d}$ & {$\left[f / l^{2}\right]$} & mobilized soil-pile adhesion \\
\hline$t_{a d, \max }$ & {$\left[f / l^{2}\right]$} & mobilized soil-pile adhesion \\
\hline$T_{y}$ & {$[f / l]$} & shear flow in direction $y$ \\
\hline $\boldsymbol{u}, \boldsymbol{u}^{*}$ & {$[l]$} & displacement field \\
\hline$U_{0}$ & {$[-]$} & maximum dimple tolerance parameter \\
\hline$U_{r}$ & {$[-]$} & out-of-roundness parameter \\
\hline
\end{tabular}




$\begin{array}{lll}u_{s d} & {[l]} & \text { scaled-down deflections } \\ \mathrm{v} & {[-]} & \text { factor of consolidation } \\ v, w & {[l]} & \text { displacements } \\ V_{y}, V_{z} & {[f]} & \text { shear forces } \\ \mathrm{W} & {[-]} & \text { exponent of deformation } \\ w_{y}, w_{z} & {[f / l]} & \text { forces transferred from sheetings to piles in combiwalls } \\ \boldsymbol{x} & {[l]} & \text { Euler coordinates } \\ \boldsymbol{X} & {[l]} & \text { Lagrange coordinates } \\ z & {[l]} & \text { depth }\end{array}$




\section{Introduction}

\subsection{General}

Mega ships of the new generation (e.g. ocean liners, freighters or tank ships) require large depths for manoeuvring due to their enormous draughts. Existing ports rarely provide these depths, leading to several planned harbour constructions. If made of steel, such harbour structures are commonly constructed using combiwalls consisting of large diameter and very long tubular piles as primary elements connected to sheeting as secondary elements (Fig. 1.1 and Fig. 1.2). The tubular piles are manufactured from hot rolled coils $(t=10-24 \mathrm{~mm}$ ) by welding (helically or longitudinally) in a diameter $D$ range of $900-2400 \mathrm{~mm}$. Steel grades with yield stresses between $350 \mathrm{~N} / \mathrm{mm}^{2}$ and $480 \mathrm{~N} / \mathrm{mm}^{2}$ (API X70) are commonly used. The usual length of the piles ranges from 20 to $30 \mathrm{~m}$. In the future, for the deeper quay walls, lengths up to $40 \mathrm{~m}$ can be provided.

Until now these tubes have been designed in Europe using mainly a verification of the bending stresses (yielding in the extreme fibre). So far no failure of a structure occurred due to buckling of such a tubular pile. Shell buckling was hardly ever taken into account because the geometrical slenderness $(D / t)$ was smaller than 100 .

\subsection{Problem}

The assessment procedure will change with the introduction of the Eurocodes. The design of retaining walls and piling will be governed by Eurocode 3, part 5: Piling (EN 1993-5) for the resistance of steel members and by Eurocode 7, part 1 (EN 1997-1) for the geotechnical side. For the determination of the design resistance of tubular members, EN 1993-5 refers to Eurocode 3, part 1-1 (EN 1993-1-1). From table 5.2 it transpires that tubes with a slenderness $D / t$ of

$$
D / t>90 \varepsilon^{2} \quad \text { with } \varepsilon=\sqrt{235 / f_{y}} \text { and } f_{y}\left[N / \mathrm{mm}^{2}\right\rfloor
$$

are class 4 members and have to be verified against shell buckling according to Eurocode 3, part 1-6 (EN 1993-1-6). The criterion leads e.g. to the following limiting $D / t$-values for the various steel grades: $f_{y}=355 \mathrm{~N} / \mathrm{mm}^{2} \Rightarrow D / t_{\text {lim }}=59.6$ and $f_{y}=480 \mathrm{~N} / \mathrm{mm}^{2} \Rightarrow D / t_{\text {lim }}=44.1$. Thus, all tubes used until now in standard combiwall projects appear to be class 4 members requiring a shell buckling verification. This verification leads to tubular piles, which are $10 \%-20 \%$ heavier than the piles used today. The reasons for this conservative design are:

- The design rules given in EN 1993-1-6 do not take into account the stiffening effect of the soil located both inside (plug effect) and outside (embedded part of the retaining wall or pile) of the driven tube; 


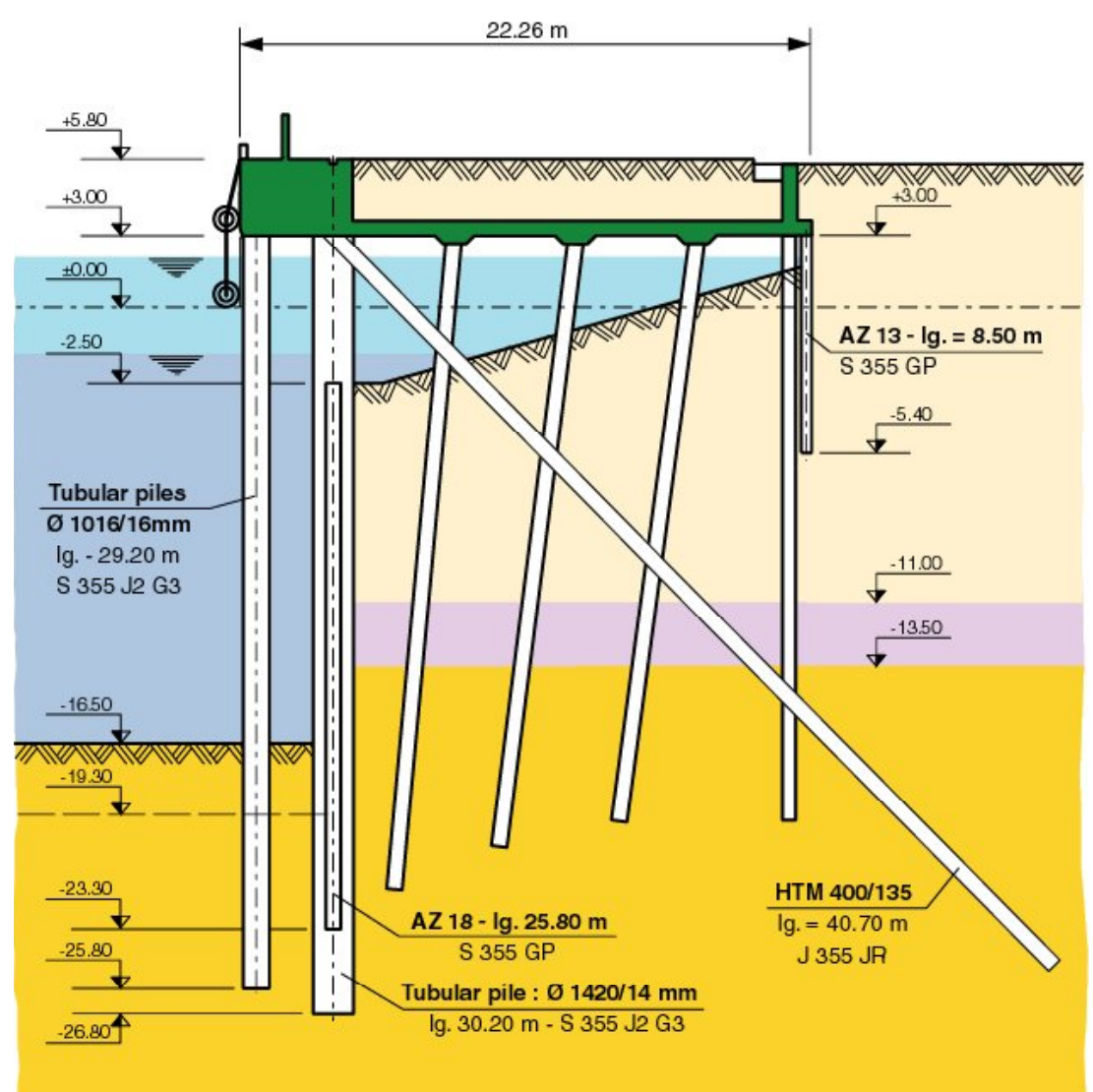

Fig. 1.1: Example for a harbour construction

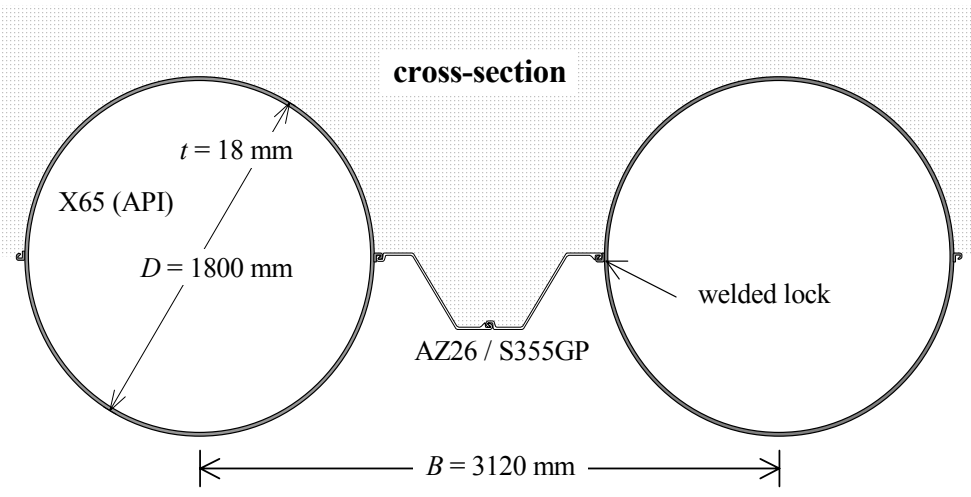

Fig. 1.2: Cross-section of an example combiwall

- The rules have been established mainly for extremely slender shells (about $D / t>300$ ) where shell buckling is prevailing, whereas the tubes used for combiwalls are more subjected to plastic bending at failure.

- The circumferential pressure due to soil and water is not constant along the perimeter (and the length) of the tube. Taking the maximum value for the verification leads again to conservative results.

- Also the approach of superposing the maximum values of the different stress components seems quite conservative for the specific stressing of tubular piles. 
- On the other side it is important to be aware that the boundary conditions considered in EN 1993-1-6 do not cover the boundary conditions prevailing in piling.

Furthermore, inconsistencies emerge in the definition of limit imperfections for the definition of quality classes between the product standard EN 10219 and the relevant design standard EN 1993-1-6. The introduction of forces resulting from earth and water pressure via infill sheeting is specific to combiwalls and has to be considered.

\subsection{Outline of the study}

The objective of this study is to establish the scientific basis for dedicated design rules of large diameter piles taking into account shell buckling. This section outlines the necessary steps in order to derive a proposal for new buckling design rules for the complex cases considered.

The problem involves the interaction of two major fields of constructional engineering, namely geotechnical engineering regarding earth pressures, soil properties, etc., and structural engineering regarding shell buckling. Both fields shall be briefly introduced covering mechanical fundamentals such as the stability of shells and the Finite Element Method (FEM), an essential tool in the investigations. This introduction shall then be followed by an overview of the state-of-the-art regarding shell buckling, soil models and approaches to soil-structure interactions for establishing a fundamental basis for the investigation. This design problem can be illustrated in a typical combiwall structure calculation according to common practical design codes. The differences between the current approach and the changes evolving with the implementation of the Eurocode shall be highlighted for detailed follow-up studies. In the next steps several issues, which are believed to significantly influence the problem, are planned to be investigated more in detail.

Since the load transfer mechanisms of a constructed combiwall are similar to that of a laterally loaded pile, it is intended to simplify it respectively for convenience and efficiency. Based on the theoretical fundamentals and the state-of-the-art summary of soil models, different soil models and soil-structure interaction models can be analysed and compared in numerical studies. Purpose is to define a model for the subsequent studies that is as simple as possible but sufficiently detailed to include all necessary parameters. Experimental tests using reduced scale models and additional backcalculations are going to be necessary to verify the predictions from this model.

Because the effect of geometrical imperfections may be different for a shell structure embedded in soil, this aspect has to be studied with special attention being paid to the influence of radial and/or uneven boundary imperfections in combination with axial stresses. A less detrimental effect of imperfections can lead to smaller reduction factors in the buckling assessment. 
The next issue is the aspect that the buckling rules in the design procedure do not consider any stiffening effect of the soil located both inside and outside of the tubular pile. Because the surrounding soil may increase the carrying capacity of cylinders, axially loaded and/or externally pressurised cylindrical shells in interaction with soil this aspect shall be investigated by numerical means. Experiments measuring the modulus (bender element tests) of sand can serve for a further validation. The intention is to adapt the results for obtaining larger buckling strengths of cylindrical shells with supporting boundary conditions.

Another issue making the tubular pile embedded in soil different from standard shell buckling problems is the complex load transfer mechanism. Investigations over the entire pile length and locally at the points of the maximum moment regarding these load transfer aspects can serve to justify the application to current design codes on shell buckling. Further, the effect of non-uniform stress distributions has to be discussed based on the results from the planned reduced scale tests.

Since it is believed that in the assessment the most unfavourable influence on the buckling strength of the tubular piles arises from the external pressure, the influence of non-uniform external pressure in circumferential and meridional direction on the shell's resistance and on the load case with respect to the Eurocode shall be investigated numerically. Additionally, experiments are planned for evaluating the buckling behaviour of very long shells for covering such exceptional cases.

Finally, a proposal for improved buckling design rules for special cases such as tubular piles in combiwalls can be developed based on the results of the previous numerical and experimental investigations. The changes need to be highlighted in a comparison between the old and the proposed new design calculation. 


\section{Mechanical fundamentals}

\subsection{Stability of shells}

A shell is defined as a curved surface structure and can be commonly found in nature as well as in classical architecture. The characteristic of using membrane stresses instead of bending stresses for transferring imposed loads makes shells more economical in comparison to e.g. plates stiff structures for transmission of loads. As a consequence of the load transfer mechanism in shells, these structures may be constructed with very small wall thickness. Shell structures appear as single curved shells (e.g. tanks, silos, columns, chimneys) or more complex as double curved shells (e.g. roofs, vehicles, water towers). Shells can be very thin-walled and are often subjected to compressive stresses making them susceptible to buckling. These structures may have difficulties coping with local loads since they induce local bending moments which have to be taken up by the far smaller bending resistance. Generally, structural members subjected to loads are prone to failure due to buckling if a small disturbance or infinitesimal load increment forces the structure to change from a stable equilibrium to a neutral or unstable equilibrium.

The classical cases as the conical shell and especially the cylindrical shell have been the topic of numerous research projects in the $20^{\text {th }}$ century. Intensive research in the field of aerospace (e.g. rockets) had a positive impact on shell buckling research with new findings to be used in constructional engineering. Beginning of the $20^{\text {th }}$ century first analytical solutions were derived and later supported by test results. From the second half of the $20^{\text {th }}$ century numerical analyses developed, providing nowadays an indispensable tool besides theoretical and experimental approaches for the investigation of shell stability. The large discrepancies found, especially for axially loaded cylindrical shells, between the results of theoretical and experimental predictions pushed researchers to investigate the influence of imperfections and to base the design on experimental results.

The aspect of stability is briefly discussed in the following. A shell subjected to a sufficiently small load is in a stable elastic equilibrium (see v. Mises, 1923). If this base state of the shell is disturbed by imposing an infinitesimal displacement or incremental load, additional external forces are needed to cope with the correlated stresses and strains evolving from the disturbance. In case such external forces appear, the system remains in a stable equilibrium. If the disturbance does not lead to additional disturbance forces, a critical load is reached (neutral equilibrium). Further loading of the system results in an unstable equilibrium related to changes of the initial position of the shell in order to find an adjacent path of equilibrium. 


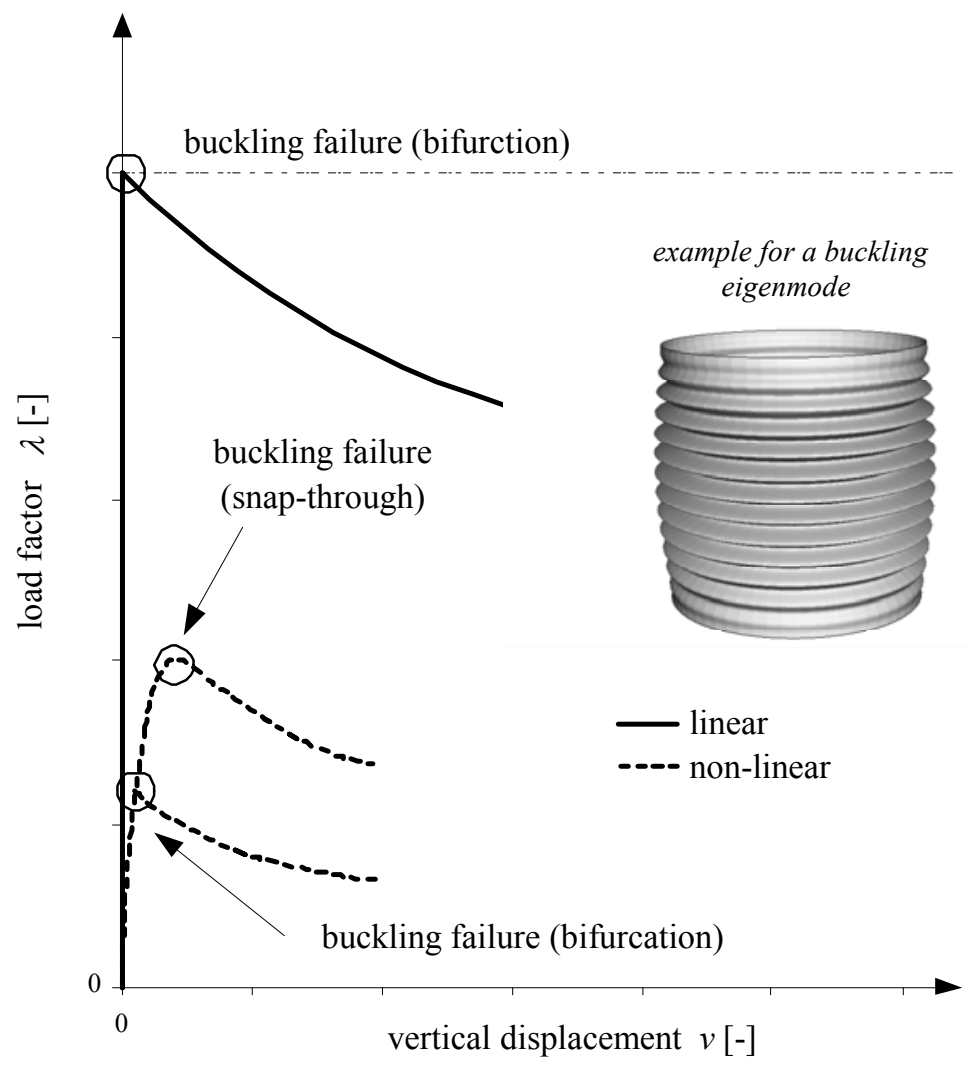

Fig. 2.1: Load-displacement curves for an axially loaded cylindrical shell (static loading)

Fig. 2.1 depicts some possible load-displacement paths for axially loaded cylindrical shells made of linear elastic material. The abscissa shows the vertical displacement and the ordinate shows the dimensionless load factor $\lambda$. The dimensionless load factor $\lambda$ corresponds to the applied load normalized over the theoretical carrying capacity, e.g. for a buckling problem the theoretical buckling stress. Beginning of the $20^{\text {th }}$ century Lorenz (1908) and Thimoshenko (1910) developed approximations for the bifurcation load of a perfect shell. Flügge (1932) derived solutions for the buckling equations (classical linear shell buckling theory).

Real shell structures may fail significantly below the theoretical buckling load of a shell (horizontal dotted line in Fig. 2.1) due to unavoidable shape deviations for real constructions. The understanding of the imperfection sensitivity is based on the pioneering work by Koiter (1945). The stability problem may be governed by a symmetric (stable or unstable) or asymmetric buckling point. Fig. 2.2 shows a loaddisplacement curve according to Fig. 2.1 (left) and the evaluation of the imperfection sensitivity for the different stability cases (right). For the unstable cases a high imperfection sensitivity can be identified, i.e. a small change of the imperfection amplitude $\Delta \varepsilon$ results in a drastic reduction of the load factor $\lambda$. The response of the asymmetric buckling point follows a parabolic curve and the function $\lambda$ for the stablesymmetric case follows locally the so-called "two-thirds" law according to Koiter (1945), i.e. $\lambda$ is a function of $\varepsilon^{2 / 3}$. Therefore, the stable-symmetric case shows lower 
imperfection sensitivity than the asymmetric case as e.g. seen for an imposed imperfection $\varepsilon_{1}$ (Fig. 2.2).

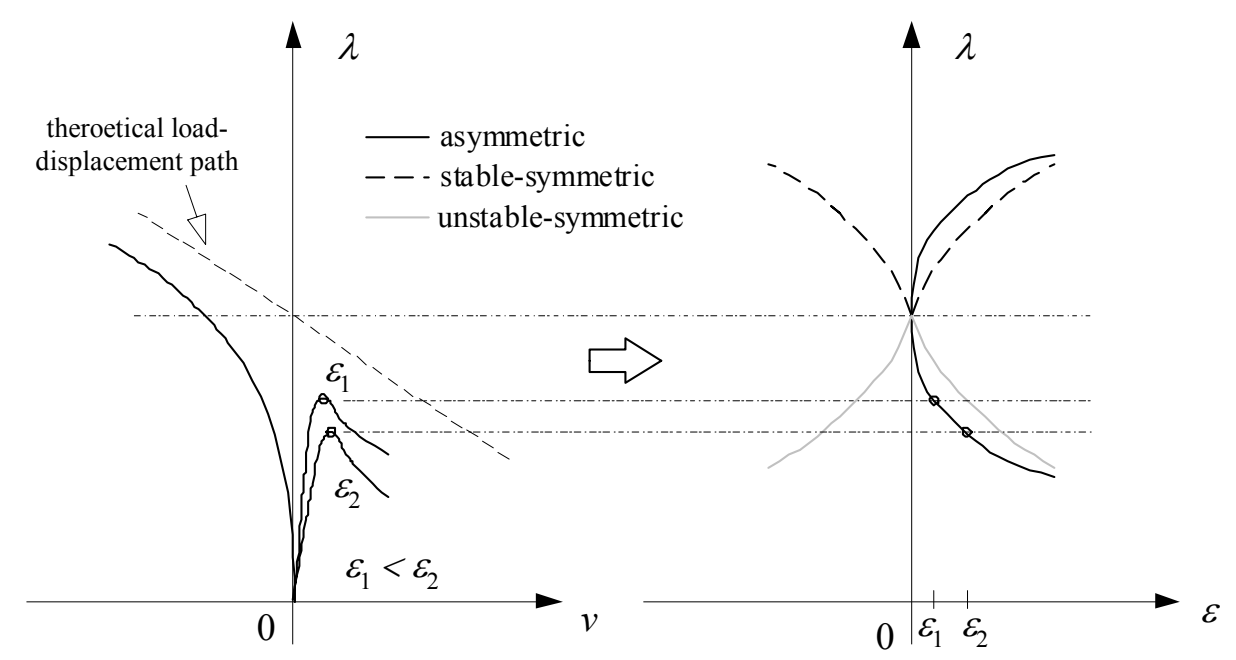

Fig. 2.2: Influence of imperfection amplitude $\varepsilon$-imperfection sensitivity

Since the axially loaded and the externally pressurized cylinder are problems with asymmetric bifurcations, the problems can be defined as extremely imperfection sensitive. The carrying capacity of a thin-walled membrane shell under compression is either limited by a buckling failure (sudden change of the shape) in form of bifurcation or a limit point buckling (snap-through). For limit point buckling the load-deflection path reaches a zero slope or continuously differentiable maximum $(d \lambda / d v=0)$. The failure mode is also referred to as snap-through failure because there is no bifurcation of the equilibrium path but for continued loading the structure may "snap" to a new equilibrium position at a different point of the load-deflection path. In case of bifurcation buckling the structure jumps to an adjacent equilibrium path which intersects with the current load-deflection curve or equilibrium path. At the bifurcation point the structure is not continuously differentiable $(d \lambda / d v \neq 0)$. For real structures, i.e. as soon as geometrical and structural imperfections are considered, the two failure modes are close together and difficult to distinguish. Bifurcation buckling is rather theoretical, since in practical design mostly snap-trough occurs or the problem turns out as a simple stress based problem (monotonically increasing load-deflection path).

\subsection{Fundamentals of continuum mechanics}

\subsubsection{General}

In this chapter some basic equations of continuum mechanics are stated because they are needed for the derivation of the finite element method (FEM). For more details the reader is referred to the work by J. and H. Altenbach (1994), Olivella and de Saracibar (2000) and Parisch (2003). Solid continua are defined as bodies with abstract 
hypothetical continuous material properties. The material point $P_{0}$ is an arbitrary point in the body in which the entire material behaviour can be represented, i.e. the material point serves as reference for all state variables. The continuum model is defined by using the three formulations: displacement-strain relationship, strain-stress-relationship and equilibrium of the continuum.

\subsubsection{Description of movement (displacement-strain relationship)}

The description of the movement in space is needed for the description of a body. Kinematics include on the one hand the rigid body motion defined as the pure change of position (new position $P_{i}$ ) and thus without influencing the stresses in the body, and on the other hand the distortion of the body directly influencing the stress state. Therefore, a description with independent objective state variables is postulated. The current geometrical position of a body is here named as configuration ${ }_{0} C$ and ${ }_{i} C$ (Fig. 2.3).

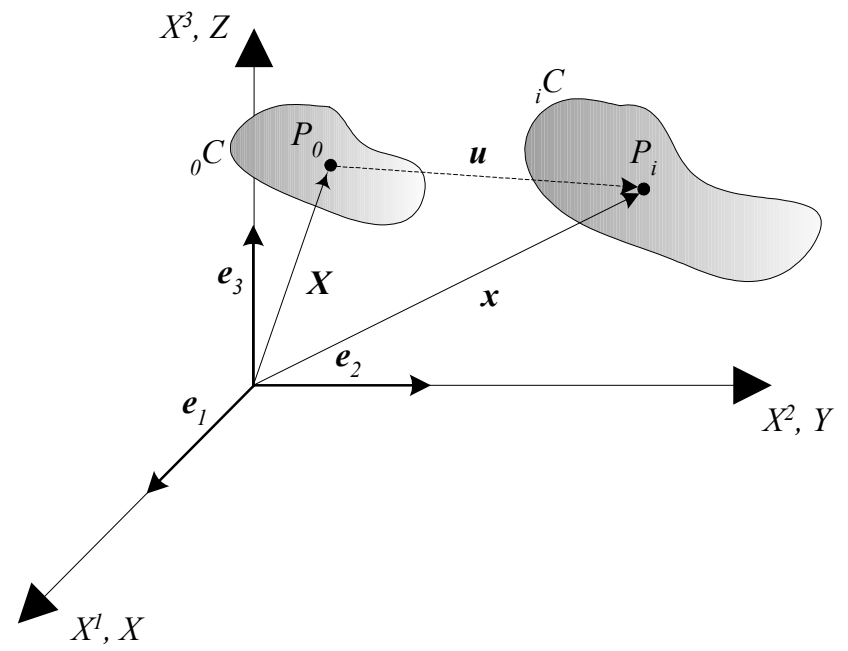

Fig. 2.3: Configurations in continuum mechanics

Based on the Cartesian coordinate system in Fig. 2.3 the position of the point $\mathrm{P}$ can be given using Lagrange coordinates (material coordinates) $\boldsymbol{X}$ or Euler coordinates (spatial coordinates) $\boldsymbol{x}$

$$
\begin{gathered}
\boldsymbol{X}=X^{1} \boldsymbol{e}_{1}+X^{2} \boldsymbol{e}_{2}+X^{3} \boldsymbol{e}_{3}=X^{i} \boldsymbol{e}_{i} \\
\boldsymbol{x}=x^{1} \boldsymbol{e}_{1}+x^{2} \boldsymbol{e}_{2}+x^{3} \boldsymbol{e}_{3}=x^{i} \boldsymbol{e}_{i}
\end{gathered}
$$

The Lagrange description is mainly used in solid continuum mechanics since a specific property (e.g. density) of a point is looked at over the course of time. The Euler formulation describes the change of property at a fixed position in space over the course of time and thus is commonly used in the field of fluid mechanics.

The Lagrange description of motion as a non-linear transformation is written as

$$
\boldsymbol{x}=\boldsymbol{x}(X, t)
$$


The spatial displacement field $\boldsymbol{u}$ of the present position and the reference for $t>0$ is

$$
\boldsymbol{u}(\boldsymbol{x}, t)=\boldsymbol{x}-\boldsymbol{X}(\boldsymbol{x}, t)
$$

For inverting the unique transformation for any point in time the Jacobian determinant $J$, which can be described with the deformation gradient $\boldsymbol{F}$ has to exist

$$
\operatorname{det}\left(\frac{\partial \boldsymbol{x}}{\partial \boldsymbol{X}}\right)=\operatorname{det}(\boldsymbol{F})=J>0
$$

The deformation gradient $\boldsymbol{F}$ contains information on the relative movement over the course of time $t$ for all material points and can be written as

$$
\boldsymbol{F} \equiv \frac{\partial \boldsymbol{x}}{\partial \boldsymbol{X}}=1+\frac{\partial \boldsymbol{u}}{\partial \boldsymbol{X}}
$$

Based on the deformation gradient $\boldsymbol{F}$ the symmetrical Green-Lagrangian strain tensor $\boldsymbol{E}$ can be defined as

$$
\boldsymbol{E}=\frac{1}{2}\left(\boldsymbol{F}^{T} \cdot \boldsymbol{F}-1\right)
$$

For a deformation gradient $\boldsymbol{F}$ equal to 1 , the Green-Lagrangian strain tensor $\boldsymbol{E}$ becomes zero. As postulated no strains appear for pure rigid body motion $(\boldsymbol{F}=1)$. Assuming the hypothesis of small or infinitesimal deformations the Green-Lagrangian strain tensor $\boldsymbol{E}$ becomes the strain tensor for small deformations $\varepsilon$. The correlation between displacements and deformations and strains for continuum mechanics is essential for back-calculating strains from displacements. For the correlation between strains and stresses further equations are needed, namely a material law or constitutive relationship.

\subsubsection{Constitutive equations (strain-stress relationship)}

Besides the geometrical description some postulates or conservative equations have to be fulfilled in continuum mechanics independent of the size of displacements or deformations. The postulates include the conservation equations of mass, momentum and angular momentum and the two fundamental theorems on thermodynamics (conservation of energy and entropy). For determining all unknowns, further equations describing the material properties are needed: the constitutive equations. For many cases the equations can be reduced to the thermo-mechanical constitutive equation relating stresses to strains and the conservation equations of mass and momentum. The strain-stress relationship for a linear elastic isotropic material (Hook's law) can be derived for small strains $\varepsilon$ as

$$
\boldsymbol{\sigma}=\frac{\mu E}{(1+\mu)(1-2 \mu)} \operatorname{Tr}(\boldsymbol{\varepsilon}) \boldsymbol{1}+\frac{E}{(1+\mu)} \boldsymbol{\varepsilon}
$$

with the Young's modulus $E$ and the Poisson ratio $\mu$. 


\subsubsection{Equations of equilibrium}

Given a body subjected to external loads and displacements, a formulation for the resultant stresses, strains and displacements is needed for which the equilibrium is maintained. Derived from the conservation equation of momentum (Cauchy equation) the equilibrium of a continuum can be expressed as boundary value problem by

$$
\nabla \cdot \boldsymbol{\sigma}+\rho \boldsymbol{b}=\rho \frac{\partial^{2} \boldsymbol{u}}{\partial t^{2}} \quad \forall \boldsymbol{x} \in V
$$

with the density $\rho$ and the mass forces $\boldsymbol{b}$ and the equation for the equilibrium for the stress boundary conditions (Neumann boundary conditions) valid over the surface $\Gamma_{\sigma}$

$$
\boldsymbol{\sigma} \cdot \boldsymbol{n}-\boldsymbol{t}^{*}=0 \quad \forall \boldsymbol{x} \in \Gamma_{\sigma}
$$

in which $\boldsymbol{t}^{*}$ represents the surface loads and $\boldsymbol{n}$ the normal unit vector. For the equations the displacement boundary conditions (Dirichlet boundary conditions) have to be fulfilled over the surface $\Gamma_{u}$

$$
\boldsymbol{u}=\boldsymbol{u}^{*} \quad \forall \boldsymbol{x} \in \Gamma_{u}
$$

i.e. all admissible displacements are defined with $\boldsymbol{u}^{*}$. The equations (2.10) to (2.12) are called the strong formulation of equilibrium for the boundary value problem because all equations are defined for each surface and volume element. The solution of this boundary value problem by using numerical approximations is the concept of the finite element method.

\subsection{Finite element method}

\subsubsection{General}

The modelling of structures in this work is performed by using the well-known commercially available program ABAQUS (Hibitt, Karlsson \& Sorensen, 1998) which uses the finite element method (FEM). The program consists of the three moduli preprocessor, solver and post-processor. For pre- and post-processing the modulus ABAQUS/CAE is applied as a simple and consistent interface for working on the modelling and displaying results calculated by ABAQUS.

\subsubsection{FEM formulation}

For most engineering cases the boundary value problem as described by equation (2.10) to (2.12) has no closed form or analytical solutions. Thus, assumptions and approximations are made in order to obtain solutions. By dividing the object continuum into discrete elements the FEM provides a tool for solving the problem by numerical means. There are several methods tackling the problem (Galerkin method, calculus of variations, principle of virtual work). In this approach the Galerkin method is applied based on the equilibrium in equation (2.10). The functional $G(x, \eta)$ is formulated for 
equation (2.10) using a so-called trial function $\eta$ which fulfils the boundary conditions from equation (2.11) and (2.12). The method is based on the assumption that the equilibrium is satisfied averaged over the integral, and thus the functional is set equal zero:

$$
G(\boldsymbol{x}, \boldsymbol{\eta}):=\int_{V}\left[\nabla \cdot \boldsymbol{\sigma}+\rho\left(\boldsymbol{b}-\frac{\partial^{2} \boldsymbol{u}}{\partial t^{2}}\right)\right] \cdot \boldsymbol{\eta} d V+\int_{\Gamma_{\sigma}}\left[\boldsymbol{\sigma} \cdot \boldsymbol{n}-\boldsymbol{t}^{*}\right] \cdot \boldsymbol{\eta} d \Gamma=0
$$

Redrafting the equation (2.13) by applying the Gauss theorem and using

$$
\boldsymbol{b}^{*}=\boldsymbol{b}-\frac{\partial^{2} \boldsymbol{u}}{\partial t^{2}}
$$

it can be written as the so-called weak formulation of equilibrium (Galerkin equation):

$$
\int_{V}[\boldsymbol{\sigma}: \nabla \boldsymbol{\eta}] d V=\int_{V} \rho \boldsymbol{b}^{*} \cdot \boldsymbol{\eta} d V+\int_{\Gamma_{\sigma}} \boldsymbol{t}^{*} \cdot \boldsymbol{\eta} d \Gamma
$$

The weak formulation of equilibrium can also be obtained by applying the principle of virtual work. As a consequence the values of the functional can be interpreted as work or energy, i.e. the internal virtual work is equal to the external virtual work. The principle has to be understood as an approximation for the differential equation (2.10) since no real energy conversion occurs. The canonical form of the Galerkin equation or the principle of virtual work can therefore be written by replacing the arbitrary trial function $\eta$ with the virtual field of displacement $\delta \boldsymbol{u}$.

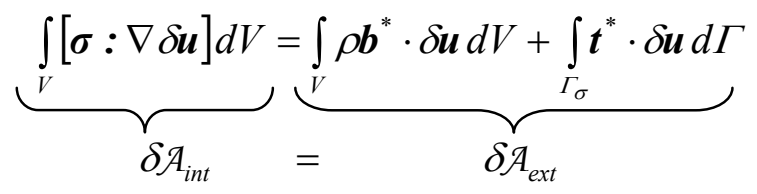

Based on the canonical form of the Galerkin equation (2.16) the stiffness matrix is introduced in the next step. Further redrafting with deriving the virtual work and discretizing the weak formulation of equilibrium (2.15) for both sides separately a general algebraic set of nonlinear equations can be written for the complete system:

$$
\delta \boldsymbol{d}^{T} \cdot(\boldsymbol{K} \boldsymbol{d})=\delta \boldsymbol{d}^{T} \cdot \boldsymbol{F}_{\text {load }}
$$

with the global stiffness matrix $\boldsymbol{K}$, the global node displacements $\boldsymbol{d}$, the virtual global node displacements $\delta \boldsymbol{d}$, and the energetically equivalent global node vector $\boldsymbol{F}_{\text {load }}$ incorporating external loads. For the case that the global stiffness matrix $\boldsymbol{K}$ does not depend on the displacements (linear case) the unique solution for the unknown nodal degrees of freedom is:

$$
\boldsymbol{d}=\boldsymbol{K}^{-1} \boldsymbol{F}_{\text {load }}
$$

For the solution of the linear matrix system different direct and indirect algorithms can be used, e.g. Gauß, Jacobi, Gauß-Seidel. A point of stability occurs if the global node vector $\boldsymbol{F}_{\text {load }}$ disappears and a homogenous set of equations remains. The nontrivial solution of this system leads to the points of stability. 
In this work different types of analyses are performed. Static analyses are used for investigating linear load-displacements and general preliminary calculations. For the stability investigations two different methods are used. On the one hand the problem can be approached using a linear eigenvalue analyses resulting in eigenvalues and eigenmodes for the investigated case. On the other hand path algorithms can be used for performing a non-linear load-displacement analysis. In the latter case imperfections are needed for perfect systems in order to initiate a failure.

\subsubsection{Eigenvalue analysis}

Eigenvalue analyses can yield good results for stiff systems responding fairly linearly up to the point of stability (see Fig. 2.1). Still, this method can provide valuable findings regarding the shape of failure for other problem. The load rendering the value zero for the determinant of the stiffness matrix can be derived from:

$$
\boldsymbol{K} \boldsymbol{d}=\mathbf{0}
$$

Expanding the equation into a Taylor-series up to a linear form leads to

$$
\left(\boldsymbol{K}_{0}+\lambda_{E V, i} \boldsymbol{K}_{\Delta}\right) \phi_{i}=\mathbf{0}
$$

where $\boldsymbol{K}_{0}$ is the material and initial stiffness matrix in which preloads may be included, and $\boldsymbol{K}_{\Delta}$ is the differential stiffness matrix arising from the incremental load increase. The corresponding eigenvalues and buckling eigenmodes are defined by $\lambda_{E V, i}$ and $\phi_{i}$. The critical buckling load $P_{c r i t}$ is the sum of the initial load $P_{0}$ and the product of the load increment $Q_{L}$ and the eigenvalue $\lambda_{E V, i}$.

$$
P_{c r i t}=P_{0}+\lambda_{E V, i} \cdot Q_{L}
$$

Since the bifurcation analysis is a linear procedure no geometrical or material nonlinearity can be taken into account. Still, this procedure can be used for investigating problems with geometrical and material non-linear response. For this type the analysis is split into two steps. In the first step a preload is applied to the structure bringing it close to the point of buckling and taking geometrical and material non-linearity into account where required. The magnitude of the preload needs to be determined by trial and error. Subsequently, in the second step a linear eigenvalue analysis is performed based on the non-linear base state calculated in the first step. For a pre-buckling state close to the critical point of stability, this so-called non-linear bifurcation analysis yields good results. For all eigenvalue analyses in this work non-linear bifurcation analyses are performed.

\subsubsection{Non-linear load-displacement analysis}

The non-linear equations arising for discrete systems from equation (2.19), cannot be solved explicitly and require special solver algorithms. The non-linearity demands the application of iterative solver algorithms consisting of an iteration method for obtaining equilibrium and an equation solver. Generally, a path-following algorithm is split into 
discrete load increments and an iteration method integrated in each load increment. ABAQUS does not use the standard solver (Newton) but the more elaborate solver by Wemper (1971) and Riks (1972). In contrast to the Newton method, the so-called arclength method includes the load level. It has the advantage that it can smoothly follow any path of equilibrium including passing points of stability. Another important aspect is that the load increments are not fixed but are automatically adjusted to the path of equilibrium and the rate of convergence. ABAQUS uses a modified Riks method based on Crisfield (1981), Ramm (1981) and Powell and Simons (1981). In ABAQUS the arc length $\Delta l$ (Fig. 2.4) is an automated parameter which can be regulated by a minimum and maximum arc length.

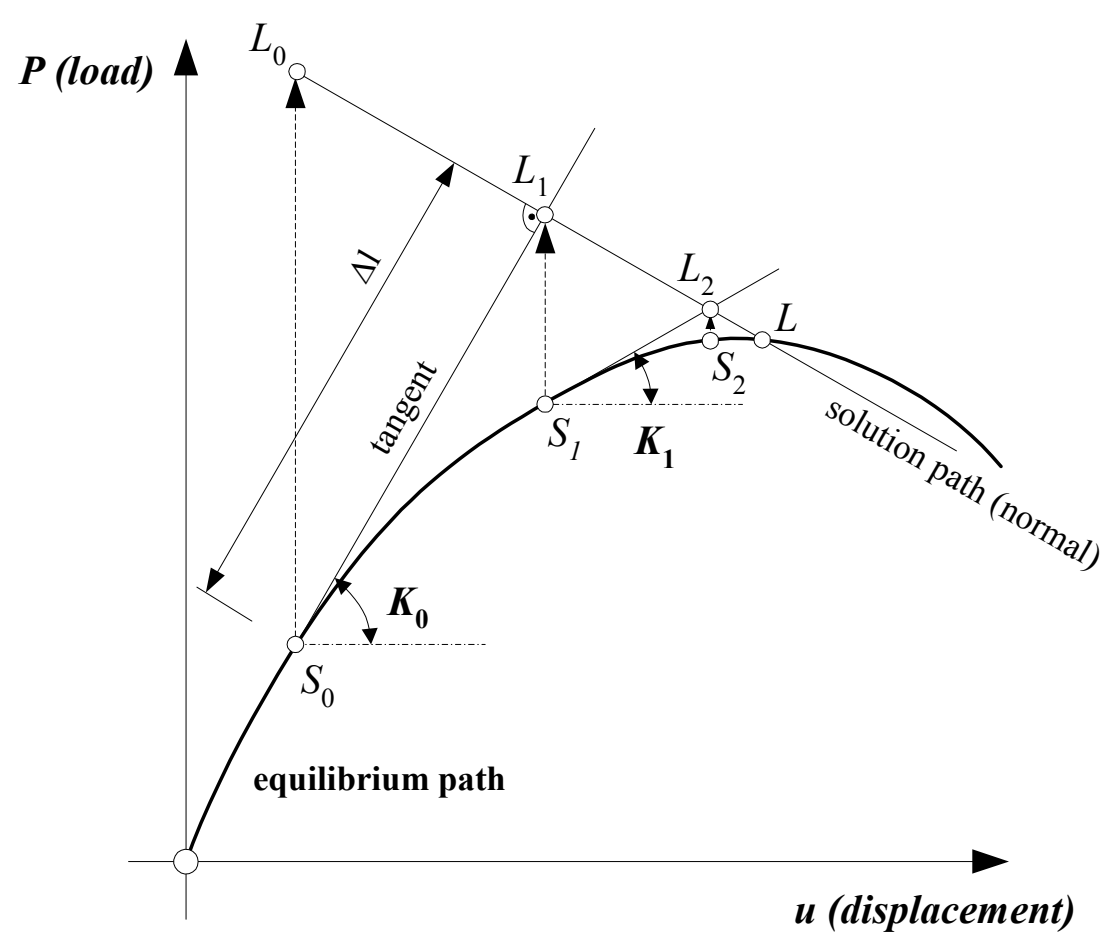

Fig. 2.4: Arc length method by Wemper and Riks for one load increment

Fig. 2.4 depicts the iteration procedure within a load increment schematically. Starting from the point $S_{0}$ an orthogonal solution path is defined by the initial tangent stiffness $\boldsymbol{K}_{0}$ and the restricted arc length $\Delta l$. Along the solution path the individual points of solution $L_{\mathrm{i}}$ of the increments can be found. The next point of solution is obtained by applying the newly determined tangent stiffness $\boldsymbol{K}_{1}$ (equivalent to the Newton-Raphson method) to the new found starting point $S_{1}$. The relating loads are defined by the implemented load increment control and do not remain constant in contrast to the standard algorithm. The iteration process is continued until a specified convergence error is satisfied. In this context the starting points coincide with the starting points according to the criteria of convergence. More detailed information on the implementation of the method in ABAQUS can be found in Hibitt, Karlsson and Sorensen (1998). 


\subsubsection{Contact modelling}

This section deals with a brief overview of the contact modelling available in ABAQUS and used in this work. More details can be found in the appropriate literature (e.g. Belytschko et al., 2003).

Two methods are available for applying interface modelling: surfaces or contact elements. Most problems (here e.g. soil-structure) can be modelled using surface-tosurface contact. But discrete contact elements such as gap elements (here e.g. imperfect shell boundaries) may in some cases be a more flexible method of modelling contact. In ABAQUS the surface based contact can use one of the three approaches: finite-, smallor infinitesimal-sliding formulation. For the investigated cases finite-sliding is assumed allowing the contact surfaces to arbitrarily separate, slide, and rotate. The other two formulations may be numerically less extensive but only allow restricted relative movement or rotation of the contact pair. The kinematics are defined using a pressureoverclosure for which currently two methods of constraint enforcement can be used in ABAQUS: the classical Lagrange multiplier method or the augmented Lagrangian method. The classical Lagrange multiplier method of constraint enforcement does not allow any penetration of the surfaces. The augmented Lagrangian method uses the penalty method during each iteration. Iterations continue until convergence of the solution is obtained with the penalty method. The penalty method applies an additional stiffness (penalty value) for enforcing the contact constraints. In the mechanical contact simulation the interaction between the contacting bodies is defined by assigning a contact property model to the contact interaction in which e.g. normal and tangential behaviour can be defined with friction and damping.

\subsubsection{Finite elements in geotechnical problems}

In this section some special aspects are raised which appear in numerical analyses while tackling geotechnical problems.

The discussed partial differential equations are of the type elliptical partial differential equations often described using the calculus of variations. In contrast to the elliptical partial differential equations appearing for steady state problems (standard constructional analyses), parabolic partial differential equations may appear in certain fields of geotechnical engineering. The latter equations appear for problems similar to steady state problems but only for transient considerations. Parabolic problems may arise in geotechnical engineering but will not be discussed in this work since all analyses are performed considering steady state conditions.

The derivation of the equations in the previous sections was mainly based on considering a conventional structural material (e.g. linear isotropic material). Steel properties exhibit a mostly linear response up to yielding and can be classified in the latter category whereas soil can hardly be included in this group. Soil and in this context especially sand is a material that changes its properties by rearrangement of particles that may interact through mainly frictional mechanisms. The far more complex material 
behaviour of soil demands a numerically more elaborate model. From the numerical standpoint the implementation of a constitutive model comprises the integration of the material state at an integration point over a time increment during a non-linear analysis. In the finite element analyses it is assumed that the material behaviour is completely defined by each material point (integration point). For the investigated cases only implicit calculations are performed and therefore the derivation of a precise material stiffness matrix is important for determining the Jacobian of the non-linear equilibrium equations. The deformation gradient $\boldsymbol{F}$ from the analyses is passed to the constitutive routines. Based on the state variables a material contribution to the Jacobian is calculated. For complex soil models the material may be defined as an incremental model and therefore implicit analyses require integration within the subroutine. Thus, besides geometrical equilibrium iterations as discussed in the previous section additional solvers are needed within the routines to perform material iterations. This additional algorithm has to be called at every integration point and leads to a significant increase in computing time. In ABAQUS a possibility of programming a subroutine is given in order to implement any mechanical constitutive model. More common material models are included in the existing library. As a consequence of the complex material models, geotechnical problems need to be tackled using three-dimensional modelling in order to cover the reaction of the soil accurately.

A further concern specific to geotechnical problems in finite element analyses is the initial stress state. Problems involving soil need an initial stress state arising e.g. from self weight to be imposed before performing an analysis. Generally, in the first step of the analysis a geostatic step with applied gravity loads is performed in order to obtain an equilibrium for the system and to produce ideally no displacements. However, for many problems it may be very difficult to obtain an exact solution because of an unknown initial stress state, and therefore the geotechnical step may not find equilibrium.

In this context the importance of the loading history needs to be emphasized as it influences the response of the soil. Mostly, it is very difficult to obtain information regarding the loading history, and even if available the problem of considering it in the analyses remains.

In defining or working on elaborate soil models several aspects need to be taken care of and are very specific to these models. Here, some aspects are mentioned for completeness. Besides the influence of the stresses on the response (incremental model, initial stress state), another complexity in dealing with soil models is the dependency of the soil's stiffness on the stresses. Here, the term stiffness of the soil or the modulus of the soil is used for describing the non-linear relationship between stresses and strains. Similarly, the resistance of the soil is highly stress sensitive. The aspects of stiffness and resistance may be sufficient for describing most material used in civil engineering. In contrast to these models, soil models essentially need to take the volumetric behaviour into account, too. At this, point especially the dilatancy of granular materials is pointed out, i.e. a change in volume due to shearing. Embedding these behaviours in finite 
element analyses are important for reproducing reliable finite element analyses in the field of geotechnical engineering. 


\section{State-of-the-art}

\subsection{General}

The summary of previous investigations is composed of the investigations on the stability of cylindrical shells, material models for soil and approaches for modelling the soil-structure interaction. These three aspects shall be combined and verified in order to use state-of-the-art research in all three parts for developing an interdisciplinary solution for the entire problem.

Contemporary methods allow the modelling and numerical investigation of soil in combination with the buckling phenomena. Empirical findings can be used for validating the results and for leading to more efficient modelling.

\subsection{Stability of loaded cylindrical shells}

\subsubsection{Axially loaded imperfect cylindrical shells}

Stability problems of thin-walled cylindrical shells made of elastic and elastic-plastic materials have been dealt with by various researchers with analytical, numerical, and experimental investigations. The major interest has always been the axially loaded cylindrical shell. Within these studies the shells were investigated by assuming classical boundary conditions which can also be found in the design codes for shells. This section outlines previous investigations in the field of shell buckling, focussed on axially loaded cylindrical shells and the interaction problem between soil/bulk material and shells as studied particularly for the case of silo structures. For classical boundary conditions shell buckling has been thoroughly studied for nearly a century (see e.g. Schmidt, 1991), and the most important developments are summarized in the following for the sake of completeness. Still, for special circumstances or boundary conditions as studied here, there are few literature references available.

Beginning of the $20^{\text {th }}$ century Lorenz (1908) and Thimoshenko (1910) were the first to study the problem of shell stability and developed the linear shell theory. Since a shell is the perfect structure for transferring loads as membrane forces, it became interesting for the construction and aerospace industry. Studies found large discrepancies between the analytically derived carrying capacities and the data gained from experiments. These differences could be traced back to geometrical imperfections (manufacturing, construction, joints, etc.), structural imperfections (residual stresses from welding, nonhomogeneous material properties, material defects, etc.), and imperfections in the boundary conditions (loading, displacements, foundation, etc.). Thereby, imperfections were commonly related to deviations in modelling. In contrast to the practical design, it 
is of no importance in research whether the deviations are unknown variables, or known variables neglected for simplifying the model.

The study by Rotter and Teng (1988) describes several effects influencing the stability of shells. The field of imperfections was emphasized because their influence on the carrying capacity of an axially loaded shell can be detrimental. The investigation was limited to the coverage of structural and geometrical imperfections with an analytical and numerical model. As a result they defined different radial geometrical shape imperfections arising from circumferential welds on cylindrical shells to be used for numerical analyses.

Most researches for axially loaded cylindrical shells are based on classical boundary conditions. In reality imperfect edges lead to a non-uniform load introduction. This effect may be just as detrimental to the carrying capacity of cylindrical structures as radial geometrical imperfections of the shell. The influence of uneven supports was revealed relatively late (Saal et al., 1979). Saal et al. (1979) performed tests with internally pressurized cylindrical steel shells. The specimens for the experiments were precisely and very carefully manufactured, and the buckling loads were compared to predictions given in codes and guidelines.

Knebel (1997) confirmed by numerical means that uneven edges of cylindrical shells (imperfect boundary conditions) lead to similar reductions of the carrying capacity as radial deviations of the geometry (geometric imperfections).

Uneven load introduction and the effect of non-uniform stress distributions of axial membrane stresses were investigated by Bijlaard and Gallagher (1959). The problem of uneven supports and the problem of a non-uniform axial stress distribution are similar but different as shown by the previously mentioned researches. They highlighted that uneven supports cannot be represented by a derived non-uniform axial stress distribution.

\subsubsection{Loaded cylindrical shells in interaction with another medium}

The first investigations dealing with the buckling phenomena of shells in combination with another medium were performed in the field of aerospace research, as this problem appeared for rockets filled with solid fuel. Federhofer (1937 and 1954) derived analytical solutions based on the general theory by Flügge (1932) for axially loaded cylindrical shells with an elastic core. In the 1950s, aerospace engineers Seide (1962) and Yao (1962) investigated cylindrical shells with elastic cores by proposing more elaborate analytical solutions based on more complex approaches. Their work was accessible to the public not until the 1960s as their findings were classified confidential. Further analytical work in the field of aerospace include studies by Zak and Bollard (1962) who took additional circumferential loads into account, and Zak and Willimas (1962) who worked with a viscoelastic core. Kempner (1954) and Almroth and Brush (1963) analysed the post-buckling behaviour of axially loaded cylindrical shells with an 
elastic core. The derived post-buckling minima were suggested to be used as lower bounds for calculating the carrying capacity, i.e. as actual buckling load.

Esslinger and Pieper (1979), Rotter et al. (e.g. 1988, 1989), and Knödel and Schulz (1988) performed experimental and numerical investigations concerning the stability of silo structures. Esslinger and Pieper (1979) highlighted the increase in carrying capacity for a granular filling (sand) in contrast to the more fluid-like filling with cement. Rotter et al. published several test results showing that for cylinders filled with granular material far larger buckling than for equivalent hydrostatically loaded cylinders can be obtained. It was shown that the actual buckling loads may exceed the theoretical buckling loads. The tests by Knödel and Schulz (1988) and their investigation on the rotationally symmetrical case with a linear elastic support of the silo wall due to the bulk cargo provided the basis for design proposal by Knödel (1994) for the assessment of axially loaded silos filled with granular material. He investigated the stability of cylindrical steel shells for a range of $r / t$ between 250 and 1000. Knödel (1994) validated his numerical analyses with additional experiments using cylindrical shells of $1.2 \mathrm{~m}$ diameter filled with sand. The interaction between soil and structure was modelled using modified Winkler springs with a tri-linear material model. The study revealed a significant increase of the carrying capacity due to the presence of the soil on the inside (supporting effect and internal pressure).

Schweizerhof and Ramm (1987) investigated the influence of follower forces, i.e. the direction of the load rotates with the rotation of the surface, on the carrying capacity of hydrostatically loaded shells. In their work the influence of displacement dependent loads was thoroughly investigated. The paper also covered comparisons with design codes regarding wind loads. Rotter and Zhang (1989) investigated the rotationally symmetrical case with a linear elastic support of the silo wall due to the bulk cargo, and provided the theoretical basis for subsequent numerical and experimental studies. Zhang and Ansourian (1991 and 1992) performed experiments with axially loaded, very small, scaled-down silo models filled with different granular materials. Their numerical comparisons have to be interpreted carefully as no measurements of the imperfections were performed. Generally, very large increases of the buckling load due to the presence of granular fillings were obtained. Formulas for relating the internal pressure to the bulk storage stiffness were proposed. A different form of soil-structure interaction was investigated by Wunderlich et al. (1994), who performed research in the field of tank structures subjected to loadings arising from earthquakes. In this context the buckling loads are mostly characterized by the foundation which was described using a linear elastic - perfectly plastic material law for the soil. Further, they introduced models for considering the unevenness of boundary conditions in numerical analyses.

Knebel and Schweizerhof (1995) generalized the problem to a not rotationally symmetric case, and showed the necessity of non-linear approaches for the interaction between bulk cargo and shells structure, but also stressed the lack of information available in this matter. 
Ummenhofer's (1997) research was based on the work by Knödel (1994), and dealt with the imperfections in thin-walled steel silos subjected to the loads arsing from bulk cargo. He performed experimental and numerical investigations on the influence of elastic foundations. Ummenhofer (1997) proposed a mechanical model for evaluating the carrying capacity by especially taking the imperfection sensitivity of axially loaded cylinders into account.

Schweizerhof et al. (1998) summarized his work from (1987) and (1995), extended the study and pointed out that with transient analyses reliable predictions can be obtained for the post-buckling area. The topic of a bulk cargo - structure interaction was not further detailed in this work.

\subsection{Material models for soil}

A major goal of research in soil mechanics has always been the search for a sophisticated material law, which would represent all relevant material properties and a realistic soil behaviour. Nowadays engineers in the field of geotechnical engineering are able to construct detailed models of the soil by means of finite element programs using solid and also infinite elements. The computational power allows the user to work with complex three-dimensional problems involving the modelling of the soil. For the latter step, the user needs an implemented material model as mentioned in the introductory sentence. Material laws with complex descriptions for modelling the soil only evolved slowly. The associated formulation for taking the volume change of soil subjected to shear into account influences the normal stresses significantly. This aspect was implemented relatively late. Collapse modes with plasticity models (e.g. MohrCoulomb or Drucker-Prager) or soil specific characteristics as dilation or flow rules were investigated in soil mechanics. In Cambridge in the 60s for example the Cam Clay theory was developed based on the elastoplastic theory (see e.g. Atkinson, 1993). More recent models consider for example objective strain rates or stress- and density dependent material behaviour.

Common material models based on continuum mechanics include the hyperelasticity, the hypoelasticity, the plastic theory, the endochrone theory, the hyperplasticity, and the hypoplasticity. The main models key features are listed in an overview in Fig. 3.5.

A material is defined as Cauchy-elastic if the deformations are independent of the loading history, and if this is valid for any coordinate system (materially objective). A special form is the hyperelasticity for which the material is assigned minimum one potential (here strain energy function) and thus is defined as conservative. The hyperelasticity is as it's name reveals based on a "too high" elasticity and combines it with a reversibility and a constancy of entropy. Materials still exhibiting elastic behaviour for very high strains can be well described using the hyperelasticity. Such a material for example is an elastomer. Other examples include formulations for clay (e.g. Niemunis and Cudny, 1998). 
In contrast to the hyperelastic material, the stresses for the hypoelasticity are path dependent, i.e. a correlation between the current and the reference configuration is needed. The theory introduced by Truesdell (1955) can model unlimited yielding, as its name implies a "too small" elasticity. In the field of tunnelling often the Duncan-Chang and the Schad material models are used which are both based on the hypoelastic material model.

In the basic form of the plastic theory frequently used for soil problems, the body is described with a scleronomic material model consisting of elastic and plastic components. Plastic deformations are non-reversible, i.e. non-conservative, and energy dissipation takes place. For soil modelling non-associated flow rules (plastic yielding is not associated with the yield surface) are necessary for describing the behaviour correctly.

The hyperplasticity arises from the potential functions in analogy to the hyperelasticity and was coined by Houlsby and Puzrin (2000). The mutual dependency of e.g. strain and stress on the deformation rate is termed incrementally non-linear. The direction of the plastic lengthening rate only depends on the current stress state, meaning that the conventional plastic strain is substituted by a continuous field of an infinite number of plastic strain components, each with a separate yield surface. This model has proven to be appropriate for modelling multi-loading sequences or cyclic loads, as for example for offshore wind turbines in Byrne and Houlsby (2003).

In reality the direction of the lengthening rate depends on the strain rate. This fact led to the introduction of the hypoplasticity. Based on the theory of Truesdell (1955) a new formulation was derived in Grenoble (Darve and Chambon, 1974 and 1979) and Karlsruhe (Gudehus, 1979; Kolymbas, 1988) which covers the elastic and the plastic behaviour within one equation. Over the years the theory was extended by various researchers (e.g. Wu, 1992; von Wolffersdorff, 1996). The material model was for example implemented in the finite element program ABAQUS (e.g. Roddeman, 1997; Fellin, 2000). The model of hypoplasticity is suitable for non-cohesive soils, and describes these soils as highly non-linear and anelastic by taking amongst others the distinct change of volume under shear deformation into account. The recent development in the field of hypoplasticity allows a relatively simple modelling of the soil in comparison to the previous approaches, and thus the implementation in standard finite element programs. Due to reasons of efficiency, in the research that does not explicitly deal with soil mechanics no or only in individual cases more complex soil models as described in this section are applied.

More constitutive models which are here not further discussed but included for completeness are for example the viscoplasticity as a time and rate dependent plasticity, the endochrone theory (see Valanis, 1971; Bazant, 1978), or the stress vertex-theory (see Rudnicki and Rice, 1975). 


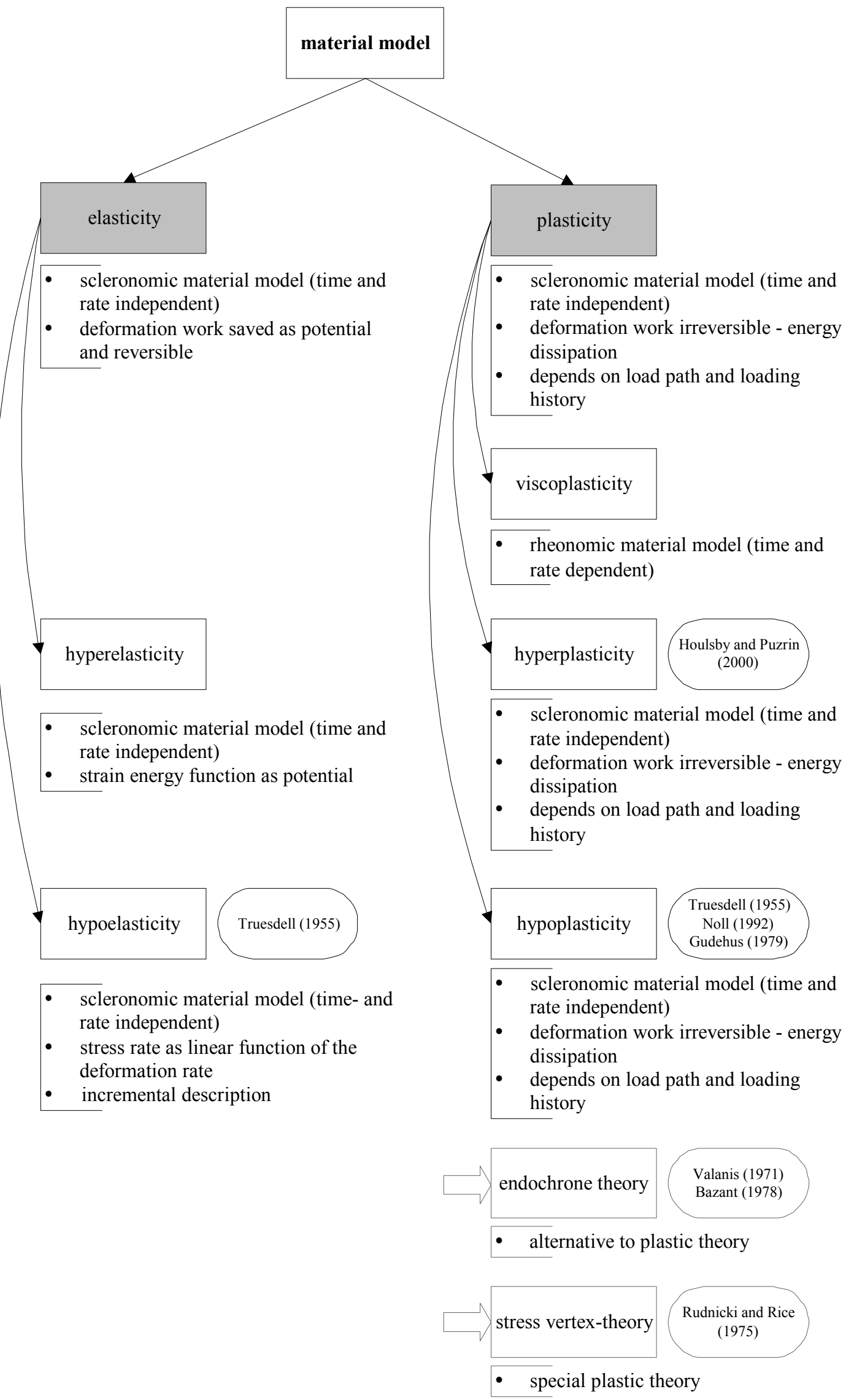

Fig. 3.5: Overview of the main soil models and constitutive laws 


\subsection{Approaches for modelling the soil-structure interaction (SSI)}

The simple and well-known method for modelling soil subjected to loads is the hypothesis using discrete springs. The Winkler foundation (Fig. 3.6) is a system consisting of discrete uncoupled springs deforming under loads. A different physical interpretation describes the parameter for the vertical spring stiffness as volume weight of a liquid and that way defines the buoyancy. Fig. 3.6 depicts the deformed soil if modelled with Winkler springs. The problem with this model is how to deduce the spring stiffness from the soil characteristics. Another critical item can be clearly seen in Fig. 3.6: the uncoupled system leads to very unrealistic soil displacements.
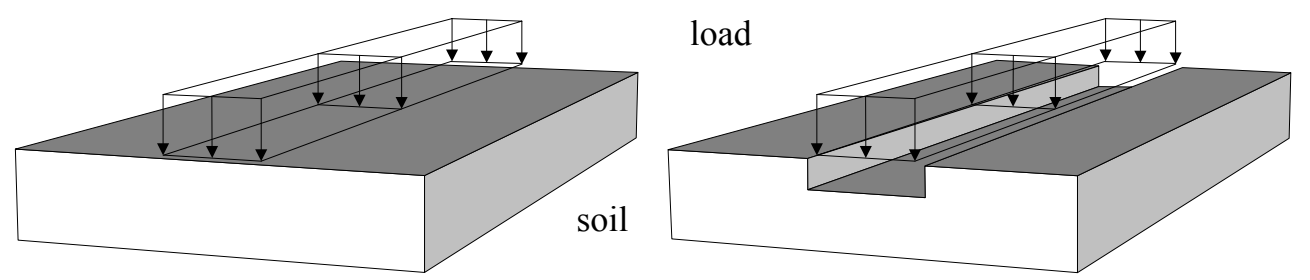

Fig. 3.6: Settlement for foundation using uncoupled springs (Winkler)
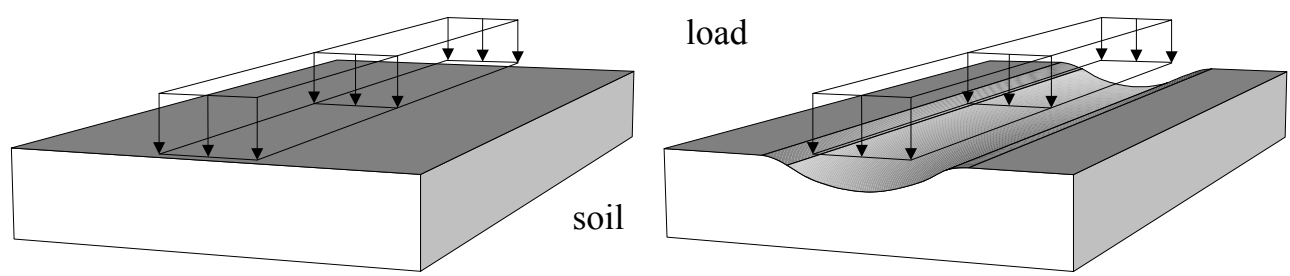

Fig. 3.7: Settlement for foundation using coupled springs (Pasternak, 1954) or continuum

Describing the soil as a coupled system in the interaction surface and hence not uncoupled at discrete points results in a different model (Fig. 3.7). Various multiparameter models were developed (e.g. Rhines, 1965: spring - plate - shear layer spring) which lead to practical results for special cases (see Horvath, 2002). Nevertheless, the major deficiency of these models is that the required input is not a measurable soil parameter but depends on size and form of the structure. Formulae for such input parameters were given by Askegaard (1961), Fuchs (1969), Kolymbas (1988), Ehlers (1979), Rotter and Zhang (1989), and Rombach (1991). Knödel (1995) compared the values for the coefficient of subgrade reaction given by several researchers for a specific range, and revealed a difference of a factor 40 for a reference case using different approaches. Burland et al. (1977) gave a summary in a state-of-theart report concerning the soil-structure interaction. His report was mainly focussed on rafts and footings. Van Langen (1991) studied the soil-structure interaction by numerical means and programmed a special 10-node interface element for the use with the finite element program PLAXIS with a constitutive equation relating the stresses to slip and compression. Horvath (1984, 1988, and 2002) compared SSI models with examples from foundation engineering regarding realistic behaviour. Horvath (2002) stated an improvement of the results for the sequence Winkler model, multi-parameter- 
model, and elastic continuum with numerous comparisons. He pointed out that the accuracy of the Winkler hypothesis is directly related to how well the spring coefficient for the assumed coefficient of subgrade reaction matches the actual.

Parallel to the modelling of the soil with multi-parameter models, research was done on describing the soil as a linear elastic and homogenous continuum for overcoming the problem of unrealistic settlement given by the Winkler spring model (e.g. Reissner, 1958 and 1967; Vlasov and Leontev, 1960). 


\section{Common practical design of combiwalls}

\subsection{General}

In harbour construction large tubes are used in conjunction with retaining wall structures (combiwalls) as primary elements. So far the assessment of these tubes was performed using stress design according to beam theory independent on the steel grade. Due to the geometrical slenderness of $D / t$ smaller than 100 , the special load carrying behaviour of shells regarding stress distributions and instability (buckling) were not taken into consideration in this context. Gresnigt (1986) extended the Dutch assessment by taking the additional influence of the secondary loads into account, which are introduced into the tubes via the intermediate sheetings. The present assessment in Europe uses stress design based on the yield stress of the tube's material. With the institution of the Eurocode EN 1993-1-1 the design procedure changes as the steel grade plays a role in the calculation of the geometrical slenderness. The high yield stresses of the steels used for tubular piles in harbour constructions cause the tubes to be categorised as thin-walled structures (class 4 cross-section). Therefore, the tubular piles have to be assessed versus shell buckling according to EN 1993-1-6.

In the following section the design procedure for a reference case is calculated according to different codes. Special attention is paid to the assumptions for boundary and loading conditions, highlighting the importance of problem specific design rules. In the next step these conservative assumptions shall be discussed and investigated in order to provide suggestions and comments for enhancing design rules for tubular piles regarding shell buckling.

\subsection{Formulation of the problem}

Fig. 4.1 and Fig. 4.2 depict a combiwall structure as commonly installed in harbour constructions subjected to working loads. Based on this standard reference case, with the system and loads shown in Fig. 4.1 and Fig. 4.2, the deformations and the resultant forces can be obtained. The crane load of $F_{V}=1200 \mathrm{kN} / \mathrm{m}$ and the spacing of the tubular piles of $3.12 \mathrm{~m}$ results in an axial force of $3750 \mathrm{kN}$ for each tubular pile. The loads acting on the structure are obtained with the program RIDO. The program RIDO is a finite element program for calculating sheet pile walls applying an elasto-plastic approach and taking the individual working stages of the construction into account. The follow-up, more detailed numerical studies performed in this paper use the general purpose, finite element package ABAQUS. In the investigations a Young's modulus of $210000 \mathrm{~N} / \mathrm{mm}^{2}$ and a Poisson ratio of 0.3 is applied for steel. 
The standard approach (Eurocode EN 1993-5) for the transfer of loads in the assessment for combiwalls assumes that the primary elements (tubular pile) bear the earth pressures and the secondary elements (Z-shaped sheetings) bear the water pressure. This approach is based on the assumption that an arch transferring the loads to the primary elements forms within the soil.

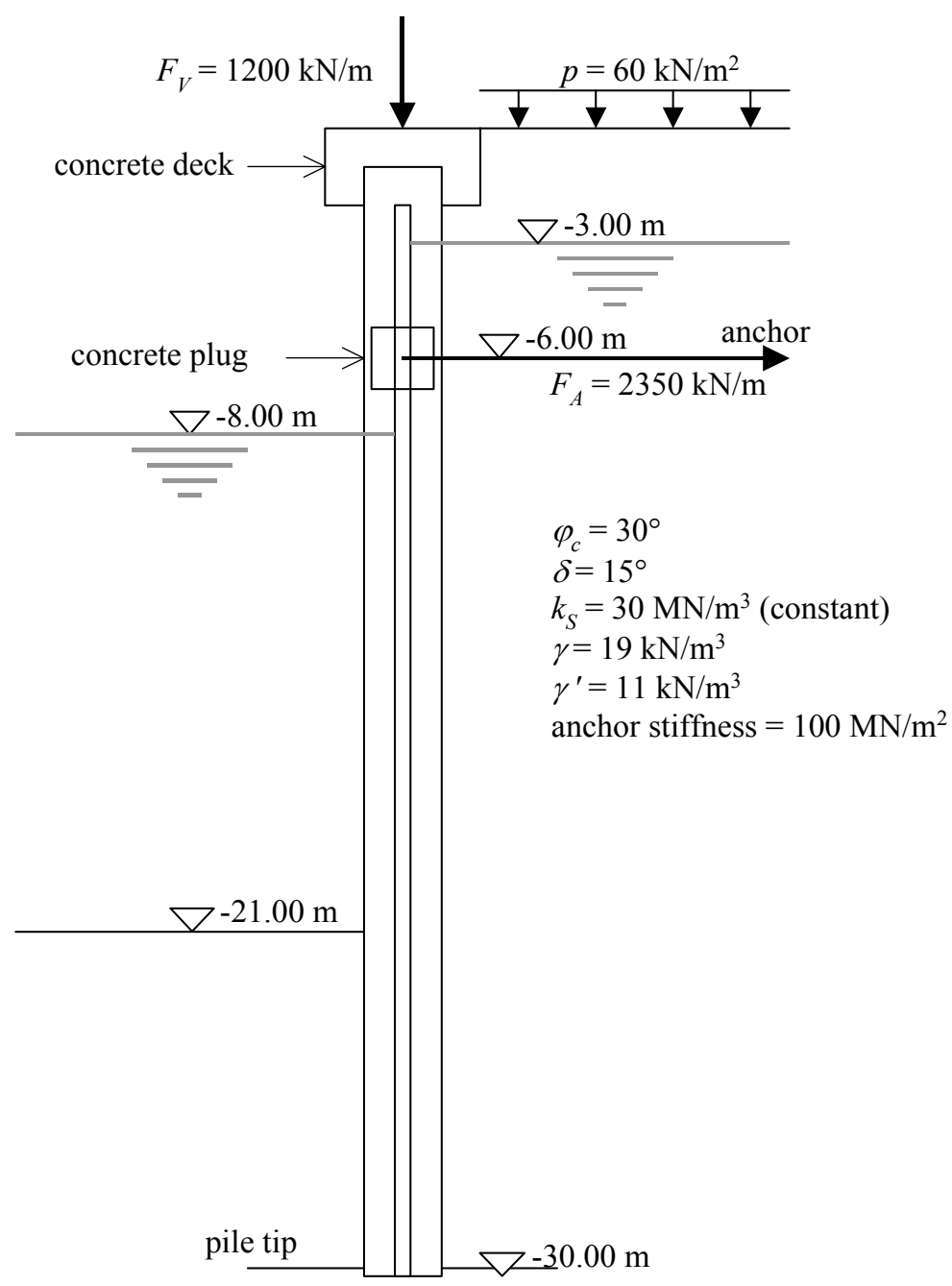

Fig. 4.1: Combiwall with loads

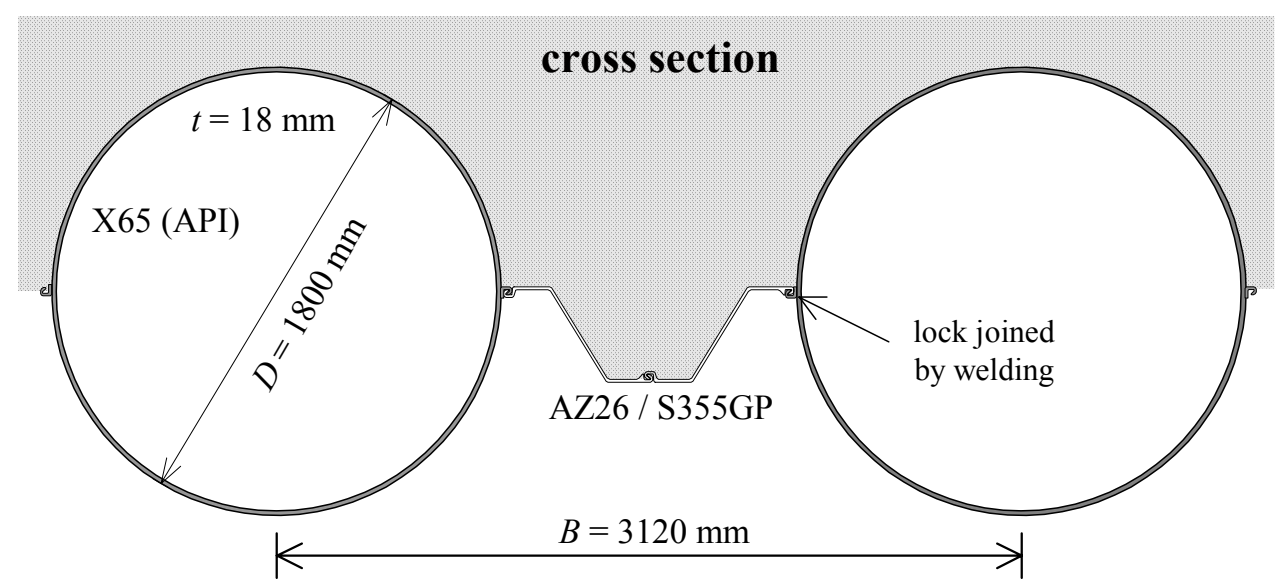

Fig. 4.2: Cross-section of the combiwall system 
The displacements and the distribution of the bending moment and the shear forces were calculated with RIDO and are shown in Fig. 4.3. The calculation assumes a constant coefficient of subgrade reaction over the entire length of the system. Additionally, comparing analyses were performed with the finite element program SPW2004 (Verruijt, 1995) which also calculates the deformations and the force resultants using an elastoplastic approach. In contrast to the analysis with RIDO, SPW2004 applies a varying coefficient of subgrade reaction depending on the depth. The results and further details for the analyses with SPW2004 can be found in Annex A: Retaining wall calculation.
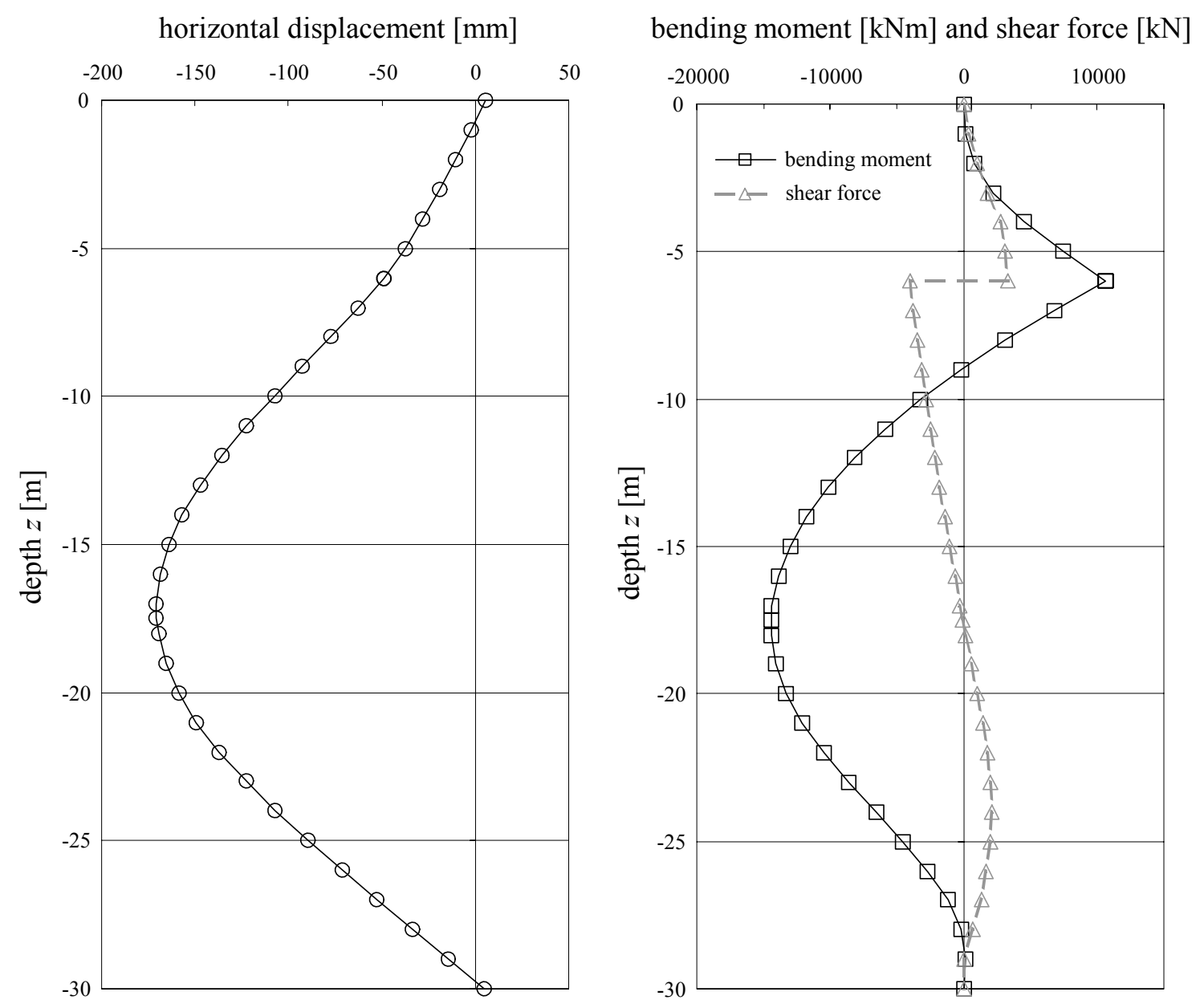

Fig. 4.3: Displacements and distribution of bending moment and shear forces for tubular pile

In Fig. 4.3 the relevant cross sections can be identified: the point of application for the anchor at $z=-6 \mathrm{~m}$, and the position of the maximum bending moment at $z=-17.5 \mathrm{~m}$. Tab. 4.1 summarizes the resultant forces for the corresponding cross sections.

In Tab. 4.2 the geometrical data and the yield stress for the tubular pile are given. The tube is made of steel X65 (API). The yield stress for this type of steel is defined as $445 \mathrm{~N} / \mathrm{mm}^{2}$ for $t \leq 16 \mathrm{~mm}$. But for the required thickness of $18 \mathrm{~mm}$ it is chosen to be $f_{y, k}=430 \mathrm{~N} / \mathrm{mm}^{2}$ as a conservative approach. 
Tab. 4.1: Summary of the resultant forces

\begin{tabular}{|l|c|c|}
\hline position & anchor $(-6 \mathrm{~m})$ & max. moment $(-17.5 \mathrm{~m})$ \\
\hline bending moment $[\mathrm{kNm}]$ & 10670 & 14460 \\
\hline axial force $[\mathrm{kN}]$ & 3750 & 3750 \\
\hline anchor force $[\mathrm{kN}]$ & 7330 & - \\
\hline shear force $[\mathrm{kN}]$ & $4025 / 3310$ & - \\
\hline max. displacement $[\mathrm{mm}]$ & - & 170 \\
\hline
\end{tabular}

Tab. 4.2: Data for tubular pile

\begin{tabular}{|c|c|c|c|c|c|c|}
\hline & $D[\mathrm{~mm}]$ & $t[\mathrm{~mm}]$ & $A\left[\mathrm{~mm}^{2}\right]$ & $I_{y}=I_{z}\left[\mathrm{~mm}^{4}\right]$ & $S_{y}\left[\mathrm{~mm}^{3}\right]$ & $f_{y, k}\left[\mathrm{~N} / \mathrm{mm}^{2}\right]$ \\
\hline tubular pile & 1800 & 18 & $1.018 \cdot 10^{5}$ & $4.123 \cdot 10^{10}$ & $1.944 \cdot 10^{7}$ & 430 \\
\hline
\end{tabular}

\subsection{Classical design}

The stress resultants given in Tab. 4.1 are sufficient for performing the classical design. The assessment is based on stress design at the appropriate positions. The axial and shear stresses are calculated according to (4.1) and (4.2).

$$
\begin{gathered}
\sigma_{x}=\frac{N}{A}+\frac{M_{y}}{I_{y}} \cdot z \\
\tau=\frac{V_{z} \cdot S_{y}}{I_{y} \cdot S}
\end{gathered}
$$

The calculations in Tab. 4.3 reveal that the verifications according to (4.1) and (4.2) satisfy the elastic assessment. The verification of the equivalent stress is abandoned since the maximum axial and shear stress appear at different positions.

Tab. 4.3: Stress design

\begin{tabular}{|l|c|c|}
\hline position & anchor $(-6 \mathrm{~m})$ & max. moment $(-17.5 \mathrm{~m})$ \\
\hline$\sigma_{x}$ acc. to $(4.1)\left[\mathrm{N} / \mathrm{mm}^{2}\right]$ & $37+233=270$ & $37+316=353$ \\
\hline$\tau$ acc. to (4.2) $\left[\mathrm{N} / \mathrm{mm}^{2}\right]$ & 53 & 0 \\
\hline verification el.-el. & $\frac{\sigma_{x}}{\sigma_{R d}}=\frac{270}{391}=0.69$ & $\frac{\sigma_{x}}{\sigma_{R d}}=\frac{353}{391}=0.90$ \\
\hline verification el.-el. & $\frac{\tau}{\tau_{R d}}=\frac{53}{226}=0.23$ & - \\
\hline & fulfilled & fulfilled \\
\hline
\end{tabular}




\subsection{Classical design with secondary effect}

Gresnigt (1986) investigated buried pipelines in areas of settlement and considered the effect of ovalization (Brazier, 1927) in his study. His results were regarded in annex G.2 of Eurocode ENV 1993-5:1998 to be applied for combiwall structures. A secondary effect in ovalising the tubular piles arises, when the primary elements are additionally loaded by the secondary elements (sheetings). This influence is approximated in annex G.2 of ENV 1993-5:1998 by using a reduced cross section for the tubular piles.

The secondary elements are taken into account for transferring the water pressure. The resultant forces $w_{x}, w_{y}$ and $m$ acting on the sheet pile wall are shown in Fig. 4.4, and are evaluated using the depicted simplified model in combination with a structural analysis program. The forces are transferred from the sheetings to the tubular piles by locks which are commonly welded to the outside of the tubes. The resultant forces which are passed on to the tubular piles for the investigated case are summarized in Fig. 4.5 and Tab. 4.4.

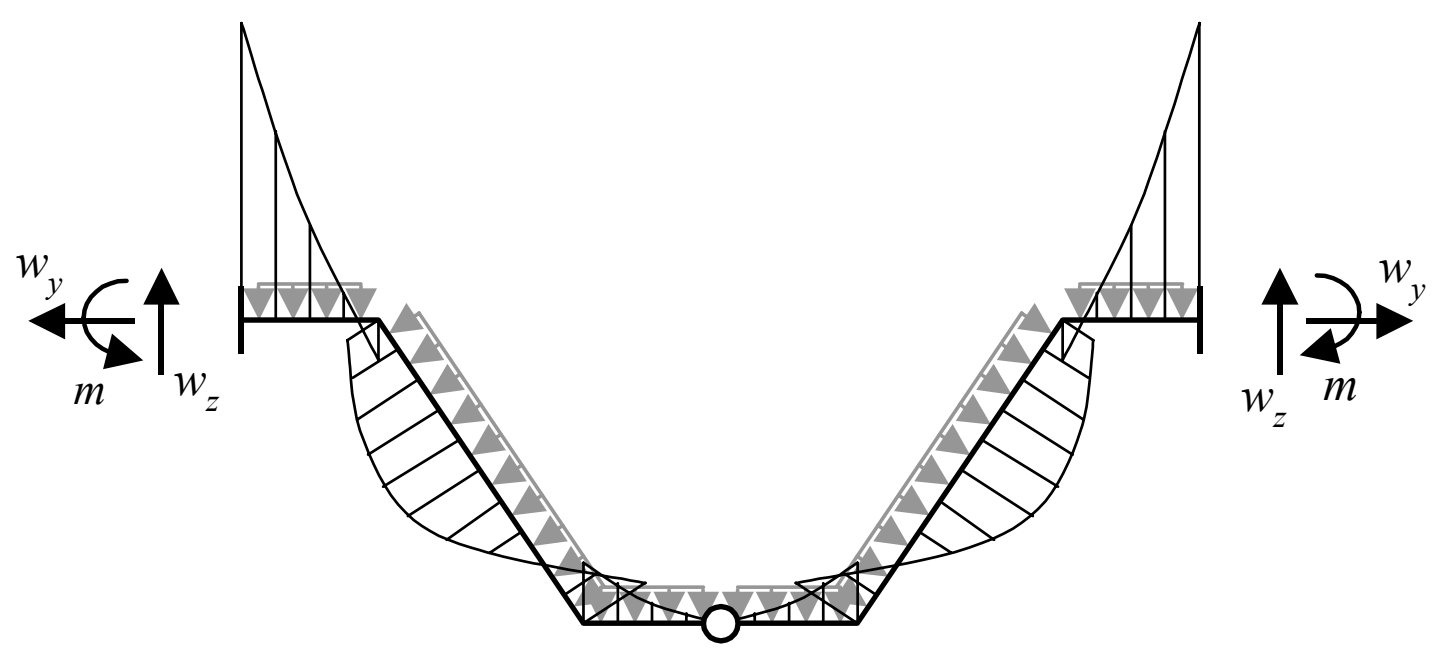

Fig. 4.4: Resultant forces and bending moment distribution for secondary elements

Tab. 4.4: $\quad$ Secondary resultant forces

\begin{tabular}{|l|c|c|}
\hline position & anchor $(-6 \mathrm{~m})$ & max. moment $(-17.5 \mathrm{~m})$ \\
\hline$w_{y}[\mathrm{kN} / \mathrm{m}]$ & 14.2 & 23.6 \\
\hline$w_{z}[\mathrm{kN} / \mathrm{m}]$ & 18.9 & 31.5 \\
\hline$m[\mathrm{kNm} / \mathrm{m}]$ & 2.6 & 4.4 \\
\hline
\end{tabular}




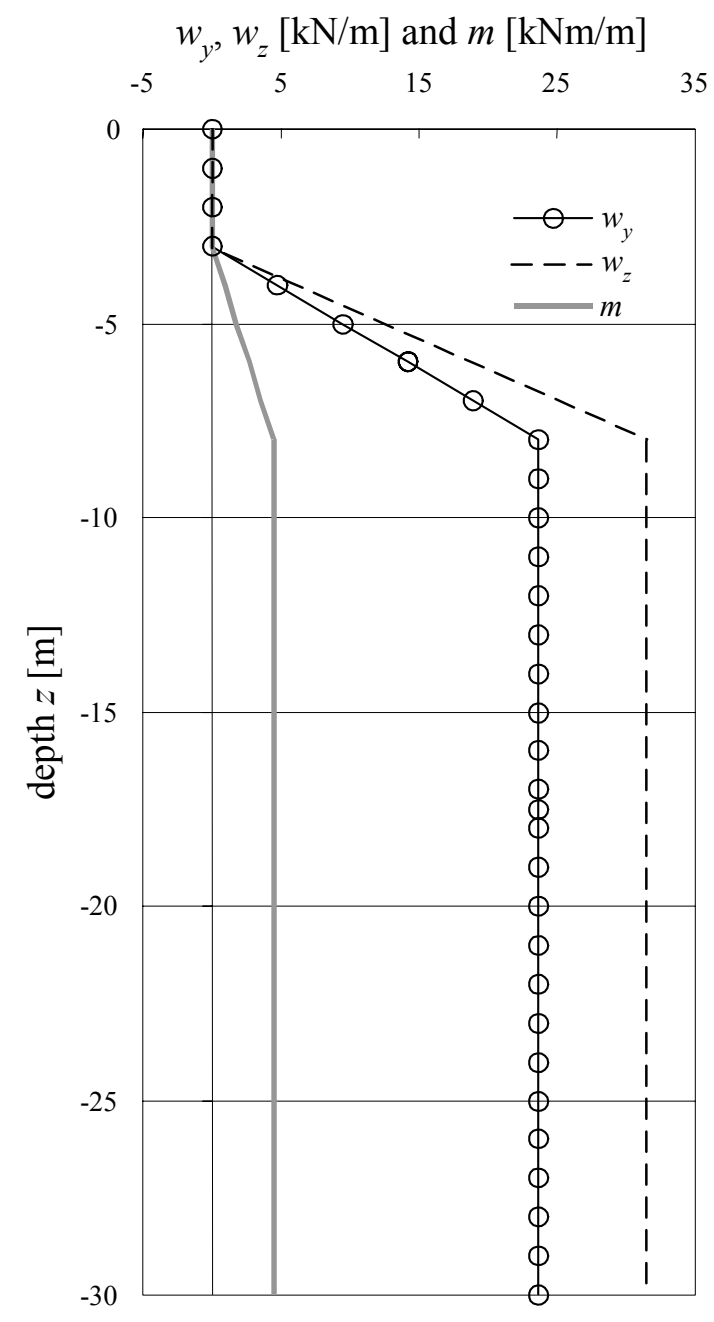

Fig. 4.5: Secondary resultant forces $w_{y}, w_{z}$ and $m$ from sheetings

Fig. 4.6 depicts the influence of the secondary resultant forces on the primary elements. The force $w_{y}$ leads to an ovalization of the tube and thus to a reduced bearing capacity versus bending moments. These forces at the locks result in a bending of the tubular pile's cross-section.

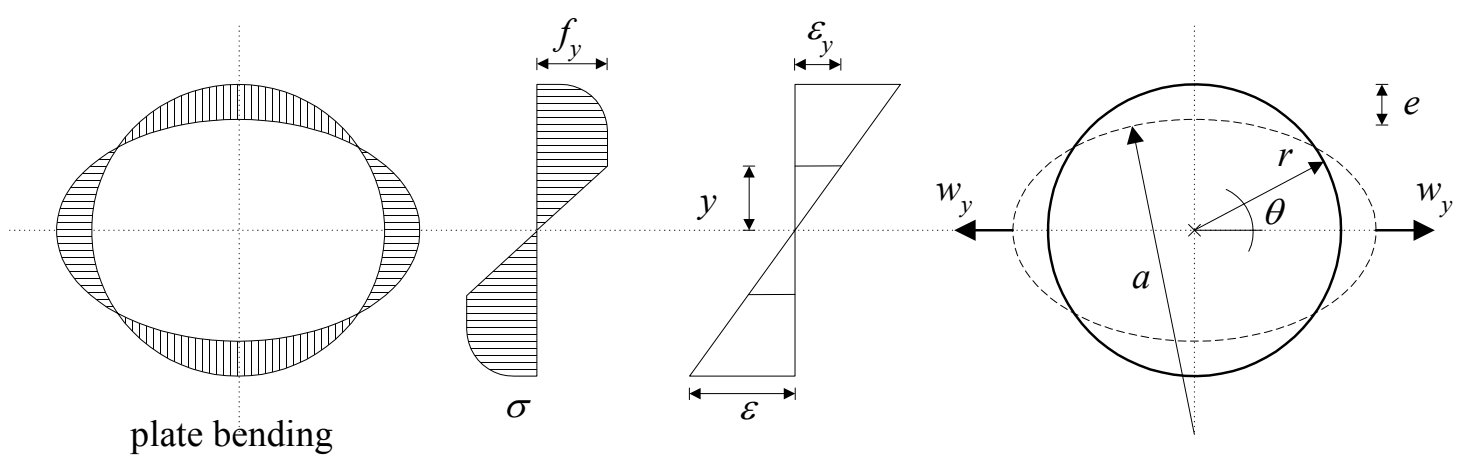

Fig. 4.6: Bending of the tubular pile - circumferential and axial bending according to ENV 19935:1998 annex $G$ 
The ENV 1993-5:1998 states formulae for calculating the reduced plastic resultant forces for the design procedure whose application is shown in the following example. For the ovalization $e$ and the radius of curvature $a$ according to Fig. 4.6 it can be written:

$$
\begin{gathered}
e=0.0684 w_{y} \frac{r^{3}}{E I} \quad \text { but } \quad e \leq 0.1 r \\
a=\frac{r}{1-\frac{3 e}{r}}
\end{gathered}
$$

The stiffness for shell bending of the tube is calculated as:

$$
E I=E \cdot \frac{t^{3}}{12}
$$

With the radius of curvature $a$ according to Fig. 4.6 the critical strain $\varepsilon$ for which buckling occurs can be evaluated as

$$
\begin{array}{cc}
\text { for } \frac{t}{a} \geq \frac{1}{60} \quad \text { use } \quad \varepsilon=0.25 \frac{t}{a}-0.0025 \\
\text { for } \frac{t}{a} \leq \frac{1}{60} \quad \text { use } \quad \varepsilon=0.10 \frac{t}{a}
\end{array}
$$

The original formulae for calculating the critical strain $\varepsilon$ given in ENV 1993-5:1998 and ENV 1993-5:2002 (Stage 32) are incorrect. The formulae (4.6) and (4.7) give the correct version according to Gresnigt (1986).

For the angle $\theta$ for which the axial stresses reach their maximum, ENV 1993-5:1998 states:

$$
\begin{gathered}
\theta=\pi / 2 \quad \text { for } \quad \varepsilon \leq \varepsilon_{y} \\
\sin \theta=1 / \mu_{\varepsilon} \quad \text { for } \quad \varepsilon>\varepsilon_{y}
\end{gathered}
$$

with

$$
\mu_{\varepsilon}=\frac{\varepsilon}{\varepsilon_{y}}=\varepsilon \cdot \frac{E}{f_{y}}
$$

The effective local design plastic moment of resistance for the shell $m_{\text {eff }}$ is distributed uniformly over the cross section according to ENV 1993-5:1998:

$$
m_{\text {eff }}=0.160 m+0.125 w_{y} \cdot r
$$

The effective local design plastic moment of resistance for the shell $m_{\text {eff }}$ is needed for the interaction factor $g_{\text {int }}$ :

$$
g_{\text {int }}=\frac{c_{1}}{6}+\frac{2}{3}
$$


with

$$
c_{1}=\sqrt{4-2 \sqrt{3} \frac{m_{e f f}}{m_{p l, R d}}}
$$

The local design plastic moment of resistance for the shell $m_{p l, R d}$ is defined as:

$$
m_{p l, R d}=\frac{1}{4} t^{2} \frac{f_{y}}{\gamma_{M 0}}
$$

According to ENV 1993-5:1998 the reduction factor $\beta_{g}$ which takes the ovalization into account is:

$$
\beta_{g}=1-\frac{2 e}{3 r}
$$

and the reduction factor $\beta_{s}$ which takes the deformation capacity into account is:

$$
\begin{gathered}
\beta_{s}=0.75 \quad \text { for } \quad \varepsilon \leq \varepsilon_{y} \\
\beta_{s}=0.625+0.125 \mu \quad \text { for } \quad \varepsilon_{y} \leq \varepsilon \leq 3 \varepsilon_{y} \\
\beta_{s}=1.0 \quad \text { for } \quad 3 \varepsilon_{y} \leq \varepsilon
\end{gathered}
$$

With the reduction factors $\beta_{g}$ and $\beta_{s}$, and the interaction factor $g$ the reduced moment of resistance $M_{\text {red,Rd }}$ according to ENV 1993-5:1998 can be calculated:

$$
M_{r e d, R d}=\frac{1}{2}\left(\frac{\theta}{\sin \theta}+\cos \theta\right) g_{i n t} \cdot \beta_{g} \cdot \beta_{s} \cdot M_{p l, R d}
$$

with the design plastic moment of resistance $M_{p l, R d}$ of the circular cross-section:

$$
M_{p l, R d}=4 r^{2} \cdot t \frac{f_{y}}{\gamma_{M 0}}
$$

The design plastic resistance to normal force $N_{p l, R d}$ of the circular cross-section is reduced in order to take the local bending of the shell into account by the interaction factor $g$ as:

$$
N_{p l, R d}=2 \pi \cdot r \cdot t \frac{f_{y}}{\gamma_{M 0}}
$$

to

$$
N_{\text {red }, R d}=g_{\text {int }} \cdot N_{p l, R d}
$$

The verification of the resultant forces of the tube can be performed with the following moment-axial force interaction criterion:

$$
\left(\frac{M_{S d}}{M_{r e d, R d}}\right)+\left(\frac{N_{S d}}{N_{r e d, R d}}\right)^{1.7} \leq 1
$$


Tab. 4.5: $\quad$ Assessment of the example according to ENV 1993-5:1998

\begin{tabular}{|c|c|c|c|c|}
\hline parameter & $e q$ & anchor $(-6 m)$ & max. moment $(-17.5 \mathrm{~m})$ & dimension \\
\hline$E I$ & $(4.5)$ & \multicolumn{2}{|c|}{$1.0206 \cdot 10^{8}$} & $\mathrm{Nmm}$ \\
\hline$e$ & $(4.3)$ & $6.94 \leq 90$ & $11.53 \leq 90$ & $\mathrm{~mm}$ \\
\hline$a$ & $(4.4)$ & 921.3 & 936.0 & $\mathrm{~mm}$ \\
\hline$t / a$ & & $0.0195 \geq 0.0167$ & $0.0192 \geq 0.0167$ & - \\
\hline$\varepsilon$ & $(4.6)$ & 2,38 & 2,31 & $\%$ \\
\hline$\mu$ & $(4.10)$ & $1.162 \geq 1$ & $1.128 \geq 1$ & - \\
\hline$\theta$ & $(4.8)$ & 1.036 & 1.090 & $\mathrm{rad}$ \\
\hline$m_{e f f}$ & $(4.11)$ & 2.01 & 3.36 & $\mathrm{kNm} / \mathrm{m}$ \\
\hline$m_{p l, R d}$ & $(4.14)$ & \multicolumn{2}{|c|}{31.67} & $\mathrm{kNm} / \mathrm{m}$ \\
\hline$c_{1}$ & $(4.13)$ & 1.94 & 1.91 & - \\
\hline$g$ & $(4.12)$ & 0.990 & 0.985 & - \\
\hline$\beta_{g}$ & $(4.15)$ & 0.995 & 0.991 & - \\
\hline$\beta_{S}$ & $(4.16)$ & 0.770 & 0.766 & - \\
\hline$M_{p l, R d}$ & $(4.20)$ & \multicolumn{2}{|c|}{$2.280 \cdot 10^{4}$} & $\mathrm{kNm}$ \\
\hline$M_{r e d, R d}$ & $(4.19)$ & $1.482 \cdot 10^{4}$ & $1.442 \cdot 10^{4}$ & $\mathrm{kNm}$ \\
\hline$N_{p l, R d}$ & $(4.21)$ & \multicolumn{2}{|c|}{$3.979 \cdot 10^{4}$} & $\mathrm{kN}$ \\
\hline$N_{\text {red,Rd }}$ & $(4.22)$ & $3.939 \cdot 10^{4}$ & $3.919 \cdot 10^{4}$ & $\mathrm{kN}$ \\
\hline \multirow[t]{2}{*}{ verif. } & $\begin{array}{c}(4.23) \\
\gamma_{M}=1.1\end{array}$ & $0.72+0.095^{1.7}=0.75$ & $1.00+0.096^{1.7}=1.02$ & - \\
\hline & & fulfilled & not fulfilled & - \\
\hline \multirow[t]{2}{*}{ verif. } & $\begin{array}{c}(4.23) \\
\gamma_{M}=1.0\end{array}$ & $0.65+0.086^{1.7}=0.67$ & $0.91+0.087^{1,7}=0.93$ & - \\
\hline & & fulfilled & fulfilled & - \\
\hline
\end{tabular}

In Tab. 4.5 the example case is calculated step-by-step regarding the equations (4.3) to (4.23). Annex G.2 of ENV 1993-5:1998 states that a water level difference of more than $4 \mathrm{~m}$ should be regarded when evaluating the interaction factor $g$. In this context a modified formula is given for calculating the interaction factor $g$. Since the influence for the investigated case is minor (deviation $<<1 \%$ ), a modification of $g$ is set aside. Further, in the design half of the outer diameter $D$ is used as the mid-radius $r$ as approximation (influence for the reference case $\approx 1 \%$ ). The verification is performed using a partial safety factor for the resistance $\gamma_{M}$ of 1.1 for the limit state design as a 
conservative approach. Additionally, the verification is performed using a partial safety factor for the resistance $\gamma_{M}$ of 1.0, since the specifications differ between versions of the Eurocode draft (EN 1993-5).

The verification taking the additional influence of the secondary forces and therefore the ovalization of the tubular pile into account is fulfilled for the investigated problem. Only, the verification applying a partial safety factor for the resistance $\gamma_{M}$ of 1.1 and not considering the supporting effect of the soil is not fulfilled by $2 \%$ for the design method according to ENV 1993-5:1998. Similar procedures which are and have been applied in different European countries may lead to slightly different results.

\subsection{Design according to EN 1993-1-6}

\subsubsection{General}

The required verifications change if the entire Eurocode concept is considered. Following EN 1993-1-1 members are grouped into different cross-section classes. This classification into four classes is made according to Table 5.2 in prEN 1993-1-1:200202. For tubular sections the value of $D / t=90 \varepsilon^{2}$ is the limit for Class 4 cross-sections. Hence, most tubes used for harbour constructions end up as Class 4 cross-sections:

$$
\text { Class } 4 \text { valid for } \quad D / t>90 \varepsilon^{2} \quad \text { with } \quad \varepsilon=\sqrt{235 / f_{y}}
$$

For the investigated case this means that the tubular pile in our example is clearly grouped into Class 4:

$$
D / t=100>90 \varepsilon^{2}=90 \cdot \frac{235}{430}=49.2
$$

As the cross-section cannot be grouped into Class 1 to 3, the Eurocode prEN 1993-11:2002-02 refers to EN 1993-1-6, which signifies an additional assessment against shell buckling according to the latter part of the Eurocode (EN 1993-1-6). Generally, an assessment with limit state buckling design of tubular piles according to the Eurocode involves the consideration of the following stress components:

- axial membrane stresses from axial forces and bending moments with limit state buckling design against axial compression

- shear stresses from transverse forces with limit state buckling design against shear

- circumferential stresses from external pressure with limit state buckling design against circumferential compression

The first part of the design procedure which includes the effect of ovalization according to ENV 1993-5:1998 has already been preformed in the previous section, and the bearing capacity verifications are fulfilled. 
As already noted in ENV 1993-5:1998 the tubular piles of combiwalls are not exempt from a buckling assessment. In a more recent version of the EN 1993-5 (prEN 19935:2004-07) a different approach is suggested which eliminates several inconsistency of the previous versions. Since the several parts of the Eurocode are still being drafted, two recent concepts (ENV 1993-5:2002 (Stage 32) and prEN 1993-5:2004-07) will be introduced for better understanding.

\subsubsection{Concept according to ENV 1993-5:2002 (Stage 32)}

The concept given in ENV 1993-5:2002 (Stage 32) is based on the approach explained in the previous sections. As the member is grouped into Class 4, design against buckling according to EN 1993-1-6 is required. The verification procedure is performed according to ENV 1993-1-6:2002-05.

The secondary effect from the resultant forces introduced via the connection to the sheetings has to be considered and results in an increased radius. In the design procedure the radius increased by the ovalization $a(=936 \mathrm{~mm})$ is used instead of the initial radius of the geometry $r(=900 \mathrm{~mm})$. The calculation of the resistances of the cross-section (Tab. 4.6) is based on the original geometry as an approximation. The stresses are calculated according to ENV 1993-1-6:2002-05 by applying membrane theory. As an approximation for evaluating the circumferential stresses only the pressure arising from the difference of the water loads is applied as uniform external pressure. The assumed uniform loading conditions for $\sigma_{x}, \sigma_{\theta}$ and $\tau$ are a conservative simplification. The stresses are calculated as:

$$
\begin{gathered}
\sigma_{x}=\frac{N}{2 \pi r t} \pm \frac{M}{\pi r^{2} t} \\
\sigma_{\theta}=\frac{\Delta p \cdot r}{t} \\
\tau=\frac{V}{\pi r t}
\end{gathered}
$$

Therefore, for a difference of the water loads of $3 \mathrm{~m}$ and $5 \mathrm{~m}$ the design stresses are summarized in Tab. 4.6.

Tab. 4.6: Design stresses

\begin{tabular}{|l|c|c|}
\hline position & anchor $(-6 \mathrm{~m})$ & max. moment $(-17.5 \mathrm{~m})$ \\
\hline$\sigma_{x}$ acc. to $(4.26)\left[\mathrm{N} / \mathrm{mm}^{2}\right]$ & $37+233=270$ & $37+316=353$ \\
\hline$\sigma_{\theta}$ acc. to $(4.27)\left[\mathrm{N} / \mathrm{mm}^{2}\right]$ & 2 & 3 \\
\hline$\tau_{x \theta}$ acc. to $(4.28)\left[\mathrm{N} / \mathrm{mm}^{2}\right]$ & 80 & 0 \\
\hline
\end{tabular}

The design buckling stresses needed for the buckling limit state design are calculated with the following formulae: 


$$
\begin{gathered}
\sigma_{x, R d}=\sigma_{x, R k} / \gamma_{M}, \quad \sigma_{\theta, R d}=\sigma_{\theta, R k} / \gamma_{M}, \quad \tau_{R d}=\tau_{R k} / \gamma_{M} \\
\sigma_{x, R k}=\chi_{x} \cdot f_{y k}, \quad \sigma_{\theta, R k}=\chi_{\theta} \cdot f_{y k}, \quad \tau_{R k}=\chi_{\tau} \cdot f_{y k} / \sqrt{3}
\end{gathered}
$$

The buckling reduction factors $\chi$ can be calculated as a function of the relative slenderness of the shell $\bar{\lambda}$ :

$$
\begin{gathered}
\chi=1 \quad \text { for } \quad \bar{\lambda} \leq \bar{\lambda}_{0} \\
\chi=1-\beta\left(\frac{\bar{\lambda}-\bar{\lambda}_{0}}{\bar{\lambda}_{p}-\bar{\lambda}_{0}}\right)^{\eta} \quad \text { for } \quad \bar{\lambda}_{0}<\bar{\lambda}<\bar{\lambda}_{p} \\
\chi=\frac{\alpha}{\bar{\lambda}^{2}} \quad \text { for } \quad \bar{\lambda}_{p} \leq \bar{\lambda}
\end{gathered}
$$

using the squash limit relative slenderness $\bar{\lambda}_{p}$ :

$$
\bar{\lambda}_{p}=\sqrt{\frac{\alpha}{1-\beta}}
$$

The elastic imperfection reduction factor $\alpha$, which depends on the fabrication quality, the plastic range factor $\beta$, and the interaction exponent $\eta$ describing the shape of the buckling curves can be found in annex D of ENV 1993-1-6:2002-05.

The relative slenderness of the shell for the different loadings can be determined using the following formulae:

$$
\bar{\lambda}_{x}=\sqrt{f_{y k} / \sigma_{x, R c}}, \quad \bar{\lambda}_{\theta}=\sqrt{f_{y k} / \sigma_{\theta, R c}}, \quad \bar{\lambda}_{\tau}=\sqrt{f_{y k} /\left(\tau_{R c} \cdot \sqrt{3}\right)}
$$

The critical buckling stresses are evaluated considering the appropriate loading case as described in annex D of ENV 1993-1-6:2002-05.

The dimensionless length parameter $\omega$ characterises the length of the shell segment:

$$
\omega=\frac{l}{\sqrt{r t}}
$$

For the example case the simplified assumption is made that the reference length is defined from pile tip to the position of the anchor. The boundary conditions for the cylinder embedded in soil cannot be described with the specified boundary conditions BC 1 (clamped) to BC 3 (free) from EN 1993-1-6 as the possibilities for displacements and rotations may not be defined explicitly. As a very conservative approximation the boundary conditions BC 2-BC 2 (pinned) are chosen. Further as a conservative approach, the boundaries restrain meridional displacements. The verification against circumferential stresses is performed using the boundary conditions BC 1-BC 3 (clamped-free) given that for these conditions the factor $C_{\theta}$ reaches a minimum.

ENV 1993-1-6:2002-05 states limits for the classification of shells into fabrication tolerance quality classes (A to $\mathrm{C}$ ). For diameters larger than $1.25 \mathrm{~m}$ the limit for the 
worst quality class is a maximum out-of-roundness parameter $U_{r}(=\Delta D / D)$ of 0.015 and a maximum dimple tolerance parameter $U_{0}$ which is evaluated with measuring gauges of 0.016. In Tab. 4.7 the requirements for Class C according to ENV 1993-1-6:2002-05 are compared with the minimum requirements according to the standard for the production of helically welded tubes EN 10219-2 which are often installed in harbour constructions.

Tab. 4.7: Comparison of the quality requirements for a tube $D=1800 \mathrm{~mm}$

\begin{tabular}{|l|c|c|}
\hline parameter & EN 1993-1-6(Class $C)$ & EN 10219-2:1997 (min.) \\
\hline $\begin{array}{l}\text { out-of-roundness } \\
\text { parameter } U_{r}\end{array}$ & $\leq 0.015$ & $\leq 0.020$ \\
\hline $\begin{array}{l}\text { maximum dimple } \\
\text { tolerance parameter } U_{0}\end{array}$ & $\leq 0.016$ & - \\
\hline $\begin{array}{l}\text { deviation of outer } \\
\text { diameter D }\end{array}$ & - & $\pm 10 \mathrm{~mm}$ \\
\hline allowable excess weld & - & $\pm 4.8 \mathrm{~mm}$ \\
\hline
\end{tabular}

The comparison reveals that tubes manufactured following EN 10219-2 do not fulfil the minimum requirements of Class C from ENV 1993-1-6:2002-05 regarding out-ofroundness. The tubular piles used in combiwalls of harbour constructions are mainly covered by EN 10219-2. In the following the tubes are classified as Class C crosssections according to ENV 1993-1-6:2002-05 for simplification.

The following three tables summarize the parameters for limit state buckling design. Tab. 4.8 lists the parameters for the design against axial compression with a partial safety factor $\gamma_{M}$ of 1.1. For the calculation of the critical buckling stress, the factor $C_{x}$ in ENV 1993-1-6:2002-05, which takes the failure with extensive buckles and thus the transition to column buckling into account, is assumed to have a value of 0.6 for the reference length. This simplified assumption is a conservative approximation for the design of the investigated case. 
Tab. 4.8: Parameters for limit state buckling design against axial compression

\begin{tabular}{|c|c|c|c|}
\hline parameter & $e q$. & axial compression & dimension \\
\hline & $(4.36)$ & as $\omega=185>0.5 r / t=26 \Rightarrow$ long cylinder & - \\
\hline$C_{x b}$ & Table D.1 (EC) & 1 & - \\
\hline$C_{x}$ & Annex D (EC) & $\begin{array}{c}C_{x}=1+\frac{0.2}{C_{x b}}\left[1-2 \omega \frac{t}{r}\right]=-0.22 \text { but } \geq 0.6 \\
\Rightarrow 0.6\end{array}$ & - \\
\hline$\sigma_{x, R c}$ & Annex D (EC) & $\sigma_{x, R c}=0.605 \cdot E \cdot C_{x} \cdot \frac{t}{r}=1466$ & $\mathrm{~N} / \mathrm{mm}^{2}$ \\
\hline $\bar{\lambda}_{x}$ & $(4.35)$ & $\bar{\lambda}_{x}=\sqrt{430 / 1466}=0.54$ & - \\
\hline$Q$ & Annex D (EC) & $16($ Class $C)$ & - \\
\hline$\Delta w_{k}$ & Annex D (EC) & $\Delta w_{k}=\frac{1}{Q} \sqrt{\frac{r}{t}} \cdot t=8.11$ & - \\
\hline$\alpha_{x}$ & Annex D (EC) & $\alpha_{x}=\frac{0.62}{1+1.91 \cdot\left(\Delta w_{k} / t\right)^{1.44}}=0.386$ & - \\
\hline factors & Annex D (EC) & $\bar{\lambda}_{x 0}=0.20, \quad \beta=0.60, \quad \eta=1.00$ & - \\
\hline $\bar{\lambda}_{x p}$ & $(4.34)$ & $\bar{\lambda}_{x p}=\sqrt{0.386 /(1-0.6)}=0.98$ & - \\
\hline$\chi_{x}$ & $(4.32)$ & $\chi_{x}=1-0.6\left(\frac{0.54-0.2}{0.98-0.2}\right)^{1}=0.74$ & - \\
\hline$\sigma_{x, R d}$ & $(4.29),(4.30)$ & $\sigma_{x, R d}=\frac{0.74 \cdot 430}{1.1}=289$ & $\mathrm{~N} / \mathrm{mm}^{2}$ \\
\hline
\end{tabular}

In Tab. 4.9 the parameters needed for the limit state buckling design against circumferential compression with a partial safety factor $\gamma_{M}$ of 1.1 are summarized. 
Tab. 4.9: Parameters for limit state buckling design against circumferential compression

\begin{tabular}{|c|c|c|c|}
\hline parameter & $e q$. & circumferential compression & dimension \\
\hline & $(4.36)$ & as $\omega=185>1.63 r / t=85 \Rightarrow$ long cylinder & - \\
\hline$C_{\theta}$ & Annex D (EC) & 0.6 & - \\
\hline$\sigma_{\theta, R c}$ & Annex D (EC) & $\sigma_{\theta, R c}=E\left(\frac{t}{r}\right)^{2}\left[0.275+2.03\left(\frac{C_{\theta}}{\omega} \frac{r}{t}\right)^{4}\right]=21$ & $\mathrm{~N} / \mathrm{mm}^{2}$ \\
\hline $\bar{\lambda}_{\theta}$ & $(4.35)$ & $\bar{\lambda}_{\theta}=\sqrt{430 / 21}=4.47$ & - \\
\hline$\alpha_{\theta}$ & Annex D (EC) & 0.5 (Class $C)$ & - \\
\hline factors & Annex D (EC) & $\bar{\lambda}_{\theta 0}=0.40, \quad \beta=0.60, \quad \eta=1.00$ & - \\
\hline $\bar{\lambda}_{\theta p}$ & $(4.34)$ & $\bar{\lambda}_{\theta p}=\sqrt{0.5 /(1-0.6)}=1.12$ & - \\
\hline$\chi_{\theta}$ & $(4.33)$ & $\chi_{\theta}=0.5 / 4.47^{2}=0.025$ & - \\
\hline$\sigma_{\theta, R d}$ & $(4.29),(4.30)$ & $\sigma_{\theta, R d}=\frac{0.025 \cdot 430}{1.1}=10$ & $\mathrm{~N} / \mathrm{mm}^{2}$ \\
\hline
\end{tabular}

Similarly in Tab. 4.10 the parameters for the cylinder subjected to shear are calculated for a partial safety factor $\gamma_{M}$ of 1.1 .

Tab. 4.10: Parameters for limit state buckling design against shear

\begin{tabular}{|l|c|c|c|}
\hline parameter & $e q$. & shear & dimension \\
\hline & $(4.36)$ & $\begin{array}{c}10<\omega=185<8.7 r / t=452 \\
\text { medium length cylinder }\end{array}$ & - \\
\hline$C_{\tau}$ & Annex D (EC) & 1 & - \\
\hline$\tau_{R c}$ & Annex D (EC) & $\tau_{R c}=0.75 E \cdot C_{\tau} \cdot \frac{1}{\sqrt{\omega}} \frac{t}{r}=223$ & $\mathrm{~N} / \mathrm{mm}^{2}$ \\
\hline $\bar{\lambda}_{\tau}$ & $(4.35)$ & $\bar{\lambda}_{\tau}=\sqrt{430 /(223 \cdot \sqrt{3})}=1.06$ & - \\
\hline$\alpha_{\tau}$ & Annex D (EC) & $0.5($ Class C) & - \\
\hline factors & Annex D (EC) & $\bar{\lambda}_{\tau 0}=0.40, \quad \beta=0.60$, & $\eta$ \\
\hline $\bar{\lambda}_{\tau p}$ & $(4.34)$ & $\bar{\lambda}_{\tau p}=\sqrt{0.5 /(1-0.6)}=1.12$ & - \\
\hline & $(4.33)$ & $\chi_{\tau}=1-0.6\left(\frac{1.06-0.4}{1.12-0.4}\right)^{1}=0.45$ & $\mathrm{~N} / \mathrm{mm}^{2}$ \\
\hline$\chi_{\tau}$ & $(4.29),(4.30)$ & $\tau_{R d}=\frac{0.45 \cdot 430}{1.1 \cdot \sqrt{3}}=102$ & \\
\hline$\tau_{R d}$ & & & \\
\hline
\end{tabular}


With the calculated buckling resistances from Tab. 4.8 to Tab. 4.10 the buckling strength verification can be carried out:

Tab. 4.11: Buckling strength verifications

\begin{tabular}{|l|c|c|}
\hline position & anchor $(-6 \mathrm{~m})$ & max. moment $(-17.5 \mathrm{~m})$ \\
\hline axial compression & $\frac{\sigma_{x}}{\sigma_{x, R d}}=\frac{270}{289}=0.93<1$ & $\frac{\sigma_{x}}{\sigma_{x, R d}}=\frac{353}{289}=1.22>1$ \\
\hline $\begin{array}{l}\text { circumferential } \\
\text { compression }\end{array}$ & $\frac{\sigma_{\theta}}{\sigma_{\theta, R d}}=\frac{2}{10}=0.20<1$ & $\frac{\sigma_{\theta}}{\sigma_{\theta, R d}}=\frac{3}{10}=0.33<1$ \\
\hline shear & $\frac{\tau_{x \theta}}{\tau_{R d}}=\frac{80}{102}=0.78<1$ & 0 \\
\hline interaction & $\left(\frac{\sigma_{x}}{\sigma_{x, R d}}\right)^{1.25}+\left(\frac{\sigma_{\theta}}{\sigma_{\theta, R d}}\right)^{1.25}+\left(\frac{\tau_{x \theta}}{\tau_{R d}}\right)^{2} \leq 1$ \\
\hline verification & $0.91+0.13+0.61=1.65>1$ & $1.28+0.25+0=1.53>1$ \\
\hline
\end{tabular}

The buckling strength verifications cannot be fulfilled following this method. Another local buckling strength verification at the position of the maximum circumferential compression stresses is performed. Fig. 4.8 depicts the pressure distribution on the primary elements according to the definition from Fig. 4.7.

earth pressure

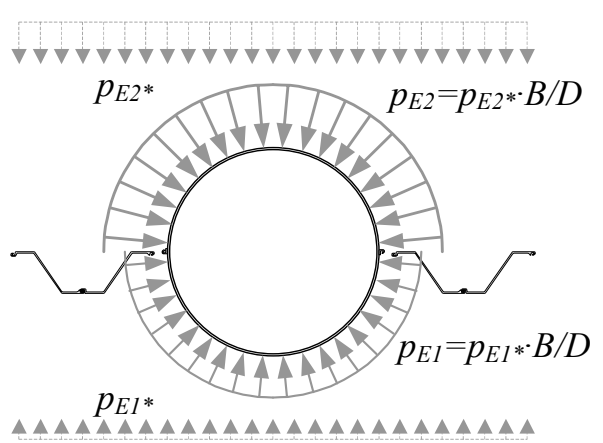

water pressure

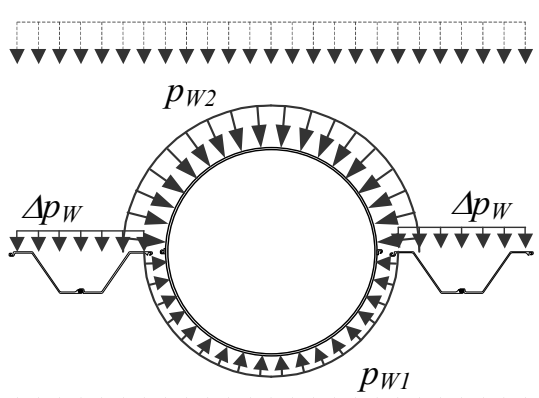

internal pressure

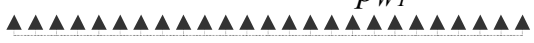

Fig. 4.7: Pressure distribution on combiwall from soil and water

The verification of the circumferential compression stresses is performed for a depth of $29 \mathrm{~m}$. In Tab. 4.12 the relevant pressures are summarized. The pressures are always defined as positive as depicted in Fig. 4.7. 
Tab. 4.12: Pressures at the depth $z=-29 \mathrm{~m}$ according to Fig. 4.7

\begin{tabular}{|l|c|c|}
\hline pressures & In front of tube (index 1) & behind tube (index 2) \\
\hline earth pressure $p_{E}\left[\mathrm{kN} / \mathrm{m}^{2}\right]$ & 733 & 203 \\
\hline water pressure $p_{W}\left[\mathrm{kN} / \mathrm{m}^{2}\right]$ & 210 & 260 \\
\hline total pressure $p_{E+W}\left[\mathrm{kN} / \mathrm{m}^{2}\right]$ & 943 & 463 \\
\hline averaged total pressure $p_{m}\left[\mathrm{kN} / \mathrm{m}^{2}\right]$ & \multicolumn{2}{|c|}{703} \\
\hline internal pressure $p_{\text {int }}\left[\mathrm{kN} / \mathrm{m}^{2}\right]$ & $160\left(\right.$ with $\left.K_{0}\right)$ \\
\hline
\end{tabular}

The internal pressure $p_{\text {int }}$ is calculated in this design with the earth pressure coefficient at rest $K_{0}$. This assumption does not include a possible plugging effect, and covers the stiffening influence of soil inside the tube as a conservative approach.

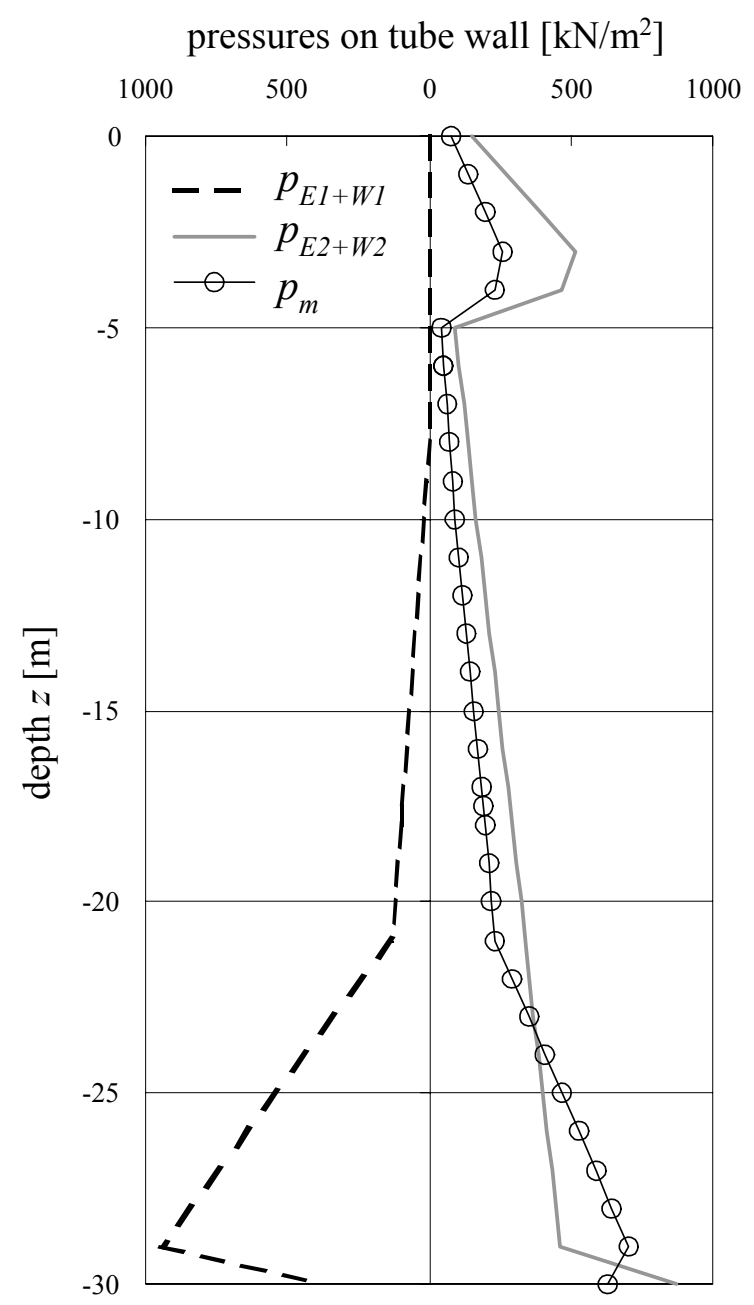

Fig. 4.8: Pressure distribution on tube as a function of the depth based on the conventions from Fig. 4.7

In Tab. 4.13 the relevant circumferential compression stresses are calculated and compared with the buckling resistances from Tab. 4.9. The comparison is based on a conservative estimation as the maximum circumferential compression stresses only appear at one position along the tube while the design buckling stress $\sigma_{\theta, R d}$ from 
ENV 1993-1-6:2002-05 is valid for a $24 \mathrm{~m}$ (pile tip to anchor) long cylinder subjected to a uniform pressure distribution. Again, the assumption of this reference length and the assumed boundary conditions are very conservative estimates.

Tab. 4.13: Circumferential stresses and verifications for the depth $z=-29 m$ with Tab. 4.12

\begin{tabular}{|l|c|}
\hline pressure & circumferential stress $\sigma_{\theta}\left[\mathrm{N} / \mathrm{mm}^{2}\right]$ \\
\hline with averaged total pressure $p_{m}$ & $\sigma_{\theta}=\frac{p_{m} \cdot r}{t}=\frac{703 \cdot 0.9}{18}=35$ \\
\hline $\begin{array}{l}\text { with averaged total pressure } p_{m} \\
\text { less internal pressure } p_{\text {int }}\end{array}$ & $\sigma_{\theta}=\frac{\left(p_{m}-p_{\text {int }}\right) \cdot r}{t}=\frac{(703-160) \cdot 0.9}{18}=27$ \\
\hline $\begin{array}{l}\text { from water pressure difference } \\
\Delta p_{W}=p_{W 2}-p_{W 1}\end{array}$ & $\sigma_{\theta}=\frac{\Delta p_{W} \cdot r}{t}=\frac{50 \cdot 0.9}{18}=3$ \\
\hline $\begin{array}{l}\text { with averaged earth pressure } \\
p_{m E}=\left(p_{E 1}+p_{E 2}\right) / 2\end{array}$ & $\sigma_{\theta}=\frac{p_{m E} \cdot r}{t}=\frac{0.5 \cdot(733+203) \cdot 0.9}{18}=23$ \\
\hline $\begin{array}{l}\text { averaged earth pressure } p_{m E} \text { less } \\
\text { internal pressure } p_{\text {int }}\end{array}$ & $\sigma_{\theta}=\frac{\left(p_{m E}-p_{i n t}\right) \cdot r}{t}=\frac{(468-160) \cdot 0.9}{18}=15$ \\
\hline $\begin{array}{l}\text { with max. total pressure } p_{E 1+W 1} \\
\text { less internal pressure } p_{\text {int }}\end{array}$ & $\sigma_{\theta}=\frac{\left(p_{E 1+W 1}-p_{\text {int }}\right) \cdot r}{t}=\frac{(943-160) \cdot 0.9}{18}=39$ \\
\hline design buckling stress $\sigma_{\theta, R d}$ & $\sigma_{\theta, R d}=10$ \\
\hline
\end{tabular}

It can be seen from Tab. 4.13 that even for the assumption of an averaged earth pressure $p_{m E}$, a maximum water pressure difference $\Delta p_{W}$ of $5 \mathrm{~m}$, and considering a internal pressure based on the earth pressure at rest with $K_{0}\left(15 \mathrm{~N} / \mathrm{mm}^{2}+3 \mathrm{~N} / \mathrm{mm}^{2}=18 \mathrm{~N} / \mathrm{mm}^{2}\right)$ the design buckling stress $\sigma_{\theta, R d}$ is exceeded $(18 / 10=1.80)$. For the other assumption the verification is even more unfavourable.

The implementation of this concept would lead to a very uneconomic design of the structure. The methods discussed in the previous sections lead to more slender structures and have been applied in the past successfully without any known collapses. Thus, it is the aim of this study to improve and to extend the existing methods in order to establish a more precise and adequate design procedure. This includes the more accurate consideration of the soil in the analyses.

\subsubsection{Concept according to prEN 1993-5:2004-07}

As a result of the difficulties shown, the concept in ENV 1993-5:2002 (Stage 32) was revised in prEN 1993-5:2004-07. In the latter version of the Eurocode (prEN 19935:2004-07) an adapted design was proposed considering the shell buckling problem of Class 4 cross-sections as used for combiwall constructions.

A new note states that the influence of shear buckling in areas of load introductions can be disregarded if theses areas are stiffened sufficiently by stiffeners or concrete fillings. 
As a consequence the shear component from the verification at the anchor $(\mathrm{z}=-6 \mathrm{~m})$ can be neglected. Moreover, the shear component does not have to be taken into account within the interaction verification. The factor $C_{x}$ is set equal to 1 without performing the calculation from ENV 1993-1-6:2002-05. According to ENV 1993-16:2002-05 the boundary conditions of case 3 in Table D-3 or D-4 from ENV 1993-16:2002-05 shall be used for circumferential compression stresses. This corresponds to $\mathrm{BC} 2-\mathrm{BC} 2$. Additionally, the tubes can be assumed to be of the fabrication tolerance quality class $\mathrm{B}$.

The main steps of the new design procedure are summarized in Tab. 4.14. The evaluation of the ovalization remains unchanged in prEN 1993-5:2004-07. Hence, the assessments are performed using the previously calculated radius of curvature $a$ $(=936 \mathrm{~mm})$ instead of the original radius $r$.

Tab. 4.14: New parameters for limit state buckling design against axial compression

\begin{tabular}{|l|c|c|c|}
\hline parameter & $e q$. & axial compression & dimension \\
\hline$C_{x}$ & $\begin{array}{c}\text { prEN 1993- } \\
5: 2004-07\end{array}$ & 1 & - \\
\hline$\sigma_{x, R c}$ & Annex D (EC) & $\sigma_{x, R c}=0.605 \cdot E \cdot C_{x} \cdot \frac{t}{r}=2443$ & $\mathrm{~N} / \mathrm{mm}^{2}$ \\
\hline $\bar{\lambda}_{x}$ & $(4.35)$ & $\bar{\lambda}_{x}=\sqrt{430 / 2443}=0.42$ & - \\
\hline$Q$ & Annex D (EC) & $25($ Klasse B) & - \\
\hline$\Delta w_{k}$ & Annex D (EC) & $\Delta w_{k}=\frac{1}{Q} \sqrt{\frac{r}{t} \cdot t=5.19}$ & - \\
\hline$\alpha_{x}$ & Annex D (EC) & $\alpha_{x}=\frac{0.62}{1+1.91 \cdot\left(\Delta w_{k} / t\right)^{1.44}}=0.470$ & - \\
\hline $\bar{\lambda}_{x p}$ & $(4.34)$ & $\bar{\lambda}_{x p}=\sqrt{0,470 /(1-0.6)}=1.08$ & $\mathrm{~N} / \mathrm{mm}^{2}$ \\
\hline$\chi_{x}$ & $(4.32)$ & $\chi_{x}=1-0.6\left(\frac{0.42-0.2}{1.08-0.2}\right)^{1}=0.85$ & \\
\hline$\sigma_{x, R d}$ & $(4.29),(4.30)$ & $\sigma_{x, R d}=\frac{0,85 \cdot 430}{1.1}=332$ & \\
\hline
\end{tabular}


Tab. 4.15: New parameters for limit state buckling design against circumferential compression

\begin{tabular}{|c|c|c|c|}
\hline parameter & $e q$. & circumferential compression & dimension \\
\hline$C_{\theta}$ & $\begin{array}{l}\text { prEN 1993- } \\
\text { 5:2004-07 and } \\
\text { Annex D (EC) }\end{array}$ & 1 & - \\
\hline$\sigma_{\theta, R c}$ & Annex D (EC) & $\sigma_{\theta, R c}=E\left(\frac{t}{r}\right)^{2}\left[0.275+2.03\left(\frac{C_{\theta}}{\omega} \frac{r}{t}\right)^{4}\right]=22$ & $\mathrm{~N} / \mathrm{mm}^{2}$ \\
\hline $\bar{\lambda}_{\theta}$ & $(4.35)$ & $\bar{\lambda}_{\theta}=\sqrt{430 / 22}=4.39$ & - \\
\hline$\alpha_{\theta}$ & Annex D (EC) & 0.65 (Class B) & - \\
\hline factors & Annex D (EC) & $\bar{\lambda}_{\theta 0}=0.40, \quad \beta=0.60, \quad \eta=1.00$ & - \\
\hline $\bar{\lambda}_{\theta p}$ & $(4.34)$ & $\bar{\lambda}_{\theta p}=\sqrt{0.65 /(1-0.6)}=1.27$ & - \\
\hline$\chi_{\theta}$ & $(4.33)$ & $\chi_{\theta}=0.65 / 4.39^{2}=0.034$ & - \\
\hline$\sigma_{\theta, R d}$ & $(4.29),(4.30)$ & $\sigma_{\theta, R d}=\frac{0.034 \cdot 430}{1.1}=13$ & $\mathrm{~N} / \mathrm{mm}^{2}$ \\
\hline
\end{tabular}

The new buckling strength verifications are calculated as:

Tab. 4.16: Buckling strength verifications

\begin{tabular}{|l|c|c|}
\hline position & anchor $(-6 \mathrm{~m})$ & max. moment $(-17.5 \mathrm{~m})$ \\
\hline axial compression & $\frac{\sigma_{x}}{\sigma_{x, R d}}=\frac{270}{332}=0.81<1$ & $\frac{\sigma_{x}}{\sigma_{x, R d}}=\frac{353}{332}=1.06>1$ \\
\hline $\begin{array}{l}\text { circumferential } \\
\text { compression }\end{array}$ & $\frac{\sigma_{\theta}}{\sigma_{\theta, R d}}=\frac{2}{13}=0.15<1$ & $\frac{\sigma_{\theta}}{\sigma_{\theta, R d}}=\frac{3}{13}=0.23<1$ \\
\hline interaction & $\left(\frac{\sigma_{x}}{\sigma_{x, R d}}\right)^{1.25}+\left(\frac{\sigma_{\theta}}{\sigma_{\theta, R d}}\right)^{1.25} \leq 1$ \\
\hline verification & $0.77+0.09=0.86<1$ & $1.07+0.16=1.23>1$ \\
\hline
\end{tabular}

It can be seen from Tab. 4.16 that the verification is fulfilled for the position of the anchor for the new calculation. Still, the verification is not fulfilled for the position of the maximum moment. Again the buckling verification cannot be made for the investigated case. In prEN 1993-5:2004-07 it is noted that non-uniform pressure distributions shall be approximated by uniform pressure distributions based on the maximum existing pressure. This assumption again makes the design in the previous section much worse. 
The verification for the secondary loads introduced via locks from the sheetings is changed in prEN 1993-5:2004-07. This modification shall not be investigated further at this point. 


\section{Discussion of the design procedures}

\subsection{General}

The application of the standards of shell buckling on the problem of combiwalls as performed in the previous section revealed several inadequacies in the assessment. The design rules have been kept so general that they may result in extremely conservative constructions, since several special influences are not taken into account.

In the following aspects of the design procedure for tubular piles are presented and conservative assumptions in the assessment of the investigated problem are pointed out. In the next step these conservative assumptions are discussed in order to provide suggestions and comments for enhancing design rules for tubular piles regarding shell buckling. These extracted problems are investigated separately in the following chapters. Arising from the previous assessment, the studies are based on several aspects governing the carrying behaviour of the investigated structure with respect to shell buckling and the global non-linear load displacement characteristic:

- simplifying the model: comparison between combiwall and single pile,

- study of the modelling of the soil and the soil-structure interaction,

- geometrical imperfections, i.e. not all shape imperfections may be relevant for the tubular piles,

- the influence of the surrounding soil on the buckling behaviour and load,

- global load transfer behaviour of a tubular pile,

- the choice of boundary conditions and loads to properly represent the real conditions (external pressure).

The aim is to provide a contribution to improved assessment rules for the given case based on the different aspects influencing the carrying behaviour in a different way.

\subsection{Comparison between combiwall and single pile}

In this first chapter a general comparison is made between a combiwall and a single pile regarding the load transfer mechanisms. It is the aim to relate the complex combiwall problem to the problem of a single pile in order use this simplified model for further studies. 


\subsection{Modelling of the soil}

This chapter deals with the modelling of the soil since its behaviour needs to the defined for the subsequent chapters. The complex problem of modelling soil is tackled for the investigated case providing a concept to be used for simple and more detailed numerical analyses.

\subsection{Geometrical imperfections}

The aspect of geometrical imperfections is investigated with the aim to determine the influence of radial shape deformations and uneven supports on the buckling load of cylinders. For this study the special problem of combiwalls is set aside and the problem is studied for the general case of a loaded cylindrical shell. The intention is to obtain a better founded knowledge of the buckling load reductions arising from uneven supports, as it is assumed that this aspect does not play a decisive role for combiwalls due to the surrounding soil.

\subsection{Influence of soil on buckling behaviour}

As seen in the design procedure the applied design codes do not provide any possibility to take a stabilizing influence of the surrounding soil on the buckling load into account. In order to evaluate the influence of the soil a set of numerical studies is performed investigating the buckling behaviour of a cylinder embedded in sand and subjected to axial loading or external pressure. The investigation is based on the soil models defined in the chapter dealing with the modelling of the soil.

\subsection{Global load transfer behaviour}

As seen in the design procedure, the understanding of the load transfer behaviour of the entire system is important. Thus, the load transfer from the tubular piles into the soil is investigated more in detail. Here it is emphasized on studying the stresses and deformations arising in the piles with respect to continuing experimental tests and the uniformness of the stress distribution. The latter case is of importance, as a non-uniform stress distribution may detrimentally influence the buckling behaviour of a shell.

\subsection{Boundary conditions and loads - external pressure}

The design procedure highlights several conservative assumptions which have to be made for assessing of the structure against the different stress components. These aspects influence especially the limit state buckling design against circumferential compression. Hence, for the case of external pressure the aspects of non-uniform loading, boundary conditions and buckling behaviour are investigated in detail. 


\section{Comparison between combiwall and single pile}

\subsection{Overview}

Since the construction of a combiwall is fairly complicated including primary elements (e.g. tubular piles) and sheetings as secondary elements between the piles connected with locks, it is intended to simplify the system to the problem of a loaded single pile for investigation. Based on a model more versatile and easier to handle, several studies can be performed allowing to obtain important information. Subsequently, the findings can be transferred back to the problem of the combiwall structures and extended by more construction-specific analyses. Here, it is indispensable that the results from the chosen system can be used for the current study and vice versa. This chapter deals with a simple comparison of load transfer mechanisms and thus stress distributions arising in combiwall structures and single piles by numerical analyses. In this context the external pressure distribution on the tubular pile of a combiwall after excavation is compared with the external pressure distribution on a laterally loaded pile. Here, it is emphasized that only the shape of the pressure distributions is investigated and compared. Due to the fact that only plane strain models are used, the real spatial load transfer mechanisms cannot be captured. Thus, the chosen load magnitudes for the two cases do not coincide as they are chosen based on different approaches. The essential question to be discussed is whether a similar pressure distribution evolves for both cases if subjected to their specific loading.

The idea of this comparison was initiated by first studies on combiwalls performed at the University of Louvain (UCL) and described in Holeyman et al. (2007). The presented results in this chapter are based on a close co-operation with these two researchers.

\subsection{Analyses and comparisons}

First, a plane strain model of a combiwall is investigated representing a horizontal cross section trough the construction. The geometry is chosen according to the reference case used in common practical design calculations (Fig. 6.1). 


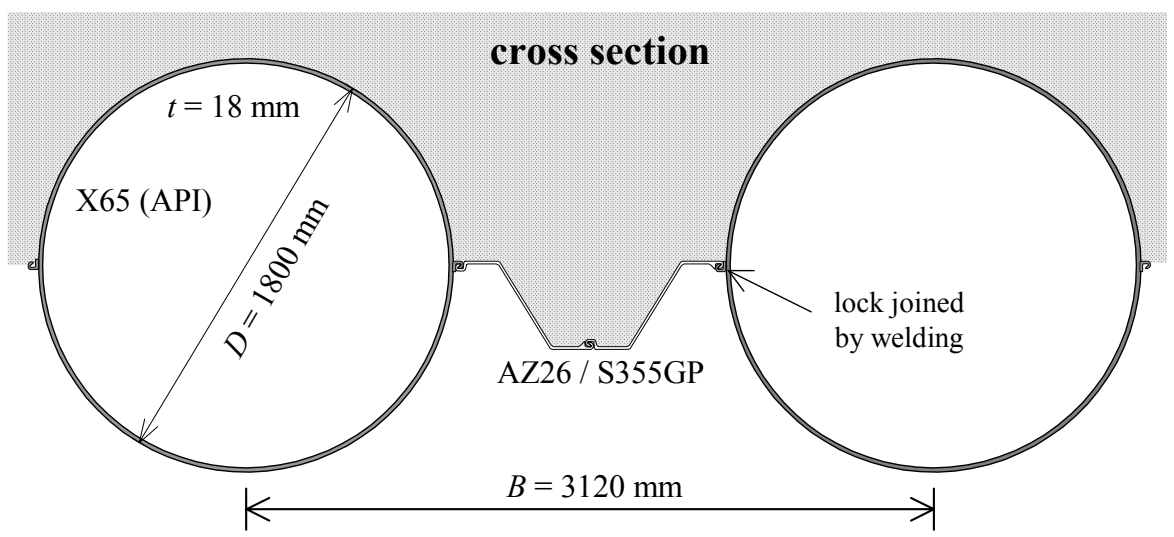

Fig. 6.1: Cross-section of the combiwall system

The investigation is based on the assumption that the sheetings serve to transfer the earth pressure acting on them to the connected tubular piles by membrane stresses only. Thus, the sheeting is substituted by a truss with no bending stiffness in the numerical model. Although the connection is executed by a lock joining the components together, the numerical model uses a hinge at this position. In order to obtain the equivalent axial rigidity or in-plane stiffness $E A$ of the sheeting a two-dimensional structural analysis program is used. The calculation according to Fig. 6.2 results in an equivalent in-plane stiffness $E A$ of $371 \mathrm{~N} / \mathrm{mm}$ or $371 \mathrm{kN} / \mathrm{m}$.

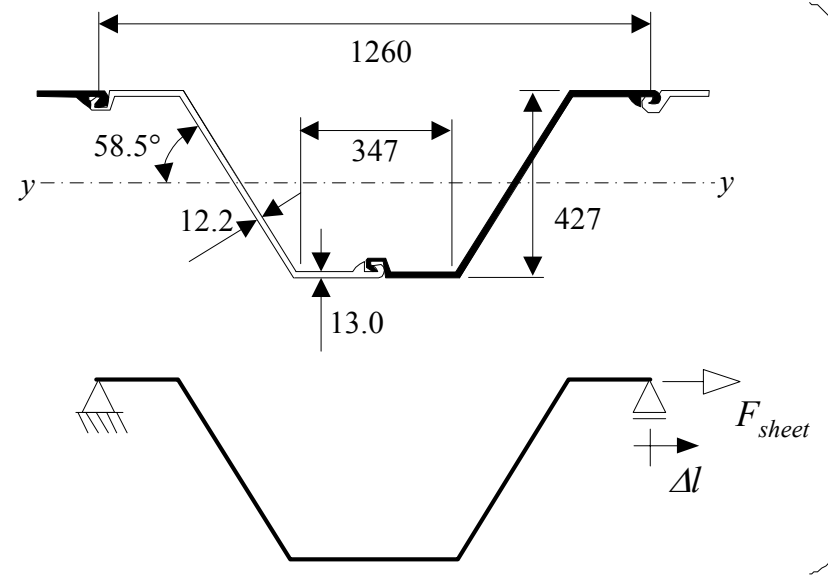

$$
\begin{aligned}
& \text { with } \\
& E=210000 \mathrm{~N} / \mathrm{mm}^{2}
\end{aligned}
$$

equivalent in-plane stiffness

$$
\begin{aligned}
& E A=F_{\text {sheet }} l / \Delta l \\
& \text { ओी } l=1320
\end{aligned}
$$

$[\mathrm{mm}]$

Fig. 6.2: Model for calculating the equivalent in-plane stiffness

Fig. 6.3 depicts the model used for the analysis of the combiwall. By applying symmetry conditions along the edges and at the tubular pile and the sheeting, the combiwall can be reduced to the depicted model. Neither the sheeting nor the tube are restrained in the direction $y$. The extent of the model (soil 1) perpendicular to the combiwall is chosen sufficiently large (total length $3.9 \mathrm{~m}$ ) to prevent a noticeable influence of the applied boundary conditions. 

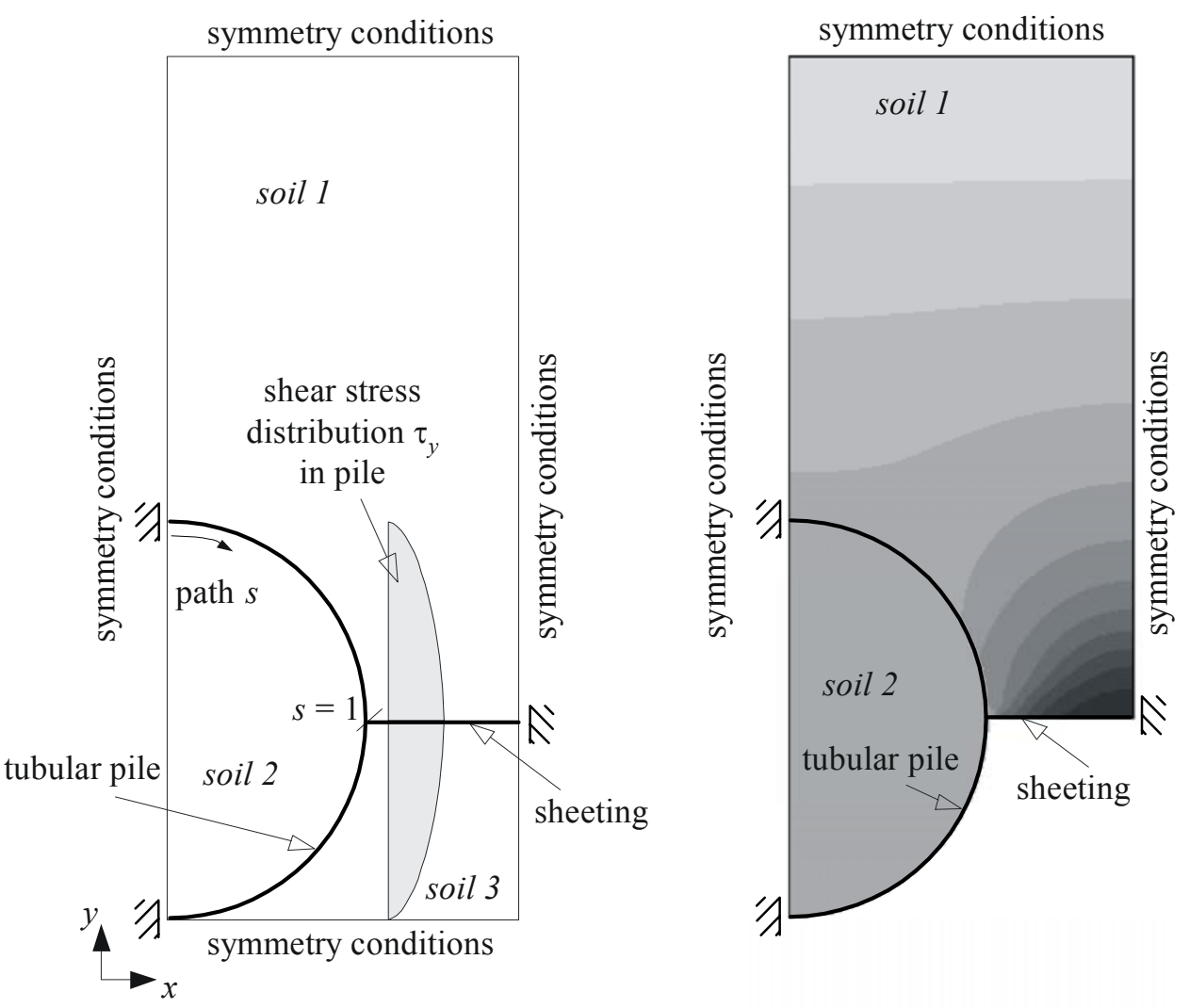

Fig. 6.3: Left: model for calculating the equivalent in-plane stiffness (plane strain). Right: displacement contour plot for hypoplasticity where dark shaded areas indicate large displacements

The initial stress conditions in the soil evolving from self-weight are simulated by imposing in-plane stress conditions of $\sigma_{1}=\sigma_{2}=-100 \mathrm{kPa}$ and out-of-plane stress conditions of $\sigma_{3}=-200 \mathrm{kPa}$ as first assumption. These parameters reflect the geostatic stress conditions for an undisturbed soil with a density of $16 \mathrm{kN} / \mathrm{m}^{3}$ corresponding to poorly graded sand at a depth of $12.5 \mathrm{~m}$ with an earth pressure coefficient at rest of $K_{0}=0.5$. For the tube and the sheeting made of steel a Young's modulus $E=210000 \mathrm{~N} / \mathrm{mm}^{2}$ and a Poisson ratio $\mu=0.3$ is assumed. For the contact surfaces between soil and sheeting/tubular pile an interface friction coefficient $\tan \delta=0.3$ is introduced by working with a Coulomb friction law. A hard contact formulation allowing the surfaces to separate is applied. The sand is modelled by using two different soil models: linear elasticity with Mohr-Coulomb plasticity, and hypoplasticity. The parameters of the soil models used in this first comparison are taken from chapter 7 , in which a set of consistent soil data is derived. No absolute values are necessary at this stage.

In the first step of the analysis, only the initial geostatic stress state is imposed on the soil (soil 1 to soil 3). In the following step the excavation is simulated by removing the soil (elements) in front of the combiwall (soil 3). During the removal step the forces of soil 3 on the combiwall are gradually ramped down to zero. Simultaneously, in order to establish equilibrium, reaction forces are required. For a thin walled circular cross section the shear stress distribution $\tau$ can be assumed to be constant and uniform. Thus, 
the component of the shear stresses $\tau_{y}$ acting in the direction $y$ according to Fig. 6.3 generating a state of equilibrium with the geostatic stress state can easily be calculated. The distribution of the shear stresses $\tau_{y}$ is depicted in Fig. 6.3. The function of the shear flow $T_{y}$ in the direction $y$ can be defined as $T_{y}=\tau_{y} \cdot t$ with the wall thickness $t$ of the tubular pile. The integration of these stresses over the path $s$ (Fig. 6.3) results in the correlating shear force $H_{c o m b i}$. All values depend on the unit thickness of the model. In the analysis, first the shear force $H_{c o m b i}$ is chosen, and then the corresponding shear flow $T_{y}$ is calculated. The shear flow $T_{y}$ is applied as a line load per unit thickness along the circumference of the tubular pile. The magnitude of $H_{c o m b i}$ cannot be identified easily because the spatial stress state is not taken into account. Since, in this step only the shape of the pressure distribution is to be compared, the shear force $H_{c o m b i}$ is calculated by deriving the gradient $\left(113 \mathrm{kN} / \mathrm{m}^{2} / 15 \mathrm{~m}=7.5 \mathrm{kN} / \mathrm{m}^{3}\right)$ of the resultant pressure $\left(p_{\text {res }}=\right.$ $\left.p_{E 1+W 1}-p_{E 1+W 1}\right)$ for the section $-5 \mathrm{~m}<z<-20 \mathrm{~m}$ from Fig. 4.8 as an approximation. By relating the gradient to the system width $B$ (Fig. 6.1) and a segment of $1 \mathrm{~m}$, the shear force per unit thickness is calculated to be $H_{c o m b i}=23.4 \mathrm{kN} / \mathrm{m}$. A displacement contour plot for the hypoplastic model is depicted in Fig. 6.3.

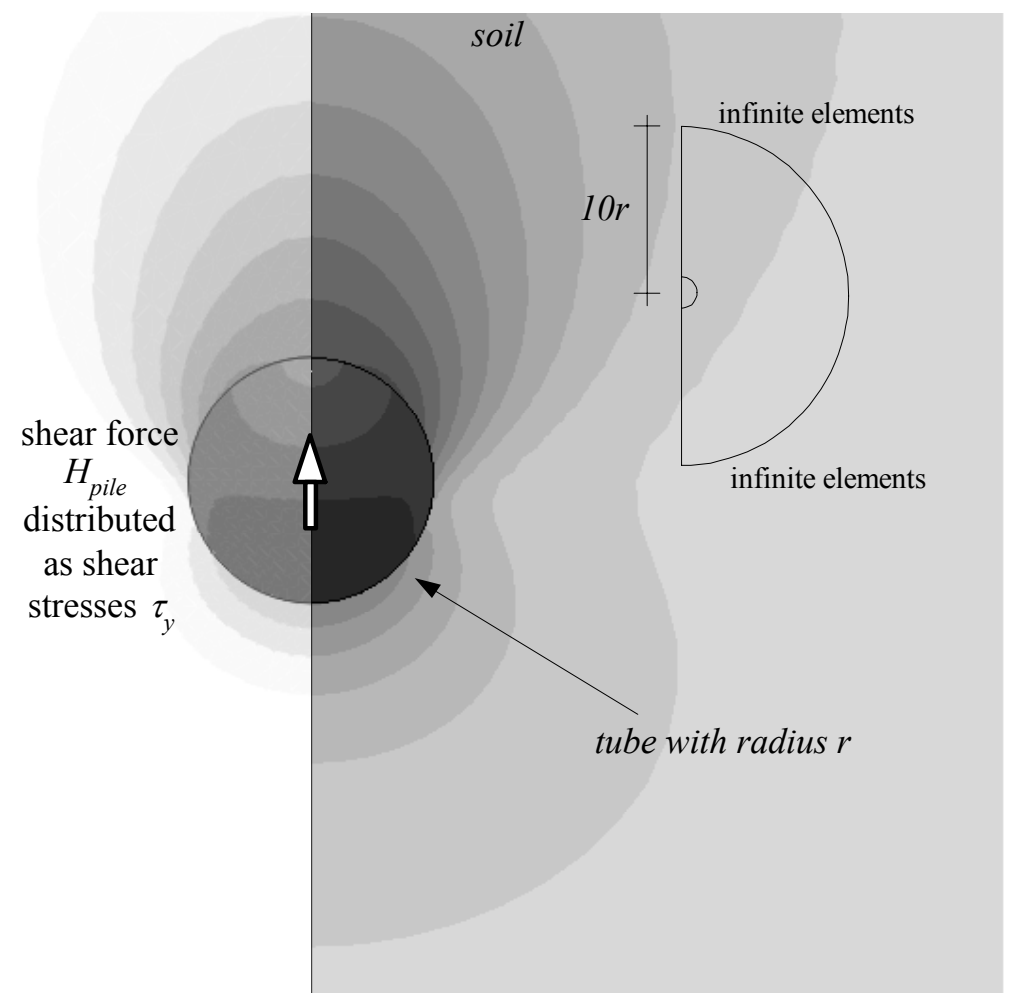

Fig. 6.4: Two-dimensional plane strain model for laterally loaded pile; displacement contour plot for hypoplasticity where dark shaded areas indicate large displacements

In the next step similarly to the combiwall model, a two-dimensional plane strain model is built for analysing a laterally loaded pile (Fig. 6.4). The model consists of a tubular pile installed in an undisturbed circular region of soil. The outer boundaries are modelled with infinite elements in order to reduce the boundary effect. Again, the geostatic stress state is $\sigma_{1}=\sigma_{2}=0.5 \sigma_{3}=-100 \mathrm{kPa}$ and the soil and contact properties 
applied follow the previous model. In the loading step the tube section is loaded inplane with a transverse or shear force $H_{\text {pile }}=1000 \mathrm{kN} / \mathrm{m}$. As a first approach, the load is chosen in the order of magnitude according to the shear force distribution of the calculated reference case from Fig. 4.3. The shear force $H_{\text {pile }}$ is not to be compared to the shear force $H_{c o m b i}$ directly as two different loading systems are considered. For loading the shear force $H$ is decomposed into shear stresses $\tau_{y}$ as described for the combiwall case. The chosen magnitude of the shear force $H_{\text {pile }}$ is not too important as in the following a variation of parameters will be discussed.

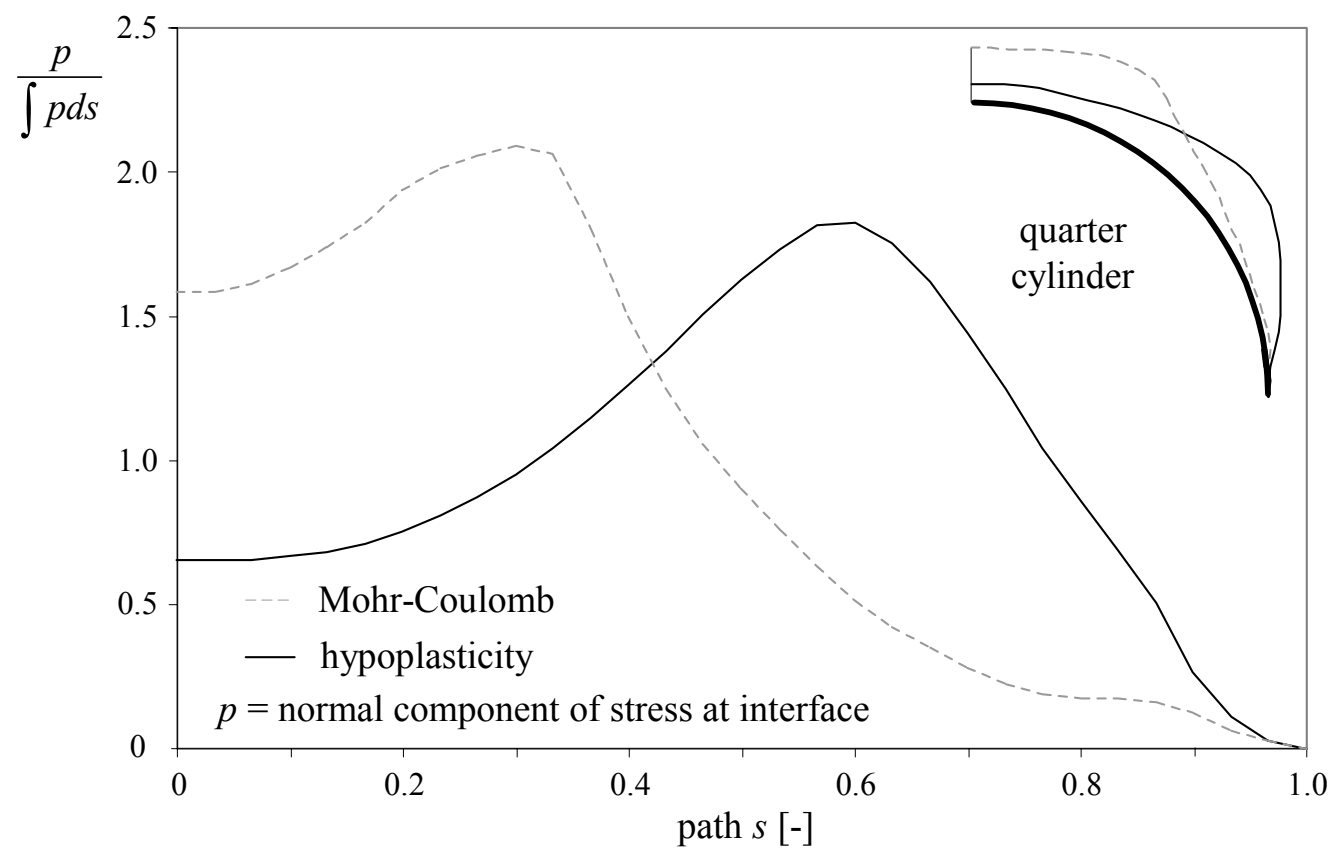

Fig. 6.5: Normalised pressure distributions for combiwall with $\sigma_{1}=\sigma_{2}=0.5 \sigma_{3}=-100 \mathrm{kPa}$

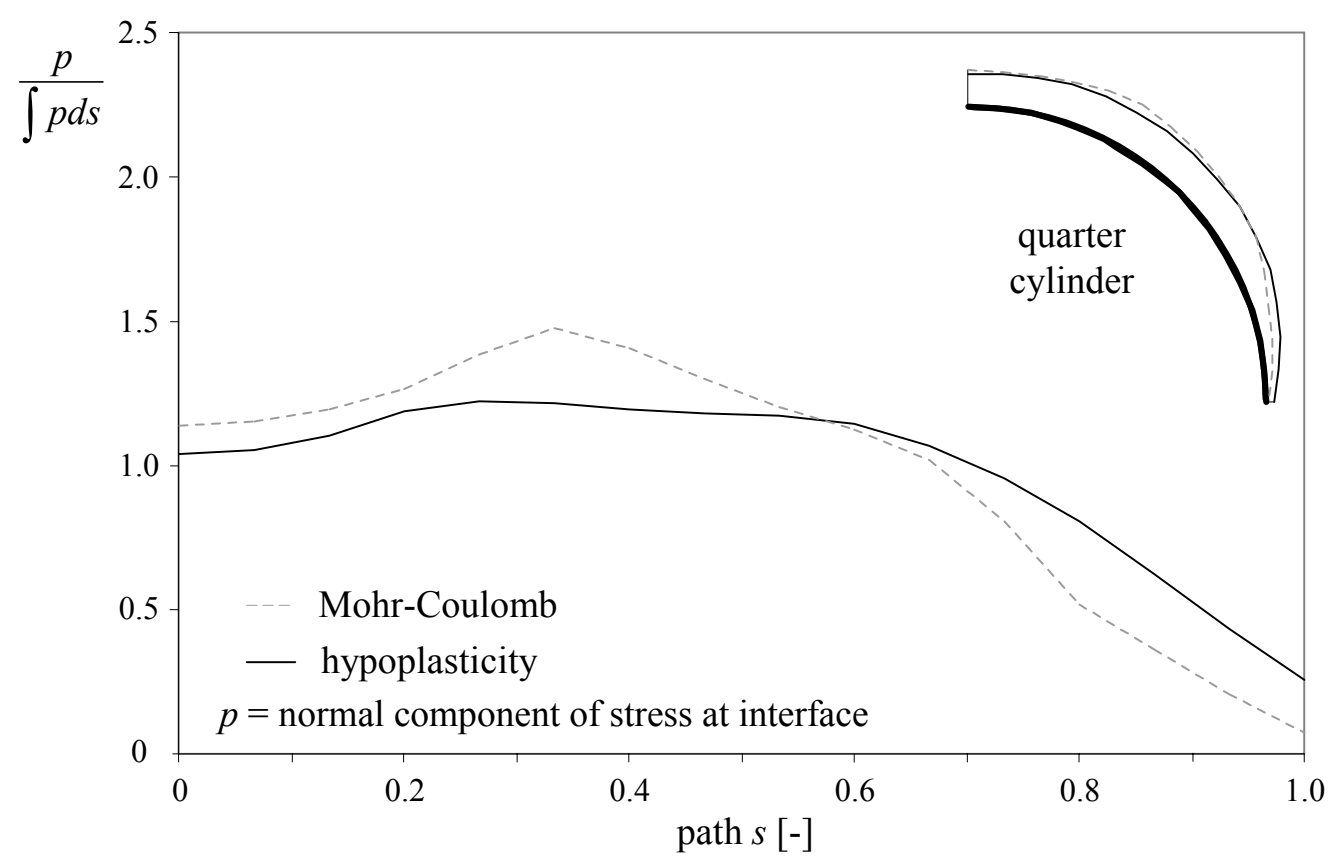

Fig. 6.6: Normalised pressure distributions for laterally loaded pile with $\sigma_{1}=\sigma_{2}=0.5 \sigma_{3}=-100 \mathrm{kPa}$ 
Fig. 6.5 and Fig. 6.6 show the normalised pressure distributions along the path $s$ as depicted in Fig. 6.3 for the combiwall. Additionally, in the upper right corner of both diagrams the normalised pressure distributions are plotted with respect to a quarter cylinder. The path $s$ is defined accordingly for the laterally loaded pile. Here, the straight line spanning from the centre of the tubular pile to the point $s=0$ coincides with the loading direction. In all cases the initially constant pressure distribution over the circumference of the tubular piles changes to a non-linear pressure distribution with decreasing pressure values for $s$ tending towards 1 . Further, a pressure peak can be identified in all analyses in the region $0.2<s<0.6$. For both systems, combiwall and laterally loaded pile, the pressure distributions obtained for the Mohr-Coulomb model exhibit larger gradients and higher maxima along the path $s$. In all analyses yielding of the soil occurred near the tubular pile. The arising forces in the locks are very small: the axial force $F_{\text {sheet }}$ in the sheeting (Fig. 6.2) reaches values about $0.1 H_{\text {combi }}$ for the calculated cases, and the component perpendicular to the latter force is negligible small $(\approx 0)$.

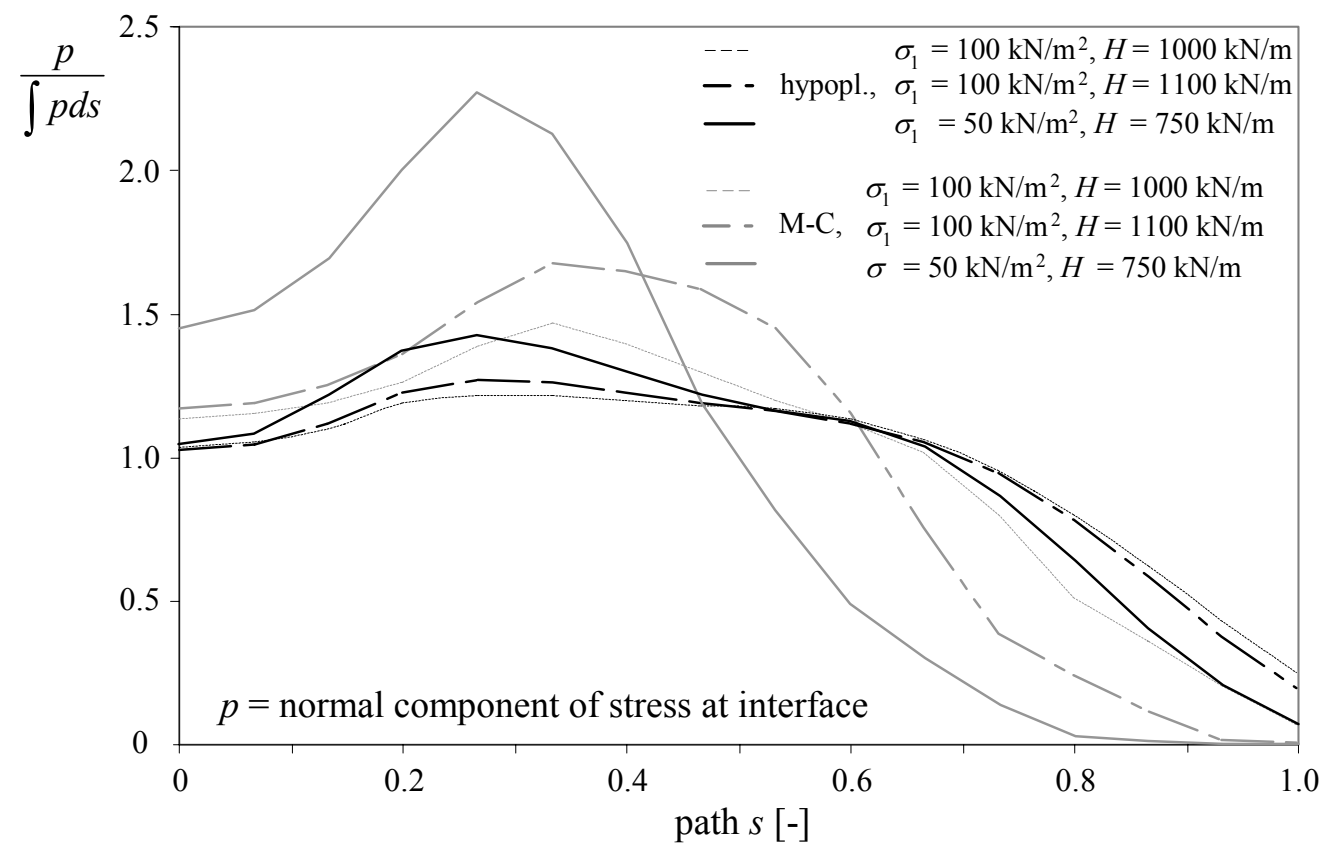

Fig. 6.7: Normalised pressure distributions for laterally loaded pile with varying parameters

Still, the non-linear behaviour is much more distinct in Fig. 6.5 in comparison to Fig. 6.6. Thus, several additional numerical studies were performed with changing parameters in order to investigate whether the described findings can be assumed to be generally valid, and whether the results of the two cases exhibit better agreement for different loadings and initial stress states. In the following the corresponding parameters are briefly discussed. The imposed friction coefficient of $\tan \delta=0.3$ was varied between 0 and 0.5 . The parametric study showed that for $\tan \delta>0.3$ the pressure maximum moves towards $s=1$, since the influence of circumferential shear stresses along the tubular pile increases. For very small friction coefficients, the pressure maximum appears close to the position $s=1$, too. This occurs due to the load introduction with a 
shear stress distribution as given in Fig. 6.3 in which large stresses are concentrated around $s=1$. In the investigated range, analyses using the Mohr-Coulomb model and $\tan \delta=0.28$ yielded the distributions with a maximum closest to $s=0$. The imposed initial geostatic stress state is based on a rough first assumption. Numerical analyses for both cases and for different initial stresses were performed. In case of the combiwall the geostatic stress state obviously influences the absolute values of the pressure distribution. Nevertheless, the normalised pressure distribution remains nearly unchanged. Two parameters have noticeable influences on the shape of the pressure distribution obtained from the analyses of the laterally loaded pile as shown in Fig. 6.7: the initial stress state and the applied in-plane load $H_{\text {pile }}$. Larger initial stresses and similarly a smaller in-plane load result in more uniform pressure distributions, i.e. more constant pressure distributions. For small initial stresses and/or large in-plane loads yielding prevails leading to very large deformations and failure of the soil. Generally, the pressure distribution is more sensitive to changes for the Mohr-Coulomb model in comparison to the hypoplastic model. Based on the parametric study it can be summarized that the general behaviour as depicted in Fig. 6.5 and Fig. 6.6 remains, if the parameters are varied as described above. Here, no specific ranges are given, as different combinations of initial stress state and applied load may result in a failure of the soil. Only the general shape of the pressure distributions are compared for proving the connection between both problems, since the derivation of consistent input parameters for the investigated plane strain models cannot be performed. Still, very similar pressure distributions can be found for the case using the Mohr-Coulomb model from Fig. 6.5 and the Mohr-Coulomb model with $H_{\text {pile }}=750 \mathrm{kN} / \mathrm{m}$ from Fig. 6.7 .

\subsection{Conclusions}

The first important result found in the comparing study is the fact that the external pressure distribution evolving due to the excavation process differs from segment-wise constant pressure distribution estimated in the reference case calculation (chapter 4). Depending on the implemented soil model and the boundary and initial conditions, the appearing pressure distributions may be highly non-linear.

The non-linearity of the loading of tubular piles used in combiwalls takes place because of an arching effect evolves in the soil. The results are based on the assumption that nearly cohesionless sand $\left(c=0.2 \mathrm{kN} / \mathrm{m}^{2}\right)$ is located around the combiwall. Fig. 6.8 depicts the principal stresses near the combiwall after the excavation process.

It can be seen that the area in contact with the sheeting is nearly stress free. At the position $s=0.6$ large principal stresses appear perpendicular to the surface of the tubular pile. The stresses can be identified in Fig. 6.5. The sand between the tubular piles forms an arch with supports on the tubular piles. This arching effect transfers the load arising from relieving the geostatic stress state by means of compressive stresses to the primary elements. The general behaviour of the load transfer mechanism for the model using Mohr-Coulomb is similar except for a slightly different shape of the arch. 
This finding validates the assumption made for the earth pressure that the intermediate sheetings mainly transfer axial loads and only very small shear or transverse forces and that the pressure may be assumed to act only on the tubular piles.
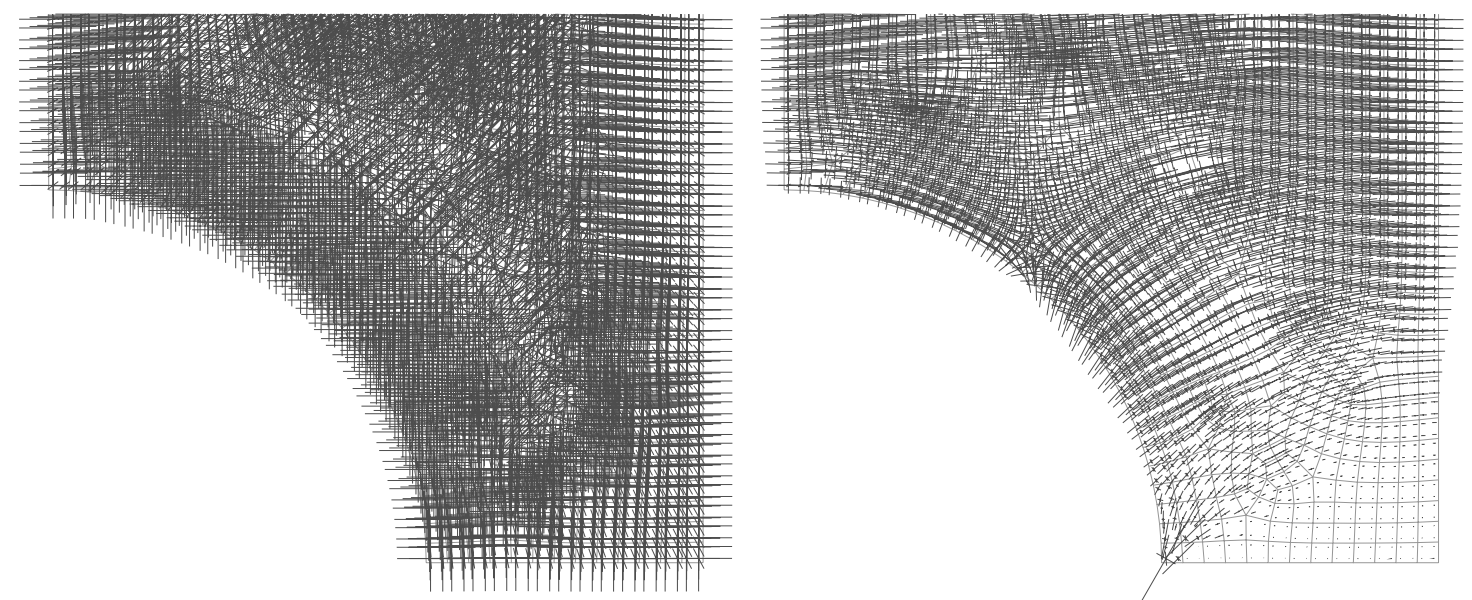

Fig. 6.8: Principal stresses in soil 1 before (left) and after (after) the excavation process for hypoplastic soil model

For the performed analyses the ovalization effect occurring for the tubular pile is slightly different. In the case of the combiwall the tensile forces $F_{\text {sheet }}$ transferred via the sheeting into the tubular pile result in an ovalization of the structure. Nevertheless, as seen in Fig. 6.8 an arching effect with compressive stresses takes place between the primary elements. This aspect counteracts the tensile forces from the sheetings resulting in a reduced ovalization effect for the investigated cases. Here, only small changes of radii were found for the unloaded and loaded tubular pile $(<1 \%)$. For the laterally loaded pile the ovalization $(<10 \%)$ is much more pronounced as the loads are significantly larger. In this context the applied friction coefficient influences the ovalization directly, as it defines the location of the pressure maximum. A pressure concentration (maximum) close to $s=0$ leads to an arching effect rather increasing the ovalization instead of preventing it. Nevertheless, in this comparison the ovalization due to bending (Brazier, 1927) is not taken into account as the spatial load transfer mechanisms need to be considered.

The presented results (Fig. 6.5 to Fig. 6.7) from the two different systems coincide fairly well and lead to the conclusion that the problem of the combiwall can be analysed by using the system of a laterally loaded pile. This system shall be used in numerical and experimental studies providing information regarding stress distributions and load transfer mechanisms. The obtained findings can then be re-transferred back to the problem of the combiwalls. 


\section{Investigation of soil and soil-structure interaction}

\subsection{General}

The behaviour of soil can be modelled using different methods. In constructional engineering often the simplified description of soil with springs is applied. This type of modelling may be sufficient for various problems, but is only able to reflect a small part of soil behaviour. Thus, in geotechnical engineering more complex models have been developed representing the soil as a continuum. In practical use such elaborate methods have not been used much in the analyses and assessment of the soil-structure interaction (SSI).

The description of soil in its simplest form with springs evolved historically due to the possibility of the calculating tools. Meanwhile, powerful computers allow to combine and analyse complex soil behaviour with non-linear reactions of structures. In this section several approaches are introduced more in detail regarding their possibilities and efficiency. The aspect of modelling the soil with springs is highlighted more in detail as it is a widely applied concept. In this context, especially attention is paid to the horizontal stiffness of soil loaded by a circular structure (vertical pile) as it is directly related to the problem of the combiwalls.

In the second step three different types of analyses are performed. First, a block of soil is loaded with a plate (settlement problem) for illustrating the general differences between the models. In the next investigation a settlement problem is analysed based on experiments by Schlegel (1985). Here, the material parameters are taken from previous investigations. The last and most complex comparison deals with a horizontally loaded pile embedded in sand based on experiments (Holeyman et al., 2006). For this case a set of consistent soil data is derived. Although there is no single solution for modelling the soil and the soil-structure interaction for all problems, it is the aim to choose a method for further investigations. Still, the choice of a convenient modelling technique may remain problem dependent. In this context the procedure is divided into two steps: First, a reference analysis of the laterally loaded pile with about $70 \%$ of the failure load is performed involving different material models. The results provide the basis for refining the numerical and experimental concepts. In the second step, the refined model is analysed for a load region far smaller than in the first step (about $10 \%$ of the ultimate load). Here, the strains should be small (elastic) and recoverable. This comparison then is used for discussing the material models. 


\subsection{Soil models}

\subsubsection{General Winkler foundation}

The first and the simplest concept for describing soil in the field of SSI is the mechanical model with one parameter. This initially purely mathematical method is nowadays known as the Winkler hypothesis (Winkler, 1876). In this approach the soil is modelled using one-dimensional, discrete, and uncoupled springs whose displacements $w$ at each point only depend on the applied load $p$ and the spring stiffness $k_{S}$.

$$
p(x, y)=k_{S}(x, y) \cdot w(x, y)
$$

The one-parameter model (7.1) which can be modelled using springs is mostly applied with a linear elastic formulation, and acts perpendicular to the surface of action. For real structures local deformations directly influence the deformation behaviour of the surrounding soil. As for the discrete, uncoupled springs only the portion of soil directly loaded is activated for transferring the forces and displacements, no realistic settlement will form. For the design it is intended to specify constant and problem related values for the spring stiffness $k_{S}$, although the spring stiffness is not a soil parameter. As a consequence there is a lot of data to be found in literature regarding the spring stiffness based on empirical and numerical approaches. The problem in this field of research is the fact that geotechnically seen the model is incorrect, and therefore the retrieval of adequate values for the spring stiffness $k_{S}$ does not make any sense. The deficiencies of the Winkler hypothesis for the assessment of settlements as already demonstrated by e.g. Terzaghi (1955), were also indicated in more recent studies by Horvath (1993) and Liao (1995). The Winkler concept was amongst others formulated as a pseudo-coupled system (Bowles, 1988). This method again does not explicitly couple the vertical springs for transferring the shear forces, but simulates this behaviour by using springs with different stiffness $k_{S}(x, y)$.

\subsubsection{Coefficient of subgrade reaction}

\subsubsection{Definition}

Many more detailed concepts are based on springs but more evolved by e.g. introducing non-linear load-displacement relations. Thus, in the following the concept is called subgrade reaction method with the coefficient of subgrade reaction $k_{S}$ which shall include foundations similar to the linear Winkler foundation. The most important factor while applying the method using springs is the definition of the magnitude of the coefficient of subgrade reaction $k_{S}$. Concerning the value of the coefficient of subgrade reaction extensive studies have been performed in the last century. Within this study two questions are of major interest which are discussed in the following: what is the magnitude of the coefficient of subgrade reaction to be used in analyses and how do we take into account that e.g. a pile embedded in soil interacts spatially with the surrounding soil? 


\subsubsection{Definition of the Young's modulus of soil}

In the field of geotechnical engineering an elastic material is described with the Young's modulus of elasticity which can be determined for example from oedometer consolidation tests as tangent or secant modulus. Since, for soil the deformations are no linear function of the stresses (Fig. 7.1) this approach is only valid to a certain extent. The stiffness depends on the range of stress and on the history of loading and therefore may vary significantly as the case arises. For better representation the compressibility index $C_{c}$ is used sometimes which corresponds to the reciprocal value of the relatively constant gradient of the compression curve in a half-logarithmic compression diagram under initial loading.

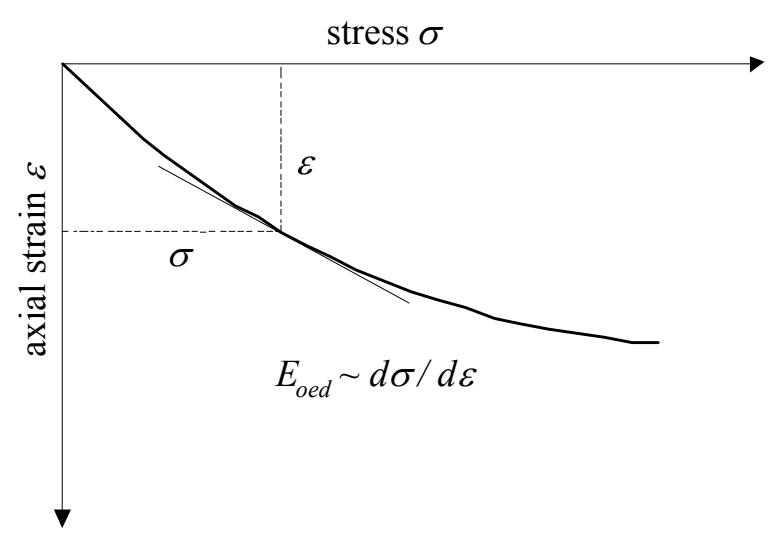

Fig. 7.1: Evaluation of the oedometer modulus $E_{\text {oed }}$ from oedometer consolidation test (tangent modulus)

In a first very rough approximation a linear dependence between stresses and strains can be assumed for non-cohesive soils. Often, the inaccuracies from determining the stiffness are larger than those arising from the linearisation. This simplified approach allows to perform analyses by applying a constant elastic modulus or compressibility index. It is very difficult to estimate the stiffness for an overconsolidated soil with a loading history which can easily reach a multiple of the value obtained for initial loading under the same vertical stresses.

The differences between the material parameters $E, E_{s}, E^{50}$, and $E_{\text {oed }}$ which are all expressed in $f / l^{2}$ [forcellength $\left.{ }^{2}\right]$ are highlighted in the following. In this work the Young's modulus for soil used for general continuum analyses and investigations is defined as $E$.

The so-called soil modulus $E_{s}$ is used for 1-D considerations involving piles. It can be interpreted as the ratio between the lateral soil reaction $p[f / l]$ and the deflection of the pile $y[l]$ and may be formulated as function of the depth $z$. According to Terzaghi's recommendations (1955) it can be written as

$$
E_{s}=k_{S} \cdot D
$$

where $D$ is the diameter of the pile. In the following mainly the coefficient of subgrade reaction $k_{S}$ is used instead of the soil modulus $E_{s}$. 
The Young's modulus $E^{50}$ is defined as the secant gradient at $50 \%$ of the maximum deviatoric stress from triaxial tests (deviatoric loading) as shown in Fig. 7.2.

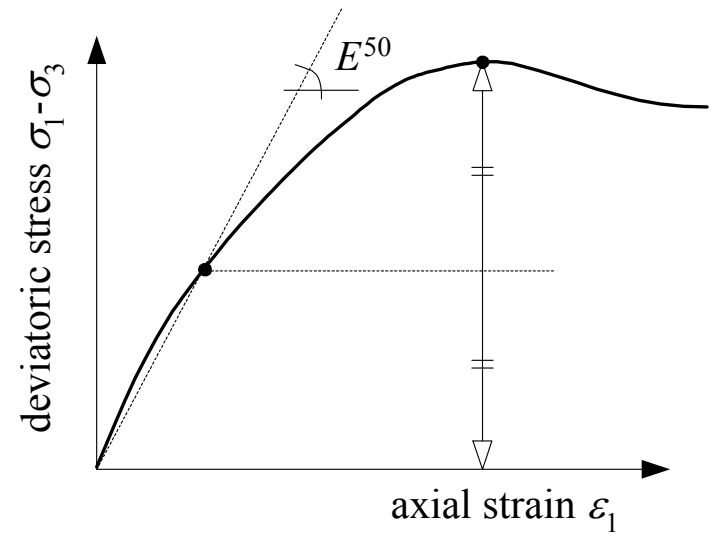

Fig. 7.2: Evaluation of the Young's modulus $E^{50}$ at $50 \%$ of the maximum deviatoric stress from triaxial tests

It can bee seen that the evaluation of the strength parameters for elastic analyses is very sensitive and leaves a wide field of interpretation since their magnitude is highly dependent on the stress level in the soil. Often the approach of applying $E=E^{50}$ is used for geotechnical investigation. In this work this method is adopted in accordance with Holeyman (2006).

The oedometer modulus $E_{\text {oed }}$ can be derived from oedometer tests according to E DIN 18135:1999-06 as:

$$
E_{\text {oed }}=-\frac{\Delta \sigma^{\prime}}{\Delta e}(1+e)
$$

The oedometer modulus is defined as secant from the pressure-void ratio-curve of an oedometric compression and interpreted as the gradient of the curve in a specific stress interval. The choice of the corresponding stress interval significantly influences the estimate of the oedometer modulus $E_{\text {oed }}$. Since the oedometer modulus $E_{\text {oed }}$ obtained considering lateral displacement restraint differs from the Young's modulus $E^{50}$ referring to a deviatoric loading and thus more or less isotropic loading, these parameters are related to each other by the Poisson ratio $\mu$ for the elastic region as follows:

$$
E_{\text {oed }}=\frac{(1-\mu) E^{50}}{(1-2 \mu)(1+\mu)}
$$

The dependence of the oedometer modulus on the vertical pressure can be taken into account according to Ohde (1951):

$$
E_{\text {oed }}=\sigma_{k} \cdot \mathrm{v} \cdot\left(\frac{\sigma_{\mathrm{v}}}{\sigma_{k}}\right)^{\mathrm{w}}
$$


where $\sigma_{k}$ is a reference stress $\left(=100 \mathrm{kN} / \mathrm{m}^{2}\right), \sigma_{\mathrm{v}}$ the effective vertical stress, $\mathrm{v}$ the factor of consolidation and $\mathrm{w}$ the exponent of deformation. As a first, very rough approximation for the effective vertical stress $\sigma_{\mathrm{v}}$ the overburden pressure arising from self-weight for an elastic and isotropic half space is used. It is calculated as the product of the unit weight $\gamma$ of the soil and the depth $z$.

\subsubsection{Coefficient of subgrade reaction for piles}

The soil-structure interaction is applied by imposing a foundation to the surface defined by the coefficient of subgrade reaction. The coefficient of subgrade reaction $k_{S}$ $\left[\right.$ forcellength $\left.{ }^{3}\right]$ defines what load or force results in the corresponding displacement for a specific area.

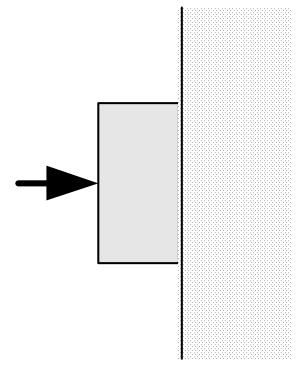

(a)

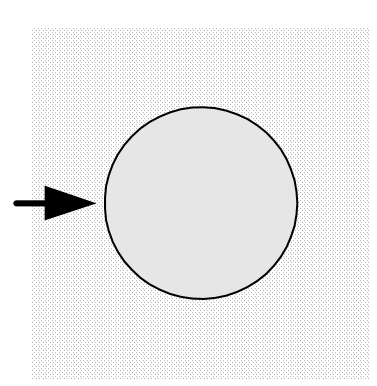

(b)

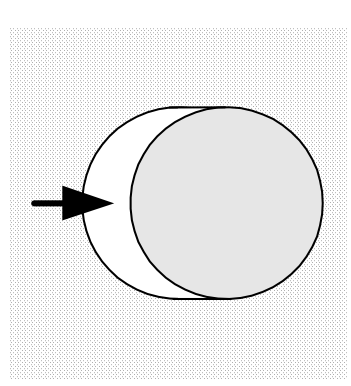

(c)

Fig. 7.3: Deformation mechanisms for soil structure interactions - after Pyke and Baikae (1984)

Many studies have dealt with the evaluation of the coefficient of subgrade reaction, but they focussed mainly on the analyses of retaining structures such as diaphragm or sheet pile walls. In this context Schmitt (1995), Dhouib (1995) or Monnet (1994) proposed approaches which are widely used in practical applications. Still, these results cannot be extrapolated easily to the problem of the vertical pile, as the case of piles is more complex due to the spatial stress distribution within the soil (Fig. 7.3).

Since the case of the laterally loaded pile is a special case concerning the evaluation of the coefficient of subgrade reaction $k_{S}$, different researchers have worked on this problem. Terzaghi (1955) was one of the first who discussed different methods for obtaining $k_{S}$. Based on the theory of elasticity he recommended to link this parameter of the soil inversely proportional to the width or the diameter $D$ of the pile. According on this recommendation the basic and most general form of the relation can be written as follows:

$$
k_{S}=C \cdot \frac{E}{D}
$$

with $C$ being a coefficient which shall take several aspects such as the shape into account. Still, for more precise analyses based on the theory of elasticity and the subgrade reaction method the results for $k_{S}$ and $E$ have to be adapted regarding the deformation behaviour. From the assumption that the displacements further away than 
three times the diameter of the pile do not influence the behaviour anymore, Terzaghi (1955) suggested the value 0.74 for $C$.

Broms (1964) investigated the lateral resistance of piles in cohesive soils based on the results from Vesic (1961) who studied an infinite horizontal beam on an elastic foundation. For different cohesive soils and piles with different stiffnesses Broms (1964) proposed values for $C$ between 0.48 and 0.9. Besides, for example Baguelin et al (1978), Skempton (1951) and Davisson (1970) proposed different values for the coefficient of subgrade reaction especially for cohesive soils which shall not be further investigated in this study. Poulos and Davis (1980) summarized several aspects in their book.

Poulos (1971) proposed a more general solution by again comparing elastic continuum solutions for loaded plates with the Winkler hypothesis. Based on this approach and the assumption of an elastic half space he suggested a value of $C=0.82$ for equation (7.4). As the surrounding soil is neglected by his approach, it was considered by Pyke and Beikae (1984) to just double the coefficient $C$ in order to take the soil of the half space on the back side of the pile into account, too.

Scott $(1980,1981)$ performed in co-operation with J.P. Bardet centrifuge tests and compared his results with the findings of Reese et al (1974) who proposed detailed and complicated approaches to calculate curves for the load-deflection behaviour of piles in sand. Therefore, Scott suggested to use a bi-linear load-displacement behaviour with an initial stiffness corresponding to a value of $C=1$ for equation (7.4). The suggested value is based on results by Baguelin et al (1977) which come from theoretical studies of a rigid cylinder which is moved horizontally in an elastic continuum. These approaches are very sensitive to the applied boundary conditions, as the distance of the pile from the support significantly influences the load-displacement behaviour and thus the coefficient of subgrade reaction. Scott proposed to use the subgrade reaction evolving from evaluating Bardet's solution for boundaries at the distance of 50 times the pile radius.

The solution proposed by Baguelin et al (1977) uses a two-dimensional plane strain system with a rigid cylinder which is displaced laterally. The load applied to the pile causes a uniform translation of the corresponding circular boundary, i.e. perfect adhesion between soil and pile. Baguelin et al (1977) solved the problem analytically by deriving the stresses from a single Airy function. The maximum radial displacements $u_{R}$ of the pile per unit thickness can be calculated as follows:

$$
u_{R}=\frac{P}{8 \pi E} \frac{1+\mu}{1-\mu}\left[(3-4 \mu) \ln \left(\frac{r_{\text {out }}}{R}\right)^{2}-\frac{r_{\text {out }}^{2}-R^{2}}{r_{\text {out }}^{2}+R^{2}}-\frac{(4 \mu-1)}{(3-4 \mu)} \frac{r_{\text {out }}^{2}-R^{2}}{r_{\text {out }}^{2}+R^{2}}\right]
$$

By relating the applied load $P$ to the radial displacements $u_{R}$ the coefficient of subgrade reaction $k_{S}$ can be calculated as a function of the Poisson ratio $\mu$ and the outside boundary $r_{\text {out }}$. Fig. 7.4 shows the coefficient $C$ calculated for different Poisson ratios $\mu$ and outer boundaries by applying the analytical solution and numerical analyses. It can 
be seen that the coefficient of subgrade reaction is relatively constant up to a Poisson ratio of around 0.35 , but then increases significantly especially for small distances to the outer boundary. Furthermore, Scott's proposed coefficient of $C=1$ for $r_{\text {out }}=50 R$ is confirmed by Baguelin's solution. Fig. 7.4 emphasizes that the problem is very sensitive to the distance to the outer boundary. Equation (7.5) makes clear that for this solution $C$ tends to zero as $r_{\text {out }}$ tends towards infinity.

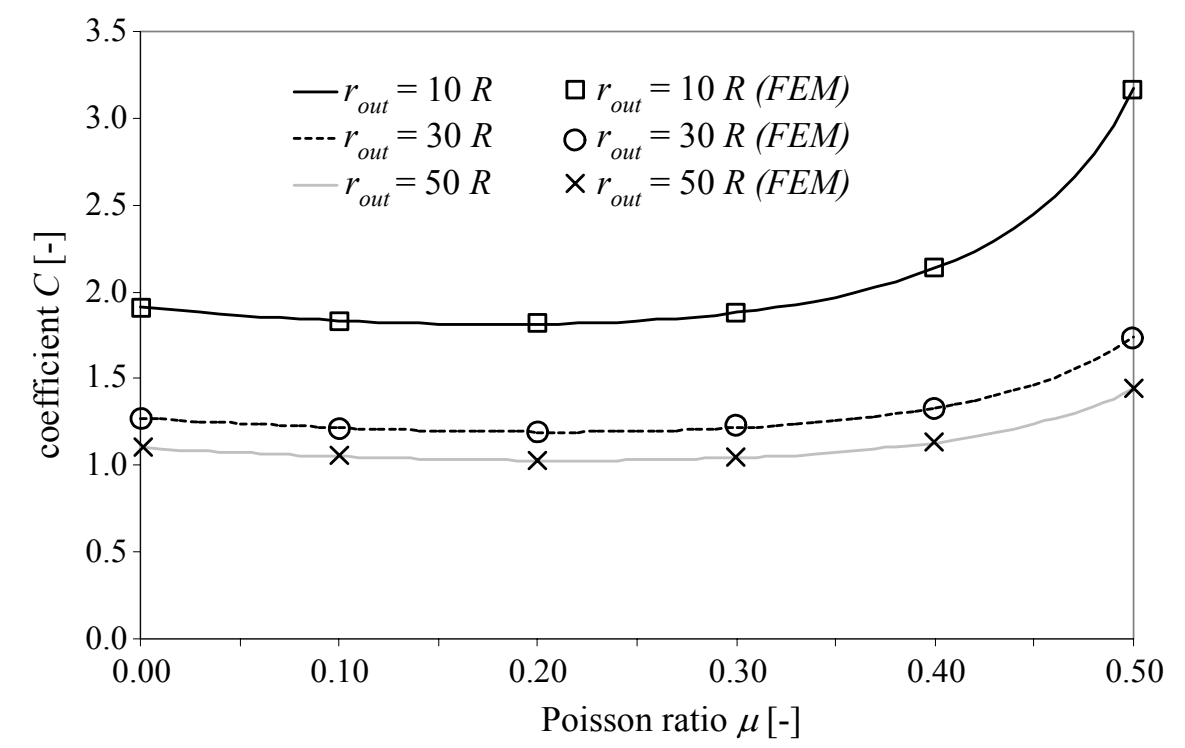

Fig. 7.4: Coefficient $C$ as a function of the Poisson ratio $\mu$ for different outer boundaries according to Baguelin et al (1977)

Pyke and Beikae (1984) investigated the problem of a laterally loaded pile idealizing it as an infinitely long rigid cylinder moving horizontally in an elastic medium. Based on real behaviour they allowed the cylinder to separate from the soil, and hence modelled in comparison to e.g. Baguelin et al (1977) a soil that is not capable of transferring tension at the back side of the pile (Fig. 7.3). Pyke and Beikae (1984) solution procedure uses Muskhelsihvili's presentation of stress in terms of potential functions. Cauchy's integral is applied to formulate the problem so that the outer boundary of the elastic medium is at infinity. By solving the equations (cfr. Beikae, 1982) and relating them to the displacements Pyke and Beikae (1984) proposed the values 2.3, 2.0, and 1.8 for the coefficient $C$ for Poisson ratio equal to $0,0.33$, and 0.5 , respectively. They suggested to use $C=2$ for practical purposes and noted that the assumption of about 10to 20-pile radii for the choice of the outer boundary is more consistent with popular conceptions of the radius of influence for laterally loaded piles. These influence radii lead according to Baguelin et al (1977) for a Poisson ratio equal to 0.3 to a value for the coefficient $C$ of 1.9 for 10-pile radii and 1.4 for 20-pile radii.

German practical applications are commonly calculated with a coefficient $C=1$. This simple approach which is valid for a straightforward pile calculation is based on experience in this field since the seventies and was also stated by Franke (1992). This elementary equation was implemented in the outdated German DIN 4014 and 
DIN EN 1536 which partially substitutes DIN 4014. Concerning the simplified assessment general information is also given in DIN 1054. The range of application for equation (7.4) is limited in DIN 1054:2005-01 to a maximum horizontal displacement that is defined as the smaller value of either $2 \mathrm{~cm}$ or $0.03 \mathrm{D}$. For diameters larger than $1 \mathrm{~m}$ the diameters should be set to $D=1 \mathrm{~m}$ in equation (7.4). This approach is restricted to practical cases where the soil is not close to a collapse. The limitation of the value for the diameter to $1 \mathrm{~m}$ can also be found in DGGT (1998) and is based on work by Schmidt (1986).

Terzaghi (1955) also proposed a more general approach using a coefficient of subgrade reaction as a function of the depth. The value of $k_{S}$ depends on the so-called constant of lateral subgrade reaction $n_{h}$.

$$
k_{S}=n_{h} \cdot \frac{z}{D}
$$

The values for $n_{h}$ are empirical coefficients depending on the density of the sand and given by Terzaghi (1955) for loose $\left(2.2 \mathrm{MN} / \mathrm{m}^{3}\right)$, medium $\left(6.6 \mathrm{MN} / \mathrm{m}^{3}\right)$, and dense sand $\left(18 \mathrm{MN} / \mathrm{m}^{3}\right)$. Other approaches for evaluating the coefficient of subgrade reaction involving depth-dependent values as for example stated by Gabr et al (1997), use the following equation:

$$
k_{S}=m_{h} z^{\omega_{G}} \zeta^{\left(1-\omega_{G}\right)}
$$

Where $m_{h}$ is the ratio between the coefficient of subgrade reaction and the depth below ground surface and $\omega_{G}$ the empirical power index equal to or greater than zero describing the distribution of $k_{S}$. The constant $\zeta$ is set to $1 \mathrm{~m}$ for maintaining the correct dimensions. Several researchers have proposed values to be used in equation (7.7) for different situations. In order to name two sets of parameters as examples the values suggested by Gabr et al (1997) include: $\omega_{G}=0.5$ and $m_{h}=34 \mathrm{MN} / \mathrm{m}^{4}$ (dense sand), and $\omega_{G}=1$ and $m_{h}=20 \mathrm{MN} / \mathrm{m}^{4}$ (medium sand).

Habibagahi and Langer (1984) proposed another solution for a modulus dependent on the depth that also is a function of the deflection. The following equation was evaluated for a friction angle $\varphi$ of $30^{\circ}$ and for the initial stiffness and is calculated for the corresponding overburden stress $\sigma_{v}$ :

$$
k_{S}=\frac{\sigma_{v}}{2.5 m m}\left(5+\sqrt{\frac{z}{D}}\right)
$$

As an example case the value for the coefficient of subgrade reaction is analysed for a specific type of pile embedded in homogeneous sand. The equations are calculated for a specific weight of $\gamma=15.25 \mathrm{kN} / \mathrm{m}^{3}$ and a pile with a diameter $D$ of $1 \mathrm{~m}$. Other values such as the factor of consolidation $\mathrm{v}$ and the exponent $\mathrm{w}$ for (7.3) are taken from Schneider (1998) and Smoltczyk (2004). The recommendations of the Arbeitsausschuss Ufereinfassungen (EAU, 1990) suggest constant values for Young's modulus $E$ to be used in common analyses. The values given for sand range from $20 \mathrm{MN} / \mathrm{m}^{2}$ to 
$150 \mathrm{MN} / \mathrm{m}^{2}$. For the different approaches using a constant coefficient of subgrade reaction in equation (7.4), a range for the coefficient of subgrade reaction is shown in Fig. 7.5. The range is chosen from $C=0.5$ to $C=2.3$ as lower and upper boundary, respectively.

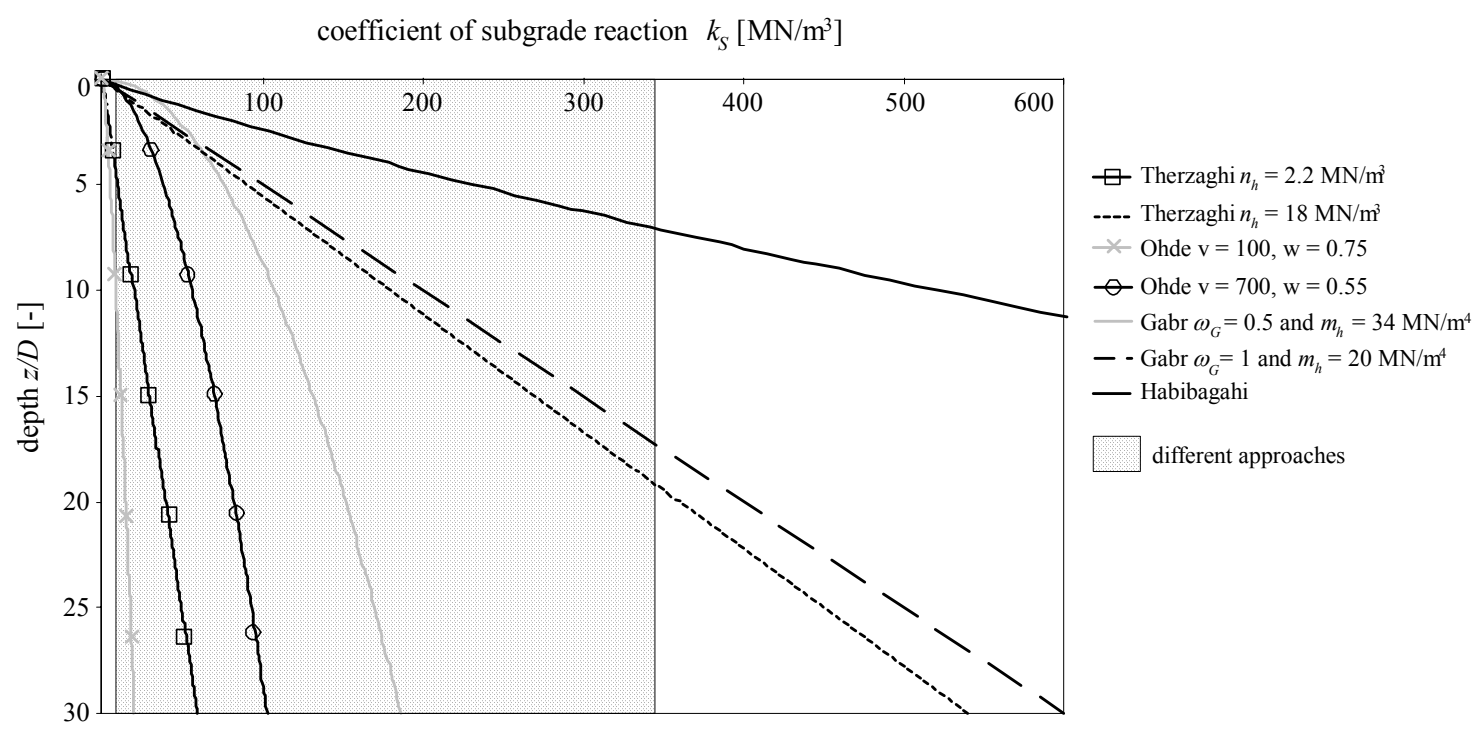

Fig. 7.5: Coefficient of subgrade reaction $k_{S}$ as a function of the depth for different sands with $\gamma=15.25 \mathrm{kN} / \mathrm{m}^{3}$

For a pile length of $10 \mathrm{~m}$ and a diameter of $1 \mathrm{~m}$ for example the coefficient of subgrade reaction reaches according to Fig. 7.5 values between $10 \mathrm{MN} / \mathrm{m}^{3}$ to $345 \mathrm{MN} / \mathrm{m}^{3}$. It can be seen that the specifications for the coefficient of subgrade reaction vary significantly and hence the calculated results. This aspect emphasizes the sensitivity of the analyses to the chosen set of parameters. For the example case of the combiwall (chapter 4) with a length of $30 \mathrm{~m}$ and a maximum bending moment in a depth of around $10 \mathrm{~m}$, values of $10 \mathrm{MN} / \mathrm{m}^{3}$ to $100 \mathrm{MN} / \mathrm{m}^{3}$ can be assumed for the coefficient of subgrade reaction $k_{S}$ as a first approximation based on conservative assumptions.

\subsubsection{Approach to evaluate the coefficient of subgrade reaction for piles}

Based on the previous proposals another solution is derived for a rigid disc loaded horizontally in an elastic medium imposing plane strain conditions. The aim is to verify the presented relations between Young's modulus and the coefficient of subgrade reaction. In the next step the analytical solutions are compared with numerical analyses.

The problem of a cylindrical pin and a plate containing a circular hole of the same or almost the same size has been studied intensively in the field of mechanical engineering by analytical means. Persson (1964) was the first one to study the problem for design purposes, as the well-known Hertz formulation (cfr. e.g. Johnson, 1985) of the problem only deals with idealized half-planes. Fig. 7.6 illustrates the problem with the corresponding explanation of the notations. 


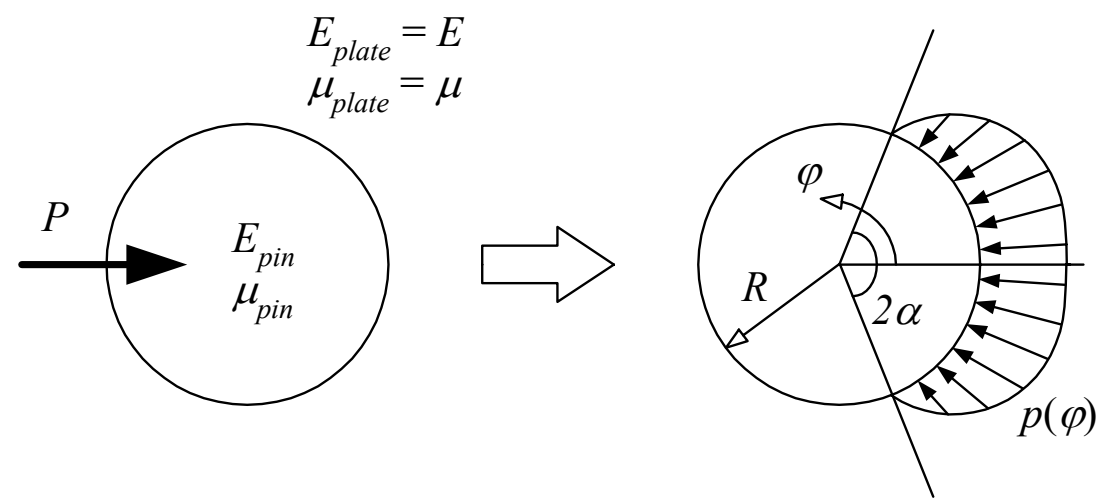

Fig. 7.6: Pin loaded horizontally in elastic medium with resulting pressure distribution

Lin et al (1997) and Hou and Hills (2001) continued the work from Persson (1964) and emphasized the influence of non-conforming holes. Lin et al (1997) and Hou and Hills (2001) both gave a good overview on the work performed by Persson (1964). The formulae given by Persson (1964) are for the case of plane stress, but can be used for the plane strain problem by simply replacing $E$ with $E^{*}=E /\left(1-\mu^{2}\right)$, and $\mu$ with $\mu^{*}=\mu /(1-\mu)$. Based on the type of loading the Airy stress function is used for describing the stress conditions which gives rise to presenting the stresses in terms of potential functions. More details can be found in publications by Persson (1964), Lin et al (1997), and Hou and Hills (2001). Relating the stress functions with the compatibility of the displaced shape of the deformed bodies over the arc of contact and using infinite boundary conditions, yields the following relation with $B_{P}$ representing a variable according to Persson (1964):

$$
B_{P}=\frac{2\left(1+n_{p p}\right)-\lambda_{p p}}{1+n_{p p}}-\frac{2 n_{p p}}{1+n_{p p}} \int_{-b}^{b} q(y) \frac{d y}{1+y^{2}}-\frac{\pi E_{p i n} \Delta R}{P\left(1+n_{p p}\right)}
$$

where $\Delta R$ is the distance of radii for non-conforming pin-hole connections, and:

$$
\begin{aligned}
& y=\operatorname{tg} \frac{\varphi}{2} \\
& b=\operatorname{tg} \frac{\alpha}{2}
\end{aligned}
$$

The approach is derived for two elastic bodies in contact. The relationship between the pin and the plate is expressed by the ratio $n_{p p}$ and the value $\lambda_{p p}$ :

$$
n_{p p}=\frac{E_{\text {pin }}}{E_{\text {plate }}} \quad \text { and } \quad \lambda_{p p}=1-\mu_{\text {pin }}-n\left(1-\mu_{\text {plate }}\right)
$$

The normalized pressure distribution $q(y)$ used in equation (7.9), which satisfies the condition of deformation at all points of the surface in contact is defined as:

$$
q(y)=\frac{R p(\varphi)}{P}
$$


and can be written according to Perrson (1964) as:

$$
q(y)=\frac{2}{\pi \sqrt{b^{2}+1}} \frac{\sqrt{b^{2}-y^{2}}}{1+y^{2}}+\frac{1}{2 \pi b^{2}\left(1+b^{2}\right)} \ln \frac{\sqrt{b^{2}+1}+\sqrt{b^{2}-y^{2}}}{\sqrt{b^{2}+1}-\sqrt{b^{2}-y^{2}}}
$$

Further, the semi-angle of contact, $\alpha$, can be related to the variable $B_{P}$ considering equation (7.11):

$$
B_{P}=\frac{2 b^{4}+2 b^{2}-1}{b^{2}\left(1+b^{2}\right)}
$$

Just as in the case of an embedded steel pile in sand where the ratio $n_{p p}$ tends to infinite $(\approx 7000)$, this problem is investigated by assuming the pile to be rigid. As a consequence, the Poisson ratio for the pin is set equal zero. For these parameters the formulae given by Perrson (1964) are evaluated, and the following equation can be written as plane strain formulation by substituting the appropriate values and equation (7.9) with (7.15):

$$
\frac{2 b^{4}+2 b^{2}-1}{b^{2}\left(1+b^{2}\right)}=3-\mu^{*}-2 \int_{-b}^{b} q(y) \frac{d y}{1+y^{2}}
$$

The last expression from equation (7.9) including $\Delta R$ and $E_{\text {pin }}$ tends to zero for large ratios $n_{p p}$ and thus does not appear in (7.16). Therefore, equation (7.16) is also valid for conforming pin-hole connections $(\Delta R=0)$ which is assumed for the investigated case and/or for $E_{\text {pin }}=0$. Since, a closed solution for the problem cannot be given the evaluation of equation (7.14) in combination with (7.16) is performed by iteration in order to obtain the semi-angle of contact, $\alpha$.

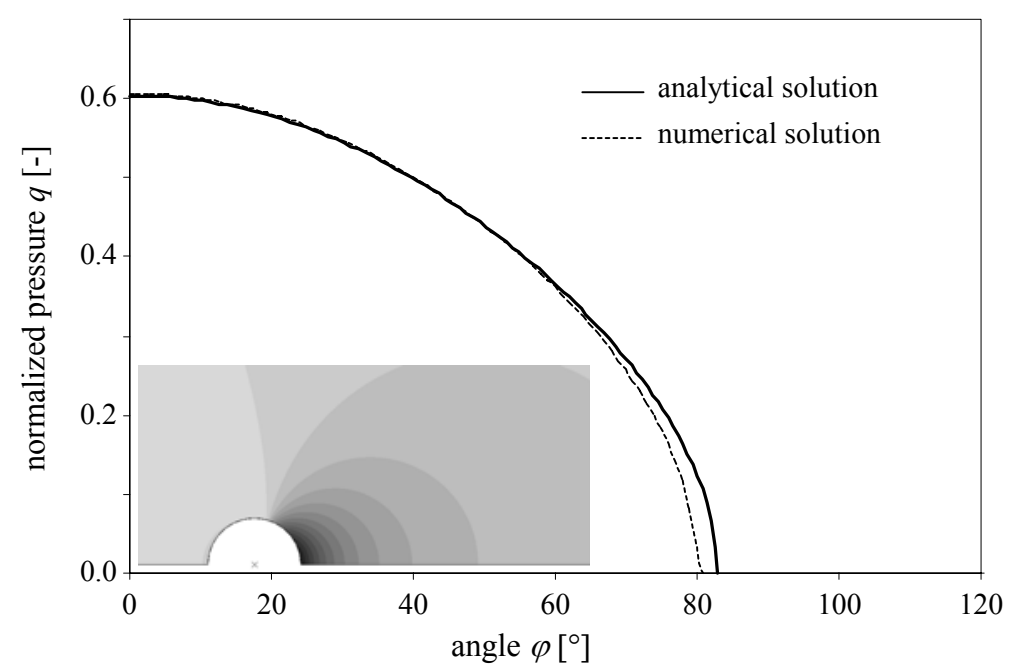

Fig. 7.7: Comparison of the normalized pressure distribution $q(\varphi)$ for $\mu=0.3$ and plot of the radial stress distribution from numerical analyses

For comparison finite element analyses for the discussed problem are performed, using a two-dimensional plane strain model with linear finite elements modelling the region 
around the pile, and with the infinite elements modelling the far-field region. Fig. 7.7 depicts the pressure distribution along the contact surface for the numerical and the analytical solution for a Poisson ratio of 0.3 . The small picture shows the radial stress distribution from the numerical analysis. The deviations between the semi-angle of contact calculated by numerical and analytical means for Poisson ratios are small and reach a maximum of $2.3 \%$ in the range of 0.3 to 0.4 .

While converged solutions for the pressure distribution around the circumference can be found at the edge of the hole, no closed solution is available for the displacements. As the loading is not self-equilibrating, the far-field displacements have the form $u=\ln (r)$ and therefore imply that the displacement approaches infinity as $r$ tends to infinity. Hence by using this approach, no unique solution can be found for numerical analyses even when using infinite elements and also no unique value for the coefficient of subgrade reaction. Another approach using a plane strain model of a plate with a rigid disc with contact is investigated with fixed boundaries. The contact between the rigid pile and the elastic continuum is modelled just as in the case described before with the augmented Lagrangian method. Similar to Fig. 7.4, Fig. 7.8 shows the coefficient $C$ calculated for different Poisson ratios $\mu$ and outer boundaries for no soil-pile adhesion. As seen in Fig. 7.8 the coefficient of subgrade reaction decreases for a Poisson ratio of for example 0.3 between $35 \%$ and $50 \%$ in comparison to the approach using a soil-pile interaction that is capable of transferring tension (complete adhesion). This significant difference of the coefficient $C$ between the solution of Baguelin et al. (1977) and the finite element analyses using "hard" contact evidently arises due to the fact that in contrast to the "adhesion solution" which may overestimate the carrying behaviour considerably for a SSI no tension forces can be transferred at the unloaded side. This effect is clear since for the case of a contact modelling a gap evolves between pin and plate. The choice of a larger radius of influence (= outside boundary $r_{\text {out }}$ ) for the laterally loaded pile does not affect the coefficient of subgrade reaction as much as for the case of the complete soil-pile adhesion.

The curves plotted in Fig. 7.9 are evaluated applying regression equations as a function of the outer radius $r_{\text {out }}$. In order to obtain comparable functions to the functions derived by Baguelin et al. (1977) for complete adhesion, the regression analyses are performed based on a logarithmic approach and as a function of an arbitrary radius $30 R$ (Fig. 7.9). The values for a Poisson ratio of 0.495 deviate considerably due to numerical reasons associated with using the latter Poisson ratio. For the two other Poisson ratios depicted a perfect match can be found with logarithmic regression lines. 


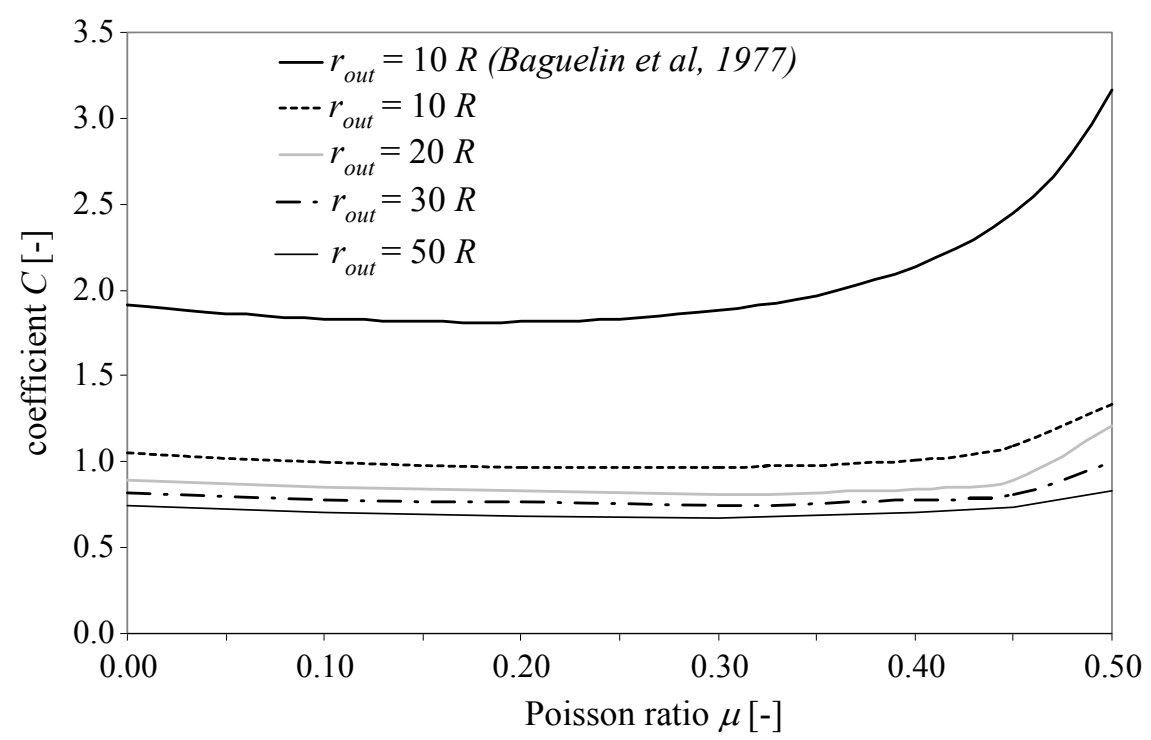

Fig. 7.8: Coefficient $C$ as a function of the Poisson ratio $\mu$ for different outer boundaries for no soil-pile adhesion

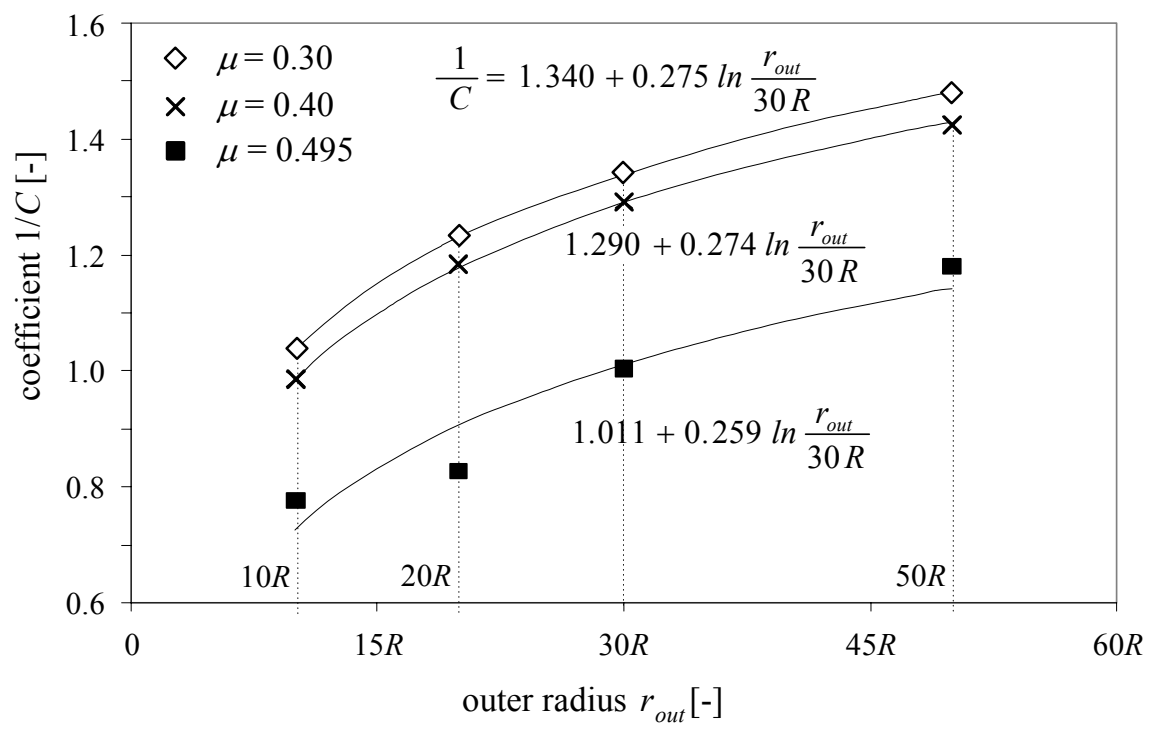

Fig. 7.9: Logarithmic regression analyses for obtaining the coefficient $C$ as a function of the outer radius $r_{\text {out }}$ for no soil-pile adhesion

A value of 1 for the coefficient $C$ for the coefficient of subgrade reaction for a common Poisson ratio of 0.3 for sand can be extracted from the analyses assuming an influenced area of about 10- to 20-pile radii from the centre of the pile. The values $C$ for relating the Young's modulus soil to the coefficient of subgrade reaction proposed by different researchers and approaches are summarized in Tab. 7.1.

The comparison reveals equations for the analytical functions for the coefficient $C$ given by Baguelin et al. (1977) with soil-pile adhesion and the calculated numerical functions without soil-pile adhesion differing mainly by an additional term. Applying friction to the interface zone would lead to coefficients between the two extreme cases. 
Comparing the calculated results to the values proposed by different researchers, similar values for the coefficient $C$ appear as the lower bound. Hence, as a conservative approach at this stage it is suggested to use $C=1$, which corresponds to $k_{S}=E / D$ for the coefficient of subgrade reaction $k_{S}$.

Tab. 7.1: Different approaches for relating the Young's modulus soil E to the coefficient of subgrade reaction $k_{S}$

\begin{tabular}{|c|c|}
\hline$C=D \cdot \frac{k_{S}}{E}$ & $C[-]$ \\
\hline approach & \\
\hline Terzaghi (1955) & 0.74 \\
\hline Broms (1964) & 0.48 to 0.90 \\
\hline Poulos (1971) & 0.82 \\
\hline Scott $(1980,1981)$ & 1.00 \\
\hline Pyke and Beikae (1984) & $2.30,2.00,1.80$ for $\mu=0,0.33,0.495$ \\
\hline \multirow{4}{*}{ Baguelin et al. (1977) } & 1 for $\mu=0.33$ \\
\hline & $\begin{array}{l}0.808+0.265 \ln \frac{r_{\text {out }}}{30 R} \\
\quad \text { for } r_{\text {out }}=30 R \Rightarrow 1.24\end{array}$ \\
\hline & 1 for $\mu=0.495$ \\
\hline & $\begin{aligned} 0.573+0.239 & \ln \frac{r_{\text {out }}}{30 R} \\
\text { for } r_{\text {out }} & =30 R \Rightarrow 1.75\end{aligned}$ \\
\hline \multirow{7}{*}{$\begin{array}{l}\text { rigid pin in hole with no soil-pile } \\
\text { adhesion (Fig. 7.9) }\end{array}$} & $1 \quad$ for $\mu=0.30$ \\
\hline & $\begin{array}{l}1.340+0.275 \ln \frac{r_{\text {out }}}{30 R} \\
\text { for } r_{\text {out }}=30 R \Rightarrow 0.75\end{array}$ \\
\hline & 1 for $\mu=0.40$ \\
\hline & $1.290+0.274 \ln \frac{r_{\text {out }}}{30 R}$ \\
\hline & for $r_{\text {out }}=30 R \Rightarrow 0.78$ \\
\hline & 1 for $\mu=0.495$ \\
\hline & $\begin{aligned} 1.011+0.259 & \ln \frac{r_{\text {out }}}{30 R} \\
\text { for } r_{\text {out }} & =30 R \Rightarrow 1.00\end{aligned}$ \\
\hline
\end{tabular}




\subsubsection{Multi-parameter models}

The deficiency of the simple Winkler model not being capable of taking shear forces into account, can be adjusted by using a multi-parameter model comprising of two or more types of physical components in contrast to the Winkler foundation. Thereby, the modelling of the SSI becomes significantly more complicated. Besides the problem of defining the values for each parameter, e.g. the spring stiffness, the multi-parameter model gives rise to the question of the correlation of the different deformation components.

The most important characteristic of these models is the additional component acting parallel to the contacting surface. This component can be either introduced using further horizontal or diagonal springs, or an incompressible "shear layer" defined as a linear elastic layer only resisting vertical shear forces (Pasternak model, 1954). The multiparameter models can be described by extending equation (7.1):

$$
\begin{aligned}
& p(x, y)-c_{p_{1}} \nabla^{2} p(x, y)+c_{p_{2}} \nabla^{4} p(x, y)= \\
& =c_{w_{1}} w(x, y)-c_{w_{2}} \nabla^{2} w(x, y)+c_{w_{3}} \nabla^{4} w(x, y)
\end{aligned}
$$

The constant coefficients $c_{p i}$ and $c_{w i}$ depend on the chosen model, and arise from the different mechanical elements used in the model: e.g. spring stiffness $k$, stiffness of the shear layer $g_{s l}$ or flexural plate stiffness $D_{p l}$. As an example for the multi-parameter models a concept proposed by Ummenhofer (1997) is used (Fig. 7.10). Shear forces can be transferred using this model.

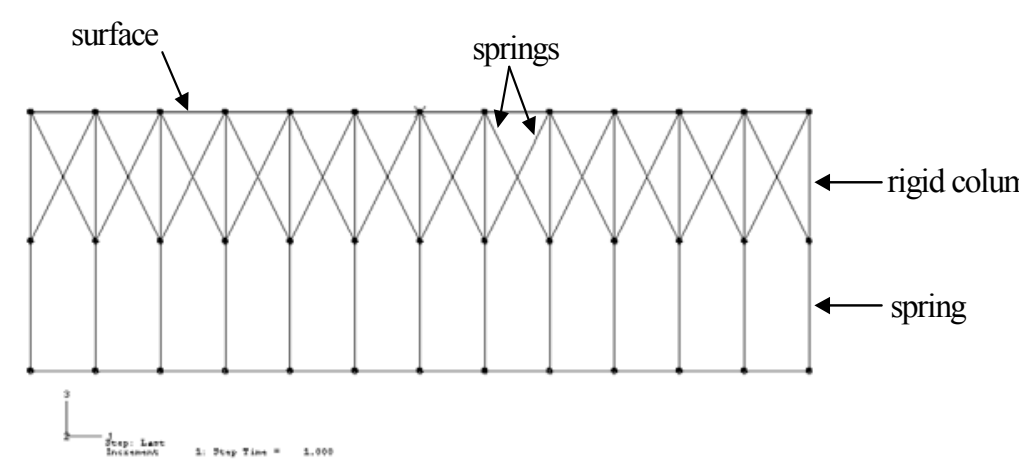

Fig. 7.10: Side view of the applied multi-parameter model

As seen for the subgrade reaction model, the derivation of appropriate parameters may be very difficult. Again, this aspect becomes more complicated when using multiparameter models.

\subsubsection{Elastic continuum}

A self-evident solution for representing the soil appears to be modelling it as a threedimensional elastic continuum. The contact between the continuum and the structure and be modelled using specific contact formulations or even connector elements with a 
defined stiffness. The interaction can be implemented to act only at pressure (no adhesion) or on tension as well (adhesion). Therefore, the interaction can be defined in a way that a gap can evolve between soil and structure. For the elastic formulation of the continuum only the Young's modulus and the Poisson's ratio of the soil are needed. A far better modelling of the soil is possible by taking non-linear soil behaviour as plasticity, e.g. Mohr-Coulomb, or dilation into account. The Mohr-Coulomb plasticity is a common failure criterion used in geotechnical engineering. It is defined as a linear relationship between normal stresses $\sigma$ and shear stresses $\tau$ at failure:

$$
\tau=c-\sigma \tan \varphi
$$

in which $c$ is the cohesion of the material and $\varphi$ the friction angle of the material. If not specified differently a smooth flow potential (hyperbola) described by the dilation angle $v$ is applied in combination with a non-associated flow rule and no hardening. With this more detailed analysis the spatial load transfer mechanisms that cannot be covered by a two-dimensional continuum can be investigated and additionally further soil properties can be taken into account.

For the case of a vertically embedded pile this approach can be first analysed modelling a pile entirely connected to an elastic continuum. The homogenous linear elastic assumption allows analytical solutions using Mindlin's closed form solutions for the soil displacement due to a point load embedded in a semi-infinite medium (Poulos, 1971).

\subsubsection{Hypoplastic continuum}

A three dimensional modelling of soil as introduced for the elastic continuum allows the user to incorporate complex material models. Since the hypoplasticity is one of the most elaborate and sophisticated soil models used in geotechnical investigations, it is considered in this evaluation of the soil models. For this study the formulation by $\mathrm{v}$. Wolffersdorf (1996) who extended the base formulation by the void ratio. Further, the model includes additional stress functions in comparison to the formulation by Kolymbas (1988). Fellin and Ostermann (2002) linked the hypoplastic formulation to the finite element program ABAQUS with a subroutine written in FORTAN.

In the following the base equations for the hypoplasticity are summarized, and the relevant parameters are explained. More details can e.g. be found in Fellin (2000) or Fellin and Kolymbas (2002). The main concept of the hypoplasticity is that it is an entirely non-linear material model and not divided into a linear part and a plastic part found for most other material models. The base equation can be written as:

$$
\stackrel{\circ}{\boldsymbol{\sigma}}=f_{b} f_{e} \frac{1}{\operatorname{tr}\left(\hat{\boldsymbol{\sigma}}^{2}\right)}\left[F^{2} \boldsymbol{D}+a^{2} \hat{\boldsymbol{\sigma}} \operatorname{tr}(\hat{\boldsymbol{\sigma}} \cdot \boldsymbol{D})+f_{d} a F\left(\hat{\boldsymbol{\sigma}}+\hat{\boldsymbol{\sigma}}^{\prime}\right)\|\boldsymbol{D}\|\right]
$$

with the tensor of the deformation rate $\boldsymbol{D}$, the Jaumann stress tensor $\stackrel{\circ}{\sigma}$, and 


$$
\begin{gathered}
\hat{\boldsymbol{\sigma}}=\frac{\boldsymbol{\sigma}}{\operatorname{tr}(\boldsymbol{\sigma})} \\
\hat{\boldsymbol{\sigma}}^{\prime}=\hat{\boldsymbol{\sigma}}-\frac{1}{3} \boldsymbol{I}
\end{gathered}
$$

and the factor $a$, which takes account of the critical friction angle $\varphi_{c}$ on the material behaviour:

$$
a=\frac{\sqrt{3}\left(3-\sin \varphi_{c}\right)}{2 \sqrt{2} \sin \varphi_{c}}
$$

The scalar stress function $F_{s f}$ is defined by $\hat{\boldsymbol{\sigma}}^{\prime}$ and Lode angle $\theta_{L}$ :

$$
F_{s f}=\sqrt{\frac{1}{8} \tan ^{2} \kappa+\frac{2-\tan ^{2} \kappa}{2+\sqrt{2} \tan \kappa \cos \left(3 \theta_{L}\right)}}-\frac{1}{2 \sqrt{2}} \tan \kappa
$$

where:

$$
\begin{gathered}
\tan \kappa=\sqrt{3}\left\|\hat{\boldsymbol{\sigma}}^{\prime}\right\| \\
\cos \left(3 \theta_{L}\right)=-\sqrt{6} \frac{\operatorname{tr}\left(\hat{\boldsymbol{\sigma}}^{\prime} \cdot \hat{\boldsymbol{\sigma}}^{\prime} \cdot \hat{\boldsymbol{\sigma}}^{\prime}\right)}{\left[\operatorname{tr}\left(\hat{\boldsymbol{\sigma}}^{\prime} \cdot \hat{\boldsymbol{\sigma}}^{\prime}\right)\right]^{3 / 2}}
\end{gathered}
$$

The following scalar functions $f_{b}, f_{e}$, and $f_{d}$ in equation (7.19) incorporate the average density and the void ratio $e_{i}$ at minimum density, $e_{d}$ at maximum density, and $e_{c}$ for the critical state:

$$
\begin{gathered}
f_{b}=\frac{h_{s}}{n}\left(\frac{e_{i 0}}{e_{c 0}}\right)^{\beta} \frac{1+e_{i}}{e_{i}}\left(\frac{3 p_{s}}{h_{s}}\right)^{1-n}\left[3+a^{2}-a \sqrt{3}\left(\frac{e_{i 0}-e_{d 0}}{e_{c 0}-e_{d 0}}\right)^{\alpha}\right]^{-1} \\
f_{e}=\left(\frac{e_{c}}{e}\right)^{\beta} \\
f_{d}=\left(\frac{e-e_{d}}{e_{c}-e_{d}}\right)^{\alpha}
\end{gathered}
$$

Thus, for this formulation of the hypoplasticity eight material parameters $\left(\varphi_{c}, h_{s}, n, e_{i 0}\right.$, $\left.e_{c 0}, e_{d 0}, \alpha, \beta\right)$ are needed. These eight parameters are described briefly. Further details can be found e.g. in the publications of Herle (1997), v. Wolffersdorf (1996) or Böhrnsen (2002).

\section{Critical friction angle $\varphi_{c}$}

The determination of the critical friction angle can be carried out by piling the cohesionless material. The angle appearing for the cone produced by pouring the material through a hopper is defined a critical friction angle 
2. Granular stiffness $h_{s}$

The granular stiffness is directly related to the compressibility of the material. The more the material can be compressed the smaller is the granular stiffness. It is derived from the pressure-void ratio curve from an oedometric compression test (Fig. 7.11). With the compression index $C_{c}$ (gradient of the compression curve) for different pressures $p_{s}$ the granular stiffness can be calculated:

$$
h_{s}=3 p_{s}\left(\frac{n \cdot e}{C_{c}}\right)^{\frac{1}{n}}
$$

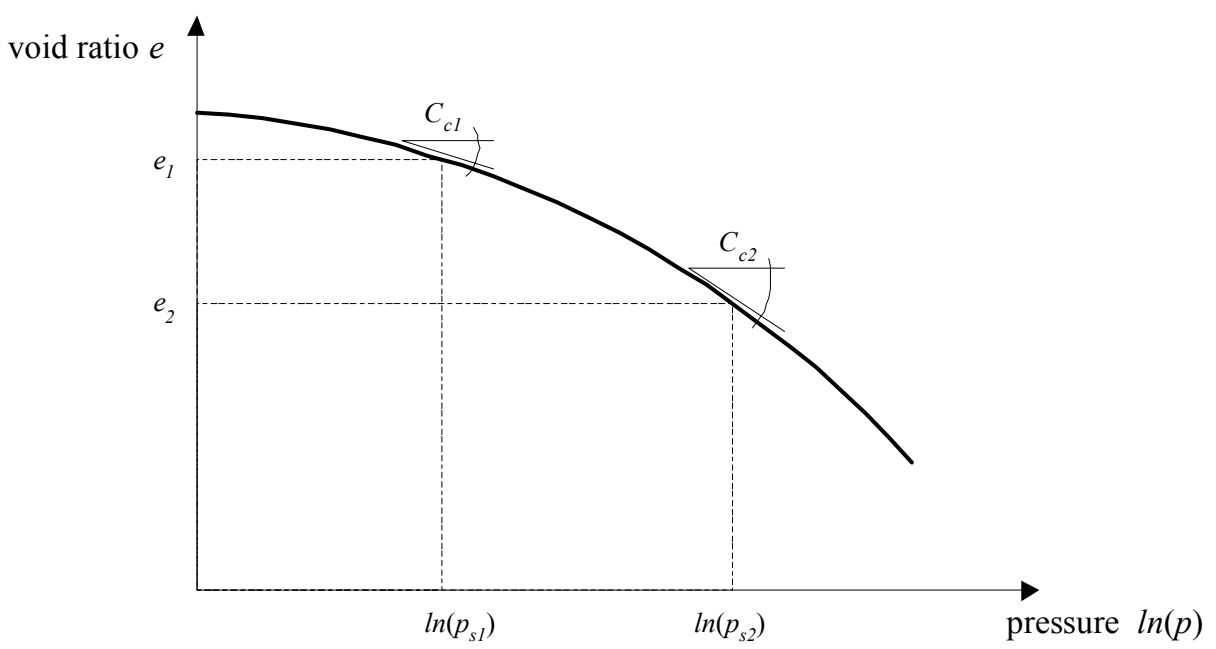

Fig. 7.11: Oedometric compression curve

The granular stiffness depends on the exponent $n$ which again depends on the active pressure. Since the granular stiffness is no material constant, the pressure range for the oedometric compression test should be chosen according the expected reaction for the investigated system.

\section{Exponent $n$}

Similar to the granular stiffness, the exponent $n$ is not a constant but a function of the applied pressure. Thus, this value has to be chosen from an oedometric compression test, and then again for a pressure range similar to the real conditions. Based on two values from the compression curve (Fig. 7.10) the exponent $n$ can be calculated:

$$
n=\ln \left(\frac{e_{1} \cdot C_{c 2}}{e_{2} \cdot C_{c 1}}\right) / \ln \left(\frac{p_{s 2}}{p_{s 1}}\right)
$$

\section{Void ratio $e_{i 0}$ at minimum density}

The void ratio $e_{i 0}$ at minimum density is derived from the maximum void ratio $e_{\max }$ at minimum density, which can be determined by experiments: $e_{i 0} \approx 1,15 e_{\max }$ 
for sand. The void ratio $e_{i 0}$ at minimum density is defined for a grain pressure of zero which cannot be reached in the gravitation field of the earth.

\section{Critical void ratio $e_{c 0}$}

The critical void ratio $e_{c 0}$ for a grain pressure of zero corresponds approximatly the maximum void ratio $e_{\max }$ at minimum density as proven by experiments.

\section{Void ratio e $e_{0}$ at maximum density}

Void ratio $e_{d 0}$ at maximum density can approximately be equated with the minimum void ratio $e_{\min }$ which can be obtained easily with experiments using a vibrating table.

\section{Exponent $\alpha$}

The exponent $\alpha$ takes the influence of the density on the friction angle into account. The value for $\alpha$ can be determined knowing the critical friction angle $\varphi_{c}$, the peak friction angle $\varphi_{p}$ and the relative void ratio $r_{e}$ from diagrams given by Herle (1997).

\section{Exponent $\beta$}

The exponent $\beta$ influences the stress rate for increasing density of the material. For most cases $\beta$ can be set equal to 1 as suggested by Herle (1997).

\subsection{Comparison of different soil models for a loaded block}

For the comparison of some SSIM a reference case is chosen arbitrarily for illustrating the general behaviour for different models. It is chosen to investigate a simple settlement problem. A system of the size $30 \mathrm{~m} \times 30 \mathrm{~m} \times 10 \mathrm{~m}$ is loaded with a rigid plate $(10 \mathrm{~m} \times 10 \mathrm{~m})$ in the centre. The vertical boundaries of the model are constrained in order to obtain a closed system. The stiffness of the top layer between springs and rigid plate which is included in the model for clarity is negligible. For the qualitative investigation of the model the vertical, axial springs are implemented with an arbitrary reference stiffness of $1 \mathrm{kN} / \mathrm{m}$, and the plate is loaded so that the each spring is subjected to an axial force of $1 \mathrm{kN}$. Fig. 7.12 depicts the deformed system subjected to the specified load. The plate displaces vertically $1 \mathrm{~m}$, and does not influence the surrounding soil as no transfer of shear forces is possible.

With extra diagonal springs (multi-parameter model) the shear stiffness of the soil can be defined as separate parameter, and a coupled system can be assembled. For the case of a spring stiffness of zero for the diagonal springs, the system responds as the model for the Winkler hypothesis. The reference case is modelled with additional diagonal springs with a spring stiffness of $k=1 \mathrm{kN} / \mathrm{m}$ for taking irregular axial displacements into account. It is clear from Fig. 7.12 that this more detailed approach results in a different settlement behaviour in comparison to the Winkler hypothesis. For the 
investigated case the maximum settlement reduces by $5 \%$. Still, it has to be stated that the value for the diagonal spring stiffness was chosen arbitrarily.

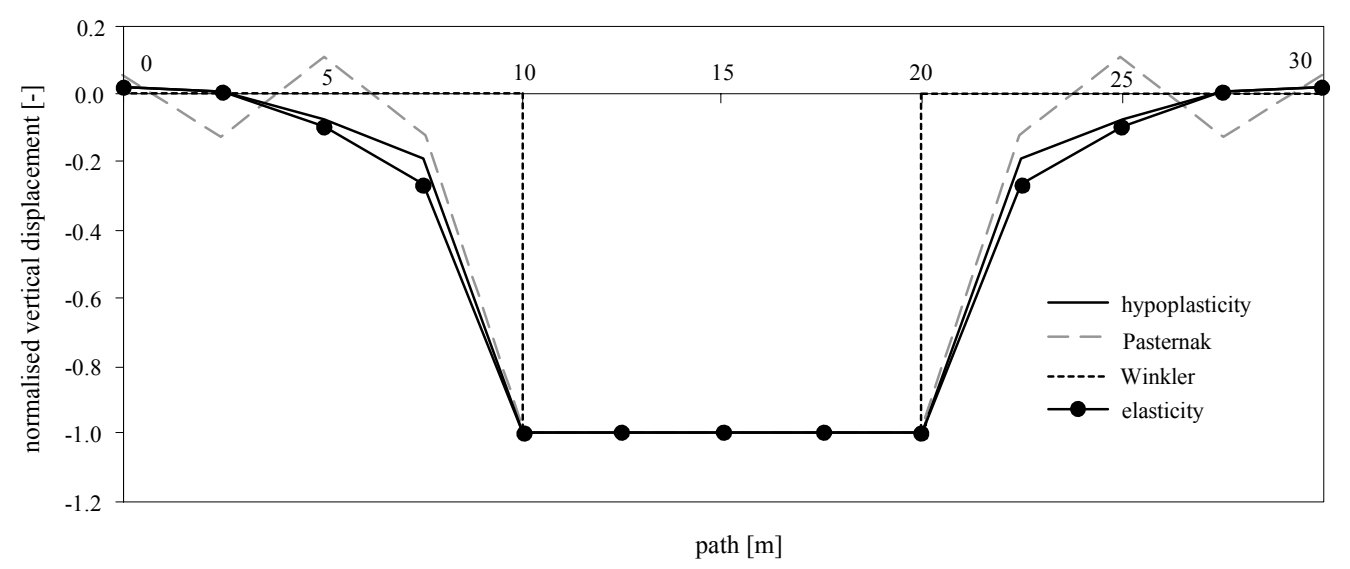

Fig. 7.12: Comparison of the shape of settlement for different soil models

For an elastic continuum the deformed body of the reference case is depicted in Fig. 7.13. A Young's modulus of $50 \mathrm{~N} / \mathrm{mm}^{2}$ and a Poisson's ratio of 0.3 were assumed for the soil. In this comparison no extensive study for harmonising the parameters of the different models was performed. The deformed structure of the reference case of the introduced hypoplastic material model does not differ much from the deformed body depicted in Fig. 7.13. The eight material parameters as listed in Tab. 7.2 were chosen according to Fellin and Ostermann (2002).

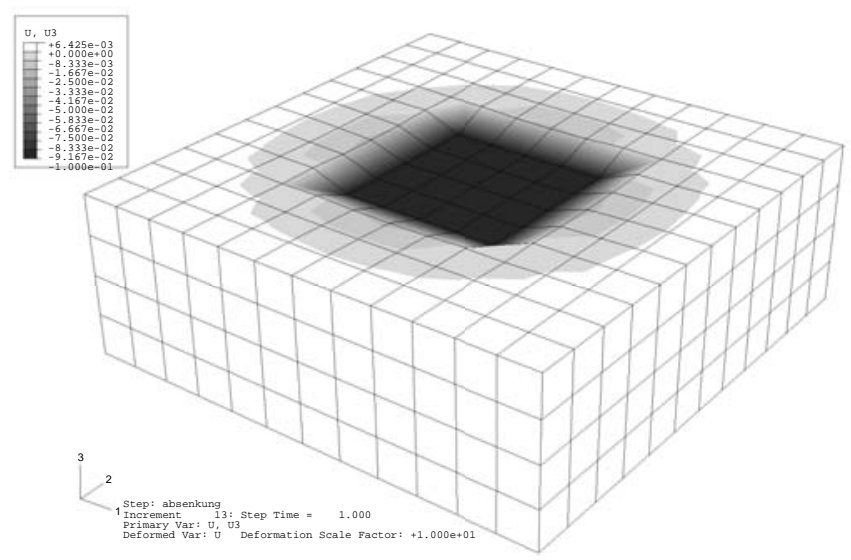

Fig. 7.13: Deformed system for foundation model using an elastic or hypoplastic continuum

The realistic settlement of the soil can be seen in the contour plot of the vertical displacements. Fig. 7.12 depicts the settlement for the different soil models as a mid section trough the symmetry plane of the model. This schematic comparison is made in order to compare the shape of the settlement and not the maximum displacements. The latter comparison can only be performed by adjusting the relevant soil parameters for all models which was not done for the discussed case but for the following comparisons. 
Tab. 7.2: Parameters for the hypoplastic material model according to Fellin and Ostermann (2002)

\begin{tabular}{|c|c|c|c|c|c|c|c|}
\hline$\varphi_{c}\left[^{\circ}\right]$ & $h_{s}[\mathrm{kPa}]$ & $n$ & $e_{i 0}$ & $e_{c 0}$ & $e_{d 0}$ & $\alpha$ & $\beta$ \\
\hline 30 & $1.9 \cdot 10^{8}$ & 0.45 & 1.19 & 0.80 & 0.40 & 0.15 & 1.0 \\
\hline
\end{tabular}

It can be seen that the deformations for the continua are similar while the shape of the settlement using springs is different. This simple comparison already shows that the approaches with springs can lead to unsatisfactory results concerning the deformation behaviour, if the settlement needs to be investigated. Nevertheless, for an non-rigid body entirely in contact with soil this aspect may not be important.

\subsection{Comparison of different soil models for a strip footing}

The following section deals with a more detailed comparison for different soil models for investigating the efficiency and the applicability of different soil models. Findings from experiments are compared with numerical analyses using an elastic and hypoplastic material model.

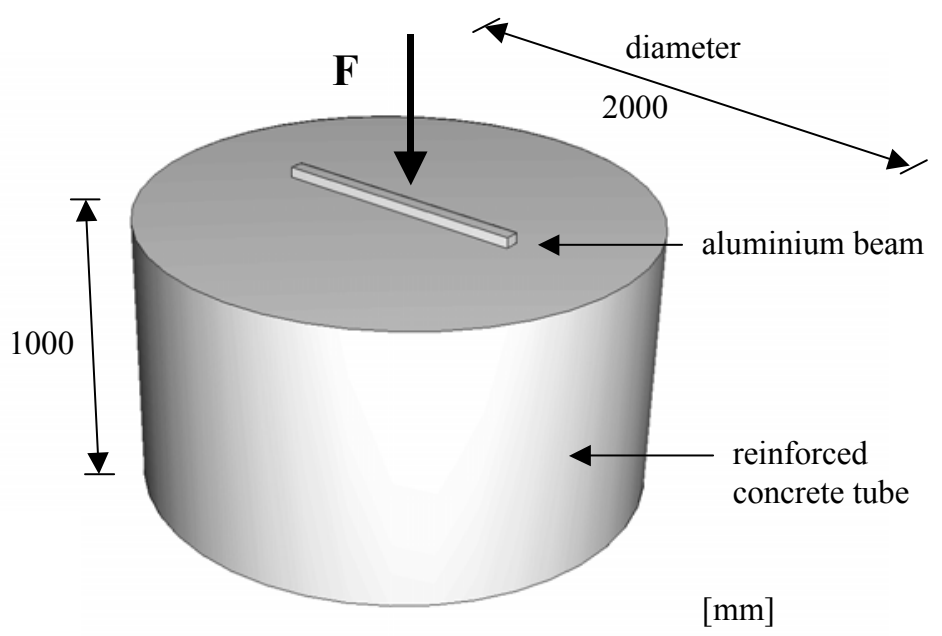

Fig. 7.14: Test set-up according to Schlegel (1985)

Schlegel (1985) performed studies with a strip footing within his work in order to derive an improved subgrade reaction model for settlement problems. The tests were performed in a cylindrical tube made of reinforced concrete with a diameter of $2 \mathrm{~m}$ and a height of $1 \mathrm{~m}$. In the tests the sand was transported with a suction device and pluviated into the reinforced concrete tube. An aluminium beam or strip of the dimensions $59 \mathrm{~mm} \times 38 \mathrm{~mm}$ and the length of $1025 \mathrm{~mm}$ is placed with the flat side downwards on top of the sand filled tube. From tensile tests the Young's modulus for the aluminium beam is evaluated with $75700 \mathrm{~N} / \mathrm{mm}^{2}$ and the Poisson ratio with 0.312 . After positioning the aluminium beam on the sand it was loaded by one or three concentrated loads. The vertical displacements and the strains arising in the aluminium beam were measured. In this study one test is selected for comparison with a single concentrated load $\mathrm{F}=0.866 \mathrm{kN}$ set in the centre of the beam. The test set-up is depicted in Fig. 7.14. 
A similar experiment by Schlegel (1985) was used for verification purposes by Fiedler (2002) who worked on settlement problems of reinforced concrete foundations. The model was chosen in accordance with the investigations performed by Fiedler (2002) who had refined his finite element mesh in order to obtain a converged mesh. By applying symmetric boundary conditions it is possible to use only a quarter of the entire system for reducing computational costs. The finite element model with a relatively coarse mesh can be seen in Fig. 7.15. With submodelling techniques it is possible to build up an additional smaller model only including the direct vicinity around the aluminium beam, and to pass the displacements obtained from the global analysis on to the submodel. This procedure allows to reduce the computational costs and leads to a better convergence. The soil is modelled using three-dimensional, isoparametric, and quadratic solid elements. The beam is modelled using 8-node shell elements. The interaction between the parts is performed by coupling the two bodies with rigid connectors in vertical direction without constraining (smooth boundary) the movement parallel to the contacting surfaces. This simplified interaction approach is chosen as the vertical displacements are relatively small and the horizontal displacements are negligible, and it leads to fast convergence and effective computing times.

Tab. 7.3: Parameters for the hypoplastic material model according to Fiedler (2002)

\begin{tabular}{|c|c|c|c|c|c|c|c|}
\hline$\varphi_{c}\left[^{\circ}\right]$ & $h_{s}[\mathrm{kPa}]$ & $n$ & $e_{i 0}$ & $e_{c 0}$ & $e_{d 0}$ & $\alpha$ & $\beta$ \\
\hline 30 & $5.8 \cdot 10^{6}$ & 0.28 & 0.53 & 0.84 & 1.00 & 0.13 & 1.05 \\
\hline
\end{tabular}

In the work of Schlegel (1985) no details are given regarding the initial conditions of the soil. For modelling a system using the hypoplastic material model the initial conditions play a significant role, as the stresses directly influence the rate dependent law and therefore the soil's reaction is by far stiffer if subjected to high pressure. Because the stresses at the surface are zero, and the implemented rate dependent law cannot cope with zero stresses due to numerical reasons, a small capillary cohesion is introduced for the top layer similar to Fiedler (2002). The material parameters are adopted from Fiedler (2002) who derived them by evaluating experiments from Bauer (1992) and Wu (1992) for „Karlsruher sand“ (Tab. 7.3). Moreover, Fiedler (2002) sets the following details for the re-calculation of the experiments:

Tab. 7.4: Parameters from experiment

\begin{tabular}{|c|c|c|}
\hline $\begin{array}{c}\text { dry density of sand } \\
\gamma_{d}\left[\mathrm{kN} / \mathrm{m}^{3}\right]\end{array}$ & $\begin{array}{c}\text { density of aluminium } \\
\gamma_{A l u}\left[\mathrm{kN} / \mathrm{m}^{3}\right]\end{array}$ & $\begin{array}{c}\text { coefficient of earth pressure at rest } \\
K_{0}\end{array}$ \\
\hline 17.15 & 27.00 & 0.28 \\
\hline
\end{tabular}

The selection of the load magnitude and the type of loading was made from the consideration that a failure of the soil must not occur. For higher loads stress concentrations (localisations) appeared close to the edge of the aluminium beam as it can be seen from the maximum strains depicted in Fig. 7.16. The high shear stresses result in an abort of the analysis as shear slips develop. Such a failure mechanism 
depends on the chosen mesh, since some shear slips can only evolve if the discretisation permits. So-called regulating measures (non-local formulation, Cosserat continuum, internal length) can be used in order to improve the situation. Given that the failure of the soil is a problem which is not dealt with in this study, no further regulating measures are considered.

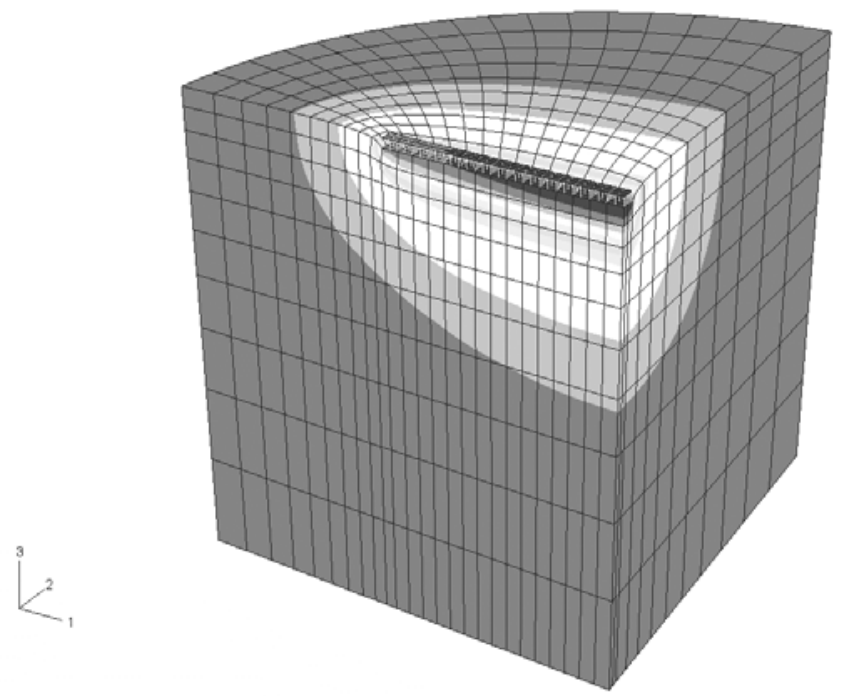

Fig. 7.15: Contour plot of the vertical displacements (scaled by factor of 30); dark areas = large displacements

Fig. 7.15 shows the vertical displacements in a contour plot with the deformations scaled by a factor of 30 . The realistic shape of the settlement and the transfer of the loads through the soil can be clearly seen. In Fig. 7.16 the Mises stresses are depicted for the loaded system. Directly below the aluminium beam stress concentrations arise.
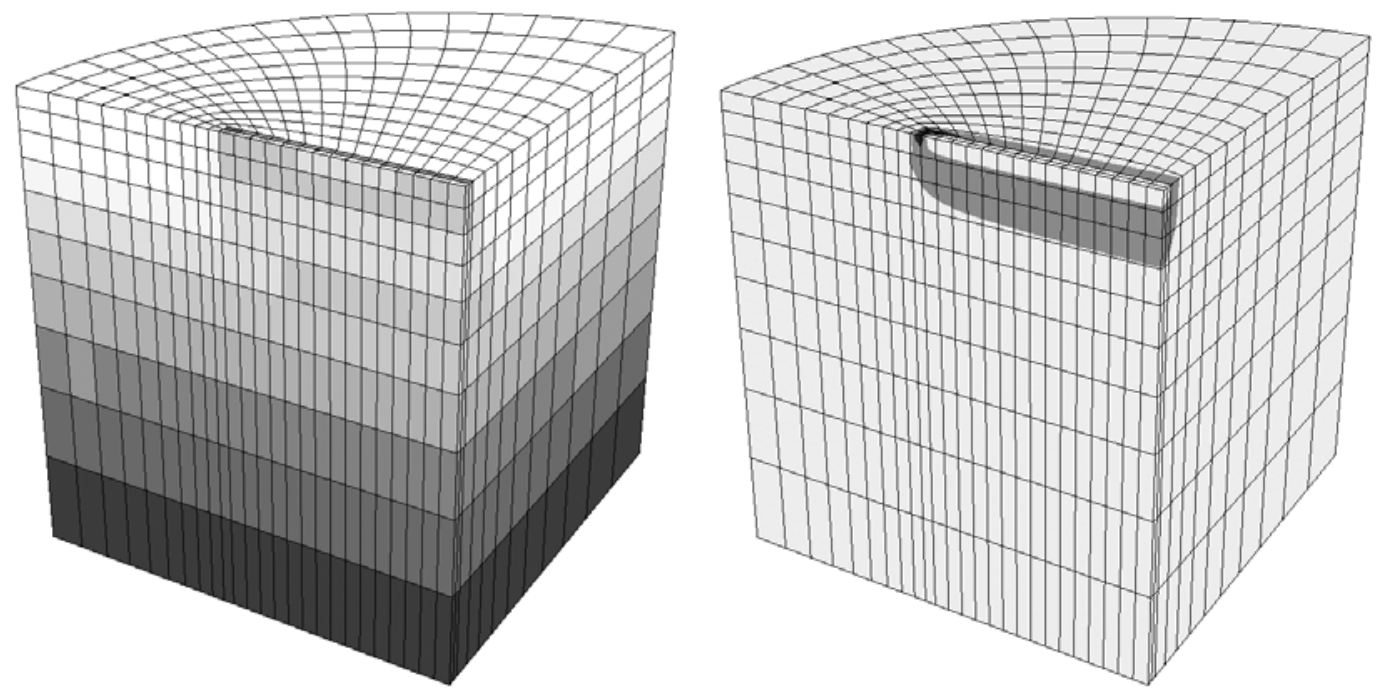

Fig. 7.16: Contour plot of the Misses stresses (left) and maximum strains (right); dark areas = high stresses and strains 
The vertical displacements of the aluminium beam are plotted along the symmetry plane in Fig. 7.17. Fig. 7.18 shows the bending moment distribution of the beam when completely loaded.

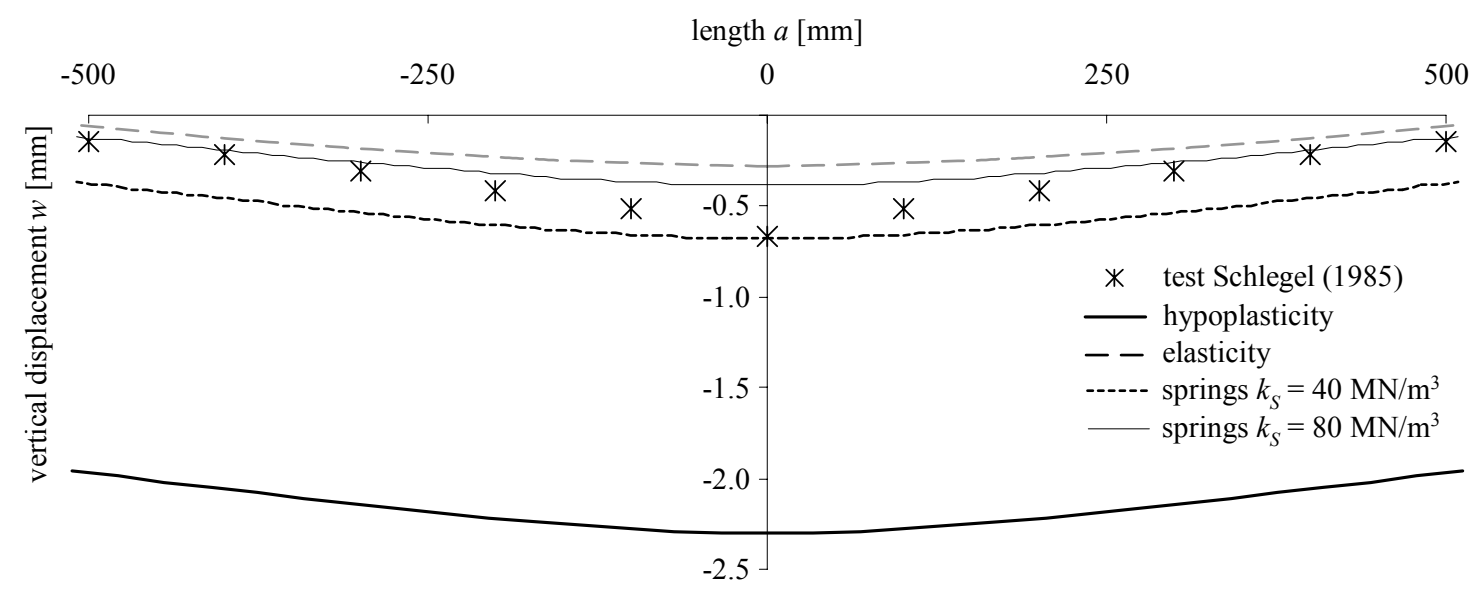

Fig. 7.17: Vertical displacements of the aluminium beam

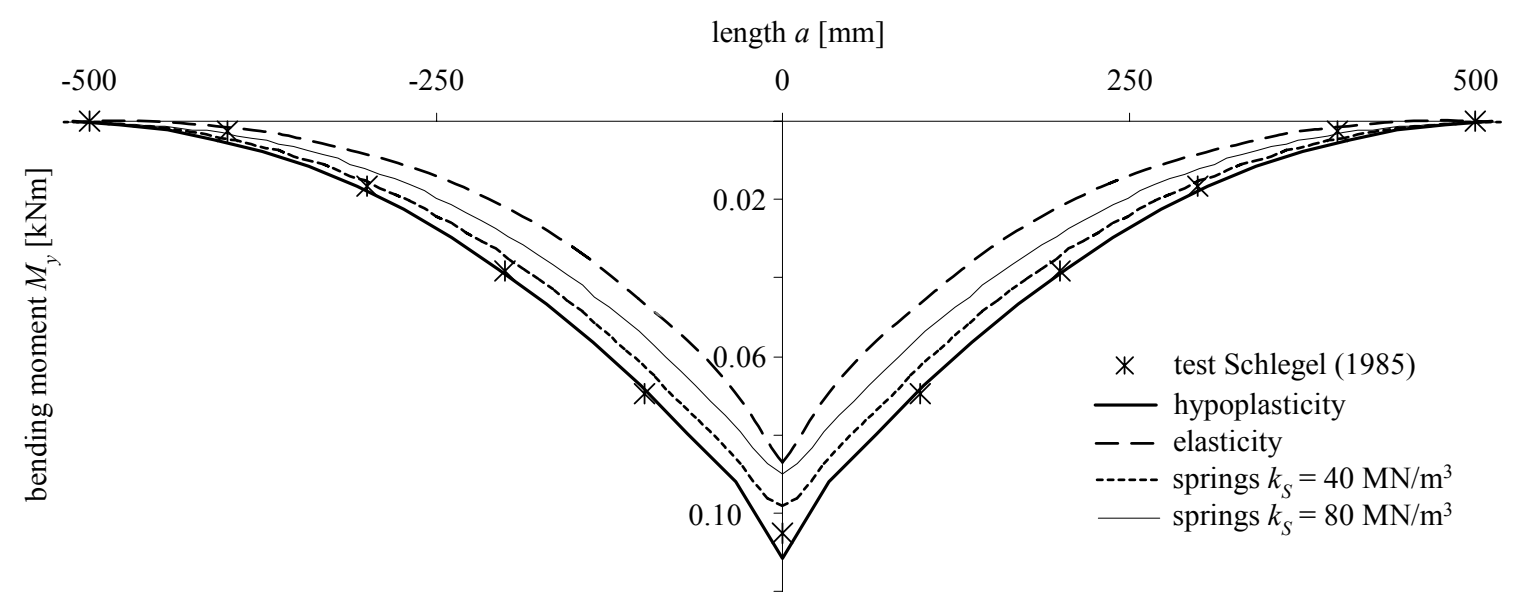

Fig. 7.18: Bending moments in aluminium beam

For comparison another analysis using a linear elastic material law for the continuum was performed. This simplified approach is based on a Young's modulus which can be determined by evaluation of the compression curve of an oedometric compression test. The magnitude of the Young's modulus is highly dependent on the chosen stress interval and the choice whether the tangent or secant modulus is used. As the stresses or applied pressure of the real system can only be assumed for most cases, the results can only deliver rough approximations. Based on the data given by Fiedler (2002) the Young's modulus is set to $7968 \mathrm{kPa}$. The results from the numerical analyses are plotted in Fig. 7.17 and Fig. 7.18. More details on evaluating an appropriate Young's modulus are given for the next comparison.

Additionally, the simple approach is modelling the soil and the SSI by linear springs is applied. Independent on the test set-up the model consists of the beam and perpendicular springs with a defined linear spring stiffness. The spring stiffness is 
approximated by using coefficients of subgrade reaction $k_{S}$ given in general design handbooks. In Schneider (1998) the values given for the coefficient of subgrade reaction $k_{S}$ range between $40 \mathrm{MN} / \mathrm{m}^{3}$ and $80 \mathrm{MN} / \mathrm{m}^{3}$ for sand in general. Thus, the numerical analyses are based on these limit values. The following comparison deals with a more detailed derivation of consistent soil data. The results are shown in Fig. 7.17 and Fig. 7.18 .

It can be seen in Fig. 7.17 that the deflections of the beam differ significantly for the different approaches. The vertical displacements for the hypoplastic model are three times as large as the displacements from the test. The displacements for the elastic continuum reach around half the magnitude of the test results. The analyses with the spring model deliver good agreement with the test results. The calculated results can be interpreted as close envelope curves for the test results. The findings for the deflection of the beam have to be handled with care, especially for the hypoplasticity, as the initial conditions significantly influence the initial stiffness and thus the settlement of the beam. Further deviations may arise due to the fact that several material parameters had to be assumed since no more details were available.

The bending moments in the beam agree well for the test and the numerical analysis using the hypoplastic material model. Still, the good agreement should not be overrated, but has to be interpreted carefully considering the assumed conditions. The results from the numerical analysis using the elastic continuum lead to maximum bending moments in the centre of the beam around $25 \%$ lower than for the other two cases. The results from the spring model lead to bending moment distributions between the other two cases, and thus to a fairly good agreement with the test results.

The results for the hypoplastic material model show that good agreement can be found for the bending moments but at the same time very large discrepancies appear for the displacements. For the application of the incremental model the input of precise initial conditions and material parameters for the soil is indispensable. Nevertheless, the task of determining the correct parameters remains. The differences in the settlement can be explained with inaccurate assumptions for the initial pressure conditions of the soil. For higher initial stresses, the soil behaves far stiffer resulting in lower displacements but only small changes of the bending moment in the beam. The issue of obtaining precise data on the initial stress state of the soil is highly difficult, making this approach hard to handle.

The aim in this study is to investigate cases concerning the SSI. For the problem of a buckling cylinder in contact with soil, the stiffness and thus the displacements play a decisive role. Nevertheless, the stresses arising in the shell as a result are very important. The comparison did not reveal satisfying findings for either of the soil models, especially as the material properties were harmonised applying simple techniques. Still, problems as localisations in form of shear slips can be neglected because the soil failure for the type of investigated structures, as e.g. combiwalls, is not of interest. The computational costs and the computing power necessary for complex 
structures in combination with the hypoplastic material model is still immense and cannot be compared with the elastic material model. Here, these computing times differed by the factor of about 10 . In this context the computing time for the spring model is incomparably small, since the soil itself is not modelled. With the very simple approach surprisingly good agreement was found.

\subsection{Comparison of different soil models for a laterally loaded pile}

\subsubsection{General}

The last example deals with a detailed analysis of a laterally loaded pile, with partial reference to Holeyman et al. (2006). In contrast to the two settlement problems discussed previously, this example involves horizontal instead of vertical contact with the soil. For the comparing case, models with non-linear springs are used. The general spring or subgrade reaction model is modified by limiting the pressure. Further, a more complex model of the $p-y$ curves is introduced in the following before deriving the consistent soil data and performing the comparing analyses.

\subsubsection{Subgrade reaction method}

The subgrade reaction method applies the coefficient of subgrade reaction as an uncoupled spring that uniformly acts as soil-pile interaction. In an assessment the bearable horizontal stress or pressure of the soil can be limited by the passive earth pressure (section 10.4.2 in DIN 1054:2005-01). The applied subgrade reaction method is based on the proposed values by Terzaghi (1955) for medium sand $\left(n_{h}=6.6 \mathrm{MN} / \mathrm{m}^{3}\right)$ and equation (7.6). In the investigated cases this restriction of the horizontal stresses is taken into account by using the passive earth pressure or the earth resistance of the spatial case according to E DIN 4085:2002-12. Due to the mobilization of the surrounding soil the earth resistance $e_{p h}$ increases. This effect is considered by introducing the representative length $l_{p g}^{E r}$ [length] instead of the actual width or length $l$ of a retaining structure for which it is implemented proportionally. In the discussed case the geometry of the pile is defined by the diameter $D$ and the embedded length $l$ and therefore the representative length is defined as the representative diameter $D_{p g}^{E r}$ [length] arising from self-weight and $D_{p c}^{E r}$ [length] arising from cohesion. In accordance with E DIN 4085:2002-12 they can be specified as:

$$
\begin{gathered}
D_{p g}^{E r}=0.55(1+2 \tan \varphi) \sqrt{D \cdot l} \quad \text { for } \quad D<0.3 l \\
D_{p g}^{E r}=D+0.6 \cdot l \cdot \tan \varphi \quad \text { for } \quad D \geq 0.3 l
\end{gathered}
$$

and

$$
D_{p c}^{E r}=1.1(1+0.75 \tan \varphi) \sqrt{D \cdot l} \quad \text { for } \quad D<0.3 l
$$




$$
D_{p c}^{E r}=0.5[D+0.9(1+\tan \varphi) \cdot h] \quad \text { for } \quad D \geq 0.3 l
$$

The passive earth resistance $e_{p h}[\mathrm{kN} / \mathrm{m}]$ can be calculated as the sum of $e_{p g h}$ and $e_{p c h}$ without external loads as a function of the depth $z$ according to E DIN 4085:2002-12 as follows:

$$
e_{p h}=e_{p g h}+e_{p c h}
$$

with

$$
\begin{gathered}
e_{p g h}=\gamma \cdot z \cdot K_{p g h} \cdot D_{p g}^{E r} \\
e_{p c h}=c \cdot K_{p c h} \cdot D_{p c}^{E r}
\end{gathered}
$$

The values for $K_{p g h}$ and $K_{p c h}$ can be obtained from tables in E DIN 4085:2002-12 for different angles of wall friction $\delta$ and the angle of internal friction $\varphi$.

The passive earth resistances from E DIN 4085:2002-12 are only valid for the general spatial case, i.e. a long wall, and thus not directly applicable to piles. Nevertheless, as a conservative approach for the pile the application of the 2D case from E DIN 4085:2002-12 may suffice. Besler (1998) summarized different models for earth resistances in his work. In his work he describes for example the models by Streck and Weißenbach for the application of spatial earth pressures acting on small pressure areas which are implemented in different pile programs (e.g. GGU-LATPILE) for calculating laterally loaded piles. As here only a brief insight is to be given for the subgrade reaction method for more details it is referred to e.g. Besler (1998).

\subsubsection{The p-y curves}

A more elaborate procedure which is based on the concept of the coefficient of subgrade reaction is the method using so-called $p-y$ curves. The lateral pile behaviour is described by the following governing equation originally presented by Hetenyi (1946):

$$
E_{p} I_{p} \frac{d^{4} y}{d z^{4}}+p=0
$$

in which $E_{p} I_{p}$ is the flexural stiffness of the pile. McClelland and Focht (1956) initially extended the subgrade reaction approach using the finite difference technique to account for non-linear soil reaction versus deflection relationships. In the first version of the method an empirical relationship between the stress-strain curves from triaxial compression tests and the real load-deflection behaviour of piles was implemented.

Based on field load tests, Reese et al. (1974) derived empirical p-y curves for sands. Their $p$ - $y$ curves are based on linear and parabolic functions defined for segments. The parameters for each function are given by the effective angle of internal friction $\varphi^{\prime}$ and the initial stiffness $E_{s, i}\left[f / l^{2}\right]$ expressed as $k_{i} \cdot z$. 
Tab. 7.5: Initial stiffness $k_{i}$ for sand (after Reese et al., 1974)

\begin{tabular}{|c|c|c|c|}
\hline relative density & loose $<35 \%$ & medium & dense $>65 \%$ \\
\hline$k_{i}\left[\mathrm{MN} / \mathrm{m}^{3}\right]$ & 6.8 & 24.4 & 61 \\
\hline
\end{tabular}

The more detailed approach given by Reese et al. (1974) uses simplified spatial failure models for the soil. The concept proposed by Reese et al. (1974), which at the beginning only included sand, states characteristic curves for the mobilized resistance of the surrounding soil $p$ [force/length] per length unit $\Delta \mathrm{l}$ (Fig. 7.19) for a corresponding horizontal displacement $y$ [length]. In this procedure the pile is divided into discrete segments that are supported at their intersections by non-linear springs. Additionally, the stiffness of the non-linear springs changes as a function of the depth $z$.
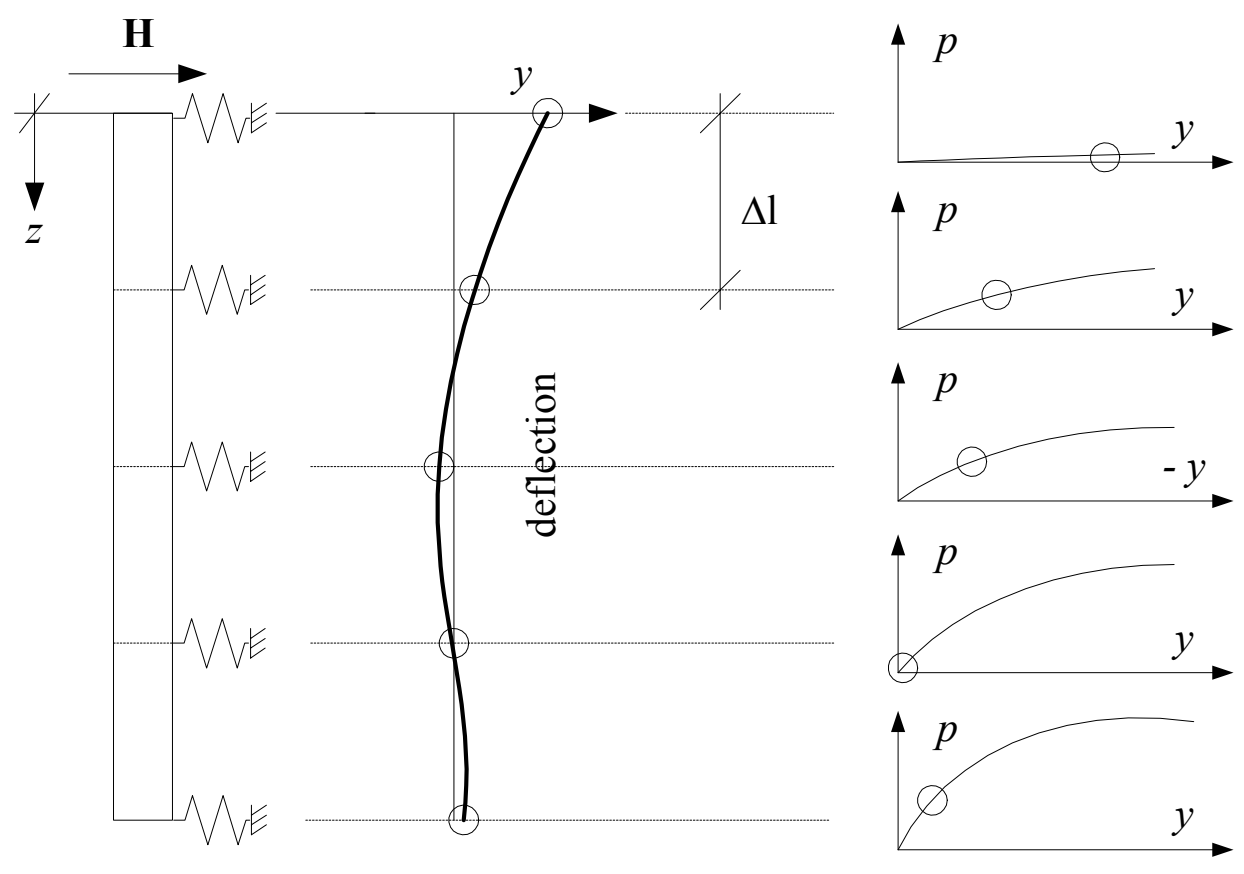

Fig. 7.19: Concept of the p-y curves for an embedded pile

The $p-y$ curves introduced by Reese et al. (1974) are based on linear and parabolic functions defined in sections. The parameters for each function are given by the angle of internal friction $\varphi$ and the initial stiffness $k_{i}$ [force/length ${ }^{3}$ ]. With the angle of internal friction the geometrical coefficients $\alpha_{G}$ und $\beta_{G}$, the earth pressure coefficient at rest $K_{0}$ and the active earth pressure coefficient $K_{a}$ for the investigated case can be calculated:

$$
\begin{gathered}
\alpha_{G}=\varphi / 2 \\
\beta_{G}=45+\varphi / 2 \\
K_{0}=1-\sin \varphi \\
K_{a}=\tan ^{2}(45-\varphi / 2)
\end{gathered}
$$


The coefficients $\alpha_{G}$ and $\beta_{G}$ correspond to the failure mechanism near the soil surface where the self-weight of the wedge of soil represents the soil resistance. The soil resistance $p_{s t}$ close to the surface is defined as the difference between the passive earth pressure according to Coulomb and the active earth pressure according to Rankine:

$$
p_{s t}(z)=\gamma^{\prime} z\left[\begin{array}{l}
\frac{K_{0} z \tan \varphi \sin \beta_{G}}{\tan \left(\beta_{G}-\varphi\right) \cos \alpha_{G}}+\frac{\tan \beta_{G}}{\tan \left(\beta_{G}-\varphi\right)}\left(D+z \tan \beta_{G} \tan \alpha_{G}\right) \\
+K_{0} z \tan \beta_{G}\left(\tan \varphi \sin \beta_{G}-\tan \alpha_{G}\right)-K_{a} D
\end{array}\right]
$$

Based on the Mohr-Coulomb theory and a plastic failure of cubic elements the soil resistance $p_{s d}$ for the deeper regions can be calculated:

$$
p_{s d}(z)=D \gamma^{\prime} z\left\lfloor K_{a}\left(\tan ^{8} \beta_{G}-1\right)+K_{0} \tan \varphi \tan ^{4} \beta_{G}\right\rfloor
$$

The minimum of $p_{s t}$ and $p_{s d}$ is adopted and defined as $p_{u}(z)$ in the following. With these coefficients, the initial stiffness $k_{i}$ [force/length ${ }^{3}$ ], and an empirical correction factor $A$, the $p$-y curves according to Reese et al. (1974) can be established. Details on the evaluation of the curve segments are for example given in Reese et al. (1974), Reese and Van Impe (2001), and Wiemann et al. (2004). The concept of the $p-y$ curves is implemented in the guidelines of the American Petroleum Institute (API) „Recommended Practice for Planning, Designing and Constructing Fixed Offshore Platforms - Working Stress Design (RP 2A-LRFD und -WSD)“(2000) and is illustrated in Fig. 7.19. Further, the model of the $p-y$ curves is introduced into the Norwegian guideline „Det Norske Veritas“ (2004), the standard procedures of the Germanischer Lloyd (1999), and the draft for the ISO/DIS 19902:2004 „Petroleum and Natural Gas Industries — Fixed Steel Offshore Structures““ (2004).

For the design procedure the concept was simplified. Thus, in more recent guidelines such as for example the „Det Norske Veritas“ (2004) the $p-y$ curves for sand are described with continuous hyperbolic tangent functions:

$$
p(y, z)=A(z) p_{u}(z) \tanh \left(\frac{k_{i} z}{A(z) p_{u}(z)} y\right)
$$

where the empirical correction factor $A(z)$, which augments the soil resistance in the region close to the surface, is linearised for the means of assessment:

$$
A(z)=3-0.8 \frac{z}{D} \geq 0.9
$$

The limitation of the maximum lateral stresses in the soil for static loads by the soil resistance $p_{u}(z)$ [force/length] from equation (7.43) and (7.44) is given in a simpler manner for all cases alike based on the parameters $K_{0}=0.4$ (loose sand) and $\alpha=\varphi / 2$ as follows:

$$
p_{u}(z)=\min \left\{\begin{array}{c}
\gamma^{\prime} z^{2} C_{1}+\gamma^{\prime} z C_{2} d \\
\gamma^{\prime} z C_{3} d
\end{array}\right\}
$$


The coefficients $C_{1}, C_{2}$, and $C_{3}$ can be taken from the diagram in Fig. 7.20 for the corresponding effective angle of friction. The value for the initial stiffness $k_{i}$ [force/length ${ }^{3}$ ] can also be taken from Fig. 7.20 according to ISO/DIS 19902:2004 or from a similar diagram as given in „Det Norske Veritas“ (2004). For a more detailed description of the procedure see Reese and Van Impe (2001), and Wiemann et al. (2002).

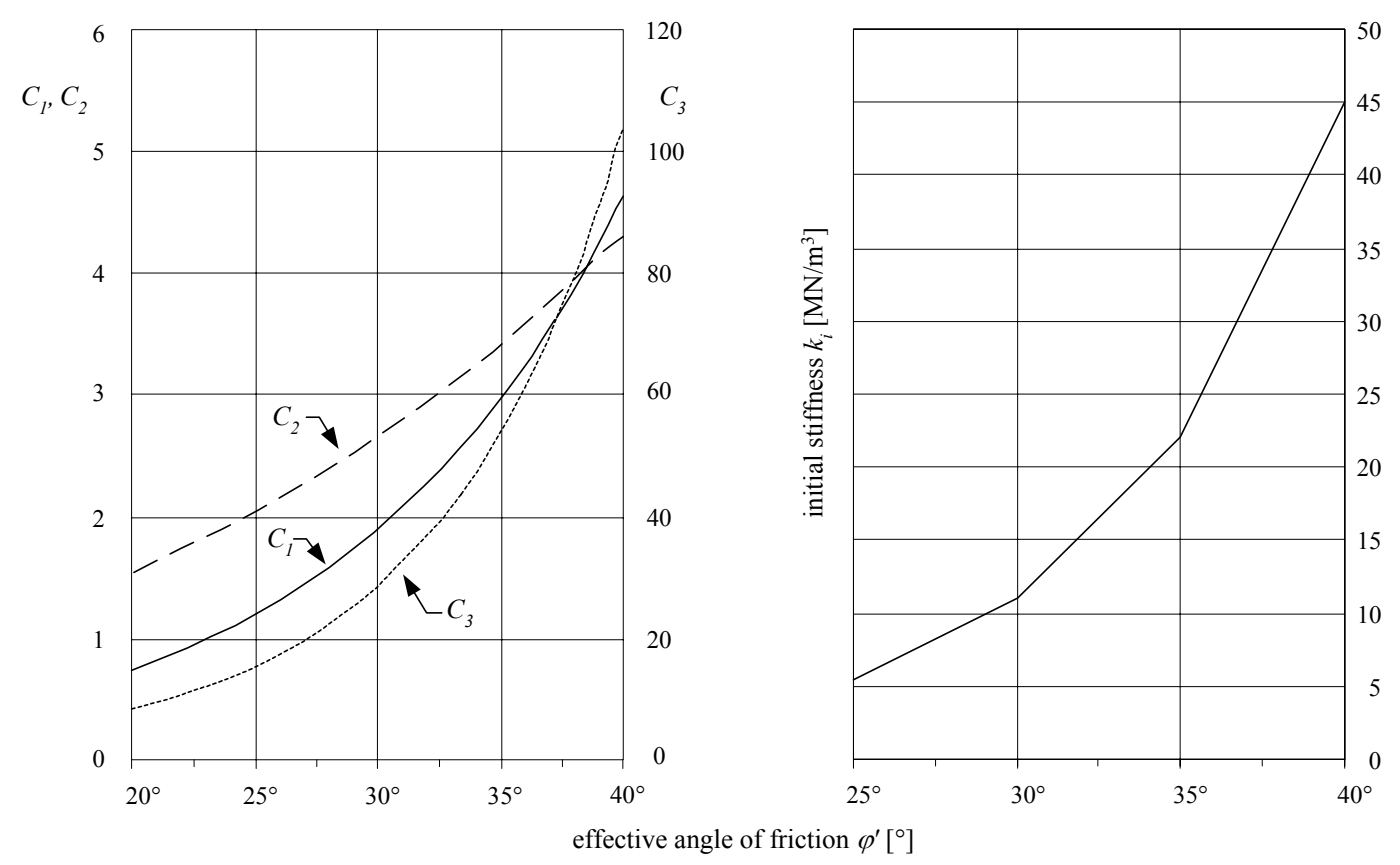

Fig. 7.20: Coefficients $C_{1}, C_{2}$, and $C_{3}$, and initial stiffness $k_{i}$ for sand below water table as a function of $\varphi^{\prime}$ according to ISO/DIS 19902:2004

For completeness it is mentioned that several more researchers have worked on producing elaborate $p-y$ curves for different soils. For example Bransby (1999) proposed a power law for the $p-y$ curves and performed finite element and finite difference calculations with two- and three-dimensional models. Other suggested $p-y$ curves are based on empirical curve fitting or full-scale tests or centrifuge model tests (cfr. Barton et al., 1983; Yan and Byrne, 1992; Dyson and Randolph, 1997).

\subsubsection{Reference case and modelling}

As for a reference case the different models for the soil and the interaction between soil and structure are calculated and the deformations are compared. Similar comparisons for the field of offshore wind energy structures were performed i.e. by Wiemann et al. (2004), Dahlhoff et al. (2003), and Grabe et al. (2005).

A $3 \mathrm{~m}$ long tube with an external diameter of $D=200 \mathrm{~mm}$ and the thickness $t=2 \mathrm{~mm}$ which is vertically embedded in dry sand is chosen as reference case (Fig. 7.21). The choice of geometry and system is made in accordance with the experimental part of the investigations. Details on the test set-up are given in Charue et al. (2007). The modelled tube is positioned vertically in a cylindrical casing and subsequently embedded in dry 
sand. Based on the previous findings it is chosen to use a casing with a diameter of $6 \mathrm{~m}$ corresponding to an influenced area of 30-pile radii from the centre of the pile as a numerical model for the comparing analyses in order to obtain more realistic comparisons between the different approaches. Initial conditions are imposed for the investigation consisting of a self-equilibrating geostatic stress field with a weightless pile and an earth pressure coefficient $K_{0}$ at rest calculated according to (7.41). Subsequently the pile head is loaded incrementally with a horizontal load of $10 \mathrm{kN}$ corresponding to a factor of safety of approximately 1.4 (Holeyman et al., 2006) and taking non-linear geometric effects into account. In this study only the vertical displacement at the top of the pile is restrained allowing it to move in the lateral direction and to rotate freely. No boundary conditions are applied to the foot of the pile. Although, the chosen boundary conditions may not represent a real situation it can provide a first basis for comparing the soil models. It has to be emphasized that due to the lack of a constraint at the bottom of the pile, large lateral forces may evolve in the sand. The boundary conditions are depicted in Fig. 7.21. The model assumes that the soil below the pile tip has no influence on the pile behaviour.

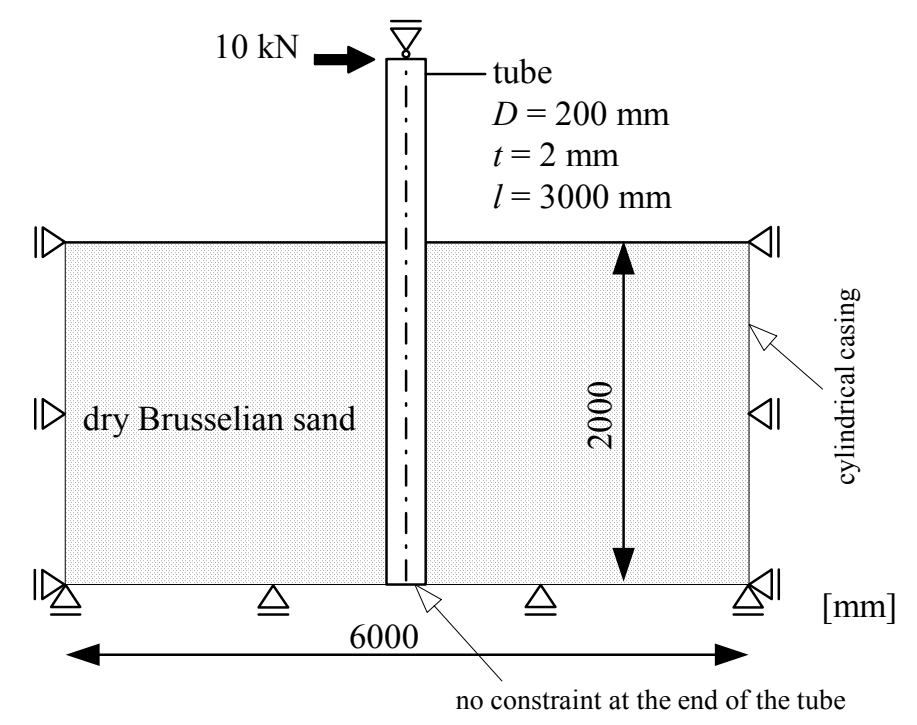

Fig. 7.21: Cross-section of the reference case with applied boundary conditions

"Brusselian Sand" is used for the study which was thoroughly investigated by Vanden Berghe (2001) with numerical and experimental means. The physical characteristics of the sand are summarized in Tab. 7.6. According to the ASTM Standard D-2487, the sand is classified as poorly graded (SP). In the analyses the sand is assumed to be installed with an initial void ratio of 0.7 at a weight of $\gamma=15.25 \mathrm{kN} / \mathrm{m}^{3}\left(D_{r}=76 \%\right)$.

Tab. 7.6: Physical parameters of the "Brusselian Sand"

\begin{tabular}{|c|c|c|c|c|c|c|}
\hline $\begin{array}{c}d_{50} \\
{[\mathrm{~mm}]}\end{array}$ & $\begin{array}{c}C_{u} \\
{[-]}\end{array}$ & $\begin{array}{c}\gamma_{\min } \\
{\left[\mathrm{kN} / \mathrm{m}^{3}\right]}\end{array}$ & $\begin{array}{c}\gamma_{\max } \\
{\left[\mathrm{kN} / \mathrm{m}^{3}\right]}\end{array}$ & $\begin{array}{c}\gamma_{s} \\
{\left[\mathrm{kN} / \mathrm{m}^{3}\right]}\end{array}$ & $\begin{array}{c}e_{\max } \\
{[-]}\end{array}$ & $\begin{array}{c}e_{\min } \\
{[-]}\end{array}$ \\
\hline 0.18 & 2.2 & 11.98 & 17.00 & 25.97 & 1.18 & 0.52 \\
\hline
\end{tabular}


For the 1-D analyses the tube and the soil is modelled with a FE-program ABAQUS by using linear beam elements in combination with linear and non-linear connector elements. For comparison analyses using the commercial program LPILE are performed.

For the 3-D approaches the modelling of the tube and the soil is performed with linear isoparametric elements. The tube is modelled using continuum shells in order to keep a shell formulation for the structure but at the same time allowing smoother contact surfaces. An important issue for simulating pile behaviour is the modelling of the area close to the contact zone between soil and pile. For laterally loaded piles, this zone is subjected to frictional behaviour with a possible separation due to lateral displacement, removing all stress transfer between soil and pile (no adhesion). Ignoring these factors can lead to a significant overestimation of the interaction forces between pile and soil. The contact is defined using a surface-to-surface contact with assigned node pairs. For the investigated case surface based contact is applied by using augmented Lagrange formulation, allowing separation after contact. In case of a material model that takes account of dilation, it has to be stressed that due to the distortions in the contact area the volume changes and hence the Coulomb friction law may only conditionally be valid (cfr. Wernick, 1978). Nevertheless, based on the suggested values of Khulaway (1991) for smooth steel piles, an angle of wall friction $\delta=0.5 \varphi$ is introduced by working with a Coulomb friction law. The friction is taken account of for the 3-D analyses since the $p-y$ approaches are based on empirical findings and therefore friction effects are incorporated. The hypoplasticity formulation implemented by Nübel and Niemunis (1999) as a FORTRAN routine in ABAQUS was used for the analyses.

\subsubsection{Deriving a set of consistent soil data}

In order to provide an indispensable basis for comparison a set of consistent soil data is derived for the investigated "Brusselian" sand. Parameters were first derived using experimental data from previous laboratory investigations (triaxial and oedometer tests) performed by Vanden Berghe (2001). Annex C deals with his experimental and numerical results for triaxial and oedometric compression tests which are compared and validated with numerical element tests applying hypoplasticity. Tab. 7.7 summarizes the consistent soil data for the investigated material models defining the soil parameters that are used for the corresponding approach. Additionally, further comments are given in the following concerning the different analyses, the modelling procedures, and the derivation of the corresponding soil parameters.

From a series of triaxial tests by Vanden Berghe (2001), the angle of friction $\varphi^{\prime}$ and the cohesion $c^{\prime}$ can be derived using Mohr's circles. The parameters can be evaluated with $33^{\circ}$ and $0 \mathrm{kPa}$, respectively (Fig. 7.22). 


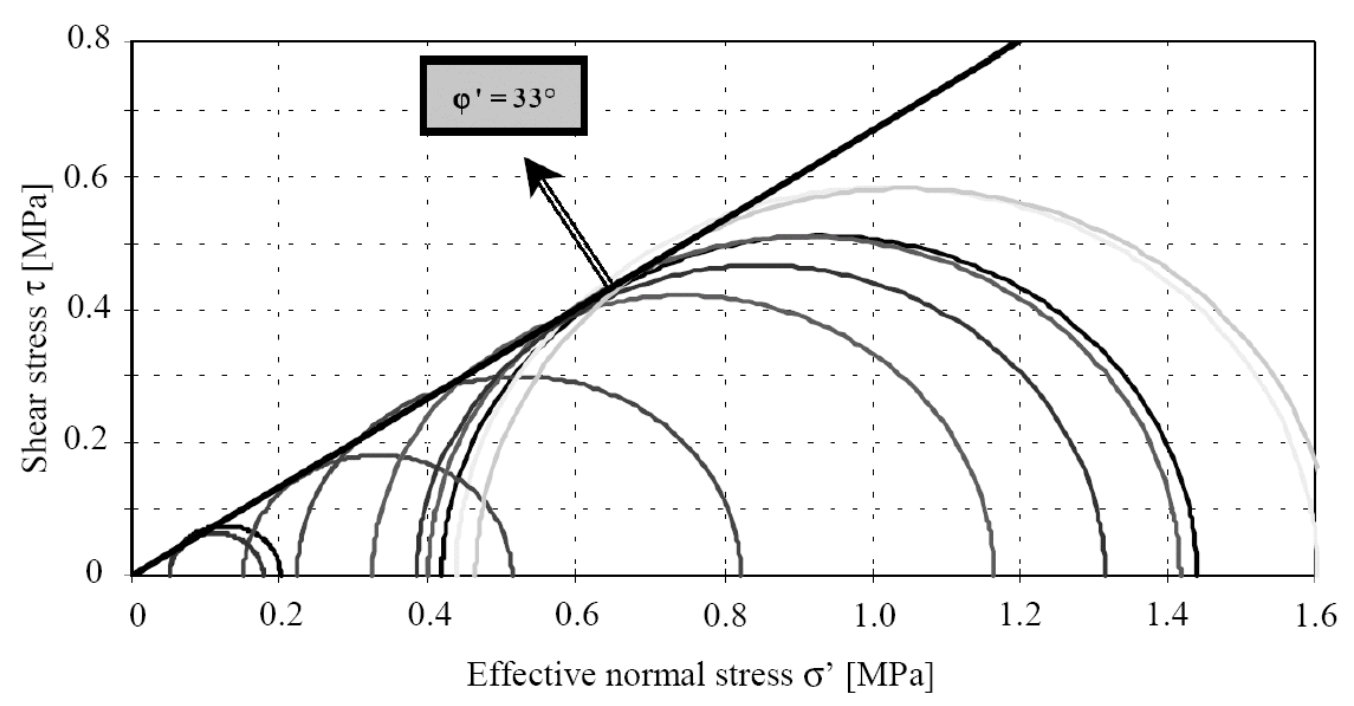

Fig. 7.22: Mohr's Circle from drained and undrained monotonic triaxial tests for "Brusselian" sand with $e=0.7$ (from Vanden Berghe, 2001)

For the p-y method according to Reese et al. (1974) with the program LPILE the coefficients based on the angle of friction $\varphi^{\prime}$ are acquired with equation (7.39) to (7.44). The (initial) stiffness $k_{i}$ can be derived as a function of $\varphi^{\prime}$ or as a function of the relative density of the sand. According to the recommendations in the API (1994) the sand is assumed to be in the state of medium density and thus the stiffness $k_{i}$ is chosen according to Tab. 7.5. Further values are taken from diagrams (cfr. Reese and Van Impe, 2001).

As stated in Holeyman et al. (2006), it should be pointed out that the stiffness $k_{i}$ represents an initial stiffness for the $p-y$ procedure while it corresponds to a secant stiffness under working load conditions for the Terzaghi (subgrade reaction method) procedure.

For the subgrade reaction method (SRM) with the program ABAQUS a routine in Visual Basic was programmed for implementing the beam model into ABAQUS, and in order to perform flexible parametric studies. For all applied subgrade reaction methods the horizontal stresses are limited to the passive earth resistance (7.35). The coefficient of subgrade reaction is applied in such a way that the integration of it over the pile length marks independent from the type of variation the same value. This approach is chosen in order to establish a mutual reference value. Still, the chosen method is not based on a research or reference but on a simple approach for referencing the values. After Terzaghi (1955) and (7.4) with $C=1$ and equation (7.6) considering a medium sand the distributions of the coefficient of subgrade reaction are defined by the following values: $k_{s, \text { const }}=66 \mathrm{MN} / \mathrm{m}^{3}, k_{s, \text { lin,pile tip }}=132 \mathrm{MN} / \mathrm{m}^{3}$ and $k_{s, \text { para,pile tip }}=99 \mathrm{MN} / \mathrm{m}^{3}$. 
Tab. 7.7: Material properties for the „Brusselian Sand“ and the different models based on Vanden Berghe (2001)

\begin{tabular}{|c|c|c|c|c|c|c|c|c|c|c|}
\hline \multicolumn{2}{|c|}{ model } & 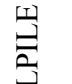 & \multicolumn{5}{|c|}{ ABAQUS } & \multicolumn{3}{|c|}{ ABAQUS } \\
\hline \multicolumn{2}{|c|}{ program } & \multicolumn{6}{|c|}{ 1D-model } & \multicolumn{3}{|c|}{ 3D-model } \\
\hline \multicolumn{2}{|c|}{ approach } & 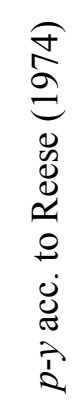 & 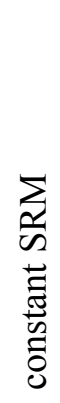 & 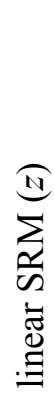 & 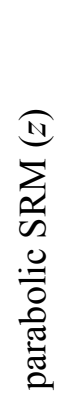 & 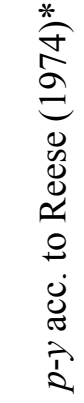 & 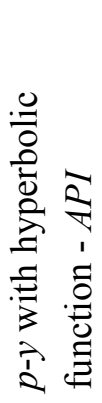 & 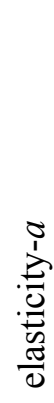 & 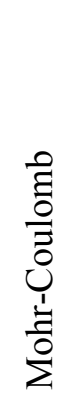 & 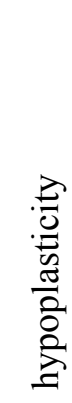 \\
\hline parameter & dim. & \multicolumn{9}{|c|}{ used } \\
\hline$\varphi=\varphi^{\prime}\left[{ }^{\circ}\right]$ & 33 & + & + & + & + & + & + & - & + & + \\
\hline \multirow{2}{*}{$k_{i}\left[\mathrm{MN} / \mathrm{m}^{3}\right]$} & 17.6 & - & - & - & - & - & + & - & - & - \\
\hline & 24.4 & + & - & - & - & + & - & - & - & - \\
\hline$n_{h}\left[\mathrm{MN} / \mathrm{m}^{3}\right]$ & 6.6 & - & \multicolumn{3}{|c|}{ provided the basis } & - & - & - & - & - \\
\hline$\gamma=\gamma^{\prime}\left[\mathrm{kN} / \mathrm{m}^{3}\right]$ & 15.25 & + & + & + & + & + & + & + & + & + \\
\hline$\overline{A_{s}}, B_{s}[-]$ & $(z)$ & $+*$ & - & - & - & $+* *$ & - & - & - & - \\
\hline$C_{1}, C_{2}, C_{3}[-]$ & $(\varphi)$ & - & - & - & - & - & $+^{\#}$ & - & - & - \\
\hline \multirow{2}{*}{$E\left[\mathrm{MN} / \mathrm{m}^{2}\right]$} & 2.14 & - & - & - & - & - & - & + & - & - \\
\hline & 4.40 & - & - & - & - & - & - & - & + & - \\
\hline$\mu[-]$ & 0.35 & - & - & - & - & - & - & + & + & - \\
\hline$v\left[^{\circ}\right]$ & 6 & - & - & - & - & - & - & - & + & - \\
\hline$\delta\left[^{\circ}\right]$ & 16.5 & - & + & + & + & - & - & + & + & + \\
\hline$c\left[\mathrm{kN} / \mathrm{m}^{2}\right]$ & 0.2 & - & + & + & + & - & - & - & + & $+*$ \\
\hline$e_{d 0}[-]$ & 0.52 & - & - & - & - & - & - & - & - & + \\
\hline$e_{c 0}[-]$ & 0.88 & - & - & - & - & - & - & - & - & + \\
\hline$e_{i 0}[-]$ & 1.21 & - & - & - & - & - & - & - & - & + \\
\hline$e_{0}[-]$ & 0.70 & - & - & - & - & - & - & - & - & + \\
\hline$n[-]$ & 0.35 & - & - & - & - & - & - & - & - & + \\
\hline$\alpha[-]$ & 0.3 & - & - & - & - & - & - & - & - & + \\
\hline$\beta[-]$ & 1.1 & - & - & - & - & - & - & - & - & + \\
\hline$h_{s}\left[\mathrm{MN} / \mathrm{m}^{2}\right]$ & 200 & - & - & - & - & - & - & - & - & + \\
\hline $\begin{array}{l}S R M=\text { subgrac } \\
*=\text { from diagr } \\
* *=\text { with appr }\end{array}$ & $\begin{array}{l}\text { reactic } \\
\text { ms acc. }\end{array}$ & $\begin{array}{l}\text { meth } \\
\text { Rees }\end{array}$ & $\begin{array}{l}\cdot \\
d V a\end{array}$ & $\begin{array}{l}\text { rov } \\
\text { se (2 }\end{array}$ & nver & $\begin{array}{l}\text { ice } \\
=d e t \\
\text { npe }(2\end{array}$ & $\begin{array}{l}{ }^{\#}=a \\
\text { belows } \\
\text { 1) with }\end{array}$ & $\begin{array}{l}\text { Fig } \\
\text { ace }\end{array}$ & & \\
\hline
\end{tabular}


For the p-y method according to Reese et al. (1974) with the program ABAQUS another routine was programmed in Visual Basic in order to link a parametric input with the program ABAQUS. Based on the specification of the angle of friction, the corresponding coefficients are calculated with equations (7.39) to (7.44). The parameters according to Fig. 7.20 are defined by linear functions in segments in order to obtain input data for the routine that is easier to handle. The stiffness $k_{i}$ is chosen according to Tab. 7.5.

For the p-y method using hyperbolic function with the program ABAQUS a conflict arises when evaluating the coefficients. The simplified curves for the coefficients $C_{1}$, $C_{2}$, and $C_{3}$ according to Fig. 7.20 are given for an earth pressure coefficient at rest of $K_{0}=0.4$ (loose sand). In contrast the calculation of the earth pressure coefficient at rest after (7.41) results for $\varphi=33^{\circ}$ in a slightly higher value of $K_{0}=0.46$ (7.44). The (initial) stiffness $k_{i}$ is taken from Fig. 7.20.

For the approaches elasticity, Mohr-Coulomb, and hypoplasticity with the program ABAQUS using a 3-D model three-dimensional solid continuum elements are used. For the analyses using the Mohr-Coulomb theory and the hypoplasticity a small capillary cohesion $c$ of $0.2 \mathrm{kN} / \mathrm{m}^{2}$ is applied. This cohesion is specified because in the hypoplastic material law divisions by the trace of the stress tensor appear, and a division by zero must not take place (i.e. pressure $=0$ ). Further, a small cohesion is needed for the Mohr-Coulomb theory in order to prevent numerical problems arising from tensile stresses.

For the homogeneous elastic model, the strategy adopted by Holeyman et al. (2006) comprising of evaluating a constant Young's modulus $E$ from Terzaghi's empirical recommendations for the soil modulus $E_{s}$ or the coefficient of subgrade reaction $k_{S}$ is applied. As shown before, the coefficient of subgrade reaction with equation (7.6) considering a medium sand can be defined as a linear function with a maximum at the tip $(z=2 \mathrm{~m})$ of $k_{s, \text { lin,pile tip }}=132 \mathrm{MN} / \mathrm{m}^{3}$ and $0 \mathrm{MN} / \mathrm{m}^{3}$ at ground level. In a preliminary study an equivalent and constant coefficient of subgrade reaction $k_{S}$ for the case of a homogeneous medium is calculated for the application in the purely elastic model. The same lateral deflection of $3.8 \mathrm{~cm}$ for the pile head, obtained from the numerical analysis using a linear distribution of the coefficient of subgrade reaction including a limiting passive earth pressure, can be calculated for a constant modulus of $13.5 \mathrm{MN} / \mathrm{m}^{3}$ of subgrade reaction without taking limiting passive earth pressure into account. The significance of the layers near the surface ground increases as the limiting earth pressure is neglected. 


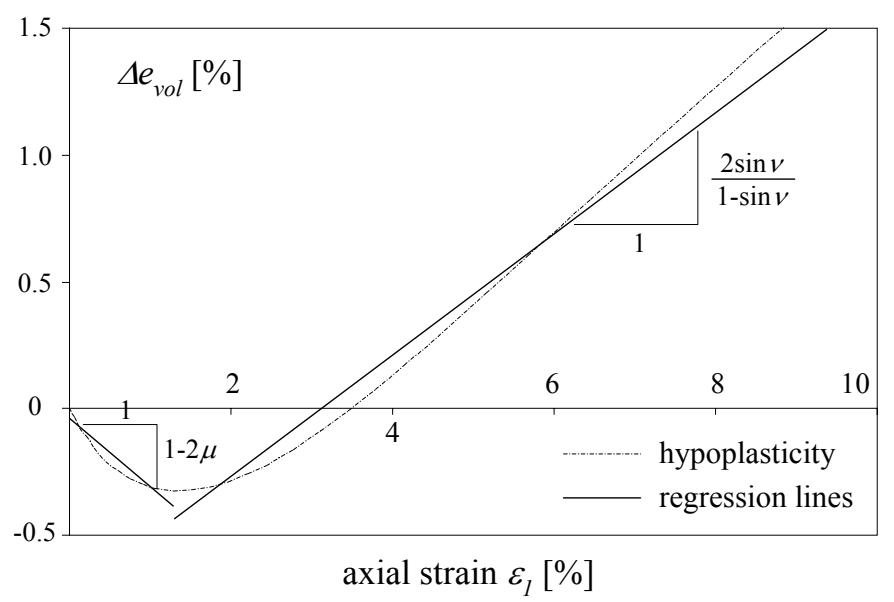

Fig. 7.23: Evaluation of the angle of dilation $v$ and the Poisson ratio $\mu$ from hypoplastic analyses of a drained triaxial compression test with $\sigma_{m, 0}=10 \mathrm{kPa}$

In the next step, the Young's modulus $E$ is back-calculated applying a relationship between the coefficient of subgrade reaction and the Young's modulus based on a 2-D plane strain system with a rigid cylinder displaced laterally in an homogenous elastic medium as summarized in Tab. 7.1. Deriving the formulas given by Baguelin et al. (1977) for the case of $r_{\text {out }}=30 R$ and $\mu=0.35$ leads to $C=1.26$ (elastic- $a$ ). Interpolating the values given in Tab. 7.1 for a rigid pin in hole with no soil-pile adhesion linearly results in $C=0.76$ (elastic- $b$ ). Considering a constant modulus of $13.5 \mathrm{MN} / \mathrm{m}^{3}$ leads to a Young's modulus $E$ of $2.14 \mathrm{MN} / \mathrm{m}^{2}$ for elastic-a and $E=3.55 \mathrm{MN} / \mathrm{m}^{2}$ for elastic-b.

The Poisson ratio for the elastic and elasto-plastic analyses is derived from comparing numerical hypoplastic analyses since no experimental data for low confining pressures are available. The Poisson ratio is evaluated from the gradient of the first part of the volumetric deformation - axial deformation curve from a drained triaxial test (Annex C) with an initial mean pressure of $10 \mathrm{kPa}$ as depicted in Fig. 7.23. The choice of the stress interval is explained in the following details on the Mohr-Coulomb approach. A linear regression line is calculated for the first section leading to a Poisson ratio $\mu=0.35$. The variation of the Poisson ratio with different stress levels (confining pressures) is rather small; e.g. for an initial mean pressure of $50 \mathrm{kPa}$ instead of $10 \mathrm{kPa}$ the Poisson ratio increases by about $2 \%$.

The elasto-plastic model is based on a materially linear behaviour before plastic flow, for which a function defines the yield surface (Mohr-Coulomb). Since the material model consists of a plastic behaviour the corresponding elastic behaviour needs to be defined differently in order to allow a comparison with the latter case. The common approach of defining the elasticity as the Young's modulus $E^{50}$ at $50 \%$ of the failure load from triaxial tests is adopted. The evaluation is performed according to Holeyman et al. (2006). Mostly, the displacements of a laterally loaded pile is governed by the soil layers near the ground level where only small confining pressures appear. A first investigation of the stress distribution for the investigated case subjected to self-weight 
(geostatic loading) reveals a mean or confining pressure $\sigma_{m}$ increasing linearly over the depth from $0 \mathrm{kPa}$ to $20 \mathrm{kPa}$ at the tip of the pile $(z=2 \mathrm{~m})$ with

$$
\sigma_{m}=\frac{1}{3}\left(\sigma_{1}+\sigma_{2}+\sigma_{3}\right)
$$

and the principal stresses $\sigma_{\mathrm{i}}$. From preliminary analyses with different material models it can be seen that the mean stresses rise up to $100 \mathrm{kPa}$ in a depth of about $0.6 \mathrm{~m}$ on one side and up to $120 \mathrm{kPa}$ at the foot of the pile on the other side (Fig. 7.24).

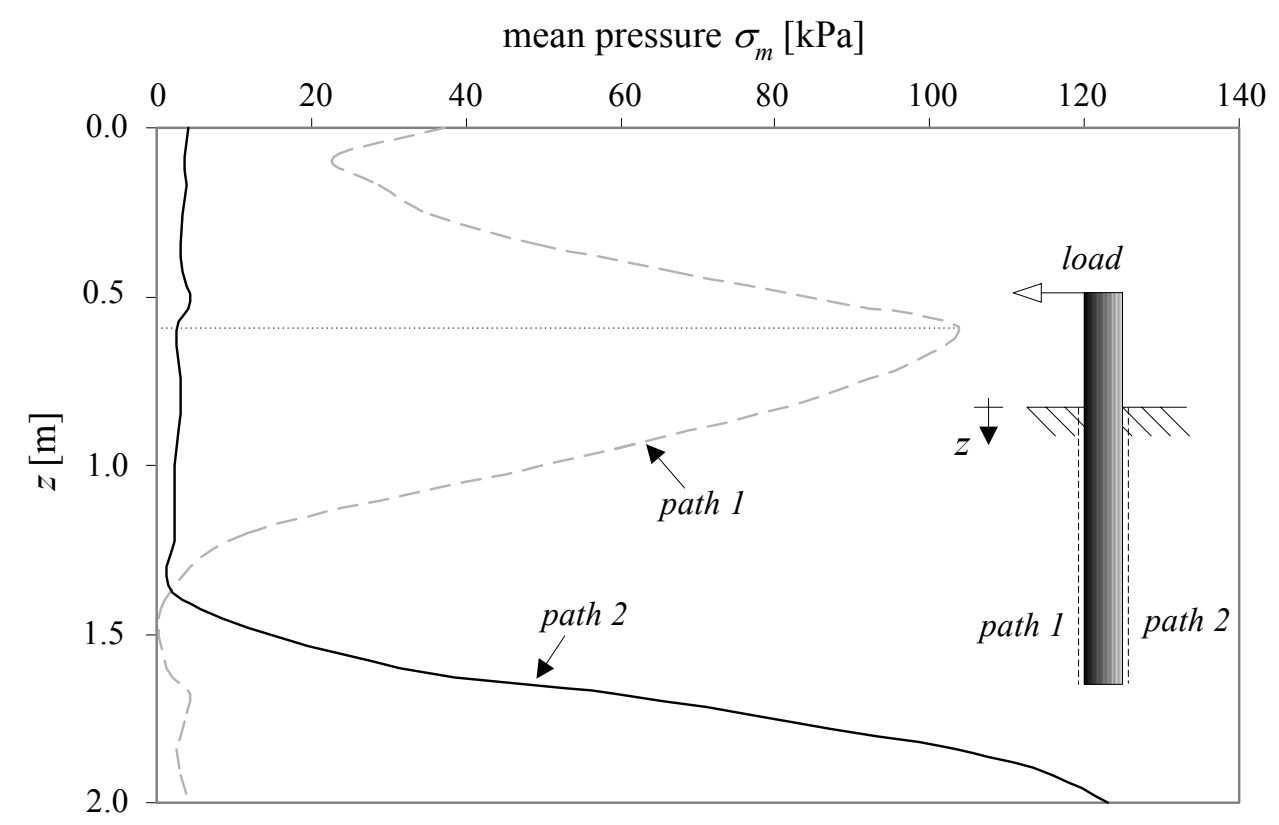

Fig. 7.24: Mean pressure distribution in the soil next to the pile along two different paths as a function of the depth $z$; evaluation for the $M-C$ model at a load of $10 \mathrm{kN}$

Because the laboratory tests performed by Vanden Berghe (2001) do not cover confining pressures of about $100 \mathrm{kPa}$ sufficiently, it is chosen to evaluate the Young's modulus by performing a regression analysis for the experimental data. The estimation of a stress dependent power function for the secant Young's modulus $E^{50}$ of the sand can be written as:

$$
E^{50}=E_{r e f}\left(\frac{\sigma_{m}}{\sigma_{r e f}}\right)^{m}
$$

with $E_{r e f}=22 \mathrm{MPa}, \sigma_{r e f}=100 \mathrm{kPa}$, and $m=0.7$. The regression line for the experiments is plotted in Fig. 7.25 in comparison with hypoplastic results as a function of the initial mean pressure $\sigma_{m, 0}$. The choice of a representative stress interval significantly influences the secant Young's modulus $E^{50}$. As an approximation and based on Holeyman et al. (2006), it is chosen to adopt $E=E^{50}=4.4 \mathrm{MPa}$ for the reference case related to an average initial mean pressure of $10 \mathrm{kPa}$. Similarly the pressure-void ratiocurve of oedometric compression tests performed with ABAQUS using the material data for the hypoplastic model can be used for deriving the Young's modulus. From the 
oedometer modulus $E_{\text {oed }}$ which is interpreted as the gradient of the curve in a specific stress interval the Young's modulus can be calculated according to equation (7.2). Calculating the oedometer modulus $E_{\text {oed }}$ with $E^{50}=4.4 \mathrm{MPa}$ and equation (7.2) leads to $E_{\text {oed }} \approx 7 \mathrm{MPa}$. Nevertheless, the evaluation of the gradient from the tests by Vanden Berghe (2001) and the numerical element tests (Fig. D.3) yield an oedometer modulus $E_{\text {oed }} \approx 2 \mathrm{MPa}$ to $4 \mathrm{MPa}$ at an initial mean pressure of $10 \mathrm{kPa}$. These differences arise due to the fact that the derived power approximation (Fig. 7.25) is based on a large scatter of test data without sufficient information for small initial mean pressure $\sigma_{m, 0}$. Further, the calculation of the gradient from an oedometer tests is highly influenced by the choice of the interpreted stress interval. Still, these differences highlight the sensitivity and difficulty of obtained well-founded soil parameters.

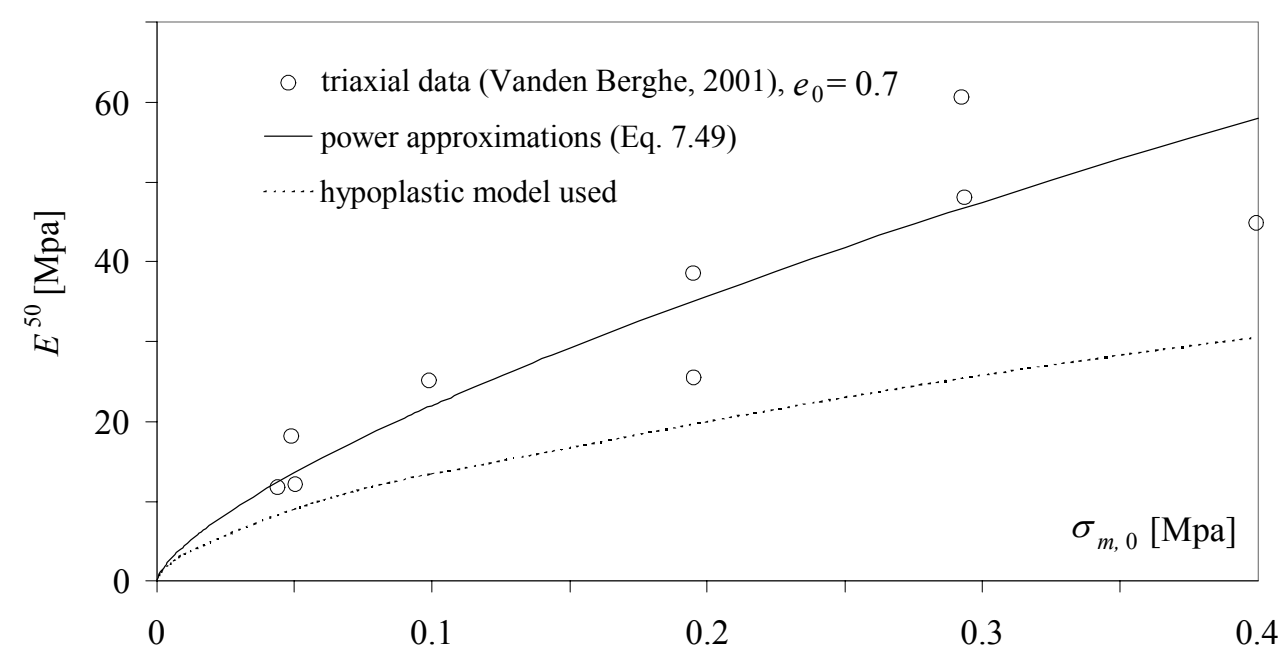

Fig. 7.25: Evaluation of Young's modulus at $50 \%$ of the failure load from triaxial tests as a function of the initial mean pressure (from Holeyman et al., 2006)

The evaluation of the angle of dilation for the numerical analyses using an elastic material model with Mohr-Coulomb theory is based on the results from the hypoplastic material model. In a study the angle of dilation for the elasto-plastic model is varied in simulations of the drained triaxial compression test (Annex C). The investigation uses the hypoplastic model as described in Tab. 7.7 with an initial void ratio $e_{0}=0.7$ corresponding to a density of $\gamma=15.25 \mathrm{kN} / \mathrm{m}^{3}$. Since the aim is to obtain a set of consistent soil data, the analyses using the Mohr-Coulomb model is based on the Young's modulus $E=4.4 \mathrm{Mpa}$ and the Poisson ratio $v=0.35$ as derived before.

The comparison of the change of volume reveals that an angle of dilation $v$ of $6^{\circ}$ can be used as approximation. The angle of dilation is derived applying the formula given in Fig. 7.23 for the gradient of the second part of the diagram which is obtained by a regression analysis. A further set of analyses was performed, demonstrating the validity of the angle of dilation for different initial confining pressures (= mean pressures) as depicted in Fig. 7.26. Nevertheless, the dilation angle varies with the stress level and therefore with the initial mean pressure. The angle of dilation $v=6^{\circ}$ seems acceptable 
for the investigated range. Still, evaluating the numerical triaxial compression tests for a confining pressure of $50 \mathrm{kPa}$ leads to an angle of dilation $v$ of about $5^{\circ}$.
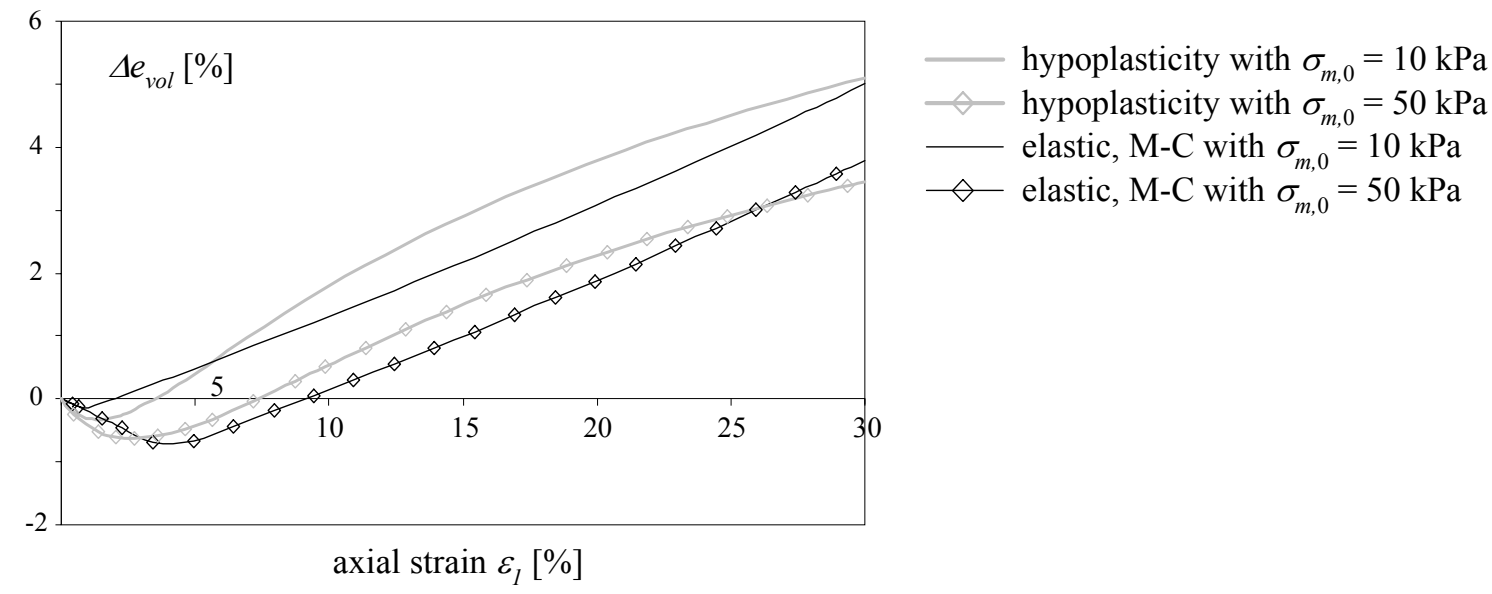

Fig. 7.26: Comparison between the hypoplastic and the elastic material model using Mohr-Coulomb theory for an angle of dilation $v=6^{\circ}$ for different initial mean pressures

Tab. 7.8: Derivation of the material properties for the „Brusselian Sand“ for the different models

\begin{tabular}{|c|c|c|c|c|c|c|c|c|c|}
\hline model & 岁 & \multicolumn{5}{|c|}{ ABAQUS } & \multicolumn{3}{|c|}{ ABAQUS } \\
\hline program & \multicolumn{6}{|c|}{ 1D-model } & \multicolumn{3}{|c|}{ 3D-model } \\
\hline approach & 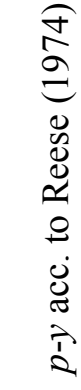 & 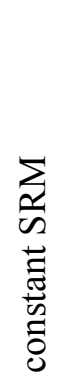 & 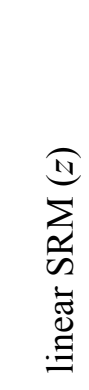 & 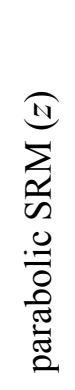 & 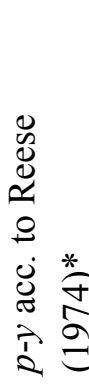 & 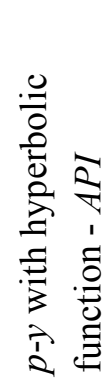 & 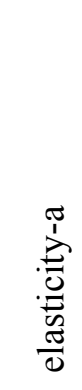 & 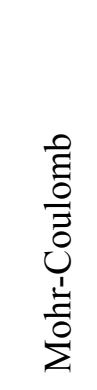 & 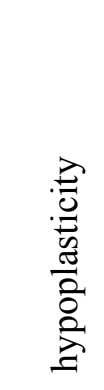 \\
\hline $\begin{array}{l}\text { empirical } \\
\text { obeservations }\end{array}$ & $k_{i}, \boldsymbol{A}$ & \multicolumn{3}{|c|}{$n_{h}, \delta, c$} & \multicolumn{2}{|c|}{$k_{i}, \boldsymbol{A}$} & $E, \delta$ & \multicolumn{2}{|c|}{$\delta, c$} \\
\hline laboratory tests & \multicolumn{6}{|c|}{$\varphi, \gamma$} & $\gamma$ & $\varphi, \gamma, E$ & $\varphi, \gamma, \boldsymbol{H}$ \\
\hline $\begin{array}{l}\text { num. analyses based } \\
\text { on hypoplasticity }\end{array}$ & & & & & & & $\mu$ & $\mu, v$ & \\
\hline classification & \multicolumn{6}{|c|}{ group-a } & & \multicolumn{2}{|c|}{ group-b } \\
\hline
\end{tabular}

$\boldsymbol{A}$... parameters $\overline{A_{s}}, B_{s}, C_{1}, C_{2}, C_{3}$

$\boldsymbol{H}$... hypoplastic parameters

For deriving a consistent set of material data different approaches were used. Tab. 7.8 summarizes the approaches and the methods of derivation. The parameters for the 1-D analyses were entirely taken from empirical relationships for cohesionless sand and combined with a limit pressure taking partially cohesion and friction into account. The 
parameters for the purely elastic analysis for the 3-D model are based on empirical findings combined with an additional analytical correlation (elastic-a) for the Young's modulus. While the parameters for the hypoplastic analysis are entirely derived from experimental tests, the values needed for the elasto-plastic approach are evaluated from laboratory tests and additional comparing numerical analyses with the hypoplastic model. For both, the purely elastic and the elasto-plastic approach, the Poisson ratio is predicted by studying the hypoplastic numerical analyses of the laboratory tests.

\subsubsection{Calculations and Results}

The 3-D continuum analyses are based on a geostatic step equilibrating the initial stress conditions from self-weight of the soil with an earth pressure coefficient $K_{0}$ at rest of $0.46\left(=1-\sin \varphi^{\prime}\right)$. Holeyman et al. (2006) confirm the validity of the 3-D model with an analytical solution based on Mindlin's closed form solution considering the evaluated Young's modulus. They come to the conclusion that the correspondence is sufficient to validate the finite element model.

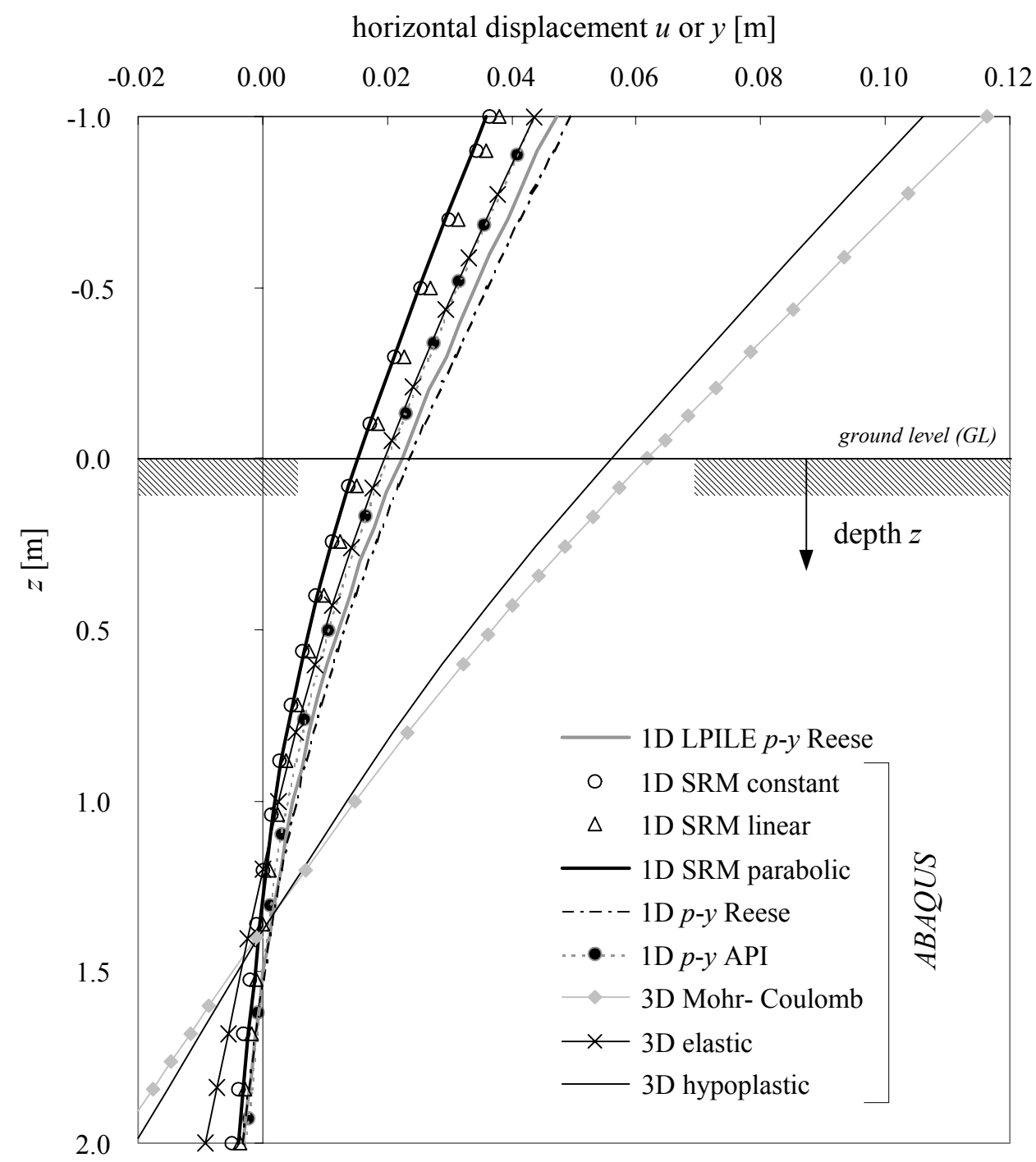

Fig. 7.27: Deflections for the reference case for different soil approaches for "Brusselian Sand" 
The deflections of the pile as a result of the different analyses are compared in Fig. 7.27 and Fig. 7.28. By far larger deformations with around twice the deflection at the pile head in comparison to most of the other approaches appear for the hypoplasticity. Although, the soil was slightly stiffer than in the approaches with smaller deflections because a capillary cohesion was added for numerical reasons, such large deflections take place. Comparing analyses with a soil featuring an even smaller capillary cohesion of $0.1 \mathrm{kN} / \mathrm{m}^{2}$ lead to an increase of the displacements of the pile head of some percentages. A more detailed investigation of this phenomenon is set aside at this stage. The soft behaviour of the soil modelled with hypoplasticity can be observed around the pile tip. In contrast to the other approaches, which exhibit deformations that die out at half of the pile length, the movement of the pile tip for the hypoplastic model is not zero, i.e. the bending moment reaches the pile tip. Similar results with an even softer behaviour can be found for the elasto-plastic approach applying the Mohr-Coulomb criterion. It can be seen that the calibration procedure based on laboratory tests seems to lead to softer results than those issued from the more empirical procedure.

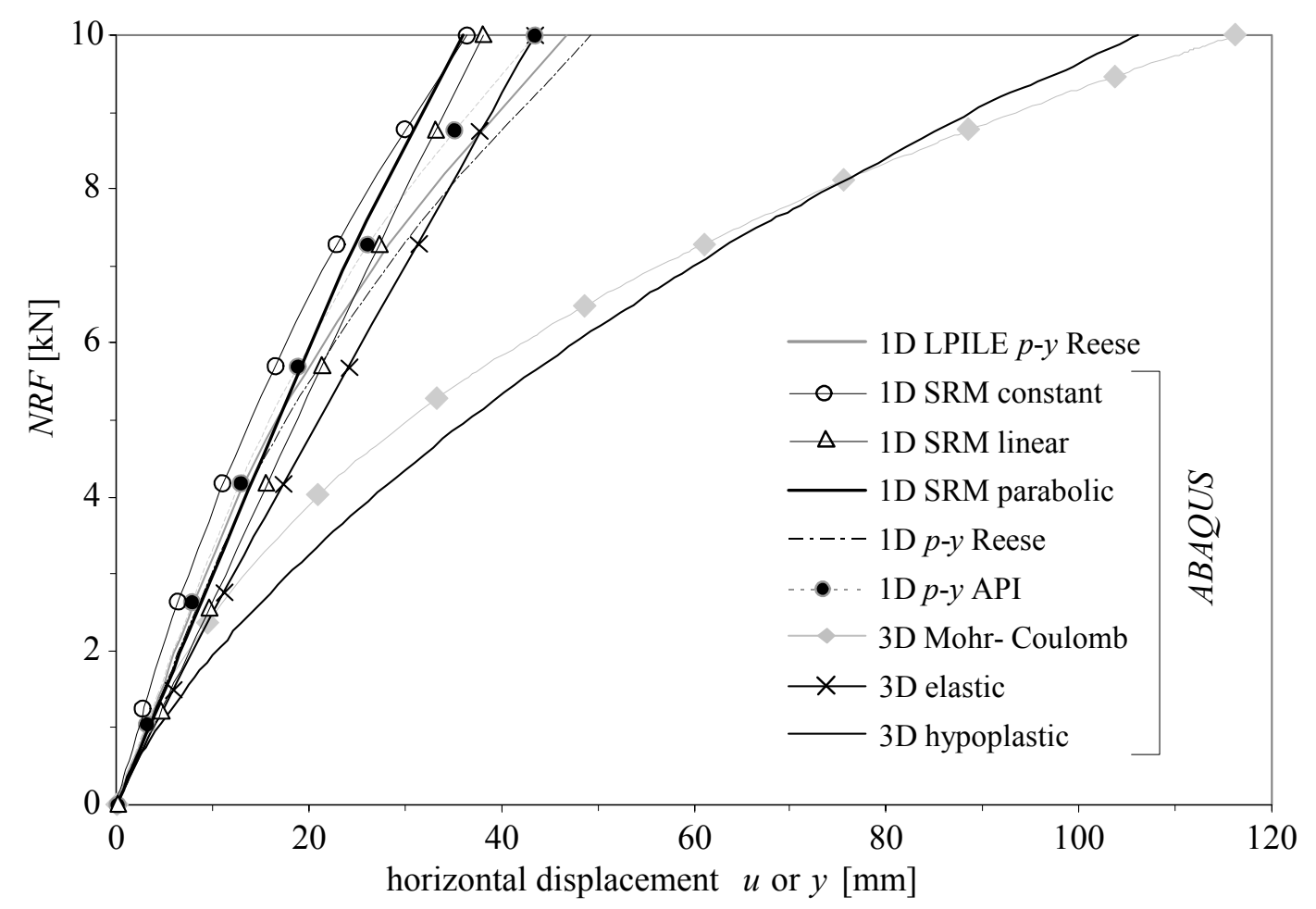

Fig. 7.28: Pile head deflection comparison for the reference case for different soil approaches for "Brusselian Sand"

The displacements given by the $p-y$ method after Reese et al. (1974) correspond well with the results from the analyses with LPILE and ABAQUS with a difference of the maximum deflection of $5 \%$. The calculations for the $p-y$ method as e.g. described in ISO/DIS 19902:2004 lead to smaller deflections than for the $p-y$ method according to Reese et al. (1974) with LPILE (8\%) and ABAQUS (12\%). The smaller deflections arise from the higher earth resistance given by the hyperbolic functions for small 
displacements - especially for the area close to the surface. Fig. 7.29 illustrates two example $p-y$ curves for different sections.

$-p-y[\tanh (z)]$ with ABAQUS

- $p-y[$ Reese] with LPILE

$p-y[$ Reese] with ABAQUS

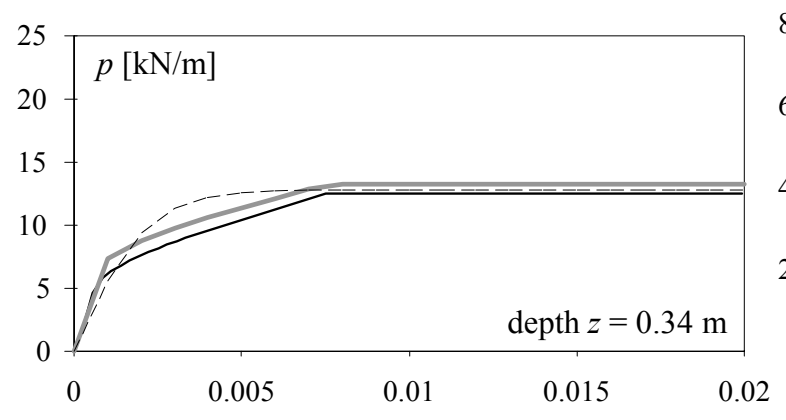

$y[\mathrm{~m}]$

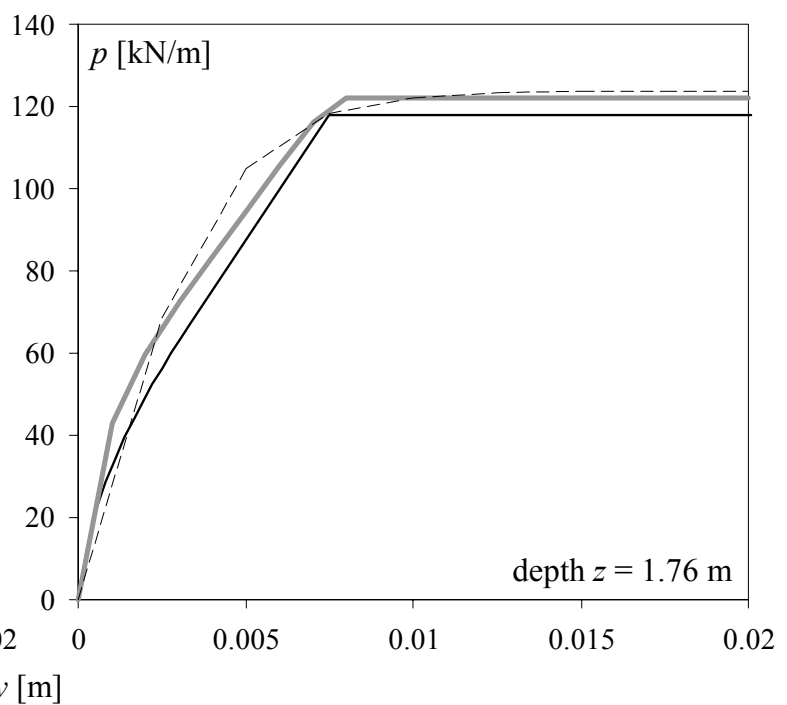

Fig. 7.29: p-y curves for two sections and different approaches

The analyses for the different approaches using the subgrade reaction method deliver very similar deflections. The results from the SRMs differ only slightly due to the fact that the limitation of the horizontal stresses is defined identically for the SRMs.

The elastic results correlate with the SRM analysis using the API method up to a depth of about $1 \mathrm{~m}$. In deeper region the deflections deviate resulting in more than 3 times larger deflections for the elastic analysis at the pile tip. The significance of an implemented plasticity can be easily identified when comparing the results for the 3-D elastic analyses with $E=2.15 \mathrm{MPa}$ and the 3-D elasto-plastic model with nearly twice the stiffness of $E=4.4 \mathrm{MPa}$. The differences in deflection emphasize the influence of a plastic zone. In Holeyman et al. (2006) the two failure mechanisms, i.e. the wedge failure and the plastic flow failure as proposed by Reese et al. (1974), are identified in the elasto-plastic analysis (Fig. 7.30).

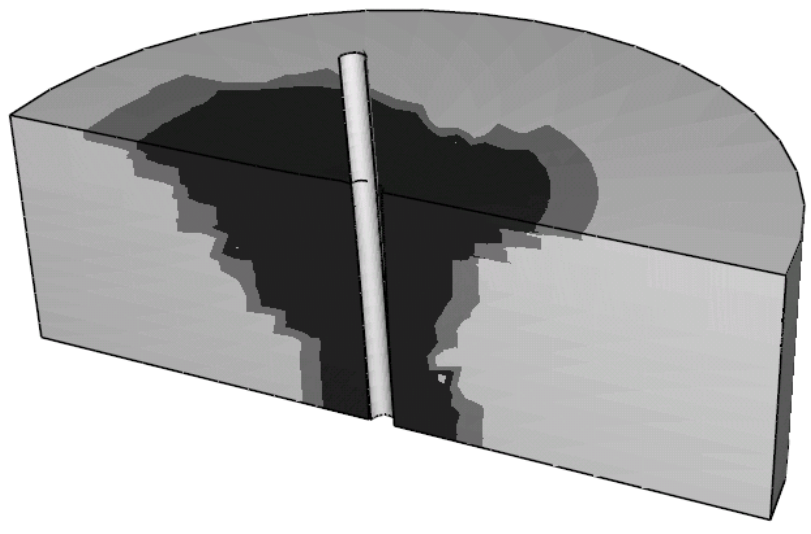

Fig. 7.30: Plastic region at lateral loading of $10 \mathrm{kN}$ for the elasto-plastic model (from Holeyman et al., 2006) 
Fig. 7.30 depicts the failure wedge near ground level with a failure angle of $50^{\circ}$ and $20^{\circ}$ in the passive and the active region. The wedge corresponds fairly well to the predictions by the Rankine theory (passive: $\beta_{G}=\pi / 4+\varphi^{\prime} / 2=61^{\circ}$, active: $\beta_{G}=\pi / 4$ $\varphi^{\prime} / 2=28^{\circ}$ ). Reese et al. (1974) proposed a shift between the two failure types at a depth of $0.7 \mathrm{~m}$. In the elasto-plastic analysis this transition can be found at a depth of about $1.3 \mathrm{~m}$.

Further analyses with the 3-D purely elastic model were performed in order to compare the results to 1-D analyses. The calculation for the material model elastic-b with $E=3.55 \mathrm{kN} / \mathrm{m}^{2}$ leads to a maximum deflection of $3.12 \mathrm{~cm}$ which is nearly $30 \%$ smaller than for elastic-a with $E=2.14 \mathrm{kN} / \mathrm{m}^{2}$. The analysis for elastic-b represents the stiffest material in the investigation resulting in the smallest deflections. The 3-D analysis of a pile completely connected to the soil and by applying the material model elastic-a leads to a maximum deflection of $2.8 \mathrm{~cm}$ which differs significantly from the predictions obtained using the SRM with discrete linear springs $(3.8 \mathrm{~cm})$. Since, the Young's modulus is derived from this approach it would be obvious to assume similar results. The large difference between the two predictions can be traced back to the fact that on the one hand side the secondary effects (non-linear geometrical calculation) are covered in the 3-D analyses and not completely included in the 1-D analyses. On the other hand the friction parameter activates the soil's resistance in the vertical direction, and thus decreases the lateral deflections. Comparing calculations show that if vertical springs of half the stiffness of the horizontal springs are added to the 1-D model similar deflections can be obtained. This aspect stresses the importance of a 3-D approach in order to cover all relevant parameters.

Fig. 7.31 depicts the deformed system and Fig. 7.32 the void ratio distribution $e_{0}$ for the hypoplastic case at maximum load. In the area close to the surface a decrease of the void ratio can be identified clearly. The size of the wedge shaped failure zone agrees quite well with the empirical predictions given by Reese et al. (1974).

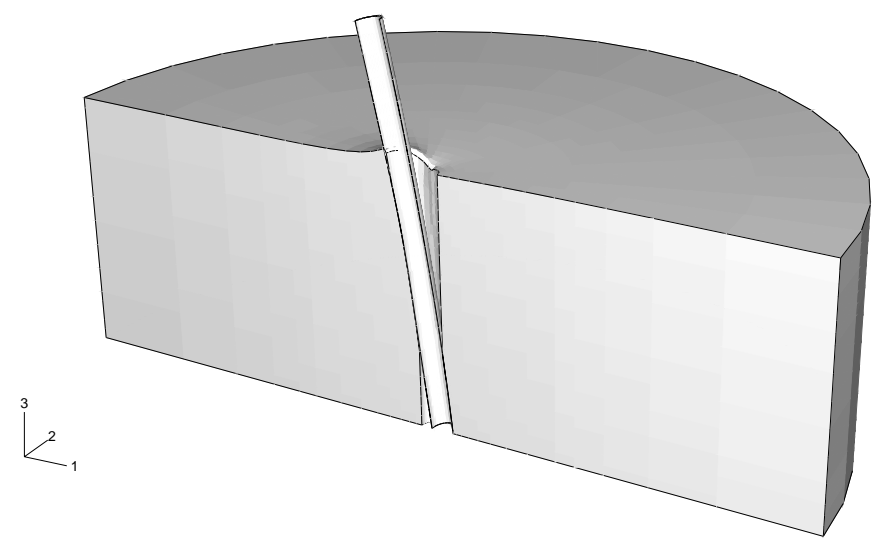

Fig. 7.31: Deformed system at maximum load for the hypoplastic model-scaled with the factor 5 


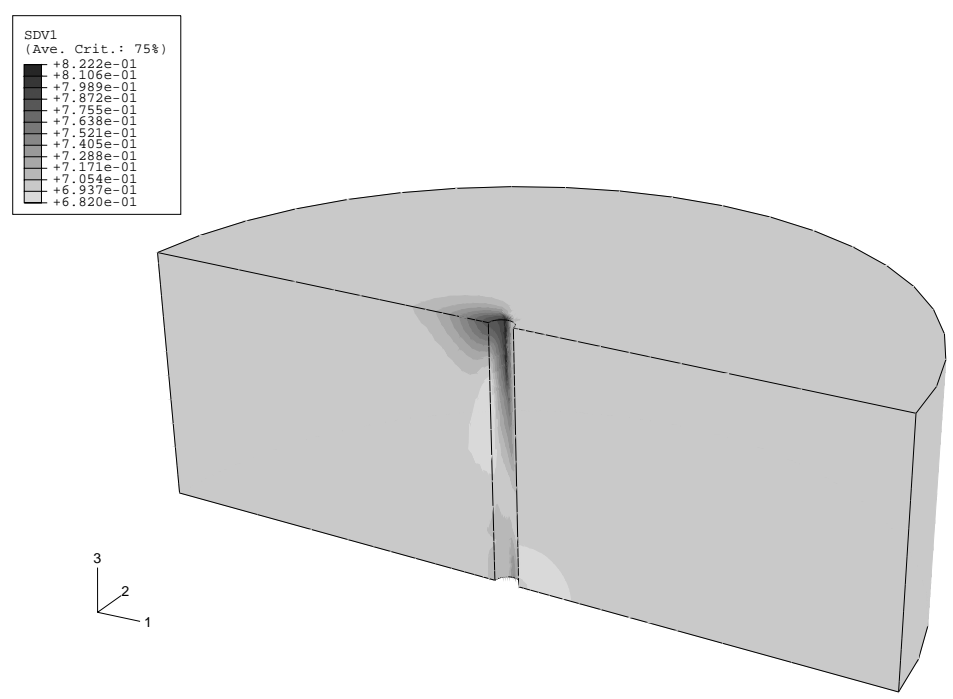

Fig. 7.32: Void ratio distribution $e_{0}$ in the soil at maximum load

The comparison reveals the small stiffness of the soil and therefore the small bending resistance for the approach using the hypoplasticity. Similar findings where stated amongst others by Wiemann et al. (2004). Other researchers as Kim et al. (2004) found large discrepancies in their results applying different approaches of $p-y$ curves. For the investigated cases the deflections for the elasto-plastic analysis are similar to those obtained for the hypoplastic approach.

For a better understanding of the load-deformation behaviour of the piles $p$ - $y$ curves are derived for some of the investigated approaches. For the 3-D approaches the curves are evaluated by integrating the contact shear and normal stresses over the circumference of the pile. The results are plotted in Fig. 7.33 for different depths up to the horizontal load of $10 \mathrm{kN}$. The constant initial stiffness for the constant SRM for the different depths can be clearly identified. For the linear SRM the initial stiffness is smaller at small depths while at the tip of the pile it is higher than for the latter case. A similar behaviour can be found for the elasto-plastic and the hypoplastic behaviour. Whereas the stiffness for the 3-D analysis with the elasto-plastic model is constant in the elastic region, the hypoplastic model covers the aspect of an increasing stiffness with increasing depths. The limit pressures for the 1-D analyses can be seen in the diagrams. Comparing calculations show that the results for a 1-D analysis with constant stiffness and no limit pressure are very similar to the proposed values for the investigated case with limit pressure. This effect is balanced as for small and for large depths the carrying behaviour is overestimated resulting in the similar deflection. The softer behaviour of the elastoplastic model in comparison to the hypoplastic approach which mainly occurs in the top layers can be seen in Fig. 7.33. It can be seen that mainly the initial stiffness governs the global deflection behaviour rather than the lateral limit pressure. 

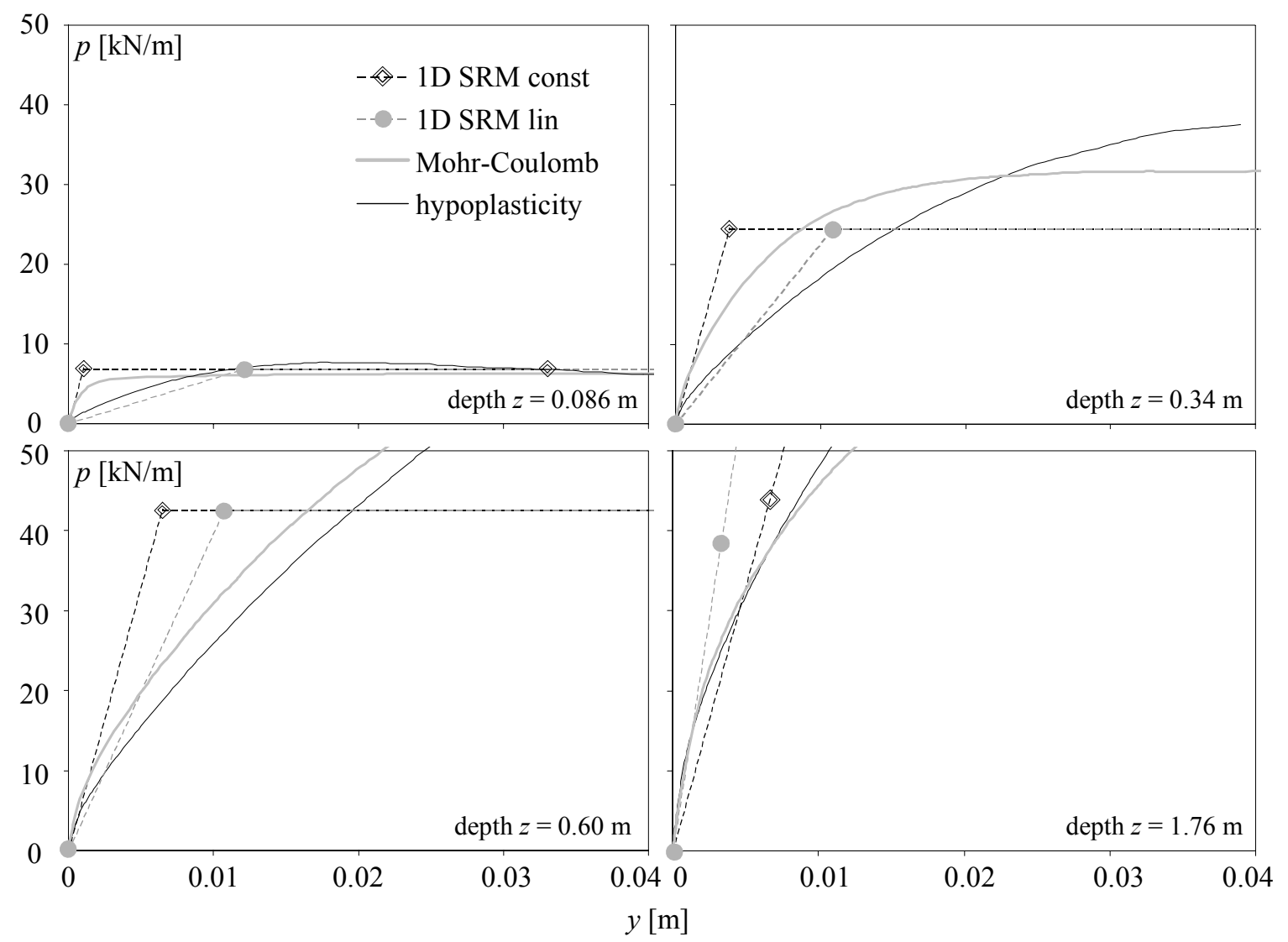

Fig. 7.33: Derived p-y curves at different depths and approaches

As the comparison shows good correspondence between the results from the $p-y$ methods, which have been confirmed by a lot of practical experience, and the results from the elastic model for the spatial investigation and the SRMs (group-a), the conclusion can be drawn that the quality of the results from the latter model appears to be fairly good for the investigated case. The findings show a good correspondence between the 3-D analysis using a simple elasto-plastic model and a more elaborated hypoplastic model (group-b) regarding the deflection of the pile and the activated lateral resistance. This match is related to the determination of the relevant soil parameters as summarized in Tab. 7.8. The soil parameters of group-a are mainly derived from empirical observations, and the parameters for group-b are chiefly obtained from laboratory tests. Both groups involve a determination of some parameters from comparing numerical analyses. Still, their influence on the load carrying behaviour is rather small in comparison to e.g. the evaluated stiffness. From the good agreement of the results within a group the conclusion can be drawn that the adopted calibration procedures seem acceptable. Although, the comparison is limited to the reference case some considerations regarding the differences between the groups are discussed. The values obtained by Vanden Berghe (2001) in laboratory tests used for the analyses of group-b are based on experiments with significantly higher confining pressures than appearing in the reduced scale test (reference case). Since the elasto-plastic parameters are the result of an extrapolation, e.g. the secant Young's modulus estimation for the 
elasto-plastic model may be inaccurate. Similarly, smaller secant stiffnesses of the system were found e.g. amongst others by Wiemann et al. (2004) for small confining pressures using the hypoplastic model. For the hypoplasticity the imposed initial void ratio has a major influence on the stiffness of the soil. Such an initial void ratio $e_{0}$ of 0.55 instead of 0.7 leads to a maximum deflection at the pile head of $3.9 \mathrm{~cm}$ instead of $10.3 \mathrm{~cm}$, i.e. a decrease of more than $60 \%$ of the lateral displacement highlighting the importance of the installation effect. Still, the deflection of the pile is only scaled and a lateral displacement at the pile tip remains. Presumably, the large displacements for the approach applying the hypoplasticity arise from neglecting the loading history and therefore the missing "memory" of the imposed material law. Fig. 7.34 depicts the compression curves from numerical oedometric simulations for hypoplasticity and the Mohr-Coulomb model using the data given in Tab. 7.7. Details are given in Annex C. It can be clearly seen that the hypoplastic material law is completely non-linear from the beginning. For the Mohr-Coulomb model a stiffness is derived which is valid for the complete analysis. It can be seen that the definition of the stiffness or the Young's modulus $E_{S}$ for the soil considerably depends on the chosen stress range. For small stress the soil reacts very soft if modelled with the hypoplasticity which can be seen by deriving the Young's modulus $E_{S}$ from Fig. 7.34. The stiffness and therefore the reaction of the soil then again differs between the two models considerably for very large axial stresses. Because of the anelastic behaviour of soil which is implemented in the hypoplastic model, a re-loaded soil reacts by far stiffer than a soil under first loading. Since a completely undisturbed soil which has not been loaded is rather improbable to encounter, the simple material models tend to cover general, load history independent problems. For the investigated case and the deflections given in Fig. 7.27. this means that the pile modelled in combination with the hypoplastic soil model may react too soft in comparison to the other models because the load history is neither known nor taken into account.

Generally, for medium to dense sands the hypoplasticity may exhibit very soft behaviour for cyclic loading as the hypoplasticity was not able to cope with the change of direction; this aspect was taken care of by introducing the intergranular strain in current hypoplastic formulations. This formulation (4 to 5 more parameters) for intergranular strain is not included in the applied routine since no cyclic loading is investigated. The parameter evaluation performed by Vanden Berghe (2001) is made according to Gudehus/Bauer (1996); newer approaches by e.g. Cudmani (2001) or Herle and Gudehus (1999) revealed significant differences for material properties evaluated by using more recent approaches. Newer approaches try to adjust the parameters using a set of parameters and a regression line and additionally use high pressure oedometers for obtaining material data. More recent sets of material data for hypoplastic models mostly tend to differ as follows: larger $n$ and $\beta$ and smaller $\alpha$ and $h_{S}$.

A further influencing aspect is the dimension of the problem. Whereas the 1-D approaches are mainly derived from full-scale laterally loaded tests with high mean pressures, the reference case uses a confined region with small mean pressure evolving. 
The results emphasize the importance of an experimental validation for evaluating the numerical results. The findings emphasize the importance of the designer's experience demand for test observations in order to calibrate the approaches.

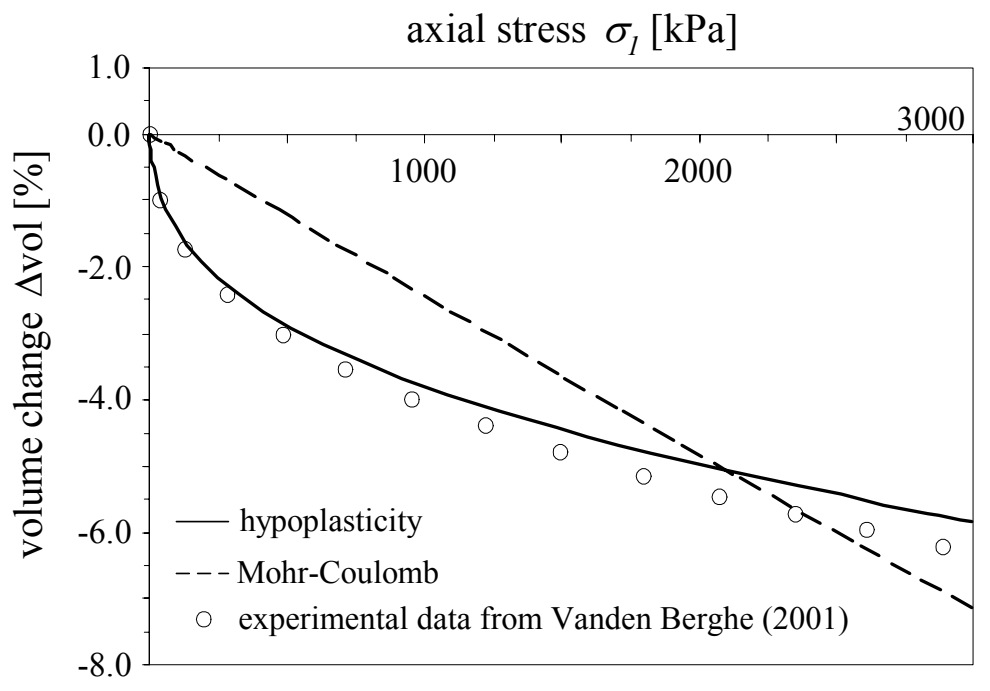

Fig. 7.34: Comparison of compression curves from numerical and experimental results

Beside the soil parameters, the interface parameters affect the pile response. In the study, a linear frictional behavior with a wall friction angle $\delta=0.5 \varphi^{\prime}$ was applied. Additional numerical analyses with the elasto-plastic model lead to a decrease of about $10 \%$ of the lateral displacement for an interface friction $\delta$ of $0.8 \varphi^{\prime}$ to $0.5 \varphi^{\prime}$.

In the following procedure four cases $\mathrm{A}$ to $\mathrm{D}$ with representative deflections are chosen, and their corresponding moment distributions along the pile are shown in Fig. 7.35. The correlation between the representative cases and the models can be found in Fig. 7.35. The maximum horizontal displacements increase in the order A to D. The maximum moments appear in the region $30 \mathrm{~cm}<z<75 \mathrm{~cm}$. The largest difference between the maximum bending moments is found for case $\mathrm{B}$ and $\mathrm{C}$ with a moment about $25 \%$ higher for $\mathrm{C}$ than for $\mathrm{B}$. These two cases also exhibit the largest difference for the position of the bending moment, even though their pile head deflection is very similar. The deflection curves indicate the softness of case B in contrast to case $\mathrm{C}$ near the pile tip, which is related to the maximum bending moment closer to the ground level. While for the cases A to $\mathrm{C}$ the bending moment at the pile tip has mostly died out, the case D still exhibits a small bending moment at the pile tip. Due to the non-linear geometrical analyses for the spatial model in the cases A to D the cross-section of the tube deforms. Therefore, the evaluation of the bending moment distribution is performed as a simple approximation based on the axial membrane stresses less the axial force components.

Due to the model and the application of no constraints at the pile foot (Fig. 7.21), very large lateral forces arise in the soil close to the pile foot as seen in the mean pressure distribution (Fig. 7.24). These large counteractive forces lead to an opposite bending moment and thus the significant change of curvature of the pile. 


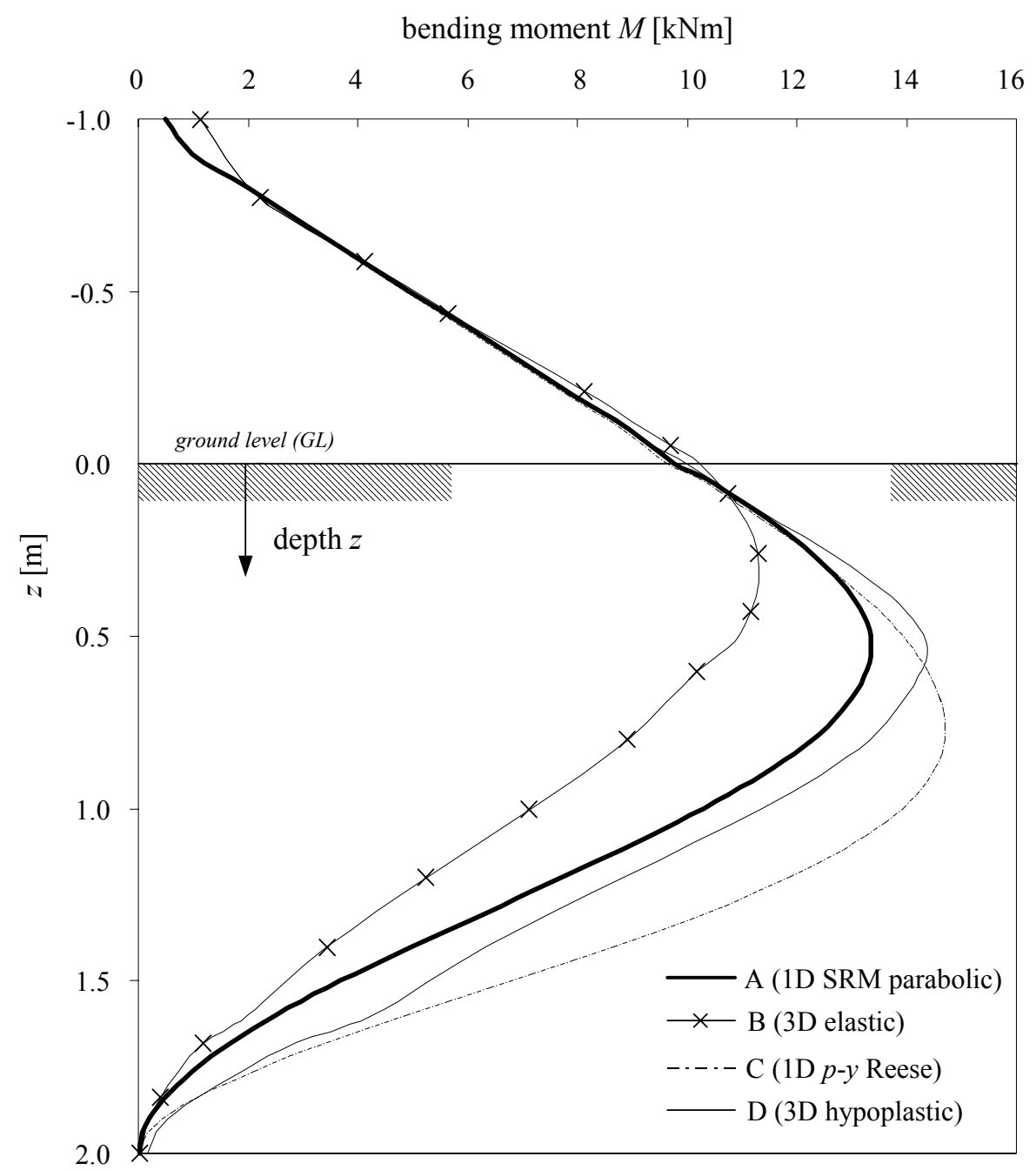

Fig. 7.35: Bending moment distribution for the reference case for applying different soil approaches for "Brusselian Sand"

\subsection{Discussion of the soil-structure interaction models}

Based on the results from the previous sections and general comparisons and considering the advantages and the disadvantages this section deals with a summary and discussion of the soil-structure interaction model (SSIM) including the soil and the contact. Tab. 7.9 gives an overview of the investigated models and their advantages and disadvantages. It is the aim to define a soil model to be used in further analyses involving soil. 
Tab. 7.9: Overview of the investigated SSIMs

\begin{tabular}{|c|c|c|c|c|c|c|c|}
\hline 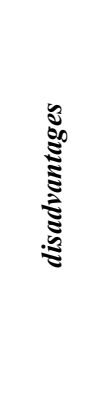 & 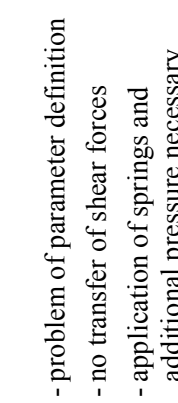 & 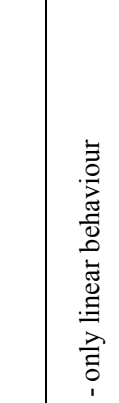 & 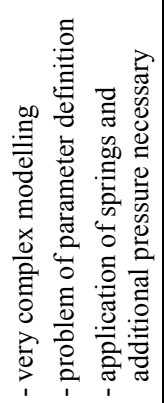 & 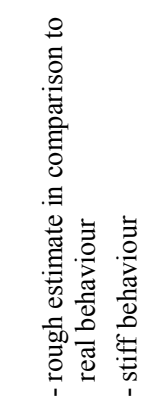 & 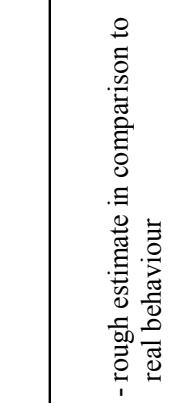 & 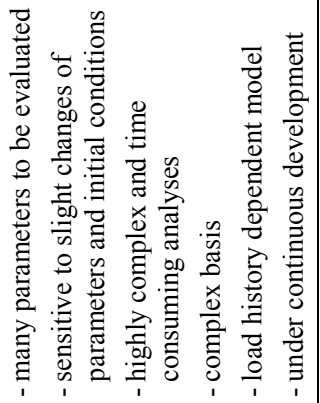 & 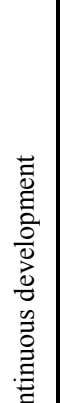 \\
\hline 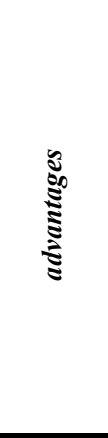 & 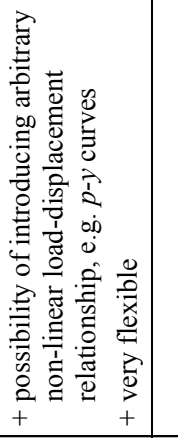 & 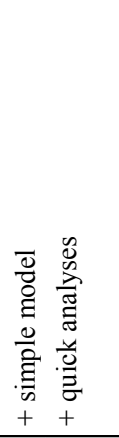 & 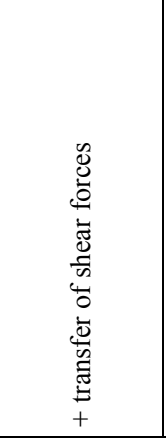 & 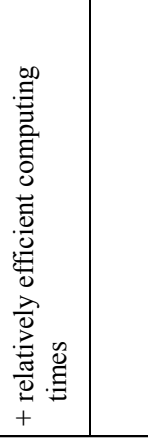 & 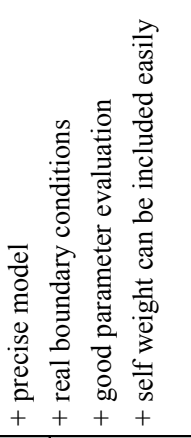 & 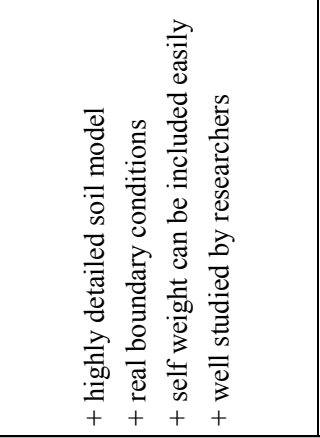 & $\begin{array}{l}0 \\
\dot{\bar{s}} \\
\Xi \\
+1\end{array}$ \\
\hline 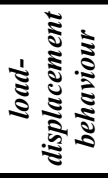 & 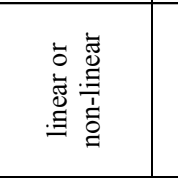 & 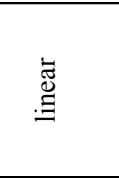 & 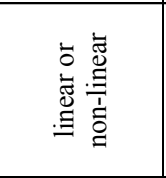 & 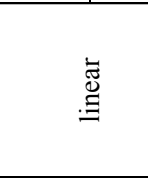 & 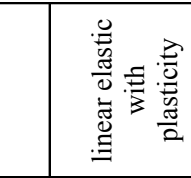 & 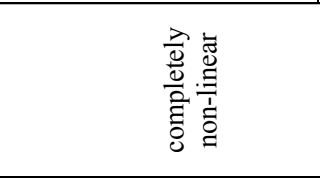 & \\
\hline 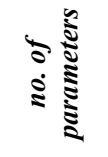 & $\vec{\wedge}$ & - & $\vec{\wedge}$ & $\sim$ & + & $\begin{array}{l}\infty \\
\wedge l\end{array}$ & \\
\hline 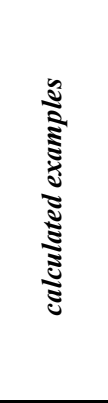 & 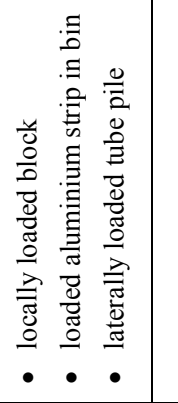 & 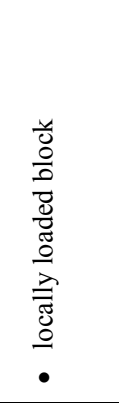 & 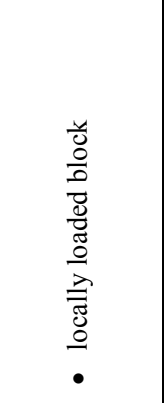 & 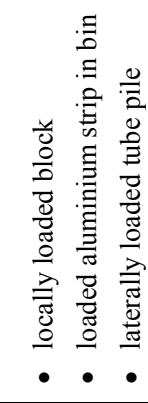 & 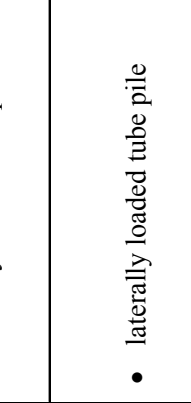 & 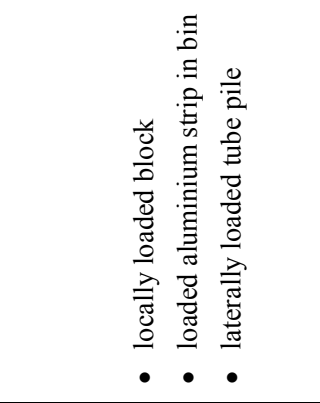 & \\
\hline 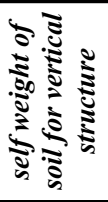 & \multicolumn{3}{|c|}{ 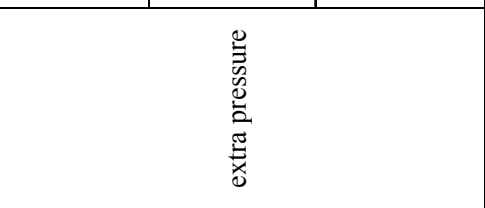 } & \multicolumn{4}{|c|}{ 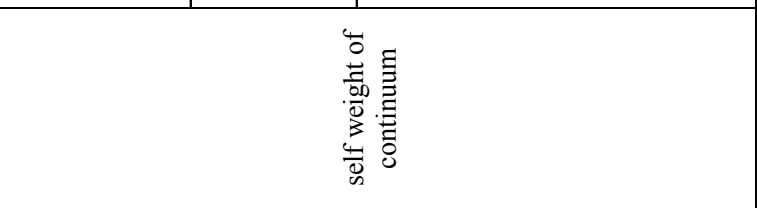 } \\
\hline 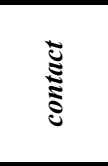 & \multicolumn{3}{|c|}{ 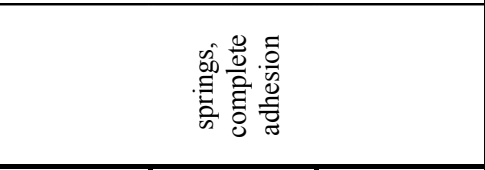 } & \multicolumn{4}{|c|}{ 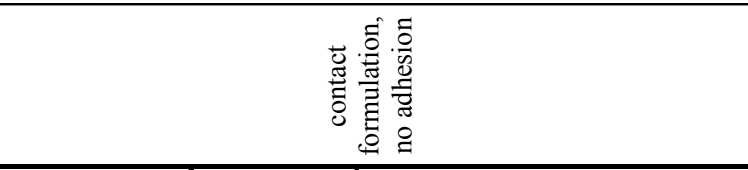 } \\
\hline $\begin{array}{l}\Xi \\
\Xi\end{array}$ & 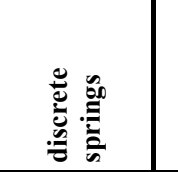 & 莺 & 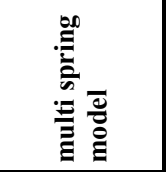 & 䒠 & 糬 & 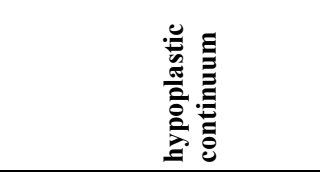 & \\
\hline
\end{tabular}


The modelling using discrete springs resulted in fairly good results in comparison with test results. The concept of discrete springs is technically seen simple, the application may lead to extensive work for modelling when working with multi-parameter models. Nevertheless, due to computing time and the obtained results it may be a very efficient tool for obtaining information on the general behaviour of structures in contact with soil. The single parameter needed for input can be easily varied. Additionally, more complex behaviour can be regarded by imposing a non-linear load-displacement relationship for the spring. Also the aspect of separation can be dealt with via the nonlinear load-displacement relationship or special gap elements. Spring elements remain a powerful tool for preliminary studies or parametric studies.

For more detailed geometrical and material non-linear analyses involving localizations or spatial soil reaction or failure, the use of complex models with a complete modelling of the soil itself in combination with a formulation taking account of the contact interaction is indispensable. The most advanced and sophisticated soil model investigated here is doubtlessly the hypoplasticity. Still, the studies have revealed that already various implemented formulations of the hypoplasticity exist for which the results may differ. For medium to dense sands the hypoplasticity may exhibit very soft behaviour for cyclic loading as the hypoplasticity is not able to cope with the change of direction; this aspect was taken care of by introducing the intergranular strain. This formulation for intergranular strain is not included in this investigation as no cyclic loading is investigated. Still, for the investigated cases applying hypoplasticity the analyses revealed in contrast to the other approaches generally a very soft behaviour of the material model.

The comparison shows that the major difficulty of the material law is that all parameters need to be defined and validated, and slight deviations of the parameters can lead to considerably different results. Another problem encountered while using the hypoplastic law is the very soft soil reaction, e.g. close to the surface for the study of laterally loaded piles. As the linear elasticity is the simplest method of introducing a material model for a continuum it is as expected not capable of describing the behaviour of sand precisely. First rough approximations for the SSI problems subjected to minor loads can be estimated concerning load transfer or global behaviour, but the model cannot be used for obtaining absolute values. Since, the reaction of the soil is non-linear from the small loads on and converges to a limit reaction this soil model is not sufficient for thorough studies. Therefore, something in-between as an elastic continuum which takes certain indispensable soil properties into account and remains simple and handy, appears to be an efficient soil model. As emphasized it is important to take a failure mechanism or limiting load carrying behaviour into account. The elastic continuum in combination with the Mohr-Coulomb theory may lead to good results but still involving only relatively simple and coherent soil parameters. Nevertheless, the correct choice of parameters and initial conditions is very important. The intention is to chose a SSIM which is able to represent the important aspects of the problem, remaining practicable and applicable outside the field of detailed research in soil mechanics. 
As a consequence it is chosen to use the simple but efficient spring model for further investigations. Due to the possibility of varying one parameter significant preliminary studies can be performed. For the use in linear bifurcation analyses linear spring models can give information about the soil's influence quickly. In this context the spring's stiffness or the coefficient of subgrade reaction can be defined within a defined parameter range depending on the soil, and thus lead to important results. In the buckling analyses the detailed soil behaviour may not be of interest. Still, the model using a continuum with an elastic material law in combination with the Mohr-Coulomb theory and a non-adhesive contact formulation shall be used for follow-up analyses including non-linear material and geometrical aspects. In these analyses special attention can be paid to imperfections and contact behaviour.

\subsection{Verification of the models}

\subsubsection{Results from reduced-scale tests}

In this section some results and evaluated results of the reduced-scale tests are presented. Details regarding the test set-up, the execution, the specimens, and the testing program are given in the Annexes $\mathrm{B}$ and $\mathrm{C}$. The evaluation of the measured strains is performed by imposing a Young's modulus $E=190000 \mathrm{~N} / \mathrm{mm}^{2}$. The value is based on tensile tests performed with coupons extracted from the test tube. The tensile tests with coupons extracted in longitudinal and circumferential direction of the test tube revealed the consistency of the Young's modulus (Annex B):

$$
E \equiv E_{L}=E_{\|} \approx E_{C}=E_{\perp}
$$

The value was verified by comparing it to measurements performed during axial loading tests. From the imposed load at the load cell and the measurements at the first strain gauges outside the soil, the proposed value was validated. The measured strains are then processed based on the equation found in the numerical studies from section 10 :

$$
\sigma_{x} \approx \frac{E}{1-\mu^{2}} \varepsilon_{l, o}
$$

for obtaining the meridional or longitudinal membrane stress $\sigma_{x}$ as an approximation based on the measured longitudinal strain $\varepsilon_{l, o}$ on the outside of the tube. Subsequently, for example the bending moment can be obtained by not taking ovalization effects into account which are negligible present for small loads for the laterally loaded pile.

The results from the test tube presented here are part of a test series performed at the laboratories of the University of Louvain (UCL) and refer to the first tested tube with less instrumentation than the second tested tube. More details can be found in Charue et al. (2007). While experiments with the first test tube were aiming for delivering first insights to the testing and the evaluation procedure with small loads and displacements, 
the experiments with the second test tube provide more detailed information on strain measurements for different cases. Still, eventually the tests with the first test tube may provide even more reliable results as the experiments are not as much disturbed by the acquiring system (strain gauges, wires, inclinometers) as in the case of the heavily instrumented second test tube. Only results from the first test tube are discussed.

\subsubsection{Back-calculation and comparison}

For comparing the results of the experimental investigation a finite element model is built according to the test set-up. The system and the applied boundary conditions is depicted in Fig. 7.36. The geometry and the system is similar to the previously investigated case depicted in Fig. 7.21. The outer diameter of the casing is based on the evaluation of the relative error of stresses in the soil as a function of the bin radius as described in Charue et al. (2007). Thus, the ratio of $D_{\text {casing }} / D_{\text {out }}$ was chosen to be about 8.5 corresponding to a relative error $<1 \%$. The chosen diameter $D_{\text {casing }}=6 \mathrm{~m}$ that was used in first modelling studies (chapter 7.5.4) was based on the evaluation of the displacements or the coefficient $C$ according to Fig. 7.8. Since the displacements of the pile are very sensitive to the outer boundary, the conservative assumption of $D_{\text {casing }}=30 D_{\text {out }}$ was used in chapter 7.5.4. Based on refinements (Charue et al., 2007) and on the limitations in the test set up, the stated the geometry as shown in Fig. 7.21 was chosen. More details on the real test set-up are given in Charue et al. (2007) and Annex D.

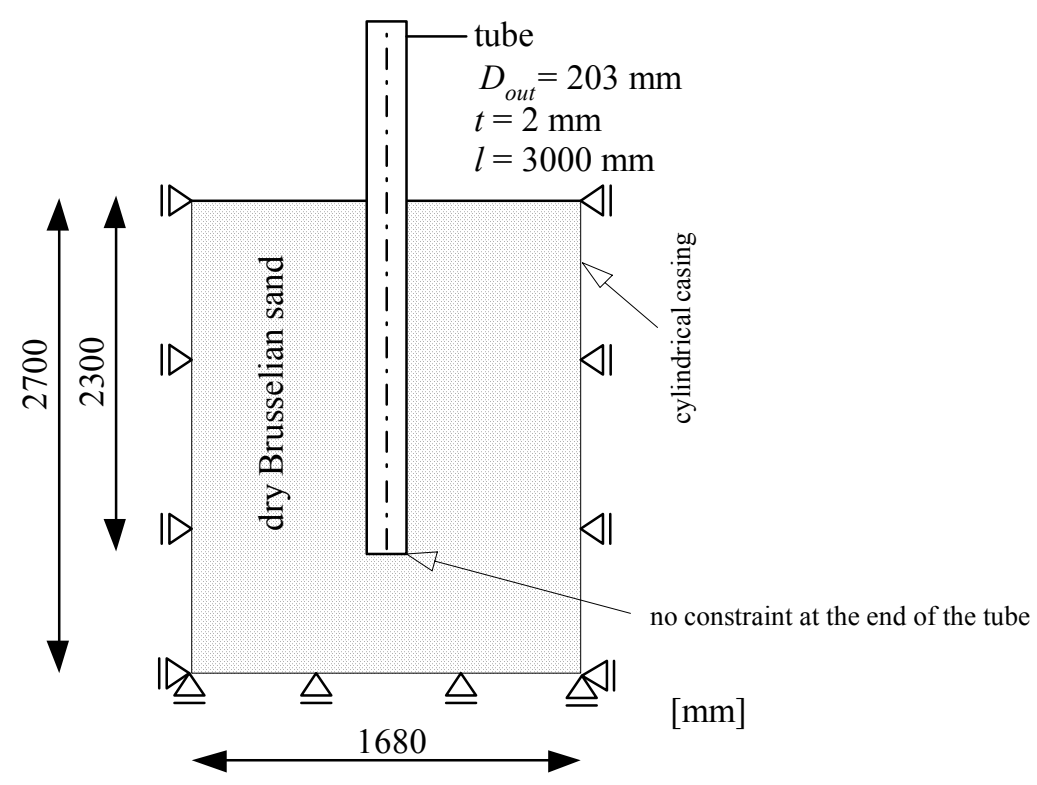

Fig. 7.36: Cross-section of the reference case with applied boundary conditions

The pile tip is not constrained. Surface contact formulations allowing separation are applied to the surface of the pile with the surrounding soil and the pile end cross section sitting on the soil below the pile. An initial stress state is imposed based on earth pressure coefficient $K_{0}$ at rest applying formula (7.41). In the first step (geostatic equilibrium step) the self-weight of the pile $\left(\gamma=78 \mathrm{kN} / \mathrm{m}^{3}\right)$ and the soil are applied. 
Since the tubular pile is not restraint vertically, it settles slightly resulting in large local stresses in the soil around the tip. The material properties are applied according to Tab. 7.7 and the previous analyses. In order to control the system more easily and to improve the contact convergence at initial loading the load is defined by a horizontal displacement of the pile head. The pile head is not constrained rotationally. For the 1Dmodel the pile is modelled as in the previous calculations from section 7.5 since the soil below the pile cannot be reflected in the analyses.

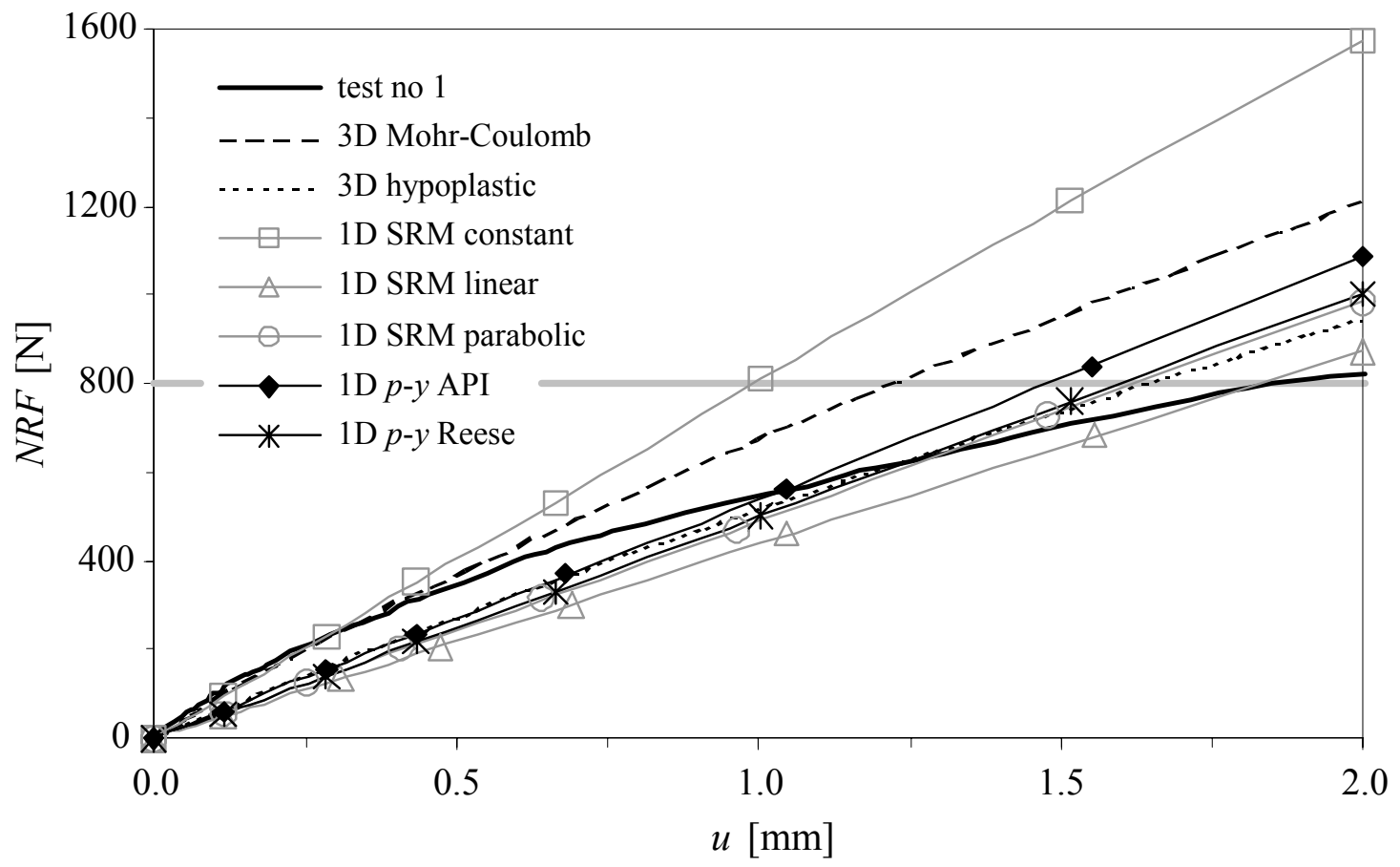

Fig. 7.37: Pile head deflection comparison for different methods and the experimental test result

For the tests the maximum applied load was taken for comparison; i.e. here the corresponding displacement of $2 \mathrm{~mm}$ was the parameter which was introduced into the numerical analyses. The corresponding force for numerically achieving the displacement is called NRF (= numerical reaction force) in the following as in the numerical analyses the boundary at the pile head was moved in the load step. In Fig. 7.37 the load-displacement plots are depicted for the different models up to a maximum displacement of $2 \mathrm{~mm}$. Additionally, a horizontal line is added indicating the displacements at a load $N R F=800 \mathrm{~N}$ corresponding to the maximum applied load in the experiment. Already for the small displacement of $2 \mathrm{~mm}$ the predicted reaction forces vary significantly between the different approaches. The 1D approach using a linear SRM results in an overestimation compared to the test of the reaction force by about $100 \%$. The other models seem to provide fairly good approximations. Generally, the models exhibit a quite linear relationship for the small loading. Nevertheless, the test results emphasize the nonlinearity of the response as seen in Fig. 7.37. Only the 3D approaches (Mohr-Coulomb and hypoplasticity) appear to be able to capture the nonlinear behaviour for small displacements. Nevertheless, generally the Mohr-Coulomb 
does not provide satisfying fits. Similar to the results from the previous analyses (Fig. 7.27), where the analyses with the $3 \mathrm{D}$ approaches revealed relatively soft response, this aspect seems to be less distinct in this case. Still, as it can be seen from Fig. 7.37 due to the nonlinearity of the response for the 3D approaches the differences between the 1D and 3D models increases with increasing loads or enforced displacements beyond $u=2 \mathrm{~mm}$. The latter issue leads for larger loads $(>1600 \mathrm{kN})$ to the largest displacements occurring for the 3D models, which can be presumed from Fig. 7.37 and is confirmed in Fig. 7.27.

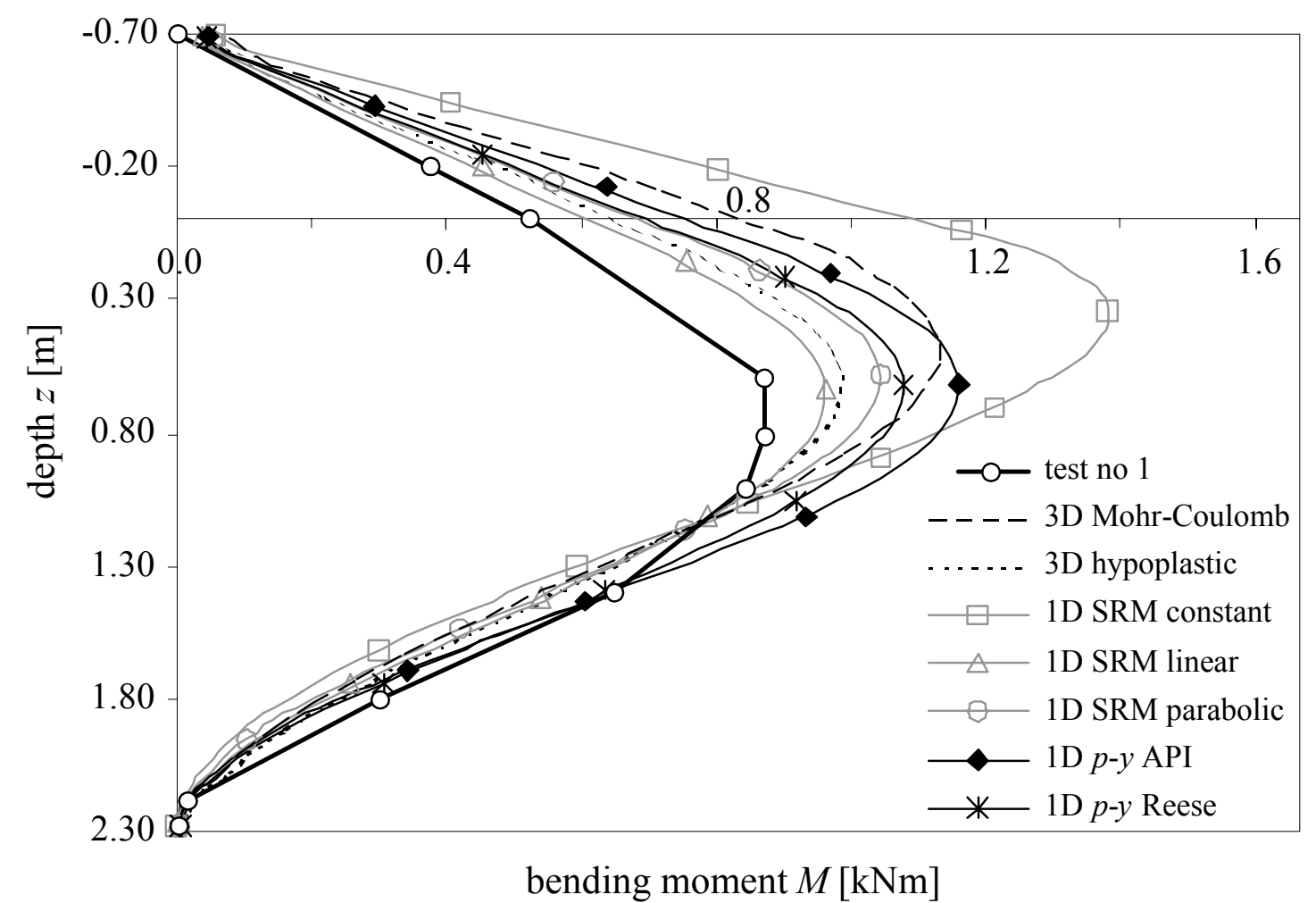

Fig. 7.38: Comparison of bending moments for different methods and the experimental test result at a displacement of $2 \mathrm{~mm}$

For the investigated range the models provide mostly an inaccurate prediction for loaddisplacement behaviour. Further, it has to be pointed out that the deviations appear to increase for larger displacements considering the curves from Fig. 7.37.

In the first calculation from section 7.5.6 (Holeyman et al., 2006) an ultimate load $(14 \mathrm{kN})$ was estimated based on limit equilibrium calculations according to Broms (1964). Consequently, the applied load $(10 \mathrm{kN})$, corresponds to a factor of safety of 1.4 , i.e. the system is loaded to $1 / 1.4 \approx 70 \%$ of the ultimate load. This value was chosen for emphasizing that the pile is not loaded close to the failure. Still, it provides a better understanding when discussing the different soil models applied. The first analyses had the purpose of establishing first model concepts which were to be refined in the second step. Here, the second set of analyses is performed for comparing the results with the experimental data. The data compared is related to a load $<1.6 \mathrm{kN}$, and thus 
corresponds roughly (different model) to a factor of safety of $14 / 1.6 \approx 9$. Still, this value indicates that the behaviour should be fairly elastic (small strains). Nevertheless, the test results from Fig. 7.37 indicate a fairly non-linear behaviour for these small loads.

The corresponding bending moments are evaluated for the investigated models and compared with the derived bending moment from the test. The results for a displacement of $2 \mathrm{~mm}$ can be seen in Fig. 7.38. The scatter of the bending moments can be explained due to the different forces applied to the pile head at the displacement of $2 \mathrm{~mm}$ (Fig. 7.37). Still, the position of the maximum moment is well predicted by most of the models except for the linear SRM model and the Mohr-Coulomb model.

In order to compare the bending moments better for specific loads it is chosen to depict the bending moments for $N R F=800 \mathrm{~N}$ as seen in Fig. 7.39. Again the largest deviations can be found for the linear SRM model and the Mohr-Coulomb model. The other models provide very close correlation with the test results. Both, the amplitude and the position of the maximum moment, are well predicted by the other models. Nevertheless, discrepancies can be found between the model predictions and the test result at ground level. Here, the results should coincide since these values are calculated without the influence of the soil. The differences probably occur due to friction. effects in the loading devices (pulley). Although the predicted bending moments agree well, the related displacement of the pile tip may differ significantly as seen at for e.g. $N R F=800 \mathrm{~N}$ indicated by the horizontal grey line in Fig. 7.37.

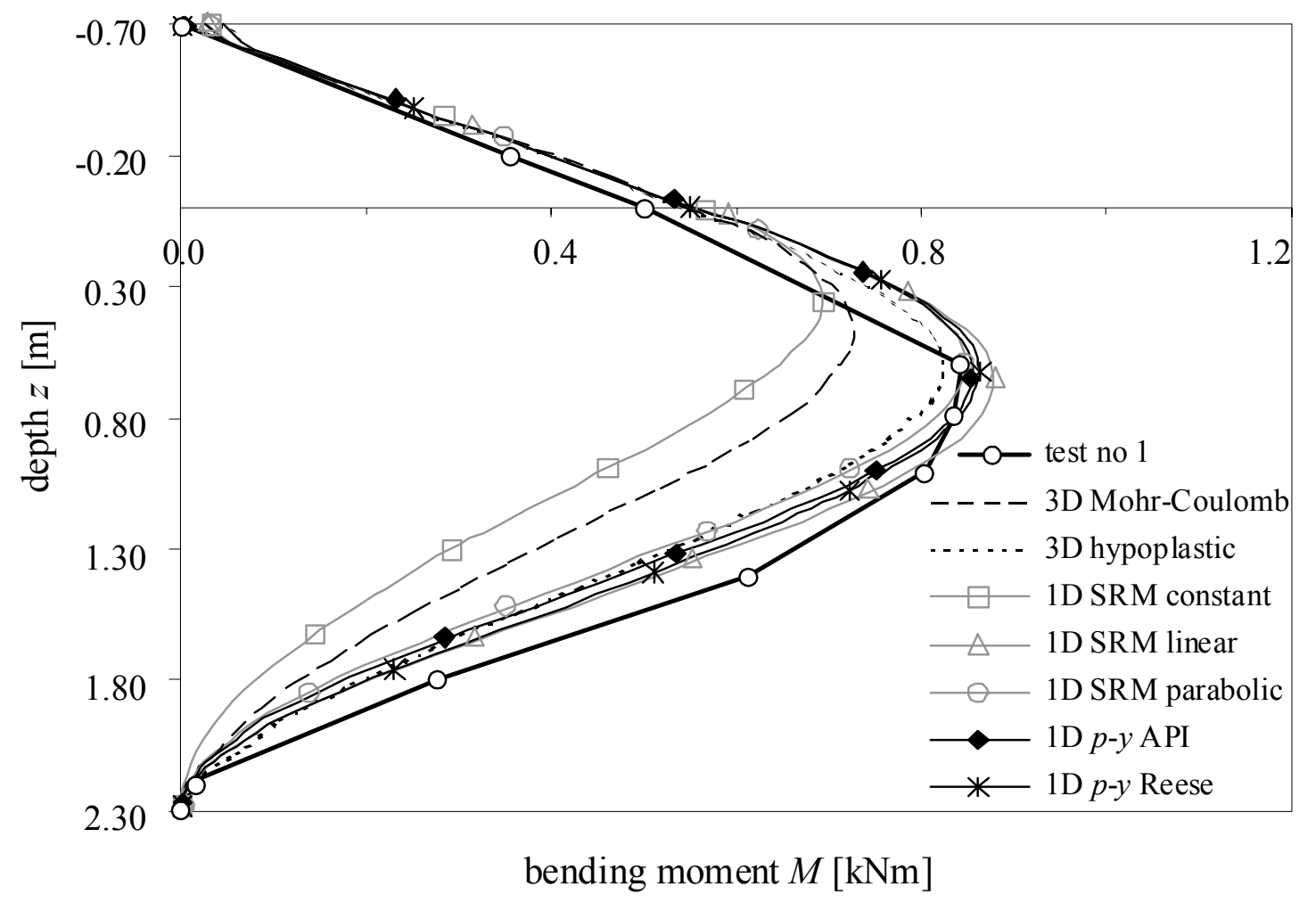

Fig. 7.39: Comparison of bending moments for different methods and the experimental test result at a lateral load of $800 \mathrm{~N}$ 
Although the results for the most complex of the applied models, the hypoplastic approach, predicts fairly good predictions the model might be prone to large deviations due to sensitivity of parameters. This issue is brought forward as the input is based on numerous values which are partly difficult to obtain. Thus, a sensitivity study for the given case is performed varying specific parameters by $\pm 10 \%$ (Tab. 7.10).

Tab. 7.10: Parameters and comparison for sensitivity study for hypoplasticity

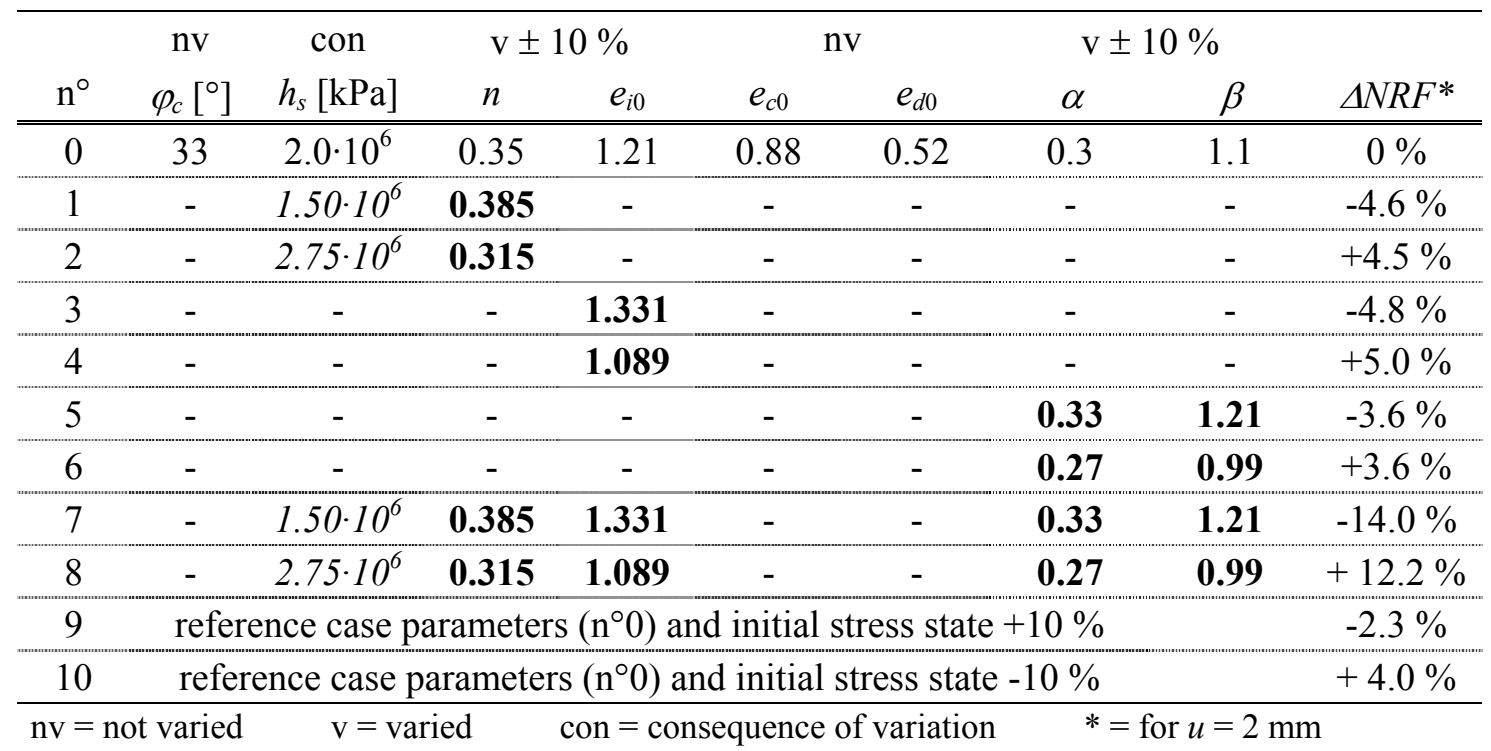

Herle (1997) performed a sensitivity study for soil parameters implemented in the hypoplastic model. Nevertheless, his study is limited to soil element tests as e.g. triaxial compression tests. Therefore, the given case is investigated by varying parameters except for for $\varphi_{c}, e_{d 0}$, and $e_{c 0}$ since they can be derived from experiments with small errors (Herle, 1997). For all cases the model is analysed by displacing the pile head by $2 \mathrm{~mm}$ and extracting the corresponding lateral or reaction force $N R F$. Based on the reference case $\left(\mathrm{n}^{\circ} 0\right)$ the deviation $\triangle N R F$ of the reaction force $N R F$ is considered and compared in Tab. 7.10.

Varying the parameters by $\pm 10 \%$ influences $N R F$ by about $\pm 5 \%$ as seen for cases $\mathrm{n}^{\circ} 1$ to $n^{\circ} 6$. Thus, the model regarding theses parameters is quite insensitive to changes for small loads. The combination of variations $\left(n^{\circ} 7\right.$ and $\left.n^{\circ} 8\right)$ results in a reduction or increase of the $N R F$ similar to the product of the individual influences. Further, two additional numerical analyses $\left(n^{\circ} 9\right.$ and $\left.n^{\circ} 10\right)$ were performed for investigating the effect of the initial stress state. Here, the imposed geostatic stress state was adopted by $\pm 10 \%$. Again, the influence on the reaction force remains small. Generally, it can be seen that for these small loads and displacements, which also occur in buckling failures the hypoplastic model appears to be fairly insensitive to parameter changes. Nevertheless, due to the non-linearity of the soil model this aspect may change significantly for larger forces or deformations close to failure. The latter issue was emphasized by some example analyses involving larger displacements. As this study is limited to small deformations this aspect is not further investigated in this work. 


\subsubsection{Discussion and conclusions}

The comparison revealed that most models provided acceptable predictions regarding the bending moments in the pile for a specific load. Nevertheless, the predicted deformations and displacements need to be interpreted carefully. This issue is well known from other numerical studies involving soil. The applicability of the well-known and widely used concept of the $p-y$ curves is confirmed as the predictions from the numerical analyses correlate well with the experimental results. Nevertheless, the complex hypoplastic model also provides good agreement with the test results. As a consequence it is concluded that for calculating and assessing tubular pile structures an analysis with the concept of the $p-y$ curves is adequate with respect to the design of the carrying capacity. The simplicity of the model makes it more valuable for applications in contrast to the complex hypoplastic model. Coefficients of subgrade reaction approaches (SRMs) which take a variation of stiffness into account provide sufficiently reliable predictions, too. Nevertheless, the $3 \mathrm{D}$ analysis with the elastic-plastic model and the constant SRM approximation differ from the other predictions and the test results. It can be stated that based on the investigated cases design calculations can be based on the simple soil models. Still, the applied programs should have the possibility of taking a varying stiffness as a function of the depth or stress state into account. The results regarding absolute values for the displacements should be used with great care. 


\section{Geometrical imperfections}

\subsection{General}

The classical buckling load can be derived for axially loaded, geometrically perfect and linear elastic cylindrical shell structures using the linear buckling theory:

$$
\sigma_{x R c}=0.605 \cdot E \cdot \frac{t}{r}
$$

with the Young's modulus $E$, the shell thickness $t$, the radius $r$ and for a Poisson ratio $\mu$ of 0.3. Since the theoretical buckling stress $\sigma_{x R c}$ cannot be reached for real structures, reduction factors are introduced for the design procedure. In the assessment against axial compression the reduction factor $\chi_{x}$ (prEN 1993-1-6:2002-05) covers the influence of geometrical imperfections (radial deviations and uneven supports) and structural imperfections (e.g. residual stresses). In the following the notation $\alpha_{x}$ is used for the linear geometric imperfection factor for axially loaded cylinders and $\chi_{x}$ is the global axial compression reduction factor.

The buckling curve proposed in DIN 18800-4 for the coefficient $\kappa_{2}$ (herein the reduction factor) incorporating the slenderness of the cylinder was derived empirically. The curve is based on more than 1200 experiments with axially loaded cylinders conducted between end of 1920 and end of 1990 and published by several researchers. Schmidt (1991) depicted the results from the experiments and the proposed reduction curves from DIN 18800-4 in one diagram. It can be seen that the experimental results scatter significantly.

The best known and most investigated parameter for the detrimental reduction of the carrying capacity in comparison to the classical buckling load is the radial deviation of the shell wall from the ideal shape. Besides this influence, there are further nonnegligible effects on the carrying capacity of an axially loaded cylinder. For example, residual stresses introduced due to welding may increase or decrease the buckling load by $30 \%$ (Hübner et al., 2003). Further aspects significantly influencing the carrying capacity of axially loaded cylinders are non-uniformly distributed axial loads along the edges or uneven supports. These effects have been investigated far less in research.

Saal et al. (1979) investigated thin-walled axially loaded cylinders with internal pressure in experiments. For three of the investigated cylinders, very non-uniform longitudinal strain distributions were measured. These non-uniform longitudinal strain distributions were ascribed to imperfect support, i.e. uneven edges of the cylinders. To prevent the obvious detrimental influence of the unevenness of the supports, the Araldit D used for joining the boundaries was enhanced with silica dust in the following experiments. 
From these experiments it is obvious that the phenomenon of imperfect supports was only discovered in the 70 s and 80 s, and thus had only been regarded to a minor degree in the reduction factors given in the design codes which are based on the numerous tests. Hence, the known values for the linear reduction factors $\alpha_{x}$ as introduced in Eurocode (prEN 1993-1-6:2002-05) comprise imperfections of the shell's geometry and non-uniform load introductions or uneven supports.

In the first step the buckling reduction factor is briefly discussed, in order to obtain an insight to the possible amount of improvement for the reduction factor in the dimensions of combiwalls. Based on the estimate, it is emphasized in the following that the influence of uneven supports is highly detrimental to the buckling behaviour of shell structures, even for small deviations of the supports. The non-uniform stress distribution arising from uneven supports only fades out slowly over the length of the cylinder. Since for combiwalls very long tubes are used (up to $40 \mathrm{~m}$ ), the disturbed stress distributions disappear over the length of the piles.

\subsection{Buckling reduction factor}

Since the part of the reduction factor due to the load reduction from uneven supports is assumed to be negligible for combiwalls, its influence is estimated. In order to evaluate this, the design procedure according to prEN 1993-1-6:2002-05 is examined for different fabrication quality tolerance classes. Fig. 8.1 and Fig. 8.2 summarize the buckling reduction factor $\chi_{x}$ for elastic-plastic effects for different cases as a function of the characteristic imperfection amplitude $\Delta w_{k}$ normalized by the thickness $t$. In Fig. 8.1 the results for a yield stress of $235 \mathrm{~N} / \mathrm{mm}^{2}$ are depicted, while in Fig. 8.2 the reduction factors are given for a yield stress of $430 \mathrm{~N} / \mathrm{mm}^{2}$. These values cover the limits for common harbour constructions. The buckling reduction factor $\chi_{x}$ is evaluated according to prEN 1993-1-6:2002-05 as described in chapter 4. The values are given for $r / t$ ratios of 30,50 , and 100 . The example calculated in chapter 4 corresponds to an $r / t$ ratio of 50 and $f_{y}=430 \mathrm{~N} / \mathrm{mm}^{2}$. The other two cases represent rough limits for possible tubular constructions. All cylinders except for $r / t=30$ and $f_{y}=235 \mathrm{~N} / \mathrm{mm}^{2}$ are classified as Class 4 cylinders according to prEN 1993-1-1:1992 and thus have to be assessed against shell buckling according to prEN 1993-1-6:2002-05.

As described previously a quality Class B in prEN 1993-1-6:2002-05 can be assumed for the design of piles in combiwalls (prEN 1993-5:1998). The increase of the reduction factor $\chi_{x}$ from the quality Class $\mathrm{B}$ to a perfect cylinder $\left(\Delta w_{k}=0\right)$ is $2.5 \%$ for the reference case from chapter 4 . This increase is related to an increase of the elastic reduction factor $\alpha_{x}$ of $25 \%$ for the same range. While the imperfection amplitude significantly affects the elastic reduction factor, the change of the elastic-plastic reduction factor is very small. The maximum theoretical increase of the reduction factor $\chi_{x}$ decreases for smaller yield stresses; here for $f_{y}=235 \mathrm{~N} / \mathrm{mm}^{2}$ only $1 \%$. This relation can also be identified for other $r / t$ ratios. A largest theoretical increase of the reduction factor $\chi_{x}$ of about $9 \%$ for $r / t=100$ and $f_{y}=430 \mathrm{~N} / \mathrm{mm}^{2}$ is still rather small. 
Thus, it can be seen that for the investigated dimensions and material properties the influence of geometrical imperfections is rather small. The comparison with a perfect cylinder according to prEN 1993-1-6:2002-05 shows that a more detailed analysis of the geometrical imperfections regarding uneven supports can only lead to a small gain with respect to the carrying capacity. Nevertheless, the investigation of this aspect is further discussed in this work as it is an important factor influencing the carrying behaviour.

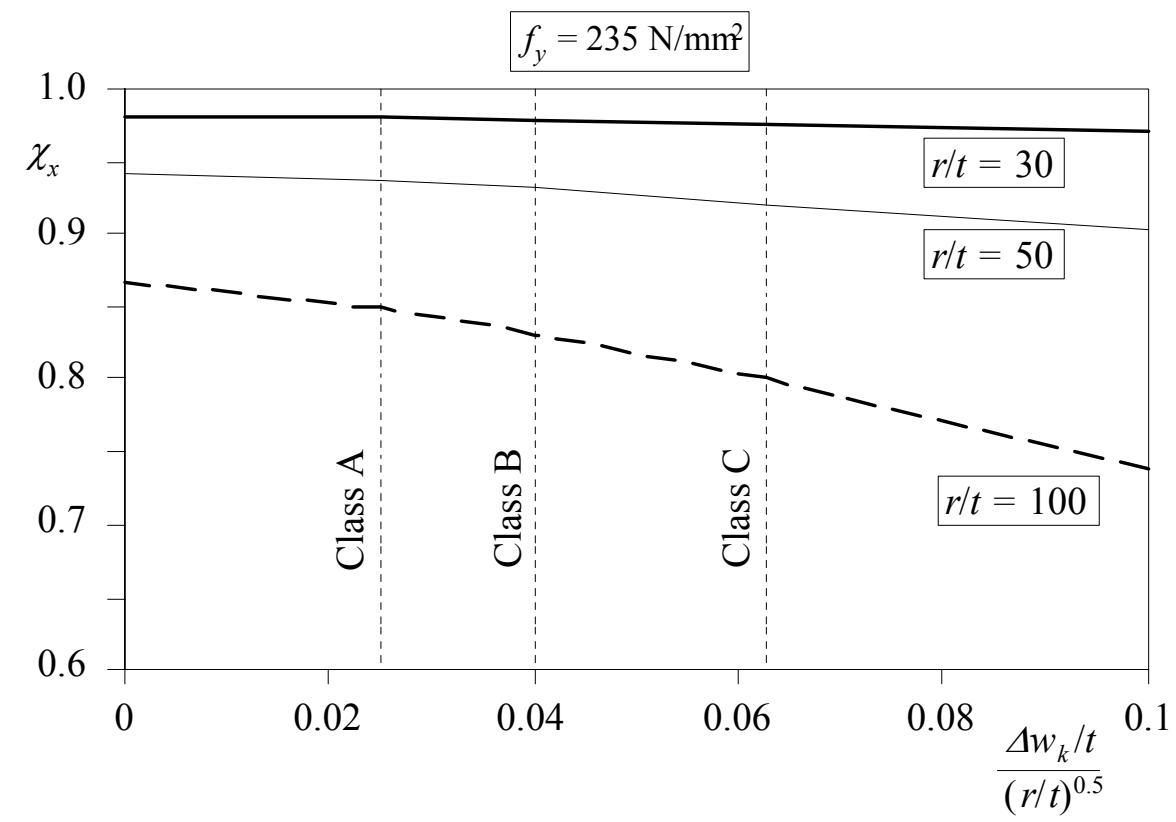

Fig. 8.1: Reduction factor $\chi_{x}$ as a function of the characteristic imperfection amplitude for a yield stress of $235 \mathrm{~N} / \mathrm{mm}^{2}$

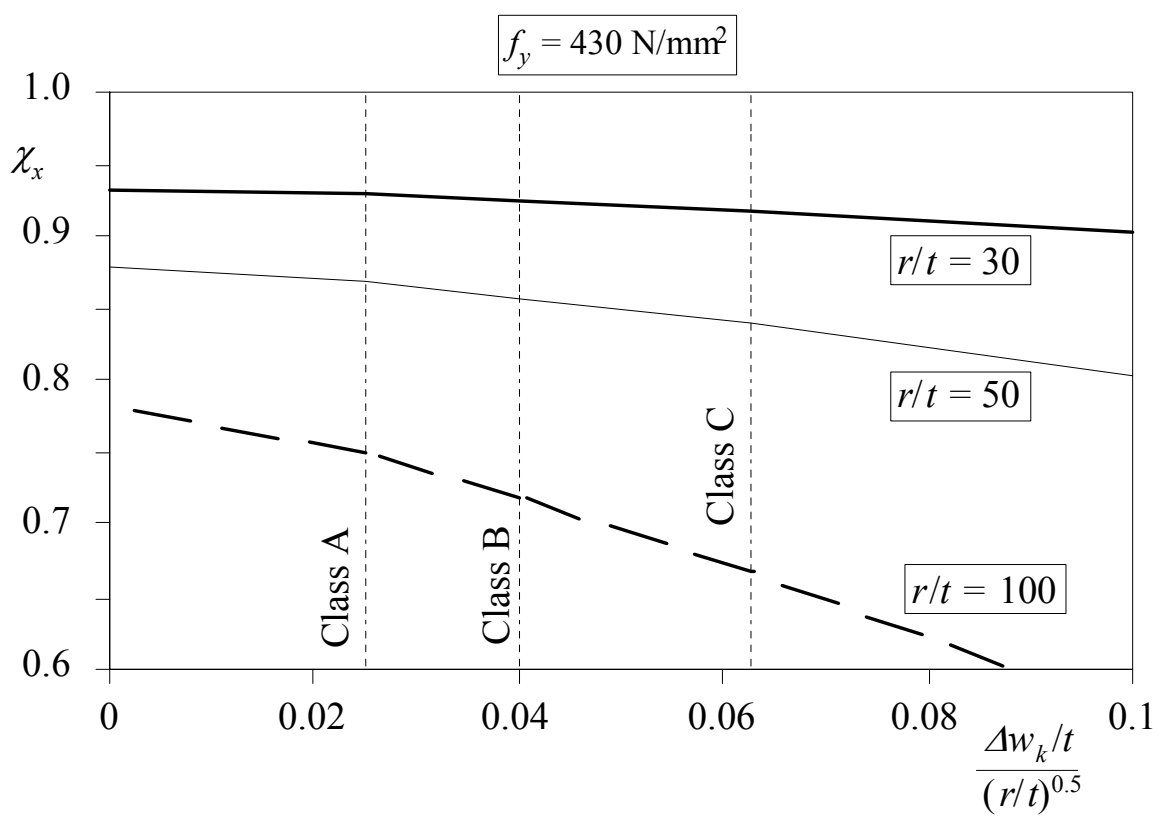

Fig. 8.2: Reduction factor $\chi_{x}$ as a function of the characteristic imperfection amplitude for a yield stress of $430 \mathrm{~N} / \mathrm{mm}^{2}$ 


\subsection{Geometrically imperfect boundary conditions}

Knebel (1997) performed preliminary studies within the framework of his research which tackled the problem of uneven supports using a quarter cylinder. The geometry is based on the cylinders investigated in the research co-operation SFB219. Knebel points out that a model involving a detailed contact formulation with interacting elements should be preferred to an approach using varying nodal loads. In the following the problem of uneven supports is investigated further based on the findings from Knebel.

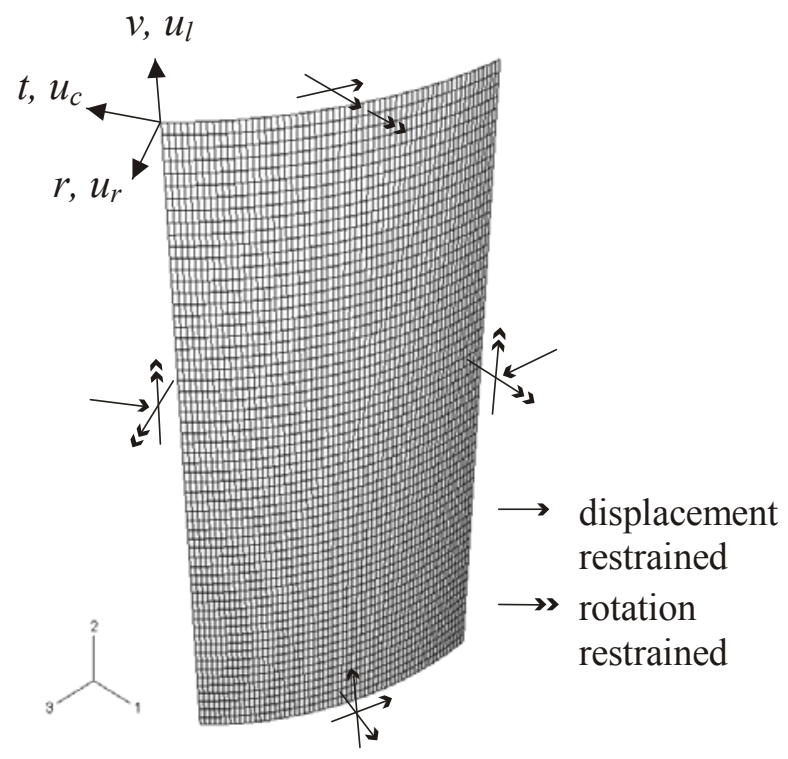

Fig. 8.3: Boundary conditions for segment from cylinder

The boundary conditions for this investigation are depicted in Fig. 8.3. First, according to the cylinders from SFB219 a $45^{\circ}$ segment of a cylinder with radius $r=625 \mathrm{~mm}$ and wall thickness $t=0.625 \mathrm{~mm}$ is investigated. A Young's modulus of $E=205000 \mathrm{~N} / \mathrm{mm}^{2}$ and a Poisson ratio of $\mu=0.3$ are used in the analyses. The imposed geometrically imperfect boundary condition is a centrally positioned cosine function over an area of $15^{\circ}$ (Fig. 8.4). Due to the symmetry of the system the gap appears eight times over the entire circumference of the cylinder. The bottom edge of the cylinder is restrained in radial and circumferential direction. The gap is modelled using interaction elements of different lengths, i.e. with different distances to the supports, acting only if subjected to axial compression and allowing the structure to elevate under tension. The implemented contact formulation is the augmented Lagrange contact formulation with the default settings concerning the penalty factor from ABAQUS. Besides this method ABAQUS Standard only provides the classical Lagrange multiplier method which can be used in combination with a ,soft" or ,hard“ formulation. Penalty contact enforcement is not supported in ABAQUS Standard but in ABAQUS Explicit. The advantage for the augmented Lagrange contact formulation is that the magnitude of the penalty factor has no influence on the result as shown by Knebel (1997). The calculations are performed as non-linear load-displacement analyses using the Riks formulation. 


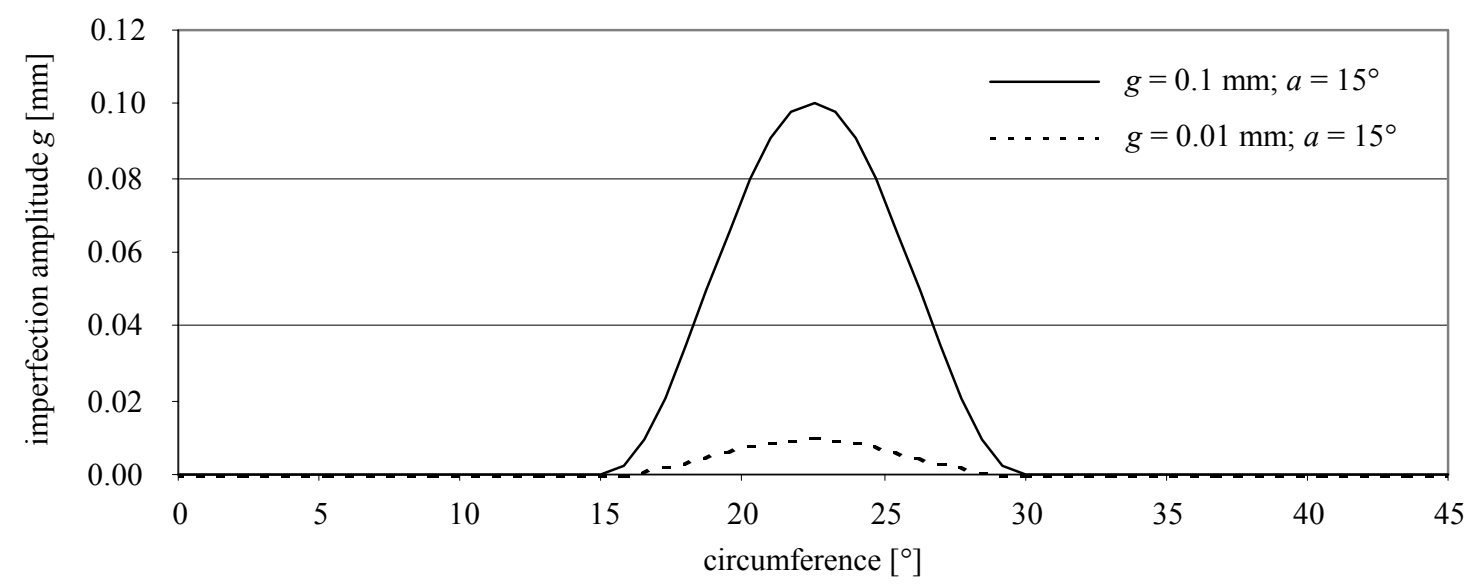

Fig. 8.4: Distribution of the geometrically imperfect boundary conditions over the circumference

In Fig. 8.5 the axial membrane stresses at the edge of the cylinder are depicted for the imperfection amplitudes $g=0.1 \mathrm{~mm}$ and $0.01 \mathrm{~mm}$ for the maximum load, which is $55 \%$ and $86 \%$ of the classical buckling load for a perfect cylinder.

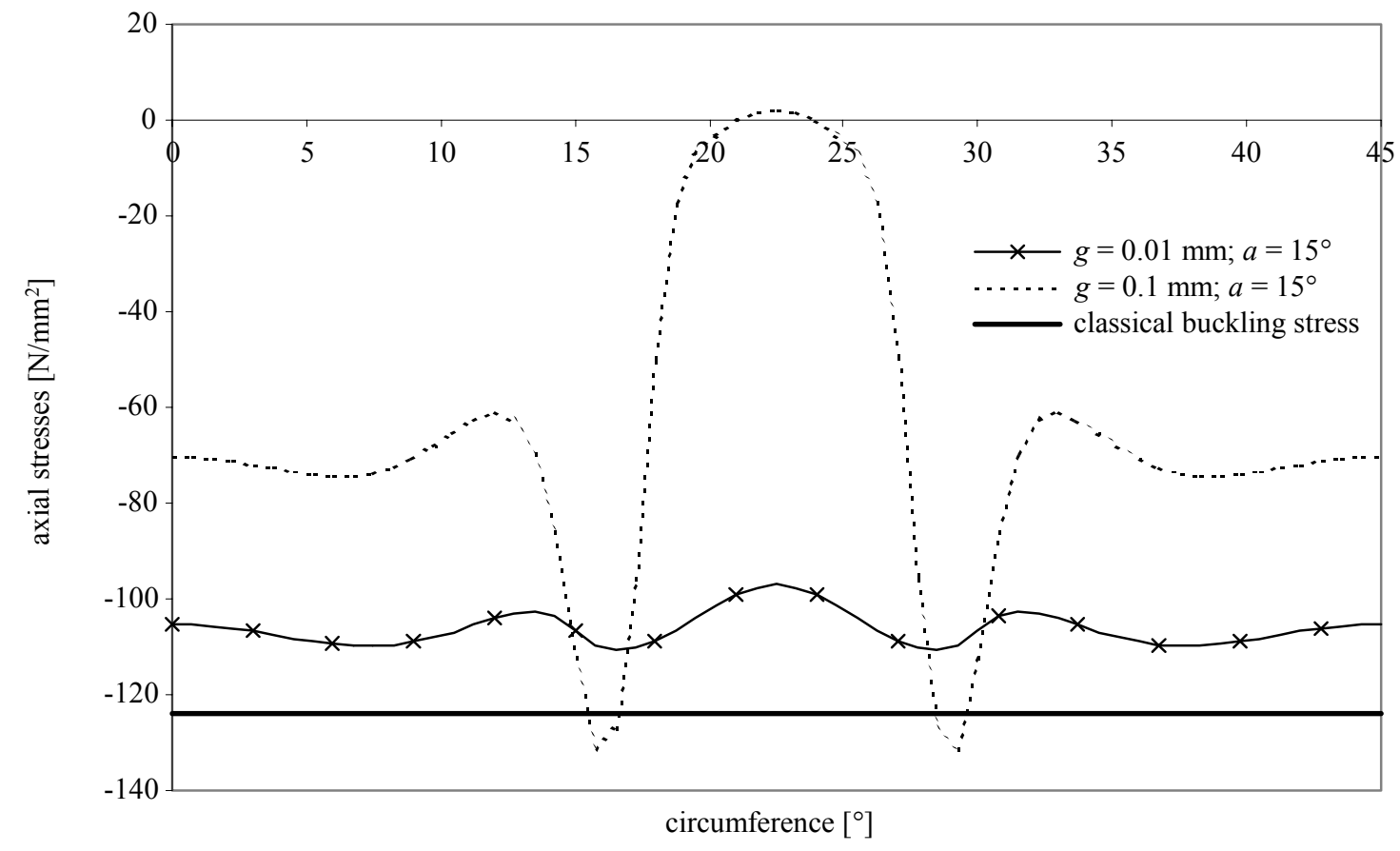

Fig. 8.5: Axial membrane stress distribution for maximum load at cylinder edge

It can be seen that the stresses at the edge of the cylinder differ considerably due to the non-uniform load introduction arising from the uneven supports. For the larger gap very high stress peaks arise in the area of the gap's ends. For smaller gaps which close before failure, the maximum axial membrane stresses do not appear at the edge of the cylinder but a little bit away from the edge. The maximum axial and circumferential membrane stresses appear in the area of the buckles forming inwards as it can be seen in Fig. 8.6. For both cases the buckles evolving from the uneven supports lead to a rapid increase of 
the bending moments in the shell and thus large radial deformations. This effect leads to the failure of the structure.

Fig. 8.6 shows the axial stress distribution for the cylinder segment as a contour plot and the deformed geometry at maximum load for an imperfection amplitude of $g=0.1 \mathrm{~mm}$.
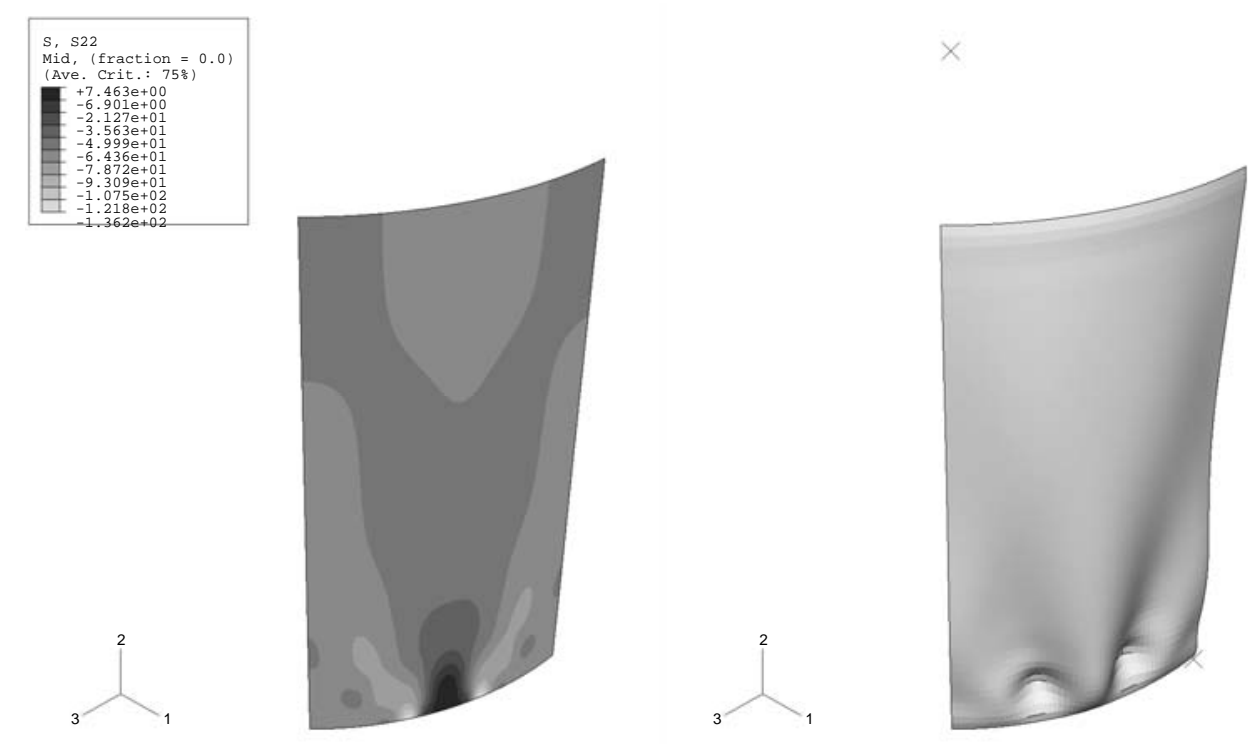

Fig. 8.6: Axial stresses and deformations scaled with a factor of 40 at maximum load for $\mathrm{g}=0.1 \mathrm{~mm}$ and $\alpha=15^{\circ}$

In the following investigation a cylinder with, a radius of $900 \mathrm{~mm}$ and a height of 1000 $\mathrm{mm}$ is chosen as a cylinder with typical diameter for combiwalls. Because the influence of the different imperfections is more noticeable for high $r / t$ ratios, in the first approach the thickness $t$ of the cylinder is chosen as a tenth of the real thickness in order to emphasize the influence of radial imperfections and uneven supports. This results in an $r / t$ ratio of 500. According to the previous case an uneven support in the shape of a cosine function is introduced, and an eigenmode affine radial imperfection is superposed. The results are summarized in Tab. 8.1 corresponding to the reference ratio of the imperfection $\Delta w_{i} / t$. The value $\Delta w_{i}$ stands for the amplitudes of radial deviations or uneven supports (gaps). For the investigation a $60^{\circ}$ segment with symmetric boundary conditions was chosen in order to be able to reflect all relevant failure modes.

These investigations reveal similar detrimental influences of both imperfection types on the load carrying capacity of the cylinder (Fig. 8.7 and Tab. 8.1). With regard to $\Delta w_{i} / t$ the decrease of the buckling load is smaller for the unevenness of the supports and it approaches a constant value at much smaller imperfections than with the radial imperfections. Thus, the radial imperfections are dominant and govern the behaviour of the cylindrical shell if both imperfection types are present. 
Tab. 8.1: Comparison of the influence of the different imperfection types $(r / t=500)$

\begin{tabular}{|c|c|c|c|c|c|c|}
\hline$\sigma / \sigma_{c l}[\%]$ & & \multicolumn{5}{|c|}{$\begin{array}{l}\text { geometrically imperfect boundary conditions } \\
\qquad 6 x \text { cosine } 15^{\circ}\end{array}$} \\
\hline \multicolumn{2}{|l|}{$\Delta w_{i} / t$} & 0 & 0.1 & 0.5 & 1.0 & 1.5 \\
\hline perfect & $\mathbf{0}$ & 100 & 64.6 & 59.9 & 58.0 & 57.6 \\
\hline \multirow{2}{*}{$\begin{array}{l}\text { eigenmode affine } \\
\text { perfect (1. EM) }\end{array}$} & 0.1 & 59.9 & 55.1 & 50.1 & 49.1 & 48.9 \\
\hline & 0.5 & 32.7 & 32.4 & 31.9 & 31.7 & 31.6 \\
\hline \multirow{2}{*}{$\begin{array}{r} \\
\\
\\
\end{array}$} & 1.0 & 29.9 & 29.8 & 29.7 & 29.2 & 29.2 \\
\hline & 1.5 & 26.1 & 26.0 & 25.6 & 25.5 & 25.5 \\
\hline \multirow{4}{*}{$\begin{array}{l}\text { eigenmode affine } \\
\text { perfect }(2 . \mathrm{EM})\end{array}$} & 0.1 & 60.5 & 56.0 & 51.1 & 50.4 & 50.2 \\
\hline & 0.5 & 36.1 & 35.6 & 35.3 & 35.0 & 34.9 \\
\hline & 1.0 & 31.0 & 30.7 & 30.5 & 30.5 & 30.5 \\
\hline & 1.5 & 26.1 & 26.5 & 26.5 & 26.5 & 26.5 \\
\hline
\end{tabular}

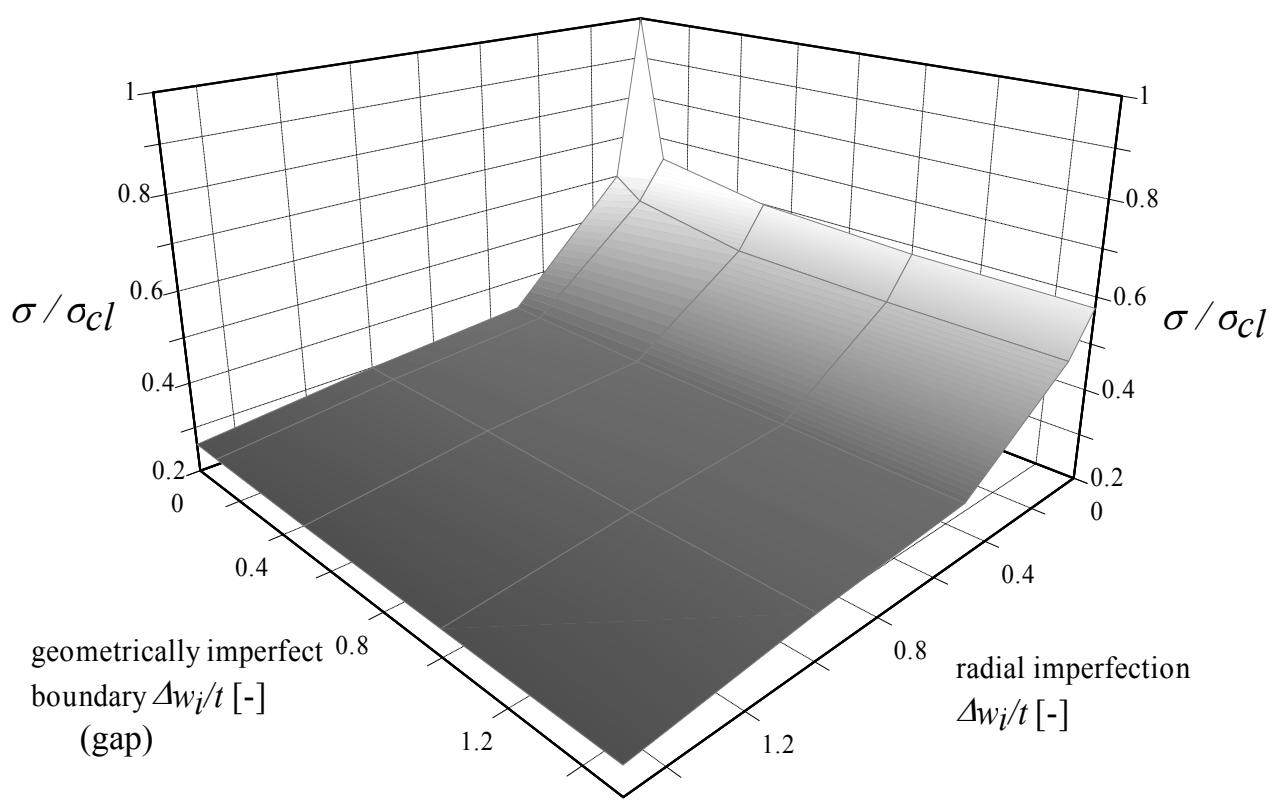

Fig. 8.7: Comparison of the influence of the different imperfection types - radial imperfections with first eigenmode $(r / t=500)$

Tab. 8.2 lists the results for the case that the uneven boundary conditions stretch over the entire circumference as a cosine function. It can be seen that the influence of the uneven boundary conditions increases significantly by the higher reduction of the 
buckling strength. Similar to the behaviour found in the previous cases for geometrically imperfect boundary conditions, it can be seen that the decrease of the carrying capacity saturates with increasing imperfection amplitude and converges to a constant value. (Tab. 8.1). The behaviour of the buckling strength tending to a limit value cannot be found for the radial imperfections.

For the investigated case of uneven support over the entire circumference of the shell, small radial imperfections lead to a slight increase of the buckling strength. The cause for the enhanced buckling strength is the fact that the eigenmode affine radial imperfection mainly comprises of ring-shaped buckles which act similarly to stiffeners against a failure due to the uneven supports. Cylinders with only geometrically imperfect boundary conditions tend to buckle with a failure mode, which consists of circumferential buckles similar to the cosine shape of the uneven supports (Fig. 8.6). Hence, both failure modes may oppose each other in some areas and lead to a beneficial instead of detrimental effect. This phenomenon is highly dependent on the imposed imperfections and thus also on the $r / t$ ratio.

Tab. 8.2: Comparison of the influence of the different imperfection types $(r / t=500)$

\begin{tabular}{|c|c|c|c|c|c|c|}
\hline$\sigma / \sigma_{c l}[\%]$ & & \multicolumn{5}{|c|}{$\begin{array}{l}\text { geometrically imperfect boundary conditions } \\
24 x \text { cosine } 15^{\circ} \text { (entire circumference) }\end{array}$} \\
\hline \multicolumn{2}{|l|}{$\Delta w_{i} / t$} & 0 & 0.1 & 0.5 & 1.0 & 1.5 \\
\hline perfect & $\mathbf{0}$ & 100 & 50.9 & 37.0 & 36.2 & 35.0 \\
\hline \multirow{2}{*}{$\begin{array}{l}\text { eigenmode affine } \\
\text { perfect (1. EM) }\end{array}$} & 0.1 & 59.9 & 54.8 & 48.7 & 48.5 & 48.3 \\
\hline & 0.5 & 32.7 & 32.7 & 32.7 & 32.7 & 32.7 \\
\hline \multirow{2}{*}{$\begin{array}{r} \\
\\
\\
\end{array}$} & 1.0 & 29.9 & 30.2 & 29.4 & 29.4 & 29.4 \\
\hline & 1.5 & 26.1 & 27.9 & 27.9 & 27.9 & 27.9 \\
\hline
\end{tabular}

Similar to the radial imperfections, the influence of uneven supports is minor for small $r / t$ ratios. As an example some results are listed in Tab. 8.3. For an $r / t$ ratio of 50, corresponding to the tubular piles used for combiwalls, the first buckling eigenmode under axial load consists of checkerboard-like buckles. In contrast to the previous cylinder the sole influence of uneven supports is very small. Whilst for example for a boundary imperfection amplitude of $\Delta w_{i} / t=1.0$ the reduction of the load carrying capacity for a cylinder with an $r / t$ ratio of 500 drops by nearly $65 \%$, the load carrying capacity for an $r / t$ ratio of 50 decreases only by about $4 \%$. The varying reductions of the buckling load arise from the different buckling eigenmodes of the thin-walled and thick-walled shell and the associated buckling half-wave lengths in comparison to the imposed imperfections. As a consequence, the main focus shall be on the relative influence of the two interaction imperfection types. 
The effect of uneven supports becomes more important for the case of combined imperfections. For the thin-walled cylinder with radial imperfections of the amplitude $\Delta w_{i} / t=1.0$ additional imperfect boundary conditions result in a slight decrease of the carrying capacity $(2 \%)$. The same case for the thick-walled cylinder leads to a reduction of $15 \%$.

Tab. 8.3: Comparison of the influence of the different imperfection types $(r / t=50)$

\begin{tabular}{|c|c|c|c|c|c|c|}
\hline \multicolumn{2}{|l|}{$\sigma / \sigma_{c l}[\%]$} & \multirow{2}{*}{\multicolumn{5}{|c|}{$\begin{array}{l}\text { geometrically imperfect boundary conditions } \\
24 x \text { cosine } 15^{\circ} \text { (entire circumference) }\end{array}$}} \\
\hline \multirow{2}{*}{\multicolumn{2}{|c|}{$\Delta w_{i} / t$}} & & & & & \\
\hline & & 0 & 0.1 & 0.5 & 1.0 & 1.5 \\
\hline perfect & $\mathbf{0}$ & 100 & 98.1 & & 90.2 & \\
\hline \multirow{2}{*}{$\begin{array}{l}\text { eigenmode affine } \\
\text { perfect }(1 . \mathrm{EM})\end{array}$} & 0.1 & & & & & \\
\hline & 0.5 & & & & & \\
\hline \multirow[b]{2}{*}{ L } & 1.0 & 77.5 & 72.4 & & 67.3 & \\
\hline & 1.5 & & & & & \\
\hline
\end{tabular}

The analyses from the first studies reveal the high dependency of the carrying capacity on the imperfection type and amplitude. The influence of uneven supports increases considerably for slender shells, but has a larger impact for thick-walled shells subjected to combined imperfections. The preliminary investigations emphasize that the influence of the imperfection types varies strongly, and depends on parameters such as dimension, imperfection pattern, amplitude, symmetry of the imperfections, and combination of different imperfection types.

For small $r / t$ ratios the elastic buckling load will not be reached since the base material yields prior to buckling. The classical buckling load for the last investigated case can be calculated for a standard steel $\left(E=210000 \mathrm{~N} / \mathrm{mm}^{2}\right)$ with $(8.1)$ as $\sigma_{x R c}=2451 \mathrm{~N} / \mathrm{mm}^{2}$. A common constructional steel e.g. S235 yields at $\sigma_{y}=240 \mathrm{~N} / \mathrm{mm}^{2}$ which is about a tenth of the classical buckling stress. Therefore, the previous case is investigated taking an elastic-perfectly plastic material behaviour into account. A yield stress $\sigma_{y}$ of $240 \mathrm{~N} / \mathrm{mm}^{2}$ is used in combination with the $J_{2}$ flow theory. The input is based on a true stress - true (logarithmic) strain relationship. In $\mathrm{v}$ some results for geometrically and materially non-linear analyses are summarized. The calculated resistances $R$ are compared with the plastic reference resistance $R_{p l}$ from a geometrically linear and materially non-linear analysis. The plastic reference resistance $R_{p l}$ corresponds to an axial membrane stress of $240 \mathrm{~N} / \mathrm{mm}^{2}$ in comparison to the classical buckling stress of $2451 \mathrm{~N} / \mathrm{mm}^{2}(\approx 9.4 \%$ of the classical buckling stress $)$. 
Tab. 8.4: Comparison of the influence of the different imperfection types $(r / t=50)$ with plasticity

\begin{tabular}{|c|c|c|c|c|c|c|}
\hline$R / R_{p l}[\%]$ & & \multicolumn{5}{|c|}{$\begin{array}{l}\text { geometrically imperfect boundary conditions } \\
24 x \text { cosine } 15^{\circ} \text { (entire circumference) }\end{array}$} \\
\hline \multicolumn{2}{|l|}{$\Delta w_{i} / t$} & 0 & 01 & 05 & 10 & 15 \\
\hline perfect & $\mathbf{0}$ & 100 & 94.7 & 88.4 & 83.0 & 81.6 \\
\hline \multirow{2}{*}{$\begin{array}{l}\text { eigenmode affine } \\
\text { perfect (1. EM) }\end{array}$} & 0.1 & 100 & 95.7 & 89.0 & 84.0 & 81.9 \\
\hline & 0.5 & 85.1 & 83.0 & 76.6 & 72.3 & 71.8 \\
\hline & 1.0 & 67.0 & 63.8 & 57.7 & 56.4 & 54.7 \\
\hline & 1.5 & 57.4 & 55.3 & 49.8 & 48.9 & 46.9 \\
\hline
\end{tabular}

For a cylinder with imperfect boundary conditions the plastic failure initiates in the area of the supports as stress peaks evolve due to the uneven supports and the restriction of deformation arising from the supports. If a large radial imperfection pattern is imposed on the cylinder high bending stresses appear in the pre-deformed structure around the buckles leading to the failure of the system. For very small radial imperfections in combination with uneven supports the study reveals a small increase of the carrying capacity, which appears due to the fact that the small radial imperfections relief the biaxial stress condition.

The overall behaviour if including the effect of plasticity for the investigated case is similar to the elastic analyses as shown in Tab. 8.3. In contrast to the elastic analyses the decrease of the carrying capacity does not saturate as quickly with increasing imperfection amplitude and no specific limit value can be obtained. For example still considerable decreases in carrying capacity can be found for increasing imperfections of uneven supports at an amplitude between $\Delta w_{i} / t=1.0$ and $\Delta w_{i} / t=1.5$.

\subsection{Bending moment and geometrically imperfect boundary conditions}

Early studies by Flügge $(1932,1960)$ presented theoretical values of critical buckling stress for cylinders subjected to bending. Flügge proposed for an investigated reference cylinder prone to pure bending and based on theoretical considerations a maximum buckling stress $30 \%$ higher than the compressive buckling stress according to (8.1). These findings were referred to by different authors without pointing out that the factor of 1.3 is only based on calculations for a particular radius-thickness ratio $\left(1 / 12 \cdot t^{2} / r^{2}=10^{-6}\right)$, for a particular longitudinal buckling half-wavelength-radius ratio $(m \pi r / l=1)$ and a Poisson ratio of $1 / 6$. 
Based on the results from Seide and Weingarten (1961) design rules were proposed by the NASA by Peterson et al. (1968) which uses identical theoretical critical buckling stresses for bending and compression in combination with a correlation factor $\gamma$ which is higher for pure bending. This is because the failure may be triggered by any imperfection for the axially loaded cylinder while for the cylinder subjected to bending the buckling generally initiates in the area of maximum compressive stresses. Later studies revealed that for real structures the pre-buckling cross-sectional ovalization has a significant influence on the buckling moment reducing it far below the latter predictions (see e.g. Karamanos, 2002).

In the following it is investigated whether geometrically imperfect boundary conditions affect the buckling behaviour of a cylinder with bending moment differently from an axially loaded cylinder. A cylinder according to the previous section is used. For a height of $1000 \mathrm{~mm}$ and an $r / t$ ratio of 50 the radius is defined as $r=900 \mathrm{~mm}$. The numerical analyses are based on a cylinder segment of $180^{\circ}$ making use of the symmetry in order to investigate the cylinder subjected to a bending moment. Boundary conditions were applied according to Fig. 8.3. The uneven support was modelled using a cosine-shaped imperfection with an amplitude of $\Delta w_{i} / t=1.0$. The cosine waves were imposed such that the position of $\sigma_{\max }$ coincides with the maximum gap spacing. Comparing analyses revealed that the influence of the position of the cosine function is rather small. The number of cosine waves in the figures refers to the entire circumference.

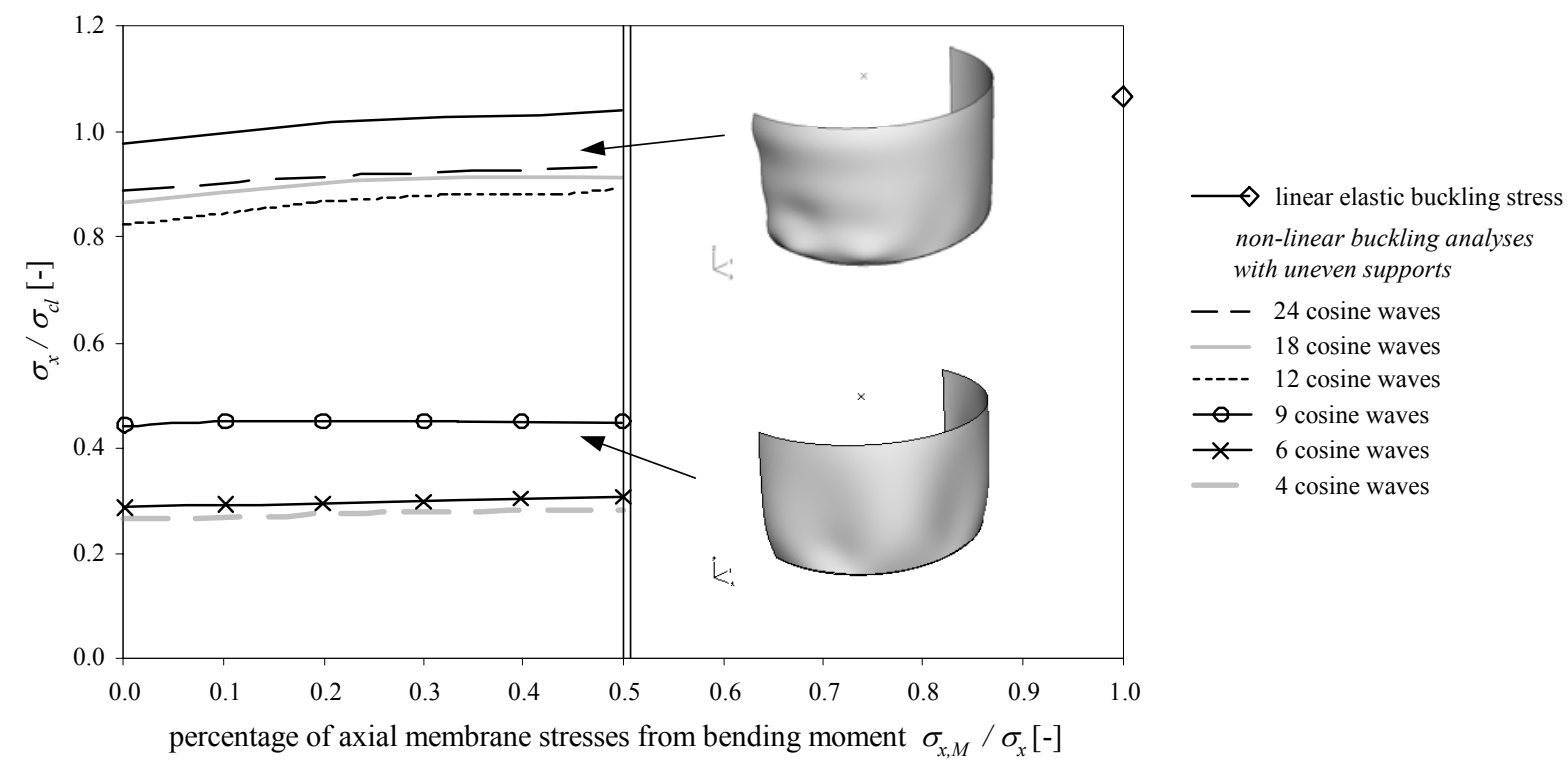

Fig. 8.8: Influence of different uneven supports with $\Delta w_{i} / t=1.0$ subjected to combined loading - cosine waves over the entire circumference

Since the contact modelling for the bottom edge of the cylinder can only transfer compression loads, initially an axial load has to be applied to the cylinder. The axial load must generate at least $50 \%$ of the compressive axial membrane stresses in order to prevent the cylinder from uplifting. Based on this approach the axial membrane stresses 
from axial loading are superposed with the axial membrane stresses arising from the applied bending moment. The buckling stresses normalized over the classical buckling stress (8.1) are depicted in Fig. 8.8 for different patterns of geometrically imperfect boundary conditions for the same imperfection amplitude. The normalized buckling stress is shown as function of the percentage of the axial membrane stresses arising from the bending moment. The value of $90.2 \%$ from Tab. 8.3 for a cylinder with only 24 cosine waves $\left(\Delta w_{i} / t=1.0\right)$ as uneven support can be found in Fig. 8.8 for a cylinder with $\sigma_{x, M} / \sigma_{x}=0$.

For the linear elastic, perfect case an increasing bending moment results in an increasing critical buckling load. For a cylinder subjected to a bending moment the classical buckling stress for solely axially loaded cylinders according to (8.1) is exceeded by $5 \%$ for the investigated case. This effect is relatively constant for the different imperfection patterns. The influence of the different imperfection patterns on the load carrying capacity is significant. Whereas for the investigated case a large number of imperfections ( 24 cosine waves along the entire circumference) only lead to a reduction of the buckling load of around $15 \%$, an imperfection pattern with a small number of imperfections (e.g. 6 cosine waves along the entire circumference) results in a decrease of the buckling load of $70 \%$. The imperfection sensitivity can be explained by how much the imperfection pattern and the buckling eigenmode are alike. The axial buckling half-wave length for a medium length cylinder with axial membrane stresses buckling in a checkerboard-like pattern can be calculated as follows (see e.g. Teng and Rotter, 2004):

$$
\lambda_{c l}=\frac{\pi}{\sqrt[4]{12\left(1-\mu^{2}\right)}} \sqrt{r t}
$$

For the reference cylinder the axial buckling half-wave length is around $440 \mathrm{~mm}$ which is similar to the length of cosine half-wave for the pattern including 6 cosine waves along the entire circumference $(470 \mathrm{~mm})$. The large decrease of the carrying capacity is confirmed in the buckling modes depicted in Fig. 8.8. The results emphasize that the small reduction of carrying capacity for thick-walled cylinders from the previous section is based on the chosen imperfection pattern and not the thickness of the shell.

In Fig. 8.10 the normalized buckling stresses are depicted for different patterns of geometrically imperfect boundary conditions for the imperfection type "block". It can be clearly seen in contrast to the results from Fig. 8.8 that the carrying capacity is considerably higher for the imperfection type "block". Still, a significant decrease of carrying capacity remains for imperfection lengths in the order of the axial buckling half-wave length as revealed in the previous case. In Fig. 8.11 results for all three imperfection types from Fig. 8.9 are shown in one diagram for the most detrimental case of 6 imperfection units ( 6 cosine waves, 6 trapeziums, 6 blocks). It can be concluded that the smaller the area which is in direct contact with the lower support (imperfection amplitude $=0$ ) is, the lower is the carrying capacity of the cylinder. The 
most detrimental case of the investigated types is the geometrically imperfect boundary condition modelled as cosine wave over the entire circumference.

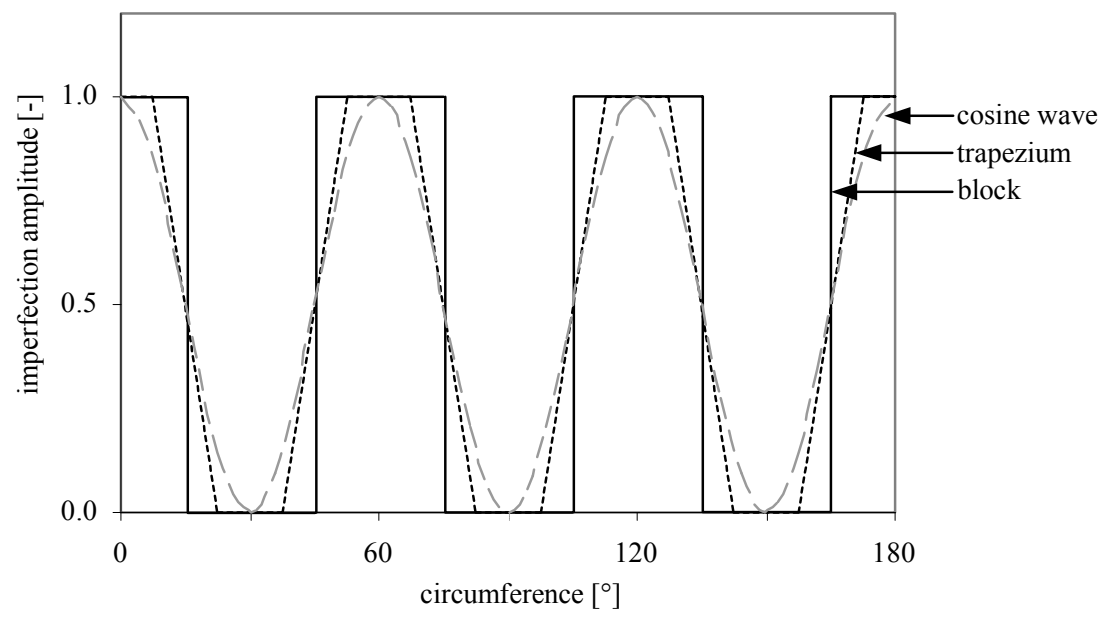

Fig. 8.9: Distribution of uneven supports for 6 cosine waves, 6 trapeziums, 6 blocks

For comparison the same problem is investigated in combination with other imperfection patterns. Fig. 8.9 shows three different imperfection patterns for uneven supports, namely cosine waves, trapeziums, and blocks. The depicted case refers to three units of the corresponding uneven support type over half of the circumference.

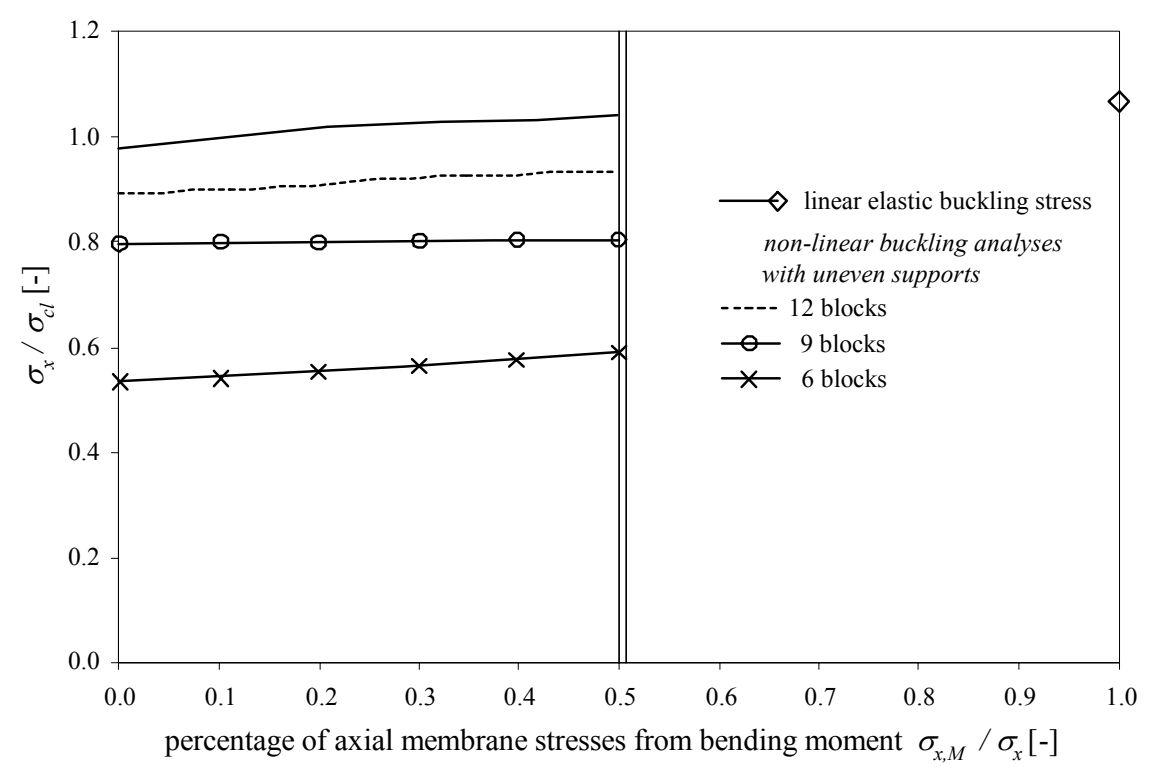

Fig. 8.10: Influence of different uneven supports with $\Delta w_{i} / t=1.0$ subjected to combined loading - blocks over the entire circumference

In the investigated case of an $r / t$ ratio of 50 and standard constructional steel the material of the cylinder will start yield before buckling elastically. Thus, the problem is investigated taking an elastic-perfectly plastic material behaviour as described in the previous section into account. The yield stress $\sigma_{y}$ is set to $240 \mathrm{~N} / \mathrm{mm}^{2}$ and the $J_{2}$ flow theory is assumed. Since the cosine wave shaped imperfection over the entire circumference delivered the smallest carrying capacity the case from Fig. 8.8 is re- 
calculated taking plasticity into account. In Fig. 8.12 the results for different uneven support types subjected to combined loading and considering plasticity are summarized. The plotted results for an imperfection shape of 3 cosine waves cover with type (b) the case of a distribution of uneven supports according to Fig. 8.9 and with type (a) the case from Fig. 8.9 shifted by $30^{\circ}$. The ordinate displays the ratio of the calculated carrying capacity $R$ and the plastic reference resistance $R_{p l}$ from a geometrically linear and materially non-linear analysis.

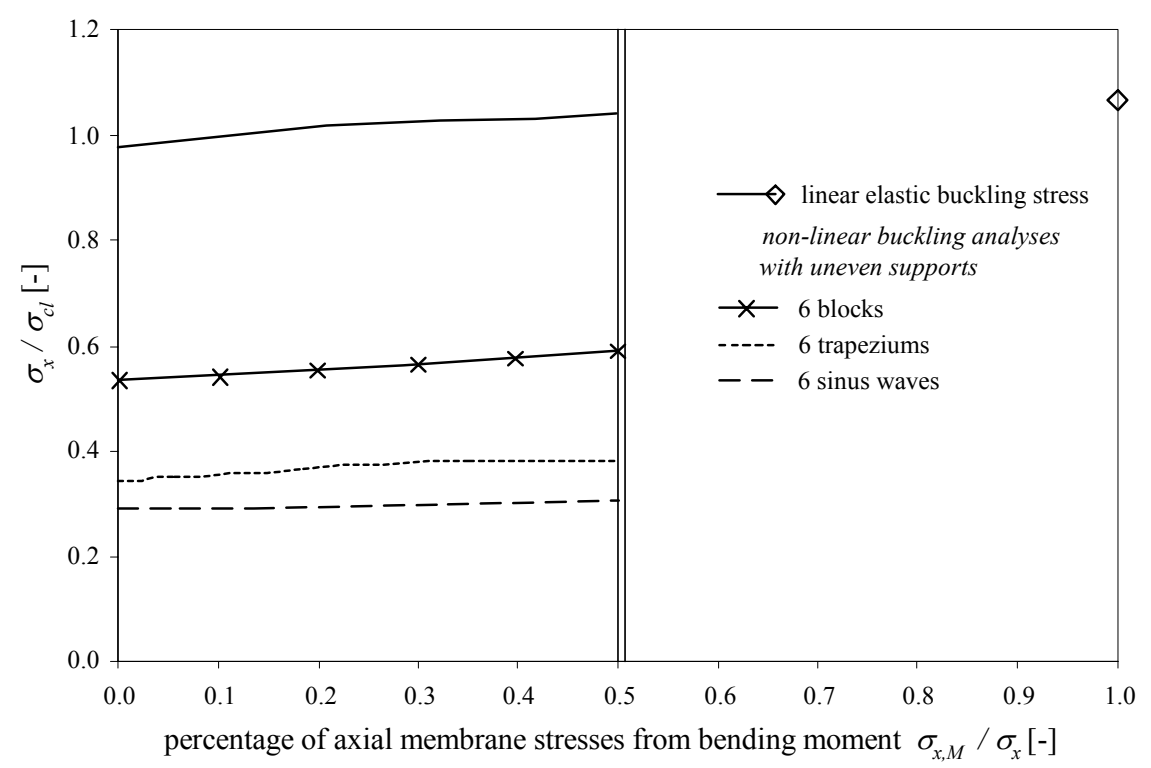

Fig. 8.11: Comparison of different uneven support types with $\Delta w_{i} / t=1.0$ subjected to combined loading

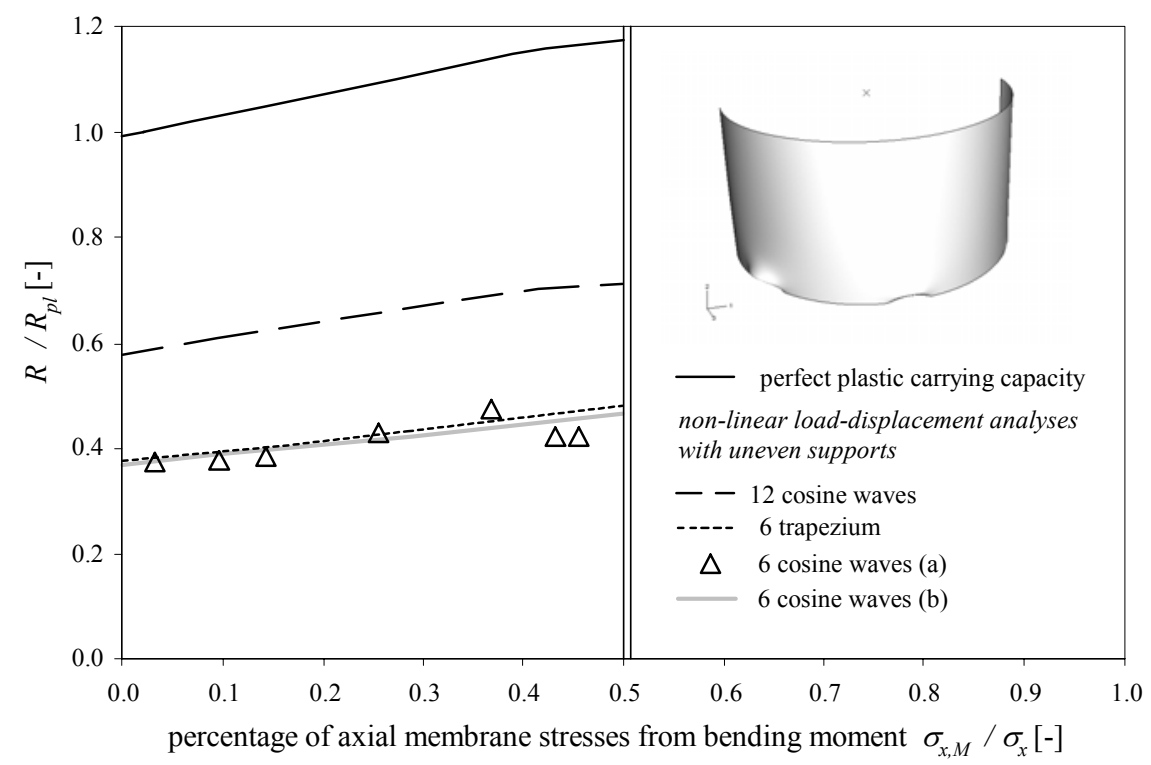

Fig. 8.12: Comparison of different uneven support types with $\Delta w_{i} / t=1.0$ subjected to combined loading considering plasticity

In the region around the local supports (imperfection amplitude zero) quickly a bi-axial stress state with large stresses evolves resulting in plastic deformations. The local stress 
concentrations lead to plastic bulges forming in these regions analogue to plastic "elephant foot" buckling (Fig. 8.12). Similar to the elastic analyses but to a more significant extent an increase of the carrying capacity can be found for larger moments. A smaller number of supports of 6 cosine waves instead of 12 cosine waves evidently leads to higher stress concentrations and a reduced carrying capacity. The difference in carrying capacity is not as distinct as found for the elastic calculations. Also the influence of the shape of the imperfection pattern is rather small as seen in Fig. 8.12. Since the failure is initiated due to plastification in the area of the supports, the influence of the shape of the supports seems negligible. Comparing the results for the cosine waves type (a) and (b) reveal that the effect of the position of the cosine function may influence the carrying capacity to a minor extent.

\subsection{Summary and conclusions}

The investigation of the geometrical imperfections due to uneven supports revealed several important issues. The findings can be grouped into three essential points:

In a first step, the current rules in design codes for geometrical imperfections were discussed. A detailed evaluation with respect to the reduction factor $\chi_{x}$ (prEN 1993-16:2002-05) for the geometrical and material range commonly used for tubular piles in combiwalls highlighted a rather small theoretical increase for perfect structures. This aspect is depicted in Fig. 8.1 and Fig. 8.2 where it can be seen that changing from Class $\mathrm{C}$ to Class A or even to a perfect structure only leads to a fairly small improvement for design purposes.

In the next step, the influence of geometrical imperfections due to uneven supports on the elastic reduction factor $\alpha_{x}$ or $\sigma / \sigma_{c l}$ was investigated. Generally, the presence of uneven supports significantly influences the load carrying behaviour of a shell subjected to axial membrane stresses (Tab. 8.1 and Tab. 8.2). The shape, the amplitude, and the length of imperfections highly affect the buckling load making it very difficult to derive simple design proposals. For rather thick-walled cylinders, uneven supports affect the carrying capacity, but their impact decreases, when radial shape imperfections are present (Tab. 8.3). Analyses with combined loading (bending moment and axial load) proved that a purely axial loading can be applied as conservative approximation.

In the last step, the connection to the design rules discussed in the first part was made by performing material elasto-plastic analyses with different geometrical imperfections regarding $R / R_{p l}$ or similarly the reduction factor $\chi_{x}$. Considering yielding does not particularly change the tendencies, but stresses a decreasing parameter sensitivity for uneven supports (Tab. 8.4). Fig. 8.1 provides a reduction factor $\chi_{x}$ of about 0.92 for $r / t=50$ and a radial imperfection amplitude $\Delta w_{i} / t=0.07(r / t)^{0.5}=0.5$. This value more or less similar in value and concept to $R / R_{p l}=0.85$ from Tab. 8.4 without uneven supports. The comparison between Tab. 8.3 and Tab. 8.4 shows the lack of considering uneven supports in design rules, but at the same time highlights the small theoretical 
gain of carrying capacity for combiwall geometries due to an application of reduced radial shape imperfections.

As the influence of plasticity appears to be small, a potential design proposal would involve a factor for increasing the linear reduction factor $\alpha_{x}$. If the influence of uneven supports on the buckling load is assumed roughly with $10 \%$ based on conservative considerations (Tab. 8.3), the increase of the design buckling stress would only be $1 \%$ or $2 \%$ for common dimensions in an assessment according to chapter 4 . 


\section{$9 \quad$ Influence of soil on buckling behaviour}

\subsection{Overview}

EN 1993-1-6 does not comment on a possible supporting effect of soil. For obtaining a better insight to the possible supporting influence of soil in contact with a buckling cylinder a simple finite element model is used for investigating the effect of the presence of soil. In the simplest case for modelling a foundation, it comprises of linear Winkler springs reacting to both tension and pressure, i.e. an adhesive foundation. In chapter 7 different coefficients of subgrade reaction $k_{S}$ for sand based on diverse derivations and references are summarized. For a maximum bending moment in a depth of around $10 \mathrm{~m}$ for combiwalls of a length of $30 \mathrm{~m}$, values of $10 \mathrm{MN} / \mathrm{m}^{3}$ to $100 \mathrm{MN} / \mathrm{m}^{3}$ can be assumed for the coefficient of subgrade reaction $k_{S}$ based on conservative assumptions. In references also values of $500 \mathrm{MN} / \mathrm{m}^{3}$ can be found for the coefficient of subgrade reaction $k_{S}$ at these depths. Different hypotheses assume e.g. linear or parabolic increase of the subgrade reaction as a function of the depth reaching a maximum at a certain depth. In a first conservative approach, if not stated otherwise, the coefficient of subgrade reaction $k_{S}$ is set constant over the depth. For the steel cylinders a Young's modulus of $210000 \mathrm{~N} / \mathrm{mm}^{2}$ and a Poisson ratio of $\mu=0.3$ are imposed in the numerical analyses.

In the following section an axially loaded and an externally pressurized cylinder are investigated with the influence of a foundation on the buckling behaviour. These two load conditions are the relevant cases appearing in the buckling assessment for tubular piles of combiwalls.

\subsection{Axially loaded cylindrical shell}

The possible supporting effect of soil against a buckling failure was investigated numerically using a small cylindrical shell with a height or length of $500 \mathrm{~mm}$. Since the model of the preliminary investigations is based on the real dimensions of combiwall constructions the $r / t$ ratio is fixed accordingly to 50 . The real dimensions are scaled down by a factor of around 10 leading to a choice for the tube diameter $D$ of $200 \mathrm{~mm}$ and a wall thickness $t$ of $2 \mathrm{~mm}$. These dimensions were chosen in correspondence with possible reduced scale laboratory tests. The axially loaded cylinder is modelled with two different segments. The upper and the lower part of the tube are designed with a thicker wall thickness $\left(t_{\mathrm{o}}=5 \mathrm{~mm}\right)$ for forcing a controlled failure to initiate in the middle section. The reinforced section of the cylinder shaded in dark grey in Fig. 9.1 is modelled with a height of $100 \mathrm{~mm}$. The investigated section in the middle has a height of $300 \mathrm{~mm}$ and a wall thickness of $t=2 \mathrm{~mm}$. 


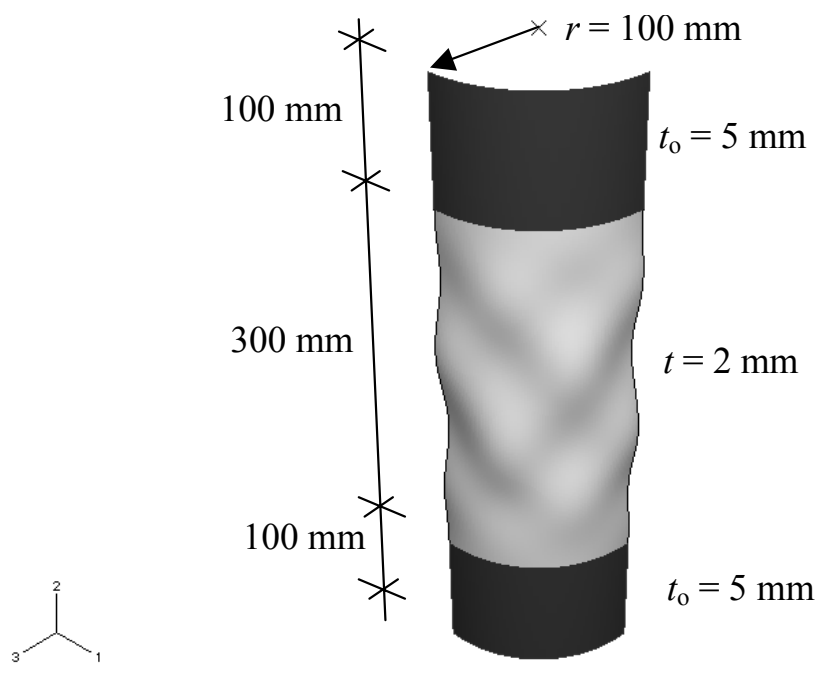

Fig. 9.1: Elastic buckling mode for axially loaded cylinder with radial foundation

The linear elastic buckling analysis using the finite element program ABAQUS for the axially loaded steel cylinder without foundation leads to an ideal buckling stress of $2541 \mathrm{~N} / \mathrm{mm}^{2}$ and the chessboard-like buckling pattern restricted to the middle section as shown in Fig. 9.1.

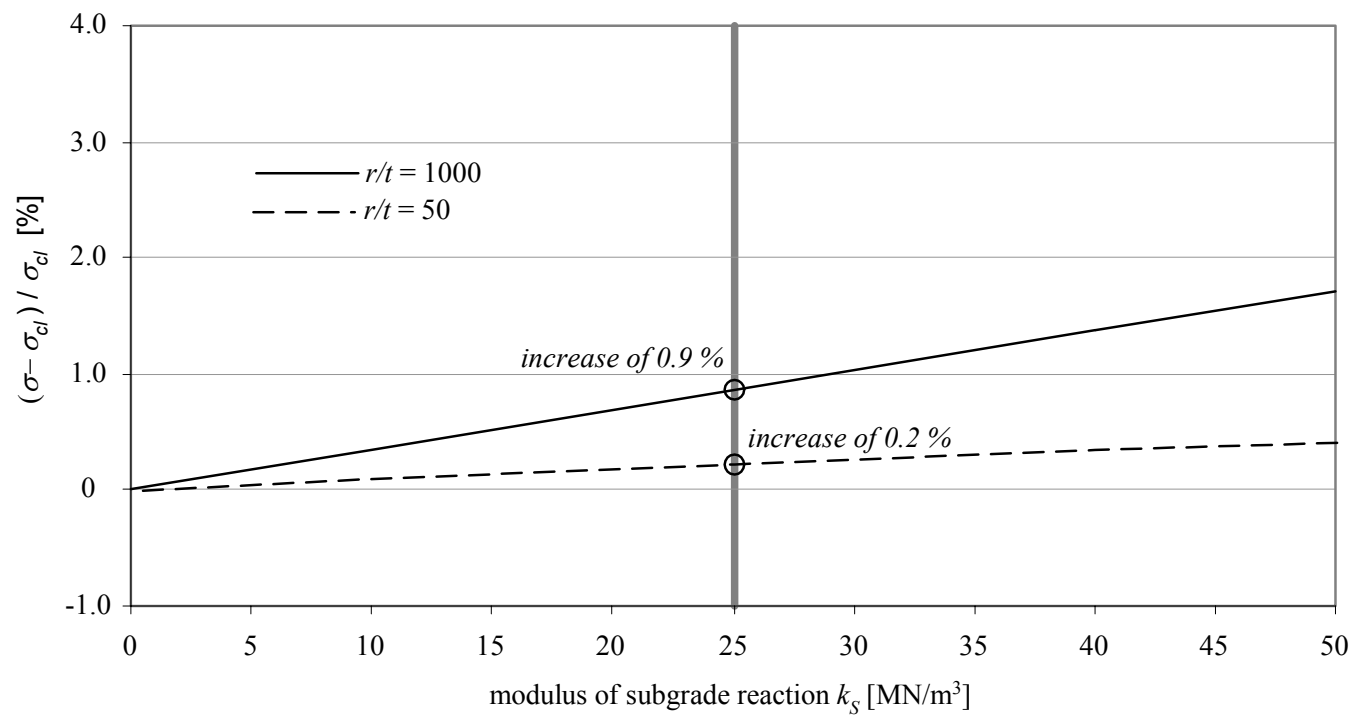

Fig. 9.2: Increase of buckling stress for different coefficient of subgrade reaction $k_{S}$

This value correlates with the result for the classical buckling load according to equation (8.1) without the term with the square root. In the next step foundation is applied in radial direction to the inside of the cylinder simulating a filling of soil. The foundation consists of linear Winkler springs reacting to both tension and pressure, i.e. an adhesive foundation. In the preliminary investigations this simple model is chosen to obtain first impressions on the supporting effect for further studies. For the investigated range for the coefficient of subgrade reaction the increase of the classical buckling load appear to be very small as e.g. depicted for $k_{S}=25 \mathrm{MN} / \mathrm{m}^{3}$ in Fig. 9.2. The linear 
bifurcation analysis using the approach of linear Winkler springs for the soil results in a nearly identical buckling load and buckling mode as found for the cylinder without foundation.

Based on the defined range between $10 \mathrm{MN} / \mathrm{m}^{3}$ to $100 \mathrm{MN} / \mathrm{m}^{3}$ and additionally by varying the $r / t$ ratio the increase of the classical buckling load is investigated for the short reduced scale cylinder analogously to Fig. 9.2. It was discovered that for small $r / t$ ratios even for very high coefficient of subgrade reaction of $500 \mathrm{MN} / \mathrm{m}^{3}$ only a very small increase of the classical buckling load was found. For the defined range up to $k_{S}=100 \mathrm{MN} / \mathrm{m}^{3}$ the increase of the bifurcation load remains below $1 \%$. In contrast, for an $r / t$ ratio of 1000 the bifurcation load increases by more than $2 \%$. Federhofer (1937 and 1954) derived based on the theory by Flügge (1932) the analytical solution for an axially loaded, elastically embedded cylinder without transfer of shear forces. The solution is based on the assumption of small deformation and can be brought down to a factor $\beta$ increasing the classical buckling load for axisymmetric failure. For the case of $\mu=0.3$ for the cylinder it can be written as:

$$
\sigma_{c r}=\sigma_{c l} \cdot \beta=0.605 \cdot E \cdot \frac{t}{r} \cdot \sqrt{1+\frac{k_{S} \cdot r^{2}}{E \cdot t}}
$$

Fig. 9.3 compares the results for two different $r / t$ ratios from the analytical solution for the axisymmetric case with the corresponding three-dimensional finite element calculations.

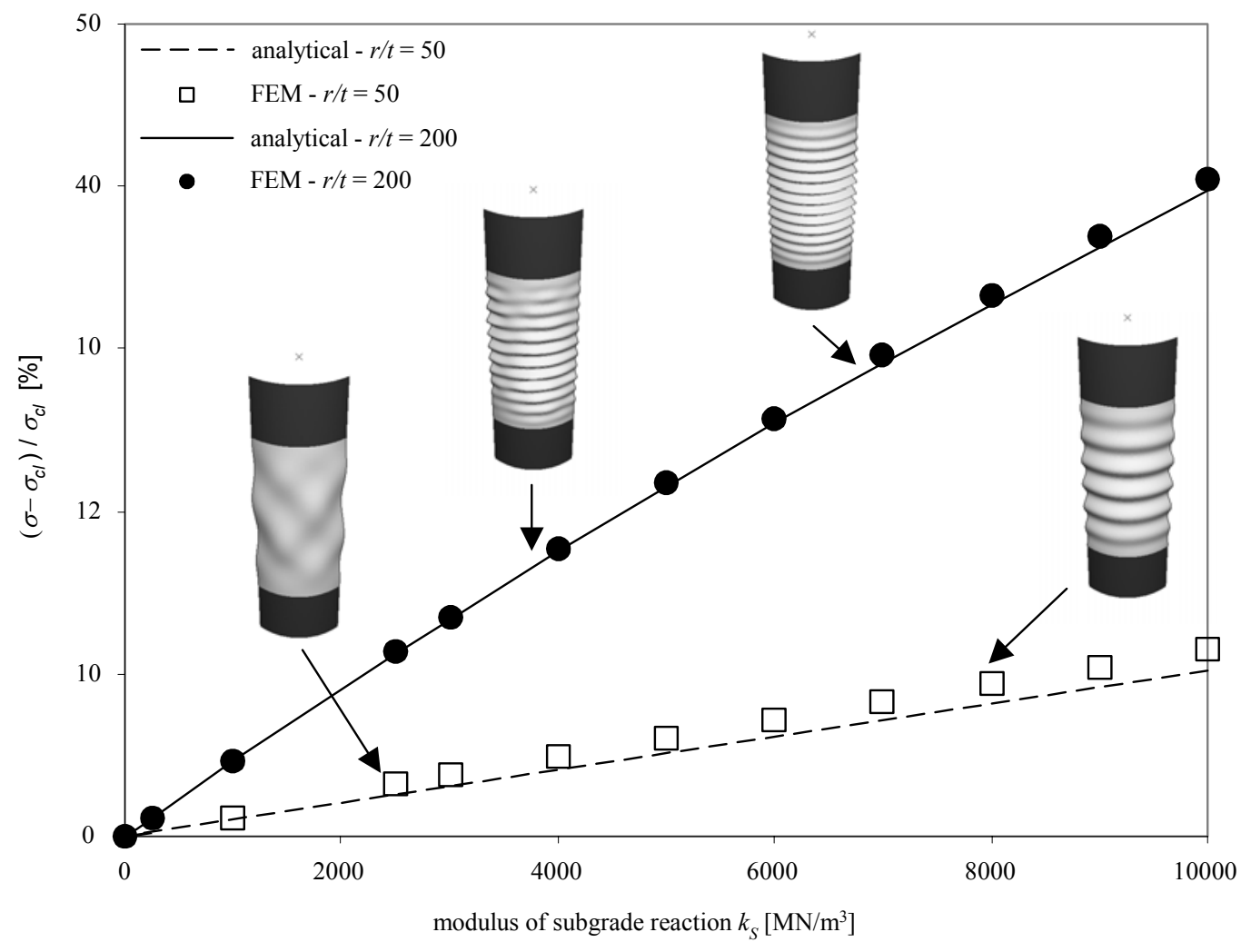

Fig. 9.3: Comparison between the analytical solution according to Federhofer and numerical analyses 
For an $r / t$ ratio of 200 the buckling mode turns to a rotation-symmetric mode (ring buckles) from small coefficients of subgrade reaction on. For an $r / t$ ratio of 50 this type of buckling mode occurs apart from a coefficient of subgrade reaction of around $2500 \mathrm{MN} / \mathrm{m}^{3}$. For lower values the chessboard-like failure mode prevails which is not covered by the equation (9.1). Still, due to the fact that the bifurcation points are clustered in a small region for the axially loaded cylinder the analytical solution by Federhofer agrees well with the numerical results.

For the $r / t$ ratio of 50 and small coefficients of subgrade reaction $\left(<500 \mathrm{MN} / \mathrm{m}^{3}\right)$ which is the relevant range for the investigation of the SSI the buckling loads drops below the classical buckling load according to Donnell because this approximation does not include the influence of reducing circumferential buckles for long cylinders. According to EN 1993-1-6 a cylinder is defined as "long" for the following expression:

$$
\omega=\frac{l}{\sqrt{r \cdot t}}>0.5 \cdot \frac{r}{t}
$$

For the reference case this value is not reached but closely approached. The so-called garland curves by Flügge (1932) which include the effect of length, show that the length effect takes place for the investigated reference case for an $r / t$ ratio of 50. In Fig. 9.3 it can be seen that the influence of the elastic foundation without shear transfer on the buckling load of a medium length cylinder in the range of realistic coefficients of subgrade reaction is very small. The supporting effect of soil increases for larger $r / t$ ratios, but does not reach design relevant magnitudes until an $r / t$ ratio of about 1000 .

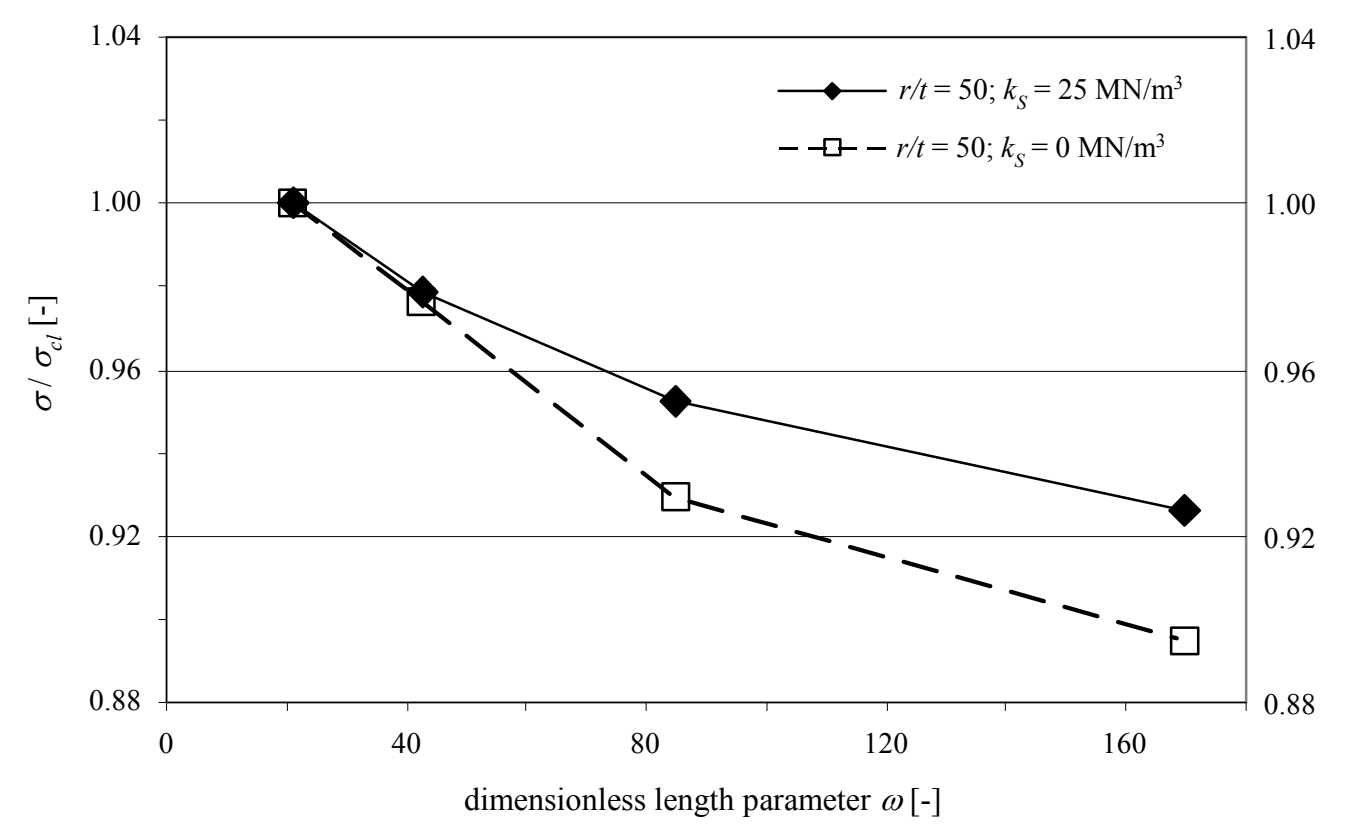

Fig. 9.4: Buckling stress for different lengths and for an elastic foundation

In the next step the influence of length is investigated. Based on the reduced scale model and a coefficient of subgrade reaction $k_{S}=25 \mathrm{MN} / \mathrm{m}^{3}$ the length of the middle section is varied. By increasing the dimensionless length parameter $\omega$ the ideal buckling 
load reduces as depicted in Fig. 9.4. This effect is directly associated with the reduction of the number of circumferential buckling waves $m$. For very long cylinders the number of circumferential buckling waves $m$ drops to 1 . The numerical analyses verify this effect included in the shell theory by Flügge (1932). Moreover, it can be seen that the supporting effect of the soil increases for longer cylinders considerably (Fig. 9.4). For a dimensionless length parameter $\omega$ of 170 , corresponding to a length of $2400 \mathrm{~mm}$ in the model and thus roughly related to the reference case scaled down by the factor of 10 , an increase of the linear buckling load of $3.5 \%$ can be found.

For the investigated $r / t$ ratios and the investigated and commonly applied steels for combiwalls the buckling stress considering imperfections is never reached. For example, the yield stress for the steel S355 or S460 lies significantly below the buckling stress calculated with a fairly small knock down factor of 0.2 to 0.3 based on large imperfections. Thus, the failure of such axially loaded tubes is governed by their plastic carrying capacity. In Fig. 9.5 the deformed plot of an axially loaded, perfect tube with an $r / t$ ratio of 50 at maximum load is depicted. For the analysis a steel S355 with a yield stress of $360 \mathrm{~N} / \mathrm{mm}^{2}$ and a linear elastic behaviour in combination with a minor hardening was implemented.

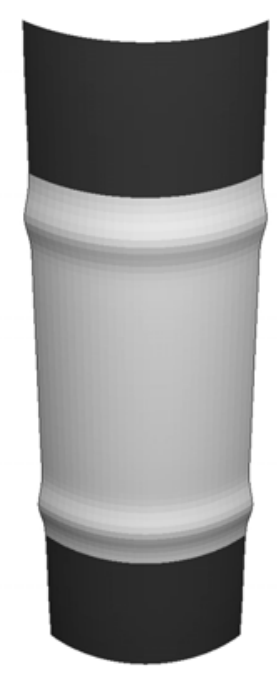

Fig. 9.5: Deformed tube axially loaded at maximum load with and without elastic foundation

For the deformed tube plastic buckles can be found in the transition to the stiffened segment (,Elephant's foot buckling”). Fig. 9.6 depicts the load-displacement curves for perfect axially loaded tubes from geometrical and material non-linear finite element calculations. The ordinate indicates the elastic knock down factor $\alpha$ defined as the ratio of the load carrying capacity and the classical buckling load. Again it can be seen that the influence of the elastic foundation on the forming of the plastic buckles is small, and no significant increase of the carrying capacity can be found. The effect of the elastic foundation becomes distinguishable along the load branch after reaching the load 
carrying capacity. For investigating the influence of soil on an imperfect cylinder, the same calculations were performed using a cylinder with two different imperfection shapes. Type A uses an imperfection shape that is affine to the first elastic buckling mode of the perfect cylinder (Fig. 9.1). For type B the deformed cylinder at the plastic carrying capacity is imposed as imperfection (Fig. 9.5). Both imperfection shapes are scaled with a maximum imperfection amplitude of $w / t=1$.

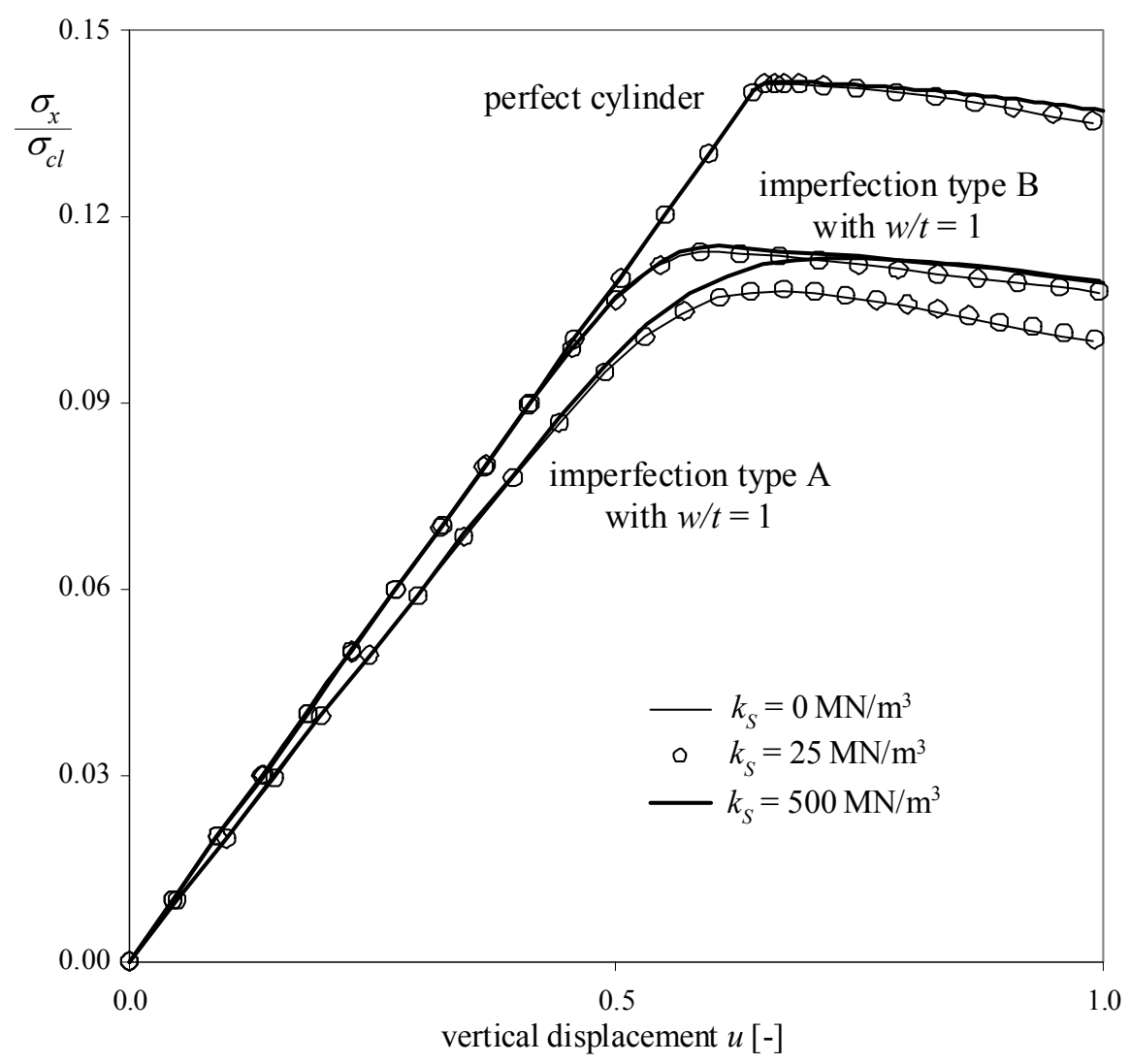

Fig. 9.6: Load-displacement curves for axially loaded cylinder for different foundations and imperfections

In contrast to the perfect cylinder it can be seen that the load-displacement curve changes, and the foundation for high coefficients of subgrade reaction gains influence for the imperfection type A. This effect is related to the fact that the plastic buckling is related to a different buckling pattern than the perfect cylinder. In Tab. 9.1 the increase of the carrying capacity of the imperfect cylinder is summarized considering geometrical and material non-linear behaviour. Similar to the case of elastic bifurcation loads the increase of the carrying capacity is fairly small for a coefficient of subgrade reaction $k_{S}$ of $25 \mathrm{MN} / \mathrm{m}^{3}$. Even for a larger coefficient of subgrade reaction the increase of the load carrying capacity remains small. The analyses were performed using a consistent imperfection amplitude of $w / t=1$.

In further studies the possible influence of load transfer mechanism parallel to the shell surface is investigated. Such load transfer mechanisms may arise for an axially loaded shell due to friction and the mobilisation of shear forces in the soil. This effect may be 
modelled by using more complex spring models. For the preliminary investigation additional springs parallel to the surface are applied with similar coefficients of subgrade reaction to the perpendicular foundation. The increase of the bifurcation load is negligible. A further increase of the carrying capacity does not evolve since the test cylinder is too short for the friction forces to develop and to support the cylinder in transferring the axial loads. The influence of tangential springs will be discussed more in detail in the subsequent sections.

Tab. 9.1: Increase of carrying capacity considering a foundation

\begin{tabular}{lcc}
\hline case & foundation $\boldsymbol{k}_{\boldsymbol{S}}$ & increase of carrying capacity \\
\hline perfect & $0 \mathrm{MN} / \mathrm{m}^{3}$ & - \\
& $25 \mathrm{MN} / \mathrm{m}^{3}$ & $+0.0 \%$ \\
& $500 \mathrm{MN} / \mathrm{m}^{3}$ & $+0.2 \%$ \\
\hline imperfect type A & $0 \mathrm{MN} / \mathrm{m}^{3}$ & - \\
& $25 \mathrm{MN} / \mathrm{m}^{3}$ & $+0.3 \%$ \\
& $500 \mathrm{MN} / \mathrm{m}^{3}$ & $+4.9 \%$ \\
\hline imperfect type B & $0 \mathrm{MN} / \mathrm{m}^{3}$ & - \\
& $25 \mathrm{MN} / \mathrm{m}^{3}$ & $+0.0 \%$ \\
& $500 \mathrm{MN} / \mathrm{m}^{3}$ & $+0.6 \%$ \\
\hline
\end{tabular}

\subsection{Cylindrical shell subjected to external pressure}

\subsubsection{Radial foundation}

A section of the tube with a length of $3250 \mathrm{~mm}$ is modelled for investigating the supporting effect of the soil. The diameter of the cylinder is $1800 \mathrm{~mm}$ and the wall thickness is $18 \mathrm{~mm}$. The preliminary investigation with a $180^{\circ}$ segment of the tube applies an elastic foundation on the in- and outside of the cylinder which refers to e.g. a formation of a plug in the region of the pile tip. Thus, a one-sided foundation with linear elastic spring acting identically under tension and compression are modelled based on Winkler springs on the inside of the cylinder. The length of the cylinder for the study was chosen based on the dimensionless length parameter $\omega$ in order that the cylinder is classified as medium-length cylinder (EN 1993-1-6) and hence both effects, the bending effect for long cylinders and the direct effect of the boundary conditions, can be neglected (see (9.3)). The pile tip shall be investigated as local buckling problem for a cylinder subjected to external pressure.

$$
20 \leq \omega=\frac{l}{\sqrt{r t}}=25.5 \leq 1.63 \frac{r}{t}=81.5
$$


The loading is applied as constant external pressure $q$. In a first step the system is investigated with two different sets of boundary conditions. In case 1 both edges are clamped and in case 2 only the top edge is clamped and the bottom edge is free following the real boundary situation. The linear buckling eigenmodes for the cylinder without foundation is depicted in Fig. 9.7. The circumferential critical buckling stress $\sigma_{\theta, R c}$ can be calculated according to EN 1993-1-6 as follows:

$$
\sigma_{\theta, R c r}=0.92 \cdot E \frac{C_{\theta}}{\omega} \frac{t}{r}
$$

The factor $C_{\theta}$ is defined from the boundary conditions in EN 1993-1-6 with 1.5 for case 1 and 0.6 for case 2. The analytical buckling loads from (9.4) are compared and confirmed with the numerically calculated linear elastic buckling loads by applying formula (9.5) which relates the critical pressure $q$ to the circumferential stress.

$$
\sigma_{\theta}=q \cdot \frac{r}{t}
$$

In Fig. 9.7 the critical action, i.e. the critical pressure $q$, is shown for the cases 1 and 2 as a function of the coefficient of subgrade reaction $k_{S}$. The action $q$ is normalized with the correspondent critical pressure $q_{c r i t}$ for a cylinder without foundation according to (9.4) and (9.5).

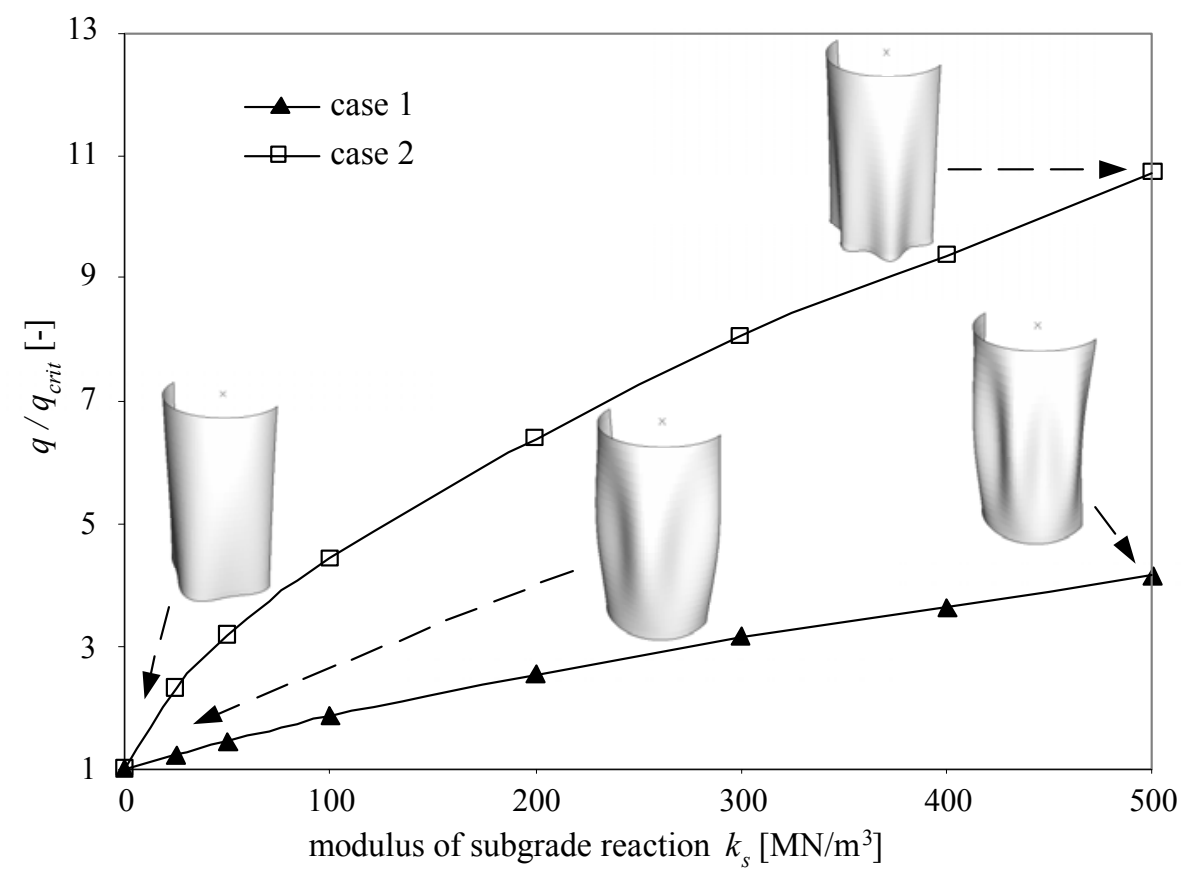

Fig. 9.7: Influence of a linear foundation on the buckling load for a medium-length cylinder subjected to external pressure

The carrying capacity of the cylinder increases significantly due to the supporting effect on the in- and outside. In case 2 and considering linear elasticity an increase of the carrying capacity by the factor of more than 10 can be seen for a coefficient of subgrade reaction of $500 \mathrm{MN} / \mathrm{m}^{3}$. The absolute increase of the carrying capacity is considerably 
larger for case 2 than for case 1 . The buckling modes change for larger coefficients of subgrade reaction to a failure mode involving higher order buckling waves. These results serve to illustrate the influence of a foundation on the buckling behaviour of a cylinder subjected to external in comparison to an axially loaded cylinder.

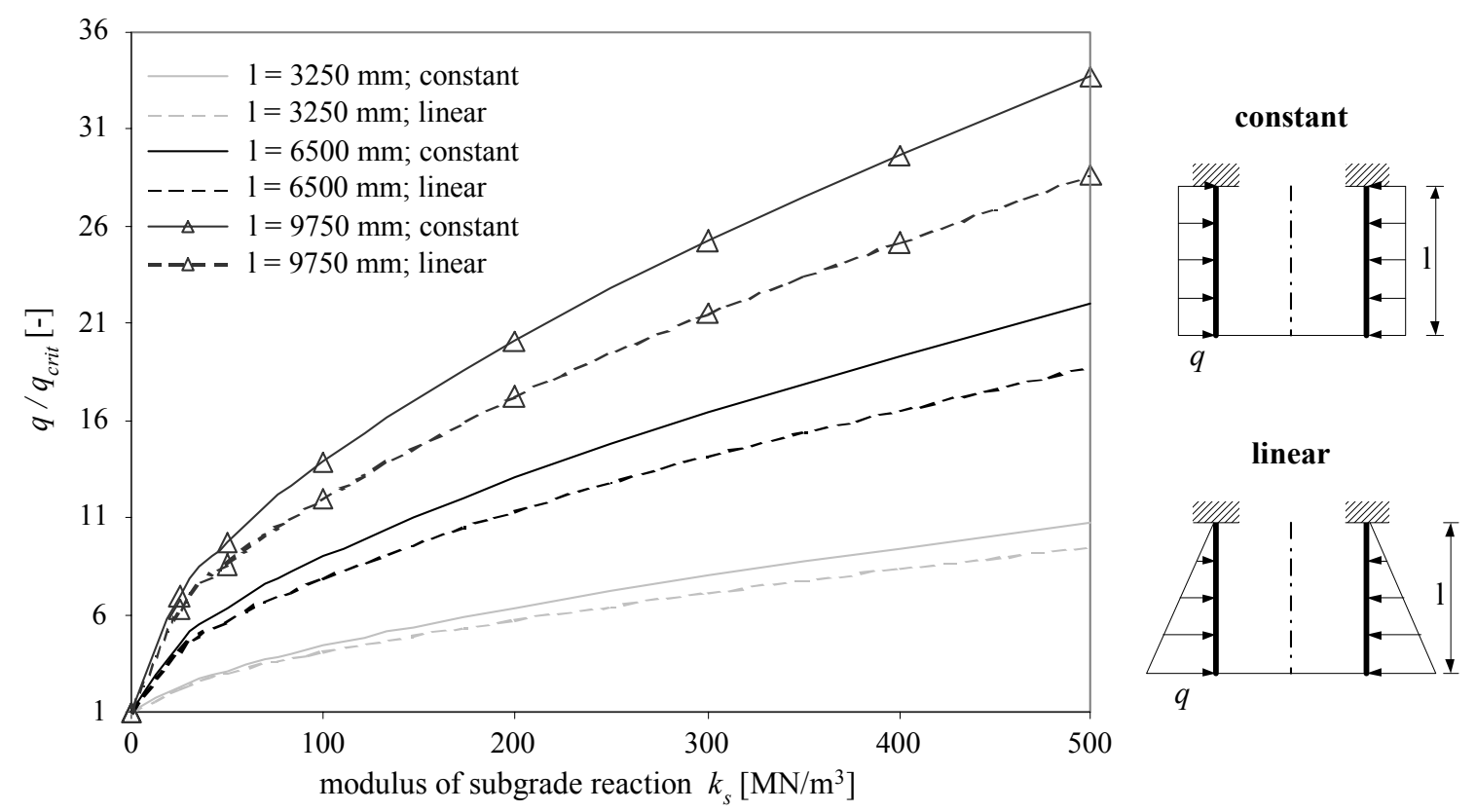

Fig. 9.8: Influence of foundation on the carrying capacity of a cylinder subjected to constant and linearly varying external pressure

In a second study the length of the cylindrical shell segment was changed, and the influence of a foundation on an external pressure distributed as a linear function of the depth was investigated. The results for the cylinder which is freely supported on one side and with the maximum external pressure at the same side are depicted in Fig. 9.8. In this context the bending of the cylinder is not considered because an external pressure constant over the circumference is applied. The circumferential critical buckling stress $\sigma_{\theta, R c}$ decreases for longer cylinders as it can be seen from equation (9.3) and (9.4). The findings show that the longer the cylinder is, the more an imposed foundation and a varying external pressure effect the critical buckling stress. The influence is smaller for a cylinder subjected to an external pressure distributed as a linear function of the depth than for a cylinder subjected to constant external pressure. The impact of the foundation for e.g. a coefficient of subgrade reaction of $25 \mathrm{MN} / \mathrm{m}^{3}$ for a short cylinder (i.e. here between $3250 \mathrm{~mm}$ and $9750 \mathrm{~mm}$ ) on the carrying capacity is large (ratio $q / q_{c r i t}=2.2$ and 6.2). Nevertheless, a further increase of the ratio for much longer cylinders (around $24000 \mathrm{~mm}$ ) is small (ratio $q / q_{\text {crit }}$ between 6.2 to 7.4 ) as seen in Fig. 9.9. The absolute increase of the carrying capacity decreases for linear instead of constant external pressure (Fig. 9.9). 


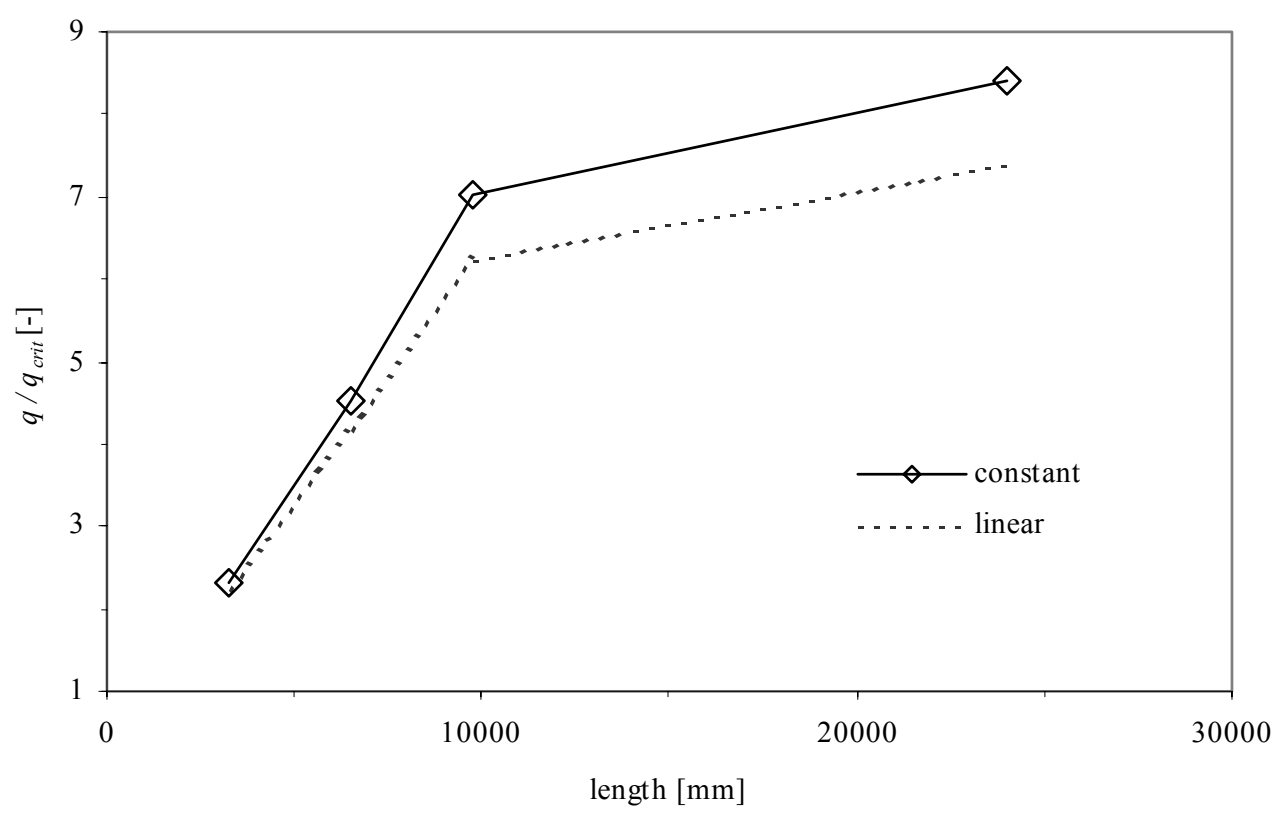

Fig. 9.9: Influence of foundation $\left(k_{S}=25 \mathrm{MN} / \mathrm{m}^{3}\right)$ on the carrying capacity of a cylinder subjected to constant and linearly varying external pressure for different lengths

\subsubsection{Tangential foundation}

The previous study did not include slip or stick friction between the shell and the soil. The effect of circumferential and/or meridional friction may influence the buckling behaviour additionally and is discussed in this section.

The buckling pattern of a cylindrical shell subjected to external pressure involves a number of buckling waves over the circumference which mainly govern the buckling case except for the applied boundary conditions. The buckling problem of an axially loaded column simply supported on both sides with infinite axial rigidity results in a buckling eigenmode with a single wave, in which all points move normal to the initial configuration (Fig. 9.10, left).

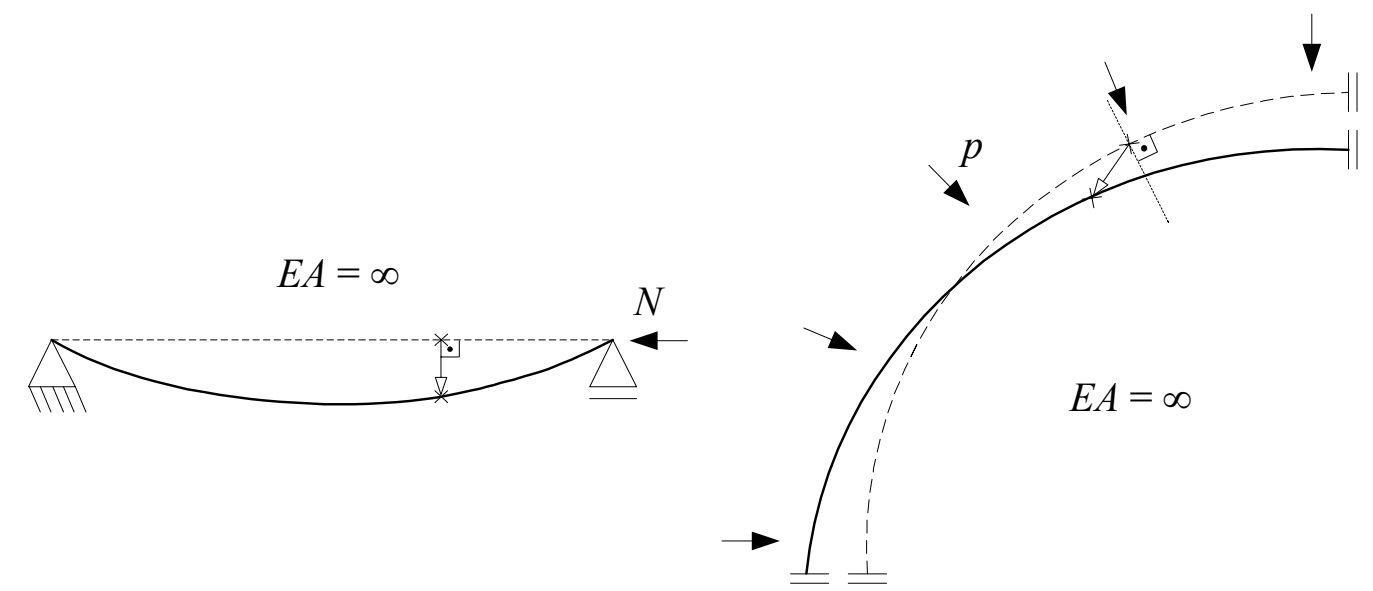

Fig. 9.10: Buckling eigenmodes for a column(left) and a ring (quarter; right) with shifted nodal position 
For this case no axial movement of the points along the column appears and thus springs arranged along the axis of the column do not influence the buckling load. A ring subjected to external pressure also fails due to column buckling. Nevertheless, for this mode the points do not move purely radially but also in circumferential direction (Fig. 9.10, right). Hence, for the buckling ring, a circumferential foundation or applying a frictional behaviour should lead to an increase of the buckling load.

In order to investigate the influence, the same cylinder from the previous chapter is used (case 1 from Fig. 9.7). At each node a connector element is attached radially acting on both, tension and compression with the same elastic spring stiffness. Additionally, two further springs are applied in the tangential plane (surface of the shell) for representing a foundation or friction in meridional and circumferential direction. This modelling represents only a rough approximation since the circumferential forces arsing from friction are a function of the normal force acting on the surface. Since in the first steps only linear bifurcation analyses are performed no non-linear stiffness or contact/separation can be used. In Fig. 9.11 some results for additional tangential springs are depicted. Based on a coefficient of subgrade reaction $k_{S}$ acting normal to the shell surface $\left(k_{S}=k_{n}\right)$ circumferential and meridional springs with the stiffness $k_{t}$ are added. The buckling load increase is evaluated with respect to the buckling load of the corresponding case without additional springs on the shell surface.

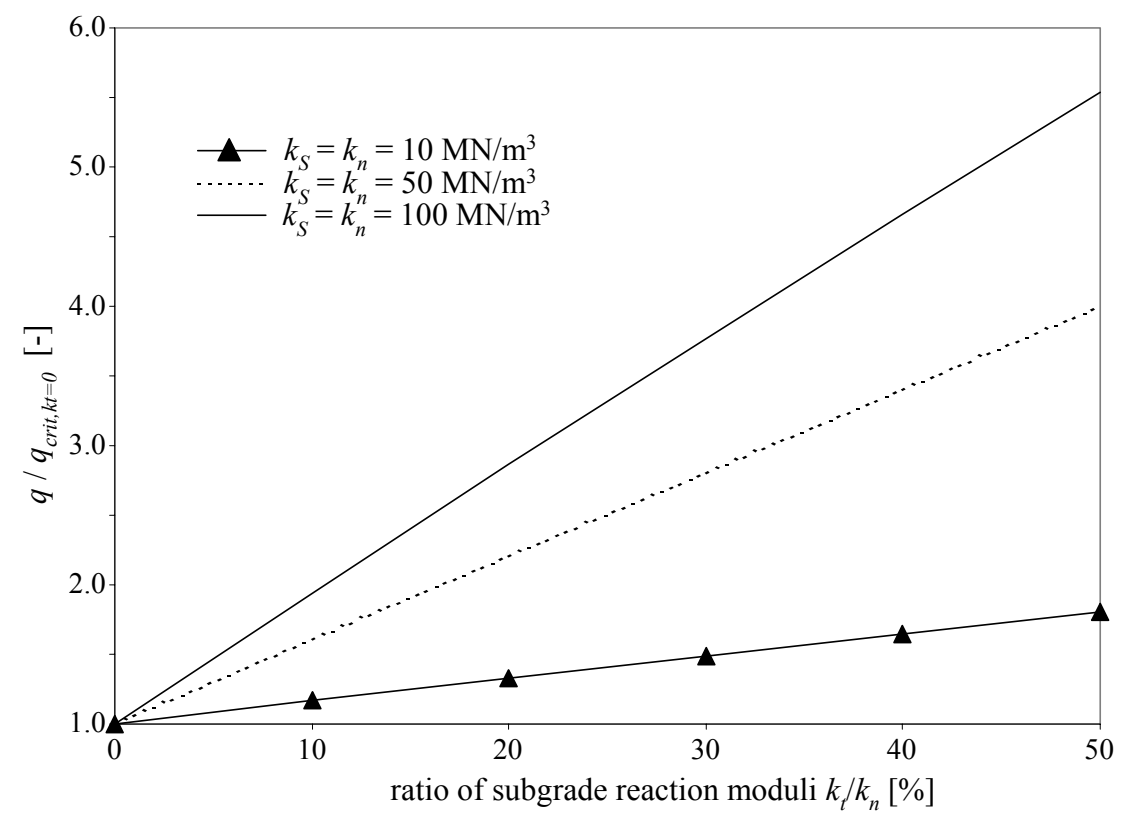

Fig. 9.11: Influence of additional tangential springs on the carrying capacity of a cylinder subjected to constant external pressure

A significant increase of the buckling load can be found for the depicted range. Already for ratio of subgrade reaction of $10 \%$, the buckling pressure rises by $20 \%$ to $90 \%$ for the investigated range. The number of buckling waves over the circumference tends to increase for larger tangential spring stiffnesses. It can be shown that the influence of the meridional springs is by far smaller than the influence of the circumferential springs. 
This finding is quite understandable as the curvature of the single buckle evolving in meridional direction is smaller than the curvature of the large number of buckling waves appearing in circumferential direction.

The present approach is only valid for the stick friction which can be modelled as a spring. As stated the slip friction needs to be coupled with the normal force acting on the shell surface. Nevertheless, the first analyses give an impression of the beneficial influence of considering friction. In the following the static coefficient of friction is set equal to the kinetic coefficient of friction and hereafter only called coefficient of friction.

Tejchman (1989) investigated the interaction between the grains of sand and structures in detail. His main aspect was the development of an interaction surface to be used in a hypoplastic model. In this context Tejchman (2001) then established a polar hypoplasticity, i.e. he extended the hypoplasticity by including polar terms (Cosserat terms), allowing to consider the rotation of grains. For these cases Tejchman (1997) derived a concept for boundary conditions (interaction surface) along structures depending on the relationship between roughness of the surface and grain size. Nevertheless, although possibilities allow to take these complex conditions in analyses into account, the applied procedure shall be kept easy to apply. Tejchman (1989) performed various experiments for obtaining information on the relationship between the ratio of the shear stresses $\tau$ in the interaction zone and the pressure/perpendicular stress $\sigma_{n}$ (= friction coefficient $\mu_{\text {fric }}$ ) depending on the displacements.

$$
\mu_{\text {fric }}=\frac{\tau}{\sigma_{n}}
$$

The experiments revealed that the friction coefficient $\mu_{\text {fric }}$ increases before reaching an asymptotic value $\mu_{\text {lim }}$ which depends on the roughness. For rough surfaces a peak (local maximum) may evolve before reaching the asymptotic value. The latter equation can be rewritten and expressed as follows:

$$
\tau=\sigma_{n} \mu_{\text {fric }}=\sigma_{n} \tan \delta
$$

where $\tau$ are the shear stresses, $\sigma_{n}$ are the normal stresses on the surface, and $\delta$ corresponds to the angle of wall friction. In the experiments using a simple shear apparatus no significant influence on $\mu_{\text {lim }}$ was found for different densities. The asymptotic values can be estimated from diagrams in Tejchman (1989) roughly as $\mu_{\text {lim }}=0.2,0.5$, and 0.8 for smooth, rough, and very rough surfaces respectively. Thus, we take as a first conservative approximation for smooth sand the values $\mu_{\text {fric }}=\mu_{\text {lim }}=0.2$. The wall friction $\mu_{\text {fric }}$ between steel and sand given literature (e.g. Bowles, 1992; Broms, 1981; ISO/DIS 19902:2004) scatters significantly $\left(0.15<\mu_{\text {fric }}<0.7\right)$ depending on the state of the soil. Khulaway (1991) states a value of $\mu_{\text {fric }}=\tan (0.5 \varphi)$ for smooth steel piles fitting well into the previously stated range for common friction angles (for $\varphi=33^{\circ}, \mu_{\text {fric }}=0.30$ ) for sand. 
The aspect of the meridional coefficient of subgrade reaction $k_{t, m}$ could be assumed to be identical to the previous case (both tangential behaviour)and thus $k_{t, m}=k_{t, c}=k_{t}$ shall be used in this work. Here, the ISO/DIS 19902:2004 is used as a basis for further considerations. In ISO/DIS 19902:2004 a method is described which is commonly used in the design of pile foundation and is based on tests. It is mentioned that more elaborate methods may lead to better approximations - but as a disadvantage, the more complex approaches demand for additional in-situ and laboratory tests. Therefore, the following considerations are based on the formulas given in ISO/DIS 19902:2004. For pipe piles in cohesionless soil the following equation is given for the skin friction $f$ :

$$
f_{s f}=\tau=\sigma_{n} \tan \delta=K \sigma_{v} \tan \delta
$$

Dimensionless coefficients $K$ of lateral earth pressure (ratio of horizontal/normal effective stress $\sigma_{n}$ over the vertical effective stress $\sigma_{v}$ ) of 0.7 to 0.8 are suggested for open-ended piles in compression with the lower value referring to loose or medium dense sand. The value $\sigma_{v}$ is the effective overburden stress at the considered position and $\delta$ is the friction angle between the soil and the pile wall (between $20^{\circ}$ and $35^{\circ}$ for sand according to ISO/DIS 19902:2004). The values for the skin friction $f$ range from $65 \mathrm{kPa}$ to $115 \mathrm{kPa}$ for sand depending on the soil's density.

The relationship between mobilized soil-pile shear transfer and local pile displacement at any depth is described using a $t_{a d}-z_{p}$ curve, in which $z_{p}$ is the local pile displacement and $t_{a d}$ is the mobilized soil-pile adhesion (in stress units). For cohesionless soil the relationship is defined as a bi-linear function, starting at the origin and having a change of gradient to zero at $z_{p}=2.5 \mathrm{~mm}$ for $t_{a d}=t_{a d, \max }=f_{s f}$. Thus, the gradient and therefore the meridional coefficient of subgrade reaction $k_{t, m}$ is defined as:

$$
k_{t, m}=\frac{t_{\max }}{z_{p}}=\frac{f_{s f}}{2.5 m m}=400 m^{-1} \cdot \sigma_{n} \tan \delta
$$

According to ISO/DIS 19902:2004 the meridional coefficient of subgrade reaction $k_{t, m}$ can be evaluated as a function of the depth $z$, e.g. for a sand with $\gamma=15.25 \mathrm{kN} / \mathrm{m}^{3}$ and

$$
\sigma_{n}=K \sigma_{v}=K \gamma z
$$

as

$$
1.5 z \leq k_{t, m} \leq 3.4 z \quad \text { with } z[\mathrm{~m}] \text { and } k_{t, m}\left[\mathrm{MN} / \mathrm{m}^{3}\right]
$$

depending on the density of the sand.

As described, the skin friction $f$ or the maximum mobilized soil-pile adhesion $t_{a d, \max }$ can be evaluated more specially for the investigated case with formula (9.9), if results of insitu tests are available. Nevertheless, the prediction of $t_{a d \text {,max }}$ remains uncertain. In the investigated case it is intended to simplify the matter as much as possible in order to provide an estimate of the magnitude of the skin friction and the supporting effect of the soil. Thus, here only two of these approaches are briefly introduced: the CUR-method (2001) and the method by Jardine and Chow (1996). Some more recent methods have 
been proposed by e.g. Kolk et al. (2005) or White (2005). For both approaches measurements from CPT tests are required.

The method developed by the CUR - Centre for Civil Engineering Research and Codes (2001) proposes the following formulas for deriving the skin friction $f_{s f}$ :

$$
f_{s f}=q_{c} 0.08\left(\frac{\sigma_{v}}{p_{\text {ref }}}\right)^{0.05}\left(\frac{h}{R_{\text {eff }}}\right)^{-0.90} \text { for } \frac{h}{R_{\text {eff }}} \geq 4
$$

and

$$
f_{s f}=q_{c} 0.08\left(\frac{\sigma_{v}}{p_{r e f}}\right)^{0.05}\left(\frac{h}{R_{e f f}}\right)^{-0.90}\left(\frac{h}{4 R_{e f f}}\right) \text { for } \frac{h}{R_{e f f}}<4
$$

with the cone resistance $q_{c}$ obtained from cone penetration tests (CPT), a reference pressure $p_{\text {ref }}=100 \mathrm{kN} / \mathrm{m}^{2}$, the effective pile radius $R_{\text {eff, }}$, and the height $h$ above the pile tip. The effective pile radius $R_{\text {eff }}$ is calculated with the outer pile diameter $D_{o}$ and the inner pile diameter $D_{i}$ :

$$
R_{e f f}=0.5 D_{o} \sqrt{1-\left(\frac{D_{i}}{D_{o}}\right)^{2}}
$$

Jardine and Chow (1996) and Jardine et al. (1998) from the Imperial College London proposed a definition of the skin friction for open-ended piles as follows:

$$
f_{s f}=\left[0.029 q_{c}\left(\frac{\sigma_{v}}{p_{r e f}}\right)^{0.13}\left(\frac{2 h}{\sqrt{D_{o}^{2}-D_{i}^{2}}}\right)^{-0.38}+2 G \frac{\Delta}{R}\right] \tan \delta
$$

where $G$ is the shear modulus of the soil, and $\Delta$ is the radial dilation of the sand at the pile-soil interface, which can be approximated by $2 \cdot 10^{-5} \mathrm{~m}$ (Jardine and Chow, 1996).

The shear modulus $G$ can be calculated as

$$
G=q_{c}\left[0.0203+0.00125 \eta-1.216 \cdot 10^{-6} \eta^{2}\right]^{-1}
$$

with

$$
\eta=\frac{q_{c}}{\sqrt{p_{r e f} \cdot \sigma_{v}}}
$$




\subsection{Evaluation of test results}

\subsubsection{Reduced scale tests}

\subsubsection{Predicted coefficients of subgrade reaction}

The reduced scale tests as described in Annex $\mathrm{C}$ involve axial and torsional loading. Purpose is to obtain results for friction relationships, which can be used in further numerical approaches.

Based on the formula by Terzaghi (1955) given in section 7 the coefficient of subgrade reaction $k_{s}$ can be calculated for loose sand as follows:

$$
k_{S}=2.2 \frac{M N}{m^{3}} \cdot \frac{z}{D}
$$

Thus, for an embedded length of $2.3 \mathrm{~m}$ and an outer diameter of the tube of $203 \mathrm{~mm}$ the coefficient of subgrade reaction becomes $k_{s}=k_{n}=25 \mathrm{MN} / \mathrm{m}^{3}$ at the bottom of the pile The meridional coefficient of subgrade reaction $k_{t, m}$ according to ISO/DIS 19902:2004 is calculated with equation (9.11) to be $3.5 \mathrm{MN} / \mathrm{m}^{3} \leq k_{t, m} \leq 7.8 \mathrm{MN} / \mathrm{m}^{3}$ at the pile tip, i.e. about $k_{t}=6 \mathrm{MN} / \mathrm{m}^{3}$ as average value.

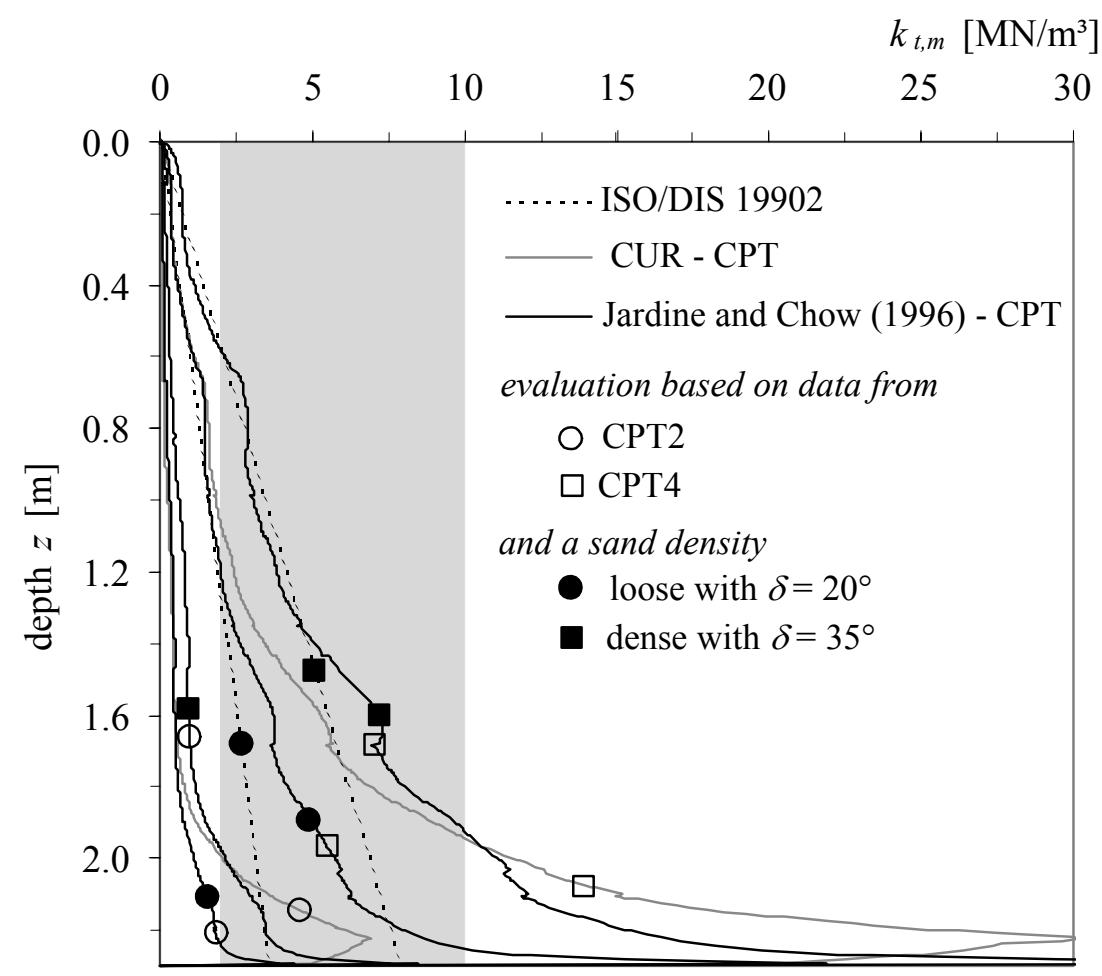

Fig. 9.12: Results of different approaches for obtaining the meridional coefficient of subgrade reaction $k_{t, m}$

In addition to these empirical predictions from literature, the meridional coefficient of subgrade reaction is evaluated by applying the approaches by CUR (2001) and Jardine and Chow (1996) as described with the formulae (9.12) to (9.15). These semi-empirical 
equations require the input of cone resistance $q_{c}$ obtained from CPTs. For the reduced scale tests according to Annex $\mathrm{C}$ four different CPTs were performed. Here, the results of the measurements from the CPT2 at zero confinement and the CPT4, which was performed at a confinement of $\sigma_{r}=30 \mathrm{kPa}$ and $\sigma_{v}{ }^{*}=6.7 \mathrm{kPa}$, are used. The fixed vertical confinement is named $\sigma_{v}{ }^{*}$, while the radial confinement, which is varied in the experiments is named $\sigma_{r}$. The results for applying the latter equations are depicted in Fig. 9.12. Additionally, the results from the evaluation according to ISO/DIS 19902:2004 are given as a function of the depth in the diagram.

CUR (2001) and Jardine and Chow (1996) yield extremely large coefficients of subgrade reaction close to the tip of the pile. For CPT4 locally values of $k_{t, m}>50 \mathrm{MN} / \mathrm{m}^{3}$ are reached (outside the scale of the diagram). These local effects are disregarded for a conservative estimate and since a global relationship is sought. Thus, based on a conservative extrapolation from the values at small depths and disregarding the local peaks we find roughly a range of $2 \mathrm{MN} / \mathrm{m}^{3} \leq k_{t, m} \leq 10 \mathrm{MN} / \mathrm{m}^{3}$ (grey shaded area in Fig. 9.12) at the pile tip. The average value of $k_{t}=6 \mathrm{MN} / \mathrm{m}^{3}$ and the range $3.5 \mathrm{MN} / \mathrm{m}^{3} \leq k_{t, m} \leq 7.8 \mathrm{MN} / \mathrm{m}^{3}$ calculated with equation (9.11) according to ISO/DIS 19902:2004 fit fairly well.

\subsubsection{Axial loading}

From the measurements of the strain gauges positioned over the test tube, the transferred load trough the pile can be plotted as a function of the pile top displacement. An example for an axial test without additional confinement is plotted in Fig. 9.13. This test is called test TD-A1-0 with TD for (dummy) test, -A1 for axial test no. 1, and -0 indicating no additional confinement.
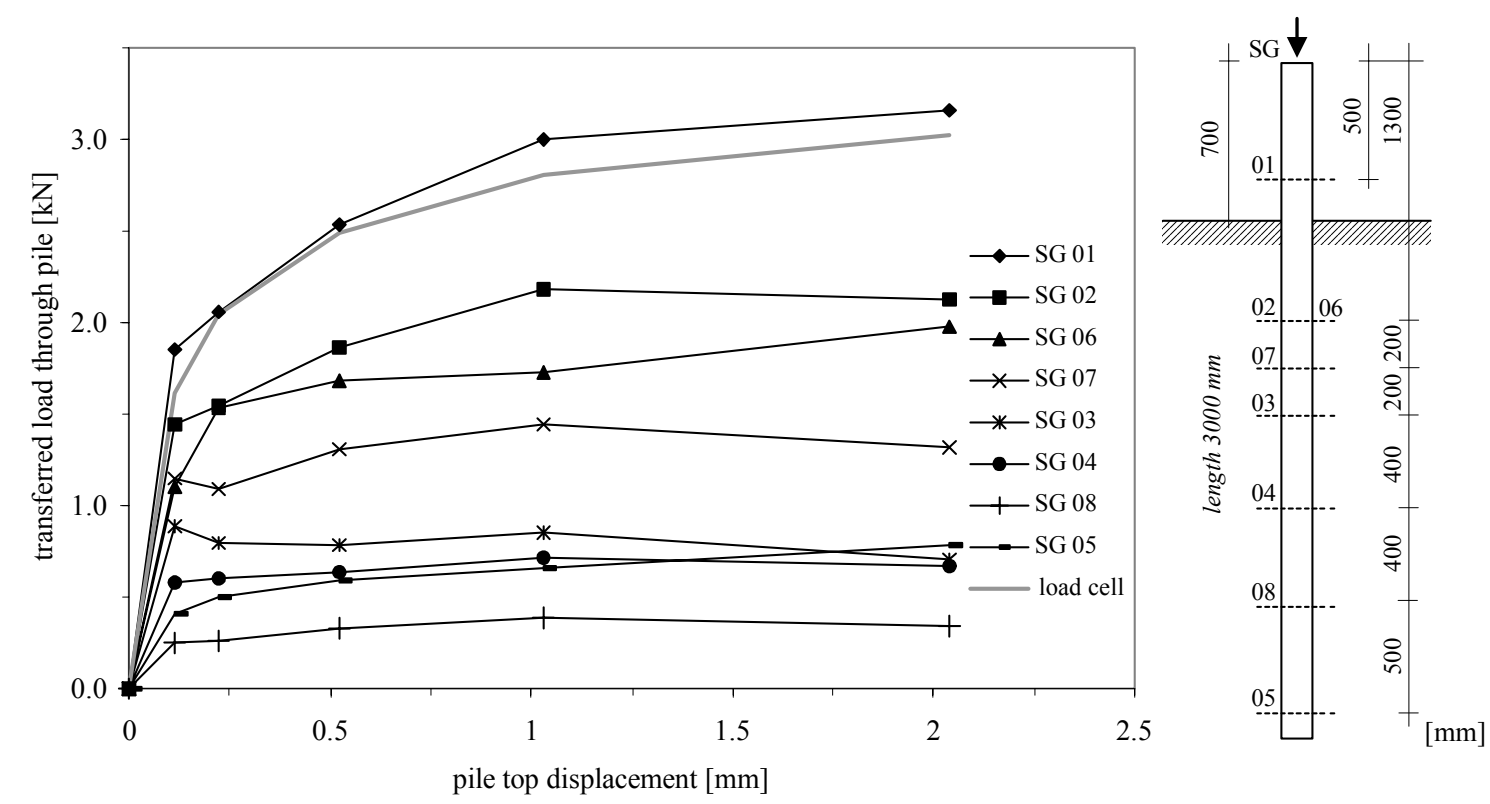

Fig. 9.13: Transferred load trough the pile as a function of the pile top displacement for axial loading for the strain gauges SG-test TD-A1-0 with positioning of the SG (from Charue and Holeyman, 2007) 
In order to separate the effect of shaft or skin friction from the influence of the tip resistance the differences are evaluated between strain gauges SG 02/06 and SG 05 close to the tip of the pile. Hence, the (tangential) meridional coefficient of subgrade reaction $k_{t, m}$ can be derived from the axial loading tests by relating the difference of the transferred load between the section to the outer area of the shaft section. The distance between two cross-sections, which are defined by two strain gauges, is named $\Delta L$. The normal force $\Delta N_{\text {seg }}$ that is in equilibrium with the shear stress integrated over the outer segment area of the pile can be calculated as:

$$
\Delta N_{\text {seg }}=E A \Delta \varepsilon_{\text {seg }}=E A\left(\varepsilon_{S G, u}-\varepsilon_{S G, l}\right)
$$

where $\Delta \varepsilon_{\text {seg }}$ is the strain difference between the strain at the upper strain gauges and the strain at the lower strain gauge. Since for first studies the buckling loads are analysed by performing linear buckling calculations the initial stiffness of the spring contributing to the buckling load is of interest. Therefore, the stiffness is evaluated for the first linear section from Fig. 9.13 (up to about $0.113 \mathrm{~mm}$ ). The cross-section of the pile is defined as area $A$. The coefficient of subgrade reaction $k_{t, m}$ is as follows:

$$
k_{t, m}=\frac{\Delta N_{\text {seg }}}{\Delta L \cdot \pi D \cdot 0.113 m m}
$$

Based on this procedure the results for test TD-A1-0 are plotted in Fig. 9.14. This test was performed without additional confinement. Averaging the coefficients of subgrade reaction over the length results in $k_{t, m}=9.1 \mathrm{MN} / \mathrm{m}^{3}$. The expected increase of stiffness as a function of the depth cannot be found (Fig. 9.14), but rather a trend of a decreasing coefficient of subgrade reaction can be seen for larger depths.

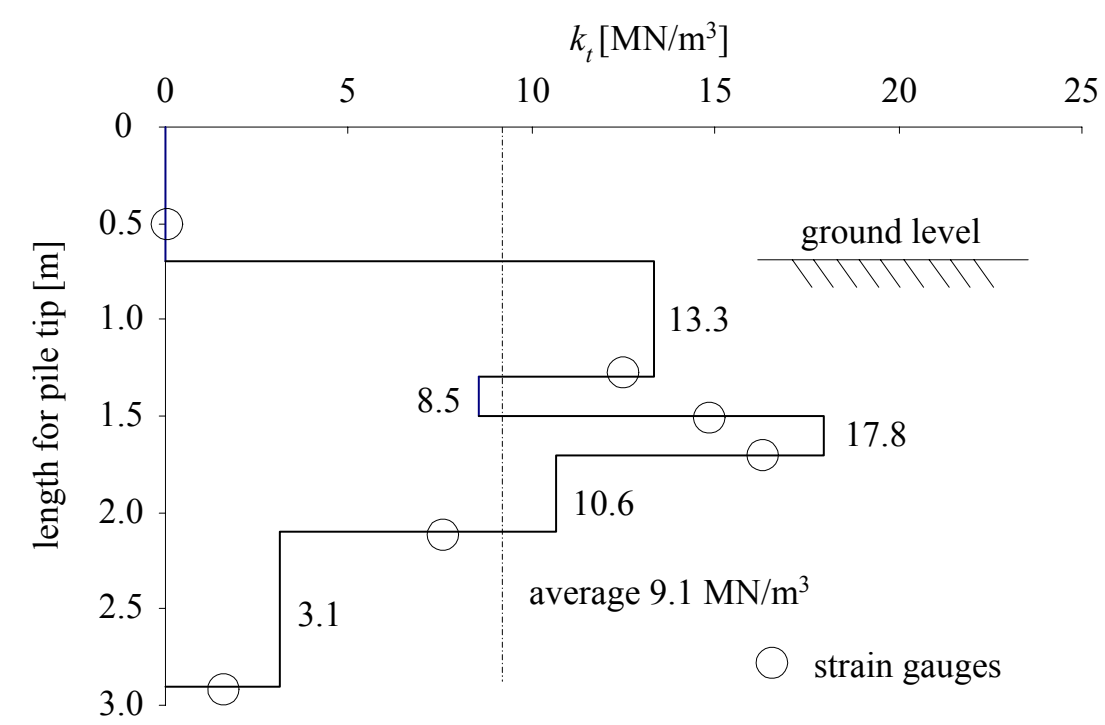

Fig. 9.14: Evaluated meridional coefficient of subgrade reaction $k_{t, m}-$ test TD-A1-0 (from Charue and Holeyman, 2007)

The evaluation of the other tests with axial loads revealed similar results. For the five tests performed with additional radial confining pressures $\sigma_{r}$ between $0 \mathrm{kPa}$ and $30 \mathrm{kPa}$ 
and an additional vertical confining pressure $\sigma_{v} *=6.7 \mathrm{kPa}$, values of $3.1 \mathrm{MN} / \mathrm{m}^{3}$ $\leq k_{t, m} \leq 79.5 \mathrm{MN} / \mathrm{m}^{3}$ can be derived, if radial confining pressures are present. Because the confining pressures are rather small and no distinct trend was discovered for the different confining pressures, the results for the meridional coefficient of subgrade reaction $k_{t, m}$ are given as a range. The averages of each test are $k_{t, m}=9.1 \mathrm{MN} / \mathrm{m}^{3}$, $37.1 \mathrm{MN} / \mathrm{m}^{3}, 35.6 \mathrm{MN} / \mathrm{m}^{3}, 33.1 \mathrm{MN} / \mathrm{m}^{3}$, and $22.0 \mathrm{MN} / \mathrm{m}^{3}$ for $\sigma_{r}=0 \mathrm{kPa}, 20 \mathrm{kPa}$, $20 \mathrm{kPa}, 30 \mathrm{kPa}$, and $30 \mathrm{kPa}$, respectively. The values are summarized in Fig. 9.15. Generally, the results are larger than the rough approximation of $k_{t, m}=6 \mathrm{MN} / \mathrm{m}^{3}$ with reference to the interpretation as given above. This emphasizes the conservativeness of the latter approximation. For the case of zero confinement the values agree well. Obviously, the tests with additional confinement yield larger coefficients of subgrade reaction. For this case the normal stress $\sigma_{n}$ is not calculated with equation (9.10) but with

$$
\sigma_{n}=K \sigma_{v}+\sigma_{r}
$$

where $\sigma_{r}$ is the radial and $\sigma_{v}$ is the vertical stress component. As the coefficient of subgrade reaction from ISO/DIS 19902:2004 increases linearly with greater depths, the coefficient of subgrade reaction $k_{t, m}$ for given confining pressures $\sigma_{r}$ can then be estimated roughly with

$$
k_{t}=400 m^{-1} \cdot \sigma_{n} \tan \delta=400 m^{-1} \cdot\left(K \gamma z+\sigma_{r}\right) \tan \delta
$$

according to (9.9).

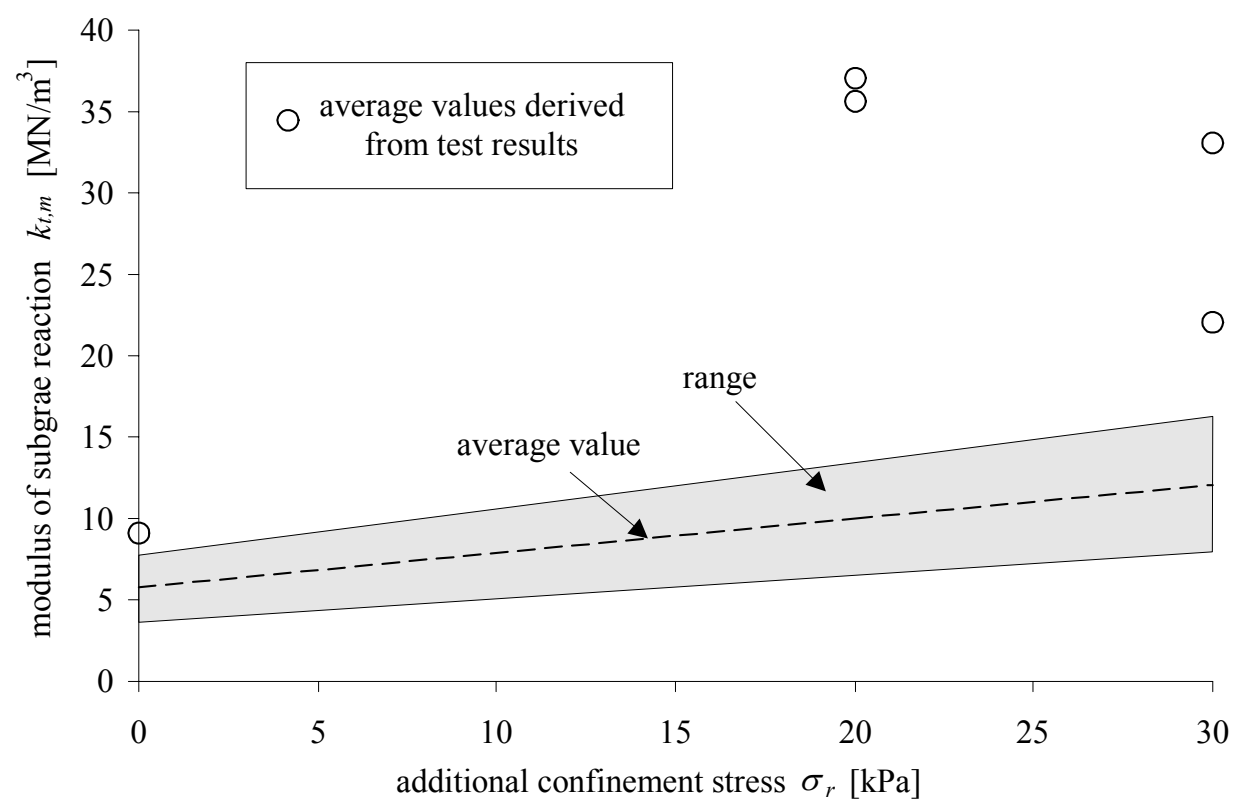

Fig. 9.15: Comparison of the evaluated meridional coefficients of subgrade reaction $k_{t, m}$ from tests and the range defined with equation (9.22) for loose to dense sand

In Fig. 9.15 the results from the tests are compared to the approach defined in equation (9.22). The given range refers the assumption of loose or dense sand as described in section 9.3.2. The values calculated with equation (9.22) provide a conservative lower 
boundary for the values derived from the experiments. Here, it has to be mentioned that equation (9.22) considers a zero gradient for a displacement greater than $2.5 \mathrm{~mm}$. The experimental results are limited to displacements smaller than $2 \mathrm{~mm}$. The evaluation of the test data is performed for a displacement of $0.113 \mathrm{~mm}$ in order to obtain the initial stiffness. Thus, the corresponding displacements according to equation (9.22) take far larger displacements into account than found in the experiments. Still, the results for $2 \mathrm{~mm}$ found in the experiments could be extrapolate to $2.5 \mathrm{~mm}$ since the stiffness appear to tend to zero (Fig. 9.13).

\subsubsection{Torsional loading}

The tangential friction defined as the opposing forces arising in the torsional loading are evaluated in a first simple approximation by using the torque - rotation relationship for the entire pile.

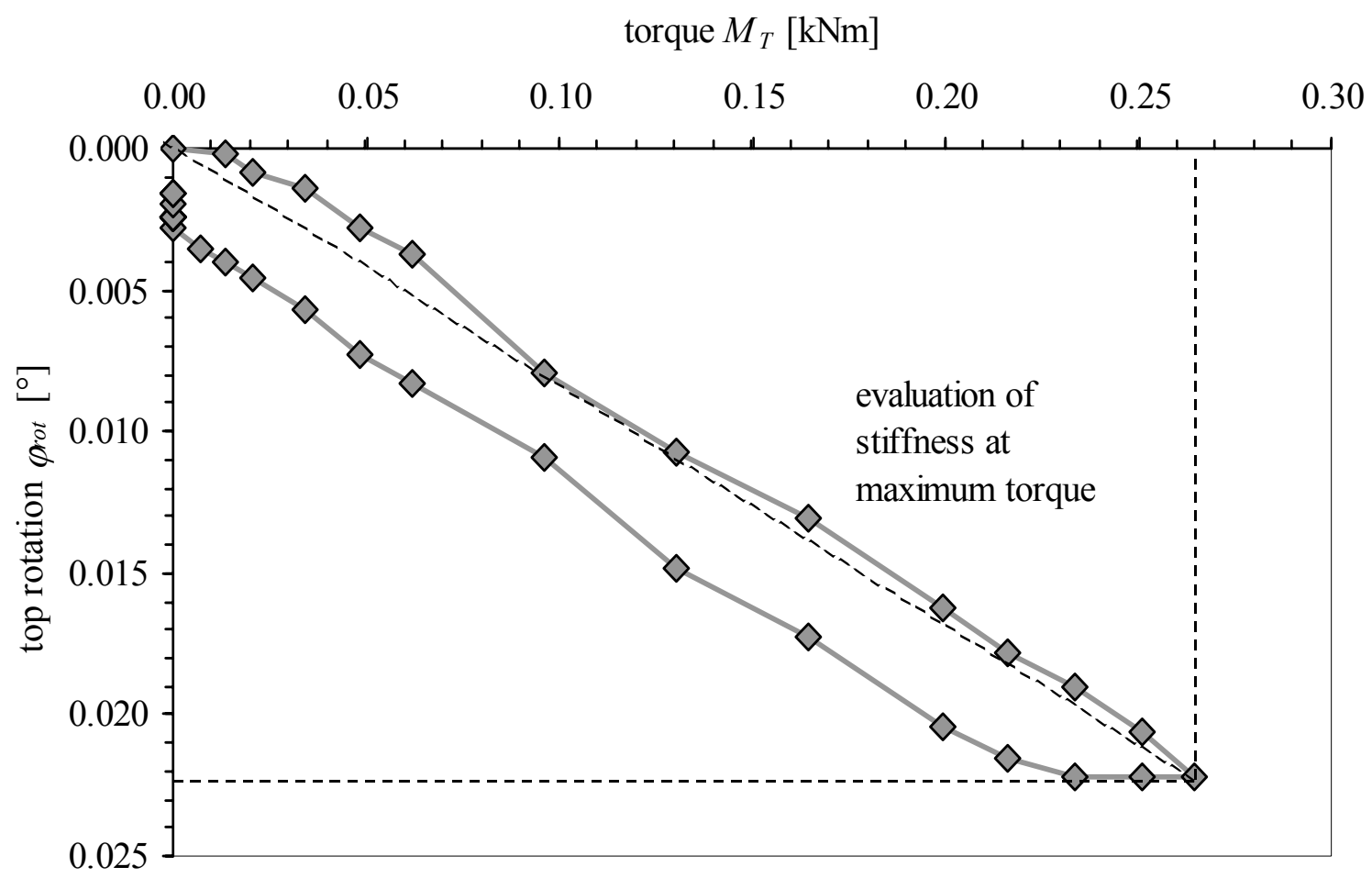

Fig. 9.16: Torque as a function of the top rotation for the entire pile with additional radial confinment $\sigma_{r}=20 \mathrm{kPa}-$ test TD-T2-20 (from Charue and Holeyman, 2007)

Assuming a uniform distribution of the shear stresses over the circumference and embedded length a stiffness can be derived according to Fig. 9.16 and then be related to the embedded shaft section. This step is performed for all seven torque loadings for obtaining a spring stiffness (tangential and circumferential coefficient of subgrade reaction $k_{t, c}$ ) with respect to small rotations similarly to the case of small displacements as seen for the axial loading. 
Since the functions torque - top rotation for the seven torque tests are rather non-linear the evaluation of the stiffness may only provide a rough approximation for the circumferential coefficient of subgrade reaction $k_{t, c}$. The values are calculated as

$$
k_{t, c}=\frac{4 M_{T}}{\pi D^{3} L_{e m b} \tan \varphi_{\text {rot }}}
$$

with the torque moment $M_{T}$, the embedded length $L_{e m b}$, and the top rotation $\varphi_{\text {rot }}$ Using the maximum applied torque, values $15 \mathrm{MN} / \mathrm{m}^{3} \leq k_{t, c} \leq 74 \mathrm{MN} / \mathrm{m}^{3}$ with a mean value of $35 \mathrm{MN} / \mathrm{m}^{3}$ are evaluated. The values correspond to a maximum displacement of $0.09 \mathrm{~mm}$. For the first test larger displacements were introduced. Nevertheless, the evaluation was limited to the latter maximum displacements. Again, no distinct increase of the stiffness was found for the tests involving additional confinement pressure $\left(\sigma_{r}\right.$ between $0 \mathrm{kPa}$ and $30 \mathrm{kPa}$ ). The predicted circumferential coefficients of subgrade reaction $k_{t, c}=k_{t, m}=6 \mathrm{MN} / \mathrm{m}^{3}$ (ISO/DIS 19902:2004) is smaller than the derived values. It has to be mentioned that the predicted value already represents a conservative approximation for the investigated cases, since the additional confining pressure is not considered. As seen in Fig. 9.15, a confining pressure of $30 \mathrm{kPa}$ leads to $k_{t, c} \approx 13 \mathrm{MN} / \mathrm{m}^{3}$. These facts indicate that the predicted values may be an applicable and conservative approximation for taking circumferential friction into account.

\subsubsection{Bender element tests}

Besides the tests with tubular piles, small scale laboratory tests on the behaviour of the investigated cohesionless sand were performed. From research performed in the field of earthquakes it is known that sand may behave fairly stiff for small strains. This issue was investigated by executing so-called Bender element tests. With these laboratory tests incorporating the shear wave theory the stiffness of soil can be derived for different confining pressures. The experiments were planned and conducted by and at the laboratories of the University of Louvain (UCL).

The bender element test applies the concept of transmitting an impulse through a soil sample from a transmitter to a receiver. In this procedure the imposed signal can be compared to the received signal for deriving the initial shear and compression modulus from the shear wave velocity. The tests were performed in the laboratories of the UCL. More details on the test set-up and the evaluation of the soil parameters are given in Charue and Holeyman (2007). For the bender test saturated Brusselian Sand was used. In the following the results of two different tests are discussed. The material properties are summarized in Tab. 9.2. Here, it needs to be stated that the relative density for the bender tests does not correspond to the relative density of the sand used in the reduced scale tests. 
Tab. 9.2: $\quad$ Soil sample characteristics

\begin{tabular}{lcc}
\hline \multicolumn{1}{c}{ parameter } & test 1 & test 2 \\
\hline sample size length $L[\mathrm{~mm}]$ & 78.7 & 80.1 \\
\hline sample size diameter $D[\mathrm{~mm}]$ & 38.2 & 38.5 \\
\hline porosity [\%] & 39.3 & 39.9 \\
\hline water content [\%] & 24.3 & 24.9 \\
\hline soil density saturated $\gamma_{\text {sat }}\left[\mathrm{kg} / \mathrm{m}^{3}\right]$ & 20.09 & 19.19 \\
\hline soil density dry $\gamma_{d}\left[\mathrm{~kg} / \mathrm{m}^{3}\right]$ & 16.16 & 16.00 \\
\hline relative density $D_{r}[\%]$ & &
\end{tabular}

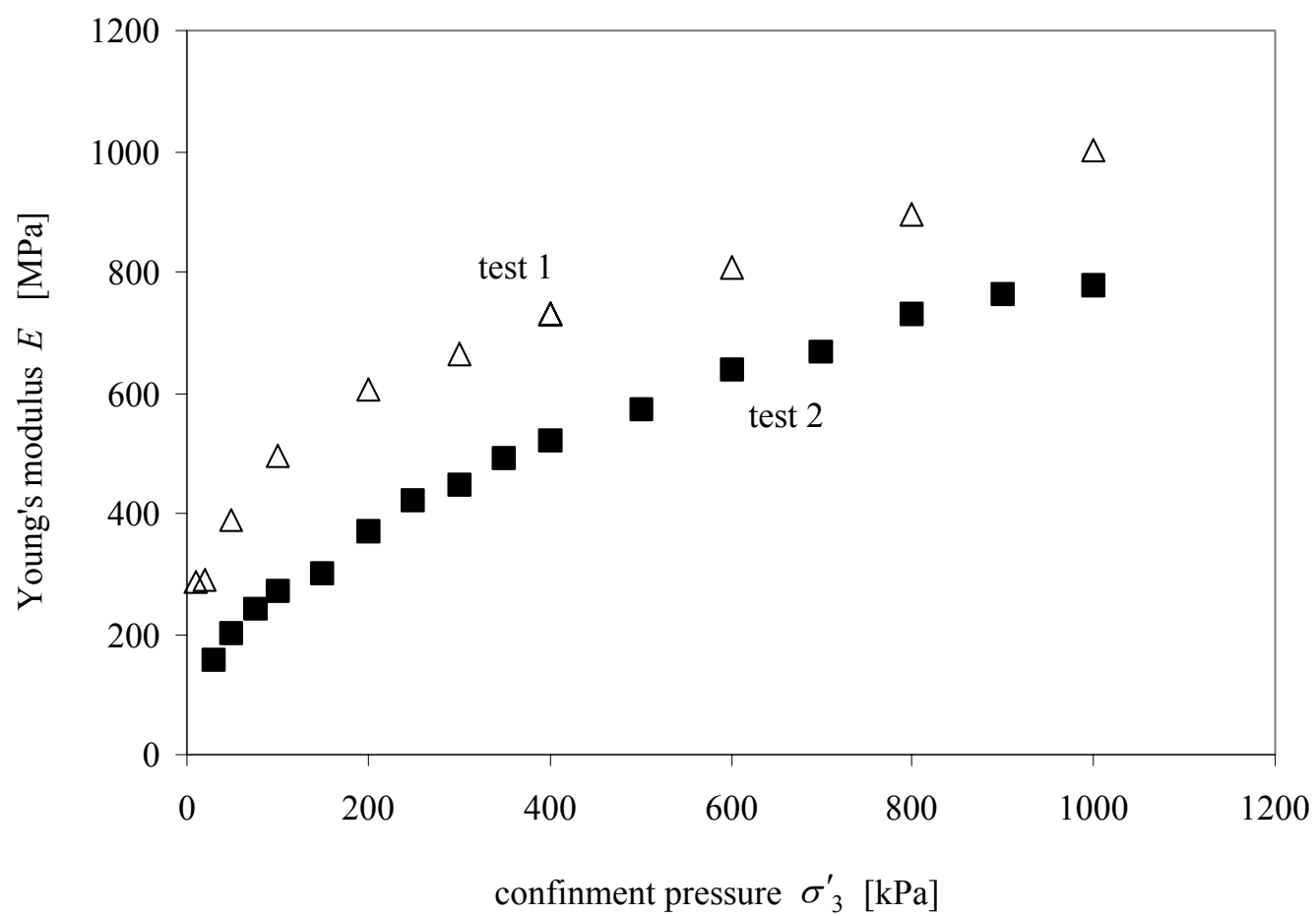

Fig. 9.17: Young's modulus $E$ as a function of the effective confinement pressure $\sigma_{3}$

It is intended to provide a relationship for the coefficient of subgrade reaction $k_{s}$ similar to equation (9.18) proposed by Terzaghi (1955). For further steps it is useful to provide $k_{s}$ as a function of the depth $z$. Thus, the relationship between the Young's modulus $E$ and the confining pressure $\sigma_{3}$ from Fig. 9.17 adapted by applying

$$
k_{S}=\frac{E}{D}
$$

based on Tab. 7.1 and assuming earth pressure at rest with

$$
\sigma_{3}=\gamma \cdot z \cdot K_{0}=\gamma \cdot z \cdot(1-\sin \varphi)
$$

and $\varphi=33^{\circ}$, and thus 


$$
z=\frac{\sigma_{3}}{\gamma(1-\sin \varphi)}
$$

For these assumptions Fig. 9.17 is re-drawn based on an assumed density of $\gamma=15.25 \mathrm{kN} / \mathrm{m}^{3}$, a non-saturated situation, and $D=1800 \mathrm{~mm}$ (Fig. 9.18). The tested configuration used saturated sand under different conditions. Although the latter assumptions are only a rough approximation, it may provide a basis for discussions and further comparisons.

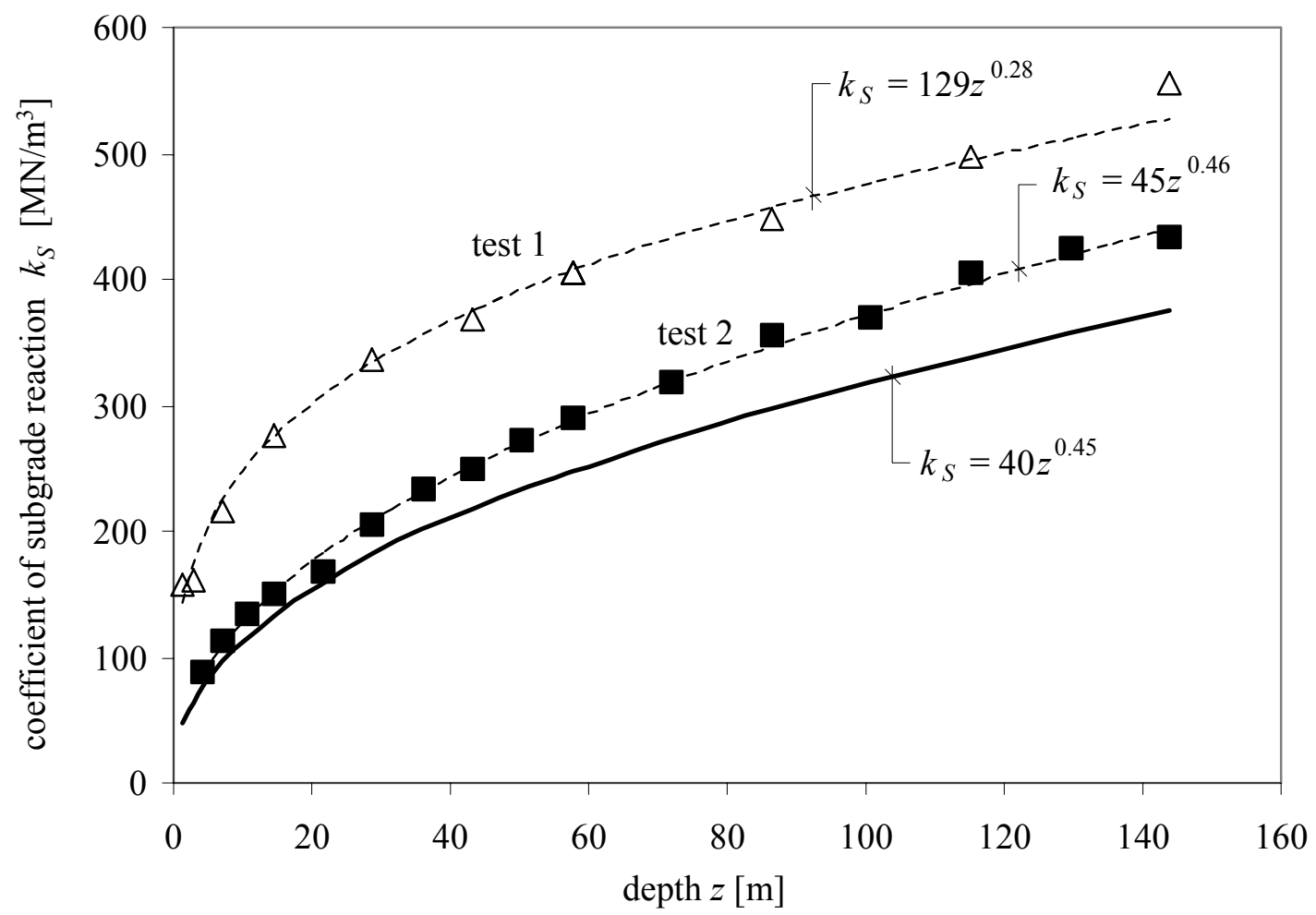

Fig. 9.18: Coefficient of subgrade reaction $k_{S}$ for $D=1800 \mathrm{~mm}$ as a function of the depth $z$

The results from the two tests are evaluated by fitting regression curves to the test results (dashed lines in Fig. 9.18). Although, the dimensionless approach of using a power function is not a convenient method for defining the relationship between the coefficient of subgrade reaction $k_{S}$ and the depth $z$, this type is applied due to coefficients of determination $R^{2}$ close to 1 . Based on these results an arbitrary and conservative lower boundary was defined as indicated by a continuous line in Fig. 9.18:

$$
k_{S}=40 z^{0.45} \quad \text { with } z \text { in }[\mathrm{m}] \text { and } k_{S} \text { in }\left[\mathrm{MN} / \mathrm{m}^{3}\right]
$$

The soil sample characteristics for the two different tests given in Tab. 9.2 appear to be very similar. Nevertheless, the evaluated results for the coefficient of subgrade reaction $k_{S}$ at e.g. a depth $z=30 \mathrm{~m}$ differ by a factor of 1.6 between the two tests. Due to these large discrepancies the results need to be considered with great care and should only provide a basis for discussions and not a design proposal. Further, the applicability of equation (9.27) is limited to the investigated sand only. Still, a comparison for e.g. a 
depth $z=30 \mathrm{~m}$ and $D=1800 \mathrm{~mm}$ between equation (9.27) and the approach by Terzaghi (1955) from equation (9.18) reveals a coefficient of subgrade reaction $k_{S}$ which is nearly 5 times larger for the calculation based on the bender element tests.

\subsection{Re-calculation for axially loaded cylindrical shell}

As a conservative first approach the evaluation according to Terzaghi (1955) for the coefficient of subgrade reaction $k_{S}$ for loose sand is applied:

$$
k_{n}=k_{S}=2.2 \frac{M N}{m^{3}} \cdot \frac{z}{D}
$$

The latter expression may provide a conservative approximation for obtaining the critical buckling load. The experimental investigations with Bender element tests provided larger values for calculating the coefficient of subgrade reaction.

Based on the previous findings the approach given in ISO/DIS 19902:2004 is used for further analyses. It was found that the predictions provide a conservative approximation for the tangential stiffness due to the surrounding soil. A further advantage is the applicability of the concept by simple means and without needing additional in-situ testing. Thus, for both, the meridional and circumferential coefficient of subgrade reaction the following equation is used:

$$
k_{t}=k_{t, m}=k_{t, c}=400 m^{-1} \cdot K \gamma z \tan \delta
$$

with $0.7 \leq K \leq 0.8$ (loose or medium dense/dense) and $20^{\circ} \leq \delta \leq 35^{\circ}$ according to ISO/DIS 19902:2004).

In the subsequent numerical analyses two approaches are used:

(a) a conservative approximation according to equation (9.28) and (9.29) and

(b) an approximation based on the evaluated Bender element tests.

For approximation (a) loose sand is considered resulting in $K=0.7$ and $\delta=20^{\circ}$. The applied values are summarized in Tab. 9.3 for an assumed density $\gamma=15.25 \mathrm{kN} / \mathrm{m}^{3}$. Similarly, the values can be derived for medium dense sand (Tab. 9.4). Since the equation given in Tab. 9.3 for (a) depends on the radius of the cylinder, a ratio $r / t=50$ with $r=900 \mathrm{~mm}$ similar to the dimensions used for tubular piles is chosen. The ends of the investigated cylinder are clamped in the global mechanical model, i.e. resulting in bending moments at the ends, but allowing the shell to rotate along the circumference at the boundaries (meridional rotation not restrained), i.e. BC 1f from EN 1993-1-6. According to EN 1993-1-6 the reduction factor is not affected by the boundary conditions for long cylinders as the reduction factor $C_{x}$ is limited to 0.6 . Further this aspect may be disregarded as according to prEN 1993-5:2004-07 a value of $C_{x}=1.0$ is to be used in the design procedure. Hence, the latter boundary conditions are used for the general investigation. 
Tab. 9.3: Summary of applied coefficients of subgrade reaction-loose sand

\begin{tabular}{lcc}
\hline appr. & $\boldsymbol{k}_{\boldsymbol{n}}$ & $\boldsymbol{k}_{\boldsymbol{t}}$ \\
\hline (a) & $1.2 \frac{M N}{\mathrm{~m}^{4}} \cdot z$ & $1.5 \frac{M N}{\mathrm{~m}^{4}} \cdot z$ \\
\hline (b) & $40 \cdot z^{0.45}\left[\frac{M N}{\mathrm{~m}^{3}}\right]$ & \\
\hline
\end{tabular}

Tab. 9.4: Summary of applied coefficients of subgrade reaction-medium dense sand

\begin{tabular}{lcc}
\hline appr. & $\boldsymbol{k}_{\boldsymbol{n}}$ & $\boldsymbol{k}_{\boldsymbol{t}}$ \\
\hline (a) & $3.6 \frac{M N}{\mathrm{~m}^{4}} \cdot z$ & $2.4 \frac{M N}{\mathrm{~m}^{4}} \cdot z$ \\
\hline (b) & $40 \cdot z^{0.45}\left[\frac{M N}{\mathrm{~m}^{3}}\right]$ & \\
\hline
\end{tabular}

Nevertheless, in the numerical analyses for axially loaded cylinders the possible supporting effect of meridional springs is disregarded as its influence on the general load transfer mechanisms is too large. The meridional springs serve directly as support over the shell and thus may significantly relief the cylinder and enhance the carrying capacity. This issue may be complex as the buckling capacity increases with increasing lengths. In order to make the investigation more general, the meridional springs are only applied for investigating the external pressure loading as a conservative approximation. In the following the assumption is applied that the linear distribution of the spring stiffness according to Tab. 9.3 can be adapted to a uniform distribution of springs over the length. Since the distribution is linear, the mean value corresponds to half of the maximum amplitude as calculated with Tab. 9.3. This conservative approximation was validated with linear buckling analyses involving calculations with uniformly embedded cylinders $\left(k_{n}\right.$ and $\left.0.5 k_{n}\right)$ and springs applied with stiffnesses increasing with depth or length up to $k_{n}$. The numerical analyses predicted smaller buckling loads for the cases "uniform $0.5 k_{n}$ " and "linear" in comparison to the case "uniform $k_{n}$ " independent of $\omega$. The differences between the cases "uniform $0.5 k_{n}$ " and "linear" were negligible emphasizing the applicability of the approximation. The purpose of this approach is to provide a general set of data from which factors for increasing the buckling strength can be derived. The approach "uniform $0.5 k_{n}$ " provides a conservative approximation for the power function from equation (9.27) given for approach (b). The calculated increase of the buckling stress with respect to the classical buckling stress is given in Fig. 9.19 for approach (a) and (b) according to Tab. 9.3. The previous findings that the supporting effect increases for increasing lengths is confirmed. Additionally, it can be seen that the beneficial influence of the surrounding soil reaches considerable levels for the investigated large diameters and long cylinders. The increase of the buckling stress evolves fairly smoothly with increasing lengths for case (a). Nevertheless, for approach (b) the springs seem to provide nearly no beneficial influence for $l<3 \mathrm{~m}$. For longer 
cylinders the buckling stress abruptly increases. This change in behaviour can be explained as for the given geometry the buckling mode turns from a rotation-symmetric mode (ring buckles) to a chessboard-like failure mode at this length. As a consequence, the tangential foundation explained in section 9.3.2 does not influence the carrying capacity for $l<3 \mathrm{~m}$. These springs are only activated for longer cylinders and then contribute to the stiffness of the system.

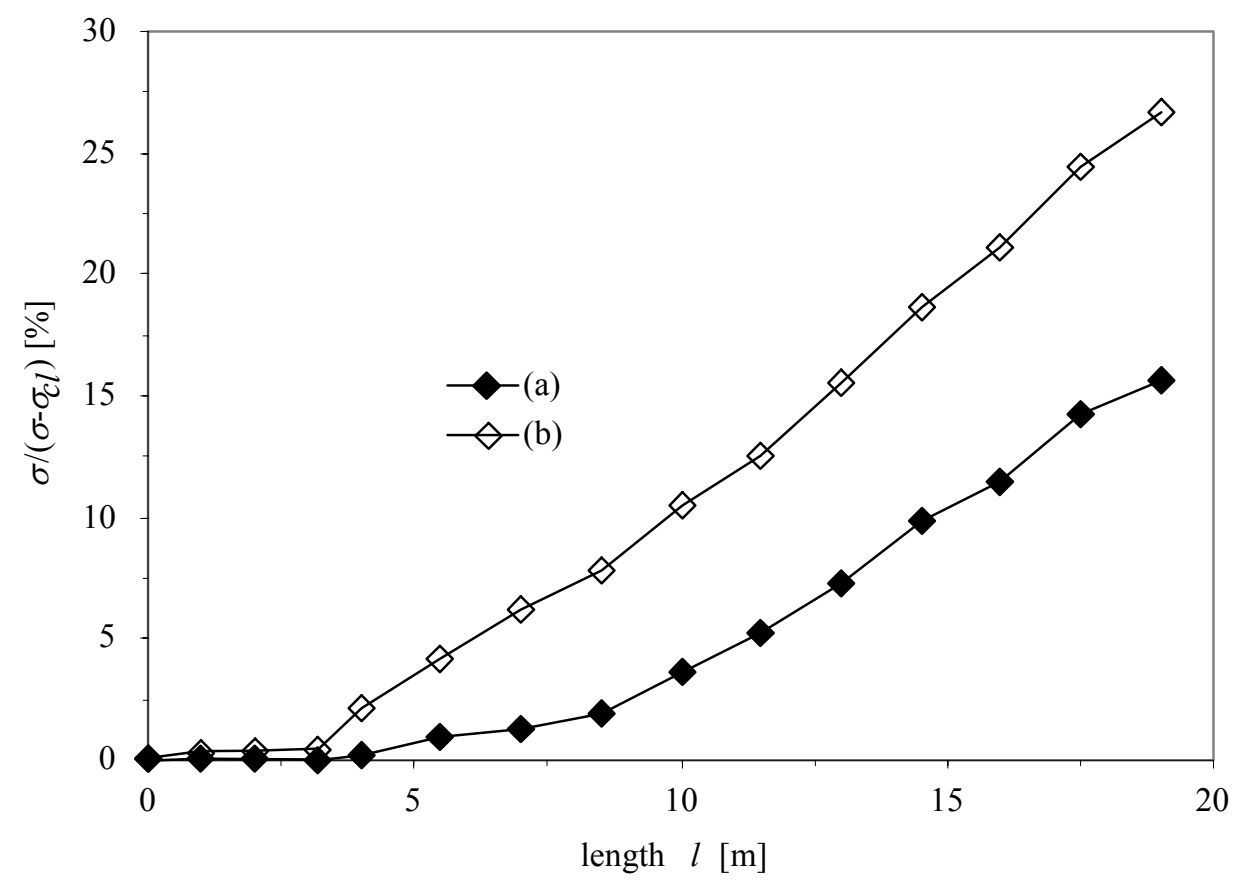

Fig. 9.19: Increase of axial buckling stress for different lengths l for type (a) and (b) with Tab. 9.3

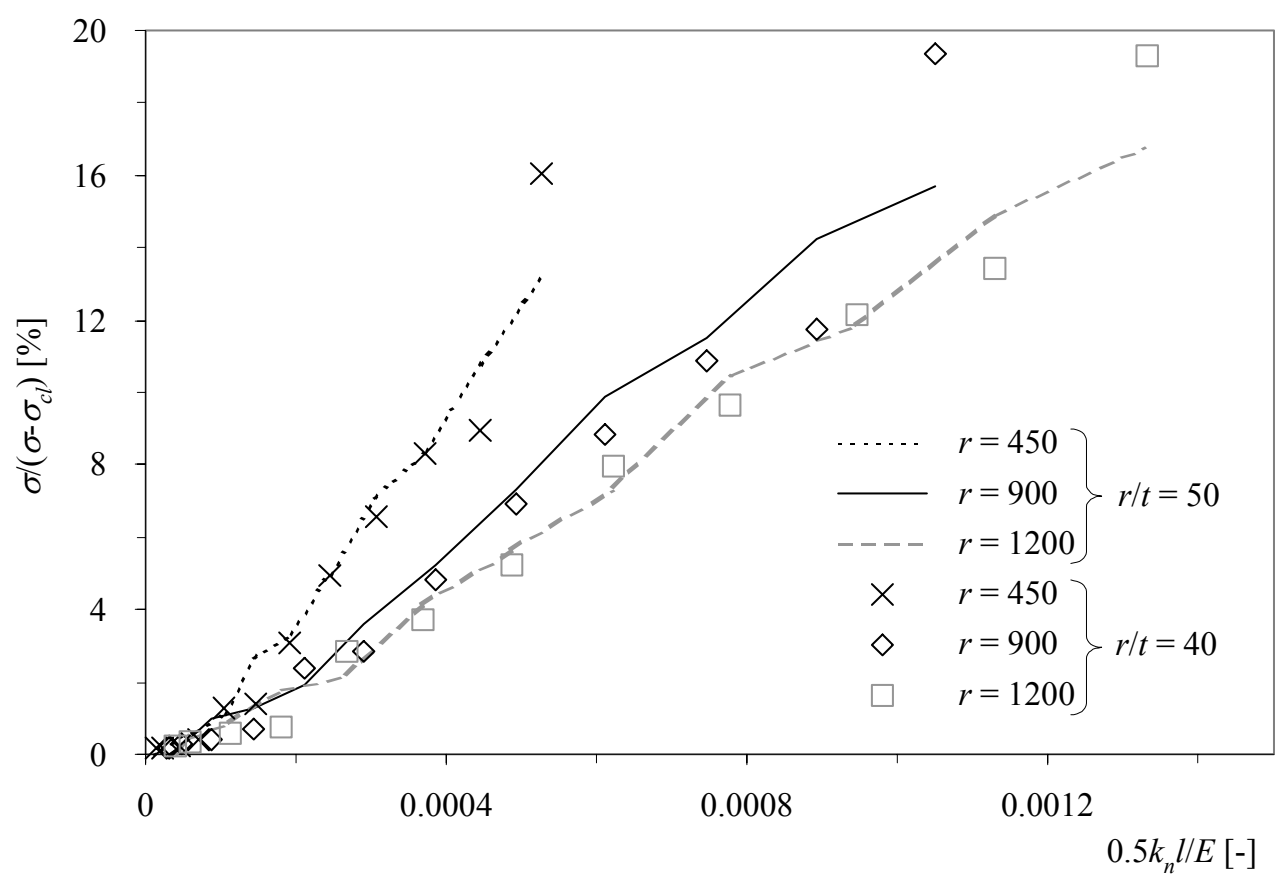

Fig. 9.20: Increase of buckling stress as a function of the dimensionless value $0.5 k_{n} l / E$ for $r / t=40$ and $r / t=50$ with varying radii $[\mathrm{mm}]-$ axial load 
In the following a parametric study based on a $180^{\circ}$ segment of a cylinder for evaluating the influence of surrounding soil based on the equations (9.28) and (9.29) is performed. This approach uses the conservative assumption of loose sand in order to provide results, which can be adapted to denser sand types. For obtaining more general information on the supporting effect of the sand, linear buckling analyses considering both radial and circumferential springs, are performed for different radii and $r / t$ ratios. Some results are depicted as a function of the dimensionless value $0.5 k_{n} l / E$ in Fig. 9.20. The results highlight similar results for the two different for the different $r / t$ ratios. In spite of the fact that deviations appear for large values of $0.5 k_{n} l / E$, the evaluation of a factor for interpreting the influence of a foundation is based on the $r / t$ ratio of 40 and should be valid for $r / t>40$. The latter aspect is taken into account in the process of deriving design proposals. Further, it can be seen that the buckling stress increases for smaller radii since equation (9.28) involves the diameter $D$ of the tubular pile. Again this aspect needs to be considered when deriving a proposal for a design procedure.

A dimensionless parameter $f$ has been derived from the results of the numerical analyses for providing a basis for design proposals. It is defined as:

$$
f=\frac{l}{D}\left(\frac{k_{n}}{n_{h, \text { loose }}}\right)^{0.25}
$$

with the constant $n_{h, \text { loose }}$ of lateral subgrade reaction for loose sand defined by Terzaghi (1955) as $2.2 \mathrm{MN} / \mathrm{m}^{3}$. The latter parameter is chosen based on a comparison of different results. Since the evaluation shall involve a general approach with larger coefficients of subgrade reaction than the approach for loose sand by Terzaghi (1955), additional numerical analyses are performed applying $n_{h}=6.6 \mathrm{MN} / \mathrm{m}^{3}$ (medium dense sand) for formula (9.28) instead of $n_{h}=2.2 \mathrm{MN} / \mathrm{m}^{3}$. The results are depicted in Fig. 9.21 as a function of the dimensionless parameter $f$. The idea is to define a linear approximation between the different parameters and the increase of the buckling load as seen in Fig. 9.21 as a conservative estimation. It can be seen that for larger $f$ the beneficial influence of the surrounding soil increases. Nevertheless, a linear function is suggested as a conservative approximation for the factor $\beta_{x, \text { sand }}$. It is derived empirically from Fig. 9.21 with a safety margin regarding the calculated results as follows:

$$
\beta_{x, \text { sand }}=1+(0.009 f-0.08)=0.92+0.009 \frac{l}{D}\left(\frac{k_{n}}{n_{h, \text { loose }}}\right)^{0.25}
$$

Similarly, to equation (9.1) the critical buckling load can be calculated as

$$
\sigma_{c r}=\sigma_{c l} \cdot \beta_{x, \text { sand }}=0.605 \cdot E \cdot \frac{t}{r} \cdot\left[0.92+0.0045 \frac{l}{r}\left(\frac{k_{n}}{n_{h, \text { loose }}}\right)^{0.25}\right]
$$

The given procedure is limited to the investigated cases: $\beta_{\text {sand }}>1, r / t \geq 40$, and $r \geq 450 \mathrm{~mm}$. The equation given in (9.32) takes into account radial and circumferential 
support from the surrounding soil. Further, the above considerations of replacing the "linear" distribution of $k_{n}$ with "uniform $0.5 k_{n}$ " are included. Thus, in the application the maximum amplitude of $k_{n}$ needs to be introduced in (9.32).

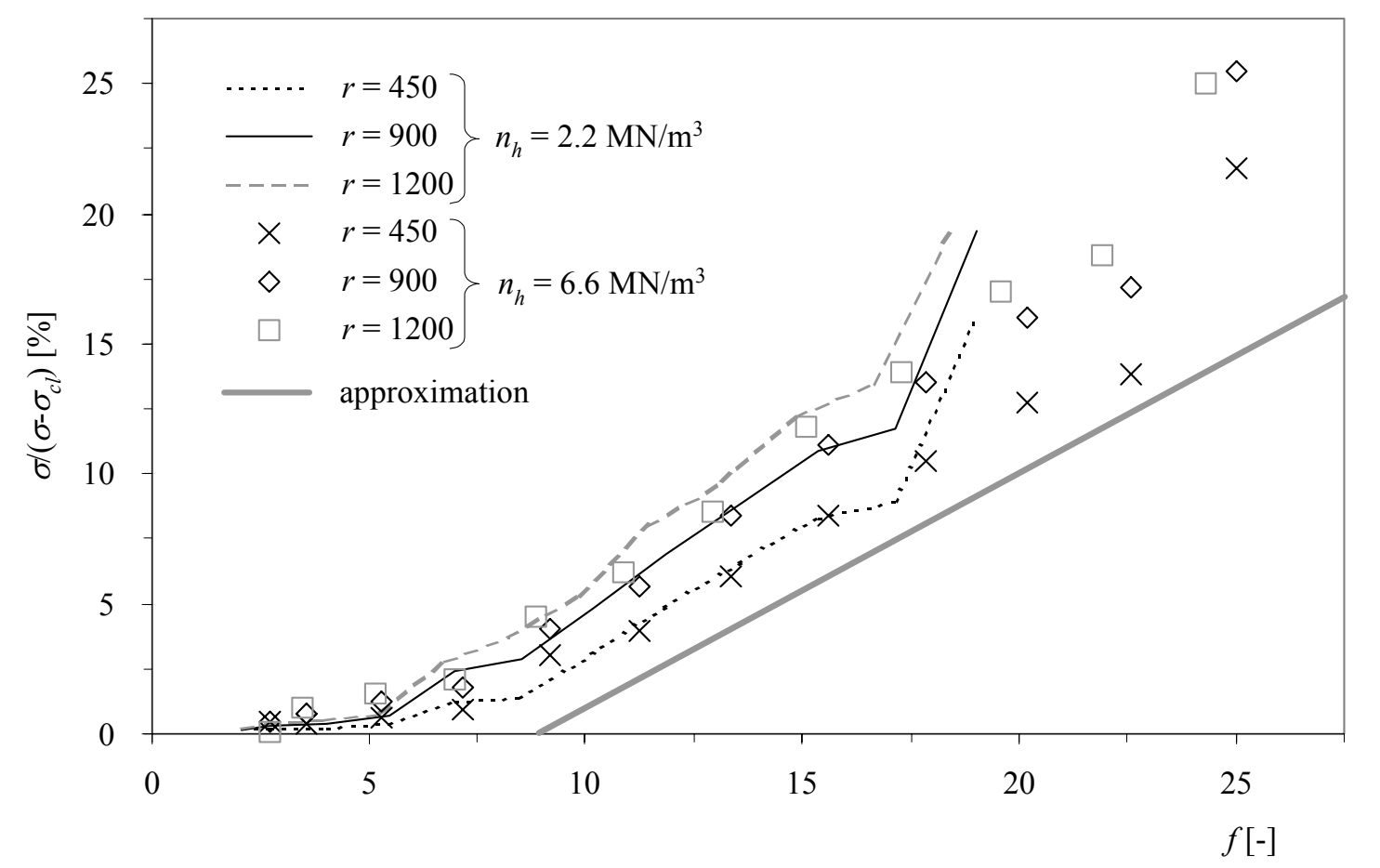

Fig. 9.21: Increase of buckling stress as a function of the dimensionless parameter ffor $r / t=40$ with varying radii $[\mathrm{mm}]$ - axial load

The investigated cases are limited to the fact that the entire cylinder is surrounded by sand or soil. Nevertheless, in real constructions of harbour walls, the tubular piles are only partly in contact with soil as the other side is exposed to the sea. Depending on the height of the wall a part is completely embedded in soil (pile footing/tip). The previous approach may provide non-conservative predictions for a tubular pile which is only partly embedded in sand. A set of additional numerical analyses in which only half of the circumference had springs attached to it ("half-embedded") showed that the increase of the buckling stress for the "half-embedded" case is larger than $50 \%$ of the completely embedded case. This finding leads to the rough but conservative proposal for deriving a factor $\beta_{\text {sand }, 0.5}$ for the "half-embedded" case:

$$
\beta_{x, \text { sand }, 0.5}=1+0.5(0.009 f-0.08)=0.96+0.0045 \frac{l}{D}\left(\frac{k_{n}}{n_{h, \text { loose }}}\right)^{0.25}
$$

Some results of the numerical studies are depicted in Fig. 9.22 along with the approximation from equation (9.33).

Further, an additional set of numerical studies was performed for validating the assumptions of the proposed approximations for large coefficients of subgrade reaction. In this context a coefficient of subgrade reaction $k_{S}=500 \mathrm{MN} / \mathrm{m}^{3}$ is chosen as arbitrary large value, which is imposed independent of the investigated length. It can be seen that 
for large coefficients of subgrade reaction the conservativeness of the approximation is maintained.

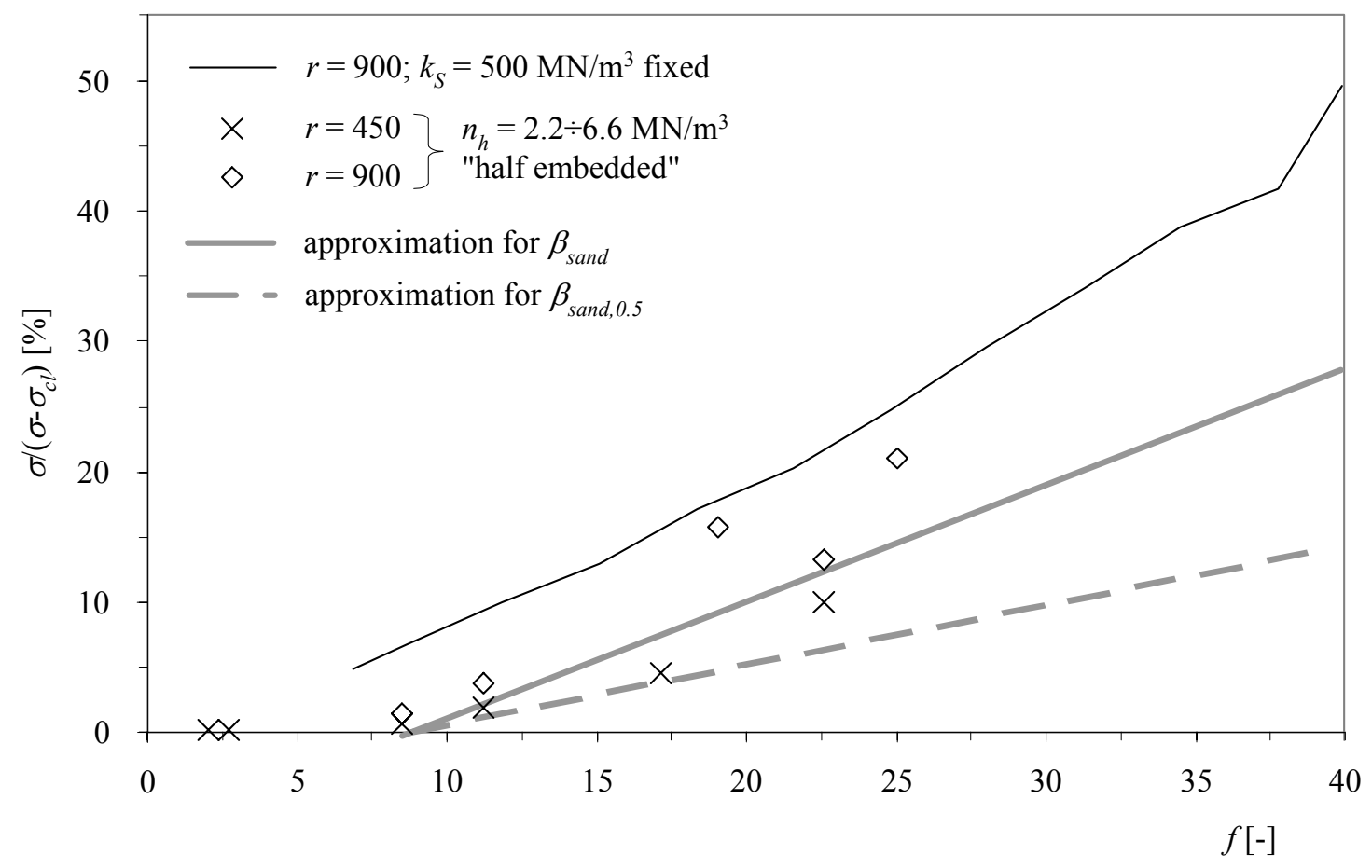

Fig. 9.22: Increase of buckling stress as a function of the dimensionless parameter f additional validating analyses with varying radii [mm] - axial load

Based on the preliminary studies it can be assumed that the behaviour may be nonlinear with a decreasing gradient (equation (9.1) and Fig. 9.3). Nevertheless, comparing studies with large coefficients of subgrade reaction, from which some results are given in Fig. 9.22, reveal even an increasing gradient. Extremely large coefficients of subgrade reaction or very long tubular piles (exceeding the investigated range of up to $30 \mathrm{~m}$ ) are not commenly used in constructions and then would need further investigations.

\subsection{Back-calculation for cylindrical shell subjected to external pressure}

According to the approach given in the previous section, the influence of the surrounding soil is investigated for external pressure. Again, the evaluation from equations (9.28) according to Terzaghi (1955) is applied for the radial foundation. The tangential spring foundation is now extended by adding meridional springs to the circumferential springs. The stiffness of these springs is calculated as given in equation (9.29). The investigated system is a cylindrical shell which is entirely restrained at one end, i.e. corresponding to BC 1r from EN 1993-1-6, while the other end is free. The investigated geometry is based on the previous numerical investigations for the axially loaded cylinder. For simplification the conservative approximation of replacing 
approaches by non-uniformly embedded cylinders (increasing stiffness with depth) with a uniformly embedded cylinder introduced in chapter 9.5 is adopted. Consistently, it is proposed to apply the average value of the linearly distributed springs.

The following parametric study uses a $180^{\circ}$ segment of a cylinder subjected to uniform external pressure for executing linear bifurcation analyses. The results for two different $r / t$ ratios are depicted as a function of the dimensionless value $0.5 k_{n} l / E$ in Fig. 9.23. It can be seen that for larger $r / t$ ratios, the supporting effect of the soil increases considerably based on the applied approach. Thus, in accordance with the study on the axially loaded cylinders, an approximation is derived by implementing the $r / t$ ratio of 40 as a lower boundary. The results can then be applied as a conservative approach for larger $r / t$ ratios. The differences between the different $r / t$ ratios is much more distinct for the case of external pressure in comparison to the axially loaded cylinder.

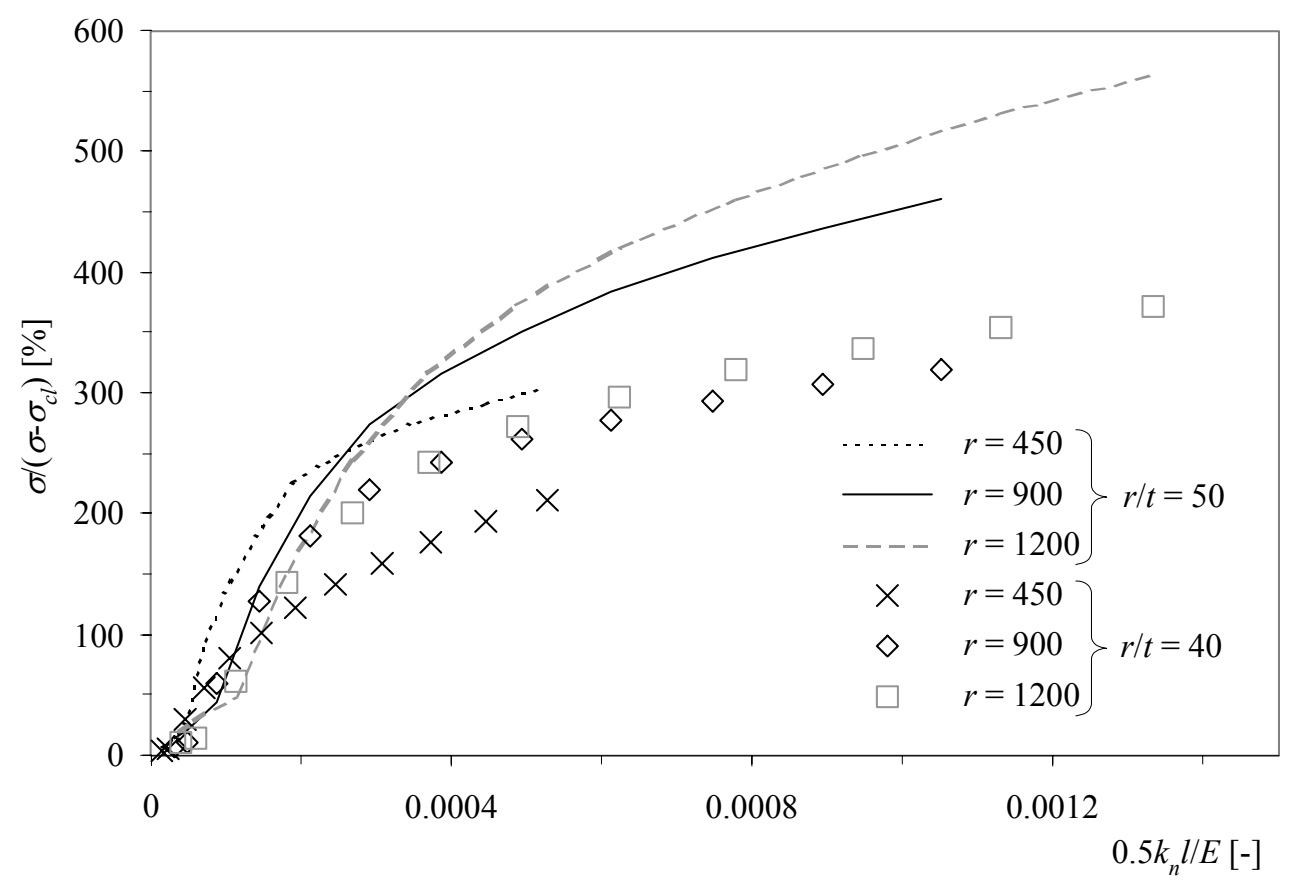

Fig. 9.23: Increase of buckling stress as a function of the dimensionless value $0.5 k_{n} l / E$ for $r / t=40$ and $r / t=50$ with varying radii $[\mathrm{mm}]$ - external pressure

The results of the different numerical analyses $\left(n_{h}=2.2 \mathrm{MN} / \mathrm{m}^{3}\right.$ and $\left.n_{h}=6.6 \mathrm{MN} / \mathrm{m}^{3}\right)$ are plotted in Fig. 9.24 over the dimensionless parameter $f$ as given in equation (9.30). Since the relationship of buckling stress and parameter $f$ is more non-linear in contrast to the axial load case for the investigated range it is suggested to used a square function for approximating the supporting behaviour of the surrounding sand as a function of the parameter $f$. A very conservative lower boundary curve is depicted in Fig. 9.24. The latter proposal underestimates the supporting influence of the soil for larger $r / t$ ratios and coefficients of subgrade reaction. Still, this conservative approach is discussed as it is simple to apply and independent on several parameters. Further, it can be seen that the application of the factor defined by the conservative lower boundary curve already results in an enormous increase of the buckling stress. 


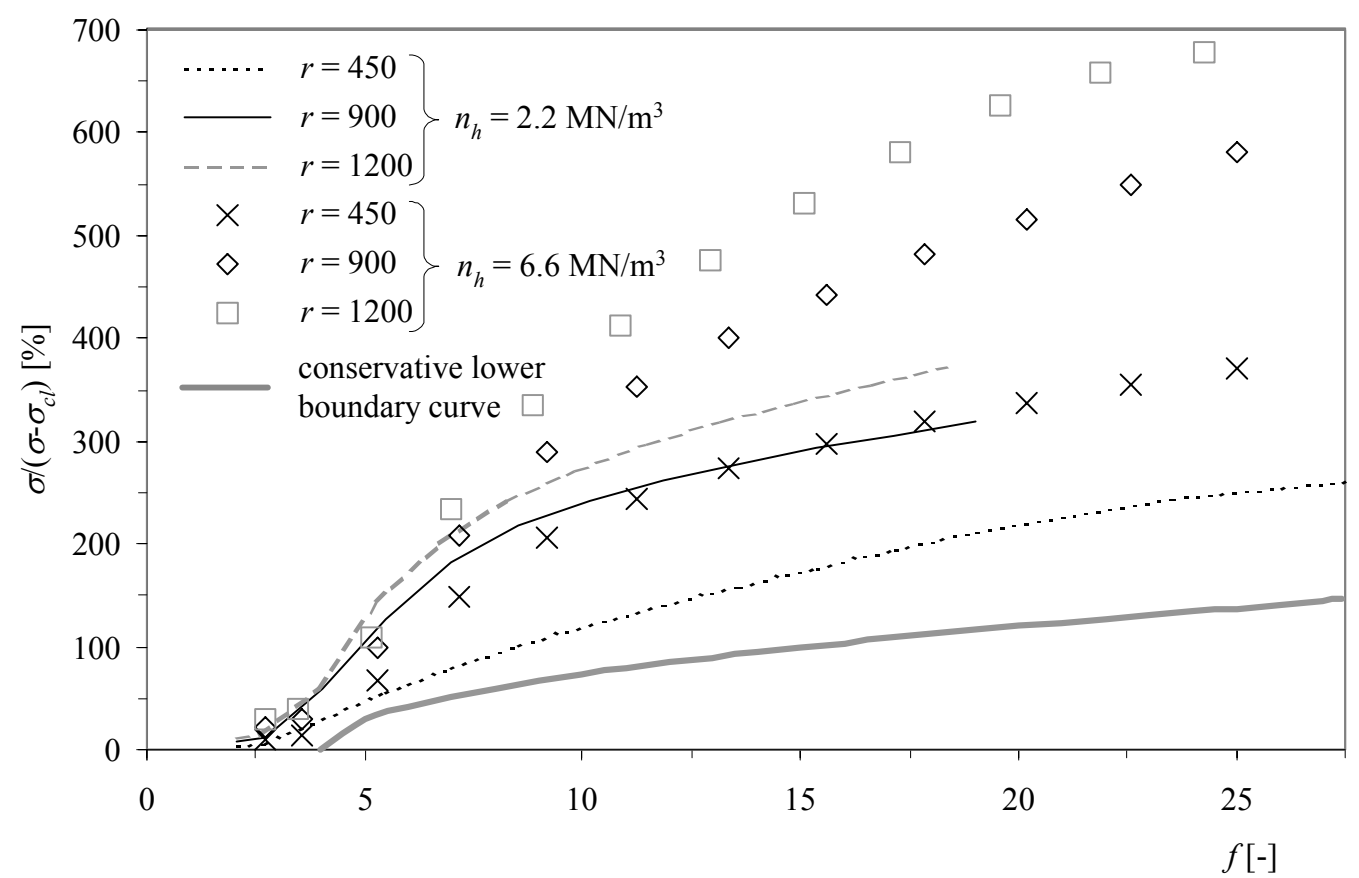

Fig. 9.24: Increase of buckling stress as a function of the dimensionless parameter ffor $r / t=40$ with varying radii [mm] - external pressure

Since it is not necessary to take account of the very large theoretical increase of buckling strength regarding practical constructions the approach with the latter boundary curve is applied as approximation and named $\beta_{\theta, \text { sand: }}$

$$
\beta_{\theta, \text { sand }}=1+(0.3 \sqrt{f-4.0})=1+0.3 \sqrt{\frac{l}{D}\left(\frac{k_{n}}{n_{h, \text { loose }}}\right)^{0.25}-4.0}
$$

is only set to be valid for $r / t \geq 40, r \geq 450 \mathrm{~mm}$, and $k_{n} \geq 2.2 \mathrm{MN} / \mathrm{m}^{3} \cdot l / D$. If the approach by Terzahi (1955) is applied, the introduced value $k_{n}$ refers to the maximum amplitude of $k_{n}$ at the pile tip. According to equation (9.33) an equation for the "half-embedded" case is proposed:

$$
\beta_{\theta, \text { sand }, 0.5}=1+0.5(0.3 \sqrt{f-4.0})=1+0.15 \sqrt{\frac{l}{D}\left(\frac{k_{n}}{n_{h, \text { loose }}}\right)^{0.25}-4.0}
$$

Again, the proposal is validated with additional numerical buckling analyses with a cylinder with only half of the circumference embedded in sand. As shown in Fig. 9.25, the proposed equation (9.35) provides a conservative approximation for the calculated results. Here it is emphasized that the given formulas are limited to $k_{n} \geq 2.2 \mathrm{MN} / \mathrm{m}^{3} \cdot l / D$ for the externally pressurized cylinder. Equation (9.34) and (9.35) may provide a nonconservative prediction for softer soils. Here, the beneficial influence of the surrounding soil should not be taken into account. 


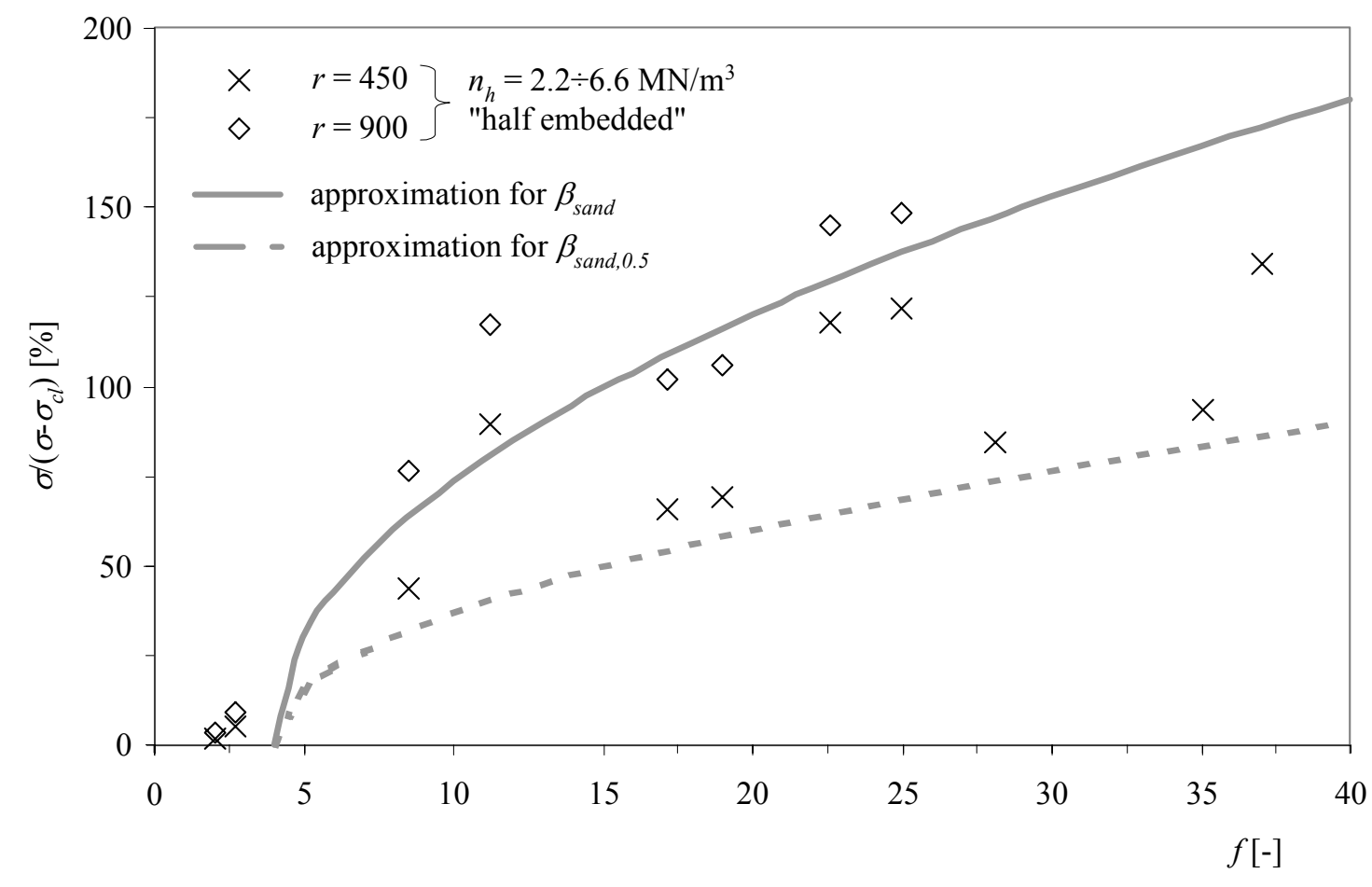

Fig. 9.25: Increase of buckling stress as a function of the dimensionless parameter f additional validating analyses with varying radii [mm] - external pressure 


\section{Global load transfer behaviour}

\subsection{Load transfer mechanisms of the bending moment over the pile length}

\subsubsection{Model}

For the investigation of the load transfer mechanisms of a partially embedded vertical pile (uniform sand) simple modelling approaches with springs are available (e.g. SRM). Initial analyses are performed with such a simple model in order to obtain more information on the load transfer mechanisms for a pile subjected to horizontal loads or moments.

In the preliminary investigation an $11 \mathrm{~m}$ long steel tube is chosen which is embedded vertically $10 \mathrm{~m}$ in sand and cantilevers $1 \mathrm{~m}$ out of the soils for a better load introduction. The cross-section is chosen in accordance with the primary elements of combiwalls from real constructions (see chapters 1 and 4). The diameter of the tubes is $1800 \mathrm{~mm}$ with a wall thickness of $18 \mathrm{~mm}$. In Fig. 10.1 the investigated case is sketched. The data for the pile is summarized in Tab. 10.1.

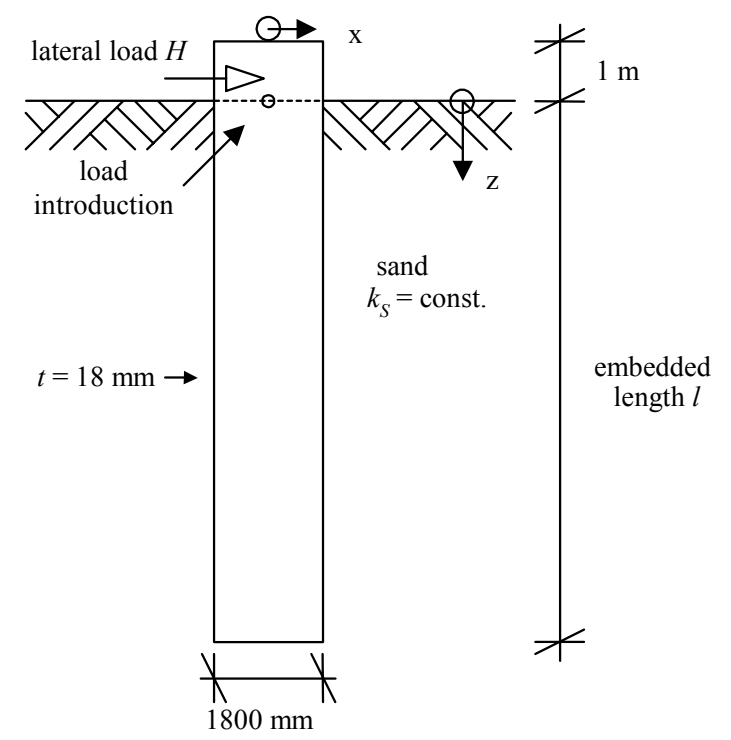

Fig. 10.1: System for the preliminary investigation

Tab. 10.1: $\quad$ Data for the analyses

\begin{tabular}{|l|c|c|c|c|c|c|c|}
\hline & $D[\mathrm{~mm}]$ & $t[\mathrm{~mm}]$ & $A\left[\mathrm{~mm}^{2}\right]$ & $\begin{array}{c}I_{y}=I_{z} \\
{\left[\mathrm{~mm}^{4}\right]}\end{array}$ & $S_{y}\left[\mathrm{~mm}^{3}\right]$ & $\begin{array}{c}f_{y d} \\
{\left[\mathrm{~N} / \mathrm{mm}^{2}\right]}\end{array}$ & $H[\mathrm{kN}]$ \\
\hline Tube & 1800 & 18 & $1,018 \cdot 10^{5}$ & $4,123 \cdot 10^{10}$ & $1,944 \cdot 10^{7}$ & $420 / \gamma_{M}$ & 20 \\
\hline
\end{tabular}




\subsubsection{Analytical solutions}

The complex three-dimensional problem of a vertically embedded, laterally loaded tube without filling can be simplified and investigated as a two-dimensional problem. For an approximation using a constant linear elastic foundation for the laterally loaded pile a closed solution can be found for the differential equation. Applying a constant coefficient of subgrade reaction $k_{S}\left[\mathrm{MN} / \mathrm{m}^{3}\right]$ in combination with the limitation of the width or diameter of the pile to $D=1 \mathrm{~m}$ the modulus of subgrade reaction $k\left[\mathrm{MN} / \mathrm{m}^{2}\right]$ for the two-dimensional case can be calculated as:

$$
k=k_{S} \cdot D
$$

For a tube according to Fig. 10.1 the following differential equation can be written:

$$
E I \frac{d^{4} x}{d z^{4}}=-k \cdot x
$$

The elastic length $L$ is introduced as a constant

$$
L=\sqrt[4]{4 \cdot \frac{E \cdot I}{D \cdot k_{S}}}
$$

and the normalized length $\zeta_{L}$ :

$$
\varsigma_{L}=\frac{z}{L}
$$

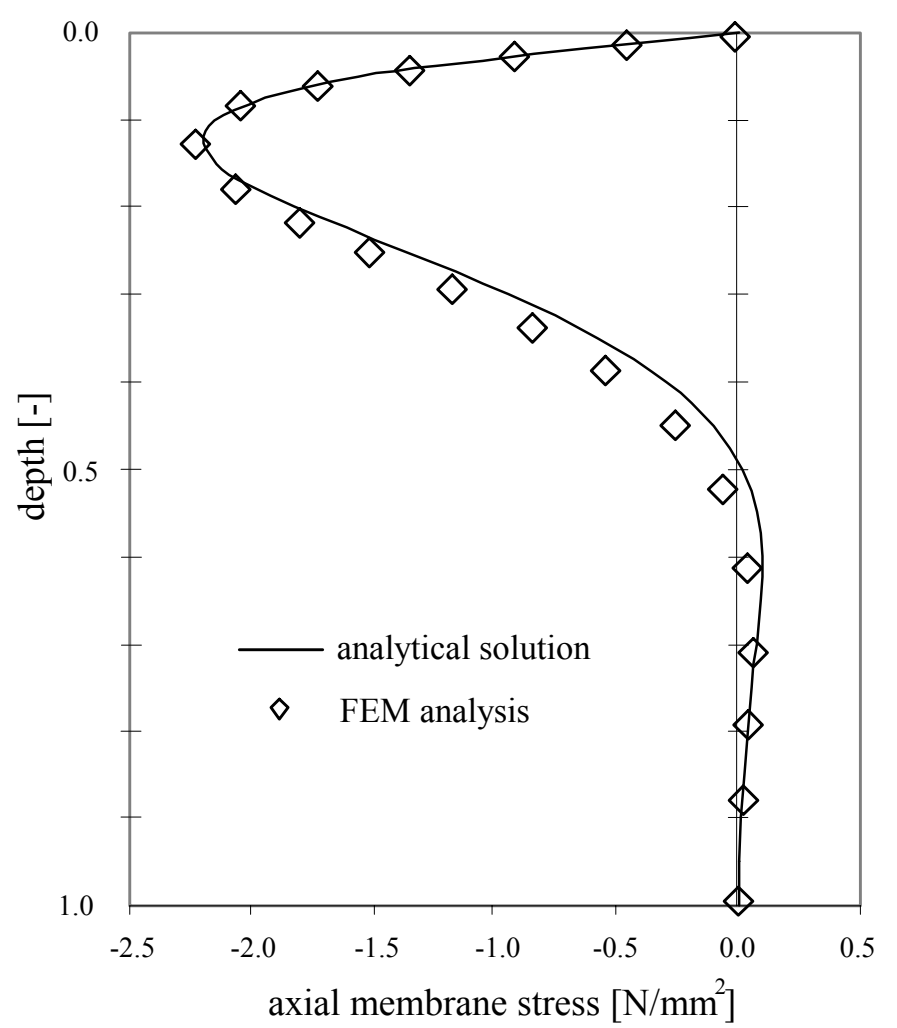

Fig. 10.2: Axial compressive membrane stresses for a laterally loaded tube $(H=20 \mathrm{kN} ; \mathrm{l}=20 \mathrm{~m}$; $k_{S}=100 \mathrm{MN} / \mathrm{m}^{3}$ ) 
For perfectly slender piles with $l / D>3$ the bending moments can be calculated with a small error $(<4 \%)$ according to the following simplified solution given by Kolymbas (1989):

$$
M=H \cdot L \cdot e^{-\varsigma_{L}} \cdot \sin (\varsigma)
$$

with the maximum bending moment

$$
M=0.3224 \cdot H \cdot L
$$

in the depth

$$
z=\pi \cdot L / 4
$$

The solution is only valid for a solely laterally loaded pile with a hinge at the pile head. Fig. 10.2 shows the results from the numerical three-dimensional analyses from section 10.1.3 and the analytical solution for the beam with elastic springs. More details and further examples can be found for example in Poulos and Davis (1980) or Kolymbas (1989).

\subsubsection{Numerical analyses}

The preliminary investigations are performed using a three-dimensional tube as a symmetric half of the tube in the finite element analysis with the imposition of symmetry conditions along the two meridional edges and a constant foundation over the outer surface. The analyses are executed as geometrical and material non-linear analyses with a lateral load at the pile head, which is introduced with a hinge. In order to prevent local stress concentrations and for reasons of a smoother load introduction a $1 \mathrm{~m}$ segment of the tube which extends over the surface is joined to a rigid body.
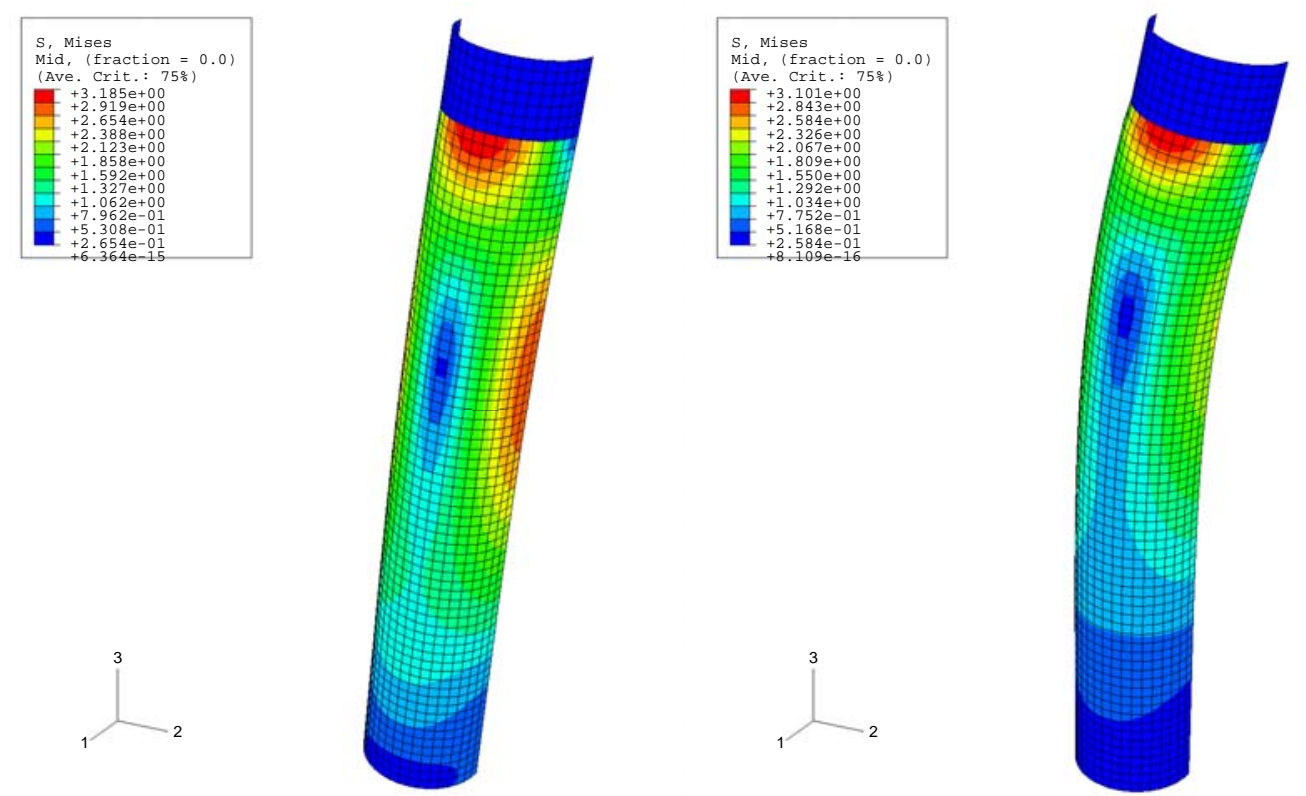

Fig. 10.3: v. Mises stresses for a short tube completely embedded with $k_{S}=10 \mathrm{MN} / \mathrm{m}^{3}$ (left) and $k_{S}=100 \mathrm{MN} / \mathrm{m}^{3}$ (right) 
Fig. 10.3 depicts the v. Mises stresses for the deformed shape of the pile with a foundation of 10 and $100 \mathrm{MN} / \mathrm{m}^{3}$ and a horizontal load at the pile head of $100 \mathrm{kN}$. For the case a coefficient of subgrade reaction of $10 \mathrm{MN} / \mathrm{m}^{3}$ the pile moves nearly as a rigid body without any significant component from bending deflection. The load introduction at the top gives rise to high shear stresses and thus also to high v. Mises stresses at the side of the tube. Additionally, due to the bending moment high compressive stresses appear about at half-length of the tube, and it is clear that the forces reach the tip of the pile as it displaces laterally and non-negligible v. Mises stresses are still present. In contrast to the latter case, the tube considerably deflects for a coefficient of subgrade reaction of $k_{S}=100 \mathrm{MN} / \mathrm{m}^{3}$. Because of the increased stiffness of the soil the stresses are re-distributed and the value of the maximum v. Mises stresses in the compression zone by far smaller than in the case of the softer soil. The bending moment is transferred to the soil at a smaller depth, and thus only a portion of the load reaches the pile tip. The lateral displacement of the pile tip is marginal.
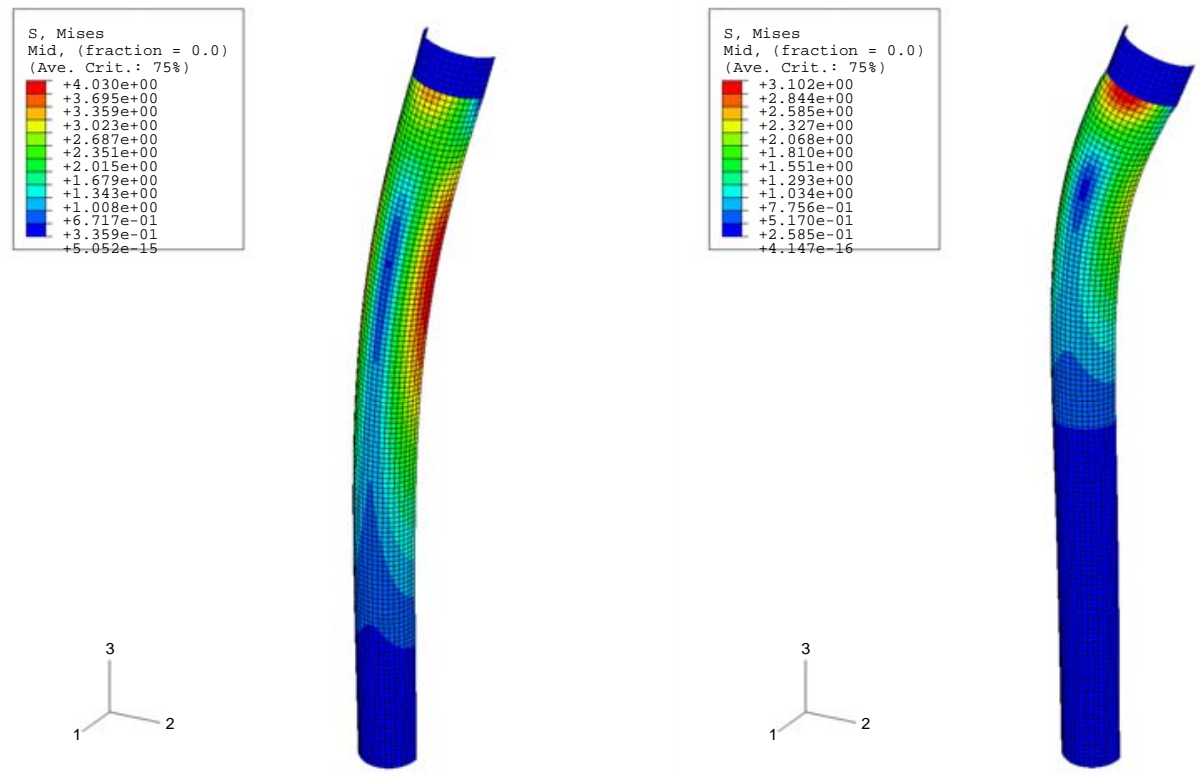

Fig. 10.4: v. Mises stresses for a long tube completely embedded with $k_{S}=10 \mathrm{MN} / \mathrm{m}^{3}$ (left) and $k_{S}=100 \mathrm{MN} / \mathrm{m}^{3}$ (right)

A pile which is embedded twice the length with $k_{S}=10 \mathrm{MN} / \mathrm{m}^{3}$ over $20 \mathrm{~m}$ (Fig. 10.4) does not exhibit a rigid body movement as seen in the previous case but deflects. For the long pile in combination with the stiffer coefficient of subgrade reaction the stresses seem to have faded out about half the way to the pile tip.

In Fig. 10.5 normalized deflections for laterally loaded short and long piles are depicted. For increasing lengths and an increasing coefficient of subgrade reaction $k_{S}$ the shape of the deflection changes and the load does not reach the tip of the pile. For a length of $20 \mathrm{~m}$ and a coefficient of subgrade reaction $k_{S}$ of $100 \mathrm{MN} / \mathrm{m}^{3}$ nearly lateral movements take place at the tip of the pile (Fig. 10.5). Such "long" piles can therefore be assumed to be infinitely long. More details on defining piles according to their bending 
behaviour and on the criterion for rigid or flexible behaviour according to different researchers can be found summarized e.g. in Kulhawy and Chen (1995).

The cases "short" and "long" piles can be defined based on the elastic length $L_{0}$. The elastic length $L_{0}$ according to Tieze (1970) for a constant coefficient of subgrade reaction is defined by the stiffness of the pile $E I$, the coefficient of subgrade reaction of the soil $k_{S}$, and the diameter of the pile $D$ :

$$
L_{0}=\sqrt[4]{\frac{E \cdot I}{D \cdot k_{S}}}
$$

The elastic length $L_{0}$ defined in equation (10.8) differs from the elastic length $L$ defined in equation (10.3) by the factor $\sqrt{2}$. In Fig. 10.6 the lateral displacements $u$ at the pile tip normalized by the maximum lateral displacement $u_{\max }$ (pile head) are shown as a function of different embedded pile lengths $l$. The pile lengths $l$ taken from the surface of the soil are normalized by the elastic length $L_{0}$ from equation (10.8).

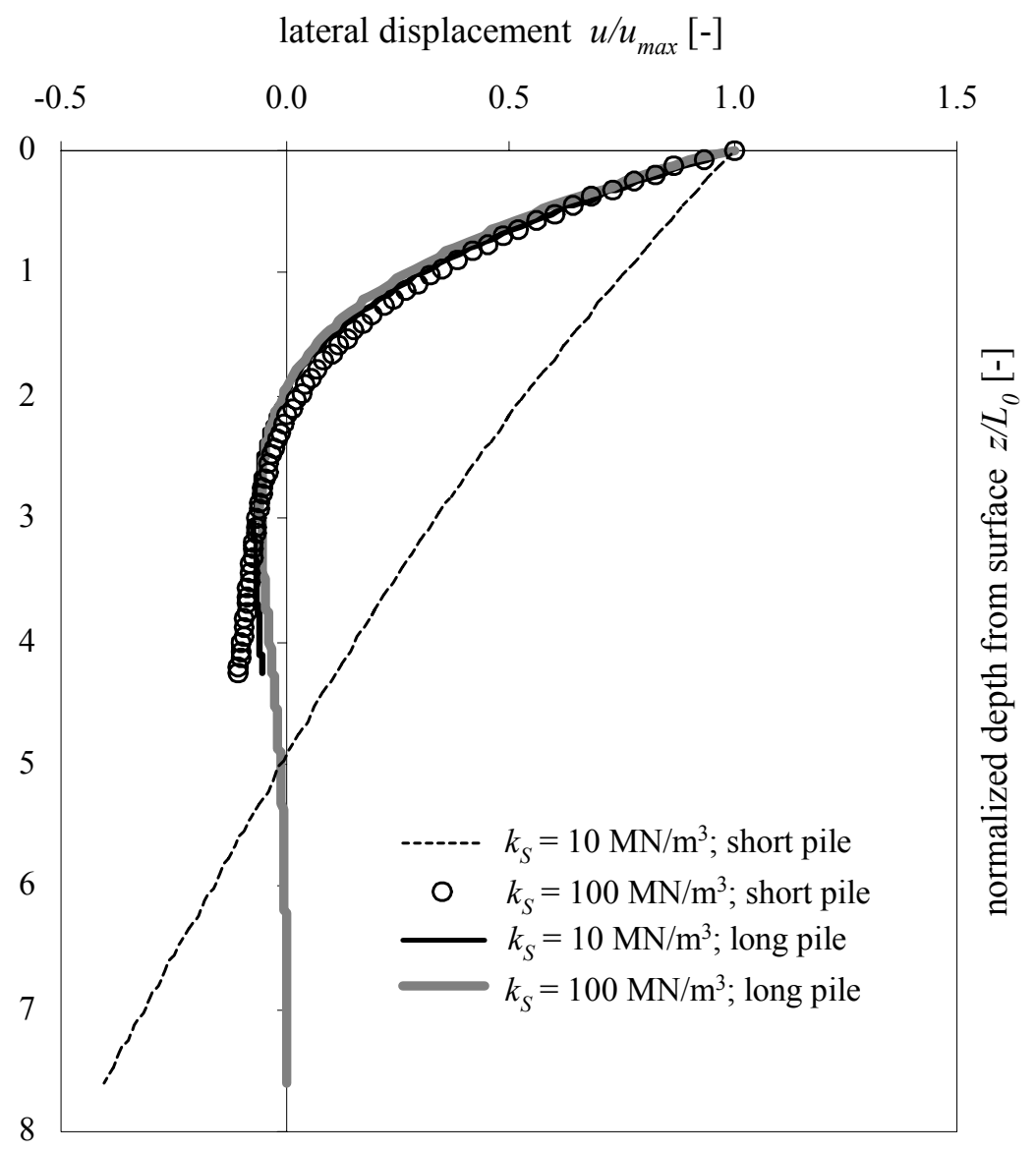

Fig. 10.5: Normalized deflections for laterally loaded piles

It can bee seen that for an embedded length $l$ larger than four times the elastic length $L_{0}$ the lateral displacements of the pile tip $u$ is smaller than $5 \%$ of the lateral displacement of the pile head (area shaded grey in Fig. 10.6). For longer piles the displacement drops below $1 \%$ of the maximum displacement. 
The tubes used as primary elements in combiwall constructions may have lengths of around $30 \mathrm{~m}$. For a coefficient of subgrade reaction of $10 \mathrm{MN} / \mathrm{m}^{3}$ and $100 \mathrm{MN} / \mathrm{m}^{3}$ for non-cohesive soils such as sands and the assumption of a constant coefficient of subgrade reaction over the length, the ratio of $l / L_{0}$ can be evaluated as 6.4 and 11.4 in a first rough approximation. Both values can be found within the grey shaded area of Fig. 10.6 .

Generally, the area of the load introduction or the boundaries is very critical for the buckling behaviour of loaded cylinders as it is very sensitive to imperfections. For the investigated tubular piles of combiwalls the influence of the area of the pile tip is rather small as the transferred loads are very small in this area for a pile subjected to lateral loads or moments. A possible unevenness of the support at the pile tip is therefore not as detrimental as for purely axially loaded cylinders. Still, the design of the tubular pile is based on the assessment in the area of the maximum bending moment and thus in combination with roughly the entire axial load, but adopting an approach which uses an assumption of global imperfections for all influencing factors seems rather conservative.

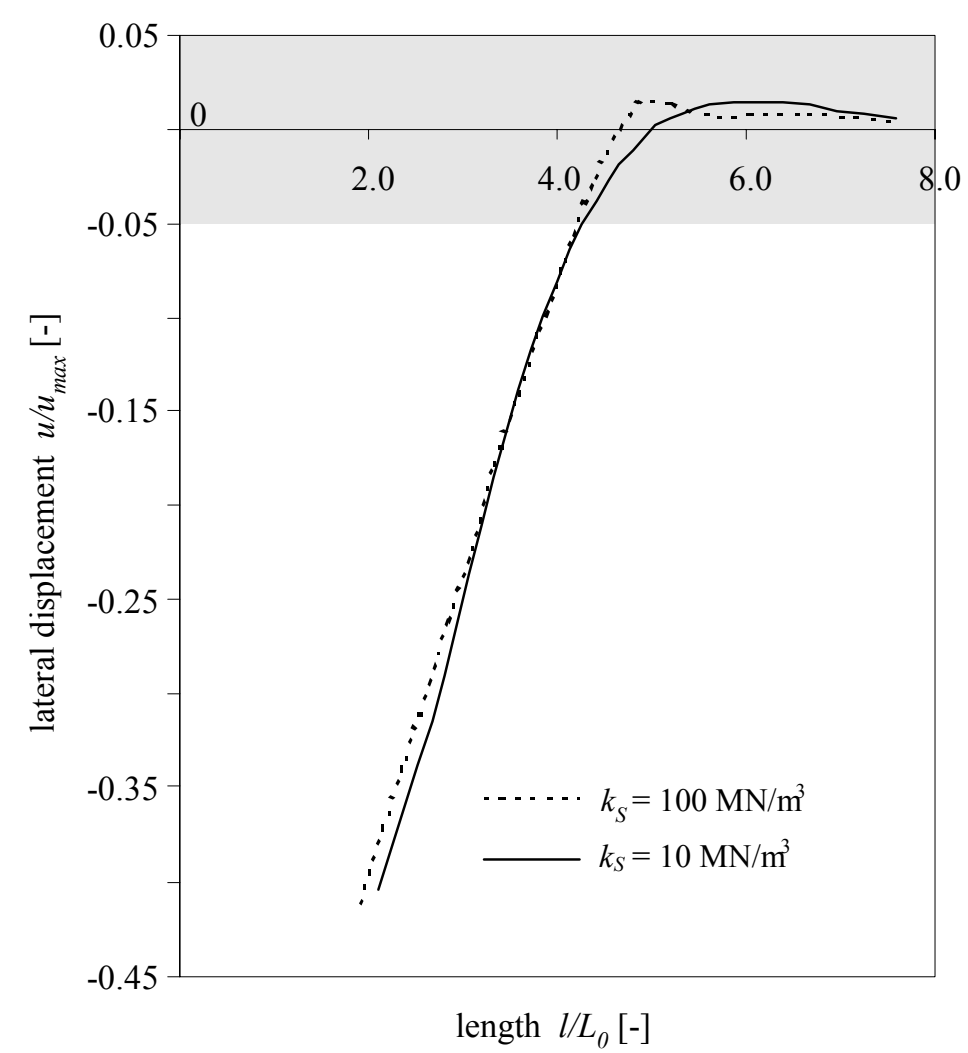

Fig. 10.6: Normalized lateral displacement $u$ at the pile toe as a function of the elastic length $L_{0}$

The previous solutions apply for so-called global fabrication tolerance classes which are assigned to the cylinder based on all different imperfection forms. Hence, possible unevenness at the boundaries is automatically taken into account when assessing the tube in the area of the maximum bending moment and as a consequence the carrying capacity is underestimated. In the area of the pile toe where unevenness of the support 
could influence the carrying capacity or the buckling resistance plugging may occur. Plugging takes place if the soil is prevented on purpose or not on purpose from entering the pile and leads to a load carrying behaviour similar to a close-ended pile. As the resistance of the end-bearing is increased by the plug resistance the influence of imperfections is even reduced. Therefore, the influence of imperfections on the buckling behaviour has to be investigated locally in two different segments and cannot be generalized for the entire tubular pile.

In a comparison considering the length effect, the results from the previous investigation regarding the horizontal deflection of a pile are studied. The different applied SRMs (constant, linear, parabolic) including a limiting passive earth pressure lead to similar maximum horizontal deflections for the investigated reference case. The validity of this aspect is further investigated regarding an additional length of piles. Hence, the comparison from 10.1 .1 for a $3 \mathrm{~m}$ pile embedded $2 \mathrm{~m}$ in sand is extended by keeping the system and only increasing the total length of the pile and by the same amount the embedded length of the pile.

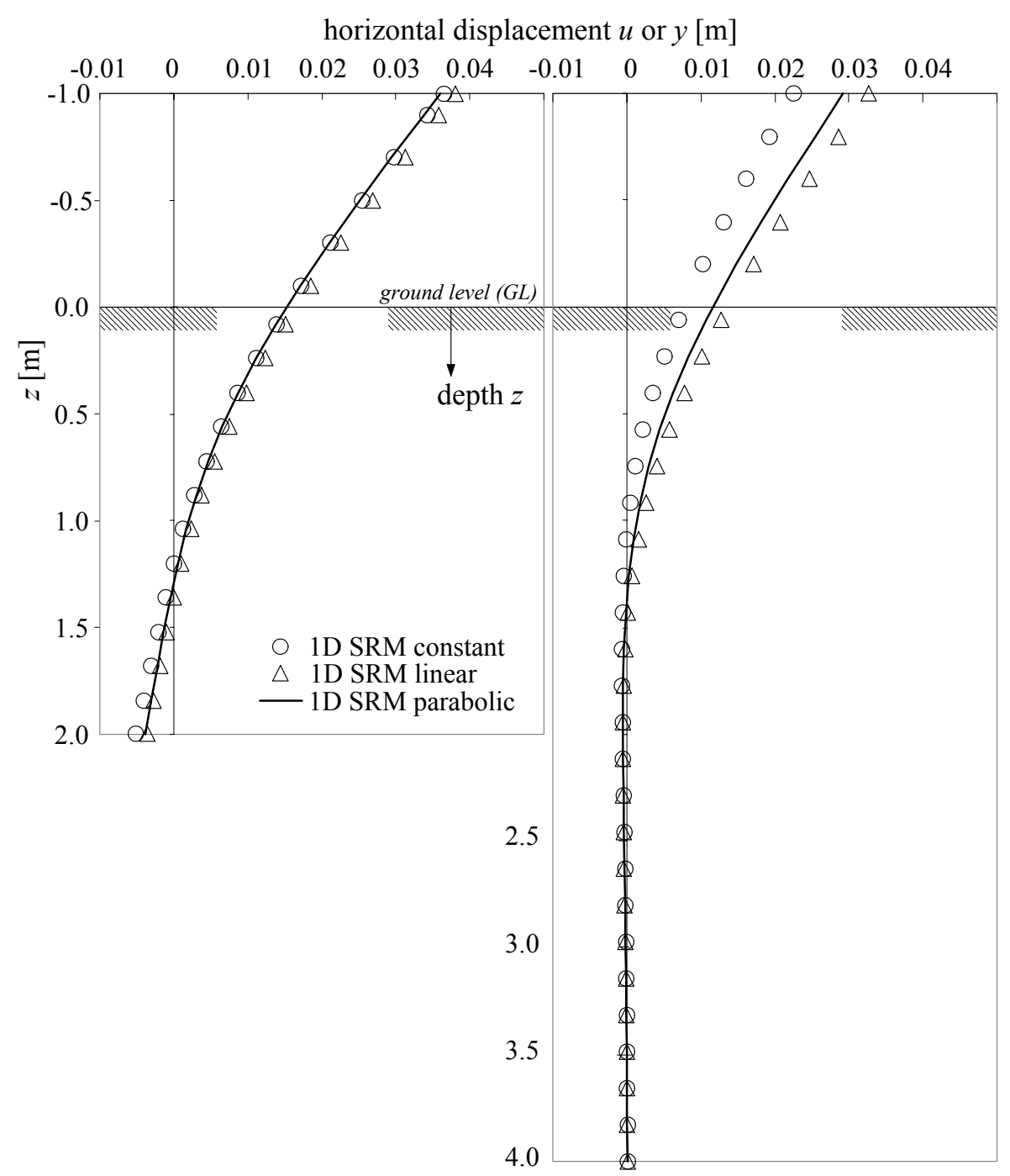

Fig. 10.7: Deflections for two different pile lengths using different SRMs 
In Fig. 10.7 the results for a pile embedded $4 \mathrm{~m}$ and $2 \mathrm{~m}$ in sand are depicted. The elastic length $L_{0}$ according to (10.8) for the reference pile is $890 \mathrm{~mm}$, leading to a normalized pile length for the $2 \mathrm{~m}$ and $4 \mathrm{~m}$ pile of $2.4 L_{0}$ and $4.8 L_{0}$, respectively. Thus, the $4 \mathrm{~m}$ pile can be classified as short pile and the $4 \mathrm{~m}$ pile as long pile according to their deflection behaviour and Fig. 10.6. For the short pile the differences between the different SRMs are marginal $( \pm 5 \%$ ) but for the long pile the differences in the maximum lateral deflection reaches a factor of 1.45 for the linear SRM in comparison to the constant SRM. For longer piles the system reacts stiffer independently on the SRM, leading to a fixed support at the pile tip. Therefore, the lateral behaviour is mainly governed by the stiffness of the upper soil layers, resulting is the largest deflections for the linear SRM and the smallest deflections for the constant SRM. For the shorter piles this aspect does not evolve since all layers still influence the deformation behaviour leading to a balancing of the upper and lower soil layers.

\subsection{Load transfer in the area of the maximum bending moment}

\subsubsection{Modelling}

For the investigation of the load transfer in the area of the maximum bending moment a different model is used. As the effect of an ovalization or flattening of the cross-section of the tube (Brazier-effect, 1927) and the influence of a pressure distribution varying over the circumference has to be taken into account, a more elaborated finite element model is used. The soil itself is represented by a three-dimensional continuum and the contact between soil and structure is modelled using an interaction solely active in compression allowing the contacting surfaces to separate under tension.

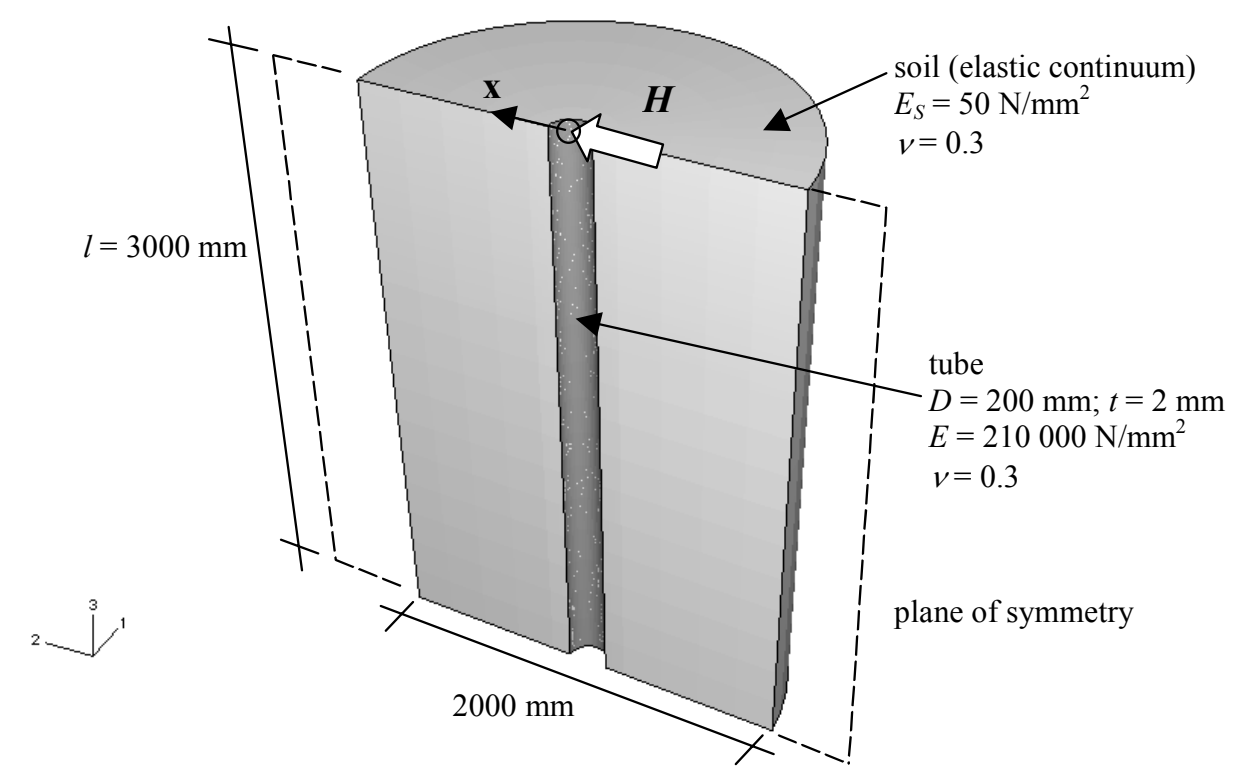

Fig. 10.8: System for investigation 
A vertically fixed steel tube $3 \mathrm{~m}$ of length entirely embedded in sand is investigated. For the first set of loading conditions the tube is empty on the inside. As the analyses are chosen to be modelled according to the experimental investigations, the geometry is based on the preliminary drafts for the experimental set-up. For the numerical analyses the tube's outer diameter of $200 \mathrm{~mm}$, a thickness of $2 \mathrm{~mm}$, and a length of $3000 \mathrm{~mm}$ are modelled using bilinear continuum shell elements. Fig. 10.8 illustrates the system and the basic material properties of both the soil and the tube. At the bottom the tube is restrained vertically. The cylinder of soil is constrained vertically at the bottom and radially on the outside while symmetrical boundary conditions are applied to the longitudinal edges. The lateral load $H=20 \mathrm{kN}$ is introduced at the pile tip without preventing the tip to rotate. The numerical analysis takes account of large deformations.

The soil is modelled simplified as a purely elastic continuum with a Young's modulus of $50 \mathrm{~N} / \mathrm{mm}^{2}$ and a Poisson ratio of 0.3 , which is not really sufficient for describing real soil behaviour. Furthermore, the model neglects the self-weight of the soil, which would lead to an interacting, initial pressure distribution on the outside of the tube. The analyses are performed with an elastic continuum as no absolute figures for stresses and displacements are of interest but the general behaviour. Moreover, in this context a soil with a rather high stiffness is used for obtaining information on the ovalization in the area of the maximum bending moment and the corresponding pressure and stress distributions, since these phenomena are more distinct for a stiffer soil. In this segment the focus is set on the longitudinal stress distribution and any deviations over the circumference and thus the load transfer. These results shall give information about the buckling resistance with respect to design codes.

\subsubsection{Unfilled tube}

First the system as shown in Fig. 10.8 is investigated as unfilled tube. Fig. 10.9 shows the deformed unfilled tube as exploded view. It is clear that the tube deflects and exhibits a flattening of the cross-section in the area of the maximum bending moment. In contrast to the analysis using an elastic foundation for modelling the soil (Fig. 10.2) the tube does not exhibit a symmetric stress distribution with reference to the centre line. The varying membrane stresses arise due to the fact that the interaction between soil and structure cannot transfer tension, and geometrical non-linearities have been taken into account in the analyses. The axial membrane stresses in the tube are depicted in Fig. 10.10 for the outer fibres on the tension and compression side. Additionally, the analytical solution for the beam on an elastic foundation according to section 10.1.1 is plotted as a reference in Fig. 10.10. For this comparison the relationship between the Young's modulus $E_{S}$ of the soil and the coefficient of subgrade reaction $k_{S}$ is taken according to equation (7.4) with a coefficient $C=1$. For a Young's modulus $E_{S}$ of $50 \mathrm{~N} / \mathrm{mm}^{2}$ and a diameter $D$ of $200 \mathrm{~mm}$ the coefficient of subgrade reaction $k_{S}$ can be calculated as $252 \mathrm{MN} / \mathrm{m}^{3}$. 


$$
k_{S}=C \cdot \frac{E}{D}
$$

It can be seen that the analyses with no adhesion between the contacting surfaces gives rise to slightly higher stresses for the compression side than given by the analytical solution. Moreover, it is clear that the assumption of $C=1$ for equation (7.4) seems to give close agreement with more elaborate numerical analyses.

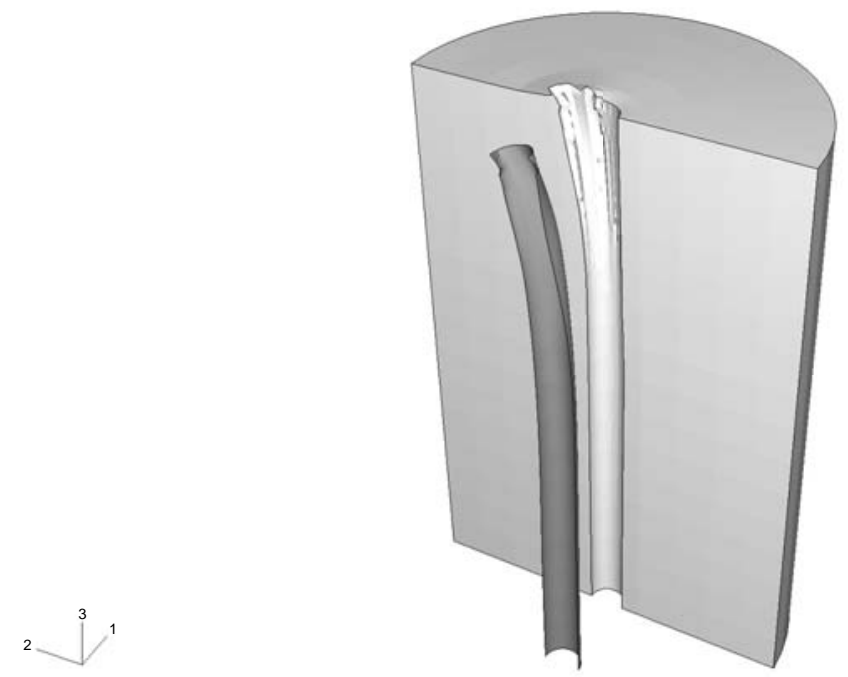

Fig. 10.9: Deformed unfilled tube at a load of $20 \mathrm{kN}$ (scaled with factor 100) as exploded view

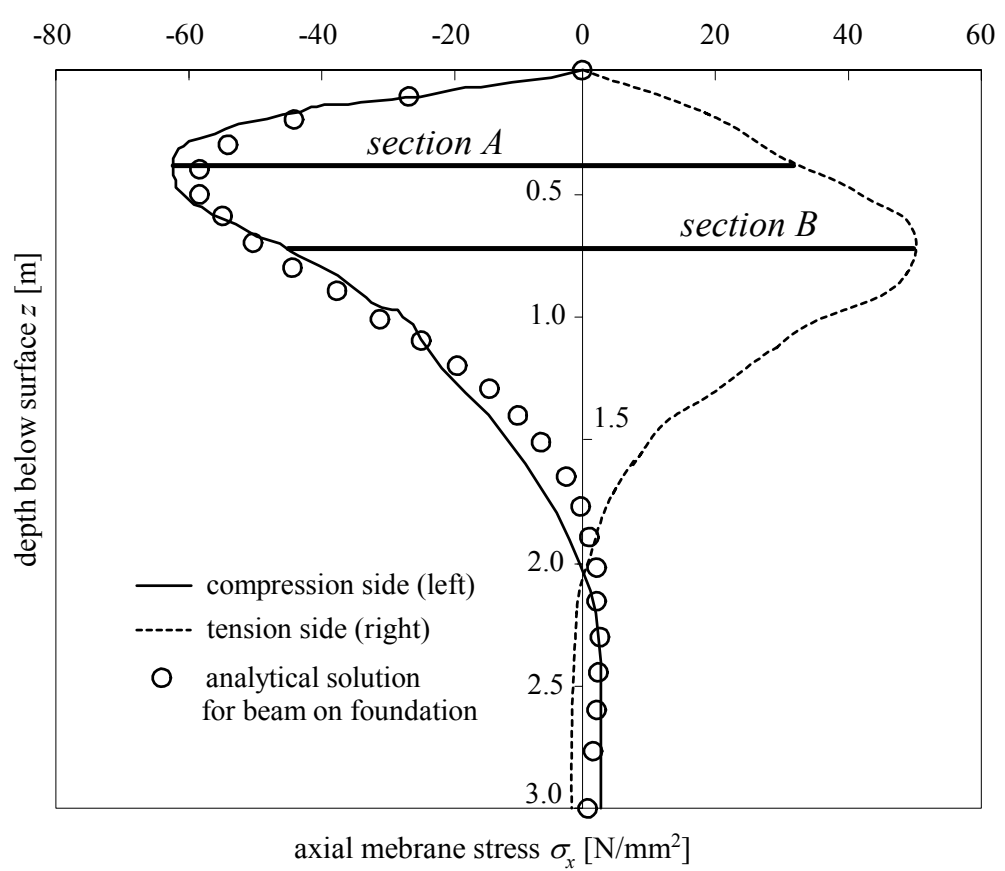

Fig. 10.10: Axial membrane stress distribution over the length of the unfilled tube for $H=20 \mathrm{kN}$

The deformations of the cross-section are compared in the sections of the maximum stresses for compression and tension (section A and B from Fig. 10.10). The largest deformations arise in section A (Fig. 10.11). The cross-section is flattened, and on the 
tension side (negative $\mathrm{x}$-axis) an inward curvature is formed. On the tension side a gap evolves between the to interacting surfaces. The cross-section in section B does not deform as much as in section A, but deforms similarly regarding the shape.

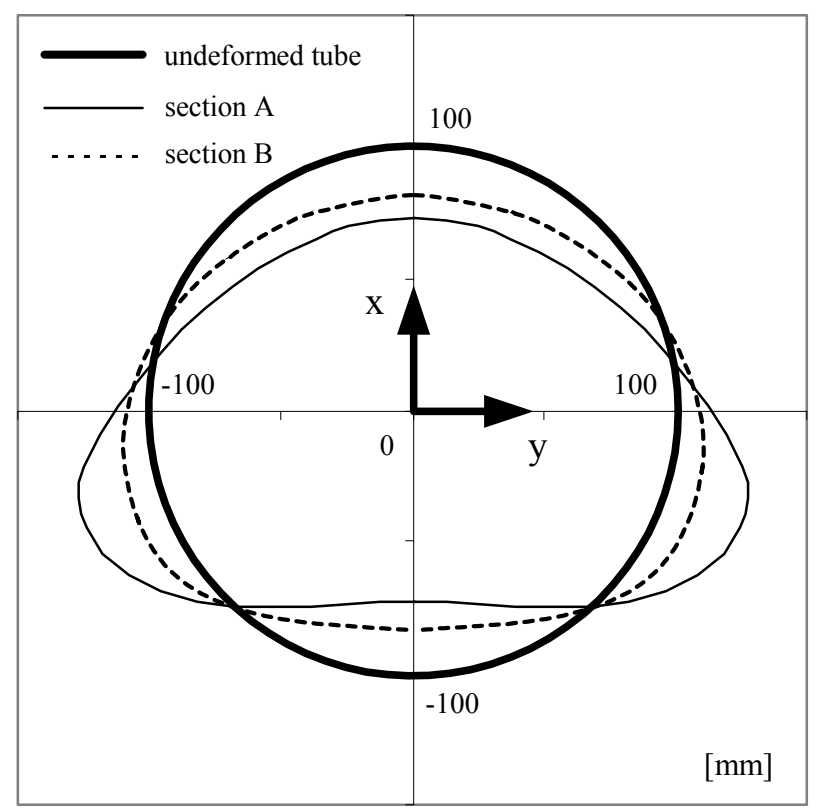

Fig. 10.11: Deformed cross-sections at section $A$ and B for the unfilled tube (scaled with factor 100) for $H=20 \mathrm{kN}$

\subsubsection{Filled tube}

In the second step the tube is filled with sand. Similar to the previous case the sand on the inside of the tube is modelled as continuum with a non-adhesion surface-to-surface contact. Fig. 10.12 depicts a deformed plot of the filled tube as exploded view. The deflection of the filled tube is similar to the unfilled tube.

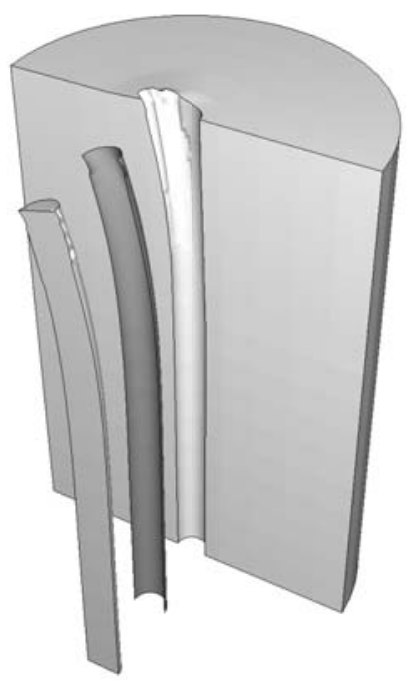

Fig. 10.12: Deformed filled tube at a load of $20 \mathrm{kN}$ (scaled with factor 100) as exploded view 
The maximum lateral displacement to be found at the pile head reduces due to the increased stiffness arising through the filling of the tube by around $2 \%$. In comparison with the previous case it can be seen that the flattening of the tube in the area of the maximum bending moment is not as significant for the filled tube as for the unfilled tube. In Fig. 10.13 the axial membrane stresses for the outer fibres of the tube are plotted over the length. Comparing Fig. 10.13 with Fig. 10.10 it is clear that the stresses are shifted to the tension side. As a consequence the maximum compressive stresses reduce by $6 \%$ and the maximum tensile stresses increase by around $7 \%$.

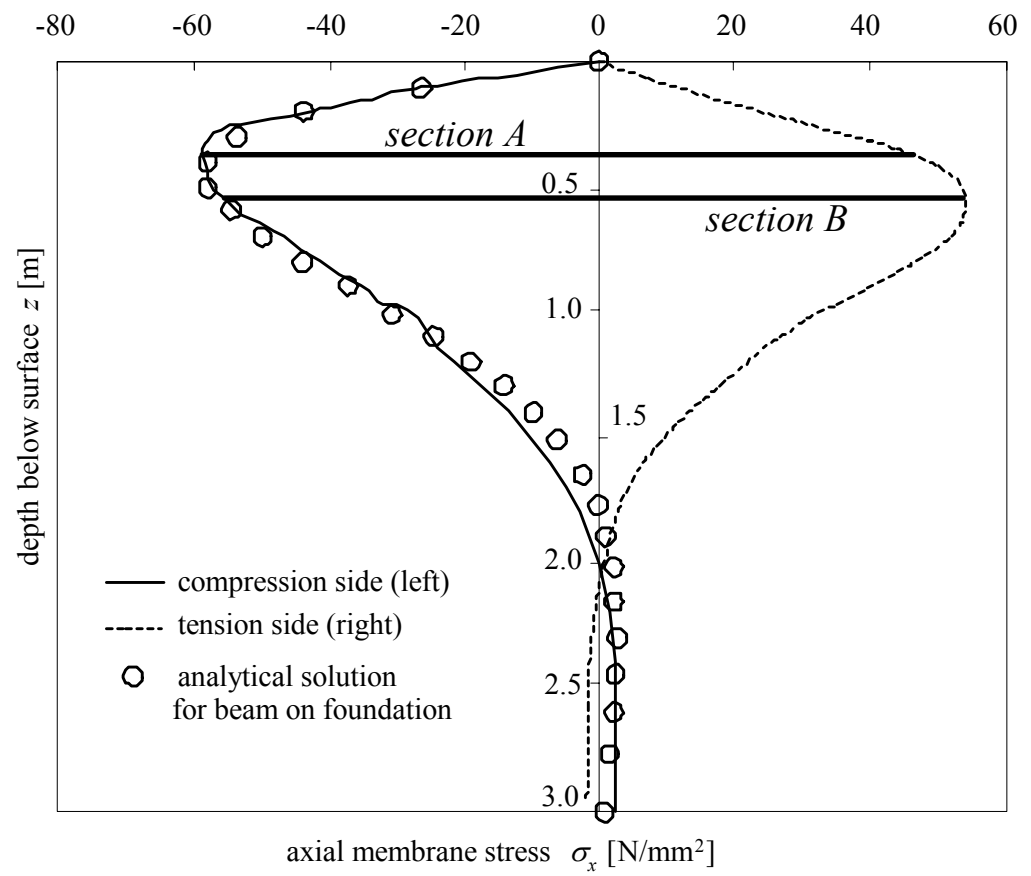

Fig. 10.13: Axial membrane stress distribution over the length of the filled tube for $H=20 \mathrm{kN}$

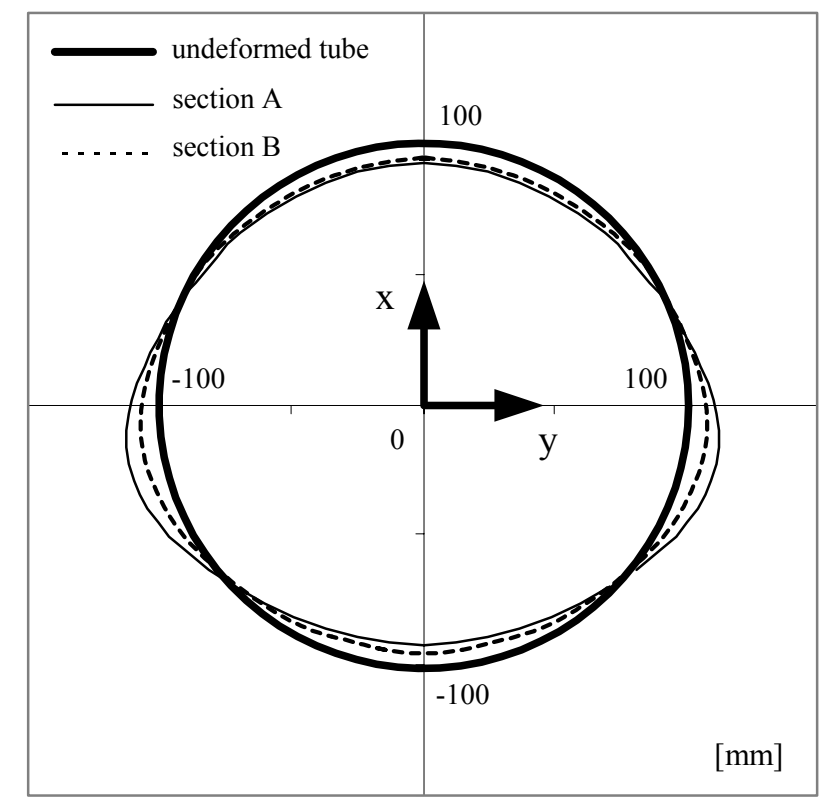

Fig. 10.14: Deformed cross-sections at section A and B for the filled tube (scaled with factor 100) for $H=20 \mathrm{kN}$ 
Due to the filling of the tube the structure behaves more like a compound-structure which results in a smaller distance between the stress maxima and a balancing of the membrane stresses. The section of the maximum tensile stress converges to the section of the maximum compressive stress, as for the case of the beam on an elastic foundation the sections coincide. Because of the sand on the inside of the tube the structure is stiffer and the deformations decrease significantly (Fig. 10.11 and Fig. 10.14). Still, the shape of the deformation in form of ovalization remains the same.

\subsubsection{Longitudinal bending stresses}

The analysis of a uniformly loaded tube on an elastic foundation calculated according to beam theory and not taking large displacements into account leads for the elastic case to a linear stress distribution over the cross-section. In Fig. 10.15 the axial membrane stress distributions for sections A and B of the filled and unfilled tube are plotted in comparison to the analytical solution for the beam theory. The numerical analyses take account of large displacements by performing geometrically non-linear analyses. Especially for the case of the unfilled tube the ovalization gives rise to non-linear axial stress distributions over the cross-section. Whereas the axial stresses in the compression zone of the tube seem to remain more or less linear, the stress distribution in the tension zone deviate notably from a linear distribution. v. Kármán (1889 and 1910) already showed early that due to flattening of the cross-section of bent tubes loaded with bending moments the maximum stresses do not appear at the outermost fibre. Comparatively, the results can be used for straight tubes, for which the equilibrium is set up for the deformed system.

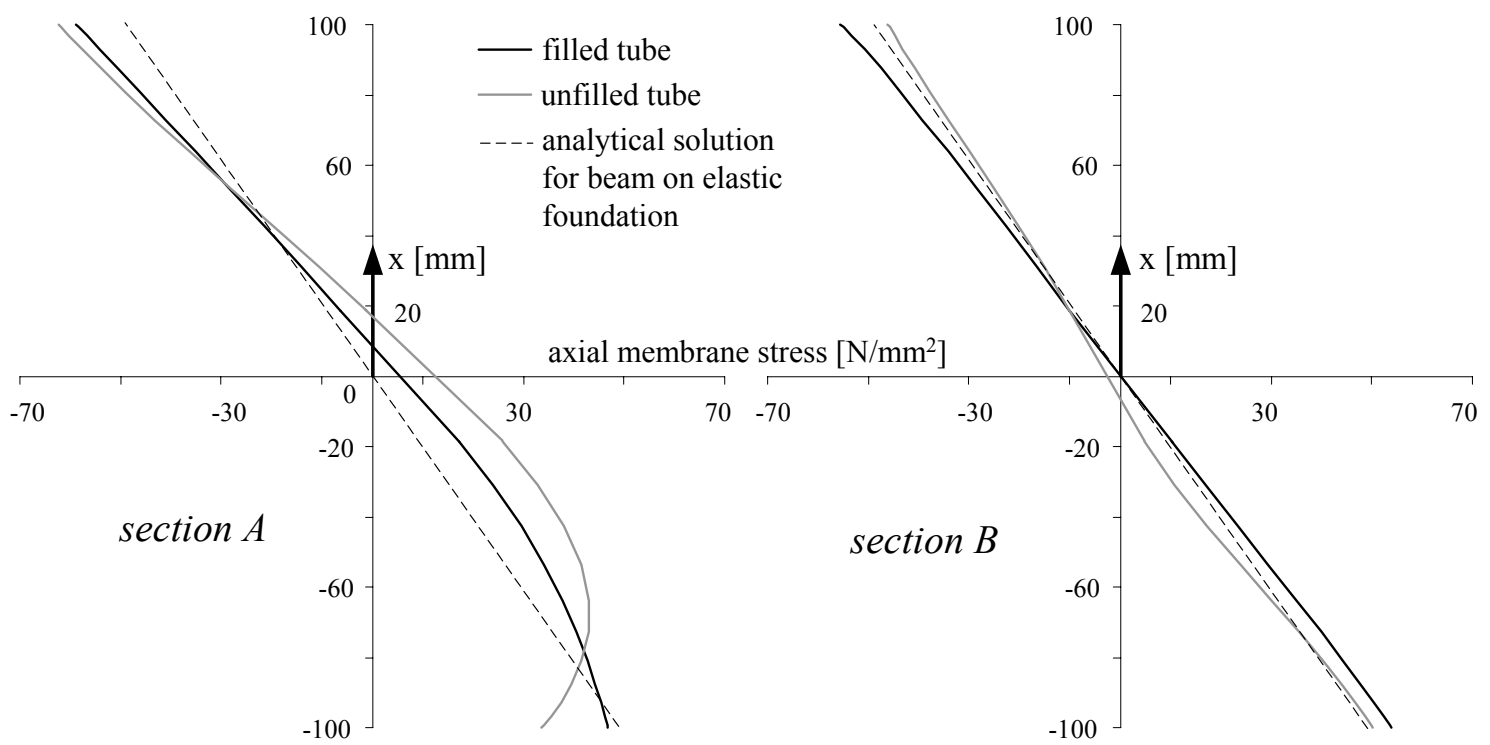

Fig. 10.15: Axial membrane stress distribution over the cross-section

The ovalization as depicted in Fig. 10.11 and Fig. 10.14 results in flattened parts especially in the tension zone of the tube. For the unfilled tube in section A the deformation of the cross-section lead to a re-distribution of the stresses from the outside 
towards the centre line (Fig. 10.15) and to maximum tensile stresses which appear not at the outermost fibre. The by far smaller deformations in the compression zone cause nearly no change in the linear stress distribution.

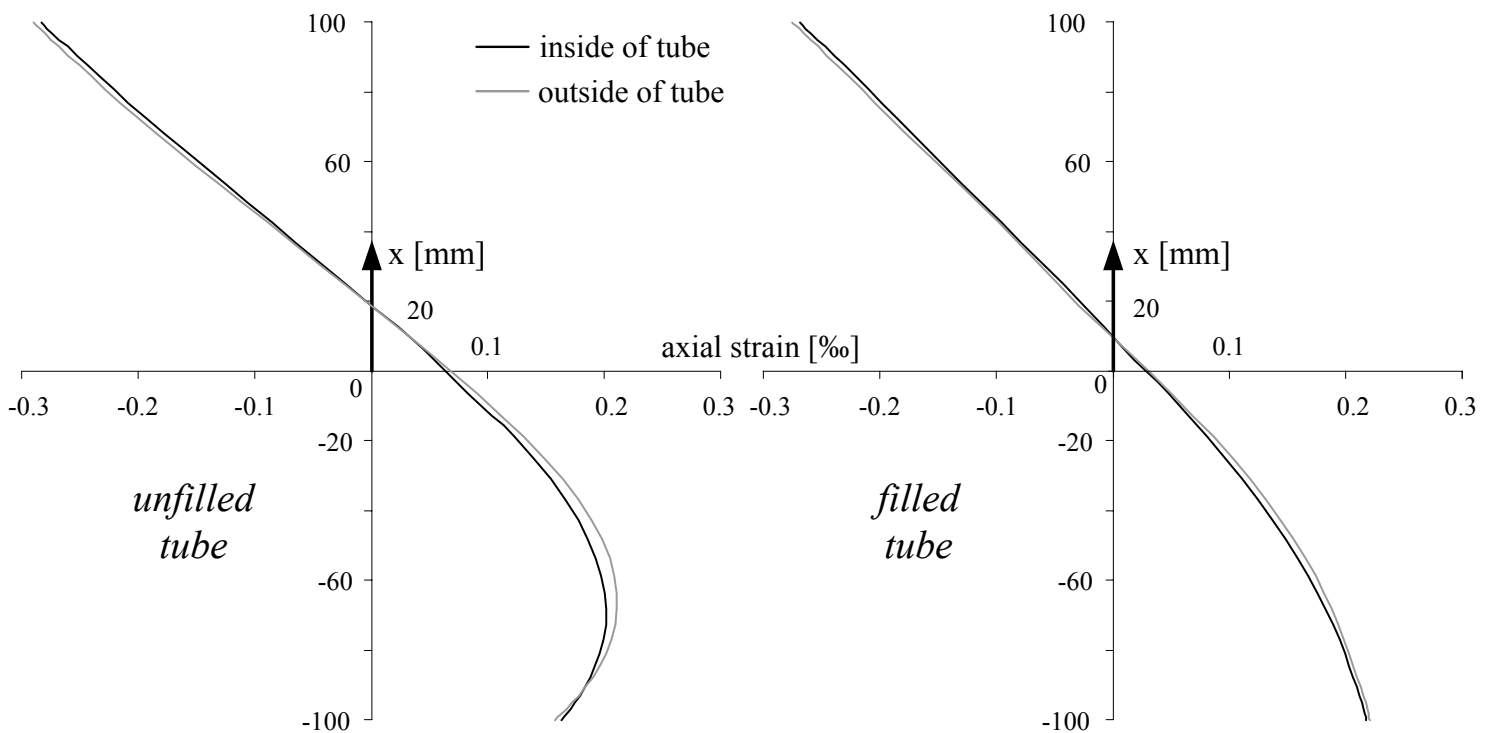

Fig. 10.16: Axial strain distribution over the cross-section on the in-and outside for section A

In view of the local deformations of the shell the forces are not only transferred by membrane stresses but also partially by bending in the plane of the shell. The stress component from the in-plane bending is of major importance for the experimental investigation because the measurement of the stresses is performed by strain gauges which are to be attached to the outside of the tube. Thus, the strains from the numerical analyses are additionally compared for the outermost fibre of the shell elements. Fig. 10.16 shows the axial or longitudinal strains on the in- and outside of the tube for section A. The strain distributions on the in- and outside of the filled tube are comparable and similar in shape to the axial membrane stresses according to Fig. 10.15. The strains on the in- and outside of the filled tube deviate by less than $2 \%$ from each other.

a)

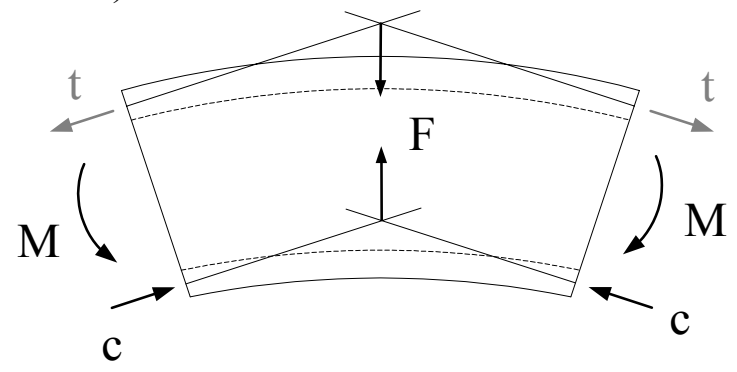

b)

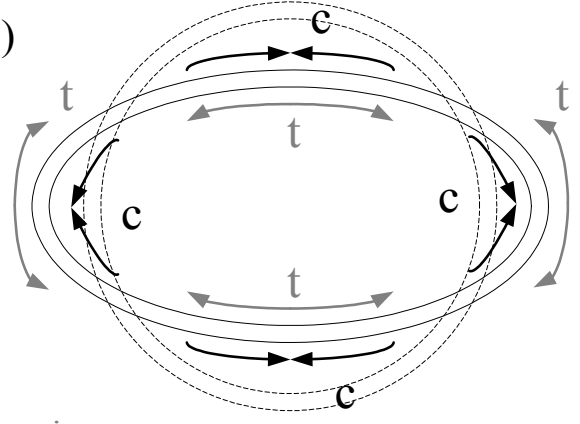

$\mathrm{t}=$ tension

$\mathrm{c}=$ compression

Fig. 10.17: Secondary effects from longitudinal bending. a) radial resultant $F$ in longitudinal section; b) ovalization with secondary in-plane bending over cross-section 
For the compression zone of the unfilled tube the strains on the in- and outside of the tube differ by up to $5 \%$ in the area of the maximum strain. It is clear that the higher strains on the outside of the tube cannot only come from the larger distance to the centre line of gravity (effect about $2 \%$ ) but also must be caused by another effect. Further away from the centre line of gravity the strain distribution inverts and the maximum tensile strain appear on the inside of the tube. In the area of large forces on the tension side of the unfilled tube the loading results in a change of curvature of the deformed cross-section. Due to this change the position of the maximum stress changes between in- and outside of the tube. In the areas with smaller strains, the strains between in- and outside differ only slightly (around $2 \%$ ). For the deformed shape the longitudinal bending gives rise to a force F directed inwards (Fig. 10.17a). The forces F compress and flatten the tube, and lead to the ovalization of the structure.

Furthermore, as the geometry changes the moment of inertia of the cross section is reduced and therefore the stresses increase. Still, this effect is in comparison of minor importance. Based on these effects the higher strains on the outside of the unfilled tube according to Fig. 10.16 can be explained.

\subsubsection{Circumferential bending stresses}

The flattening of the tube causes circumferential in-plane bending stresses which according to their position on the in- or outside result in tensile or compressive circumferential stresses (Fig. 10.17b). Again, the influence of the circumferential strain distribution on the outside of the tube is of interest as in the experiment these components are measured with strain gauges.

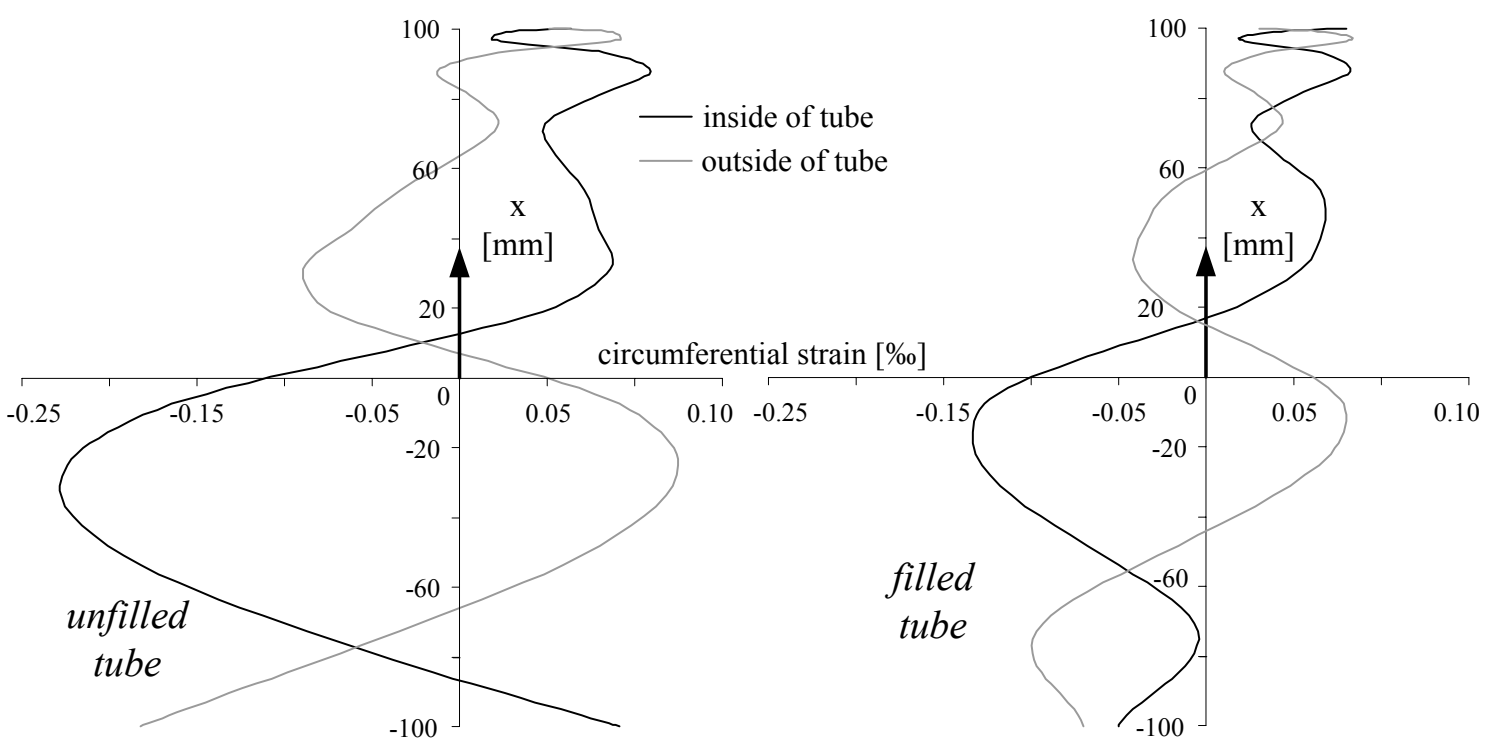

Fig. 10.18: Circumferential strain distribution over the cross-section on the in-and outside for section A

In Fig. 10.18 the circumferential strain distribution over the cross-section on the in- and outside for section A is shown. The circumferential strains for the unfilled tube exceed the maximum strains for the filled tube by $85 \%$. The circumferential strains correspond 
to the deformed cross-sections (Fig. 10.11 and Fig. 10.14). The compression-tensioncompression pattern for the outer fibre of the tube as shown in the sketch in Fig. 10.17b can be easily identified in Fig. 10.18. The maximum strains evolve at the sides of the tube. Likewise, on the tension side for the case of the unfilled tube large strain components appear due to the additional change in curvature in the cross-section at $z=-100 \mathrm{~mm}$. Evidently, based on the relatively symmetric strain distribution regarding the ordinate on the in- and outside, it can be concluded that only small circumferential membrane stresses are present. The maximum circumferential strains reach in the investigated case up to $50 \%$ of the axial strains in section $\mathrm{A}$.

These results are important for the strain measurement in the experiments. Generally, in an experimental test set-up strains are measured in different directions using strain gauges which are then evaluated according to stress components of interest. The membrane stresses of the tube are the relevant component for further studies. Due to the geometry of the object it will not be possible to measure stresses on the inside of the tube. This fact poses the question to what extent the measurement of the strains is influenced by the secondary in-plane bending around the circumference. A strain measurement on the outside of the tube at the maximum bending moment in longitudinal and circumferential direction can deliver the strains $\varepsilon_{l, o}$ and $\varepsilon_{c, o}$.

Based on the assumption of a homogenous isotropic body and applying the Hook's law with a constant Young's modulus and a constant Poisson ratio the axial stress on the outside of the tube can be calculated with the following equation:

$$
\sigma_{x, o}=\frac{E}{1-\mu^{2}}\left(\varepsilon_{l, o}+\mu \cdot \varepsilon_{c, o}\right)
$$

The equation (10.10) is valid for the assumption of a plane stress condition which is acceptable for the investigated case as the stresses in direction of the thickness of the tube wall is negligible. The equation (10.10) can be written identically for the centre or middle of the tube wall, i.e. for the membrane stresses:

$$
\sigma_{x}=\frac{E}{1-\mu^{2}}\left(\varepsilon_{l, m}+\mu \cdot \varepsilon_{c, m}\right)
$$

The circumferential strains $\varepsilon_{c, m}$ in the centre of the tube wall are negligible as deformation is prevented geometrically and the effect of the secondary bending only influences the surfaces of the tube. As the curvature $\kappa_{x}$ in the longitudinal direction is very small it can be assumed that the component of the longitudinal strains $\varepsilon_{l, o, b e n d i n g}$ arising from global bending is zero. This conclusion is drawn according to the assumptions made for the extended technical bending theory (Schardt, 1989) which set the shear strains $\gamma_{x \varphi}$ and the strains in the circumferential direction $\varepsilon_{\varphi}$ to zero. Based on this approximation it can be stated that:

$$
\varepsilon_{l, o} \approx \varepsilon_{l, m}
$$

and the equation (10.11) can be written as: 


$$
\sigma_{x} \approx \frac{E}{1-\mu^{2}} \varepsilon_{l, o}
$$

In Fig. 10.19 the axial membrane stresses for section A are plotted evaluated according to equation (10.13).

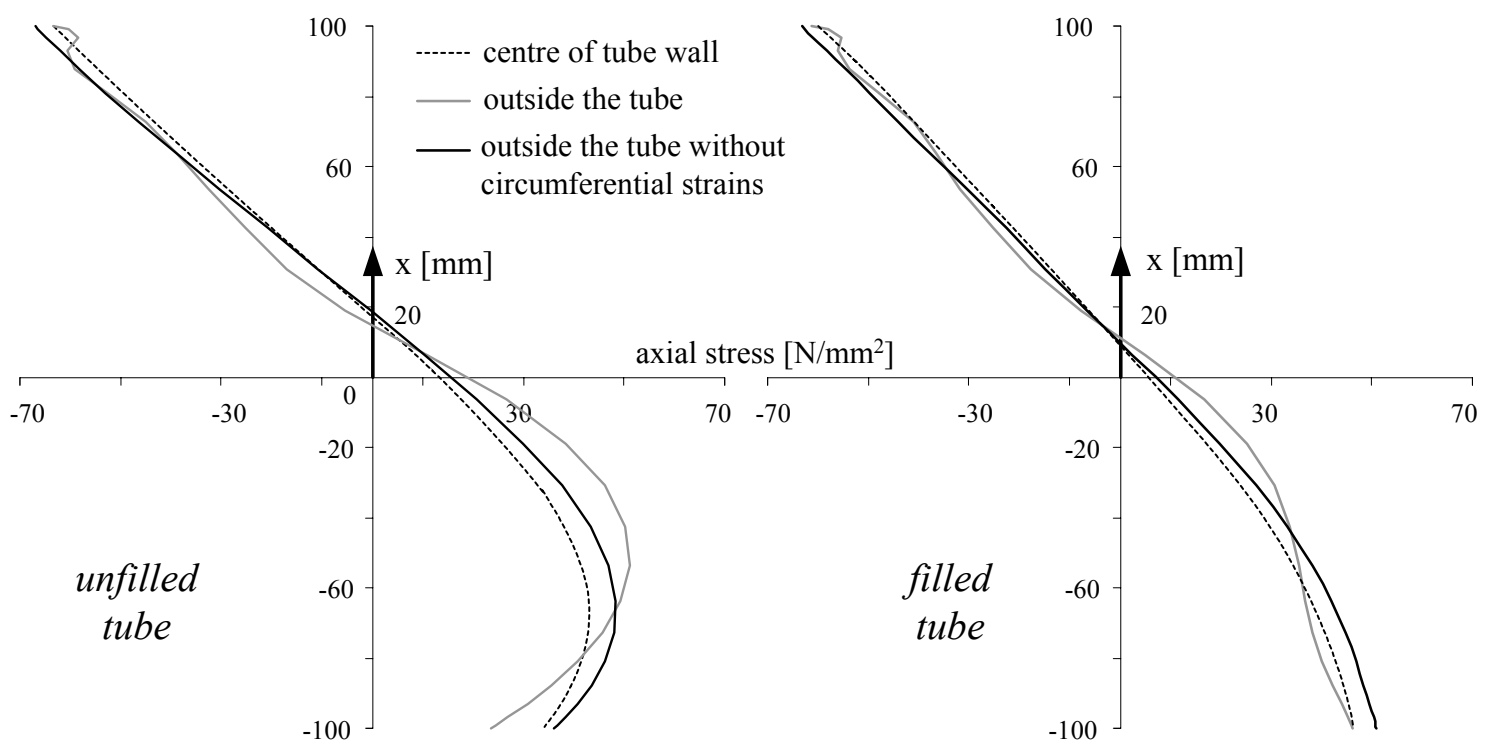

Fig. 10.19: Longitudinal bending stress distribution over the cross-section on the outside and the axial membrane stress for section A calculated from strains according to (10.13)

Reading the longitudinal strain $\varepsilon_{l, o}$ and the circumferential strain $\varepsilon_{c, o}$ at section A for the outside of the tube from the numerical analyses allows to calculated the axial stresses $\sigma_{x, o}$ at the outside according to equation (10.10). Fig. 10.19 depicts the results from the calculation corresponding to the stresses from the numerical analyses as shown in Fig. 10.16. The results differ in the area of the maximum compression by about $10 \%$ and in the area of the maximum tension by about $30 \%$ from the axial membrane stress $\sigma_{x}$. The intention is to obtain a good approximation for the axial membrane stresses $\sigma_{x}$ with the measured strains $\varepsilon_{l, o}$ and $\varepsilon_{c, o}$. The approach given in equation (10.13) leads to a good approximation with a constant deviation of around $10 \%$.

\subsubsection{Effect of friction}

For the investigation to what extent additional friction forces arising between soil and structure may influence the load transfer behaviour of the lateral load, the studied cases of the filled and unfilled laterally loaded pile are further investigated. Generally, the friction forces are of major interest when investigating axially loaded piles as the external forces have to be transferred to the soil by the shaft.

Simplified the static coefficient of friction is set equal to the kinetic coefficient of friction and hereafter only called coefficient of friction. As indication for the coefficient of friction $\mu_{\text {fric }}$ the tangent of the angle of wall friction $\delta$ for steel piles is used. Established values for the angle of wall friction $\delta$ of sand in interaction with steel vary 
between $10^{\circ}$ and $25^{\circ}$ (e.g. Bowles, 1992; Broms, 1981) depending on the consistency of the sand. As rough estimate a static and kinetic coefficient of friction $\mu=0.3$ (corresponds to $\delta=16.7^{\circ}$ ) is chosen independent of the contact pressure and the relative displacements.

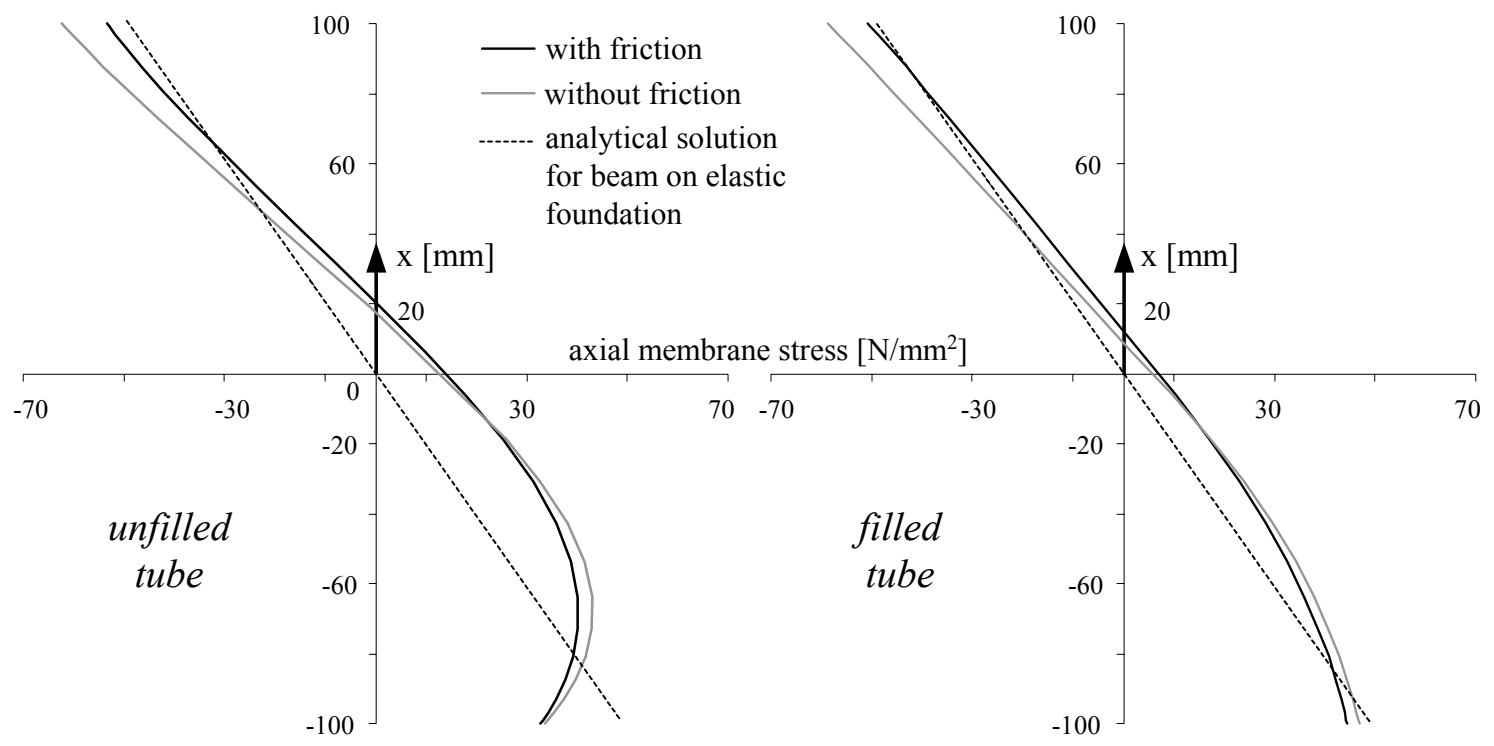

Fig. 10.20: Axial membrane stress distribution over the cross-section including friction

Applying a friction to the shaft of the tube decreases the maximum lateral displacements for both the filled and unfilled tube by around $11 \%$. The possibility of transferring parts of the loads through shear forces results in a more compound-structure-like behaviour, i.e. an increase of stiffness for the tube. Fig. 10.20 depicts the axial membrane stresses arising from longitudinal bending for the section $\mathrm{A}$ of the filled and unfilled tube with and without considering a coefficient of friction. Including friction in this example leads to a reduction of the maximum axial membrane stresses by about $15 \%$ for both cases. The stress distributions tend towards a linear stress distribution. The circumferential bending stresses decrease while considering a coefficient of friction for both investigated cases by $11 \%$. Evidently, parts of the lateral force and the resulting moment are transferred to the soil by shear stresses and hence lead to a reduction of the stress components in the tube. As the reduction of the stresses does not differ between the unfilled and the filled tube, it can be concluded that the sand core within the filled tube does not contribute any considerable stress reduction when taking friction into account. 


\section{Boundary conditions and loads - external pressure}

\subsection{General}

The design procedure (chapter 4) highlighted several conservative assumptions which have to be made for assessing of the structure against the different stress components. These aspects influence especially the limit state buckling design against circumferential compression.

In the example calculation the circumferential compressive stresses for the buckling verification of the tubes subjected to external pressure were calculated and compared with the circumferential buckling resistance $\sigma_{\theta, R d}$ according to EN 1993-1-6 in chapter 4. Based on EN 1993-1-6 a local buckling verification was performed at the position of the maximum circumferential compressive membrane stresses. Fig. 11.1 depicts the simplified pressure distribution along the circumference for the region of the tubular pile tip.

earth pressure

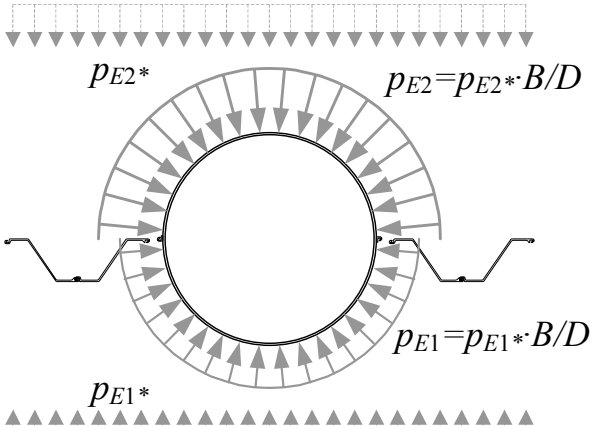

water pressure

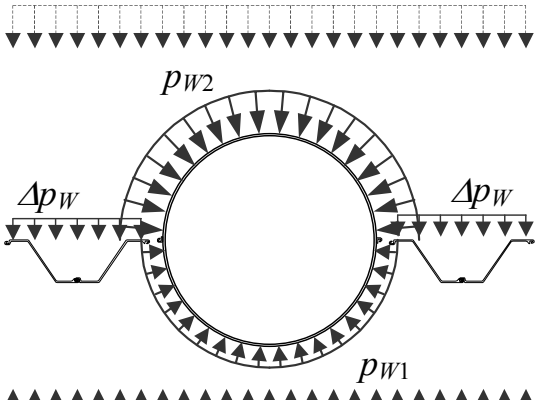

internal pressure

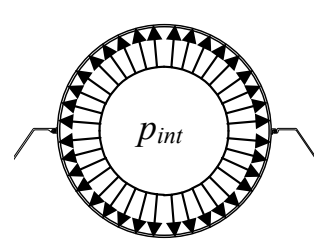

Fig. 11.1: Pressure distribution on combiwall from water and soil

The calculated circumferential compressive stresses exceed the circumferential buckling resistance $\sigma_{\theta, R d}$ significantly (factor 3.0, chapter 4 ). The assessment procedure was based on the following simplified assumptions:

- $\quad$ choice of the shell's length of $24 \mathrm{~m}$ (pile tip to anchor) for evaluating the circumferential buckling resistance $\sigma_{\theta, R d}$

- $\quad$ boundary conditions BC1-BC3 (clamped - free) according to EN 1993-1-6 for evaluating the circumferential buckling resistance $\sigma_{\theta, R d}$

- $\quad$ maximum value of the non-uniform external pressure distribution assumed constant in longitudinal direction 
- $\quad$ maximum value of the non-uniform external pressure distribution constant in circumferential direction

This approach for the combiwall example is defined as reference or initial case in the following. From these approximations a very conservative design arises, since in reality the circumferential compressive stresses only occur at one position along the length of the tube. In the calculation for the circumferential buckling resistance $\sigma_{\theta, R d}$ according to EN 1993-1-6 a constant external pressure along the entire length of shell was imposed. In this section, based on simplified assumptions and conditions in real structures the resistance and the loading, are studied and compared on the basis of the reference case.

\subsection{Investigation of the shell's resistance for external pressure}

In the stress based buckling verification according to EN 1993-1-6 neither varying external pressure distributions in longitudinal nor in circumferential direction are covered sufficiently for the investigated problem. In Fig. 11.1 and Fig. 11.2 the varying pressure distribution on the tube wall is shown for the longitudinal and the circumferential direction. In EN 1993-5 for the assessment against circumferential buckling it is annotated that in case of a non-uniform pressure distribution over the circumference the circumferential stresses should be calculated based on a constant pressure distribution using the maximum appearing pressure. EN 1993-1-6 allows to user to calculate an equivalent constant pressure for cylinders subjected to non-uniform pressure distributions from wind loading. Nevertheless, this approach cannot be transferred to the investigated case due to the significantly different shape of pressure distribution, e.g. wind loading involves suction. Thus, for a cylinder subjected to a constant pressure on half of the cross-section and unloaded on the other half of the cross-section, the assessment should be performed assuming a constant pressure distribution over the entire cross-section. The boundary conditions and the influence of length appearing in reality can hardly be adapted to the cases given in Eurocode EN 1993-1-6.

The critical circumferential buckling stress is calculated for the reference case from chapter 4 . Based on this value an investigation of the influence of boundary conditions and varying external pressure distribution on the carrying behaviour of the tubular piles is performed. The aim is to obtain differentiated results for the carrying behaviour of tubular piles by applying more detailed modelling of the system (boundary conditions and loading). The procedure is based on the evaluation of the critical circumferential buckling stress in order to limit the complexity and to facilitate the interpretation of the findings.

The pressure distributions according to Fig. 11.2 are the reactions calculated from an elasto-plastic analysis with the program RIDO taking all loadings into account (Annex B). The modelling in ABAQUS was performed by using half of the tube (cross section) over the entire length of $30 \mathrm{~m}$. Symmetric boundary conditions are imposed to the 
longitudinal edges. Additionally, at the top edge of the tube all three translational degrees of freedom are constraint. At the depth of the anchor the nodes are coupled in order to maintain the shape of the cross-section and supported by a spring modelling the anchor stiffness. The anchor stiffness is chosen in the magnitude of the input for the analyses performed with RIDO. The tubular pile is loaded applying the non-uniform pressure distribution in longitudinal and circumferential direction as shown in Fig. 11.1 and Fig. 11.2. The loading not only results in circumferential compressive stresses but also bending moments leading to axial stresses. Nevertheless, this stress component is not relevant for the buckling failure in the investigated case and thus is neglected in the following considerations.

The loading depicted in Fig. 11.2 corresponds to a load factor LF of 1 . The loading by the non-uniform pressure distribution and the stiffness of the anchor from the RIDO calculations had to be adapted slightly for the three-dimensional ABAQUS analyses in order to obtain equilibrium with equivalent displacements and force resultants. A Young's modulus of $210000 \mathrm{~N} / \mathrm{mm}^{2}$ and a Poisson ratio of 0.3 is used for the analyses.

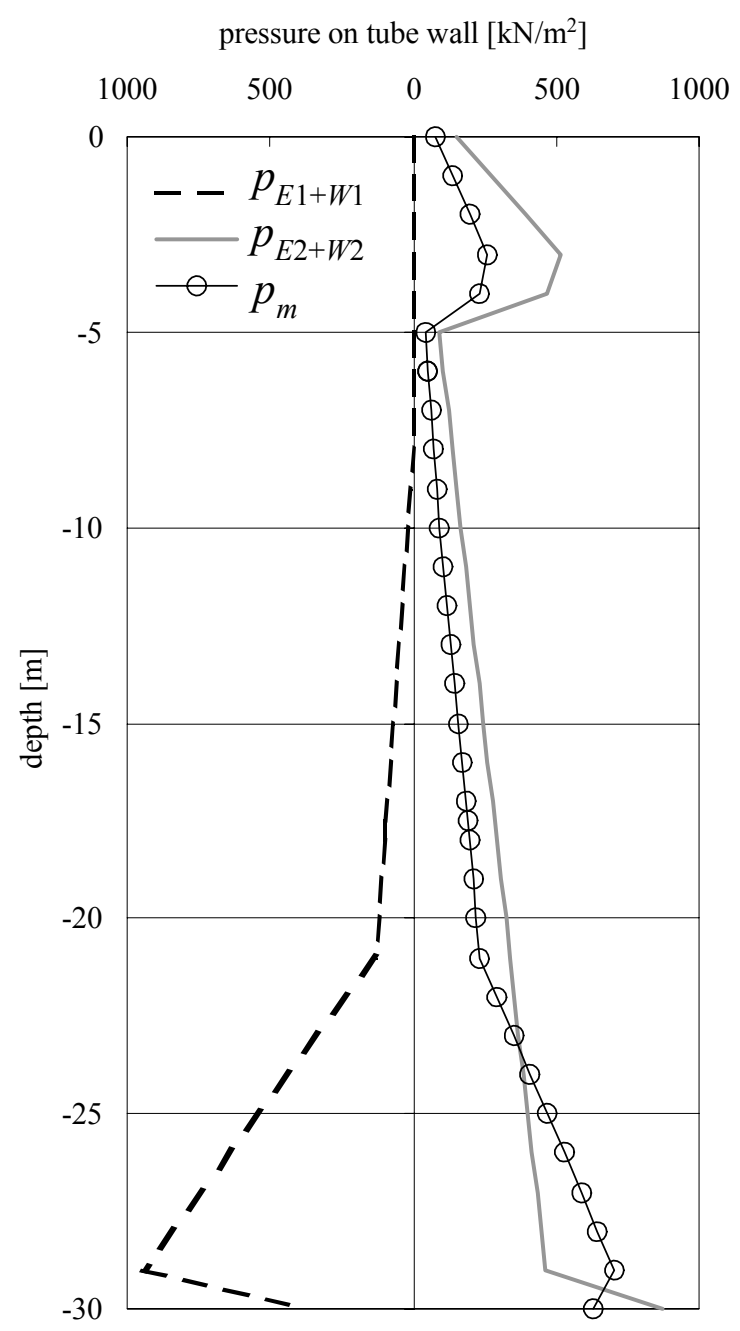

Fig. 11.2: Pressure distributions on tube as a function of the depth according to Fig. 11.1 with $p_{m}=\left(p_{E 1+W 1}+p_{E 2+W 2}\right) / 2$ 
Fig. 11.3 shows the first buckling eigenform from a linear bifurcation analysis for the non-uniform loading. The evaluation of the linear elastic buckling load results in a load factor LF of 0.85 and a buckling mode with a very small number of circumferential buckles. The maximum circumferential membrane compressive stresses in the tube wall can be calculated with a geometrical linear follow-up calculation imposing the linear buckling load calculated before as loading. The analysis reveals that for a load factor LF of 0.85 the maximum circumferential compressive membrane stresses appear in the region of the pile tip. The maximum arising compressive membrane stress of $40 \mathrm{~N} / \mathrm{mm}^{2}$ is used as correspondent circumferential critical buckling stress $\sigma_{\theta, R c}$ according to EN 1993-1-6. This more detailed modelling of the loading situation and the boundary conditions leads to an increase of the circumferential critical buckling stress from $21 \mathrm{~N} / \mathrm{mm}^{2}$ to $40 \mathrm{~N} / \mathrm{mm}^{2}$. Based on this circumferential critical buckling stress $\sigma_{\theta, R c}$ the circumferential buckling resistance $\sigma_{\theta, R d}$ is calculated in Tab. 11.1 following EN 1993$1-6$.

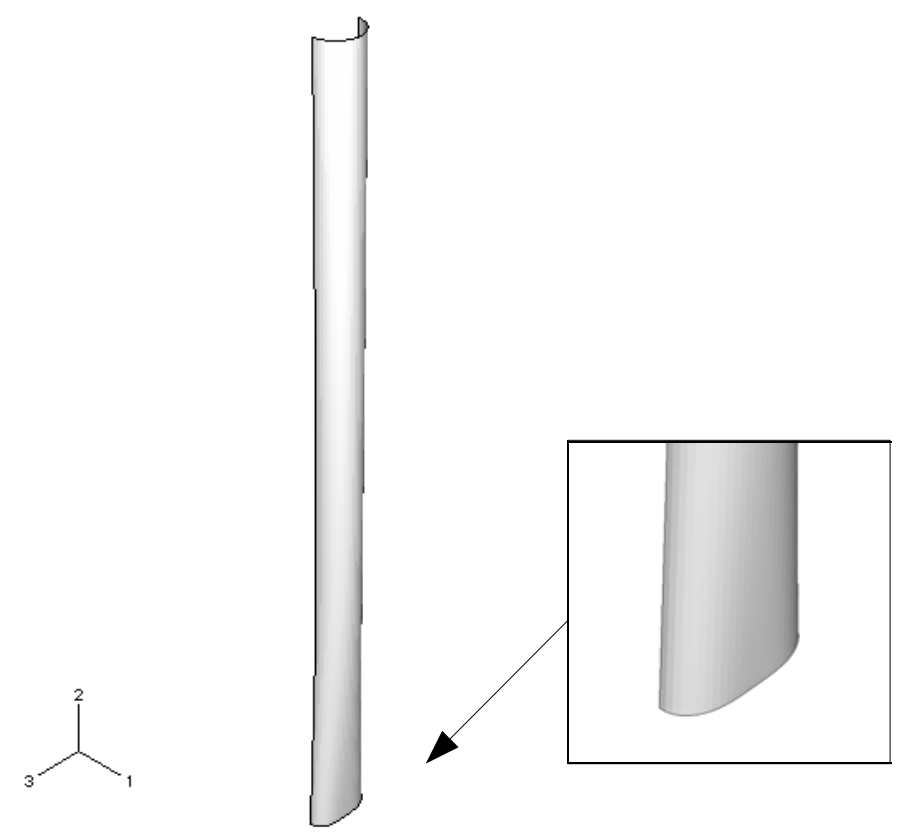

Fig. 11.3: Buckling mode for the reference tube subjected to non-uniform external pressure

The calculation according to Eurocode EN 1993-1-6 and the simplified assumptions for loading and boundary conditions (reference or initial case) using the circumferential critical buckling stress of $21 \mathrm{~N} / \mathrm{mm}^{2}$ results in a circumferential buckling resistance of $10 \mathrm{~N} / \mathrm{mm}^{2}$ for fabrication tolerance quality class $\mathrm{C}$ which is around $45 \%$ smaller than the circumferential buckling resistance evaluated in Tab. 11.1 (see chapter 4). The circumferential buckling resistance increases for the investigated case from $18 \mathrm{~N} / \mathrm{mm}^{2}$ to $24 \mathrm{~N} / \mathrm{mm}^{2}$ and for the simplified case from $10 \mathrm{~N} / \mathrm{mm}^{2}$ to $13 \mathrm{~N} / \mathrm{mm}^{2}$ if a fabrication tolerance quality class of B is assumed instead of C. Since fabrication tolerance quality class of B is proposed in EN 1993-5 to be used for tubular piles, it is applied in the following if not stated differently. 
Tab. 11.1: Parameters for limit state buckling design against circumferential compression

\begin{tabular}{|l|c|c|c|}
\hline parameter & $e q$. & circumferential compression & dimension \\
\hline$\sigma_{\theta, R c}$ & FE analysis & 40 & $\mathrm{~N} / \mathrm{mm}^{2}$ \\
\hline $\bar{\lambda}_{\theta}$ & $(4.35)$ & $\bar{\lambda}_{\theta}=\sqrt{430 / 40}=3.26$ & - \\
\hline$\alpha_{\theta}$ & Annex D (EC) & $0.5($ Class $C)$ & - \\
\hline factors & Annex D (EC) & $\bar{\lambda}_{\theta 0}=0.40, \quad \beta=0.60, \quad \eta=1.00$ & - \\
\hline $\bar{\lambda}_{\theta p}$ & $(4.34)$ & $\bar{\lambda}_{\theta p}=\sqrt{0.5 /(1-0.6)}=1.12$ & - \\
\hline$\chi_{\theta}$ & $(4.33)$ & $\chi_{\theta}=0.5 / 3.26^{2}=0.047$ & - \\
\hline$\sigma_{\theta, R d}$ & $(4.29),(4.30)$ & $\sigma_{\theta, R d}=\frac{0.047 \cdot 430}{1.1}$ & $\mathrm{~N} / \mathrm{mm}^{2}$ \\
\hline
\end{tabular}

\subsection{Investigation of the loading for external pressure}

In Tab. 11.2 the relevant circumferential compressive stresses are calculated applying

$$
\sigma_{\theta}=q \cdot \frac{r}{t}
$$

for different approximations for the loading. It can be seen that the design buckling stress $\sigma_{\theta, R d}$ of $13 \mathrm{~N} / \mathrm{mm}^{2}$ (Class B) calculated in chapter 4 based on simplified assumptions is exceeded considerably. Even, the buckling stress $\sigma_{\theta, R d}$ of $24 \mathrm{~N} / \mathrm{mm}^{2}$ (Class B) from the more detailed analysis from section 11.2 is surpassed.

Tab. 11.2: Circumferential stresses and verifications for the depth $z=-29 \mathrm{~m}$

\begin{tabular}{|l|c|}
\hline pressure & circumferential stress $\sigma_{\theta}\left[\mathrm{N} / \mathrm{mm}^{2}\right]$ \\
\hline with averaged total pressure $p_{m}$ & $\sigma_{\theta}=\frac{p_{m} \cdot r}{t}=\frac{703 \cdot 0.9}{18}=35$ \\
\hline $\begin{array}{l}\text { with averaged total pressure } p_{m} \\
\text { less internal pressure } p_{\text {int }}\end{array}$ & $\sigma_{\theta}=\frac{\left(p_{m}-p_{\text {int }}\right) \cdot r}{t}=\frac{(703-160) \cdot 0.9}{18}=27$ \\
\hline $\begin{array}{l}\text { from water pressure difference } \\
\Delta p_{W}=p_{W 2}-p_{W 1}\end{array}$ & $\sigma_{\theta}=\frac{\Delta p_{W} \cdot r}{t}=\frac{50 \cdot 0.9}{18}=3$ \\
\hline $\begin{array}{l}\text { with averaged earth pressure } \\
p_{m E}=\left(p_{E 1}+p_{E 2}\right) / 2\end{array}$ & $\sigma_{\theta}=\frac{p_{m E} \cdot r}{t}=\frac{0.5 \cdot(733+203) \cdot 0.9}{18}=23$ \\
\hline $\begin{array}{l}\text { averaged earth pressure } p_{m E} \text { less } \\
\text { internal pressure } p_{\text {int }}\end{array}$ & $\sigma_{\theta}=\frac{\left(p_{m E}-p_{\text {int }}\right) \cdot r}{t}=\frac{(468-160) \cdot 0.9}{18}=15$ \\
\hline $\begin{array}{l}\text { with max. total pressure } p_{E 1+W 1} \\
\text { less internal pressure } p_{\text {int }}\end{array}$ & $\sigma_{\theta}=\frac{\left(p_{E 1+W 1}-p_{\text {int }}\right) \cdot r}{t}=\frac{(943-160) \cdot 0.9}{18}=39$ \\
\hline
\end{tabular}


In order to use the circumferential membrane stresses arising in real constructions for the assessment, elaborate non-linear numerical analyses which take all relevant influences (e.g. sequence of constructing the combiwall) into account are required. In the following a simplified approximation is used.

For the example a numerical stress analysis in ABAQUS with a model according to section 11.2 is performed as a first approximation. For the internal pressure, only the component arising from water pressure difference is applied. The water level in the tube is put to the level of the free water level which is $5 \mathrm{~m}$ below the ground-water level (see Fig. 4.1). The internal pressure acts opposite to the earth pressure applied to the tube according to Fig. 11.2. Since the influence of second order theory without considering axial forces is marginal (few percentages), a more detailed stress determination is disregarded in this first comparison and the equilibrium is evaluated for the undeformed system. The maximum circumferential stress from the linear stress analysis considering internal pressure can be calculated for the investigated case with $34 \mathrm{~N} / \mathrm{mm}^{2}$ in the region of the pile tip. The analysis results in an about $15 \%$ smaller circumferential compressive stress than the value given in Tab. 11.2 for the approach with the maximum total pressure $p_{E 1+W 1}$ less the internal pressure $p_{\text {int }}$ and applying equation (11.1) with $39 \mathrm{~N} / \mathrm{mm}^{2}$. Nevertheless, the stress of $34 \mathrm{~N} / \mathrm{mm}^{2}$ for the reference case based on the more detailed investigation exceeds the circumferential buckling resistance of $24 \mathrm{~N} / \mathrm{mm}^{2}$ by nearly $70 \%$.

\subsection{Summary and comparison of previous analyses}

In Tab. 11.3 the results of the previous analyses are summarized. In the last column the reciprocal of the utilization factor is given for the different assessments.

Tab. 11.3: Summary and comparison

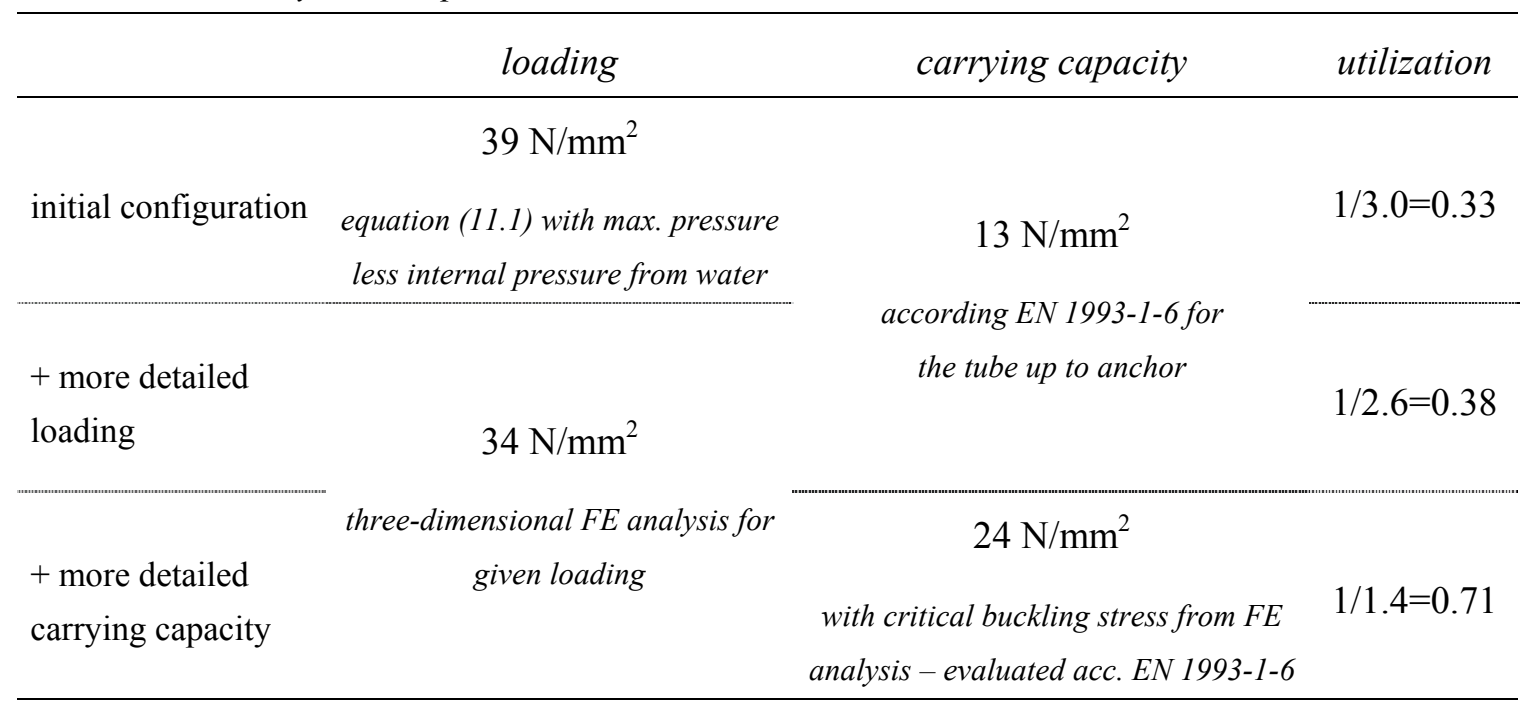

For the initial configuration or the reference case the carrying capacity is exceeded by the factor of 3.0. The evaluation of stresses in the shell by performing numerical 
analyses and considering the non-uniform pressure distribution over the length and circumference improves the situation. Still, the carrying capacity of the tubular pile is exceeded by $160 \%$. A significant drop can be found if the carrying capacity or the buckling resistance $\sigma_{\theta, R d}$ is derived by investigating the entire model numerically instead of using the simplified approaches of the reference or initial case. As a consequence the carrying capacity is only exceeded by $40 \%$. It can be seen that by a more differentiated analysis of the actions and the boundary conditions, on the one side the loading can be decreased and on the other side the carrying capacity can be increased. The beneficial influence of the surrounding soil is not taken into account in the comparison and is discussed in a different section.

\subsection{Defining an equivalent loading case}

\subsubsection{General}

The loading of the tubular pile regarding the external pressure distribution is neither constant in the circumferential nor in the meridional direction. In order to calculate the carrying capacity for limit state buckling design against circumferential compression according to EN 1993-1-6, an equivalent pressure distribution needs to be assumed. The simplest approach is to take into account the meridional stresses arising from bending and to additionally replace the non-uniform pressure distribution with a constant pressure over the circumference and length applying the maximum pressure amplitude. Since this approach is assumed to yield highly conservative results, a numerical parametric study based on common dimensions of tubular piles is performed. First, the influence of the pressure distribution over the circumference is analysed, followed by a study with reference to the non-uniform external pressure over the length.

\subsubsection{Non-uniform external pressure over circumference}

For the investigation of the influence of non-uniform external pressure over the circumference it is important to define a system for which the influence of the circumferential stresses and the merdional stresses can be separated clearly. Since many patterns of external pressures yield a resultant force which then needs a counterpart in terms of shear forces resulting in bending and hence in merdional stresses, modelling an entire cylindrical shell may not be convenient. Investigating a cylinder will always bring up the problem of longitudinal stresses. Nevertheless, analysing a reduced system as e.g. a pressurized ring does not allow to take account of all relevant failure modes as certain buckling eigenmodes are enforced.

Thus, for the investigation of the influence of non-uniform external pressure over the circumference cylinders based on two different systems as depicted in Fig. 11.4 are used. For type A one end is entirely restrained, i.e. it corresponding to $\mathrm{BC} 1 \mathrm{r}$ from EN 1993-1-6. The ends of the tubular pile from type B are clamped in the global mechnical model, i.e. resulting in bending moments at the ends, but allowing the shell 
to rotate along the circumference at the boundaries (meridional rotation not restrained), i.e. BC 1f from EN 1993-1-6.
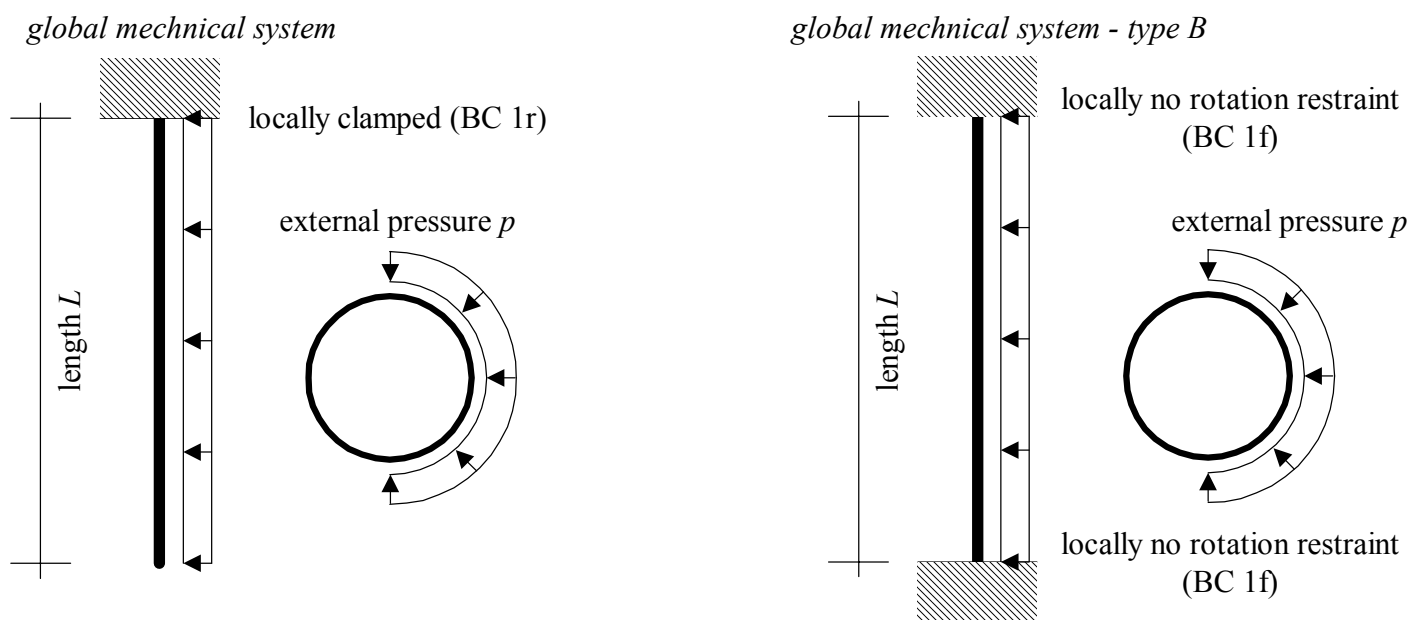

Fig. 11.4: Investigated mechanical systems for non-uniform external pressure over circumference

A parametric study is performed for cylinders according to type A and B of various lengths and for the $r / t$ ratios 40, 50, 60, and 75 comparing the linear buckling loads for the cylinders loaded as shown in Fig. 11.4 ("half-pressure") and with a constant external pressure ("full-pressure"). The results are depicted in Fig. 11.5 and Fig. 11.6 as a function of the length parameter $\omega$ and the ratio of the linear buckling pressure $p_{\text {crit, } 0.5}$ for "half-pressure" over the linear buckling pressure $p_{\text {crit } 1.0}$ for "full-pressure". In order to cover all possible buckling modes the complete cylinders are modelled. The standard two-waves buckling mode is depicted in Fig. 11.5.

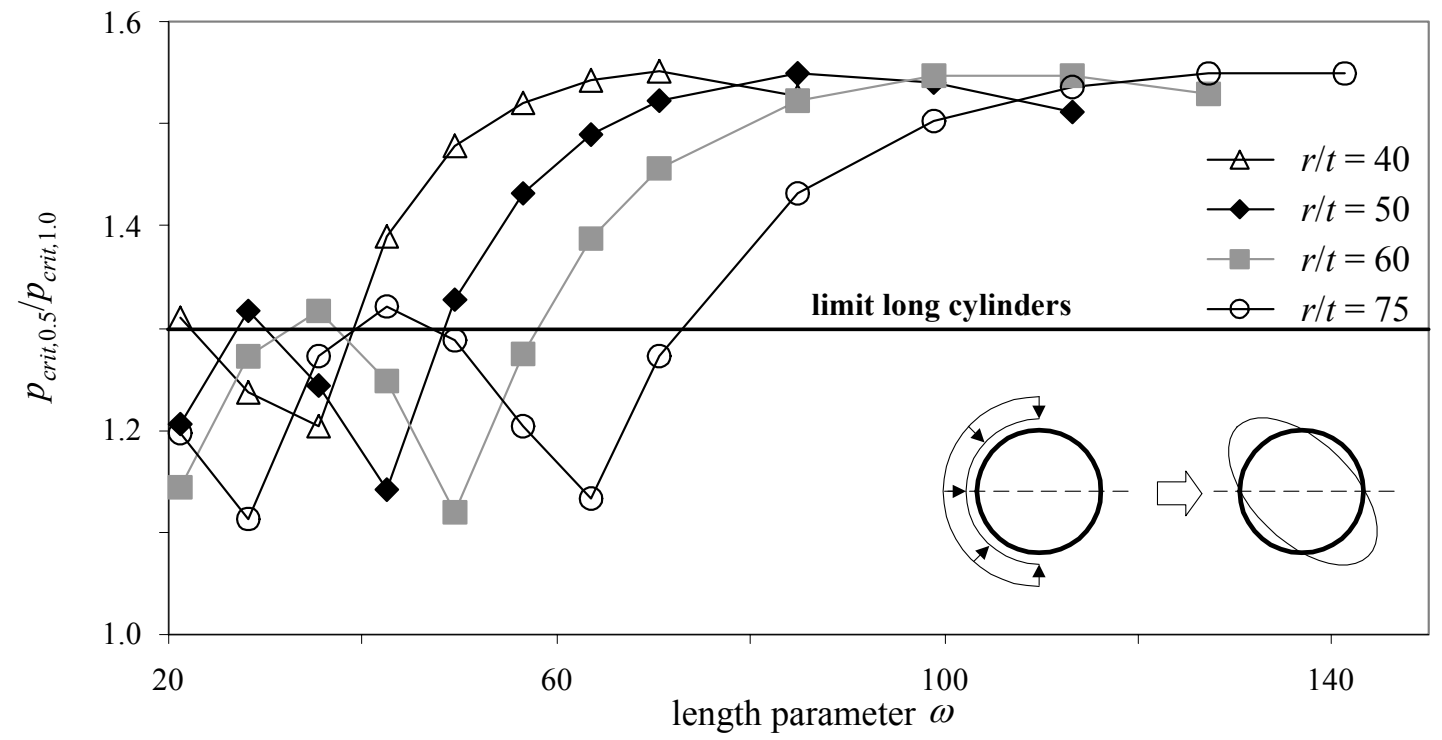

Fig. 11.5: Ratios of buckling pressures for "half-pressure" over "full-pressure" for different lengths and system $A$

A ratio $p_{\text {crit }, 0.5} / p_{\text {crit } 1.0}>1.0$ means that "full-pressure" is more detrimental for the cylinder than "half-pressure". For all investigated cases this aspect is fulfilled. The 
numerical analyses show that for short to medium length cylinders $\left(\leq 1.63 C_{\theta} r / t\right.$ with $C_{\theta}=0.6$ for type $\mathrm{A}$ and $C_{\theta}=1.5$ for type B) according to the definition given in EN 1993-1-6 the ratio oscillates. The results for long cylinders $\left(>1.63 C_{\theta} r / t\right)$ can be separated from the results of the shorter cylinders as seen in Fig. 11.5 and Fig. 11.6.

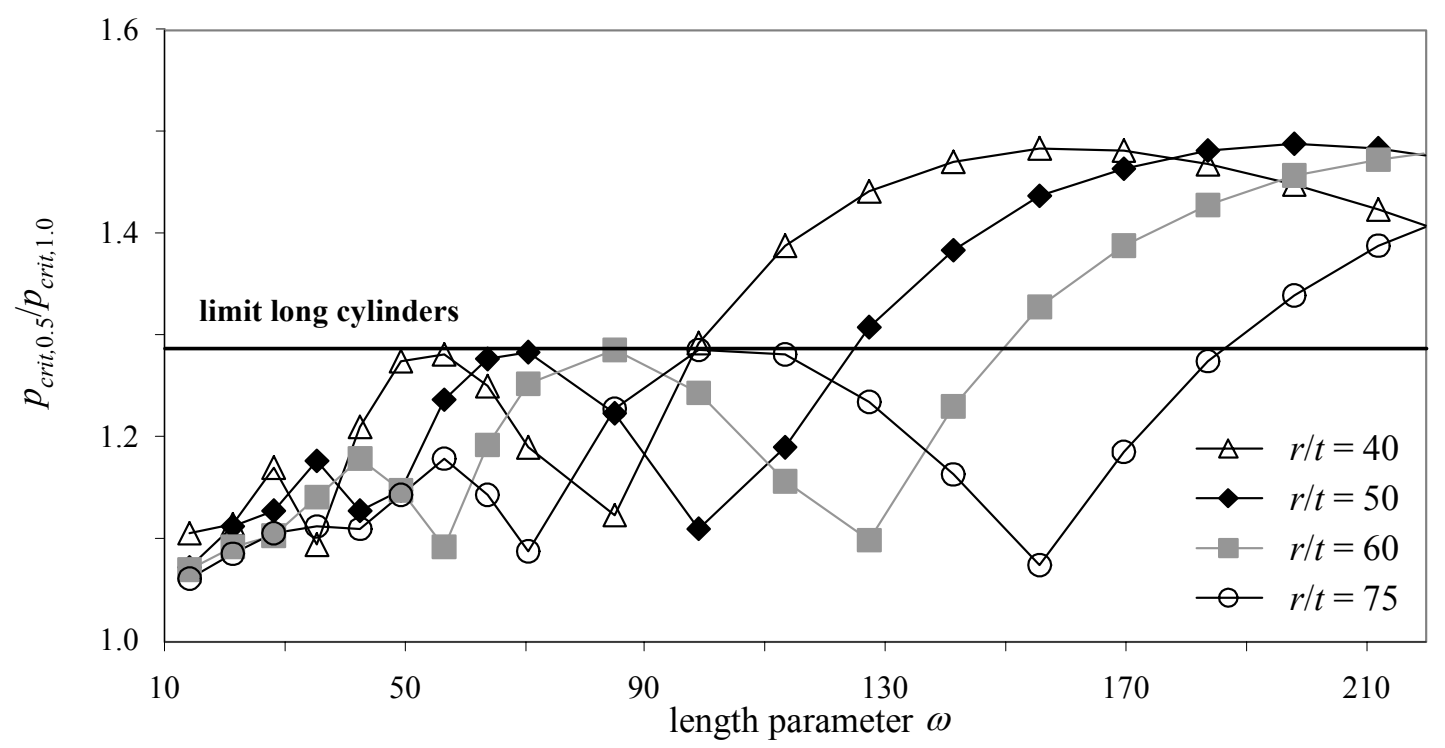

Fig. 11.6: Ratios of buckling pressures for "half-pressure" over "full-pressure" for different lengths and system $B$

For a better understanding of the oscillating aspect from Fig. 11.5 and Fig. 11.6 the numerator and the denominator from the numerical analyses (ordinate) in Fig. 11.5 (system A) are plotted separately in Fig. 11.7 for $r / t=60$. Here, the data for "halfpressure" corresponds to $p_{\text {crit }, 0.5}$ and the data for "full-pressure" corresponds to $p_{\text {crit }, 1.0}$.

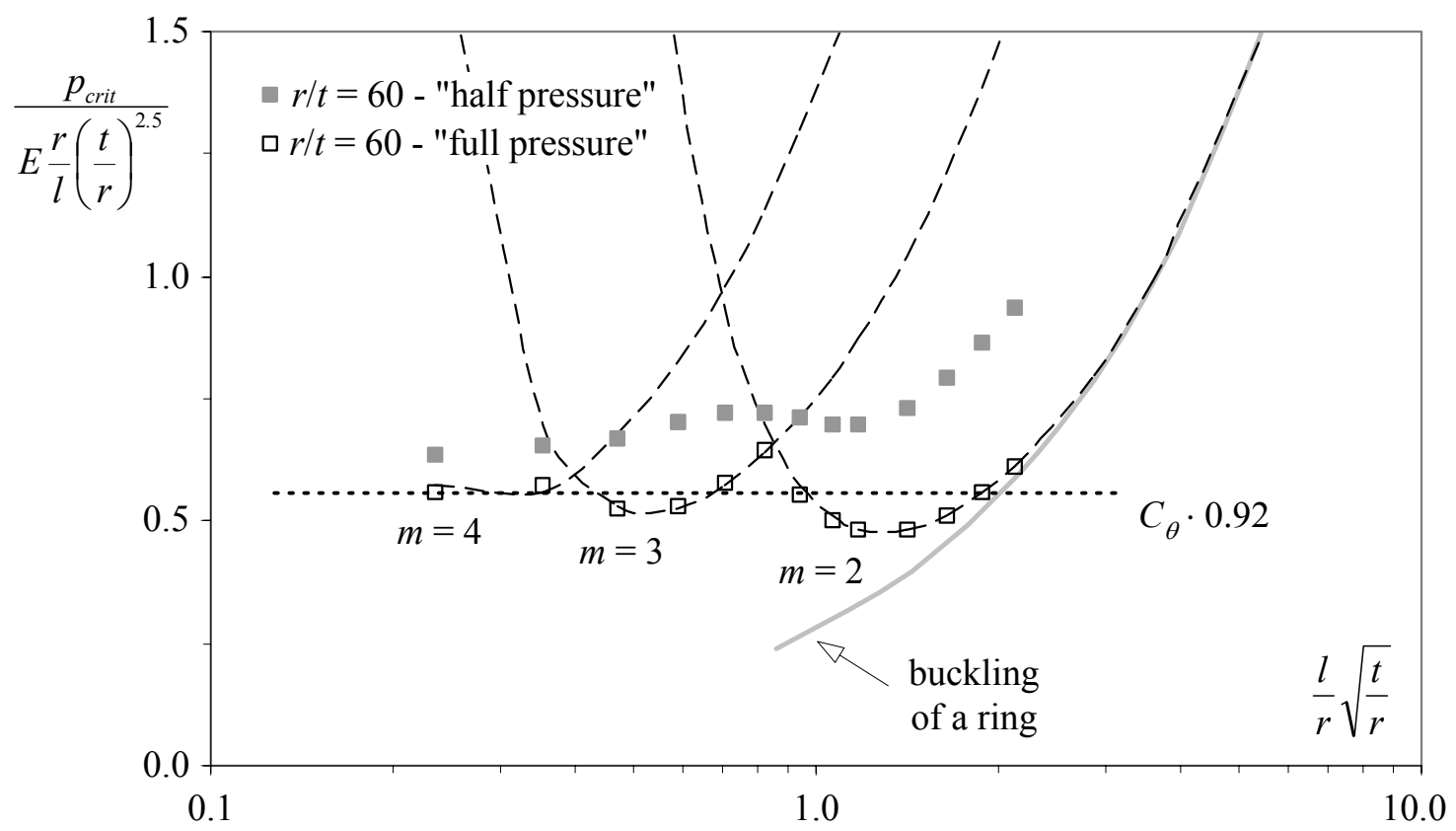

Fig. 11.7: Buckling pressures for "half-pressure" over "full-pressure” for different lengths and system A 
Additionally, Fig. 11.7 includes the results of the analytical solutions for externally pressurized cylindrical shells with circumferential buckling waves $m=2,3$, and 4 . The knuckling pressures tend towards the results for buckling of a ring (analytical solution). The horizontal line at $C_{\theta} \cdot 0.92$ indicates the buckling pressure for medium length cylindrical shells. The analytical solutions and similar figures can be found in e.g. Beuth-Kommentare (1998). It can be seen that the numerical results for "full-pressure" coincide as expected with the analytical solutions. In this context the analytical solutions need to be minimized with respect to the buckling pressure. A similar behaviour can be found for the "half-pressure" case; again, the oscillations occur due to changes in the buckling mode (circumferential buckling waves). Nevertheless, as this effect is not as distinct for the "half-pressure" case, the ratio is governed by the "fullpressure" case resulting in the observed oscillations found in Fig. 11.5 and Fig. 11.6. For system B and for other $r / t$ ratios the effect remains the same.

A constant function at 1.30 for type $\mathrm{A}$ and 1.28 for type $\mathrm{B}$ as derived from the results is added in the figures indicating the boundary between buckling ratios for long cylinders (right of the intersection point of $r / t$ ratio curve and the constant function) and shorter cylinders (left of the intersection point of $r / t$ ratio curve and the constant function). The study revealed coinciding buckling ratios for the different $r / t$ ratios for each system type leading to constant functions. Results are not depicted for all length parameters as seen in Fig. 11.5, e.g. not for $r / t=40$ and $\omega>85$. For these cases the buckling failure does not occur at the free end (type A) or at midspan (type B) from external pressure but at the clamped ends of the tubular piles due to axial compression in combination with external pressure. Obviously, the influence of the axial membrane stresses from bending increases for long cylinders since the moment increases by the power of two, respectively. This aspect can be observed as the buckling load starts to decrease for very long cylinders. Nevertheless, the preformed analyses only provide results for circumferential and meridional stresses interacting.

The intention is to replace the non-uniform external pressure distribution over the circumference having maximum amplitude $p$ by an equivalent constant pressure of amplitude $p^{*}$ without neglecting the axial membrane stresses arising from bending. The aspect of the decreasing buckling ratio for long cylinders can be disregarded because this effect is based on the bending moment, which is to be considered separately in the assessment procedure. The results for short cylinders tend to oscillate due to the influence of the boundary conditions. For these cases the definition of an equivalent pressure may be difficult. Thus, and because the major field of interest are long cylinders, the following considerations are limited to the case of long cylinder with:

$$
\omega>1.63 C_{\theta} \frac{r}{t}
$$

The investigated cases type $\mathrm{A}$ and $\mathrm{B}$ refer to external pressure buckling factors $C_{\theta}=0.6$ and 1.5, respectively. All investigated long cylinders yielded buckling ratios of about 
1.3 as seen in the figures. Thus, it appears appropriate as a first approximation to define an equivalent pressure $p^{*}$ independent of the boundary conditions with

$$
p^{*}=C_{L} \cdot p=\frac{1}{1.3} p
$$

for cylinders with $r / t<75$ fulfilling (11.2). The value $C_{L}$ is defined as equivalent pressure correction factor.

This proposed equivalent pressure represents a conservative approximation and is limited by the investigated range. Nevertheless, comparing analyses with larger $r / t$ ratios lead to similar results.

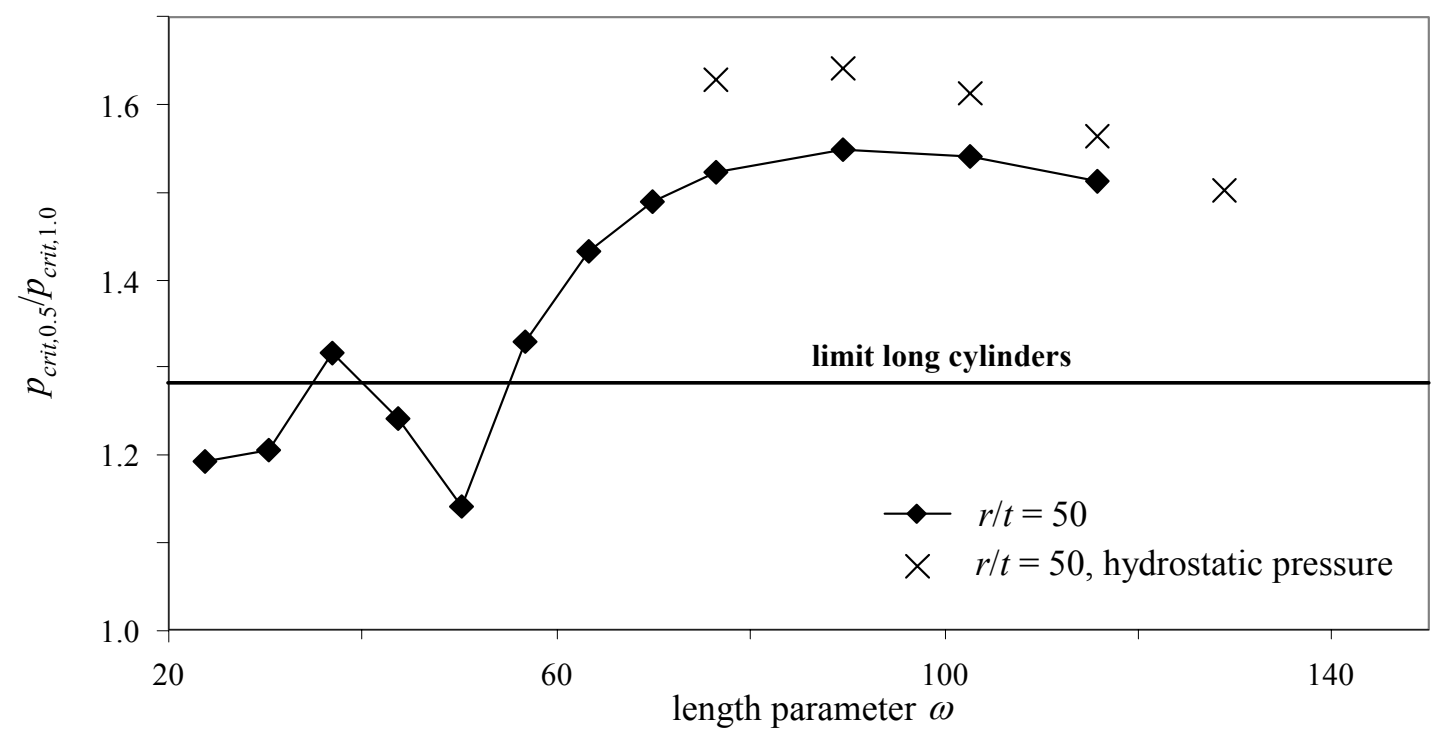

Fig. 11.8: Ratios of buckling pressures for "half-pressure" over "full-pressure" for different lengths and system A for linear (hydrostatic) pressure loading

The following sections will attempt to elaborate the simple formula (11.3) by giving more detailed factors for the equivalent pressure with reference to boundary conditions and $r / t$ ratios. It needs to be emphasized that if a "half-pressure" is replaced by a "fullpressure", the moment arising due to the resultant force still needs to be regarded. Thus, in the subsequent design procedure an interaction check is needed. In Fig. 11.8 some comparing analyses are depicted in order to validate the previous assumptions. Here, the load is not applied constantly over the length but linearly increasing towards the free end (hydrostatic $=$ linear pressure). The results show that for non-uniform pressures over the length the situation may even be better.

It can be seen from the previous study that the equivalent buckling pressure is nearly constant for the investigated $r / t$ ratios if the limit for long cylinders is considered. Thus, the approach of applying the results of analyses based on a cylinder referring to the limit case (long cylinder) generally to cylinders subjected to non-uniform external pressure (over circumference) can be adopted as conservative approach. Although, the previous analyses revealed less conservative findings, the limit case (according to (11.2)) is chosen as it is assumed that the influence of longitudinal stresses is rather small. Hence, 
in the next numerical study a cylinder is subjected to different external pressure patterns. The patterns are defined by a load angle $\varphi_{\text {load }}$ referring to the segment which is loaded by a constant pressure, i.e. the load shown in Fig. 11.5 corresponds to $\varphi_{\text {load }}=180^{\circ}$. It is the aim to provide equivalent pressures as in (11.3) for different cases. For this approach the equivalent pressure correction factor $C_{L}$ is defined as:

$$
C_{L}=\frac{p_{\text {crit }, 360^{\circ}}}{p_{\text {crit }, \text { pload }}}
$$

In Fig. 11.9 the results for the limit case (long cylinder) are depicted. The reciprocal value of the equivalent pressure correction factor $1 / C_{L}$ is given as a function of the load angle $\varphi_{\text {load }}$ for the system type A and B as defined in Fig. 11.4. It can be seen that the analyses for system type B yield slightly more conservative results (up to $4 \%$ ) in comparison to type A. This aspect is based on the fact that for system B the section where the buckling initiates (mid-span) is subjected to longitudinal stresses arsing from bending. Theses stresses are rather small for type A at the free end.

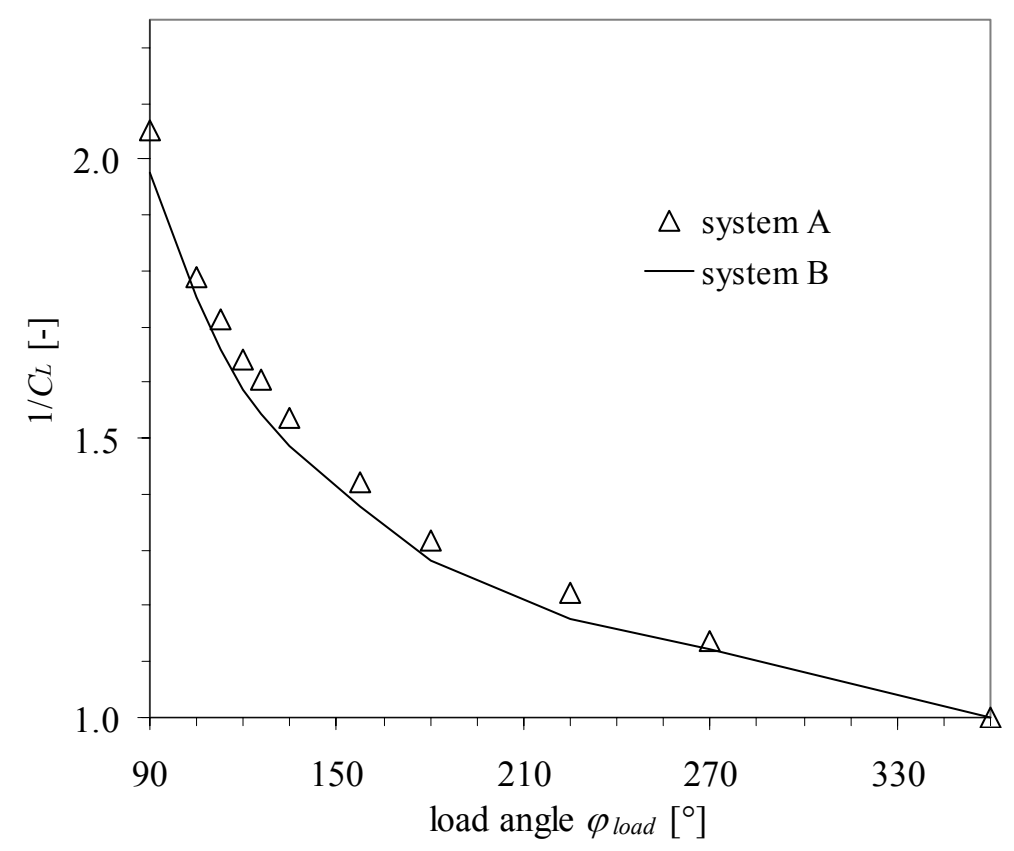

Fig. 11.9: reciprocal value of equivalent load correction factor $C_{L}$ for system $A$ and $B$ as a function of the load angle $\varphi_{\text {load }}$

Nevertheless, the analyses provide similar results and thus it is proposed to apply the equivalent pressure correction factor $C_{L}$ of system $\mathrm{B}$ as defined in Fig. 11.9 for obtaining an equivalent pressure $p^{*}$ independent on the boundary conditions:

$$
p^{*}=C_{L} \cdot p
$$

and as a function of the loaded segment, i.e. dependent on $\varphi_{\text {load. }}$. Although this approach also involves the influence of longitudinal stresses it is expected to provide a conservative but still adequate approximation for general application. Since the analyses 
were only limited to $r / t<100$ and $\varphi_{\text {load }}>90^{\circ}$ the application of Fig. 11.9 shall be limited to these values and to cylinders fulfilling (11.2). For practical application it may be convenient to adopt the curve given in Fig. 11.9 by a function. Based on a regression analyses for the results of system $B$ with a regression coefficient of $R^{2}=0.995$, a conservative approximation is derived with an additional safety factor of 1.05 :

$$
C_{L}=0.33 \ln \left(\frac{\varphi_{\text {load }}}{360^{\circ}}\right)+1
$$

The approximated curve is depicted in Fig. 11.10 with some results from the numerical analyses.

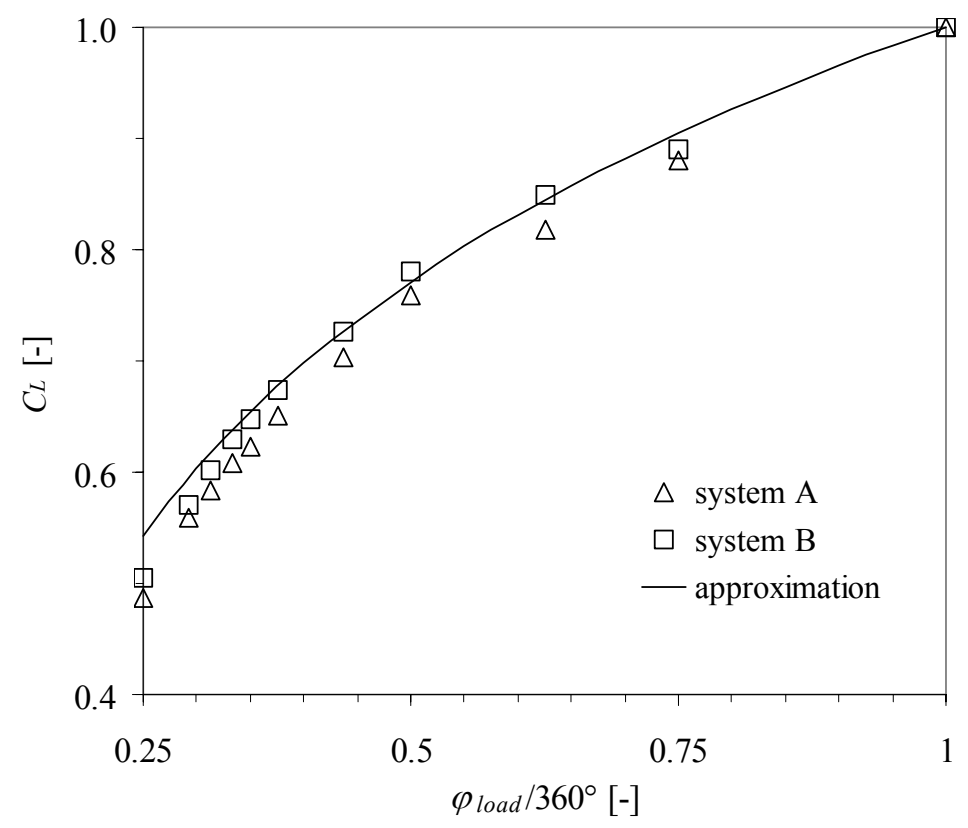

Fig. 11.10: $\quad$ Equivalent load correction factor $C_{L}$ according to approximation from (11.6)

The results need to be adopted to a more general case. In this context it is of interest to obtain information on the equivalent pressure correction factor $C_{L}$ if segments are loaded with different amplitude pressures as seen e.g. in Fig. 11.1 with $p_{E 1} *$ and $p_{E 2 *}$. In the following it is assumed that the "loaded segment" $\left(\varphi_{\text {load }}\right)$ is subjected to the maximum external pressure amplitude $p_{\max }$ (e.g. in Fig. $11.1 p_{\max }=p_{E 2 *}$ ) while the remaining circumference $\left(360^{\circ}-\varphi_{\text {load }}\right)$ is subjected to the external pressure amplitude $p_{\text {min }}$. Then, the load fraction ratio $a_{f}$ is defined as:

$$
a_{f}=\frac{p_{\min }}{p_{\max }}
$$

Hence, the results depicted in Fig. 11.9 and Fig. 11.10 refer to $a_{f}=0$. The corresponding linear buckling are calculated for different load fraction ratios and the system A and B, and some results are depicted in Fig. 11.11. 


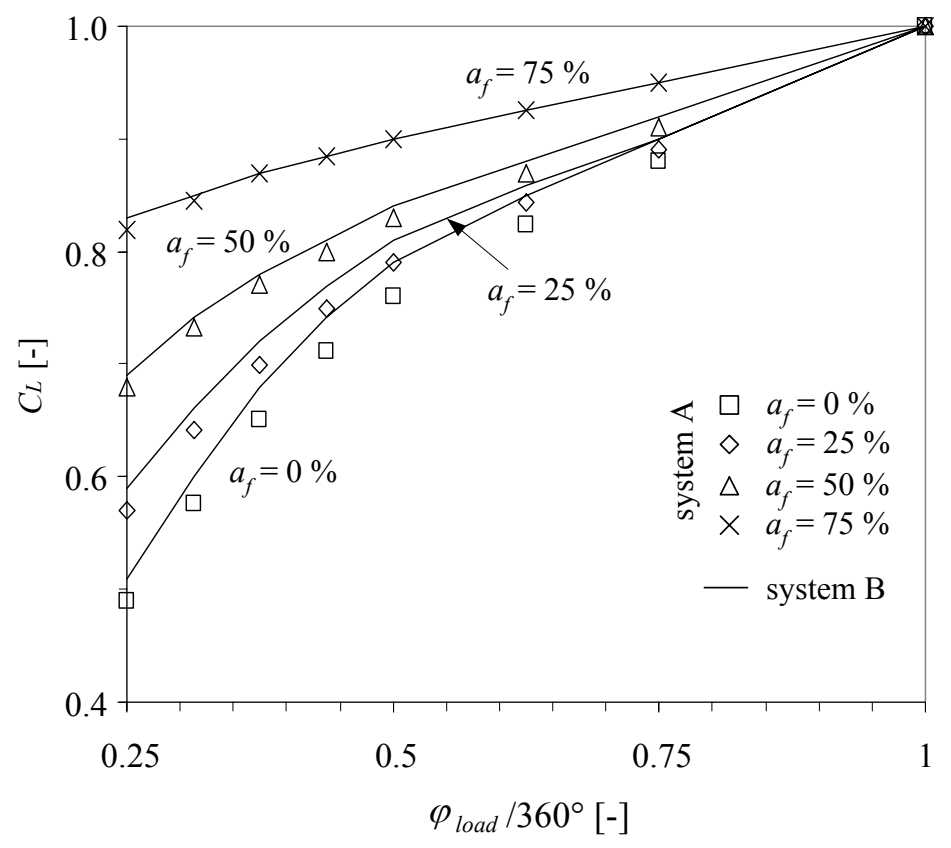

Fig. 11.11: Equivalent load correction factor $C_{L}$ for system $A$ and $B$ as a function of the load angle $\varphi_{\text {load }}$ and for different load fraction ratios $a_{f}$

Again, the results for different $r / t$ ratios smaller than 100 almost coincide and thus the findings can be interpreted independent on the $r / t$ ratio. As the equivalent pressure correction factor $C_{L}$ is similar for the boundary cases A and B but revealing slightly larger values for system $B$, the evaluation is based on the system B. According to the interpretation performed for formula (11.6) regression analyses are executed for the different load fraction ratios $a_{f}$. For all ratios a logarithmic approximation was applied. Since for consistency the derived equations need to yield $C_{L}=1$ for "full pressure", the only varying parameter is the factor multiplying the logarithmic function; e.g. 0.33 in formula (11.6). In order to establish a general equation similar to formula (11.6), regression analyses are performed for the different load fraction ratios $a_{f}$ with an additional safety factor of 1.05. The factors from the analyses are then adopted again in a regression analyses with a polynomial approximation as seen in Fig. 11.11. Finally, the general formula for the equivalent pressure correction factor $C_{L}$ valid for all boundary conditions, $r / t<100, a_{f} \leq 1$, and $\varphi_{\text {load }}>90^{\circ}$ can be written as:

$$
C_{L}=\left[-0.165\left(a_{f}^{2}+a_{f}-2\right)\right] \ln \left(\frac{\varphi_{\text {load }}}{360^{\circ}}\right)+1
$$

or

$$
C_{L}=1-0.165\left(a_{f}^{2}+a_{f}-2\right) \ln \left(\frac{\varphi_{\text {load }}}{360^{\circ}}\right)
$$




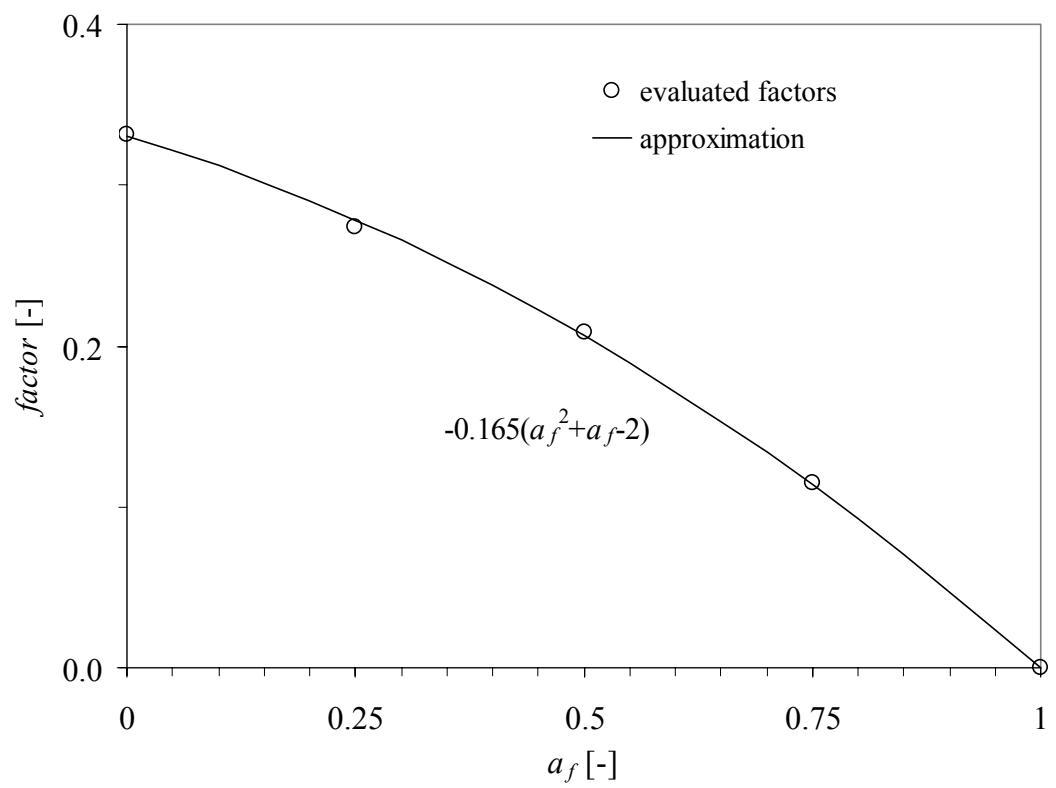

Fig. 11.12: Evaluation of the scaling factor for different load fraction ratios $a_{f}$

\subsubsection{Non-uniform external pressure over length}

The previous analyses lead to the assumption that an external pressure constant in circumferential and longitudinal direction is more detrimental to the buckling strength than an external pressure distribution which is non-uniform. The following investigation of the non-uniformity in longitudinal direction assumes uniformity over the circumference as this aspect of variability was dealt with in the previous section. It is intended to obtain an equivalent dimensionless length parameter $\omega^{*}$ for a cylinder subjected to external pressure non-uniform over the length to be used for obtaining a corresponding critical circumferential buckling stress.
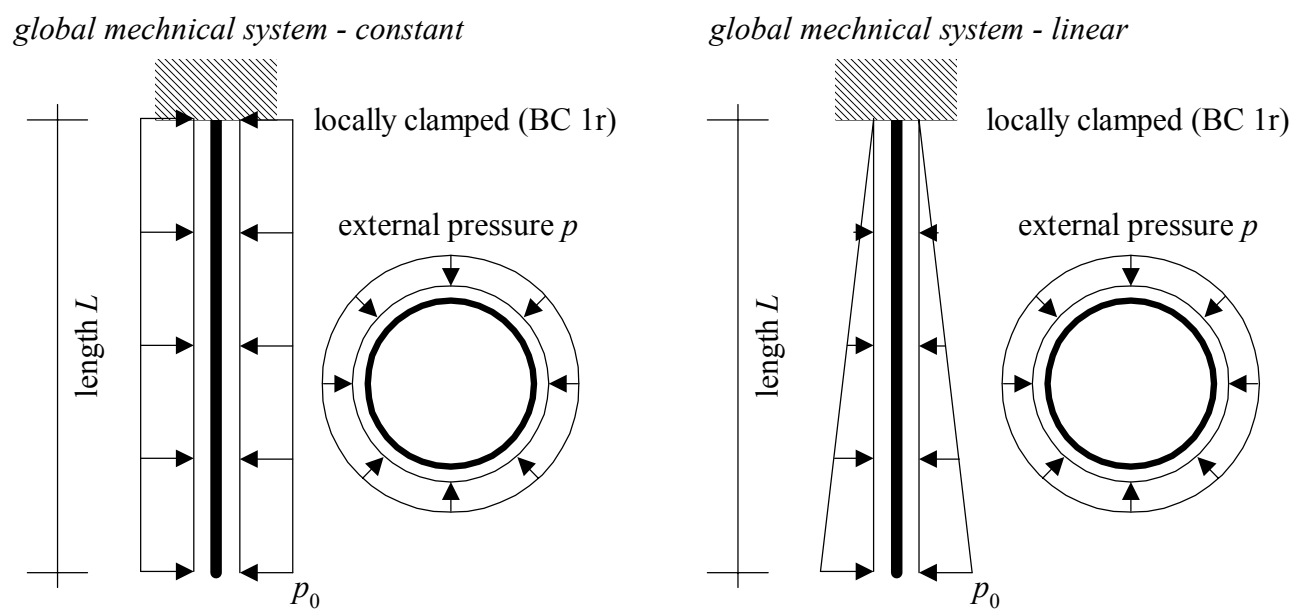

Fig. 11.13: Investigated mechanical systems for external pressure non-uniform over length

The first numerical study is based on a tubular pile clamped at one end subjected to external pressure of the type "constant" or "linear" with the same maximum amplitude 
$p_{0}$ as depicted in Fig. 11.13. At the clamped end all displacements and rotations are constraint, i.e. corresponding to BC 1r from EN 1993-1-6. The most conservative approach would be to replace the linear system by the constant system without introducing changes. For comparison both systems are investigated in detail. Linear bifurcation analyses are performed with for different $r / t$ ratios and lengths.

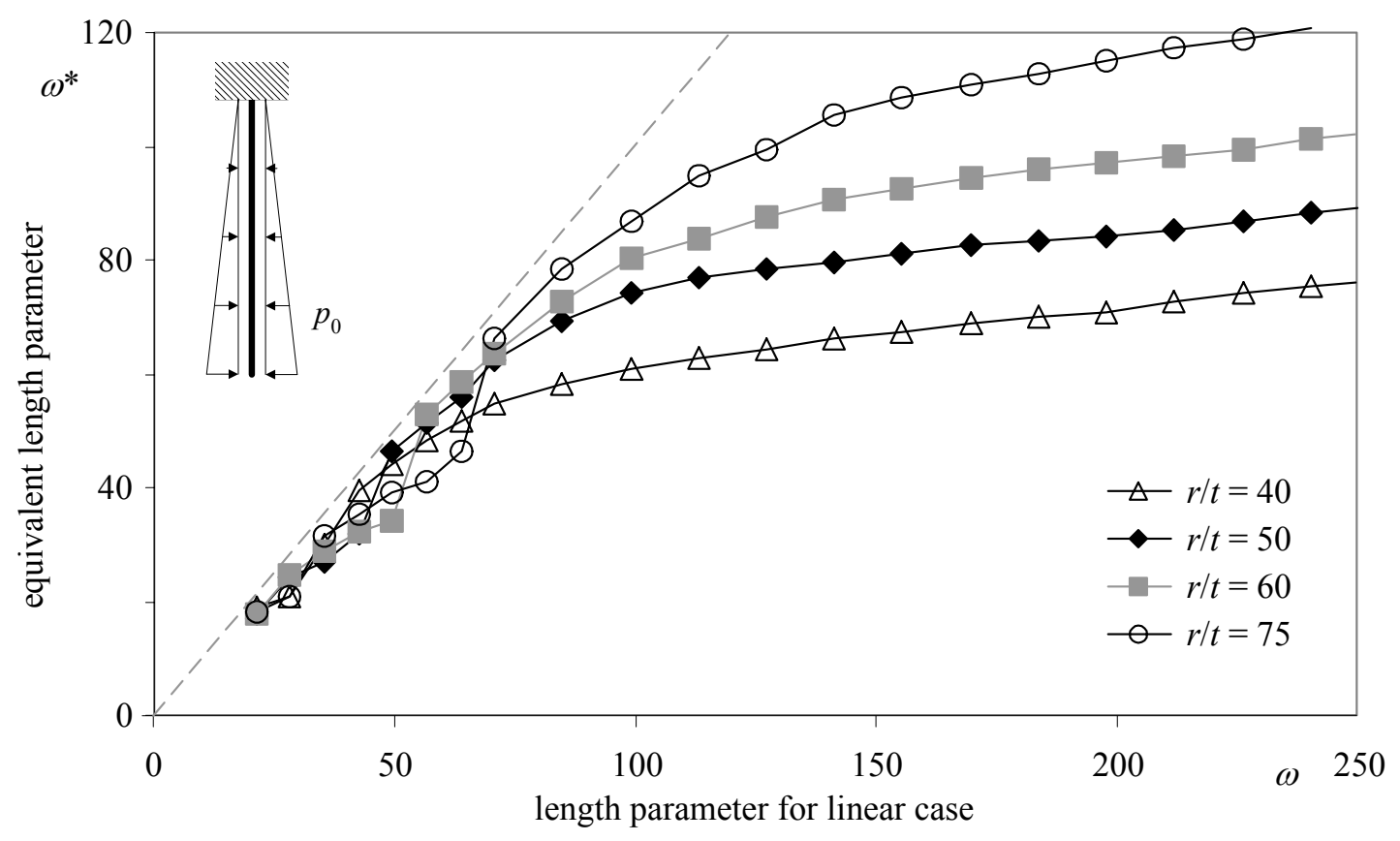

Fig. 11.14: Relationship between the length parameter for the "linear" system and the corresponding equivalent length parameter for a "constant" system

Based on the buckling loads the length parameters are interpreted. Here, for the "linear" (pressure) system with a certain length parameter $\omega$ the corresponding "constant" (pressure) system with the same buckling pressure and the equivalent length parameter $\omega^{*}$ is derived. The evaluated results are depicted in Fig. 11.14; e.g. for $r / t=50$ the buckling pressure for a "linear" system with $\omega=200$ is equal to the buckling pressure for a "constant" system with $\omega^{*}=85$. Fig. 11.14 shows that the results for small length parameters oscillate and seem to follow smoother pattern for increasing length parameters. All results are below the dotted grey line, i.e. $\omega^{*}<\omega$ for all calculated cases. Thus, for all analyses the approach of replacing the "linear" system by the "constant" system $\left(\omega^{*}=\omega\right)$ would yield conservative results.

Based on the previous findings it is chosen to approach the problem of an equivalent length by a defining mechanical model from which the corresponding values can be adopted. In this context the buckling eigenmode is investigated in more detail. Since only long cylinders according to EN 1993-1-6 are dealt with, the number of circumferential buckling waves $m=2$. In the longitudinal direction, half a buckling wave evolves for the system depicted in Fig. 11.13. In order to define a mechanically based dependency between "constant" and "linear" pressure the buckling waves in longitudinal direction are compared. The idea is to find a buckling eigenmode for a 
"constant" pressure which corresponds to the buckling mode of a "linear" pressure. This step is performed in Fig. 11.15 for $r / t=50$ and some length parameters. The eigenforms of some example cases ("linear" system) are shown as a function of the length $L$ with the deflections normalized with respect to the maximum deflection. Shifted eigenforms of "constant" systems are superposed in the figure which match the deflections of the "linear" systems well. This matching is performed by hand. It can be seen that the equivalent "constant" system yield good correlations with the "linear" systems.

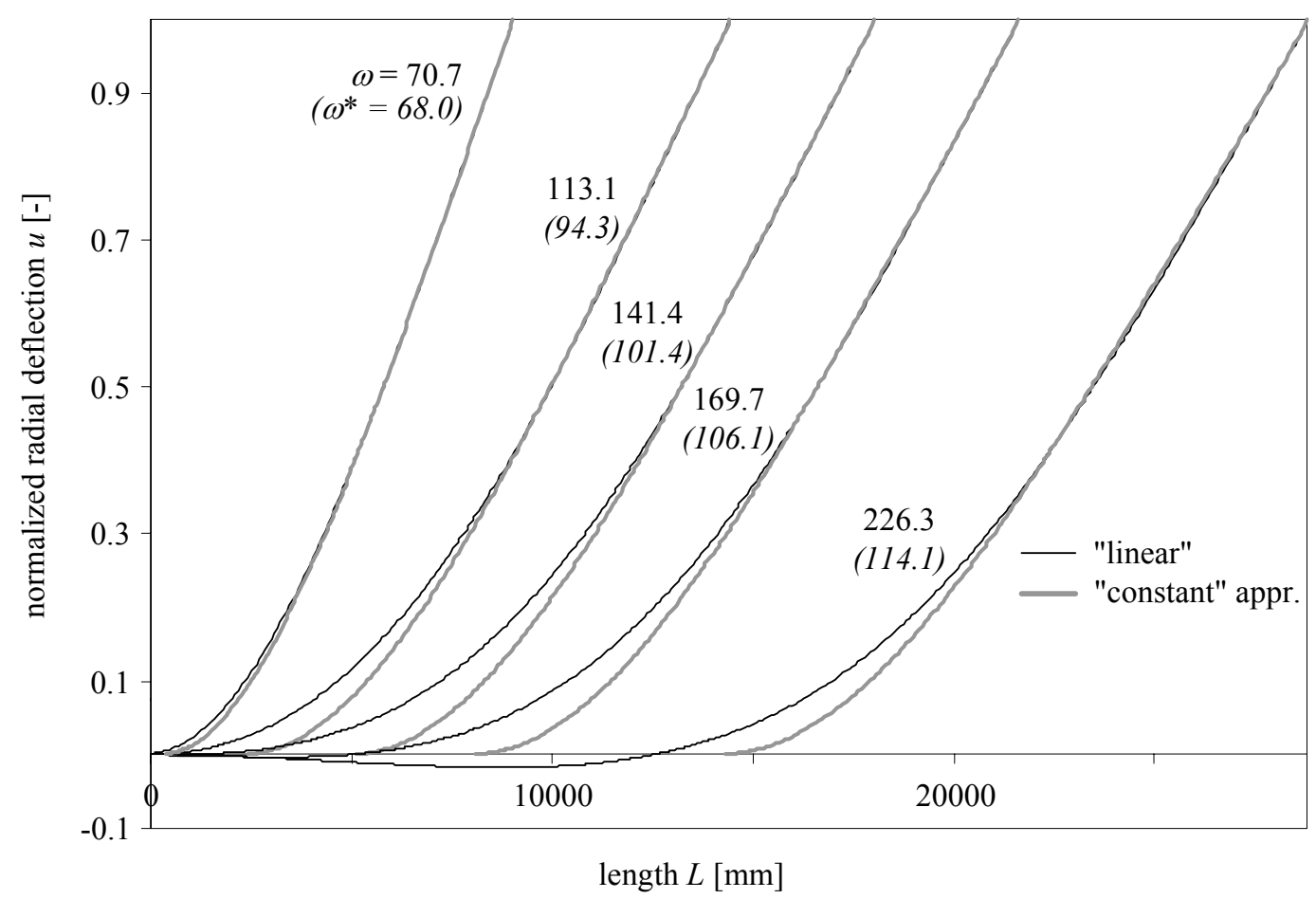

Fig. 11.15: Matching "constant" systems to "linear" systems based on the eigenmodes for $r / t=50$

The previous procedure is performed for different $r / t$ ratios and different lengths. The results are evaluated and summarized in Fig. 11.16 with the corresponding results for the buckling loads as depicted in Fig. 11.14. 


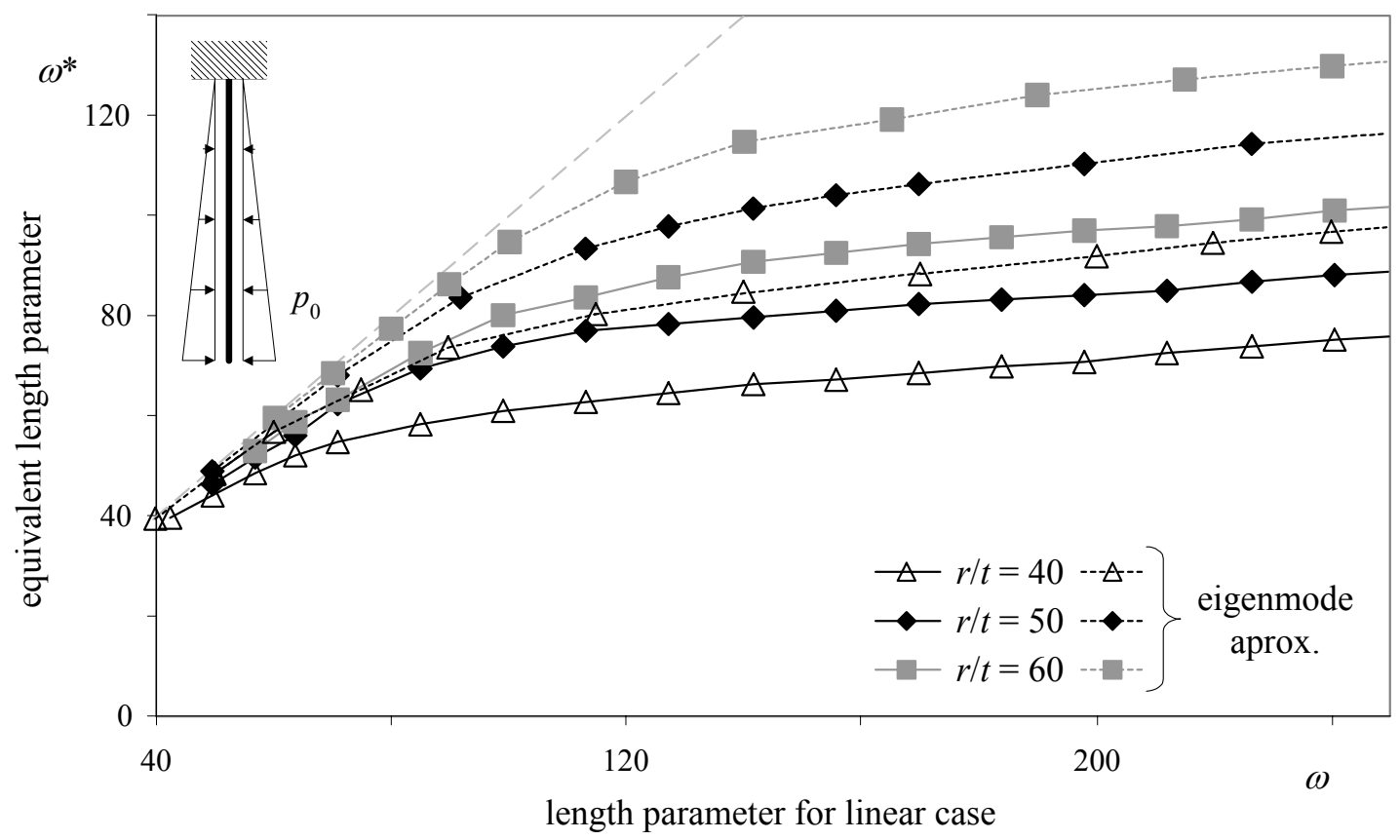

Fig. 11.16: Relationship of length parameter for "linear" system and corresponding equivalent length parameter for "constant" system for $\omega \geq \omega_{\text {long }}$ with results from eigenmode approximations

The results from Fig. 11.16 emphasize the large safety margin present when applying the eigenmode approximation. A possible solution for adopting closer agreement with the results could be to use a scaled-down "constant" eigenmode for matching in Fig. 11.15 ; i.e. the matching is performed with scaled-down deflections $u_{s d}$ adopted e.g.:

$$
u_{s d}=(u+1 / 9) \cdot 0.9
$$

instead of using $u$.

For the pressure distribution ("linear") the mean pressure value is half the pressure amplitude for the "constant" system. This relationship is defined as the load factor $b$ :

$$
b=\frac{\int_{0}^{L} p(z) d z}{p_{0} L}
$$

which is 0.5 for the latter case. The most detrimental load case with $b=0.5$ is a constant pressure "block" extending over half of the length as depicted in Fig. 11.17 as the buckling initiates in the middle. The results are shown as already depicted in Fig. 11.16 for the "linear" case for the "block" load in Fig. 11.17. Again, the approximation with the eigenmode adoption serves as a reliable method for obtaining conservative results for an equivalent length parameter. 


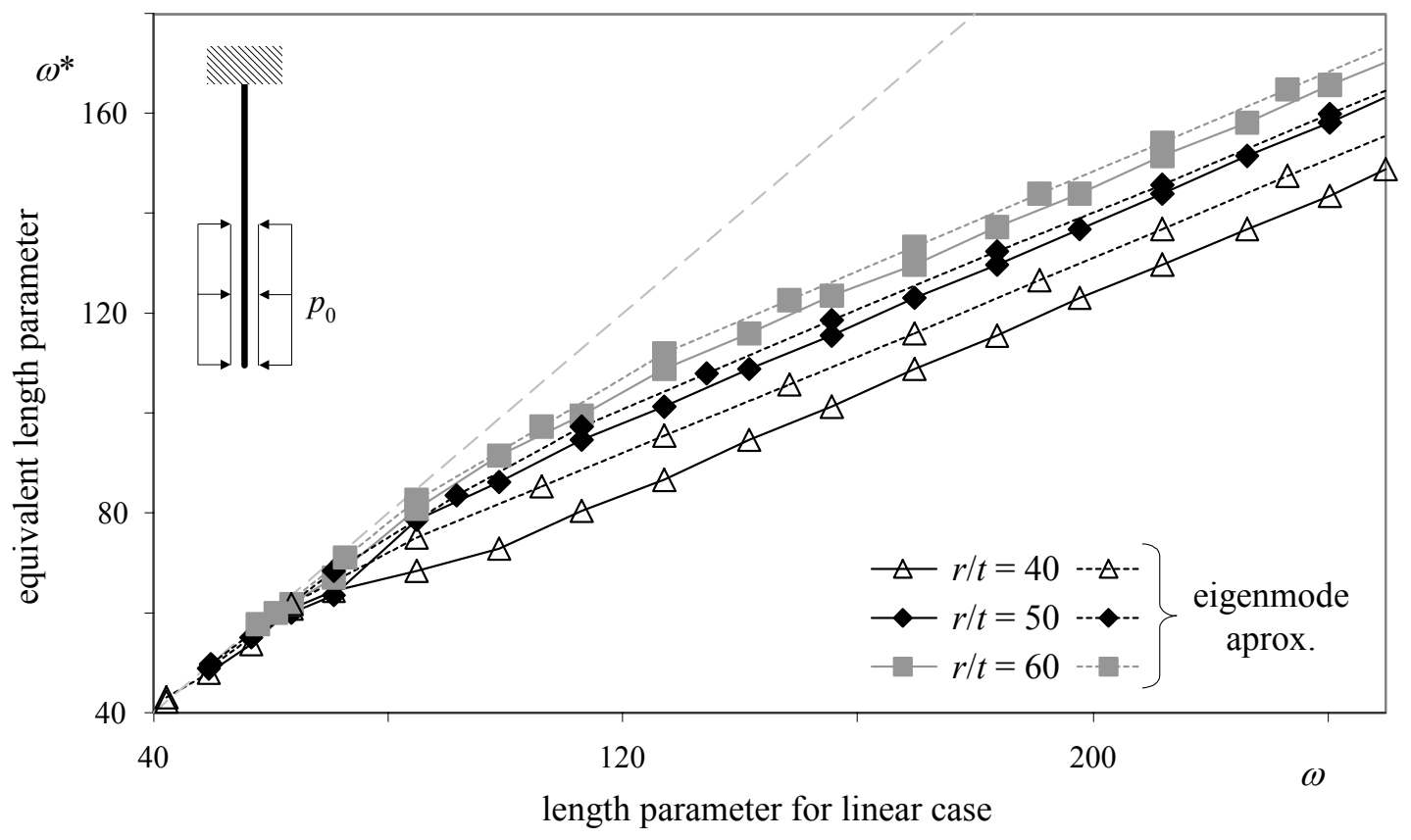

Fig. 11.17: Relationship of length parameter for "block" system and corresponding equivalent length parameter for "constant" system for $\omega \geq \omega_{\text {long }}$ with results from eigenmode approximations

This procedure can be applied for numerous pressure distribution types and load factors $b$. For theses systems individual relationship curves can be derived and plotted. Here, only the two latter cases are re-sketched in Fig. 11.18 and Fig. 11.19 in order to provide a clearer figure for practical application.

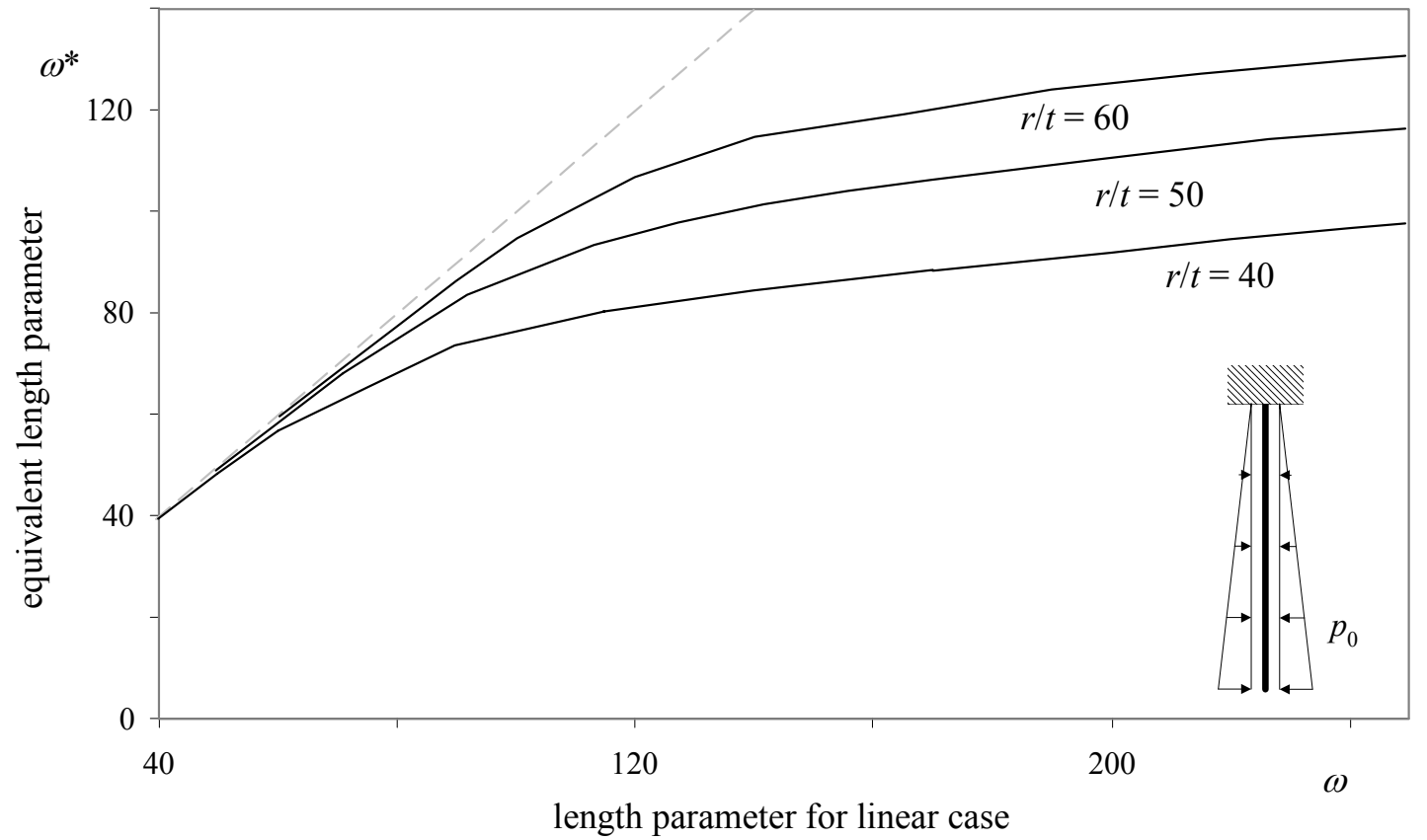

Fig. 11.18: Derived relationship of length parameter for "linear" system and corresponding equivalent length parameter for "constant" system for $\omega \geq \omega_{\text {long }}$ with $b=0.5$ 


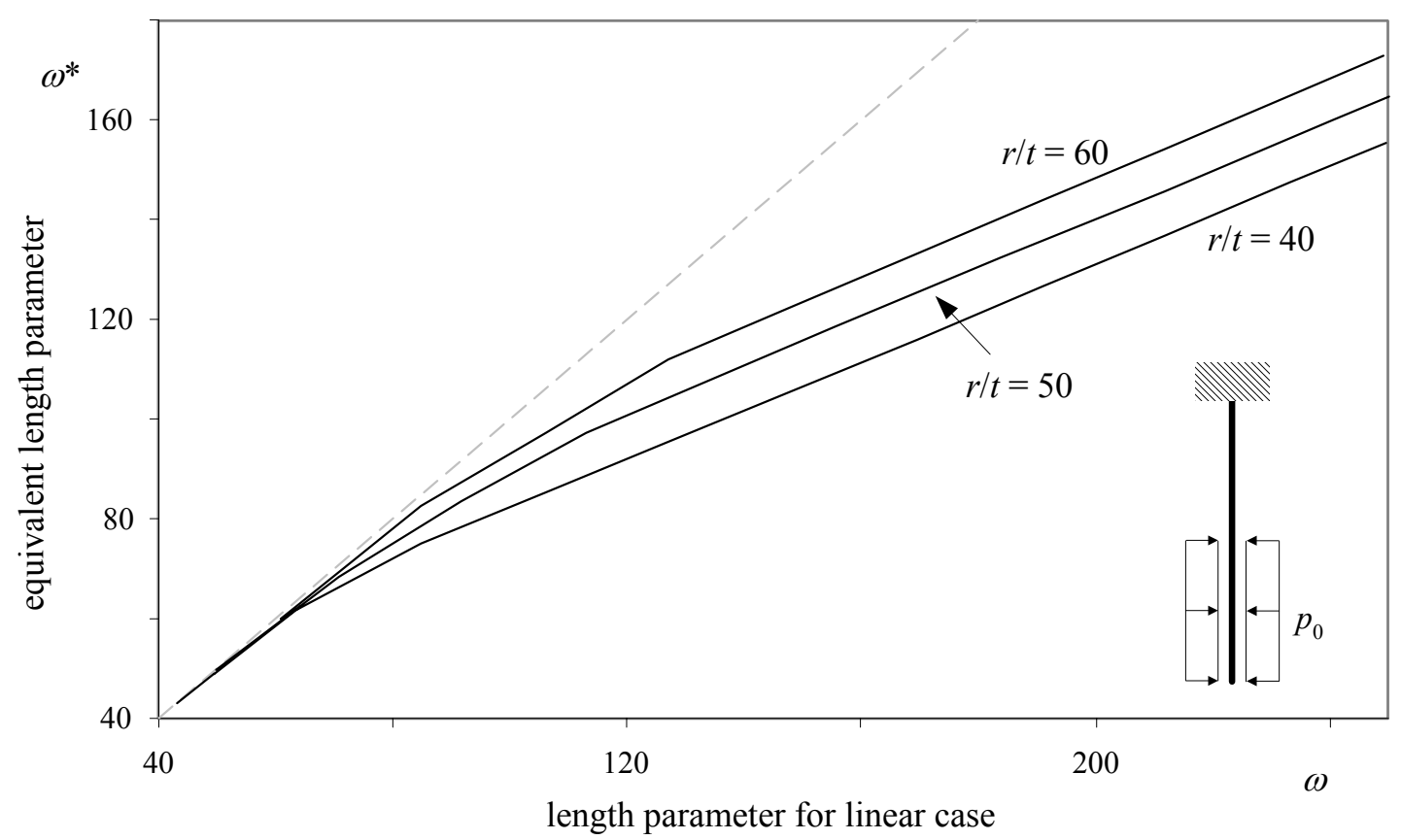

Fig. 11.19: Derived relationship of length parameter for "block" system and corresponding equivalent length parameter for "constant" system for $\omega \geq \omega_{\text {long }}$ with $b=0.5$

\subsection{Evaluation of the elastic buckling reduction factor}

\subsubsection{Definition and origin}

From the previous analyses different ideal buckling loads were obtained for a cylinder subjected to external pressure. For example a more detailed modelling of the loading situation and the boundary conditions leads to a circumferential critical buckling stress of $40 \mathrm{~N} / \mathrm{mm}^{2}$, which is used as circumferential critical buckling stress $\sigma_{\theta, R c}$ from EN 1993-1-6. Based on this value the circumferential buckling resistance $\sigma_{\theta, R d}$ is derived by applying the buckling reduction factor $\chi_{\theta}$. The calculation of $\chi_{\theta}$ depends on slenderness of the shell $\bar{\lambda}_{\theta}$ which is defined as:

$$
\bar{\lambda}_{\theta}=\sqrt{\frac{f_{y, k}}{\sigma_{\theta, R c}}}
$$

with a common yield stress $f_{y, k}$ between $235 \mathrm{~N} / \mathrm{mm}^{2}$ and $430 \mathrm{~N} / \mathrm{mm}^{2}$ and a circumferential critical buckling resistance $\sigma_{\theta, R d}$ between $13 \mathrm{~N} / \mathrm{mm}^{2}$ and $24 \mathrm{~N} / \mathrm{mm}^{2}$ as seen in the different analyses (Tab. 11.3), the slenderness of the shell $\bar{\lambda}_{\theta}$ ranges from 3.1 to 5.8 . The plastic limit relative slenderness $\bar{\lambda}_{\theta p}$ is:

$$
\bar{\lambda}_{\theta p}=\sqrt{\frac{\alpha_{\theta}}{1-\beta}}
$$


where the parameter $\alpha_{\theta}$ is between 0.5 and 0.75 depending on the quality class of the shell and $\beta$ is 0.6 . This leads to a plastic limit relative slenderness $\bar{\lambda}_{\theta p}$ ranging from 1.12 to 1.37 . Since the slenderness of the shell $\bar{\lambda}_{\theta}$ is larger than the plastic limit relative slenderness $\bar{\lambda}_{\theta p}$ for all extreme values, which applies for the investigated range of $r / t$ ratios, the buckling reduction factor is $\chi_{\theta}$ linearly dependent on the circumferential elastic imperfection factor $\alpha_{\theta}$ :

$$
\chi_{\theta}=\frac{\alpha_{\theta}}{\bar{\lambda}_{\theta}^{2}}
$$

i.e. the circumferential critical buckling stress $\sigma_{\theta, R c}$ is directly reduced by the circumferential elastic imperfection factor $\alpha_{\theta}$ depending on the imperfection class in order to obtain the circumferential design or characteristic buckling resistance $\left(\sigma_{\theta, R k}\right.$ or $\left.\sigma_{\theta, R d}\right)$.

$$
\sigma_{\theta, R d}=\frac{\sigma_{\theta, R k}}{\gamma_{m}}=\alpha_{\theta} \frac{\sigma_{\theta, R c}}{\gamma_{m}}
$$

The circumferential elastic imperfection factors $\alpha_{\theta}$ proposed in EN 1993-1-6 result in a reduction from 0.50 to 0.75 depending on the fabrication quality class.

The reduction factor $\alpha_{\theta}$ also appears in the predecessors of the Eurocode. The first general code which commented on the buckling assessment of shells based on buckling rules from different specific codes (e.g. DIN 15018 for cranes) was an amendment to DIN 4114 in 1973 (“Ergänzungserlaß zu DIN 4114”). The code only contained vague and very conservative comments on the buckling assessment due to the lack of experience. As a consequence several buckling investigations were performed. In the first place the pioneering work by Esslinger $(1975,1977)$ needs to be mentioned. Esslinger performed experimental and numerical analyses of cylinders subjected to different loads. Her investigations regarding the postbuckling behaviour of shells formed amongst others the basis for the following design codes. From experiments with Mylar-cylinders loaded with hydrostatic pressure, Esslinger (1969) derived that the ratio of the characteristic buckling load (= postbuckling minimum) and the theoretical buckling load lies between 0.60 and 0.70 , independent on the length of the cylinder. Esslinger $(1971,1976)$ fixes the reduction factor to 0.65 for isotropic cylinders.

Based on the newly obtained knowledge a German guideline appeared: the DAStRichtlinie 013 (1980). The new buckling assessment procedure described by Bornscheuer (1981) is based on the numerous experimental investigations (mainly by Esslinger and e.g. summarized by Schulz, 1981) and uses the $\alpha$-concept, i.e. a reduction factor with a purely empirical relationship between ideal buckling load and buckling resistance. The proposed elastic imperfection reduction factor $\alpha_{\theta}$ for cylinders subjected to external pressure is assumed to be 0.70 for all shells. The reduction factor varies significantly between different guidelines. In the British Standard BS 5500 (1997) and the ECCS-Recommendations (1988) a factor of 0.50 is proposed. A far higher reduction 
factor of 0.80 can be found in the ASME-Code according to the Beuth-Kommentare (1998). The differences arise as all values were fixed empirically based on experiments and some numerical analyses. The experimental results differ due to the influence of boundary conditions and the interaction with axial loading (external pressure on top of cylinder). In DIN 18800-4 (1990) a value of 0.65 is used on the basis of the investigations by Esslinger (cfr above) and by Stracke (1987), as e.g. described by Bornscheuer (1985). Finally, in prEN 1993-1-6:2002-05 three reduction factors 0.75, 0.65 , and 0.50 are given depending on the fabrication quality class $\mathrm{A}, \mathrm{B}$, and $\mathrm{C}$ of the shell, respectively, as stated above.

\subsubsection{Influence of length on buckling load and imperfection sensitivity}

First, two different load cases have to be identified. Since, for the execution of tests a loading with a hydrostatic pressure to the entire cylinder which is sealed with a top and bottom plate is by far easier than applying external pressure to the shell surface only, this case was mostly used for testing. When evaluating test results this aspect needs to be taken care of as the loading type influences the carrying capacity. The hydrostatic load case obviously results in a smaller carrying capacity because the cylinder is subjected to an additional axial loading due to the pressure on the top and bottom plate.

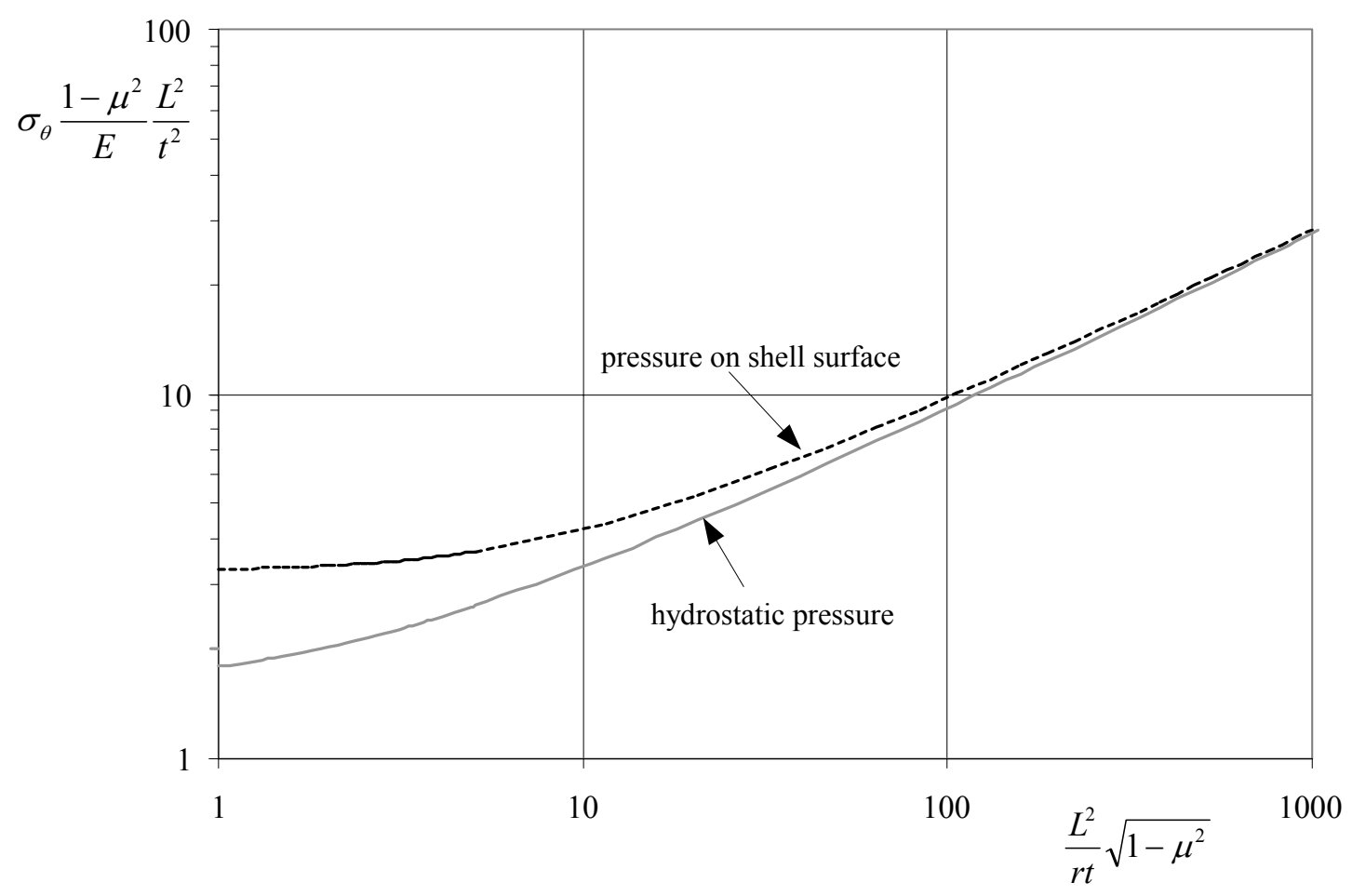

Fig. 11.20: Buckling mode for the reference tube subjected to non-uniform external pressure

Batdorf (1947) derived analytical solutions for the two cases based on linear shell theory. Both solutions for the critical buckling loads are depicted in Fig. 11.20 for increasing length parameters. Here, $\sigma_{\theta}=\sigma_{\theta, R c \text {, lin }}$ corresponds to the linear critical buckling stress. It can be seen that for length parameters $L^{2} /(r t)$ greater than 16 the 
differences between the solution drops below $20 \%$. For longer tubes the difference disappears. This phenomenon can be explained with the fact that the axial buckling load is independent of the length while the circumferential buckling load depends on the boundary conditions. Thus, the pressure on the top and bottom of the cylinder is far more detrimental to the carrying behaviour for shorter cylinders. Further, the diminishing influence of the axial membrane stress for increasing length parameters $L^{2} /(r t)$ for a cylinder subjected to hydrostatic loading can be explained as for these cases the circumferential compressive stress for inducing buckling is by far smaller than the axial compressive stress for inducing buckling. Nevertheless, the relationship between axial and circumferential stress is defined for hydrostatic pressure due to the loading with $\sigma_{x}=\sigma_{\theta} / 2$. The reference case representing a common combiwall structure with $r=900 \mathrm{~mm}$ and $t=18 \mathrm{~mm}$ already reaches the described difference of $20 \%$ for a length of $500 \mathrm{~mm}$. If the choice of the shell's length is $24 \mathrm{~m}$ (pile tip to anchor), the parameter for the abscissa according to Fig. 11.20 can be evaluated with 31000 . Thus, it can be seen that the aspect of the loading type is negligible.

Budiansky and Amazigo (1968) investigated the post-buckling behaviour of externally pressurized cylinders analytically and compared the results to experimental data. They proposed a relationship for the influence of an initial radial imperfection with the amplitude $\Delta w$ on the buckling load as follows:

$$
\alpha_{\theta}=\frac{\sigma_{\theta, R k}}{\sigma_{\theta, R c, \text { lin }}}=1+b\left(\frac{\Delta w}{t}\right)^{2}
$$

in which $\sigma_{\theta, R c \text {, lin }}$ corresponds to the linear critical buckling stress $\sigma_{\theta}$ from Fig. 11.20 for a cylinder without imperfections and to the critical buckling stress $\sigma_{\theta, R c}$ from (11.15) as well, and $b$ is the coefficient defining the influence of the imperfection as a function of the length parameter $L^{2} /(r t)$. They noted that for a length parameter $L^{2} /(r t)>1000$ and reasonably small radial imperfections in relation to the shell thickness the classical critical buckling stress $\sigma_{\theta, R c \text {, lin }}$ for a perfect cylinder ought to be a reliable approximation for the buckling stress of a real structure, i.e. $b \approx 0$ and thus $\alpha_{\theta} \approx 1$. Nevertheless, they emphasized the detrimental effect of imperfections on the buckling load for length parameters in the region $1<L^{2} /(r t)<10$. As described earlier the common combiwall structures tend to fall into the category of very long cylinders $\left(L^{2} /(r t)>1000\right)$ and thus the reduction of the buckling load arising from imperfections seems very small.

In Fig. 11.21 the equations by Batdorf (1947) are compared to the results from experiments performed by Dow (1965) and Windenburg and Trilling (1934) for hydrostatic pressure. Dow (1965) did not comment on the presence of imperfections, while Windenburg and Trilling (1934) measured the maximum out-of-roundness or eccentricity $\Delta w / t$ for the specimens lying between 0.06 and 0.73 . The $r / t$ ratio for cylinders investigated by Dow (1965) was between 70 and 270, and between 260 and 420 in Windenburg and Trilling (1934), respectively. Cleaver (1956) investigated the buckling behaviour of a set of very small and thick-walled steel cylinders. Nevertheless, some results for cylinders with $r / t=51$ are added in Fig. 11.21. The comparison 
depicted in Fig. 11.21 reveals that experimental buckling stresses smaller than the analytical values are obtained for small length parameters $L^{2} /(r t)$. For an increasing length parameter $L^{2} /(r t)$ the experimental values approach the theoretical values and correlate well. In the region of very long tubes the experimental values exceed the theoretical values by up to $40 \%$. This aspect maybe can be explained as the measured buckling stresses are related to the maximum bearable load while the theoretical buckling stress is defined as the stress where buckling initiates. Nevertheless, the comparison emphasizes the small influence of imperfections on long cylinders.

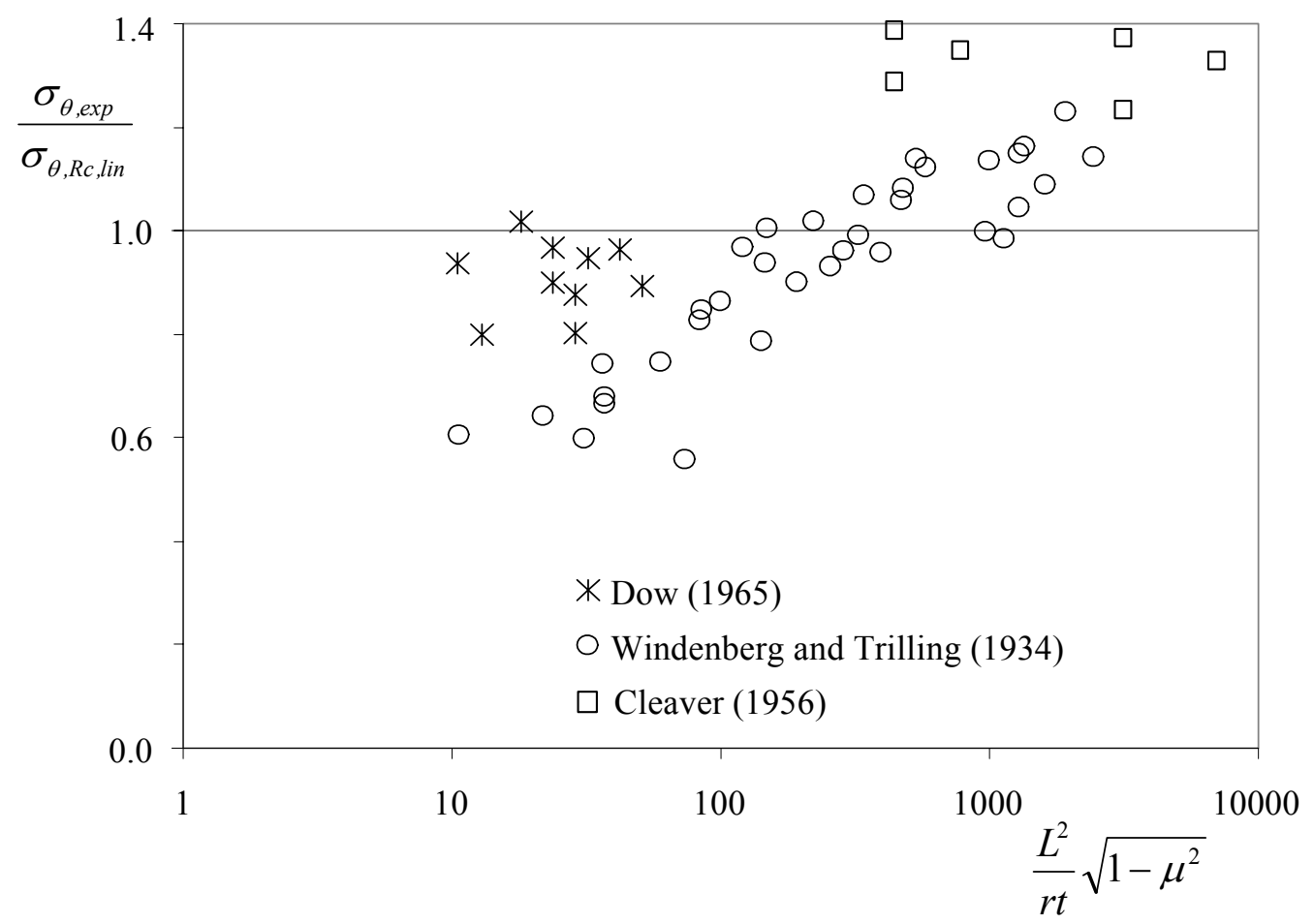

Fig. 11.21: Comparison of analytical and experimental results for hydrostatically pressurized cylinders

Pflüger (1966) investigated the buckling behaviour of cylinders with external pressure only on the shell surface analytically and with experiments. Similar to Budiansky and Amazigo (1968) he based his investigations on the equations by Donnell (e.g. Dierks, 1965) which are only valid for an $l / r<10$. For longer tubes and thus involving lower numbers of buckling waves, the application of the equations by Donnell may lead to overestimations by up to $33 \%$ as described by Kollár and Dulácska (1975). Nevertheless, the results by Pflüger (1966) can be used if they are based on a correctly obtained buckling stress for long cylinders. Based on an imperfection amplitude of $\Delta w=r / 200$ he derived the following function for the reduction factor $\alpha_{\theta}$ for an imperfect cylinder:

$$
\alpha_{\theta}=\frac{\sigma_{\theta, R k}}{\sigma_{\theta, R c, l i n}}=\frac{1}{3}+\frac{2}{3+\sqrt{r^{2} / 100 L t}}
$$


For a cylinder with $r / t=50$ (reference case) the chosen imperfection amplitude can be written as $\Delta w / t=0.25$, and the reduction factor $\alpha_{\theta}=0.97$ for $L=24 \mathrm{~m}$ (pile tip to anchor), $t=18 \mathrm{~mm}$, and $r=900 \mathrm{~mm}$. Similar to the results from Budiansky and Amazigo (1968) it can bee seen that the influence of imperfections seems to be small for the investigated case. Pflüger (1966) compared his proposed solution with test results from Cleaver (1956) involving elastic buckling loads with $1.8<r^{2} /(L t)<17$ and his own test results with $125<r^{2} /(L t)<1600$ leading to satisfactory correlations. Still, it has to be mentioned that the specimens investigated by Cleaver (1956) were very small, i.e. with a maximum radius of about $30 \mathrm{~mm}$. Here, it is also of interest to refer to the work by Schneider and Brede (2005) who proposed to use a single longitudinal dent instead of eigenform affine shape imperfections. They investigated externally pressurized cylinders by numerical means and found out that for different boundary conditions and large imperfection amplitudes the reduction factors as given in prEN 1993-1-6:2002-05 cannot be reached. It is emphasized that shells are currently assessed with highly conservative reduction factors. Further, the research revealed an significantly decreasing effect of geometric imperfections on the buckling load for increasing lengths of cylinders, i.e. slender cylindrical shells.

Additionally, a numerical study is performed. Based on a $90^{\circ}$ segment of a cylinder, the imperfection sensitivity of an externally pressurized shell is investigated for different length parameters $L^{2} /(r t)$. In order to obtain information on the influence of imperfections on the buckling load a fixed imperfection is imposed to the shell independent of the length of the cylinder. The geometry and the boundary conditions are depicted in Fig. 11.22. The shell segment (quarter cylinder) is clamped along both circumferential boundaries. The upper boundary is allowed to move in direction 3 (vertically) freely. An inward dimple or bulge with the amplitude $\Delta w / t=1.0$ is modelled in the centre of the shell segment. The extent of the bulge is chosen according to the gauge length $l_{g}$ given in prEN 1993-1-6:2002-05 for the dimple tolerances.

$$
l_{g x}=4 \sqrt{r t}
$$

if meridional compressive stresses are present and

$$
l_{g \theta}=2.3\left(L^{2} r t\right)^{0.25} \quad \text { but } l_{g \theta} \leq r
$$

if circumferential compressive stresses are present. Evaluating (11.18) for a constant radius $r=900 \mathrm{~mm}$ and a constant thickness $t=18 \mathrm{~mm}$ results in the gauge length $l_{g x} \approx 500 \mathrm{~mm}$. It is intended to investigate the influence of a length-independent imperfection on the buckling load of cylinders with varying lengths. As the gauge length $l_{g \theta}$ from (11.19) is $820 \mathrm{~mm}$ for $L=1000 \mathrm{~mm}$ and increases with increasing shell lengths, and the imperfection size shall be fixed, the extent of the bulge $b_{b} \times h_{b}$ is set to $500 \mathrm{~mm}$ x $500 \mathrm{~mm}$ (bulge A) based on the axial gauge length $l_{g x}$. 


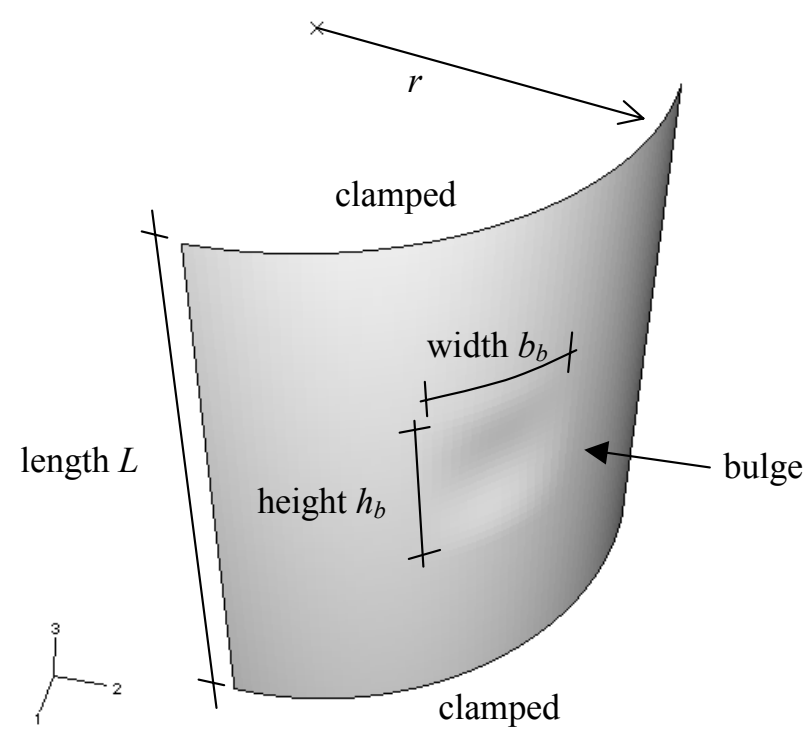

Fig. 11.22: Geometry and boundary conditions for the numerical study with bulge

For the shape of the bulge a complete cosine wave is used in both directions. The position and the dimension of the bulge are kept for all lengths. Due to the symmetry of the system the cylinder posses four bulges in total. A Young's modulus of $210000 \mathrm{~N} / \mathrm{mm}^{2}$ and a Poisson ratio of 0.3 is used for the analyses. In a first step the circumferential buckling stresses $\sigma_{\theta, p e r f}$ are obtained for perfect cylinders. Buckling modes involving even and uneven numbers of buckling waves were taken account of by varying the boundary conditions along the longitudinal edges (symmetric and asymmetric).

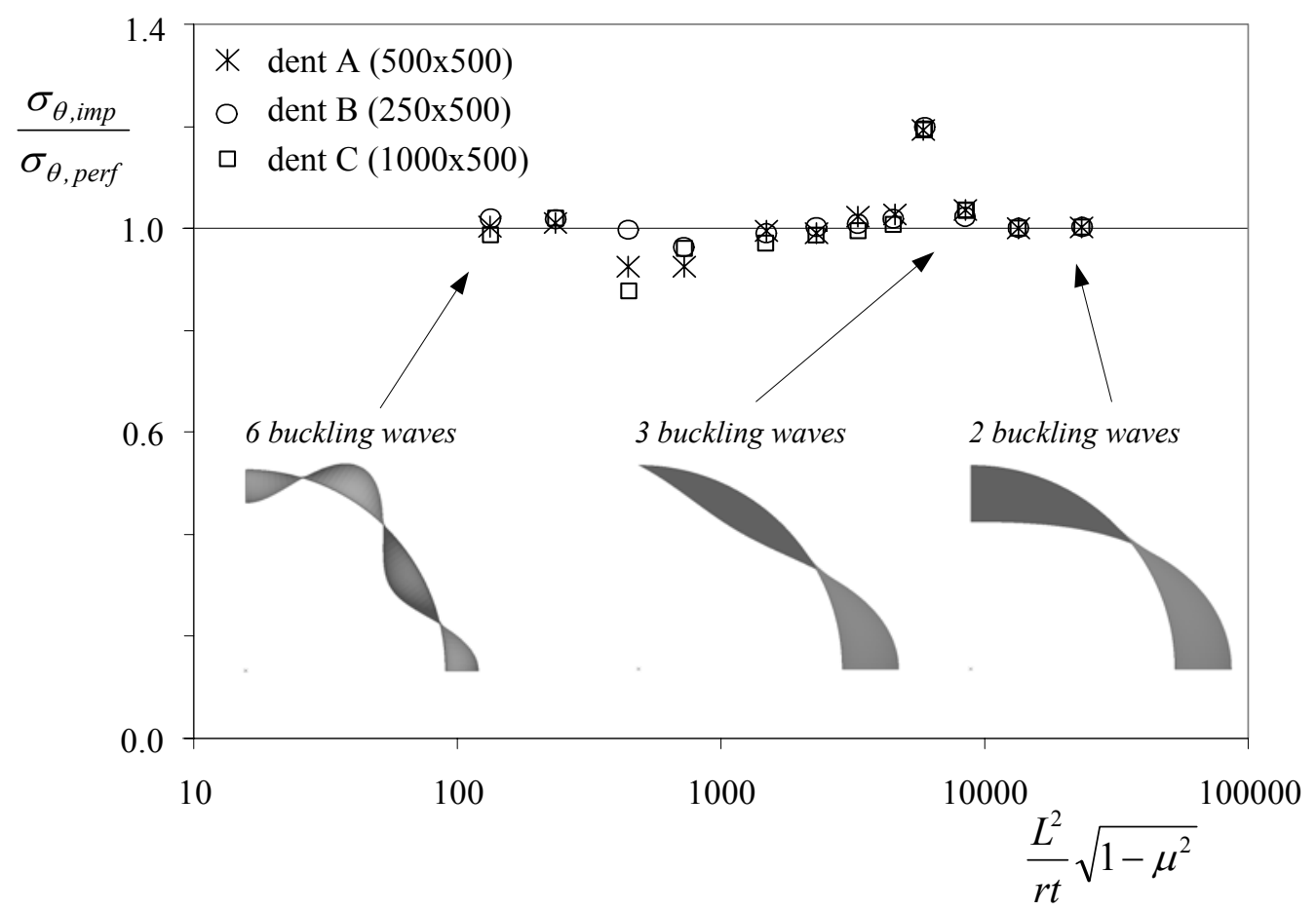

Fig. 11.23: Comparison of numerical results for different imperfection patterns 
The projected buckling modes (quarter cylinder) of three linear bifurcation analyses are depicted in Fig. 11.23. In Fig. 11.23 the perfect buckling stresses $\sigma_{\theta, p e r f}$ are compared to the imperfect buckling stresses $\sigma_{\theta, \text { imp }}$ obtained from non-linear load-displacement analyses as a function of the length parameter $L^{2} /(r t)$. Fig. 11.23 includes results for a narrower (bulge B) and a wider bulge (bulge C).

For cylinders with large imperfections no snap trough occurs for the cylinders investigated in the study. The load deflection curves increase continuously and the equilibrium does not become instable. For these cylinders the carrying capacity is not limited by failure due to buckling but by excessive displacements. Similar results were found for large initial imperfections by e.g. Thielemann and Esslinger (1967).

No significant influence of the initial imperfection can be seen in Fig. 11.23. The results for the different imperfection shapes do not differ much. Since the critical number of buckling waves over the circumference changes with the length, the carrying capacity of the cylinders is differently influenced by the initial bulge. In some cases the extension and the position of the bulge may amplify the critical buckling wave pattern and in other cases they may force the cylinder to fail in a different buckling pattern, and thus increase the carrying capacity. Generally, the non-linear analyses with the imperfect cylinders yield similar results to the linear bifurcation analyses. A slight tendency of increasing buckling stresses appears for increasing lengths as no reductions (ratio < 1.0) occur for longer cylinders.

In order to investigate the influence of a fixed imperfection on the carrying capacity of a cylinder subjected to a non-uniform external pressure distribution, the loading from the previous example is changed. The non-uniform external pressure distribution is chosen according to the results obtained from chapter 6 . In the example the pressure is distributed in the shape of a cosine function. Two different types are used: cosine function with and without inflexion point. The load is applied symmetrically maintaining the system in equilibrium (Fig. 11.24).
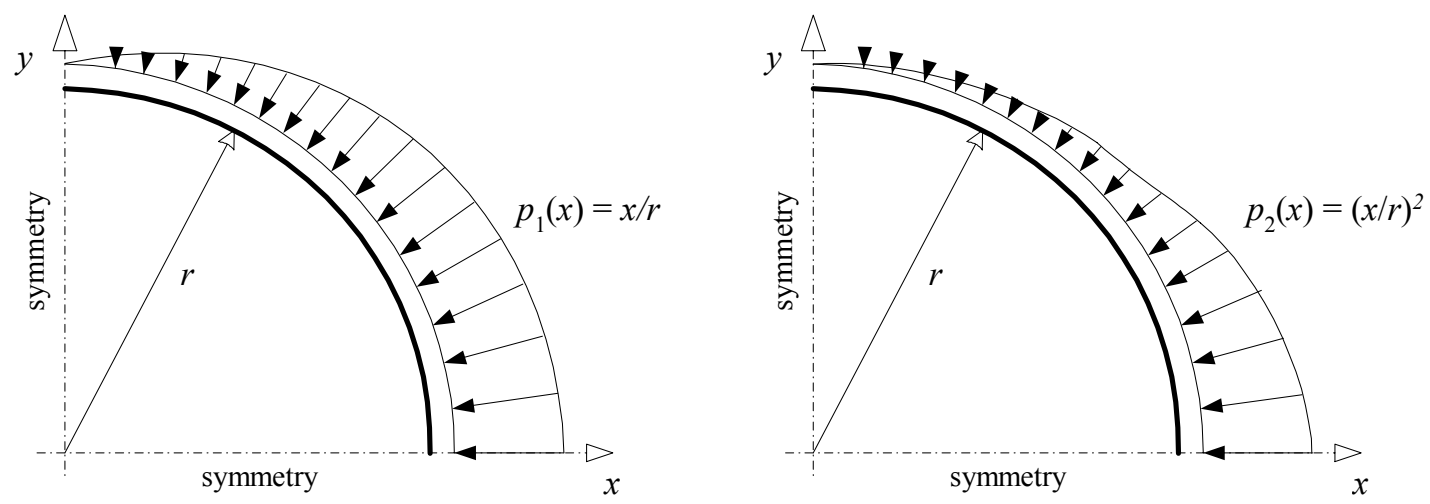

Fig. 11.24: Non-uniform loadings $p_{1}$ and $p_{2}$ of the quarter cylinder applying a cosine distribution 


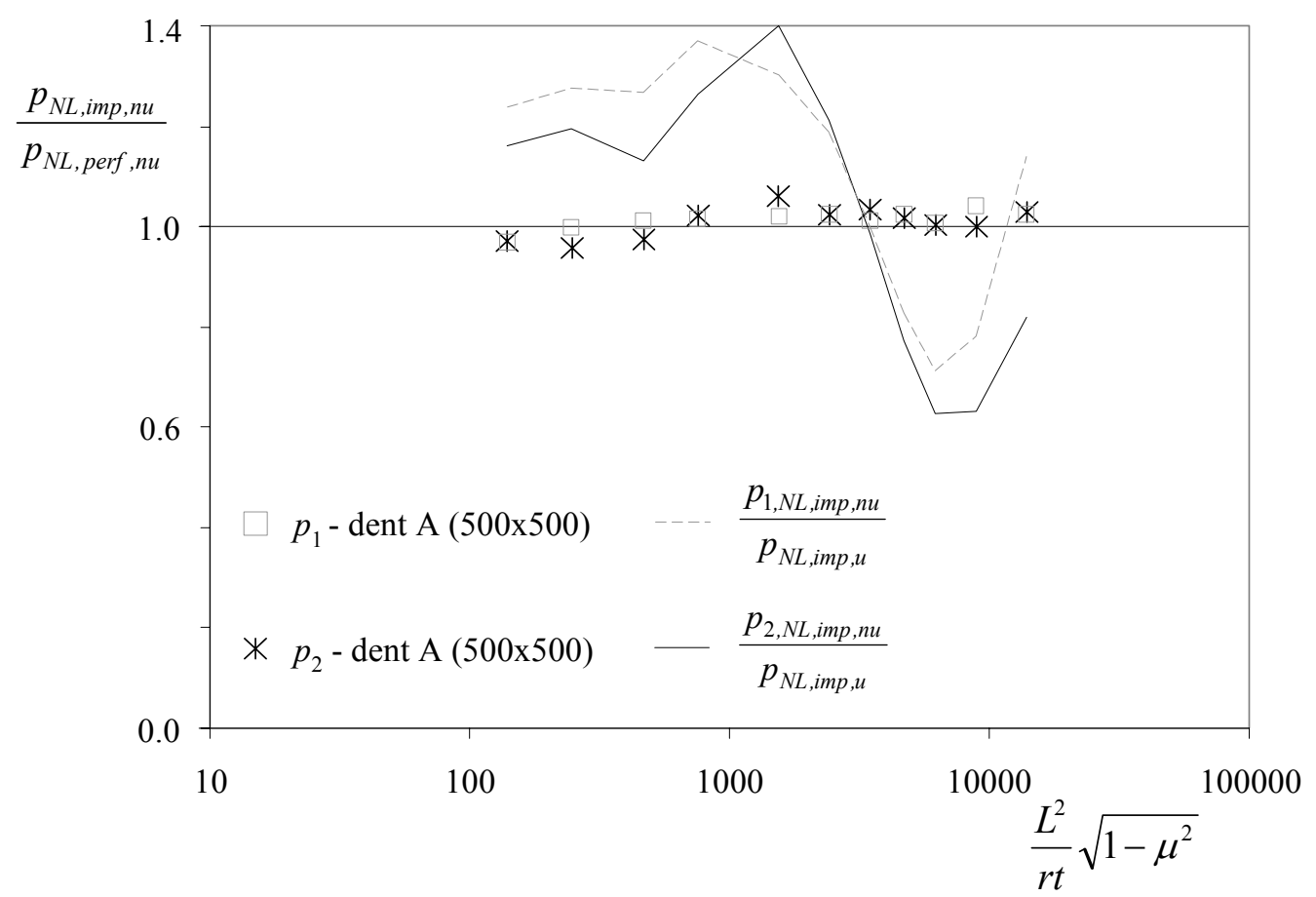

Fig. 11.25: Comparison of numerical results for different imperfection patterns with non-uniform pressure

In Fig. 11.25 the results for the different non-uniform loadings are depicted for dent A $\left(500 \mathrm{~mm} \times 500 \mathrm{~mm}\right.$ ) as a function of the length parameter $L^{2} /(r t)$. The ordinate gives the ratio of the carrying capacity (external pressure $p$ ) obtained from a geometrical nonlinear load displacement analysis for an imperfect shell $\left(p_{N L, i m p}\right)$ over the carrying capacity for a perfect shell $\left(p_{N L, p e r f}\right)$. In case of the non-uniform pressure distribution the maximum ordinates of the pressure distributions, i.e. perfect and imperfect, are compared. For the non-uniform loading it is chosen to use the latter ratio as the carrying behaviour is highly influenced by the non-uniformness of the pressure distribution in combination with an analyses calculating the equilibrium of the system based on the deformed structure.

Additionally, Fig. 11.25 depicts the ratio of the geometrical non-linear buckling pressure for an imperfect cylinder subjected to non-uniform pressure $(n u)$ over the geometrical non-linear buckling pressure for an imperfect cylinder subjected to uniform pressure $(u)$ as shown in Fig. 11.23. As expected the analyses for the uniform pressure yield lower buckling pressures for length parameters $L^{2} /(r t)<3500$. Long cylinders with $L^{2} /(r t)>3500$ fail with buckling modes involving only 3 or 2 buckling waves (Fig. 11.23). Thus, shape and size of the buckling waves correlate with the applied pressure distribution, resulting in a detrimental influence for these cases. Here, smaller carrying capacities are found for the non-uniform than for the uniform pressure distribution based on the same maximum amplitude.

Similar to the analyses with uniform pressure, the influence of the imposed bulge is fairly small. Again, a slight increase of the pressure ratio can be seen for increasing 
length parameters $L^{2} /(r t)$. The findings emphasize the small influence of an arbitrary imperfection on the buckling load for long and very long cylinders in contrast to the known large imperfection sensitivity for short cylinders. The results for the different non-uniform loadings do not differ much. No results are given for the longest investigated cylinder in contrast to Fig. 11.23 as no snap trough occurred in the analyses but the limit load is defined by the plastic limit load.

\subsubsection{Structural imperfections}

The reduction factors for external pressure found in design codes take into account the influence of geometrical imperfections as discussed in section 11.6.2, and the influence of structural imperfections. Besides inconsistencies of material properties the latter aspect covers as the major issue incorporated residual stresses in the steel shells. These residual stresses may appear due to the plate rolling procedure needed for constructing cylindrical shells and the process used for joining the curved plates or panels. Even the manufacturing of seamless tubes induces a residual stress state. The presence of residual stresses may decrease the buckling load of the shells as stated by Hübner (2006).

In order to investigate the influence of residual stresses on the carrying capacity of externally pressurized cylinders a numerical parametric study is performed. Based on the study by Schneider and Brede (2005) a cylinder with $r=2000 \mathrm{~mm}, r / t=400$, and a slenderness ratio $h / r=3.0$ is chosen. The steel is assumed to be elastic-perfectly plastic with an elastic modulus $E=210000 \mathrm{~N} / \mathrm{mm}^{2}$, a yield stress $f_{y}=240 \mathrm{~N} / \mathrm{mm}^{2}$ and a Poisson's ratio $\mu=0.3$. Warping-free boundary conditions $\mathrm{BC} 1 \mathrm{r}-\mathrm{BC} 1 \mathrm{r}$ according to prEN 1993-1-6:2002-05 are imposed. It is assumed that the cylinder is manufactured by bending two plates and subsequently joining the straight edges by welding. Here, two different types of residual stress states are investigated: residual stresses from (a) the bending process and (b) from the welding process. In this step no additional geometric imperfections are taken into account. Nevertheless, an additional static step resulting in deformations is needed in the numerical analyses in order to obtain equilibrium after imposing the initial stress state. Only a $90^{\circ}$ segment of the cylinder is modelled by taking advantage of symmetry conditions.

First, residual stresses (a) from the bending process are investigated. Research is mostly performed in the area of rectangular hollow-sections which are manufactured from circular tubes. Here, the rolling process induces additional residual stresses. Nevertheless, as even the rolling process of the plate from which cylindrical steel shells are constructed lead to residual stresses, it can be seen that the residual stress distribution arising from the manufacturing of a steel tube or cylindrical steel shell depends a lot on the process and the geometry - even without considering the joining. Thus, in this investigation it is intended to assume reasonable but simple stress distributions in order to study the influence of residual stresses on the buckling behaviour. Therefore, the residual stress distribution is derived by considering the gradual plastification of the cross section subjected to a bending moment. Since the 
radius $r$ is defined, the corresponding curvature of the bent plate must be equal to the plastic (residual) curvature $\kappa_{p l}$ remaining after the elastic unloading of the plate subjected to the bending moment:

$$
\kappa_{p l}=\frac{1}{r}
$$

The derivation of the moment-curvature relationship for a rectangular cross-section given in equation (11.21) in shown in Fig. 11.26 can be found in Petersen (1997) in detail.

$$
\frac{\kappa_{c}}{\kappa_{e l}}=\frac{1}{\sqrt{3\left(1-\frac{M}{1.5 M_{e l}}\right)}}
$$

With the arbitrary curvature $\kappa_{c}$, the elastic curvature $\kappa_{e l}$, the arbitrary moment $M$, and the elastic limit moment $M_{e l}$. The normalized length $\eta_{L}$ can be calculated with:

$$
\eta_{L}=\frac{\kappa_{e l}}{\kappa_{c}}
$$

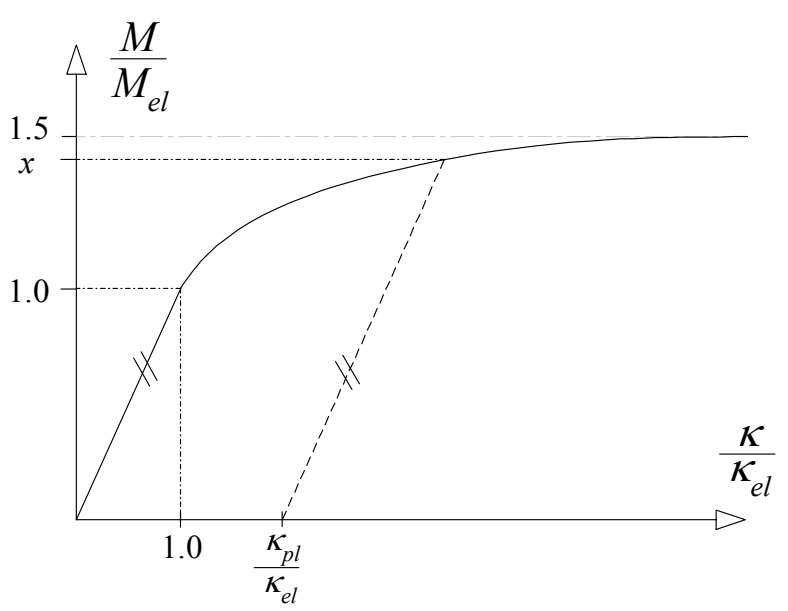

Fig. 11.26: Moment-curvature curve for rectangular cross-section

For calculating the corresponding moment ratio $x$ the intersection of the linear elastic unloading and the moment-curvature curve needs to be calculated as seen in Fig. 11.26.

Thus, the residual stress distributions can be calculated based on the previous equations for the example case with bending and unloading. Consequently, this approach results in tensile stresses at the inside and compressive stresses at the outside of the curved plate. The calculated residual stress distributions for further application are summarized in Fig. 11.27. Additionally, the hypothetical case $400^{*}$ is defined using the geometry of $r / t=400$ but the theoretical residual stress distribution corresponding to full plastification and unloading. 


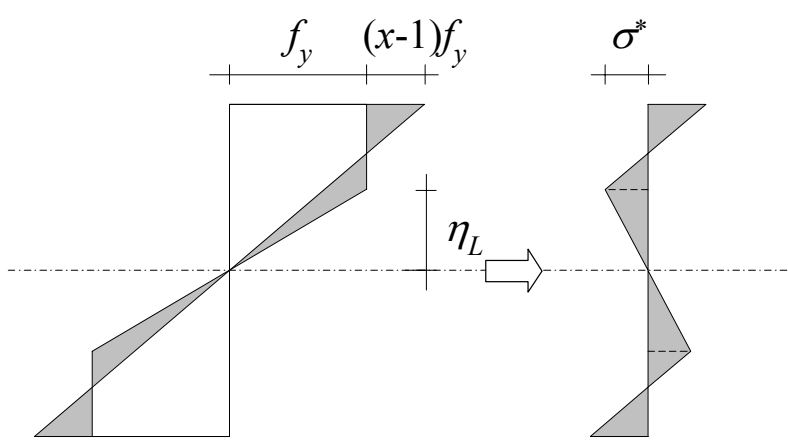

\begin{tabular}{|l|c|c|c|c|}
\hline$r / t$ & - & 400 & $400 *$ & 800 \\
\hline$(x-1) f_{y}$ & $\mathrm{~N} / \mathrm{mm}^{2}$ & 101 & 120 & 87 \\
\hline$\sigma^{*}$ & $\mathrm{~N} / \mathrm{mm}^{2}$ & 104 & 240 & 69 \\
\hline$\eta_{L}$ & - & 0.40 & 0.00 & 0.52 \\
\hline
\end{tabular}

Fig. 11.27: Residual stress distributions for different $\mathrm{r} / \mathrm{t}$ ratios with $f_{y}=240 \mathrm{~N} / \mathrm{mm}^{2}$

In the following step, the buckling analysis is performed. It consists of three steps. In the first step a linear bifurcation analysis (LA) is performed providing the critical buckling pressure $p_{c r, L A}$. In the second step, the eigenform extracted from this analysis is used as geometrical imperfection with an amplitude of $\Delta w / t$. Additionally, the residual stress distribution is introduced requiring a static equilibrium calculation. In the last step (geometrical and material non-linear), the cylindrical shell is loaded with external pressure for obtaining the buckling pressure $p_{\text {GMNIA }}$.

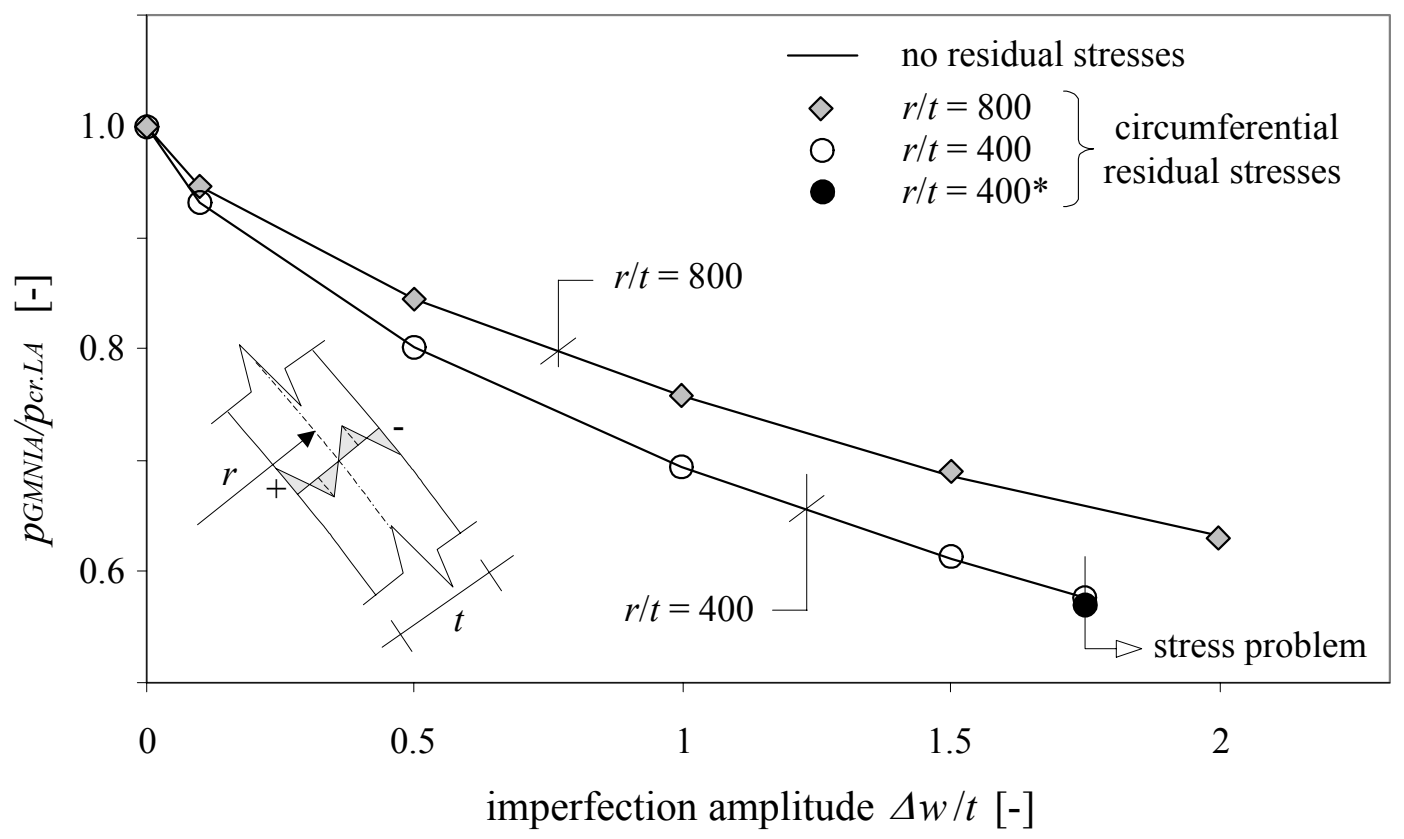

Fig. 11.28: Influence of circumferential bending residual stresses (a) on buckling load of an externally pressurized cylinder for different $r / t$ ratios and imperfection amplitudes $\Delta w / t$ with $h / r=3.0$

In Fig. 11.28 the results of several numerical analyses are summarized for different imperfection amplitudes and the additional $r / t$ ratio 800. For large imperfection amplitudes the problem changes from a buckling problem to a stress problem; as e.g. described by Schneider and Brede (2005), too. It can be seen that the applied stress distribution leads to a negligible reduction of the buckling load for all investigated cases. For $r / t=800$ and $\Delta w / t=2.0$ the analysis considering residual stresses leads to a buckling load $0.3 \%$ smaller than without residual stresses. Even for the limit case $400 *$ 
the reduction of the buckling pressure is smaller than $1 \%$ for $\Delta w / t=1.75$, since the residual stresses at the outer fibres remain far below the yield stress. Based on the performed analyses it can be assumed that the influence of circumferential residual stresses may play a noticeable role for thin-walled cylindrical shells with large imperfections and large residual stress amplitudes. Nevertheless, it appears that even for these cases the influence of the residual stresses on the buckling pressure is very small.

To investigate the influence of residual stresses (b) due to joining/welding two different approaches are used. The studied case consists of a $90^{\circ}$ segment of the cylindrical shell with one weld along one of the straight longitudinal edges. Thus, considering the symmetry conditions, the investigated cylinder comprises two half-shells joined by two longitudinal welds. In the approach type A the block strain approach described in Hübner et al. (2006) is applied. Here, an area extending $4 t$ to each side of the weld centre is subjected to a shrinkage strain representing the effect of the welding process. The arising deformations and residual stresses are then used in the next step of the analysis as geometrical and structural imperfections. The amplitude of the geometrical imperfection can be defined by controlling the magnitude of the imposed strain. In this context only two different strain amplitudes are calculated. The comparison of the buckling pressure for the analyses $(r / t=400)$ with and without residual stresses can be found in Fig. 11.29.

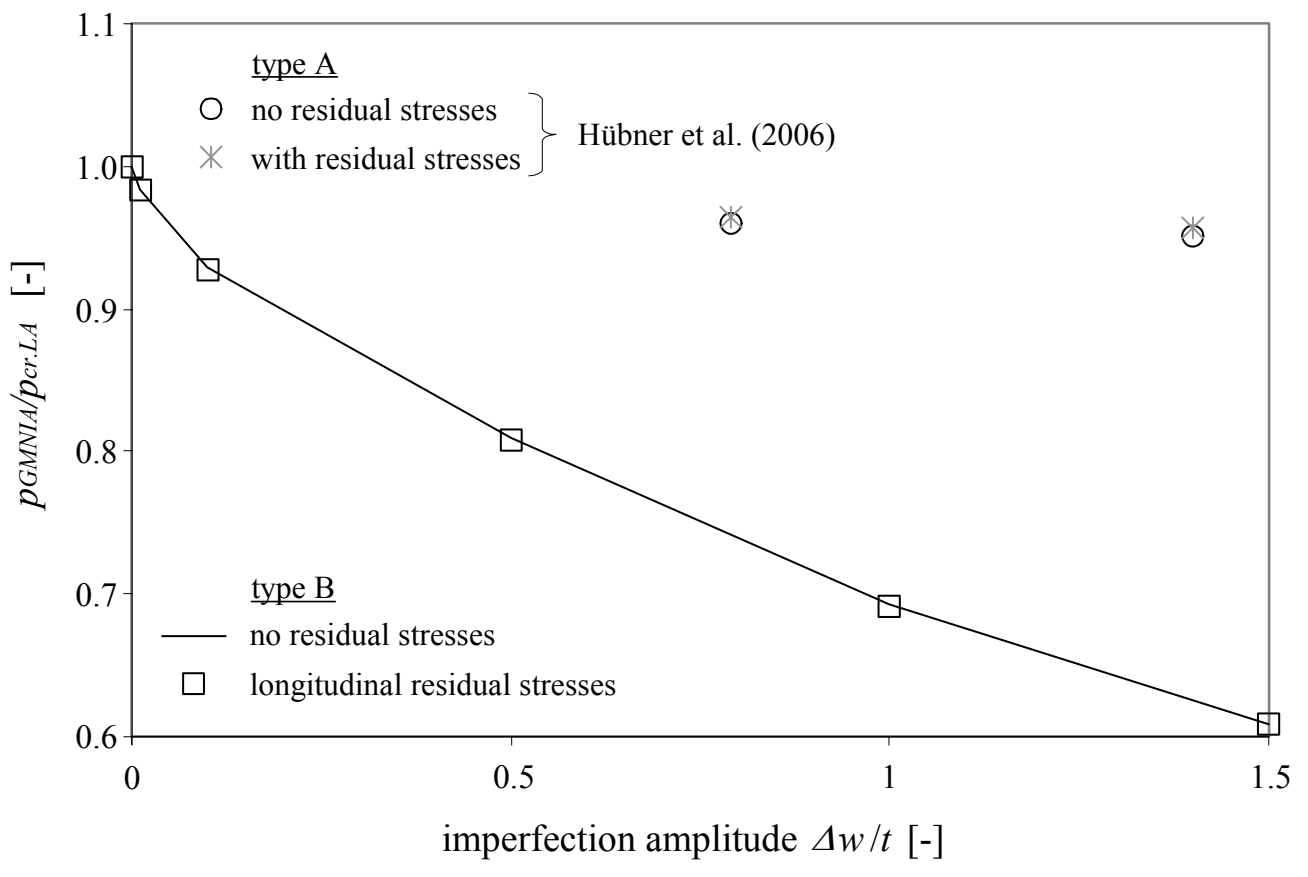

Fig. 11.29: Influence of welding residual stresses (b) on buckling load of an externally pressurized cylinder for $r / t=400$ and different imperfection amplitudes $\Delta w / t$ with $h / r=3.0$

For the investigated cases a minor increase of the buckling load can be found if residual stresses are taken into account. The analyses found in Hübner et al. (2005) indicated a considerable beneficial influence of the residual stresses in the buckling analyses. In the latter research it was pointed out that residual stresses may sometimes lead to an 
increase of the buckling load. Nevertheless, for the cases from Fig. 11.29 the influence appear to be insignificant. Here, it needs to be mentioned that both geometries and the imposed weld patterns differ and thus affect the buckling behaviour. Further, the studies show how large the influence of the geometrical imperfections in combination with the geometry of the cylindrical shell is. A second set of calculations is based on the approach type B. Here, the geometrical imperfections are chosen as eigenform affine and the structural imperfections are induced by imposing a stress field on the cylindrical shell. The stress state is chosen according to the proposal by different researchers (Hübner et al., 2006). Since, in this context no detailed study of residual stresses is to be performed, a simple distribution is chosen: longitudinal tensile stresses extending $4 t$ to each side of the weld centre with an amplitude $\sigma=f_{y}$; for equilibrium compressive longitudinal stresses at both sides in the area $4 t$ to $20 t$ from the weld centre with $\sigma=0.25 f_{y}$ are imposed. In the first step of the numerical analyses a static step without loads is introduced for obtaining equilibrium for the initial stress state. Again, no significant reduction or increase of the buckling load can be found if residual stresses are taken into account (Fig. 11.29).

In this study the influence of residual stresses has been briefly investigated and discussed for an example case found in Schneider and Brede (2005). The specimens used in experimental investigations are prone to geometrical and structural imperfections. Generally, the main focus in interpreting the results and deriving reduction factors for design is applied to the geometrical imperfections. It can be seen from the latter study and the investigations by Hübner et al. (2006) that the presence of residual stresses may reduce the carrying capacity of cylindrical steel shells: particularly for thin-walled cylinders and for residual stresses induced due to rolling/forming of plates. Nevertheless, it appears that for standard cases and external pressure the effect on the buckling pressure is rather small. Still, the aspect of residual stresses requires more detailed research for different loadings. Current considerations of residual stresses found in design codes may be not sufficiently detailed for these cases; e.g. the reduction factor $\alpha_{x}$ from EN 1993-1-6 includes an additional safety factor $\gamma=0.75$ originating from the ECCS-Recommendations (1998) and explained by Rotter (1998) for covering non-geometric imperfections as irregular loading, boundary imperfections, and structural imperfections. The influence of residual stresses on externally pressurized cylinders appears to be negligible for the geometry range of tubular piles used in combiwalls. Already the change from $r / t=800$ to $r / t=400$ reduces the small influence of the residual stresses nearly to zero as seen in Fig. 11.28 for the investigated stress amplitudes. Thus, it is assumed that the effect of residual stresses for common tubular pile structures $(r / t \approx 50)$ does not play a significant role.

\subsubsection{Limit case - buckling of a ring}

The buckling mode of the entire system (Fig. 11.3) and the previous analyses give rise to the question whether the buckling failure of the system is more the buckling of a circular ring than the buckling of a shell with external pressure. Hence, some comparing 
analyses and investigations are performed. The comparison is based on the given geometry of the reference case with the increased radius by ovalization $a(=936 \mathrm{~mm})$. For a circular ring subjected to a uniform pressure $p$ an analytical solution (cfr. Young, 1989, or Pflüger, 1964) is available reflecting the failure due to column buckling:

$$
q=\frac{3 E I}{r^{3}}
$$

where $I$ is the moment of inertia for the ring's cross section per unit of length. The analytical solution from (11.23) is valid for a load remaining normal to the surface. This loading type appears for the investigated case. For loads which maintain their direction the factor 3 in equation (11.23) is replaced by the factor 4 . In Tab. 11.4 the results for the circumferential critical buckling stress $\sigma_{\theta, R c}$ for different shell lengths and loadings are summarized for $r / t=936 / 18$.

Tab. 11.4: Comparison of circumferential critical buckling stress $\sigma_{\theta, R c}$

\begin{tabular}{|c|c|c|c|c|c|c|}
\hline \multirow{2}{*}{ No. } & \multicolumn{2}{|c|}{ pressure in direction } & \multirow{2}{*}{ method } & \multirow{2}{*}{$\begin{array}{c}\text { length } \\
{[\mathrm{m}]}\end{array}$} & \multirow{2}{*}{$\begin{array}{c}\sigma_{\theta, c r} \\
{\left[N / \mathrm{mm}^{2}\right]}\end{array}$} & \multirow{2}{*}{ comment } \\
\hline & axial & circumferential & & & & \\
\hline 1 & \multirow{6}{*}{\multicolumn{2}{|c|}{ constant }} & analytical & \multirow{4}{*}{ ring } & \multirow{2}{*}{19.3} & \multirow{2}{*}{$\mu=0$} \\
\hline 2 & & & FEM & & & \\
\hline \multirow[b]{2}{*}{4} & & & analytical & & \multirow{2}{*}{21.2} & \multirow{2}{*}{ considering $\left(1-\mu^{2}\right)$} \\
\hline & & & FEM & & & \\
\hline \multirow[b]{2}{*}{6} & & & EN 1993-1-6 & $\infty$ & 21.3 & $\omega=\infty$ \\
\hline & & & \multirow{5}{*}{ FEM } & \multirow{2}{*}{24} & 21.8 & \multirow{4}{*}{$\mathrm{BC}$ pinned-free } \\
\hline 7 & linear & constant & & & 24.5 & \\
\hline 8 & \multicolumn{2}{|c|}{ constant } & & \multirow{2}{*}{50} & 21.7 & \\
\hline 9 & linear & constant & & & 23.2 & \\
\hline 10 & \multicolumn{2}{|c|}{ varies (real) } & & $24+6$ & 40.0 & complex BC \\
\hline
\end{tabular}

The comparison shows the influence of the Poisson ratio of 0.3 for the cases 1 to 4 which does not affect the 1D problem but takes place for shells. Deriving the critical buckling stress according to EN 1993-1-6 for an infinite cylinder results in nearly the same value. For uniform pressure on the reference case $(24 \mathrm{~m})$ the ideal buckling stress differs only slightly from the previous cases. This aspect confirms that the shell buckling behaviour of the reference case is rather a column buckling problem of a circular ring than a shell buckling problem. The influence of a pressure distribution depending linearly on the depth seems rather small and decreases for longer cylinders. The application of the more detailed boundary conditions and a pressure distribution depending on depth and circumferential position, results in a significant increase of the buckling load. This emphasizes the importance of considering the variation of the 
external pressure over the circumference. The buckling eigenform may slightly differ between the complex case and the ring subjected to external pressure. For the complex situation inflexion points arise resulting in a symmetric two-wave mode (shaped as an " 8 ") as depicted in Fig. 11.3. For the ring the inflexion points may not be as distinct as for the latter case, i.e. it may rather appear to be an ellipse shaped buckling mode.

Since the failure mode of the externally pressurized ring or shell is similar to the column buckling problem, the design procedure is compared to the assessment of a compressed member prone to failure due to buckling according to the German code for constructional steelworks DIN 18800-2. In this context the assessment for an arch truss following section 6 (centric compression) in DIN 18800-2 is used. The general check is performed as:

$$
\frac{N}{\kappa \cdot N_{p l, d}} \leq 1
$$

with the axial resultant $N$ in the compressed member, the reduction factor $\kappa$ according to the European buckling curves, and the design plastic resistance $N_{p l, d}$ to normal forces. For determining the reduction factor $\kappa$ the equivalent slenderness parameter $\bar{\lambda}_{K}$ needs to be evaluated:

$$
\bar{\lambda}_{K}=\sqrt{\frac{N_{p l, d}}{N_{K i}}}
$$

The formula can be re-drafted as:

$$
\bar{\lambda}_{K}=\sqrt{\frac{f_{y, d} \cdot A}{\sigma_{c r, d} \cdot A}}=\sqrt{\frac{f_{y}}{\sigma_{c r}}}
$$

for $\gamma_{M}=\gamma_{F}=1.0$ and the critical buckling stress $\sigma_{c r}$. Based on (11.23) the critical buckling stress $\sigma_{c r}$ of an externally pressurized ring can be writing as:

$$
\sigma_{c r}=\frac{E}{4\left(1-\mu^{2}\right)}\left(\frac{t}{r}\right)^{2}
$$

considering the Poisson ratio. As the first factor is fix for steel constructions $(E$ and $\mu)$ a range can be determined for $\sigma_{c r}$ regarding common combiwall structures. The equivalent slenderness parameter $\bar{\lambda}_{K}$ is calculated for different $r / t$ ratios and materials according to (11.26) and (11.27) in Tab. 11.5. 
Tab. 11.5: Equivalent slenderness parameter $\bar{\lambda}_{K}$ for different $r / t$ ratios and materials

\begin{tabular}{|c|c|c|c|c|c|}
\hline \multicolumn{2}{|c|}{$\bar{\lambda}_{K}$} & 40 & 50 & 70 & 100 \\
\cline { 1 - 2 } material & $\mathrm{r} / \mathrm{t}$ & & & & \\
\cline { 1 - 2 }$S 235, f_{y}=240 \mathrm{~N} / \mathrm{mm}^{2}$ & $(2.58)$ & 3.22 & 4.51 & 6.45 \\
\cline { 1 - 4 }$S 355, f_{y}=360 \mathrm{~N} / \mathrm{mm}^{2}$ & 3.16 & 3.95 & 5.53 & 7.90 \\
\cline { 1 - 3 }$S 460, f_{y}=460 \mathrm{~N} / \mathrm{mm}^{2}$ & 3.57 & 4.46 & 6.25 & 8.93 \\
\hline
\end{tabular}

Except for the combination S235 and $r / t=40$ the equivalent slenderness parameter $\bar{\lambda}_{K}$ is greater than 3.0, and buckling in the completely elastic region. For $\bar{\lambda}_{K}>3.0$ the simplified equation for obtaining the reduction factor $\kappa$ according to DIN $18800-2$ is:

$$
\kappa=\frac{1}{\bar{\lambda}_{K}\left(\bar{\lambda}_{K}+\alpha\right)}
$$

with parameter $\alpha$ depending on the applied buckling curve " $a$ ", " $b$ ", " $c$ ", or " $d$ " with $0.21,0.34,0.49$, or 0.76 , respectively. The denomination $\alpha$ for the buckling curve parameter from DIN 18800-2 is misleading as it does not correspond to the elastic imperfection factor $\alpha$ from prEN 1993-1-6. For the limit case (complete utilization) in (11.24) and introducing (11.25) and (11.26) the axial force resistance can be written as:

$$
N=\frac{1}{1+\alpha / \bar{\lambda}_{K}} N_{K i}
$$

The first term (fraction) represents the linear elastic reduction factor regarding the ideal linear elastic buckling load $N_{K i}$ which is named $\alpha_{\text {column }}$ in the following. Thus, this value directly corresponds to the circumferential elastic imperfection factor $\alpha_{\theta}$ for the externally pressurized shell from (11.15). The section of the ring can be identified as solid rectangular cross-section, and hence be classified to the buckling curve " $c$ ". Evaluating the linear elastic reduction factor $\alpha_{\text {column }}$ according to (11.29) for the values given in Tab. 11.5 results in a range from 0.87 to 0.95 . For an increasing equivalent slenderness parameter the factor $\alpha_{\text {column }}$ reaches the limit value of 1.0, i.e. no reduction.

The results give rise to the question, why in the design of the externally pressurized shells an additional elastic imperfection factor of about 0.6 ( 0.5 to 0.75 according to EN 1993-1-6) has to be taken into account while such a large reduction factor does not need to be applied to the system if it is assessed as a ring buckling problem, e.g as shown here between 0.87 and 0.95 . For the analysed cases a design as a column may reach a resistance nearly twice as large as for applying the shell buckling design procedure. This aspect leads to the question which method is correct for the assessment of the given cases. 


\subsubsection{Discussion}

In Tab. 11.6 the reduction factors $\alpha_{\theta}$ for the reference case are summarized. The large reductions given in prEN 1993-1-6 are mainly based on the evaluation of the postbuckling loads (post-buckling minimum) as described earlier. In comparison, the mentioned authors from Tab. 11.6 only calculated the initial post-buckling behaviour with the equations by Donnell. Additionally, the post-buckling minimum is given according to Budiansky and Amazigo (1968) by applying the relationship given by Koiter (1963)

$$
\left(1-\alpha_{\theta}\right)^{3 / 2}=\frac{3 \sqrt{3}}{2}\left(\frac{\Delta w}{t}\right) \sqrt{-b_{c}} \cdot \alpha_{\theta}
$$

for the stated value of $b_{c}=-0.025$. It can be seen that the reduction factors given in EN 1993-1-6 reduce the carrying capacity by far more than all other stated values. Here, of course the initial post-buckling values yield a higher buckling load than considering the post-buckling minimum.

Tab. 11.6: Reduction factors $\alpha_{\theta}$ for the reference case

\begin{tabular}{|c|c|c|c|c|}
\hline \multicolumn{2}{|l|}{ approach } & $\Delta w / t$ & $\alpha_{\theta}$ & system \\
\hline \multirow[t]{3}{*}{ EN 1993-1-6 } & Qual. A & $<0.17$ & $0.75^{*}$ & \multirow{3}{*}{$r=900 \mathrm{~mm}, r / t=50, l=24 \mathrm{~m}$} \\
\hline & Qual. B & $<0.28$ & $0.65 *$ & \\
\hline & Qual. C & $<0.45$ & $0.50 *$ & \\
\hline \multirow{2}{*}{\multicolumn{2}{|c|}{ DIN $18800-2\left(\operatorname{ring} \alpha_{\text {column }}=\alpha_{\theta}\right)$}} & \multirow{2}{*}{$\begin{array}{l}l /(200 t) \\
=1.57\end{array}$} & 0.87 & $\mathrm{~S} 235, r=900 \mathrm{~mm}, r / t=50$ \\
\hline & & & 0.95 & $\mathrm{~S} 460, r=900 \mathrm{~mm}, r / t=100$ \\
\hline \multirow{5}{*}{\multicolumn{2}{|c|}{ Budiansky and Amazigo (1968) }} & 0.10 & 1.00 & \multirow{2}{*}{$L^{2} /(r t)=1048 \Rightarrow b=-0.025$} \\
\hline & & 0.50 & 0.99 & \\
\hline & & 0.17 & $0.85 *$ & \multirow{3}{*}{$\begin{array}{l}L^{2} /(r t)=1048 \Rightarrow b=-0.025 \\
\text { and }(11.30) \text { from Koiter }(1963)\end{array}$} \\
\hline & & 0.28 & $0.80 *$ & \\
\hline & & 0.45 & $0.74 *$ & \\
\hline \multirow{2}{*}{\multicolumn{2}{|c|}{ Pflüger (1966) }} & \multirow{2}{*}{0.25} & 0.88 & $r=900 \mathrm{~mm}, r / t=50, l=1 \mathrm{~m}$ \\
\hline & & & 0.97 & $r=900 \mathrm{~mm}, r / t=50, l=24 \mathrm{~m}$ \\
\hline
\end{tabular}

* = post-buckling minimum

The table confirms the detrimental influence of the reduction factors given in EN 19931-6 for the characterized imperfection amplitudes. Based on the similarity to the case of a buckling ring and the rather small imperfection sensitivity due to the length of the investigated cylinder, it could be proposed to apply smaller reduction factors for long cylinders, e.g. in the assessment of tubular piles in combiwalls. 
Fig. 11.30 depicts experimental results and different proposals for calculating a reduction factor. The equation (11.17) by Pflüger (1966) is given for two different $r / t$ ratios as the reduction factor depends on the latter parameter. The equation (11.16) proposed by Budiansky and Amazigo (1968) is calculated for two different imperfection amplitudes. Additionally, a proposal is made for calculating an adopted reduction factor $\alpha_{\theta}^{*}$ according to EN 1993-1-6 for long cylinders for which the influence of imperfections decreases and simultaneously the buckling strength increases. Since the three quality classes given for externally pressurized cylindrical shells in EN 1993-1-6 only depend on the imperfections and not on the geometry, the calculation applying the corresponding reduction factors may yield very conservative results for long cylinders, i.e. as applied to tubular piles in combiwalls of harbour structures. Thus, it is proposed to implement an adopted reduction factor $\alpha_{\theta}^{*}$ instead of $\alpha_{\theta}$ for cylinders with $L^{2} /(r t)=\omega^{2}>100$ :

$$
\alpha_{\theta}^{*}=\alpha_{\theta}+\left(\alpha_{\text {column }}-\alpha_{\theta}\right)\left(1-\frac{100}{\omega^{2}}\right) \quad \text { for } \quad \omega^{2}>100
$$

with

$$
\alpha_{\text {column }}=\frac{1}{1+0.257 \frac{t}{r} \sqrt{\frac{E}{f_{y}}}} \quad \text { for } \frac{r}{t} \sqrt{\frac{f_{y}}{E}}>1.57
$$

based on a Poisson ratio $\mu=0.3$.

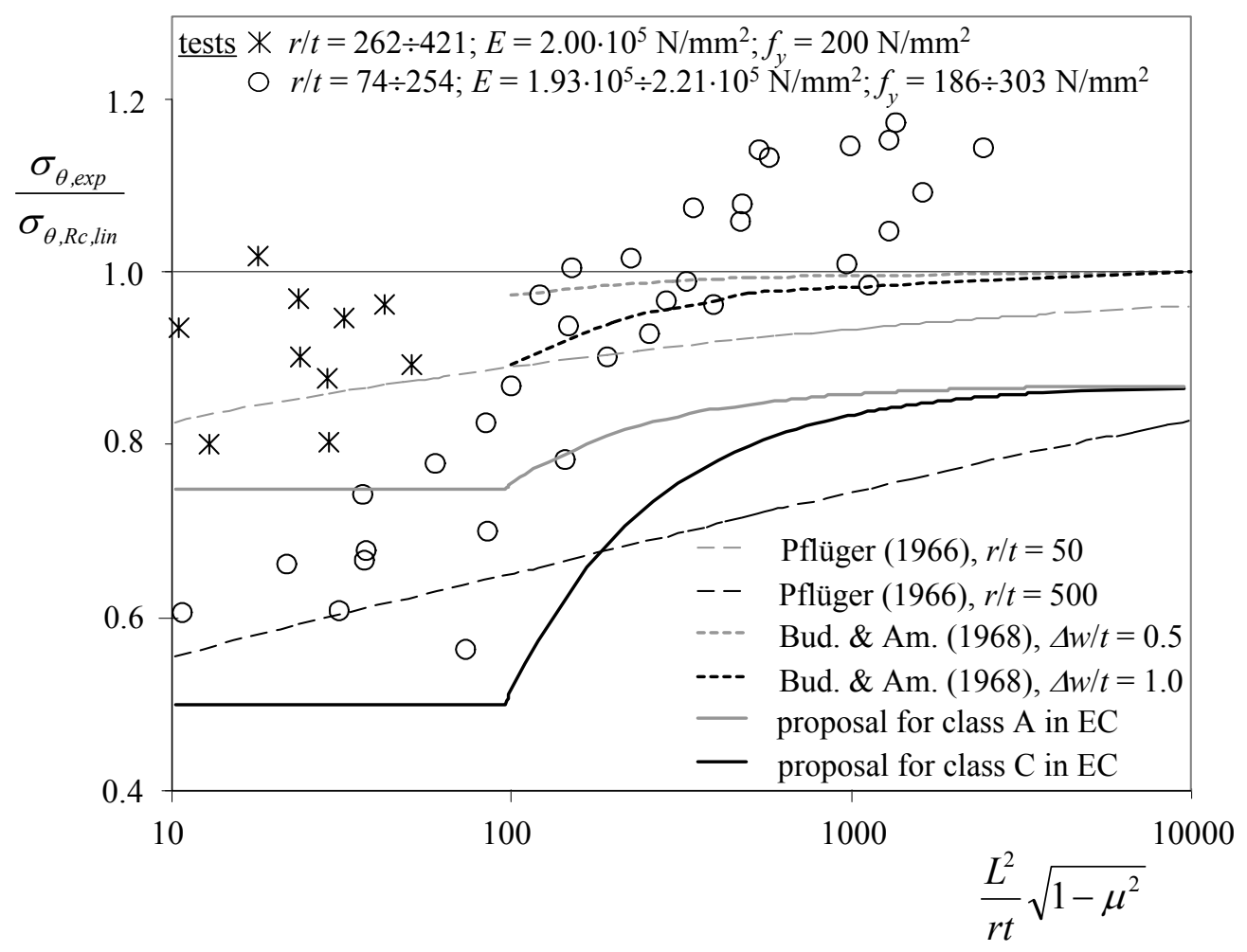

Fig. 11.30: Analytical and experimental results for hydrostatically pressurized cylinders and different proposals for the reduction factor 
Equation (11.32) can be confirmed with the example cases calculated in Tab. 11.6. As examples in Fig. 11.30 the curves for cylinders made of constructional steel S235 classified regarding their quality class as $\mathrm{A}$ and $\mathrm{C}$ with $r / t=50$ are shown. The curves are chosen according to the test results depicted in Fig. 11.21. For increasing length parameters $L^{2} /(r t)$ the curves converge asymptotically to the reduction factor for column or ring buckling as seen in equation (11.31) and Fig. 11.30 and given in equation (11.32).

\subsubsection{Experimental verification}

Experiments were planned and performed at the Universität Karlsruhe to provide a basis for verifying the proposed approach of more beneficial reduction factors from section 11.6.5.
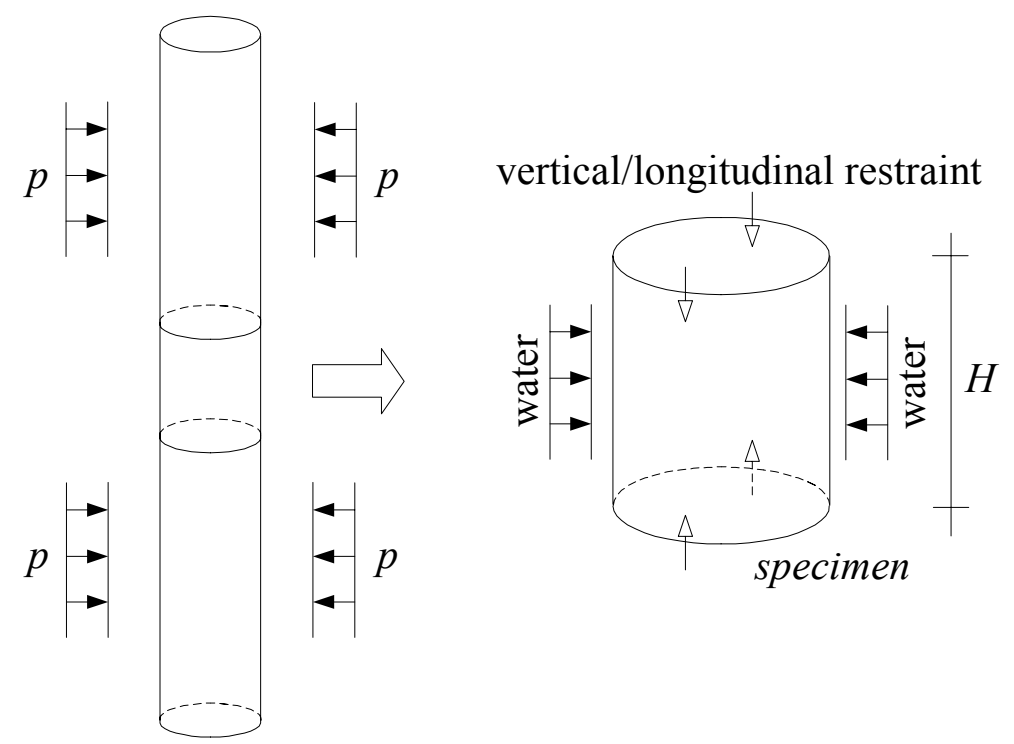

Fig. 11.31: Concept of external pressure tests

The concept of the experiments is to investigate cylindrical shells with very large length parameters $L^{2} /(r t)$ subjected to external pressure. The reduction factor for the very long cylinders are to be evaluated and compared to the reduction factors found in design codes. Due to practical and economical reasons it is very difficult to execute tests with length parameters $L^{2} /(r t)$ corresponding to real tubular piles. Thus, a test set-up was developed simulating infinitely long cylinders subjected to uniform external pressure. This is realized by taking a segment of the infinitely long cylinder and applying symmetry boundary conditions at the circumferential edges: vertical/longitudinal displacement restraint only, and free meridional rotation and radial displacement (Fig. 11.31). 


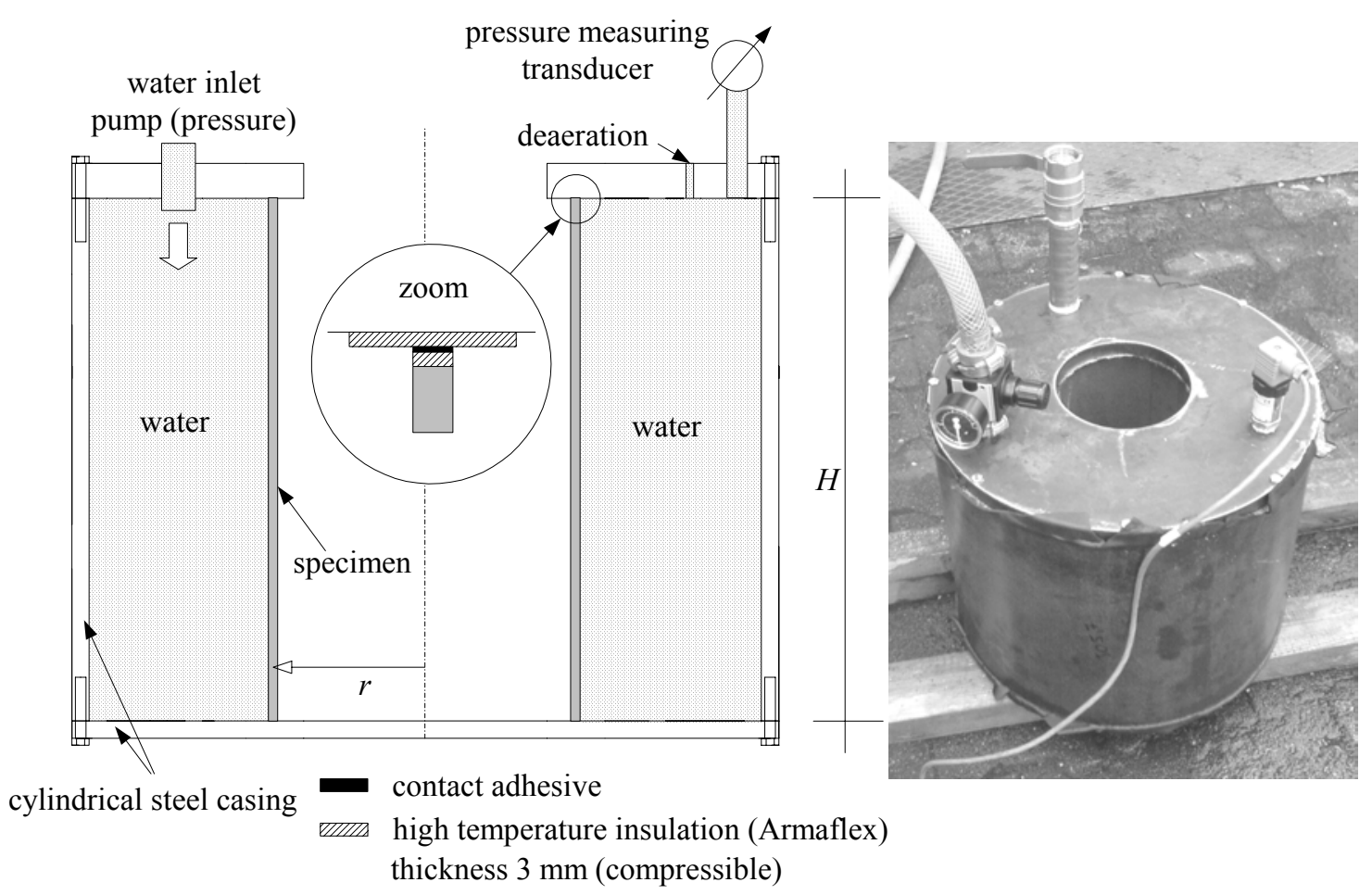

Fig. 11.32: Vertical section through center of test set-up for external pressure tests (cylindrical system) and photograph of test set-up

In Fig. 11.32 the test set-up is depicted as vertical section through the center. The photograph on the right in Fig. 11.32 shows the test set-up. The specimen is positioned in the center of a cylindrical steel casing with a steel lid at the top and bottom. The lids are connected with bolts to the casing. During the test, the space between specimen and casing is filled with water. The water is brought in by means of a pump (max. 6 bar) through an inlet in the lid resulting in a uniform pressure on the specimen. The water pressure is constantly measured by an electronic pressure measuring device attached to the top lid. The maximum applied pressure amplitude is recorded in the data acquisition system. The contact between specimen and lids is manufactured with special care, since it is important to allow the edges of the cylindrical shell to move freely in radial direction. In order to provide these conditions, two layers of a high temperature insulation (Armaflex(C) is glued to the shell edges and the lids. The insulation is soft, compressible, and has negligible shear stiffness. Thus, no radial constraint is provided at the point of buckling failure. During the loading step, this aspect can be disregarded because the external pressure is applied uniformly and simultaneously leading to a state of self-equilibrium. Additionally, the layers of Armaflex $\mathbb{C}$ are joined with a contact adhesive in order to prevent penetrating water and consequently a pressure reduction. 
Tab. 11.7: Measurements and test results

\begin{tabular}{|c|c|c|c|}
\hline specimen & $\begin{array}{c}\text { out-of-roundness } U_{r}[\%] \\
U_{r}=\frac{D_{\max }-D_{\min }}{D_{\text {nom }}}\end{array}$ & $\begin{array}{c}\text { quality class according to } \\
\text { EN 1993-1-6 }\end{array}$ & $\begin{array}{c}\text { experimental buckling } \\
\text { pressure } p_{\text {exp }}[\mathrm{bar}]\end{array}$ \\
\hline tube & $0.44 / 0.44 / \mathbf{0 . 7 9} / 0.54 / 0.64$ & & - \\
\hline S1 & $\mathbf{0 . 5 4} / 0.44 / 0.39$ & & 4.45 \\
\hline S2 & $\mathbf{0 . 4 9 / 0 . 3 9 / 0 . 2 9}$ & \multirow{3}{*}{$* 1.4 \% \Rightarrow$ Class A } & 4.85 \\
\hline S3 & $0.39 / 0.35 / 0.39$ & & 4.81 \\
\hline S4 & $0.64 / 0.59 / 0.99$ & & 4.39 \\
\hline L1 & $0.44 / 0.49 / 0.44$ & & 4.49 \\
\hline L2 & $\mathbf{0 . 5 4 / 0 . 3 9 / 0 . 4 4}$ & & 4.35 \\
\hline
\end{tabular}

The testing is executed by increasing the pressure slowly using a pressure regulator between pump and inlet until failure of the specimen. Two different geometries were tested: $H=300 \mathrm{~mm}$ (4 short specimens: $\mathrm{S} 1$ to $\mathrm{S} 4)$ and $H=600 \mathrm{~mm}(2$ specimens: L1 and L2). The specimens were segments cut from the tube used for preliminary investigations (section Annex B.1). The geometry, description of geometrical imperfections and the measured material properties are given in Annex B. The investigation of the geometrical imperfections (out-of-roundness) revealed very small imperfections resulting in a classification of quality class A according to EN 1993-1-6. Nevertheless, for the six specimens the shape deviations were re-measured. The geometrical imperfections are summarized in Tab. 11.7. For each specimen several measurements were performed determining the out-of-roundness $U_{r}$ at different crosssections. The maximum $U_{r}$ is written in bold. This indicates that all tested specimens are quality class A cylinders according to EN 1993-1-6. The maximum water pressures (= buckling pressure $p_{\text {exp }}$ ) in the tests are given in Tab. 11.7. The failure occurred suddenly resulting in a leakage of water with a pressure drop to zero. The failure mode for the externally pressurized cylinders was consistently an elliptical cylindrical two-wave shape (Fig. 11.33). This type of failure proves that there was no radial restraint of the upper and lower edges of the cylinder. The latter aspect validates the test set-up by justifying the assumption of a segment taken from an infinite cylinder (Fig. 11.31). Thus, the experimental buckling pressures $p_{\exp }$ are evaluated with respect to the theoretical buckling pressure $p_{\text {theo }}$ from EN 1993-1-6 for an infinite cylinder

$$
p_{\text {theo }}=\frac{t}{r} \sigma_{\theta}=E\left(\frac{t}{r}\right)^{3}\left[0.275+\left(\frac{C_{\theta}}{\omega} \frac{r}{t}\right)^{4}\right] \stackrel{\omega \rightarrow \infty}{\longrightarrow} 0.275 E\left(\frac{t}{r}\right)^{3}
$$

Here, the theoretical buckling pressure $p_{\text {theo }}$ is (a) based on the Young's modulus $E$ extracted from material property testing (Annex B: $190000 \mathrm{~N} / \mathrm{mm}^{2}$ ), and (b) the common value of $E=210000 \mathrm{~N} / \mathrm{mm}^{2}$ used for constructional steel in most design 
codes. The buckling pressure calculated with equation (11.33) yields the same results as equation (11.23) considering the Poisson ratio $\mu=0.3$.
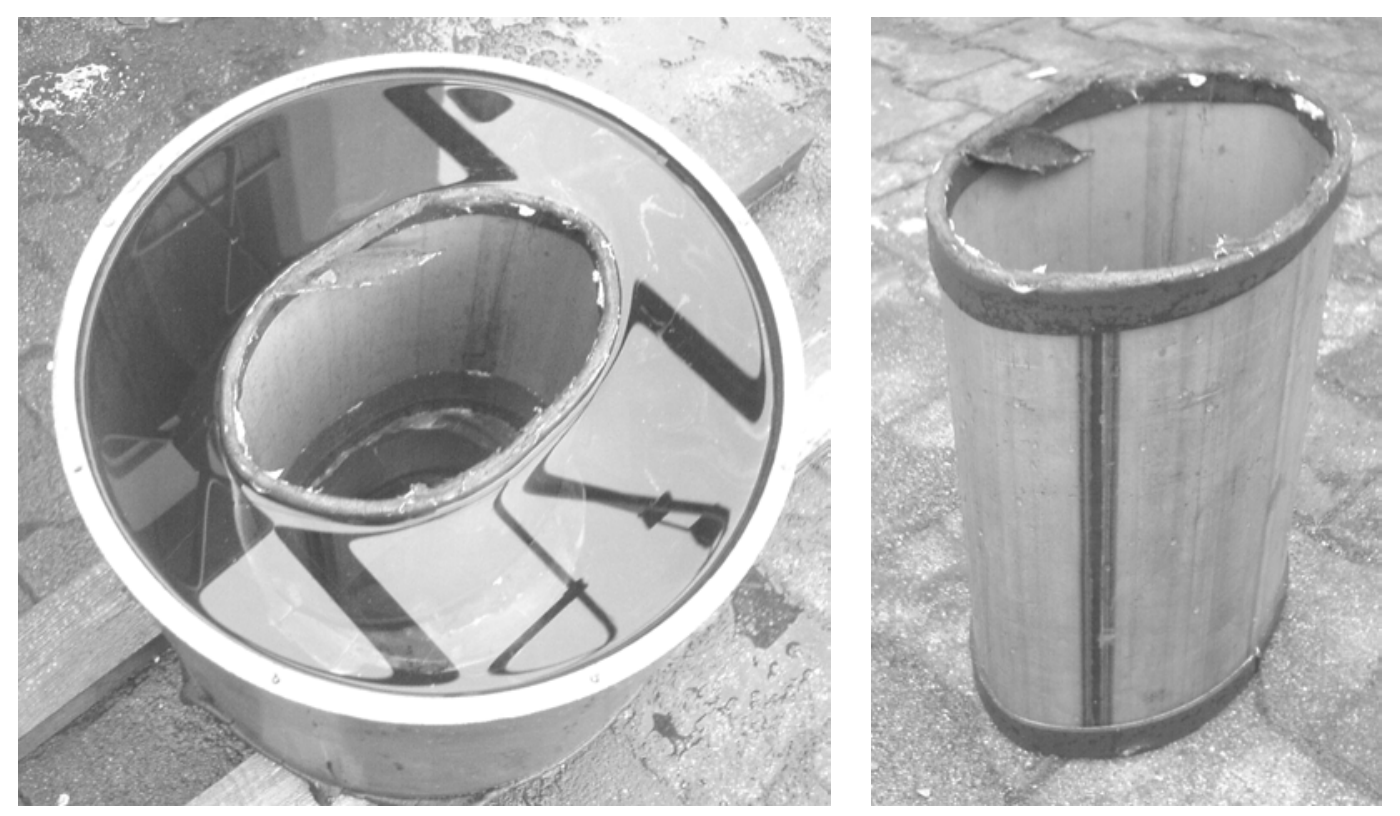

Fig. 11.33: Buckling mode of specimen S1 after unloading

The evaluated results are summarized in Tab. 11.8. Generally, all reduction factors are close to 1.0 , indicating no significant deviation of the buckling pressure from the theoretical buckling pressure due to geometrical or structural imperfections. Further, no large differences can be found between the two different test lengths.

Tab. 11.8: Evaluated test results

\begin{tabular}{|c|c|c|c|c|c|}
\hline \multirow{2}{*}{ specimen } & \multirow{2}{*}{$\begin{array}{c}\text { experimental } \\
\text { buckling pressure } \\
p_{\exp }[\mathrm{bar}]\end{array}$} & \multicolumn{2}{|c|}{ theoretical buckling pressure } & \multicolumn{2}{|c|}{ reduction factor $p_{\text {exp }} / p_{\text {theo }}[-]$} \\
\hline & & $p_{\text {theo, } a}[\mathrm{bar}]$ & $p_{\text {theo, }, b}[\mathrm{bar}]$ & $\begin{array}{c}\text { (a) } E=190 \mathrm{GPa} \\
\text { measured }\end{array}$ & $\begin{array}{l}\text { (b) } E=210 \mathrm{GPa} \\
\text { design code }\end{array}$ \\
\hline S1 & 4.45 & \multirow{7}{*}{4.12} & \multirow{7}{*}{4.55} & 1.08 & 0.98 \\
\hline S2 & 4.85 & & & 1.18 & 1.07 \\
\hline S3 & 4.81 & & & 1.17 & 1.06 \\
\hline S4 & 4.39 & & & 1.07 & 0.96 \\
\hline L1 & 4.49 & & & 1.09 & 0.99 \\
\hline $\mathrm{L} 2$ & 4.35 & & & 1.06 & 0.96 \\
\hline mean & 4.56 & & & 1.11 & 1.00 \\
\hline
\end{tabular}

In Fig. 11.34 experimental results found in publications are compared with the newly obtained reduction factors from Tab. 11.5. The results from the current buckling tests are positioned beyond the maximum length parameters $L^{2} /(r t)$ in order to emphasize that the tested cylinders correspond to infinitely long cylinders. Independently of the fact whether (a) the measured Young's modulus or (b) the Young's modulus from design 
codes is used for defining the theoretical reference buckling pressure, the experiments yield reduction factors $\alpha_{\theta}$ around 1.0. The experimental results validate the assumptions given in section 11.6.5 for defining a larger reduction factor for long cylindrical shells. An assessment based on EN 1993-1-6 would require a reduction factor of 0.75 (Class A), while the present investigation in agreement with the tests from previous researches for length parameters larger than 600 that there is no reduction factor necessary.

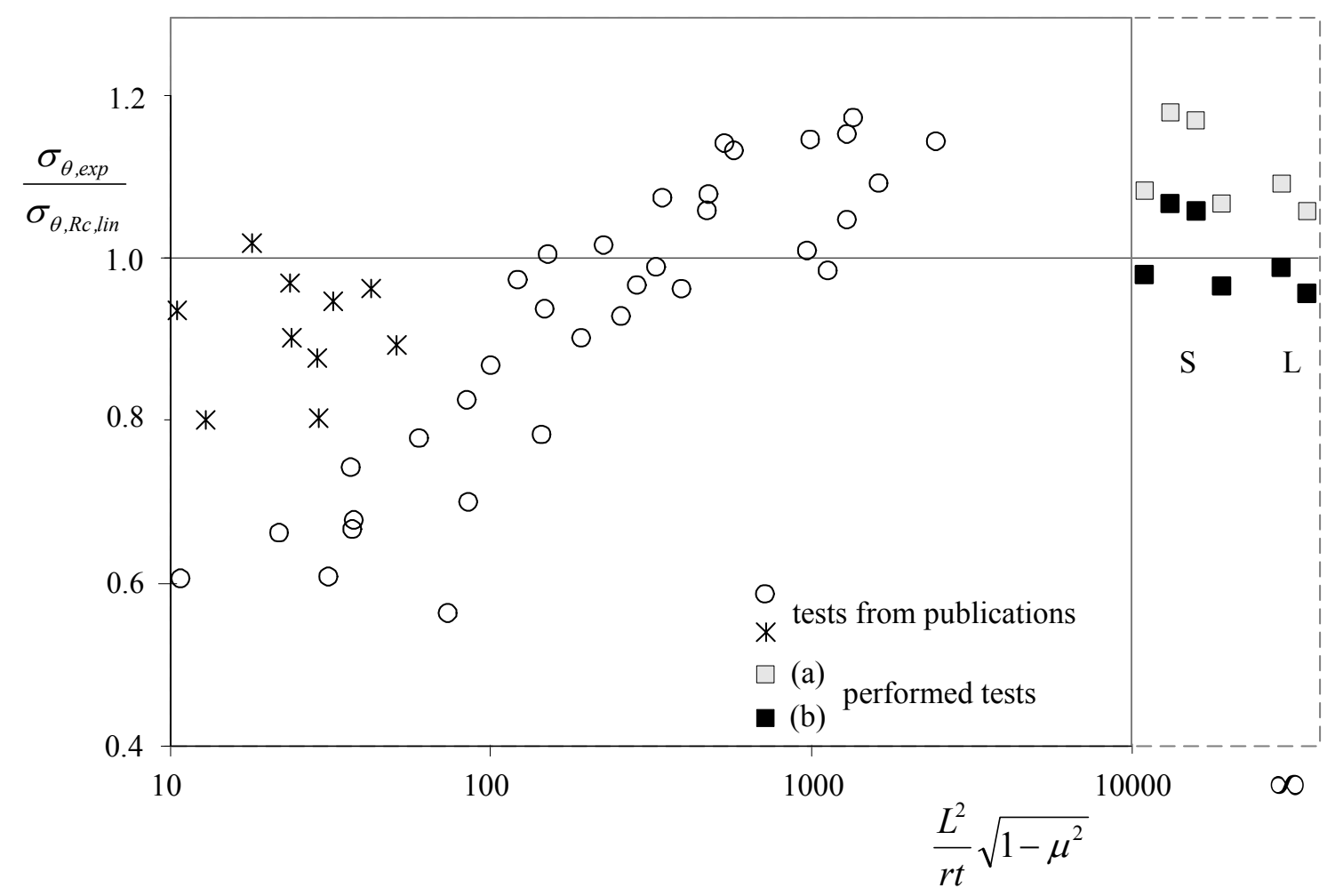

Fig. 11.34: Experimental test results for externally pressurized cylinders for the reduction factor 


\section{Summary and proposals for design codes}

\subsection{General}

In this work aspects influencing the carrying capacity of large tubular piles as used in combiwalls have been discussed and investigated. In this context the global problem was seperated into several issues as explained in chapter 4 based on common practical approaches introduced in chapter 4 . In the following the diverse influences are reviewed and summarised in new design proposals for the application in construction codes. The proposed changes are then applied to the example case from chapter 4 in order to emphasize the positive effect of using the improved design considerations.

The different aspects discussed in this work are summarized according to the corresponding chapters.

\subsection{Applicability}

In chapter 6 a comparison between combiwalls and single piles was performed. Based on the results from the numerical analyses and considerations regarding the load transfer mechanisms it was concluded that the results from the subsequent studies on single piles are applicable to the problem of combiwalls.

Further, the studies from chapter 7 involving the investigation of the soil and the soil structures interaction provided a basis for the successive numerical analyses applying different soil concepts. Here, it was important to evaluate the soil models and to define the models appropriate for further considerations.

The latter analyses served as a basis for the next steps and confirm the applicability of the various models. Additionally, the investigation of the soil and the soil structures interaction gives the engineer a deeper insight into the application of different models. Again it needs to be emphasized that the use of detailed soil models (e.g. hypoplasticity) requires great expertise in this area and a large amount of field data in order to provide an acceptable basis for design calculations. Further, the constructing engineer is advised to perform sensitivity studies regarding a scatter of parameters for attaining information on different possibilities. Nevertheless, it is proposed to use standard and common applications (e.g. RIDO) for calculating the resultant forces. The proposed design procedure comes into play after the latter step by giving possibilities for introducing more adjusted parameters for design. Studying the entire structure by numerical means involves complex models and necesitates considerable information on the boundary conditions and material data. The presented approach desists from this approach by providing the engineer a convenient and easy-to-apply tool. 


\subsection{Geometrical imperfections}

In chapter 8 the different influences of radial shape imperfections and uneven boundaries have been studied. Generally, it can be seen that for the common range of $r / t$ ratios used for combiwalls the influence of geometrical imperfections is rather small. The intention of the study was to separate the reduction of the carrying capacity arising from radial shape imperfections and uneven boundaries. It can be seen that for thicker shells, i.e. smaller $r / t$ ratios the influence of uneven boundaries increases. For the case of tubular piles as used in combiwalls the latter influence may be neglected since no direct contact with the substructure is given which may introduce non-uniform meridional stresses. Yielding does not change the behaviour considerably. For a combined loading of an axial load and a bending moment the influence of uneven boundaries increases.

As described in chapter 8 it may be proposed to apply a factor to increase the linear reduction factor $\alpha_{x}$ and/or the reduction factor $\chi_{x}$ for small $r / t$ ratios. The numerical analyses are limited due to the complexity of the influencing parameters regarding the geometrical imperfections. Here, a factor of 1.1 may be established for the linear reduction factor $\alpha_{x}$. From the numerical analyses involving plasticity an increase of the reduction factor $\chi_{x}$ can be derived. Since the effect of this increase of the linear reduction factor $\alpha_{x}$ is rather marginal (a factor of 1.01 or 1.02 for the design buckling stress in a common range of $r / t$ ratios for tubular piles), no additional and more detailed studies were performed. As a consequence it is suggested to neglect the beneficial influence of not considering the imperfection of uneven supports for large tubular piles as a conservative approach due to the marginal effect. Nevertheless, it is emphasized that this aspect has considerable influence when investigating thinner shells (larger $r / t$ ratios) as e.g. used for tank or silo structures.

\subsection{Influence of soil on buckling behaviour}

\subsubsection{Meridional critical buckling stress}

In chapter 9 factors for increasing the buckling load due to a foundation effect of the surrounding soil are derived. The evaluation is semi-empirical and based on several numerical and experimental studies. For obtaining a general practical formula in a first approach, the investigation uses a conservative approximation. The values for the applied coefficient of subgrade reaction are based on the proposed values by Therzaghi (1955) and the approaches given in ISO/DIS 19902:2004.

The critical buckling load $\sigma_{x, R c}$ * considering the beneficial influence of surrounding sand can be calculated as:

$$
\sigma_{x, R c} *=\beta_{x, \text { sand }} \cdot \sigma_{x, R c}
$$

with 


$$
\beta_{x, \text { sand }}=0.92+0.0045 \frac{l}{r}\left(\frac{k_{S}}{n_{h, \text { loose }}}\right)^{0.25}
$$

with $n_{h, \text { loose }}=2.2 \mathrm{MN} / \mathrm{m}^{3}$ and for $\beta_{x, \text { sand }}>1, r / t \geq 40$, and $r \geq 450 \mathrm{~mm}$. As a conservative approximation it is proposed to calculate $k_{S}$ according to Terzaghi (1955) for loose sand with:

$$
k_{S}=2.2 \frac{M N}{m^{3}} \cdot \frac{z}{D}
$$

For the latter case equation (12.2) can be redrafted as:

$$
\beta_{x, \text { sand }}=0.92+0.009\left(\frac{l}{D}\right)^{1.25}
$$

which is valid if the embedded length $l$ corresponds to the depth $z$. For the "halfembedded" case the factor $\beta_{x, \text { sand, } 0.5}$ can be calculated as:

$$
\beta_{x, \text { sand }, 0.5}=0.96+0.0023 \frac{l}{r}\left(\frac{k_{S}}{n_{h, \text { loose }}}\right)^{0.25}
$$

Again, it is emphasized that the influence of the supporting soil is rather small for typical dimensions of tubular piles used in combiwalls. Although the increase of the theoretical buckling stress may appear significant for large coefficients of subgrade reaction, this influence decreases during the assessment procedure as it can be seen in the recalculation of the reference case.

\subsubsection{Circumferential critical buckling stress}

In accordance with section 12.4.1 factors for increasing the circumferential critical buckling stress are given in chapter 9. Again, the values are based on Terzaghi (1955) and the ISO/DIS 19902:2004.

The critical buckling load $\sigma_{\theta, R c}{ }^{*}$ considering the beneficial influence of surrounding sand can be calculated as:

$$
\sigma_{\theta, R c} *=\beta_{\theta, \text { sand }} \cdot \sigma_{\theta, R c}
$$

with

$$
\beta_{\theta, \text { sand }}=1+0.3 \sqrt{\frac{l}{D}\left(\frac{k_{S}}{n_{h, \text { loose }}}\right)^{0.25}-4.0}
$$

for $\quad r / t \geq 40, \quad r \geq 450 \mathrm{~mm}, \quad n_{h, \text { loose }}=2.2 \mathrm{MN} / \mathrm{m}^{3}, \quad$ and $k_{S} \geq 2.2 \mathrm{MN} / \mathrm{m}^{3} \cdot l / D$. A first conservative approximation can be made with equation (9.28). for the "half-embedded" case the factor $\beta_{\theta, \text { sand }, 0.5}$ is: 


$$
\beta_{\theta, \text { sand }, 0.5}=1+0.15 \sqrt{\frac{l}{D}\left(\frac{k_{S}}{n_{h, \text { loose }}}\right)^{0.25}-4.0}
$$

considering the previous conditions. It is pointed out that the latter approximation may be very conservative for most cases. Nevertheless, even larger factors $\beta_{\theta \text {,sand }}$ will not influence the design of practical constructions.

\subsection{Global load transfer behaviour}

In chapter 10 the investigation of the load transfer behaviour of the entire system is described. In the analyses the uniformness of the stress distribution and the ovalization was studied in detail by numerical and experimental means. The main interest was to obtain information on the latter aspects in order to validate the applied approaches in the design of such structures. The investigation revealed that the stress distribution over the circumference remains fairly smooth, i.e. without any significant stress peaks. Still, it needs to be mentioned that for some cases rather non-uniform stress distributions may arise (e.g. Fig. 6.5 or Fig. 6.6). Nevertheless, based on the results it is assumed that the results are compatible with given buckling design approaches. Considerable stress changes or noticeable ovalizations would influence the carrying behaviour detrimentally and raise the question whether the standard design rules can be adopted to the case of tubular piles. Another aspect regarding the uniformness of stress arises along with the investigations summarized in section 12.3. The smoothness of the stress distributions as verified in numerical and experimental analyses would emphasize the possibility of increasing the linear reduction factor $\alpha_{x}$ used in the design procedure. Nevertheless, as stated in section 12.3 it was decided to desist from proposing to implement a factor with respect to the geometrical imperfections, and thus this aspect is not considered in the design proposal.

\subsection{External pressure}

\subsubsection{Overview}

The first presented numerical analyses and designs based on a more detailed evaluation of the shell's resistance and the loading for external pressure revealed a large beneficial influence for the more precise considerations. Hence, a more complex and workintensive approach for the designing engineer may be the possibility to analyse the system regarding the circumferential stresses by elaborate numerical analyses.

The subsequent studies were then performed for defined simple tools and parameters for the design procedure to be used for external pressure. In this context the non-uniform pressure was investigated with respect to the circumference and the length. Another set of analyses and an addtional series of experiments dealt with a discussion of the buckling reduction factor. 


\subsubsection{Non-uniform external pressure over circumference}

In section 11.5.1 different cases for boundary conditions and $r / t$ ratios were studied for deriving an equivalent load correction factor $C_{L}$ to be used independently of the boundary conditions and the $r / t$ ratio. Based on a regression analysis it is proposed to calculate an equivalent pressure $p^{*}$ as a function of the loaded segment $\varphi_{\text {load }}$ :

$$
p^{*}=C_{L} \cdot p
$$

with

$$
C_{L}=1-0.165\left(a_{f}^{2}+a_{f}-2\right) \ln \left(\frac{\varphi_{\text {load }}}{360^{\circ}}\right)
$$

where the load fraction ratio $a_{f}$ is defined as the ratio of two different segment-wise constant pressures $p_{\max }$ and $p_{\min }$ :

$$
a_{f}=\frac{p_{\min }}{p_{\max }}
$$

for $r / t<100, a_{f} \leq 1$, and $\varphi_{\text {load }}>90^{\circ}$, which then can be used as a constant pressure over the circumference in the next steps of the design procedure.

\subsubsection{Non-uniform external pressure over length}

The studies in section 11.5.3 revealed a beneficial influence on the linear buckling load for a cylinder subjected to external, non-uniform pressure over the length in comparison to a cylinder subjected to a constant external pressure based on the maximum amplitude. Thus, equivalent dimensionless length parameters $\omega^{*}$ were obtained for cylinders subjected to external pressure non-uniform over the length to be used for obtaining a corresponding critical circumferential buckling stress.

Based on numerous numerical analyses and an interpretation considering the buckling lengths for the different systems, the evaluation can be applied to numerous pressure distribution types and load factors $b$ :

$$
b=\frac{\int_{0}^{L} p(z) d z}{p_{0} l}
$$

For these systems equivalent length diagrams can be derived. Fig. 12.1 depicts the relationships for an example system to be applied in the design procedure of an externally pressurized cylinder. 


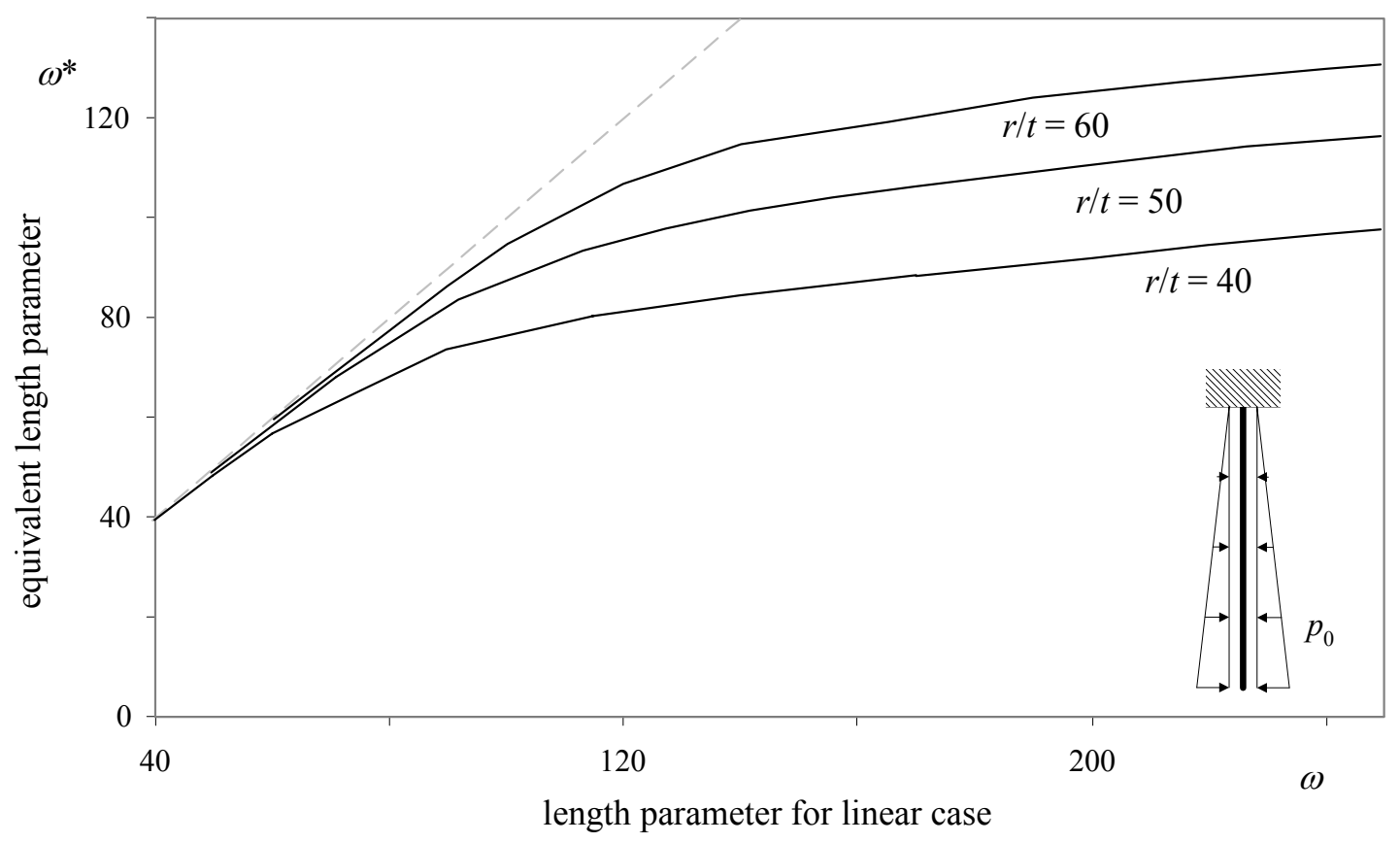

Fig. 12.1: Derived relationship of length parameter for "linear" system and corresponding equivalent length parameter for "constant" system for $\omega \geq \omega_{\text {long }}$ with $b=0.5$

For the subsequent steps the length $l$ is then replaced by the equivalent length $l^{*}$ :

$$
l^{*}=\omega * \sqrt{r t}
$$

\subsubsection{Evaluation of the elastic buckling reduction factor}

In section 11.6 the aspect of the elastic buckling reduction factor is discussed in detail. Based on an extensive literature study on experiments performed with externally pressurized cylinders and comparisons with design codes it was revealed that the elastic buckling reduction factor given in EN 1993-1-6 may lead to very conservative results for the buckling loads of long thick-walled cylinders. Additional numerical studies with perfect and imperfect cylinders emphasized this issue. Further, the similar behaviour of a long externally pressurized cylinder and a ring subjected to external pressure resulted in the assumption that the design concept should be similar. However, design codes do not allow the engineer to apply the same safety considerations for shells as for columns. Based on the investigation it is proposed to implement a modified reduction factor $\alpha_{\theta}{ }^{*}$ instead of $\alpha_{\theta}$ for cylinders with $l^{2} /(r t)=\omega^{2}>600$ :

$$
\alpha_{\theta}^{*}=\alpha_{\theta}+\left(\alpha_{\text {column }}-\alpha_{\theta}\right)\left(1-\frac{600}{\omega^{2}}\right) \quad \text { for } \omega^{2}>600
$$

with

$$
\alpha_{\text {column }}=\frac{1}{1+0.257 \frac{t}{r} \sqrt{\frac{E}{f_{y}}}} \quad \text { for } \frac{r}{t} \sqrt{\frac{f_{y}}{E}}>1.57
$$


based on a Poisson ratio $\mu=0.3$ in the design of externally pressurized cylinder in EN 1993-1-6. In contrast to section 11.6 the range of application is changed from $\omega^{2}>100$ to $\omega^{2}>600$ for the design proposal as mentioned in section 11.6. This step is made in order to limit the application of the increased reduction factors to slender and long non-standard constructions. The limit is chosen according to the results from the experimental investigation as given in in section 11.6.6.

\subsection{Recalculating the standard reference case}

In the following the design procedure for the example reference case from chapter 4 is recalculated applying the proposed design results described in the previous sections. The calculation is performed in brief since the procedure is explained in detail in chapter 4 . The changes in comparison to chapter 4 are highlighted by shading the corresponding lines in grey. The assessment is based on ENV 1993-1-6:2002-05 and prEN 1993-5:2004-07. In Tab. 12.1 the design stresses are summarized:

Tab. 12.1: Design stresses

\begin{tabular}{|l|c|c|}
\hline position & anchor $(-6 \mathrm{~m})$ & max. moment $(-17.5 \mathrm{~m})$ \\
\hline$\sigma_{x}\left[\mathrm{~N} / \mathrm{mm}^{2}\right]$ & $37+233=270$ & $37+316=353$ \\
\hline$\sigma_{\theta}\left[\mathrm{N} / \mathrm{mm}^{2}\right]$ & 2 & 3 \\
\hline$\tau_{x \theta}\left[\mathrm{N} / \mathrm{mm}^{2}\right]$ & 80 & 0 \\
\hline
\end{tabular}

The reference length is defined from pile tip to the position of the anchor. The factor $C_{x}$ is set equal to 1 without performing the calculation from ENV 1993-1-6:2002-05. According to prEN 1993-5:2004-07 the boundary conditions of case 3 in Table D-3 or D-4 from ENV 1993-1-6:2002-05 shall be used for circumferential compression stresses. This corresponds to BC2-BC2. In Fig. 12.2 and Tab. 12.2 the external pressure distributions and approximated distributions for the assessment procedure are depicted based on the amplitudes at a depth of $-29 \mathrm{~m}$. The stresses for the assessment are calculated in Tab. 12.3.

Tab. 12.2: Pressures at the depth $z=-29 \mathrm{~m}$ according to Fig. 12.2

\begin{tabular}{|l|c|c|}
\hline pressures & In front of tube (index 1) & behind tube (index 2) \\
\hline earth pressure $p_{E}\left[\mathrm{kN} / \mathrm{m}^{2}\right]$ & 733 & 203 \\
\hline water pressure $p_{W}\left[\mathrm{kN} / \mathrm{m}^{2}\right]$ & 210 & 260 \\
\hline total pressure $p_{E+W}\left[\mathrm{kN} / \mathrm{m}^{2}\right]$ & 943 & 463 \\
\hline averaged total pressure $p_{m}\left[\mathrm{kN} / \mathrm{m}^{2}\right]$ & \multicolumn{2}{|c|}{703} \\
\hline internal pressure $p_{\text {int }}\left[\mathrm{kN} / \mathrm{m}^{2}\right]$ & $160\left(\right.$ with $\left.K_{0}\right)$ \\
\hline
\end{tabular}




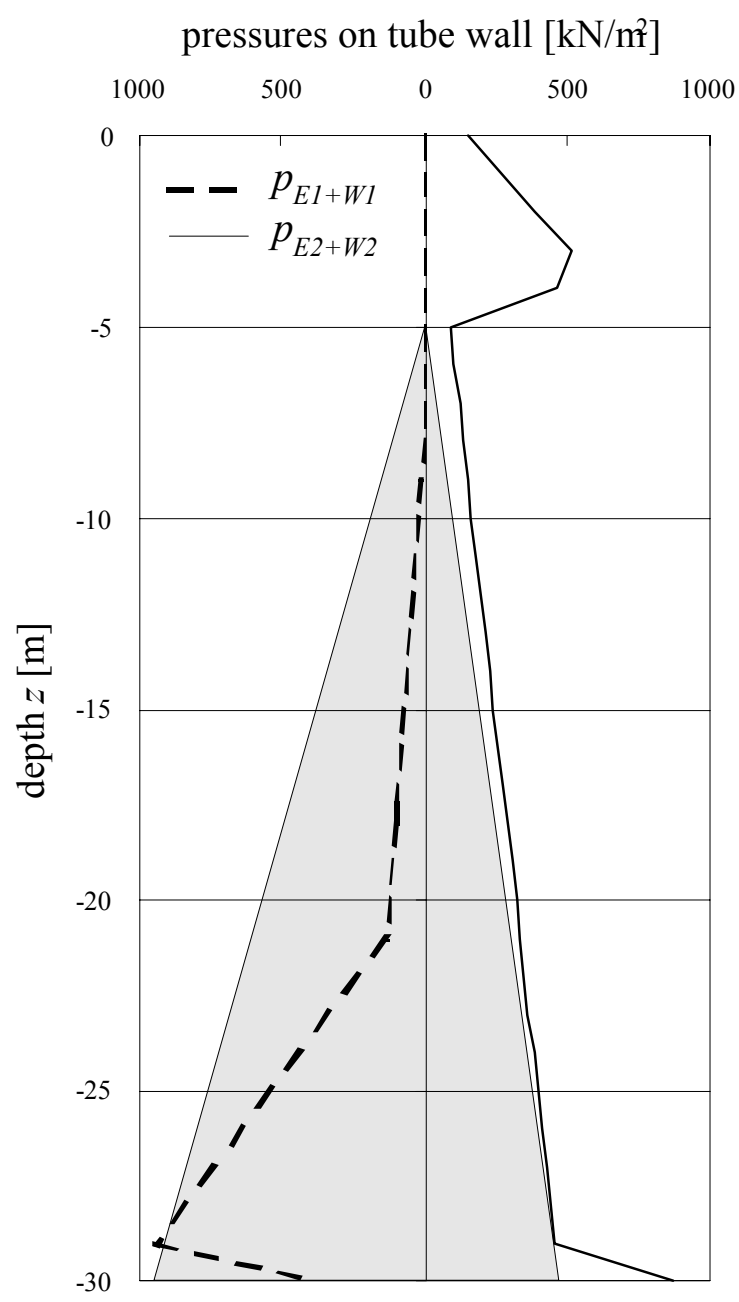

Fig. 12.2: Pressure distribution on tube as a function of the depth and approximated distribution (grey)

Tab. 12.3: Recalculation - circumferential stresses for limit state buckling design against circumferential compression at $z=-29 m$

\begin{tabular}{|l|c|c|c|}
\hline parameter & eq. & circumferential compression & dimension \\
\hline$a_{f}$ & - & $\frac{463-160}{943-160}=0.39$ & - \\
\hline$\varphi_{\text {load }}$ & - & 180 & $\circ$ \\
\hline$C_{L}$ & $(12.10)$ & $1-0.165\left(0.39^{2}+0.39-2\right) \ln 0.5=0.83$ & - \\
\hline$p^{*}$ & - & $p^{*}=0.83 \cdot(943-160)=650$ & $\mathrm{kN} / \mathrm{m}^{2}$ \\
\hline$\sigma_{\theta}$ & - & $\sigma_{\theta}=\frac{\left(p^{*}+\Delta p_{W}\right) \cdot r}{t}=\frac{(650+50) \cdot 0.9}{18}=35$ & $\mathrm{~N} / \mathrm{mm}^{2}$ \\
\hline
\end{tabular}

The following tables summarize the parameters for limit state buckling design. The coefficient of subgrade reaction is given for the calculated problem with $k_{S}=30 \mathrm{MN} / \mathrm{m}^{3}$. 
Tab. 12.4: Recalculation - parameters for limit state buckling design against axial compression

\begin{tabular}{|c|c|c|c|}
\hline parameter & $e q$. & axial compression & dimension \\
\hline$r / t$ & - & 52 & - \\
\hline$l$ & - & 24 & $\mathrm{~m}$ \\
\hline$\beta_{x, \text { sand }, 0.5}$ & $(12.5)$ & $\begin{aligned} \beta_{x, \text { sand }, 0.5}=0.96 & +0.0023 \frac{24}{0.936}\left(\frac{30}{2.2}\right)^{0.25} \\
& =1.07\end{aligned}$ & - \\
\hline$C_{x}$ & - & 1 & - \\
\hline$\sigma_{x, R c}$ & $(12.1)$ & $\sigma_{x, R c}=0.605 \cdot E \cdot C_{x} \cdot \frac{t}{r} \cdot \beta_{x, \text { sand }, 0.5}=2614$ & $\mathrm{~N} / \mathrm{mm}^{2}$ \\
\hline $\bar{\lambda}_{x}$ & - & $\bar{\lambda}_{x}=\sqrt{430 / 2614}=0.41$ & - \\
\hline$Q$ & - & 25 (Klasse B) & - \\
\hline$\Delta w_{k}$ & - & $\Delta w_{k}=\frac{1}{Q} \sqrt{\frac{r}{t}} \cdot t=5.19$ & $\mathrm{~mm}$ \\
\hline$\alpha_{x}$ & - & $\alpha_{x}=\frac{0.62}{1+1.91 \cdot\left(\Delta w_{k} / t\right)^{1.44}}=0.470$ & - \\
\hline $\bar{\lambda}_{x p}$ & - & $\bar{\lambda}_{x p}=\sqrt{0.470 /(1-0.6)}=1.08$ & - \\
\hline$\chi_{x}$ & - & $\chi_{x}=1-0.6\left(\frac{0.41-0.2}{1.08-0.2}\right)^{1}=0.86$ & - \\
\hline$\sigma_{x, R d}$ & - & $\sigma_{x, R d}=\frac{0.86 \cdot 430}{1.1}=336$ & $\mathrm{~N} / \mathrm{mm}^{2}$ \\
\hline
\end{tabular}


12 Summary and proposals for design codes

Tab. 12.5: Recalculation - parameters for limit state buckling design against circumferential compression

\begin{tabular}{|c|c|c|c|}
\hline parameter & eq. & circumferential compression & dimension \\
\hline$r / t$ & - & 52 & - \\
\hline$l$ & - & 24 & $\mathrm{~m}$ \\
\hline$\omega$ & - & 185 & - \\
\hline$\omega^{*}$ & Fig. 12.1 & 108 & - \\
\hline$l *$ & (12.13) & $l^{*}=108 \sqrt{0.936 \cdot 0.018}=14$ & $\mathrm{~m}$ \\
\hline$\beta_{\theta, \text { sand,0.5 }}$ & $(9.35)$ & $\begin{array}{c}\beta_{\theta, \text { sand }, 0.5}=1+0.15 \sqrt{\frac{14}{1.8}\left(\frac{30}{2.2}\right)^{0.25}-4.0} \\
=1.50\end{array}$ & - \\
\hline$C_{\theta}$ & - & 1 & - \\
\hline$\sigma_{\theta, R c}$ & - & 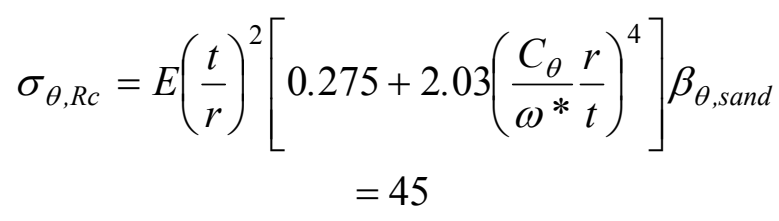 & $\mathrm{N} / \mathrm{mm}^{2}$ \\
\hline \multirow[t]{3}{*}{$\bar{\lambda}_{\theta}$} & - & $\bar{\lambda}_{\theta}=\sqrt{430 / 45}=3.09$ & - \\
\hline & $(12.15)$ & $\frac{r}{t} \sqrt{\frac{f_{y}}{E}}=2.35>1.57 \quad$ ok & - \\
\hline & $(12.14)$ & $\omega^{2}=11664>600 \quad \mathrm{ok}$ & - \\
\hline$\alpha_{\text {column }}$ & $(12.15)$ & $\alpha_{\text {column }}=\frac{1}{1+\frac{0.257}{2.35}}=0.90$ & - \\
\hline$\alpha_{\theta}$ & - & 0.65 (Class B) & - \\
\hline$\alpha_{\theta}^{*}$ & (12.14) & $\alpha_{\theta}^{*}=0.65+(0.9-0.65)\left(1-\frac{600}{108^{2}}\right)=0.89$ & - \\
\hline factors & - & $\bar{\lambda}_{\theta 0}=0.40, \quad \beta=0.60, \quad \eta=1.00$ & - \\
\hline $\bar{\lambda}_{\theta p}$ & - & $\bar{\lambda}_{\theta p}=\sqrt{0.89 /(1-0.6)}=1.49$ & - \\
\hline$\chi_{\theta}$ & - & $\chi_{\theta}=0.89 / 3.09^{2}=0.093$ & - \\
\hline$\sigma_{\theta, R d}$ & - & $\sigma_{\theta, R d}=\frac{0.093 \cdot 430}{1.1}=36$ & $\mathrm{~N} / \mathrm{mm}^{2}$ \\
\hline
\end{tabular}

The buckling strength verifications based on the proposed design procedures are calculated as: 
Tab. 12.6: Buckling strength verifications at anchor level and maximum moment

\begin{tabular}{|l|c|c|}
\hline position & anchor $(-6 \mathrm{~m})$ & max. moment $(-17.5 \mathrm{~m})$ \\
\hline $\begin{array}{l}\text { axial } \\
\text { compression }\end{array}$ & $\frac{\sigma_{x}}{\sigma_{x, R d}}=\frac{270}{336}=0.80<1 \quad(0.81<1)$ & $\frac{\sigma_{x}}{\sigma_{x, R d}}=\frac{353}{336}=1.05>1 \quad(1.06>1)$ \\
\hline $\begin{array}{l}\text { circumferential } \\
\text { compression }\end{array}$ & $\frac{\sigma_{\theta}}{\sigma_{\theta, R d}}=\frac{2}{36}=0.06<1 \quad(0.15<1)$ & $\frac{\sigma_{\theta}}{\sigma_{\theta, R d}}=\frac{3}{36}=0.08<1 \quad(0.23<1)$ \\
\hline interaction & $\left(\frac{\sigma_{x}}{\sigma_{x, R d}}\right)^{1.25}+\left(\frac{\sigma_{\theta}}{\sigma_{\theta, R d}}\right)^{1.25} \leq 1$ \\
\hline verification & $0.76+0.03=0.79<1 \quad(0.86<1)$ & $1.06+0.04=1.10>1 \quad(-)$ \\
\hline
\end{tabular}

Tab. 12.7: Buckling strength verifications at maximum external pressure

\begin{tabular}{|l|c|}
\hline position & maximum external pressure $(-6 \mathrm{~m})$ \\
\hline $\begin{array}{l}\text { axial } \\
\text { compression }\end{array}$ & $\frac{\sigma_{x}}{\sigma_{x, R d}}=\frac{37}{336}=0.11<1 \quad(0.11<1)$ \\
\hline $\begin{array}{l}\text { circumferential } \\
\text { compression }\end{array}$ & $\frac{\sigma_{\theta}}{\sigma_{\theta, R d}}=\frac{35}{36}=0.97<1 \quad(3.00>1)$ \\
\hline interaction & $\left(\frac{\sigma_{x}}{\sigma_{x, R d}}\right)^{1.25}+\left(\frac{\sigma_{\theta}}{\sigma_{\theta, R d}}\right)^{1.25} \leq 1$ \\
\hline verification & $0.06+0.96=1.02 \approx 1 \quad(-)$ \\
\hline
\end{tabular}

It can be seen Tab. 12.6 and Tab. 12.7 that the verifications are fulfilled mostly. Only the verification at the position of the maximum moment is exceeded by $10 \%$. This is due to the aspect that the meridional stresses dominate the assessment for this position. Regarding the axial stresses the derived beneficial influence is limited to the surrounding soil which then again is fairly small for axially loaded cylinders. The values from the reference case calculation without considering the new design proposals are given in brackets. Still, the assessment according to Tab. 12.6 has improved in contrast to the common practical design calculations from chapter 4 by $10 \%$ to $20 \%$. The influence of the proposed design rules has an obvious influence on the buckling strength verifications involving external pressure (Tab. 12.7). Here, the design buckling stress was exceeded significantly in the design procedure from chapter 4 . For the approach considering the design proposals the verification is fulfilled (Tab. 12.7). Here it needs to be emphasized that in the assessment very conservative assumption were made since the supporting influence of the soil was imposed only to half of the circumference for the entire tubular pile. 


\section{References}

Altenbach J, Altenbach H; Einführung in die Kontinuumsmechanik, Teubner Studienbücher Mechanik, Stuttgart 1994.

American Petroleum Institute (API); Recommeded Practice for Planning, Designing and Constructing Fixed Offshore Platforms - Working Stress Design; RP 2A-LRFD und -WSD, 2000.

Askegaard V; Measurement of Pressure between a Rigid Wall and a Compressible Medium by means of Pressure Cells. Acta Polytechnica Scandinavia. Civil Engineering an Construction Series n11, Danish Contribution n14, Copenhagen, 1961.

Baguelin F, Frank R, Said Y H; Theoretical study of lateral reaction mechanism of piles, Geotechnique, v27, n3, pp. 405-434, 1977.

Baguelin F, Jézéquel J F, Shields D H; The Pressuremeter and Foundation Engineering, Trans Tech Publications, 1978.

Barton Y O, Finn W D L, Parry R H G, Towhata I; Lateral pile response and p-y curves for centrifuge tests, Proc. of $15^{\text {th }}$ OTC, Houston, Texas, 1983.

Bauer E; Zum mechanischen Verhalten granularer Stoffe unter vorwiegend ödometrischer Beanspruchung, Veröffentlichungen des Institutes für Bodenmechanik und Felsmechanik der Universität Fridericiana in Karlsruhe, Heft 130, 1992.

Beikae M; A new solution for the stresses and displacements caused by lateral loading of a pile; Technical note TAGA 82-02, Telegraph Avenue Geotechnical Associates, Berkeley, California, Nov 1982.

Besler D; Wirklichkeitsnahe Erfassung der Fußauflagerung und des Verformungsverhaltens von gestützten Baugrubenwänden, Schriftenreihe des Lehrstuhls Baugrund-Grundbau der Universität Dortmund, Heft 22, Dortmund, 1998.

Bijlaard P P; Gallagher R H; Elastic Instability of a Cylindrical Shell Under Arbitrary Circumferential Variation of Axial Stress, J. Aero. Sci., v27, n11, pp. 854858,866, 1959.

Böhrnsen J U; Dynamisches Verhalten von Schüttgütern beim Entleeren aus Silos, Braunschweiger Schriften zur Mechanik, n45, 2002.

Bornscheuer B-F; Stabilitätsnachweise für Platten und Schalen in gleicher Darstellung wie für Stäbe. Der Stahlbau, v12, pp. 364-368, 1985.

Bornscheuer FW; Beulsicherheitsnachweis für Schalen (DASt-Richtlinie 013). Die Bautechnik, v58, pp. 313-317, 1981. 
Bowles J E; Foundation analysis and design, McGraw-Hill Book Company, New York, N.Y., USA, 4th edition, pp. 1004, 1988.

Bowles, J.E.; Engineering properties of soils and their measurement, McGraw Hill, 1992.

Bransby M F; Selection of $p-y$ curves for the design of single laterally loaded Piles, International Journal for numerical and analytical methods in geomechanics, v23, pp. 1909-1926. 1999.

Brazier L G; On Flexure of Thin Cylindrical Shell and Other Thin Sections, Proceedings of the Royal Society of London, Ser. A, 116, pp. 104-114, 1927.

Broms, B B; Lateral Resistance of Piles in Cohesive Soils, Proceedings of the ASCE, v90, SM2, pp. 27-63, 1964.

Broms, B B; Precast piling practice, Telford, 1981.

Budiansky B, Amazigo J C; Initial Post-buckling of Cylindrical Shells Under external Pressure, Journal of mathematics and physics, v47, pp. 223-235, 1968.

Burland J B, Broms B B, de Mello V F B; Behavior of foundations and structures, Proceedings of the Ninth International Conference on Soil Mechanics and Foundation Engineering, Japanes Society of Soil Mechanics and Foundation Engineering, Tokyo, Japan, v2, pp. 495-546, 1977.

Byrne B W, Houlsby G T; Foundations for offshore wind turbines, Philosophical Transactions: Mathematical, Physical and Engineering Sciences; v361, n1813, pp. 2909-2930, 2003.

Charue N, Holeyman A, Hübner A, Saal H, Tomboy O; Design, build up and control of a large scale experimental set-up for laterally and vertically loaded piles in dry sand, Proceedings, ISOPE Lisbon, 2007.

Charue N, Holeyman A; Technical Report No. 4 and 5, Project "ETIB - Enhanced Economy of Tubular Piles by Improved Buckling Design", supported by the Research Fund for Coal and Steel of the European Commission (contract number RFS-CR04041), 2007.

Cleaver P C; The Strength of Tubes under Uniform External Pressure, SRC Technical Report No. 253, Ministry of Supply - Aeronautical Research Council, 1956.

Crisfield M A; A Fast Incremental/Iteration Solution Procedure that Handles Snap, Computers and Structures, v13, pp. 55-62, 1981.

Dahlhoff P, Steck M, Taferner J; Chancen und Grenzen von Monopiles - Erste Erkenntnisse aus dem Forschungsvorhaben Opti-Pile; Chancen und Grenzen von Monopiles 2.Tagung Offshore Wind Energie, Hamburg, 2003.

Davisson M T; Lateral Load Capacity of Piles, High. Res. Rec., n333, pp.104-112, 1970. 
Dhouib A; Methods based on subgrade reaction coefficients used in France to design retaining walls: parameter study. Revue Francaise de Geotechnique, Fasicule, v72, pp. 11-21, 1995.

Dierks K; Zur Kreiszylinderschale unter Manteldruck, Abhandlungen der Braunschweigischen Wissenschaftlichen Gesellschaft XVIII, pp. 139, 1965.

Dow D A; Buckling and postbuckling tests of ring-stiffened circular cylinders loaded to failure by uniform external air pressure, NASA Center for AeroSpace Information (CASI), NASA-TN-D-3111, Nov 1, 1965.

Dyson G J, Randolph M F; Load transfer curves for pile in calcareous sand, Proc. $8^{\text {th }}$ Int. Confer. on the Behaviour of Offshore Structures, Delft, v3, pp. 245-258, 1997.

Ehlers K; Beiträge zur Ermittlung der Schüttgutkonstanten und deren Einfluss auf Silolasten, Dissertation, Universität Braunschweig, 1979.

Esslinger M, Geier B, Wood JGM; Some complements to the ECCS design code concerning isotropic cylinders. Stability of Steel Structures, Liege 13.-15. April, pp. 589-598, 1977.

Esslinger M, Geier B; Buckling and Postbuckling Behavior of Thin-Walled Circular Cylinders. Deutsche Luft- und Raumfahrt, Forschungsbericht 69-99, 1969.

Esslinger M, Geier B; Calculated postbuckling loads as lower limits for the buckling loads of thin-walled circular cylinders. Buckling of structures - Proceedings of the Symposium, Cambridge, Mass, 17-21 June 1974. pp. 274-290. 1976.

Esslinger M, Geier B; On the buckling and postbuckling behavior of thin-walled circular cylinders. Laboratoires d'Essais et de Recherches sur les Materiaux et les Constructions, Reunion Internationale, Buenos Aires, Argentina, 13-18 Sept, pp. 97124, 1971.

Esslinger M, Geier B; Postbuckling Behavior of Structures. CISM Courses and Lectures No. 236, Springer Verlag New York - Wien, 1975.

Fellin W, Kolymbas D; Hypoplastizität für leicht Fortgeschrittene, Bautechnik, v79, n12, pp. 830-841, 2002.

Fellin W, Ostermann A; Consistent tangent operators for constitutive rate equations. International Journal for Numerical and Analytical Methods in Geomechanics, v26, pp.1213-1233, 2002.

Fellin W; Hypoplastizität für Einsteiger. (Hypoplasticity for beginners), Bautechnik, v77, n1, pp. 10-14, 2000.

Fiedler M; Nichtlineare Berechnungen von Plattenfundamenten - Nonlinear Analysis of Mat Foundations, Advances in Geotechnical Engineering and Tunneling, v6, Logos Verlag Berlin, 2002. 
Flügge W; Die Stabilität der Kreiszylinderschale, Ingenieur-Archiv, v3, pp. 463-506, 1932 .

Franke E; Pfähle. Grundbautaschenbuch, 4. Auflage, Teil 3, pp. 230-236, Verlag Ernst \& Sohn, Berlin, 1992.

Fuchs G; Zum Tragverhalten von kreisförmigen Doppelsilos unter Berücksichtigung der Eigensteifigkeit des Füllguts, Dissertation, Technische Universität Braunschweig, 1969.

Gabr M A, Wang J J, Zhao M; Buckling of Piles with General Power Distribution of Lateral Subgrade Reaction, Journal of Geotechnical and Geoenvironmental Engineering, v123, February 1997.

Grabe J, Dührkop J, Mahutka K-P; Monopilegründungen von Offshore Windenergieanlagen- Zum Ansatz der Bettung; Bautechnik, v82, n1; 2005

Habibagahi K, Langer J A; Horizontal subgrade modulus of granular soils. Laterally loaded deep foundations: Analysis and performance; ASTM STP 835, pp. 21-34, 1984.

Herle I; Hyoplastizität und Granulometrie einfacher Korngerüste, Veröffentlichungen des Institutes für Bodenmechanik und Felsmechanik der Universität Fridericiana in Karlsruhe, Heft 142, 1997.

Hibbitt, Karlsson, Sorensen; ABAQUS Theory-Manual 6.3, Inc., Pawtucket, RI 02860-4847, USA, 2002.

Holeyman A, Hübner A, Saal H, Tomboy O; Comparison of different methods for simulating laterally loaded pile behaviour, Proceedings ISOPE, San Francisco, 2006.

Horvath J S; Laterally Loaded Deep Foundations: Analysis and Performance, Special Technical Publication No. 835, American Society for Testing and Materials, Philadelphia, Pa.,USA, pp. 112-121, 1984.

Horvath J S; Numerical Analysis of Beams and Beams-Columns with Linear and Non-Linear Spring Supports using Finite Differences, Research Report No. CE/GE22-1, Manhattan College, Civil Engineering Department, Bronx, N.Y., USA, 1988.

Horvath J S; Subgrade modeling for soil-structure interaction analysis of horizontal foundation elements, special joint meeting of the Structural and Geotechnical groups of the American Society of Civil Engineers Metropolitan Section, New York, N.Y., USA, 1993.

Hou J P, Hills D A; Contact between a pin and a plate with a hole under interferencefit and clearance-fit conditions, Proceedings of the I MECH E Part C Journal of Mechanical Engineering Science, v215, n6, pp. 629-639, 2001. 
Houlsby G T, Purzin A M; A thermomechanical framework for constitutive models for rate-independent dissipative materials, International journal of plasticity, v16, n9, pp. 1017-1047, 2000

Hübner A, Saal H; Tubular piles - buckling design for a complex situation. Proceedings SDSS, Lissabon, 2006.

Hübner A, Teng J G, Saal H; Buckling Behaviour of Large Steel Cylinders with Patterned Welds, Int J Pres Vess Pip, v83, n1, pp. 13-26, 2006.

Jardine R J, Chow F C; New design method for offshore piles, MTD Publication 96/103, Marine Technology Directorate, London, 1996.

Jardine R J, Overy R F , Chow F C; Axial capacity of offshore piles in dense north sea sand, Journal of Geotechnical and Geoenvironmental Engineering, ASCE, v124, n2, pp. 171-178, 1998.

Johnson K L; Contact Mechanics, Cambridge University Press, Cambridge, UK, 1985.

Karamanos, S A; Bending Instabilities of Elastic Tubes, International Journal of Solids \& Structures, v39, n8, pp. 2059-2085, 2002.

Kim B T, Kim N-K, Lee W J, Kim Y S; Experimental Load-Transfer Curves of Laterally Loaded Piles in Nak-Dong River Sand, Journal of geotechnical and geoenvironmental engineering, v130, n4, pp. 416-425, 2004.

Knebel K, Schweizerhof K; Buckling of Cylindrical Shells Containing Granular Solids, Thin-Walled Structures, v23, pp295-312, 1995.

Knebel K; Stabilität von Stahlzylindern mit unilateralen Randbedingungen bei statischer und dynamischer Beanspruchung, Dissertation, Universität Karlsruhe, Februar 1997.

Knödel P; Stabilitätsuntersuchungen an kreiszylindrischen stählernen Siloschüssen, Dissertation, Universität Karlsruhe, Januar 1994.

Kolk H J, Baaijens A E, Senders M; Design criteria for pipe piles in silicia sand, Proc. Int. Syp. in Frontiers in Offshore Geotechnics, Perth, 2005.

Kollár L, Dulácska E; Schalenbeulung, Werner-Verlag, Düsseldorf, 1975.

Kolymbas D; Eine konstitutive Theorie für Böden und andere körnige Stoffe. Habilitationsschrift,. Veröffentlichung des Instituts für Bodenmechanik und Felsmechanik an der Universität Fridericiana in Karlsruhe, Heft 109, Karlsruhe, 1988.

Kolymbas D; Pfahlgründungen; Springer, 1989.

Langen H v; Numerical analysis of soil structure interaction, Delft, Techn. Univ., Diss., 1991. 
Liao S S C; Estimating the coefficient of subgrade reaction for plane strain conditions, Geotechnical Engineering, London, U.K., v113, n3, pp. 166-181, 1995.

Lin S, Hills D A, Nowell D; Stresses in a flat plate due to a loose pin pressing against a cracked hole, The Journal of Strain Analysis for Engineering Design, v32, n2, pp. 145-156, 1997.

Lorenz R; Achsensymmetrische Verzerrungen in dünnwandigen Hohlzylindern, VDI Zeitschrift, v52, n43, pp. 1706-1713, 1908.

Monnet A; Subgrade reaction modulus, decompression ratio, about parameters used for elasto-plastic computation of retaining walls, Revue Francaise de Geotechnique, Fasicule v66, pp. 67-72, 1994.

Niemunis A, Cudny M; On Hyperelasticity for Clays, Computers and Geotechnics, v23, pp. 221-236, 1998.

Ohde J; Grundbaumechanik, Hütte, BD III, Aufl. 27, 1951.

Olivella X O, de Saracibar Bosch C A; Mecánica de medios continuos para ingenieros, Edicions UPC, Barcelona, 2000.

Parisch H; Festkörperkontinuumsmechanik, B.G. Teubner Stuttgart, 1. Auflage 2003.

Pasternak P L; On a new method of analysis of an elastic foundation by means of two foundation constants, Gos. Izd. Lit. po Strait I Arkh, Moskow, the Soviet Union (in Russian), 1954.

Persson A; On the Stress Distribution of Cylindrical Elastic Bodies in Contact, Doctoral dissertation, Chalmers University of Technology, Göteborg, Sweden, 1964.

Petersen C; Stahlbau: Grundlagen der Berechnung und baulichen Ausbildung von Stahlbauten, Braunschweig, Vieweg, 1997.

Pflüger A; Stabilitätsprobleme der Elastostatik, Berlin, Heidelberg, Springer, 1964.

Pflüger A; Zur praktischen Berechnung der Kreiszylinderschale unter Manteldruck, Der Stahlbau, v5, n8, pp. 249-252, 1966.

Pieper K; Der Standsicherheitsnachweis von Zylinderschalen aus Stahl als Silos, In: Esslinger, M (Hrsg.): Schalenbeultagung Darmstadt, Vorträge und Diskussionsbeiträge. DFVLR, Braunschweig, 1979.

Poulos H G, Davis E H; Pile foundation analysis and design, Series in geotechnical engineering, Eds. T.W. Lambe, R.V. Whitman, John Wiley and sons, 1980.

Poulos H G; Behaviour of laterally loaded piles: 1 - Single piles. Journal of the soil mechanics and foundations division, ASCE, Vol 97, pp. 711-731, 1971.

Powell G, Simon J; Improved Iterative Strategy for Nonlinear Structures, International Journal for Numerical Methods in Engineering, vol. 17, pp.1455-1467, 1981. 
Pyke R, Beikae M; A new Solution for the Resistance of Single Piles to Lateral Loading, in Laterally Loaded Deep Foundations: Analysis and Performance, Philadelphia, 1984.

Rajashree S S, Sitharam T G; Nonlinear Finite-Element Modeling of Batter Piles under Lateral Load, Journal of Geotechnical and Geoenviromental Engineering, v127, n7, pp. 604-613, 2001.

Ramm E; Strategies for Tracing the Nonlinear Response Near Limit Points, Nonolinear Finite Element Analysis in Structural Mechanics, edited by E. Wunderlich, E. Stein, and K.J. Bathe, Springer Verlag, Berlin, 1981.

Reese L C, Cox W R, Kopp F D; Analysis of Laterally Loaded Piles in Sand, Offshore Technologie Conference, Dallas Texas, paper OTC 2080, 1974.

Reese L C, Impe W F v; Single piles and pile groups under lateral loading. A.A. Balkema, Rotterdam. 2001.

Reissner E; A Note on Deflections of Plates on a viscoelastic Foundation, Journal of Applied Mechanics, v80, pp. 144-145, 1958.

Reissner E; A note on the formulation of the Deflection of Plates on an Elastic Foundation, Acta mechanica, v4, pp. 88-91, 1967.

Rhines W J; Foundation Models for Continuously Supported Structures, Thesis presented to New York University, Bronx, N.Y., U.S.A., in partial fulfilment of the requirements for the degree of Doctor of Philosophy, 236 pp., 1965.

Riks E; The application of Newton's method to the problem of elastic stability, Trans. ASME, J. Appl. Mech. 1060-1065,1972.

Rombach G A; Schüttguteinwirkungen auf Silozellen : exzentrische Entleerung, Universität Karlsruhe, Dissertation, 1991.

Rotter J M, Teng J G; Elastic Stability of of Cylindrical Shells with Circumferential Weld Depressions under Axial Compression, Research Report R564, School of Civil and Mining Engineering, University of Sydney, 1988.

Rotter J M, Zhang Q; Elastic Buckling of Imperfect Cylindrical Shells Containing Granular Solids, Research Report No. R589, The University of Sydney, 1989.

Rotter J M; Development of Proposed European Design Rules for Buckling of Axially Compressed Cylinders, Advances in Structural Engineering, v1, n4, pp. 273286, 1998.

Rotter J M; Elastic Stability of Cylindrical Shells with Weld, Journal of structural engineering, v115, n5, pp1244-1263, 1989.

Saal H, Kahmer H, Reif A; Beullasten axial gedrückter Kreiszylinderschalen mit Innendruck - Neue Versuche und Vorschriften, Der Stahlbau, v48, pp. 262-269, 1979. 
Schardt R; Verallgemeinerte technische Biegetheorie: lineare Probleme; Springer, 1989.

Schlegel T; Anwendung einer neuen Bettungsmodultheorie zur Berechnung biegsamer Gründungen auf Sand, Veröffentlichung des Instituts für Bodenmechanik und Felsmechanik an der Universität Fridericiana in Karlsruhe, n98, 1985.

Schmidt H; Großversuch zur Ermittlung des Tragverhaltens von Pfahlreihen unter horizontaler Belastung, Mitteilungen des Instituts für Grundbau, Boden und Felsmechanik der TH Darmstadt, Heft 25, 1986.

Schmidt H; Stabilität von Kreiszylinderschalen - Imperfektionsempfindlichkeit und Bemessung. In: Berechnung, Gestaltung und Fertigung von Schweisskonstruktionen im Zeitalter der Expertensysteme, DVS-Bericht 133, 1991.

Schmitt P; Estimating the coefficient of subgrade reaction for diaphragm wall and sheet pile wall design, Revue Francaise de Geotechnique, Fasicule v71, pp. 3-10, 1995.

Schneider K-J; Bautabellen für Ingenieure, Werner Verlag, Düsseldorf, 13. Auflage, 1998.

Schneider W, Brede A; Consistent equivalent geometric imperfections for the numerical buckling strength verification of cylindrical shells under uniform external pressure, Thin-Walled Structures, v43, n2, pp. 175-188, 2005.

Schulz U; Stabilitätsnachweise bei Schalen. Bericht der Versuchsanstalt für Stahl, Holz und Steine der Universität Karlsruhe, Heft 2, 1981.

Schweizerhof K, Hauptmann R, Knebel K, Rottner T, Raabe M; Statische und dynamische FE-Stabilitätsuntersuchungen an Siloschalen mit ungleichförmiger Schüttgutfüllung, Proc. Finite Elemente in der Baupraxis FEM'98, ed. Wriggers, Meißner, Stein, Wunderlich, Darmstadt 1998.

Schweizerhof K, Ramm E; Follower Force Effects on Stability of Shells under Hydrostatic Loads, Journal of Engineering Mechanics, v113, n1, pp.72, 1987.

Scott R F; Analysis of centrifuge pile tests: Simulation of pile driving, Research Rep. OSAPR Project 13, American Petroleum Institute, Washington, DC., 1980.

Scott R F; Foundation Analysis, Prentice-Hall, Inc., Englewood Cliffs, NJ 07632, 1981.

Seide P; The Stability Under Axial Compression and Lateral Pressure of CircularCylindrical Shells With a Soft Elastic Core, Structures Session IAS 30th AM, New York, pp. 851, 1962.

Skempton A W; The Bearing Capacity of Clays, Build. Res Congress, London, Inst. Civ. Engrs., div. I; pp. 180, 1951 
Stracke M; Stabilität kurzer stählerner Kreiszylinderschalen unter Außendruck. Diss. Universität GH Essen, Schweißtechnischer Forschungsbericht Band 12, Düsseldorf, 1987.

Teng J G, Rotter J M; Buckling of Thin Metal Shells, Taylor \& Francis Group, 2004.

Terzaghi K; Evalution of coefficients of subgrade reaction. Geotechnique, v5 , pp. 297-326, 1955.

Thimoshenko S P; Theory of Elastic Stability, McGraw-Hill Book Company,2nd Edition, Leipzig, 57,pp. 337-385, 1910.

Ummenhofer T; Stabilitätsverhalten imperfekter zylindrischer Stahlsiloschalen experimentelle und numerische Untersuchungen, Dissertation, Universität Karlsruhe, Juli 1996.

v. Wolffersdorff P A; A hypoplastic relation for granular materials with a predefined limit state surface, Mechanics of Cohesive-Frictional Materials, v1, pp. 251-271, 1996.

Vanden Berghe J-F; Sand Strength degradation within the framework of vibratory pile driving; Doctoral Thesis, UCL, 2001.

Verruijt A; Computational Geomechanics, Kluwer Academic Pub, 1995.

Verruijt A; Program SPW2004, Delft, 2004.

Vesic A B; Bending of beams resting on isotropic elastic solids, J. Eng. Mech. Div., ASCE 87, pp. 35-53, 1961.

Vlaslov V Z, Leontev N N; Beams, Plates and Shells on Elastic Foundations (Balki, Plity I Obolochki na upragom osnovanii) Gosudratstvennoe Izdatel'stvo, FizikoMatematicheskoi Literatury, Moscow, U.S.S.R.; translated from Russian, published 1966 by Israel Program for Scientific Translations, The National Aeronautics and Space Administration, The National Science Foundation, Washington, D.C., U.S.A., 1960.

Weingarten V I, Wang Y S; Stability of Shells Attached to Elastic Core, Journal of the Engineering Mechanics Division, v102, nEM5, 1976.

Wempner G; Discrete approximation related to nonlinear theories of solids, International Journal of Solids and Structures, v7 pp.1581-99, 1971.

Wernick E; Tragfähigkeit zylindrischer Anker in Sand unter besonderer Berücksichtigung des Dilatanzverhaltens, Veröffentlichung des Institutes für Bodenmechanik und Felsmechanik, Universität Karlsruhe, 75; 1978.

White D J; A general framework for shaft resistance for displacement piles in sand, Proc. Int. Syp. in Frontiers in Offshore Geotechnics, Perth, 2005. 
Wiemann J; Lesny K; Richwien W: Anwendbarkeit von Standardverfahren zur Bemessung von Pfahlgründungen für Offshore-WEA. 3. Symposium OffshoreWindenergie Bau- und umwelttechnische Aspekte, Hannover, 16. März 2004.

Wiemann J; Lesny K; Richwien W: Gründung von Offshore-Windenergieanlagen Gründungskonzepte und geotechnische Grundlagen. Mitteilungen aus dem Fachgebiet Grundbau und Bodenmechanik der Universität Essen, Heft 29, Hrsg.: Prof. Dr.-Ing. W. Richwien, Verlag Glückauf, Essen, 2002.

Windenburg D F, Trilling C; Collapse by instability of thin cylindrical shells under external pressure, Trans. ASME, II, pp. 819-825, 1934.

Winkler E; Die Lehre von der Elasticitaet und Festigkeit, Prag, Dominicus, 1876.

Wu W; Hypoplastizität als mathematisches Modell zum mechanischen Verhalten granularer Stoffe, Veröffentlichungen des Institutes für Bodenmechanik und Felsmechanik der Universität Fridericiana in Karlsruhe, Heft 129, 1992.

Wunderlich W, Schäpertöns B, Temme C; Dynamic stability of nonlinear shells of revolution under consideration of the soil-fluid-structure-interaction. In: International Journal for Numerical Methods in Engineering, v37, pp2679-2697, 1994.

Yan L, Byrne P M; Lateral pile response to monotonic pile head loading, Can Geotech. J., v29, pp. 955-970, 1992.

Yao J C; Buckling of Axially Compressed Long Cylindrical Shell With Elastic Core, Journal of Applied Mechanics, pp. 329-334, 1962.

Young W C; Roark's formulas for stress and strain, New York, McGraw-Hill, 1989.

Zak A R, Bollard R J H; Elastic Buckling of Cylindrical Thin Shells Filled with an Elastic Core, ARS Journal, pp. 588-593, 1962.

Zhang Q, Ansourian P; Axial Load Buckling of Steel Cylinders with Particulate Soil Content, Structural Stability Research Council, Annual Technical Session, Earthquake Stability Problems in North America, Pittsburgh, Pennsylvania, 1992.

Zhang Q, Ansourian P; Stability of Silo Wall Stiffened by Particulate Solids, Civil Engineering Transactions, IEAust, CE33, v2, pp. 137-141, 1991.

\section{$\underline{\text { Standards/Codes/Commentaries }}$}

Beuth-Kommentare; Stahlbauten: Erläuterungen zu DIN 18800-1 bis -4, hrsg. J. Lindner, J. Scheer, H. Schmidt, 1998.

British Standard BS 5500: Specification for unfired fusion welded pressure vessels, 1997. 
Centre for Civil Engineering Research and Codes - CUR: Bearing capacity of steel pipe piles, Gouda, Netherlands, 2001.

Deutscher Ausschuss für Stahlbau: DASt-Richtlinie 013: Beulsicherheitsnachweise für Schalen., 1980.

Deutschen Gesellschaft für Geotechnik (DGGT); Empfehlungen für statische und dynamische Pfahlprüfungen des Arbeitskreises 2.1 der DGGT, Schriftenreihe des IGB·TUBS, Institut für Grundbau uns Bodenmechanik der TU Braunschweig.1998.

Deutsches Institut für Bautechnik (DIBt); Mitteilungen des DIBt, Ergänzungserlaß zu DIN 4114, v3, 1973.

DIN 1054: 2005-01; Baugrund - Sicherheitsnachweise im Erd- und Grundbau.

DIN 4085: 2002-12; Baugrund - Berechnung des Erddrucks.

DIN 4114: Stahlbau. Stabilitätsfälle (Knickung, Kippung, Beulung), 1952.

DIN 18800-4: Stahlbauten; Stabilitätsfälle, Schalenbeulen, 1990.

DIN 18135:1999-06; Baugrund - Untersuchung von Bodenproben Eindimensionaler Kompressionsversuch.

ENV 1993-5:2002; Eurocode 3: Design of Steel Structures. Part 5: Piling, Brussels, European Committee for Standardisation, 2004.

prEN 1993-1-1:2003-12; Eurocode 3: Design of Steel Structures. Part 1-1: General rules and rules for buildings, Brussels: European Committee for Standardisation, 2003.

prEN 1993-1-6:2002-05; Eurocode 3: Design of Steel Structures. Part 1-6: Strength and stability of shell structures, Brussels, European Committee for Standardisation, 2002.

prEN 1993-5:2004-07; Eurocode 3: Design of Steel Structures. Part 5: Piling, Brussels, European Committee for Standardisation, 2004.

European Recommendations - Buckling of Steel Shells, ECCS - Technical Committee 8 - Structural Stability, Technical Working Group 8.4, Fourth Edition, 1988.

ISO/DIS 19902:2004; Petroleum and Natural Gas Industries — Fixed Steel Offshore Structures.

Det Norske Veritas- 2004; Norwegian Code. 


\section{Annex A: Retaining wall calculation}

\section{A.1 General}

For the calculation of the deformations and especially the resultant forces of a combiwall different investigations are performed in this research project. For the design of a reference case the resultant forces required for the assessment according to different design codes were calculated by means of finite element programs.

In geotechnical engineering various methods are used for analysing structures. The applied finite element programs are based in the beam theory considering elasto-plastic foundations. The evaluation of the resultant forces is performed with the finite element program RIDO. In Annex A the concept of the program is described briefly. Further, a comparison based on the reference case from chapter 4 between the results from RIDO and another finite element program for calculating retaining walls (SPW2004) is performed.

\section{A.2 Analysis with RIDO}

The finite element program RIDO (2004) was programmed by Fages in 1974 and improved over the years. The finite element program calculates the elasto-plastic equilibrium for retaining walls and piles subjected to loads. Besides the geometry of the problem the data given in Tab. A.1 is needed as input for calculating the reference case of the combiwall.

Tab. A.1: Input data for RIDO

\begin{tabular}{|c|c|c|c|c|c|}
\hline$\varphi_{c}\left[^{\circ}\right]$ & $\delta\left[^{\circ}\right]$ & $k_{S}\left[\mathrm{MN} / \mathrm{m}^{3}\right]$ & $\gamma\left[\mathrm{kN} / \mathrm{m}^{3}\right]$ & $\gamma^{\prime}\left[\mathrm{kN} / \mathrm{m}^{3}\right]$ & $\begin{array}{c}\text { anchor stiffness } \\
{\left[\mathrm{MN} / \mathrm{m}^{2}\right]}\end{array}$ \\
\hline 30 & 15 & 30 & 19 & 11 & 100 \\
\hline
\end{tabular}

The analyses of the resultant forces and the soil reaction is performed by a calculation of equilibrium from the different installation steps of the combiwall. The approaches of Rankine, Boussinesq and Jaki are implemented in the program for evaluating the active and passive earth pressure coefficients and the earth pressure coefficients at rest, which are needed as limits for the plastic deformation. The elastic deformations are defined by a constant coefficient of subgrade reaction which is distributed uniformly along the combiwall for simplification. 


\section{A.3 Analysis with SPW2004}

The finite element program SPW2004 by Verruijt (2004) is programmed in Turbo Pascal available as shareware is based on the concept of a beam supported by Winkler springs. The reaction of the soil on both sides of the beam consists of each of two parts. Subjected to loads the soil behaves linear and deforms proportionally to the load. The deformations are limited by the active and the passive earth pressure as a lower and upper boundary, respectively. The elasto-plastic reaction of a cohesionless soil is depicted in Fig. A.1. This spring model is only valid for deformations in positive direction (here to the right). For deformations in the opposite direction an accordingly modified model is used.

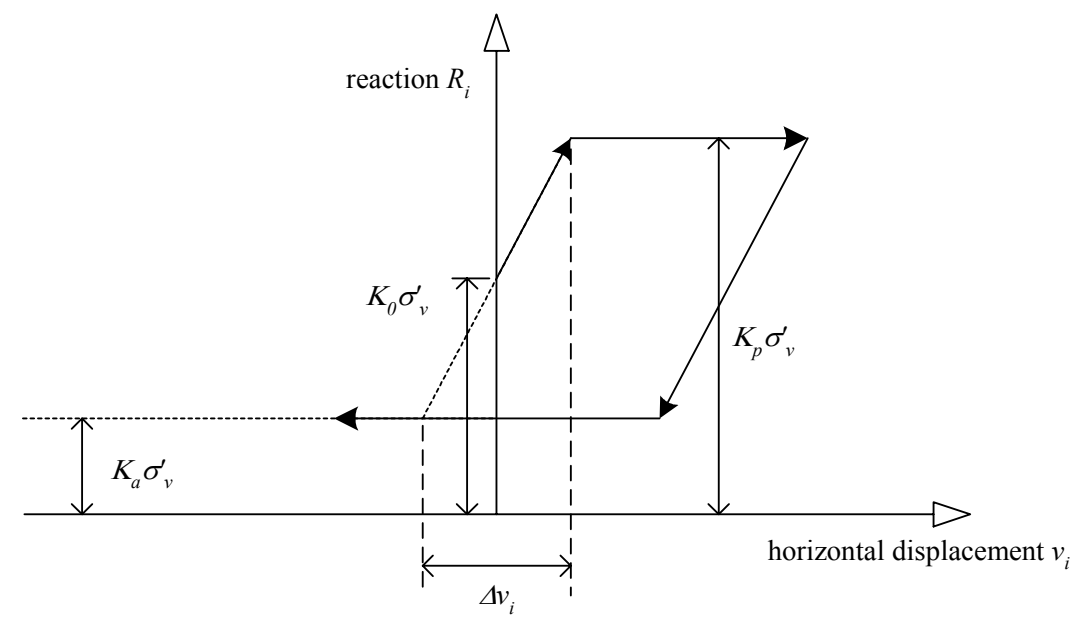

Fig. A.1: Elasto-plastic reaction for a cohesionless soil

For calculating the combiwall from the reference case the input data listed in Tab. A.2 are used besides the geometry of the system.

Tab. A.2: Input data for SPW2004

\begin{tabular}{|c|c|c|c|c|c|}
\hline$K_{a}[-]$ & $K_{b}[-]$ & $K_{0}[-]$ & $\gamma\left[\mathrm{kN} / \mathrm{m}^{3}\right]$ & $\gamma^{\prime}\left[\mathrm{kN} / \mathrm{m}^{3}\right]$ & $\Delta v_{i}[\mathrm{~mm}]$ \\
\hline 0.291 & 4.810 & 0.500 & 19 & 11 & 20 \\
\hline
\end{tabular}

The gradient of the soil reaction from Fig. A.1 is defined as the spring stiffness. In contrast to RIDO in which this value is constant, the soil stiffness changes in SPW2004 dependent on the effective stresses. In this approach the length $\Delta v_{i}$ is kept constant and thus the stiffness increases with the depth. The input parameters were adjusted to the input data for RIDO as far as possible.

\section{A.4 Comparison RIDO - SPW2004}

In Fig. A.2 and Fig. A.3 the results from the calculations with RIDO and SPW2004 for the reference case are compared. Diagrams for the horizontal displacements, the bending moment and shear force distributions, and the resulting pressure distribution on 
the tube wall are included. Since SPW2004 cannot differ the pressure on the two sides of the wall, the difference between both sides is compared.
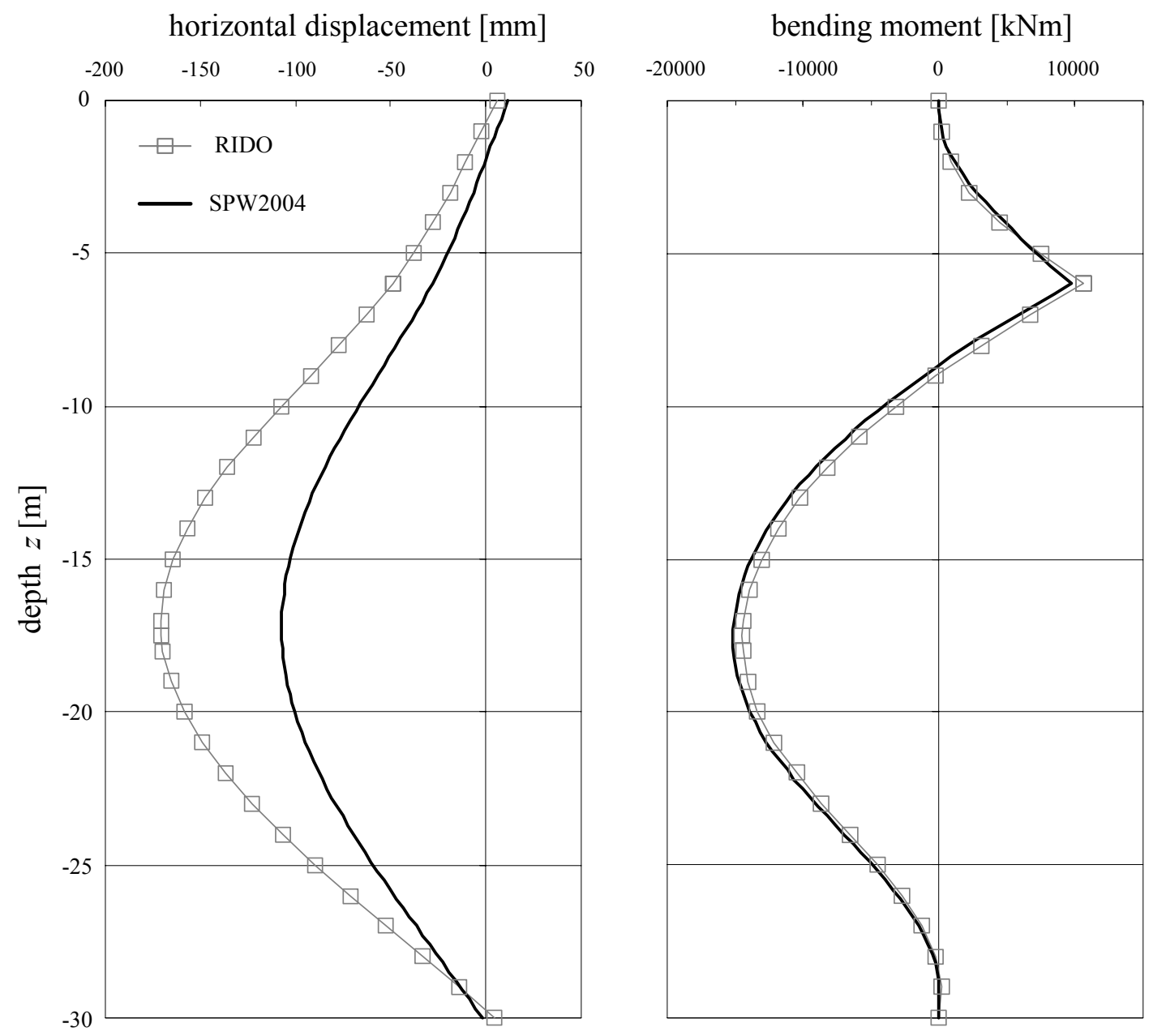

Fig. A.2: Displacements and bending moment distribution for the tubular piles

The results for the bending moment distribution exhibit slight deviations in the area of the maximum moment. The displacement at the position of the maximum moment is $60 \%$ larger in the calculation from RIDO than in the analysis from SPW2004. The pile tip moves a little bit to the left if calculated with RIDO. In contrast the calculation with SPW2004 reveals a slight displacement to the right. This difference takes place due to the different pressure distributions at the pile tip as seen in Fig. A.3. The bending moment and shear force distributions are very similar. The pressure distributions differ in the area of the pile tip and the anchor point. 


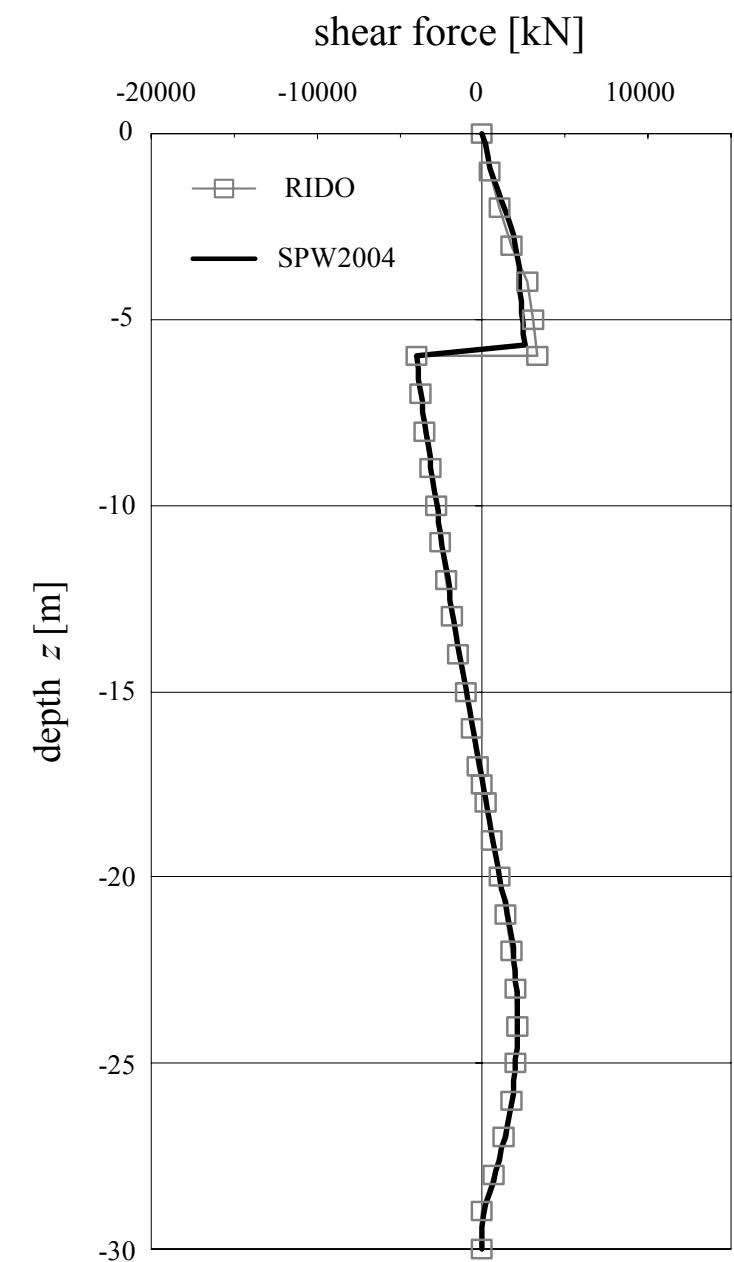

pressures on tube wall $\left[\mathrm{kN} / \mathrm{m}^{2}\right]$

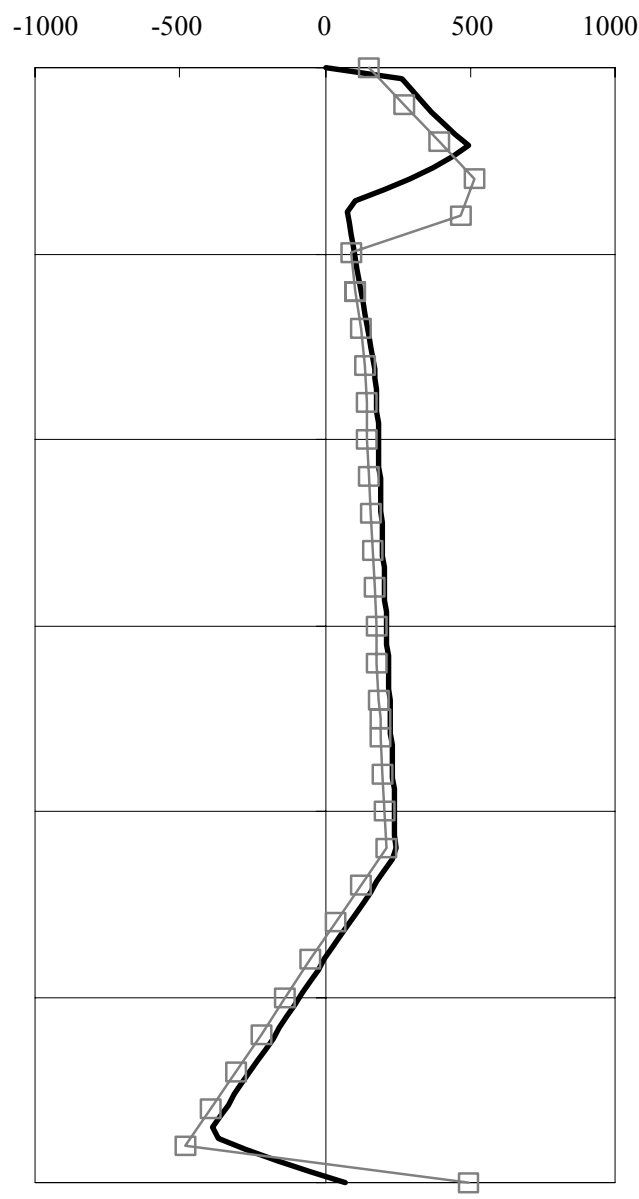

Fig. A.3: Shear force and pressures on tube wall distribution for the tubular piles 


\section{Annex B: Measurement of the specimens}

\section{B.1 Preliminary investigation of specimens made of non-alloyed steel}

\section{B.1.1 General}

For the specimens required for the reduced scale tests preliminary investigations were performed with a tube made of non-alloyed constructional steel detailed in the following:

- $\quad$ longitudinally welded tube without heat treatment

- $\quad$ mild steel

- $\quad$ material: non-alloyed constructional steel S235

- $\quad$ plane ends

- $\quad$ tolerances according to DIN 1626

- $\quad$ dimensions in mm: $203 \times 2 \times 3000$ (outer diameter $D_{\text {nom }}$ x wall thickness x length)

- $\quad$ supplier: Frank + Warnecke Industriebedarf GmbH Braunschweig

The data was compared and verified with the inspection certificate 3.1.B for the coil used for manufacturing the tubes. The steel plates running off the coil are continuously rolled to a tube (Fig. B.7). The next step in the production line is the joining of the plate's edges by a longitudinal weld (induction welding, HF) as shown in Fig. B.9. After welding the plates the tube is cut by a saw into the desired lengths.

Geometrical measurement

Measurement of the geometry according to Fig. B.1:

- $\quad$ thickness measurement at both edges (section 0 and 6) at four positions each by means of a micrometer gauge

- $\quad$ measurement of the diameter $D$ in section 1 to 5 at six different positions by means of a calliper gauge

The results of the measurement are summarized in Tab. B.1 and Tab. B.2. The tolerances are chosen according to DIN 1615, DIN 18800-4, and EN 1993-1-6. 


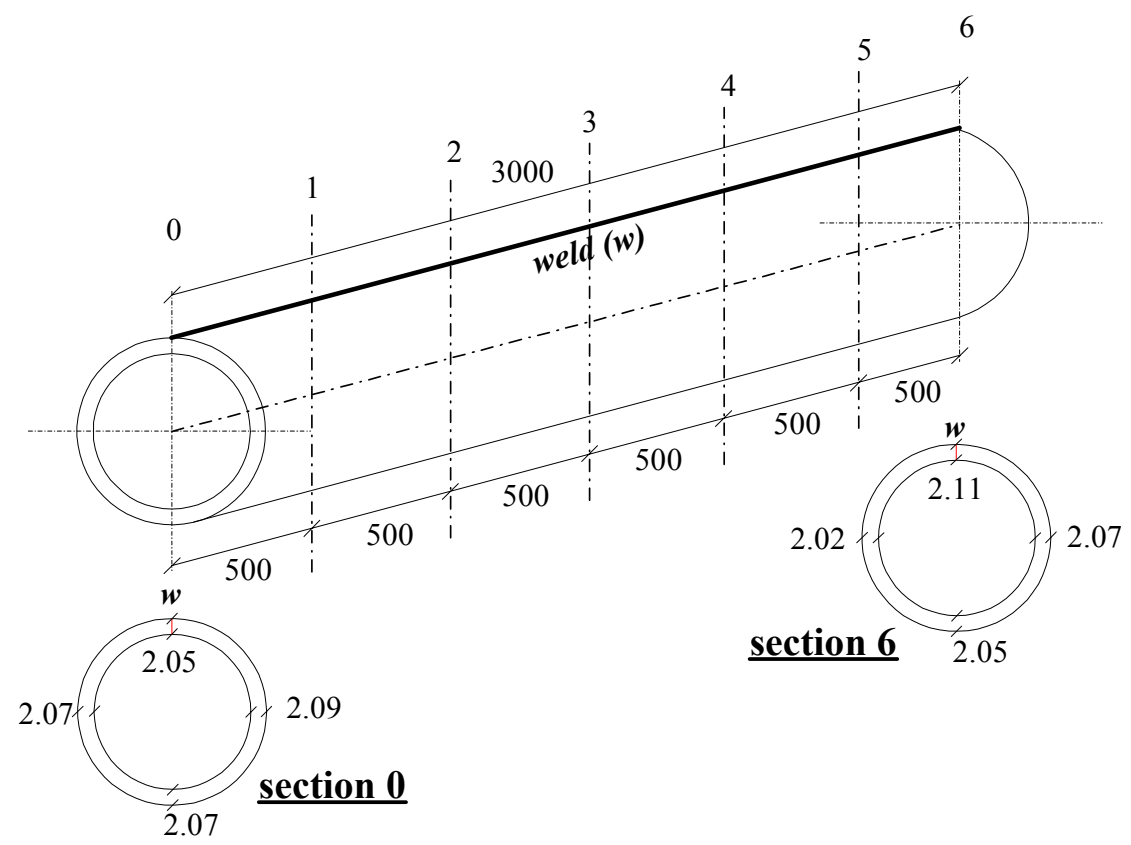

$\underline{\text { section } 1}$

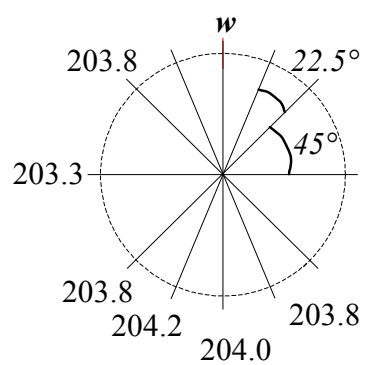

section 2

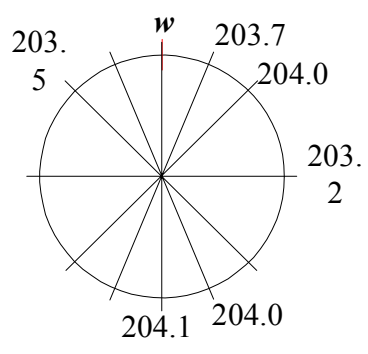

$\underline{\text { section } 3}$

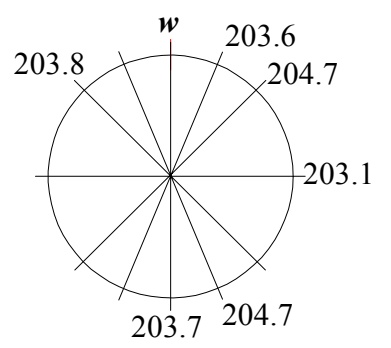

$\underline{\text { section } 4}$

$\underline{\text { section } 5}$

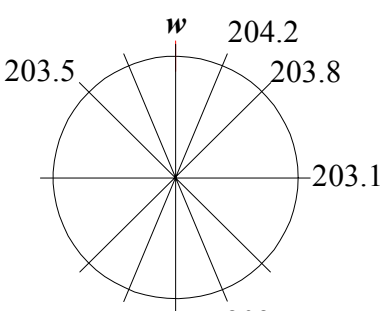

$203.7 \quad 203.5$

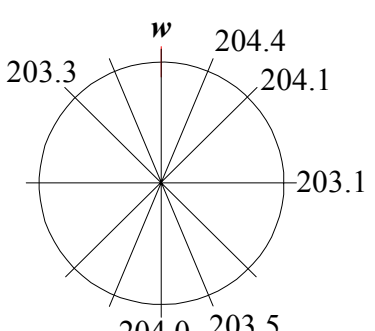

204.0203 .5 nominal diameter

$D_{\text {nom }}=203 \mathrm{~mm}$

Fig. B.1: Measurement of the geometry of the cylinder made of non-alloyed steel

The roundness $R$ from DIN 1615 equal to the out-of-roundness $U_{r}$ from EN 1993-1-6 is defined as:

$$
R=U_{r}=\frac{D_{\max }-D_{\min }}{D_{\text {nom }}}
$$

The out-of-roundness $U$ from DIN $18800-4$ is defined as:

$$
U=2 \frac{D_{\max }-D_{\min }}{D_{\max }+D_{\min }}
$$


The outer diameter $D$, the roundness $R$, and the wall thickness $t$ satisfy the tolerances given in DIN 1615. The minimum requirements for the roundness $U_{r}$ from EN 1993-1-6 for Class A are satisfied. Further, the out-of-roundness requirement for $U$ from DIN 18800-4 is satisfied.

In Fig. B.2 and Fig. B.3 the cylinder is depicted.

Tab. B.1: Deviations from nominal values - outer diameter D

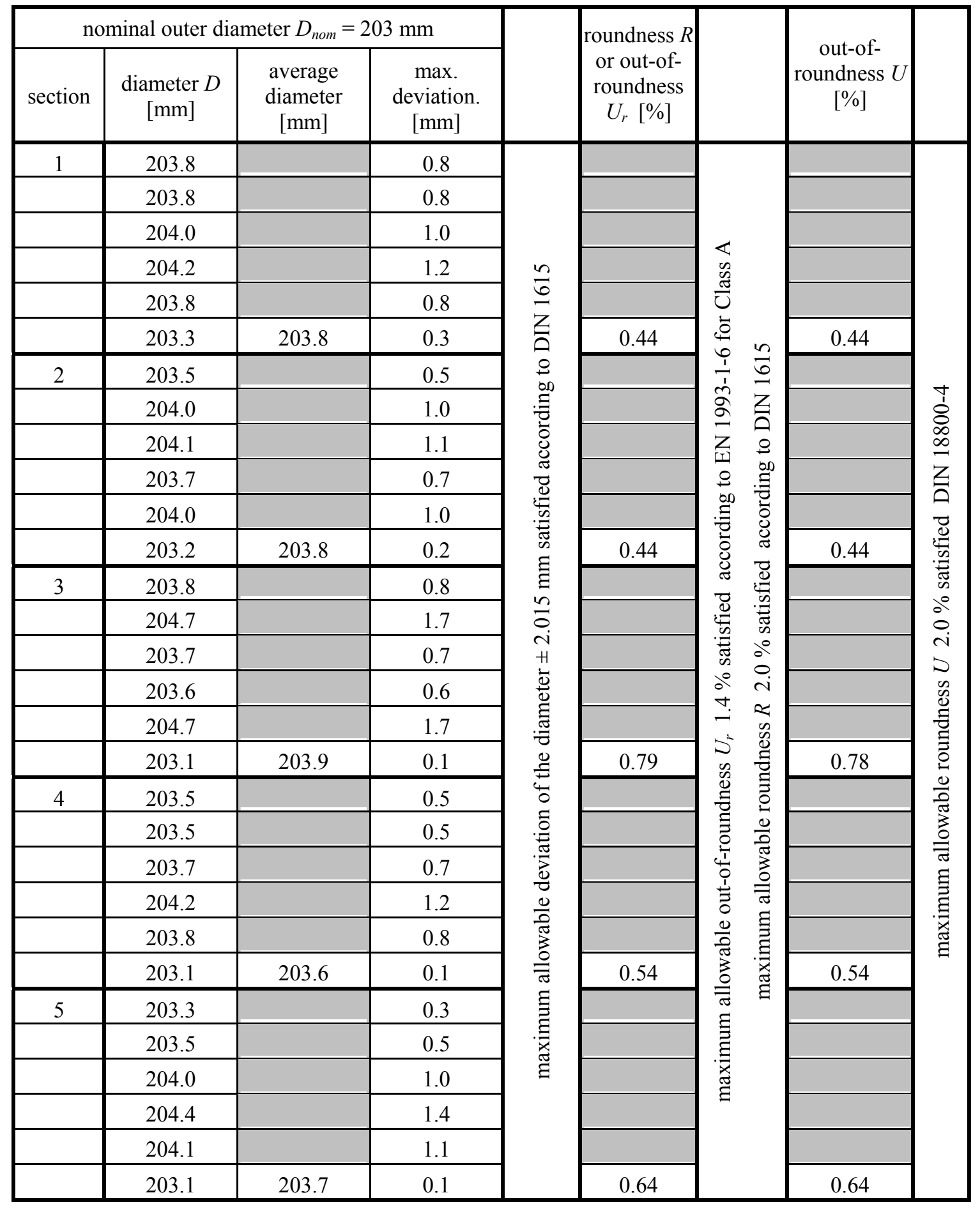


Tab. B.2: Deviations from nominal values - wall thickness t according to DIN 1615

\begin{tabular}{|c|c|c|c|c|}
\hline \multicolumn{4}{|c|}{ nominal wall thickness $t_{\text {nom }}=2 \mathrm{~mm}$} & \\
\hline section & $\begin{array}{c}\text { wall thickness } t \\
{[\mathrm{~mm}]}\end{array}$ & $\begin{array}{c}\text { average } \\
\text { thickness [mm] }\end{array}$ & $\begin{array}{c}\text { max. deviation. } \\
{[\mathrm{mm}]}\end{array}$ & \\
\hline 0 & 2.05 & & 0.05 & \multirow{8}{*}{$\begin{array}{c}\text { maximum } \\
\text { allowable } \\
\text { deviation } \\
+0.30 \mathrm{~mm} \\
-0.25 \mathrm{~mm} \\
\text { satisfied }\end{array}$} \\
\hline & 2.09 & & 0.09 & \\
\hline & 2.07 & & 0.07 & \\
\hline & 2.07 & 2.07 & 0.07 & \\
\hline \multirow[t]{4}{*}{6} & 2.11 & & 0.11 & \\
\hline & 2.07 & & 0.07 & \\
\hline & 2.05 & & 0.05 & \\
\hline & 2.02 & 2.06 & 0.02 & \\
\hline
\end{tabular}
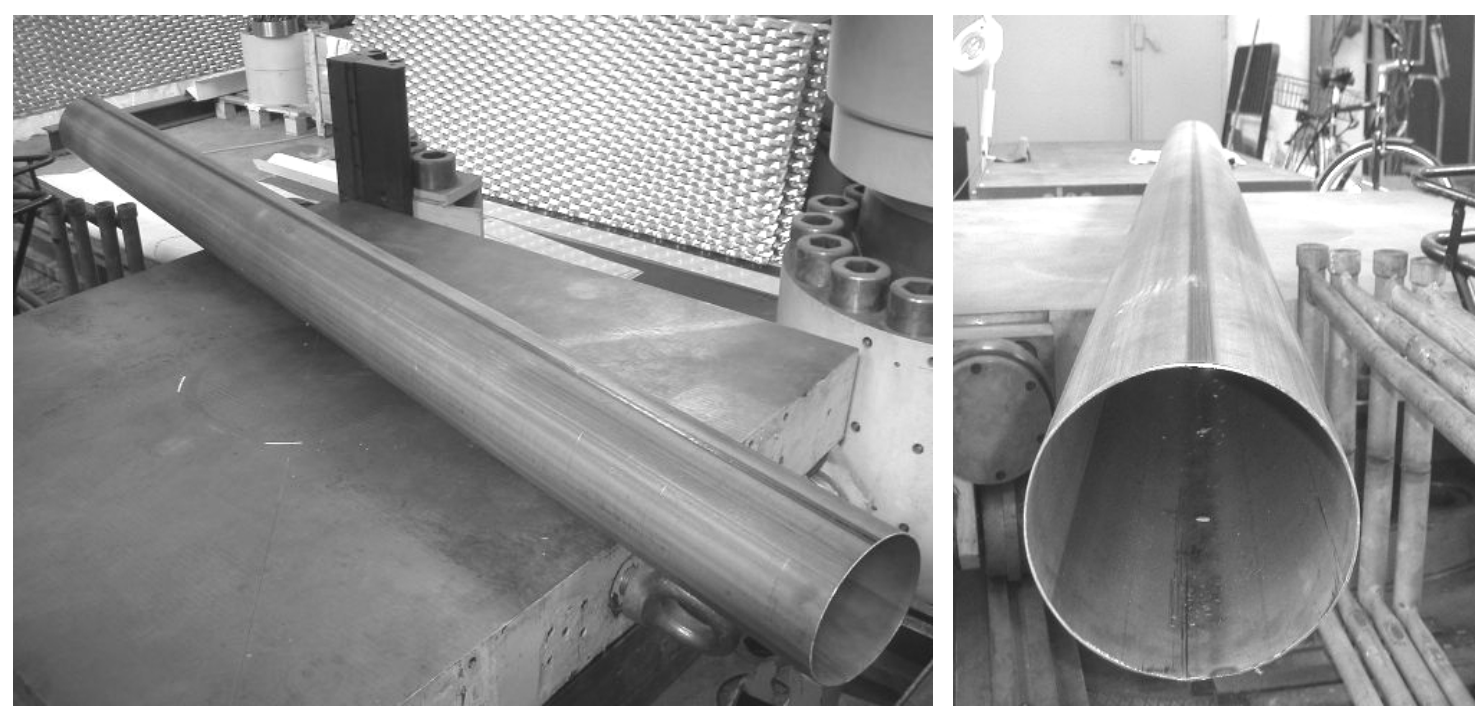

Fig. B.2: Tube made of non-alloyed constructional steel

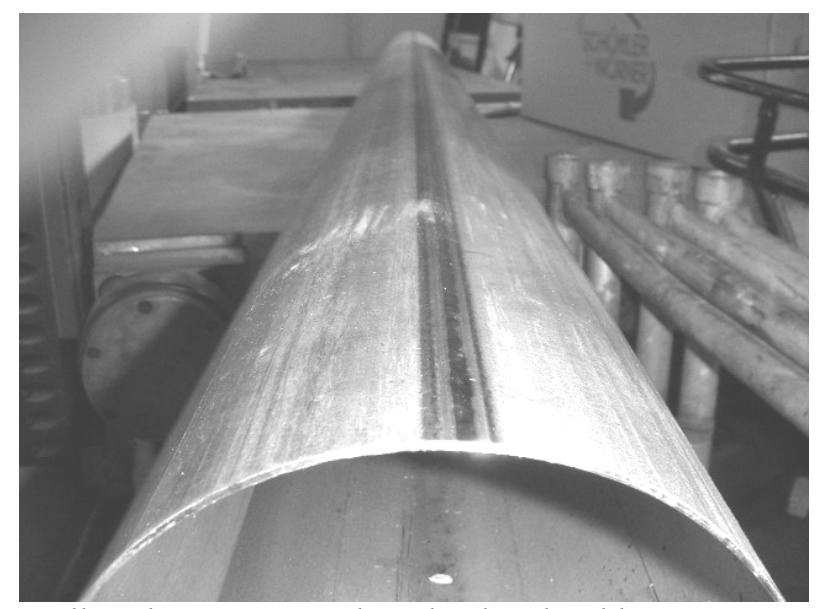

Fig. B.3: Tube made of non-alloyed constructional steel-detail weld 


\section{B.1.2 Material properties}

The base material of the cylinder was investigated with four tensile tests (extraction points shown in Fig. B.4) and a hardness test according to Vickers (HV10) over the weld area.

The results of the tensile tests are depicted as stress-strain curves in Fig. B.5. The initial measuring length $L_{0}$ was chosen with $50 \mathrm{~mm}$. The test coupons were small nonproportional coupons according to DIN EN 10002. It can be seen that the stress-strain curves for the tensile tests B 1, B 2, and B 3 hardly differ, and up to a stress level of $180 \mathrm{~N} / \mathrm{mm}^{2}$ the coupons exhibit a linear material behaviour. Due to the strain hardening in the heat affected zone of the weld the tensile test $\mathrm{W}$ results in an higher tensile strength and a lower ultimate strain. The Young's modulus of the coupon W lies within the stray area of the other tensile tests (Fig. B.5). The Young's modulus was evaluated based on the hysteresis loop.

The investigation of the Vickers hardness in the area of the weld reveals that the strain hardening only spans over a minor region around the weld (Fig. B.6).

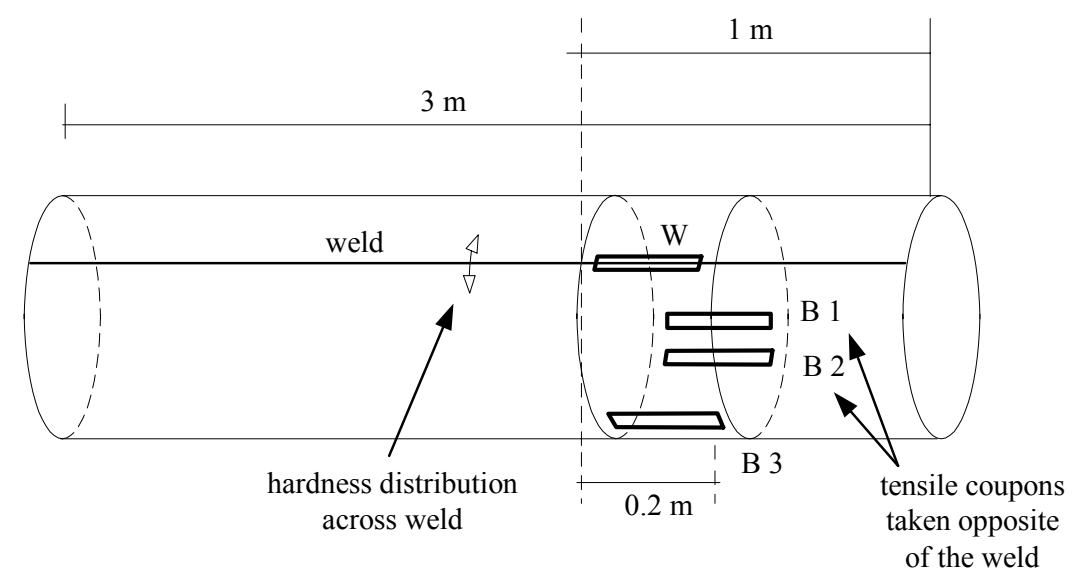

Fig. B.4: Extraction points of the coupons

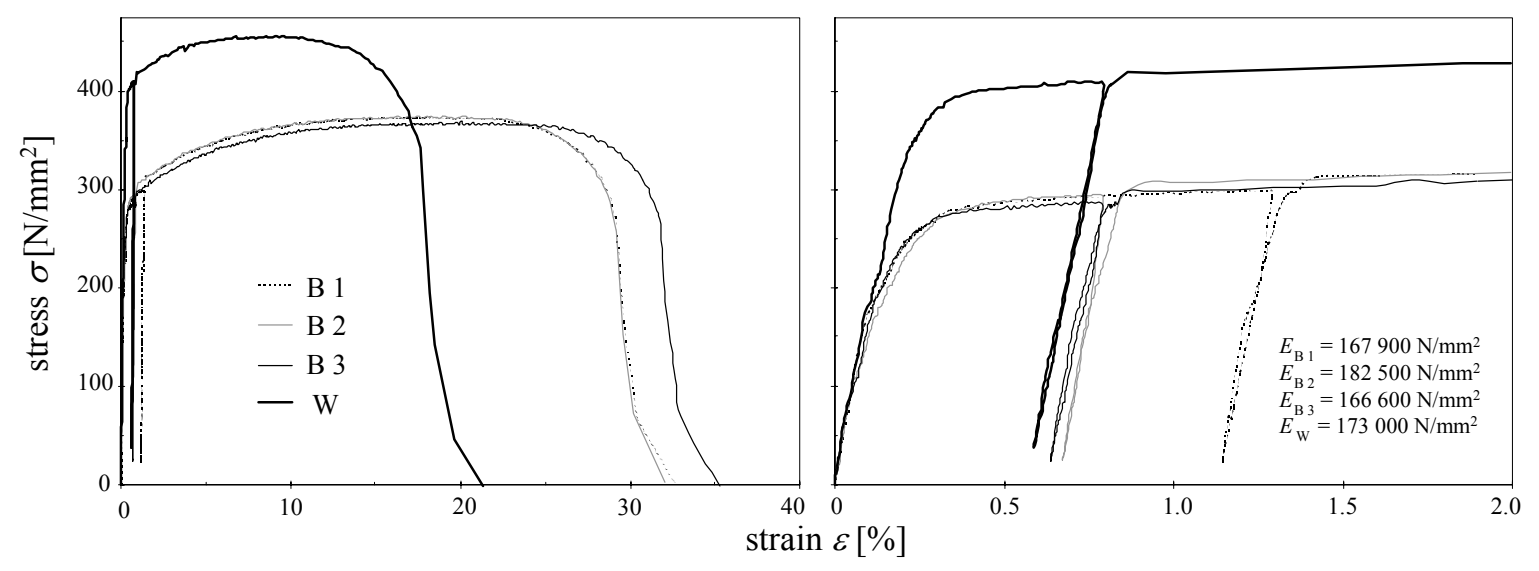

Fig. B.5: Stress-strain curves for the tensile tests for the base material 


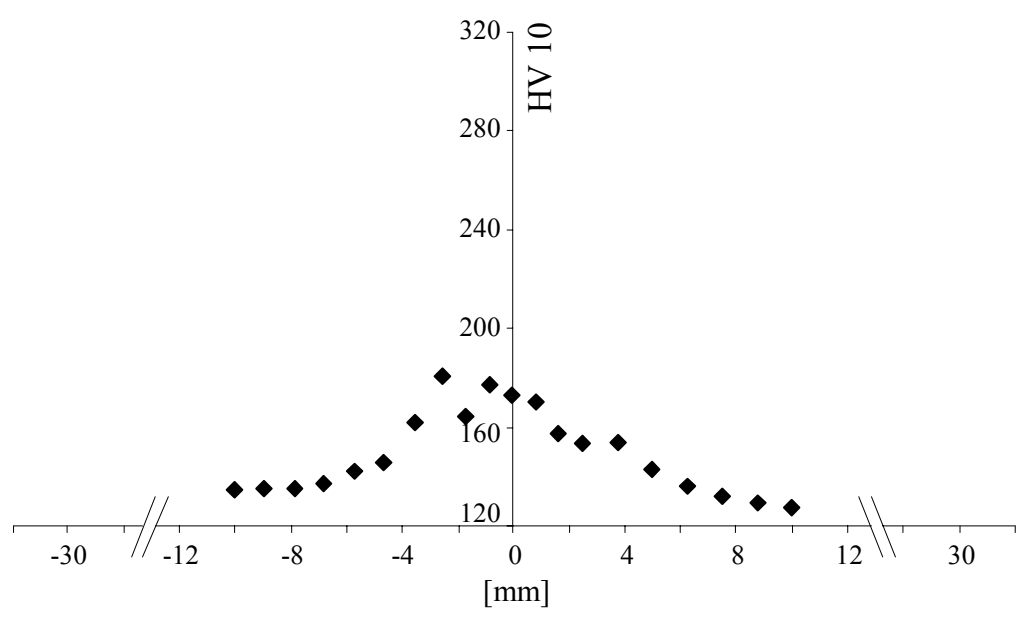

Fig. B.6: Vickers hardness distribution across the weld

\section{B.2 Investigation of test tube for the reduced scale tests}

\section{B.2.1 General}

For the reduced scale tests it was chosen to take a tube of the type investigated in the previous section in preliminary tests. The tubes were ordered for the experiments and measured more thoroughly. For completeness the specifications are repeated:

- $\quad$ longitudinally welded tube without heat treatment

- $\quad$ mild steel

- $\quad$ material: non-alloyed constructional steel S235

- $\quad$ plane ends

- $\quad$ tolerances according to DIN 1626

- $\quad$ dimensions in mm: 203 × 2 × 3000 (outer diameter $D_{\text {nom }}$ x wall thickness x length)

- $\quad$ supplier: Frank + Warnecke Industriebedarf GmbH Braunschweig

The data was compared and verified with the inspection certificate 3.1.B for the coil used for manufacturing the tubes. The steel plates running off the coil are continuously rolled to a tube (Fig. B.7). The next step in the production line is the joining of the plate's edges by a longitudinal weld (induction welding, HF) as shown in Fig. B.9. After welding the plates the tube is cut by a saw into the desired lengths. 

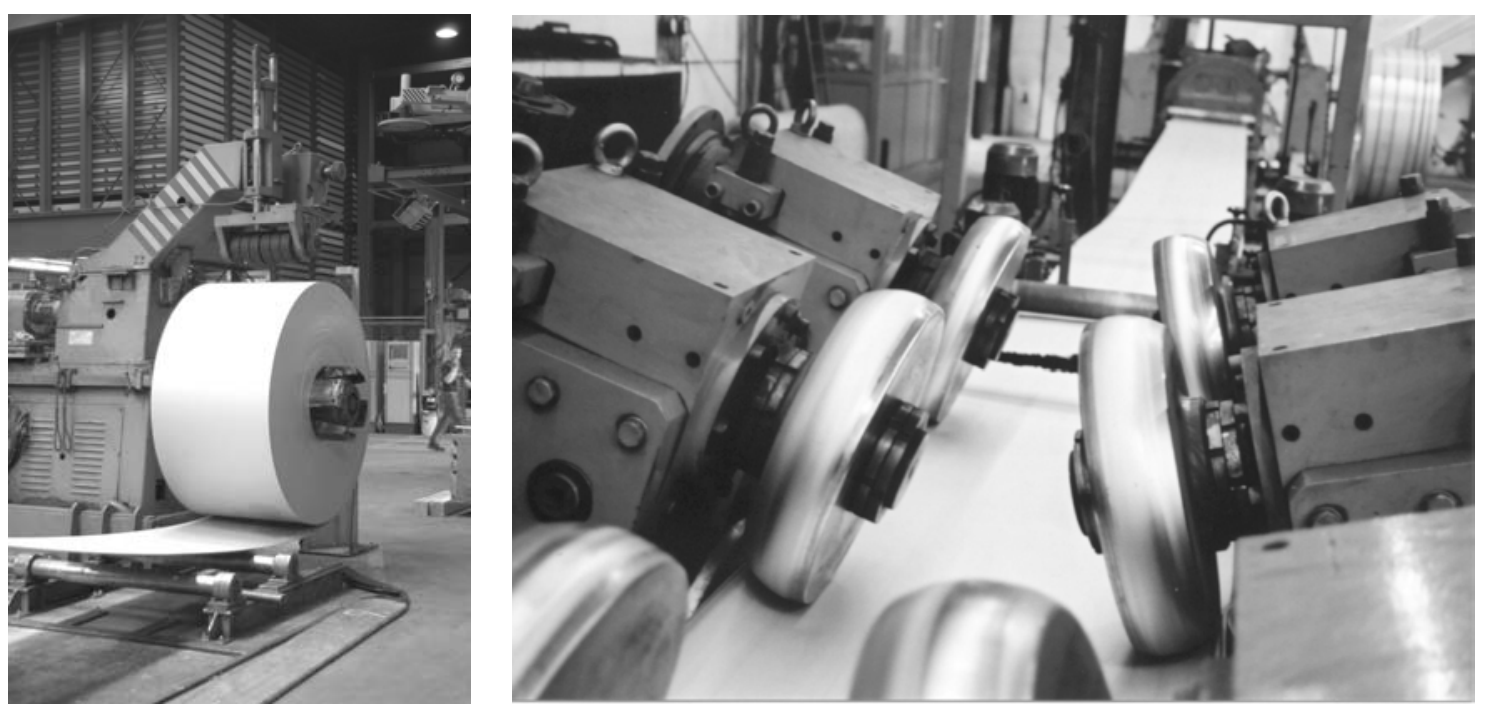

Fig. B.7: Rolling of the tubes from coil

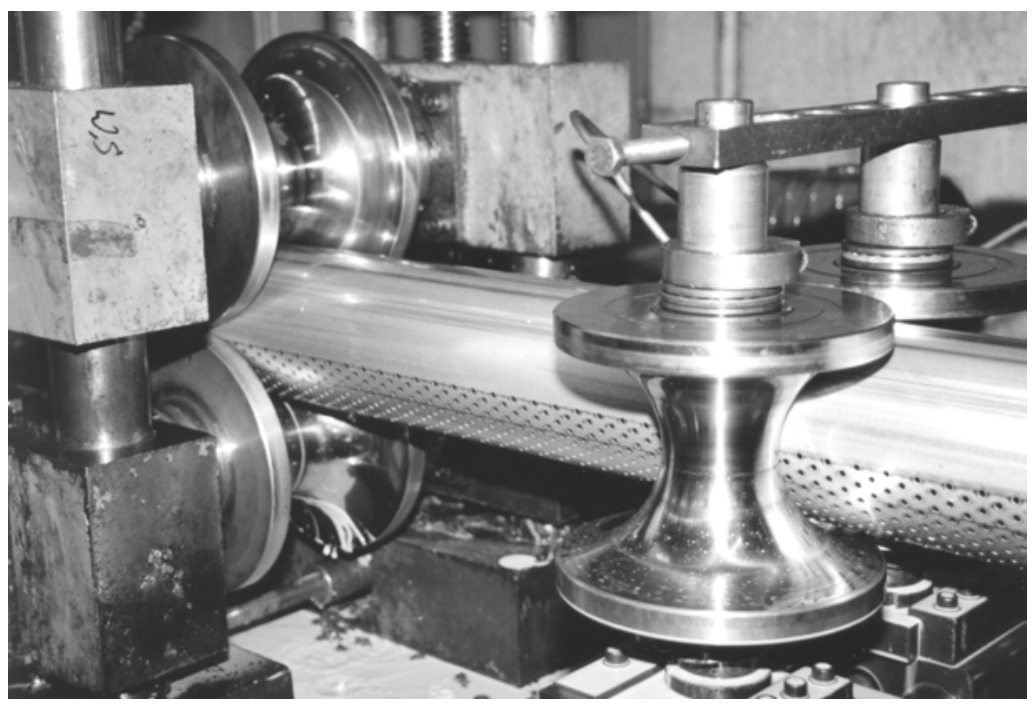

Fig. B.8: Rolling of the tubes

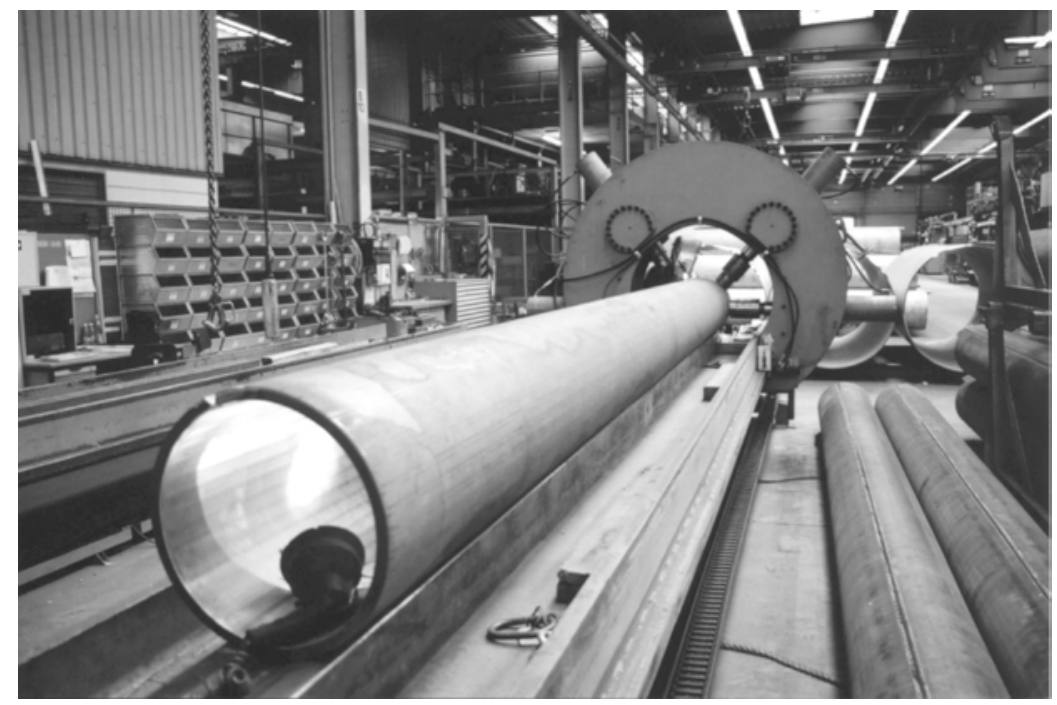

Fig. B.9: Welding of the tube 


\section{B.2.2 Geometrical measurement}

Measurement of the geometry as follows:

- thickness measurement over the entire length of the tube by means of an ultrasonic measuring device (USIP 12) in combination with wall thickness measuring module DTM12 and a straight beam probe of the company Krautkrämer; calibration and verification with micrometer gauge

- measurement of the test tube with the high-end 3D-digitization system optoTOP-L300-HE425/30

$\bigcirc$ resolution $<0.015 \mathrm{~mm}$

$\circ$ accuracy of measurement, local $<0.04 \mathrm{~mm}$, global $<0.1 \mathrm{~mm}$

$\circ$ measuring grid approx. $0.3 \mathrm{~mm}$

○ image diagonal approx. $425 \mathrm{~mm}$ : measurement with approx. 120 fields of view

○ contour dependent thinning out of measuring points: chord error $5 \mu \mathrm{m}$ $10 \mu \mathrm{m}$, grid distance approx. $2.5 \mathrm{~mm}-5 \mathrm{~mm}$

○ topometry; miniaturised projection technique (MPT)

○ combination of GrayCode- and Phaseshift-methods

○ Pass-Points-Matching, Pass-Points are calibrated photogrammetrically and the fields of view positioned automatically using the photogrammetric metrology system DPA-PRO

Fig. B.10 depicts the used ultrasonic measuring device. The results are summarized in Tab. B.3. The thickness is about $1.5 \%$ larger than the nominal value. In Fig. B.11 and Fig. B.12 the test tube is shown during the scanning process. During the scanning process the weld was positioned at the bottom.

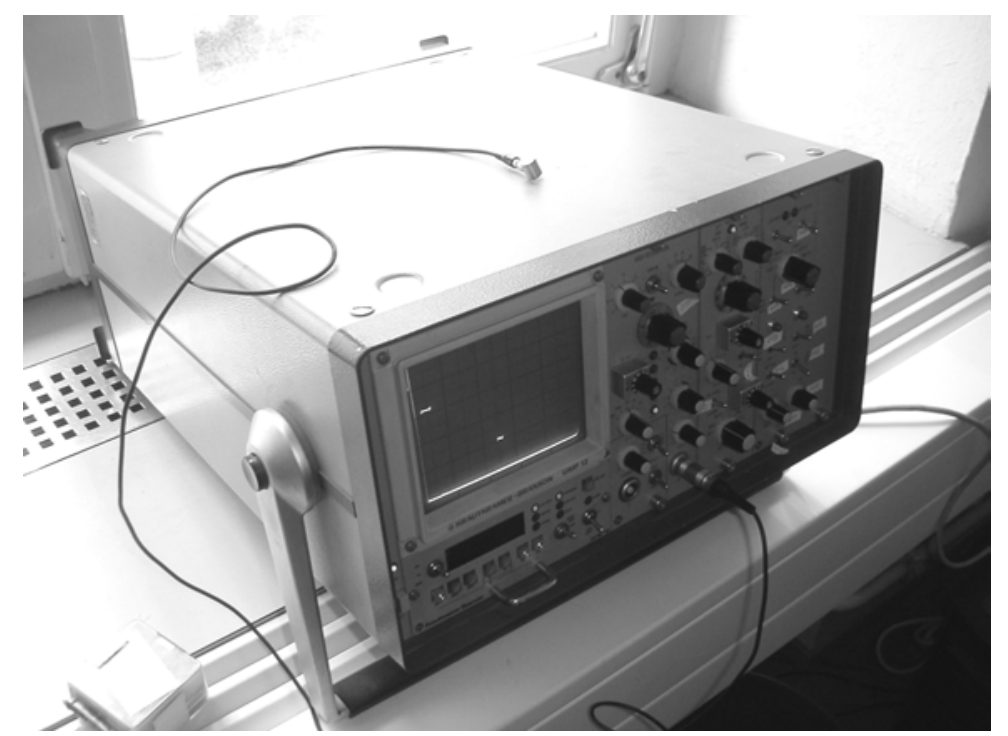

Fig. B.10: Ultrasonic measuring device USIP 12 with DTM12 


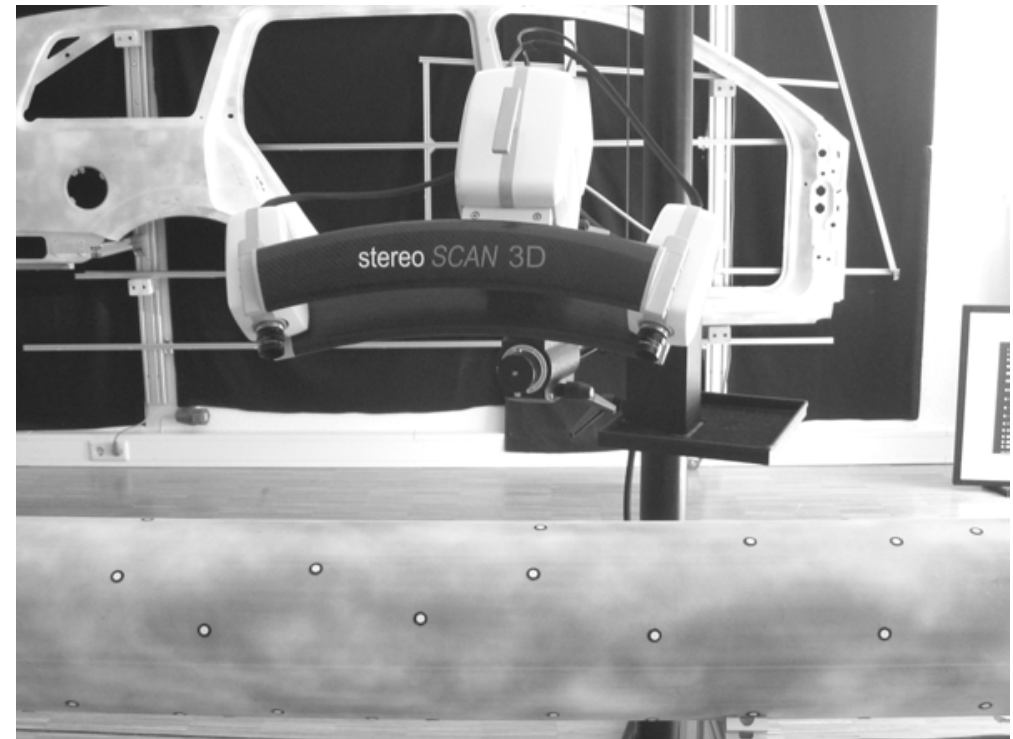

Fig. B.11: Measuring system

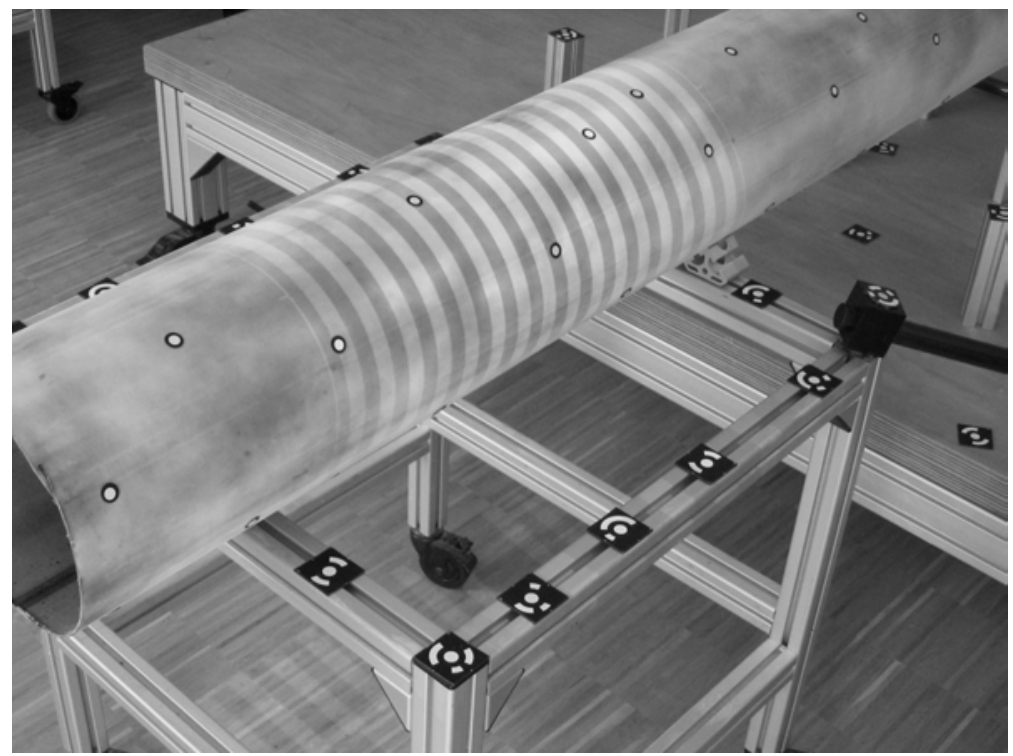

Fig. B.12: Scanning process

Tab. B.3: Deviations from nominal values - wall thickness t according to DIN 1615

\begin{tabular}{|c|c|c|c|c|c|}
\hline \multicolumn{7}{|c|}{ nominal wall thickness $t_{\text {nom }}=2 \mathrm{~mm}$} & \multirow{2}{*}{ section } \\
\hline measurements & $\begin{array}{c}\text { average } \\
\text { thickness [mm] }\end{array}$ & $\begin{array}{c}\text { standard } \\
\text { deviation }[\mathrm{mm}]\end{array}$ & $\begin{array}{c}\text { max. deviation } \\
{[\mathrm{mm}]}\end{array}$ & \\
\hline weld & 10 & 2.03 & 0.02 & +0.08 & maximum allowable \\
\hline rest & 60 & 2.03 & 0.01 & +0.05 & $\begin{array}{c}\text { deviation }+0.30 \mathrm{~mm} \\
-0.25 \text { mm satisfied }\end{array}$ \\
\hline
\end{tabular}


The results of the scanning are depicted in the following. The data set of spatial coordinates from the measurement included more than 1'000'000 measuring points along the test tube. The data set was imported in Autocad with a macro programmed in Visual Basic.

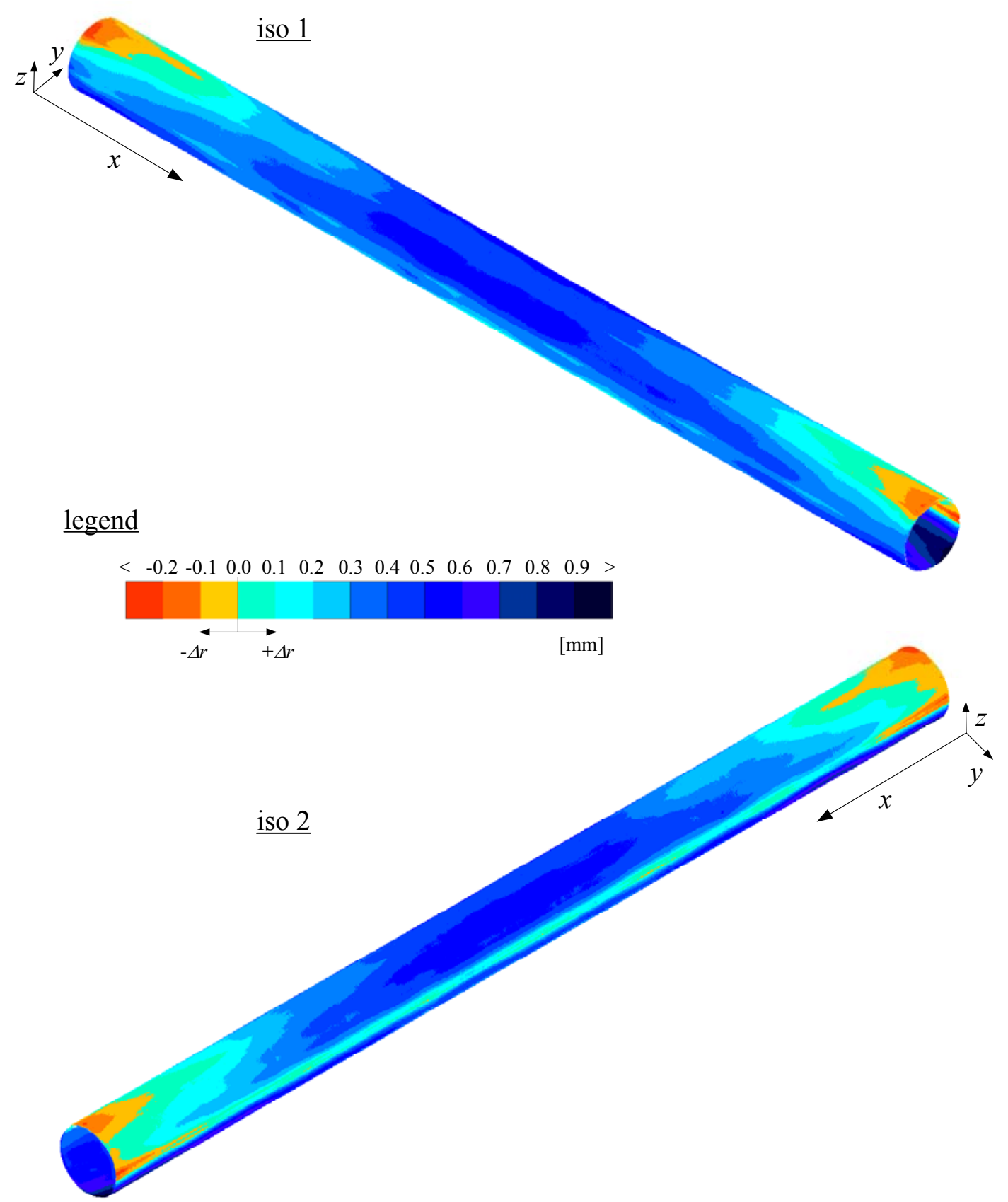

Fig. B.13: Isometries of scanned test tube with radial deviations relative to the best-fit-cylinder

The alignment of the measured data was performed by first evaluating all measured points with the best-fit-cylinder solution. This implies a re-positioning of the points based on the nominal cylinder in order to obtain the minimum sum of radial deviations. From the data a vector is defined as the longitudinal axis of the best-fit-cylinder with which a plane is constructed using an arbitrary point on the shell. The other planes are 
chosen respectively. A macro was programmed for bringing out the deviations between the measured radius and the nominal radius in terms of colour as shown in two isometries in Fig. B.13 and in two views in Fig. B.14. The deviations are based on a global coordinate system which is set according to the position of the tube during the scanning process.

The weld position can be identified clearly in iso 2 from Fig. B.13 and in view 2 from Fig. B.14 as the turquoise area stretching along the tube's length. 


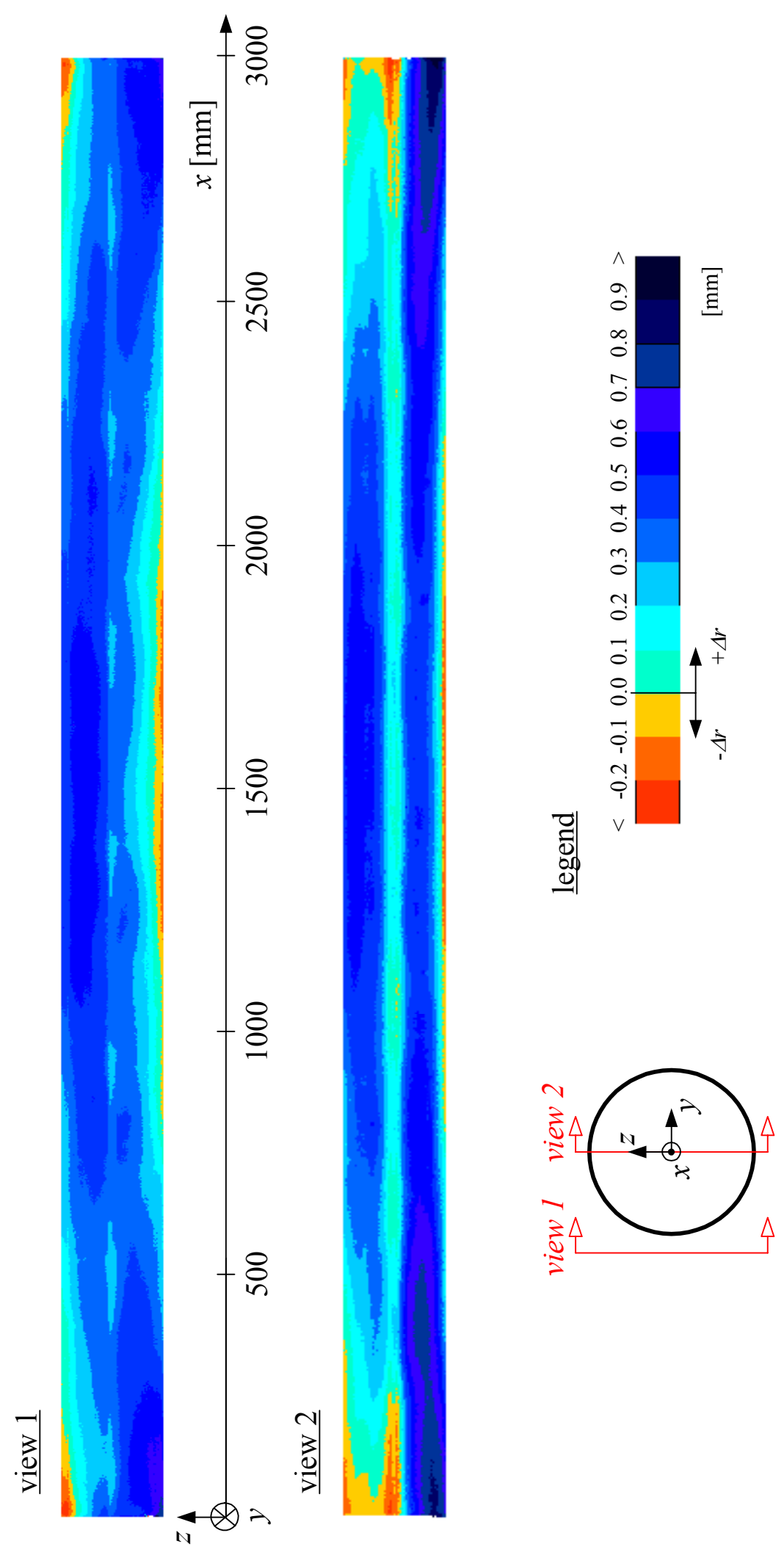

Fig. B.14: Views of scanned test tube with radial deviations relative to the best-fit-cylinder 
legend
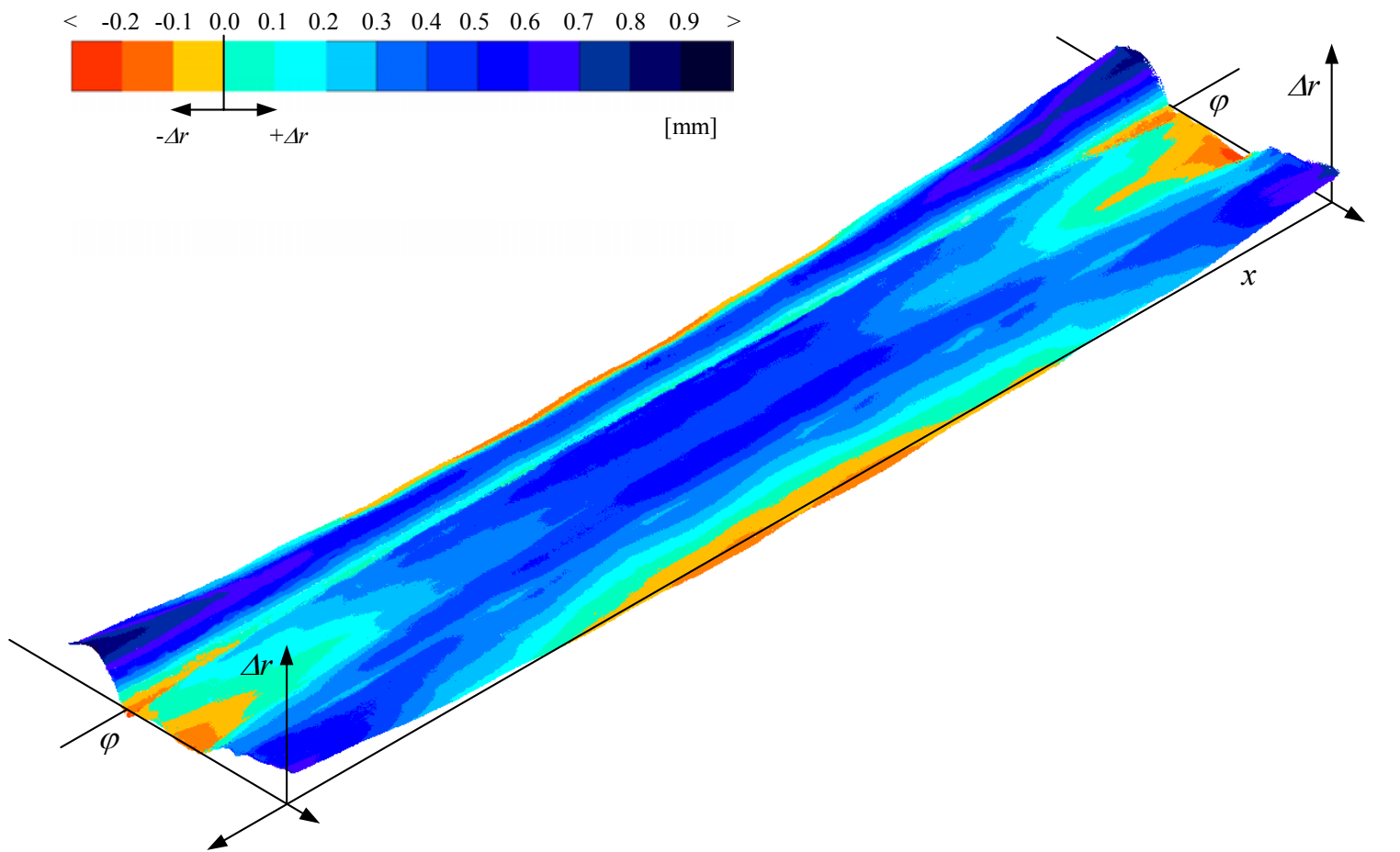

Fig. B.15: Unrolled shell of scanned test tube with radial deviations scaled by factor 100 relative to the best-fit-cylinder

Fig. B.15 depicts the unrolled shell of scanned test tube. The radial deviations $\Delta r$ are scaled with a factor of 100 .

Additionally, the test tube was investigated concerning the out-of-roundness. The relevant diameters were taken from evaluating the measuring points for specific crosssections. The data was interpreted at seven different cross-sections: $x=50 \mathrm{~mm}$, $500 \mathrm{~mm}, 1000 \mathrm{~mm}, 1500 \mathrm{~mm}, 2000 \mathrm{~mm}, 2500 \mathrm{~mm}$, and $2950 \mathrm{~mm}$. The results of the corresponding calculations of the out-of-roundness parameters according to (B.1) and (B.2) are summarized in Fig. B.16 to Fig. B.22 of the cross-section. As a reference a circle with the nominal outer diameter is added for every cross-section independently in the figures. In contrast to the reference used in Fig. B.13 and Fig. B.14 the reference diameter is positioned as centric as possible because the emphasis is on the minimum and maximum diameter and not the radii (best-fit-cross-section). The measuring points were evaluated covering an area of $\pm 1 \mathrm{~mm}$ away from the relevant cross section.

In all cross-sections the weld is positioned on the right hand side, and it can be clearly identified due to the welding-induced shrinkage deformations. For all investigated cross-sections the out-of-roundness and roundness requirements are easily satisfied, and the test tube can be classified as Class A tube according to the fabrication quality tolerance class from EN 1993-1-6. 


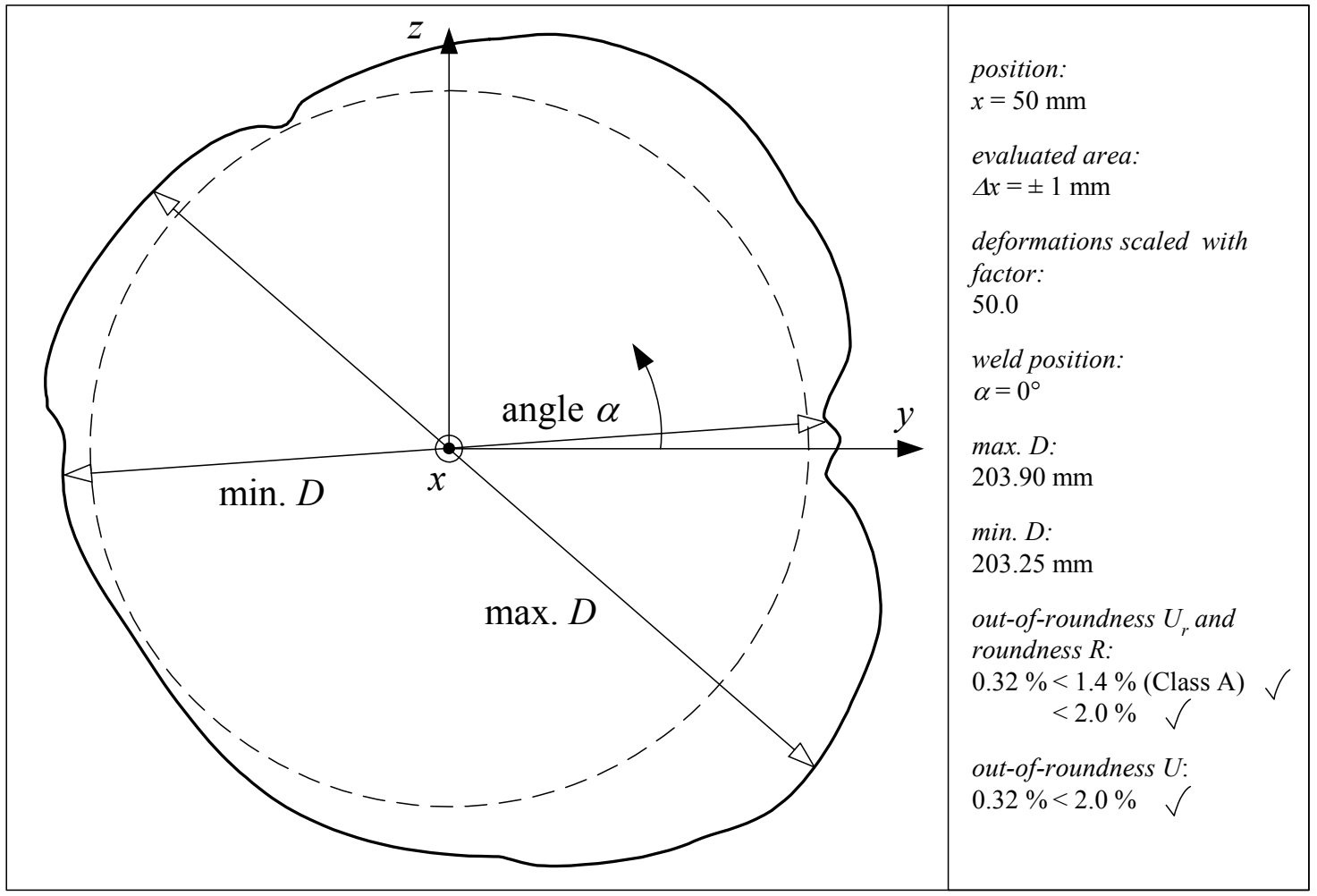

Fig. B.16: Fabrication tolerance-out of roundness at $x=50 \mathrm{~mm}$

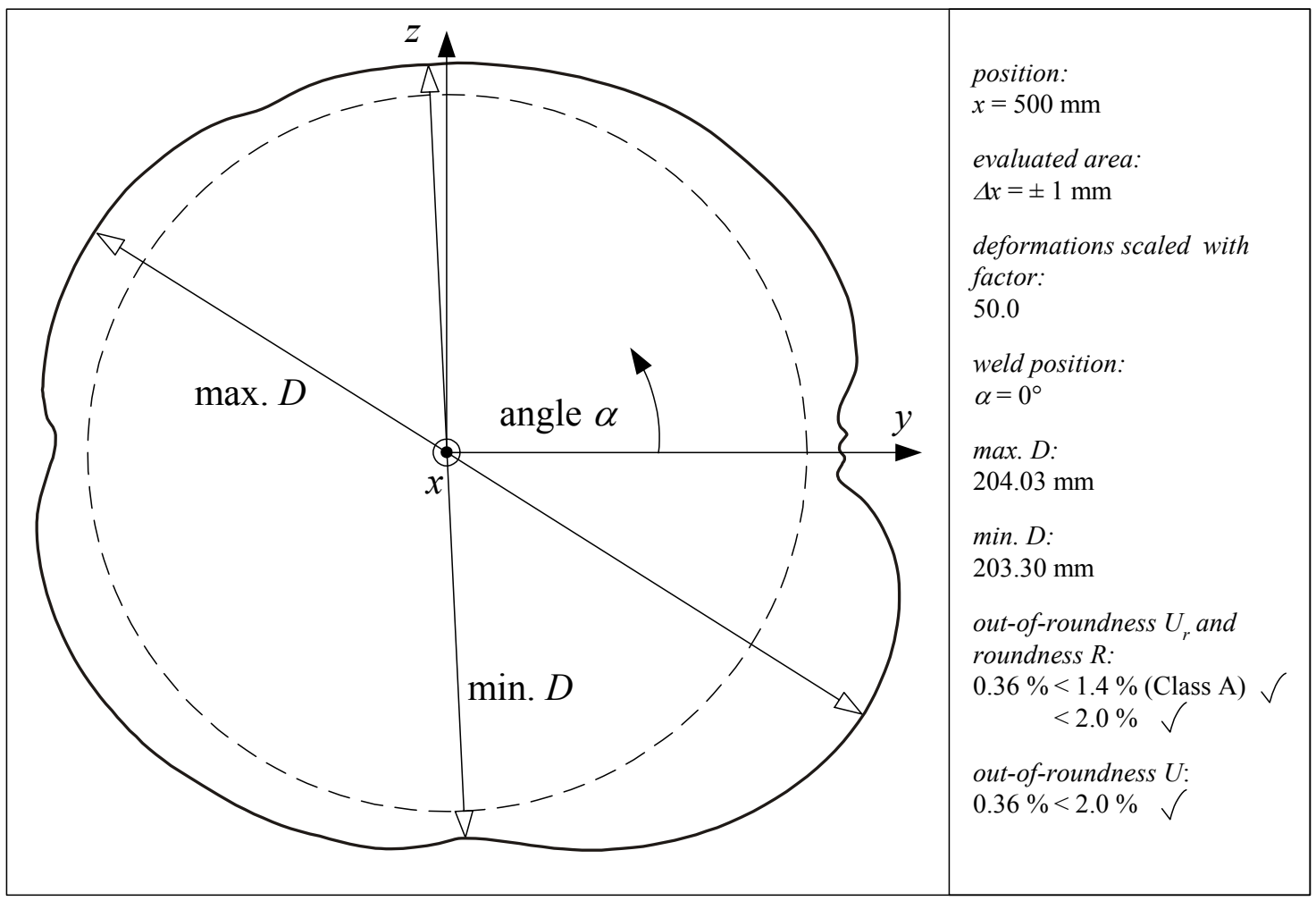

Fig. B.17: Fabrication tolerance - out of roundness at $x=500 \mathrm{~mm}$ 


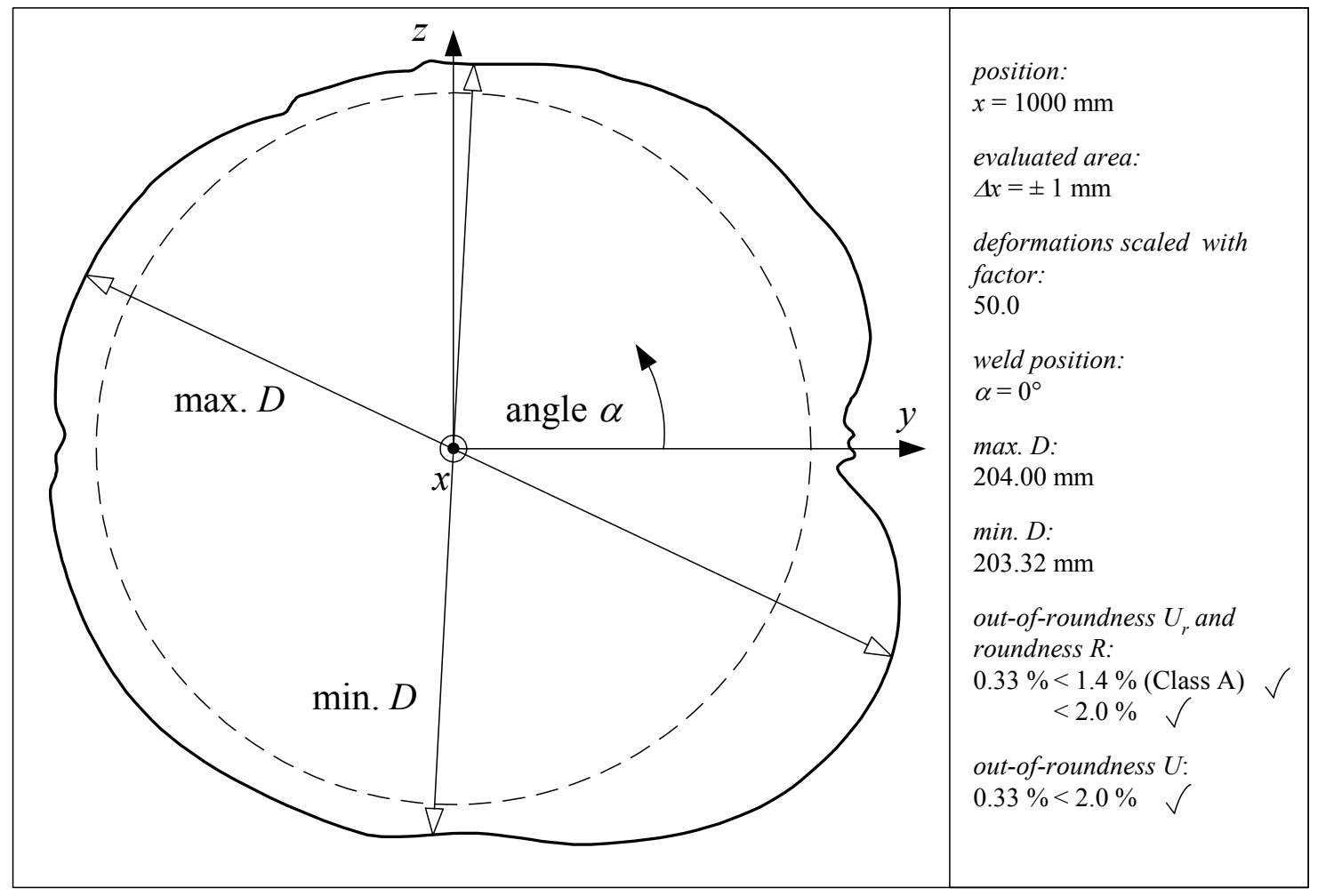

Fig. B.18: Fabrication tolerance - out of roundness at $x=1000 \mathrm{~mm}$

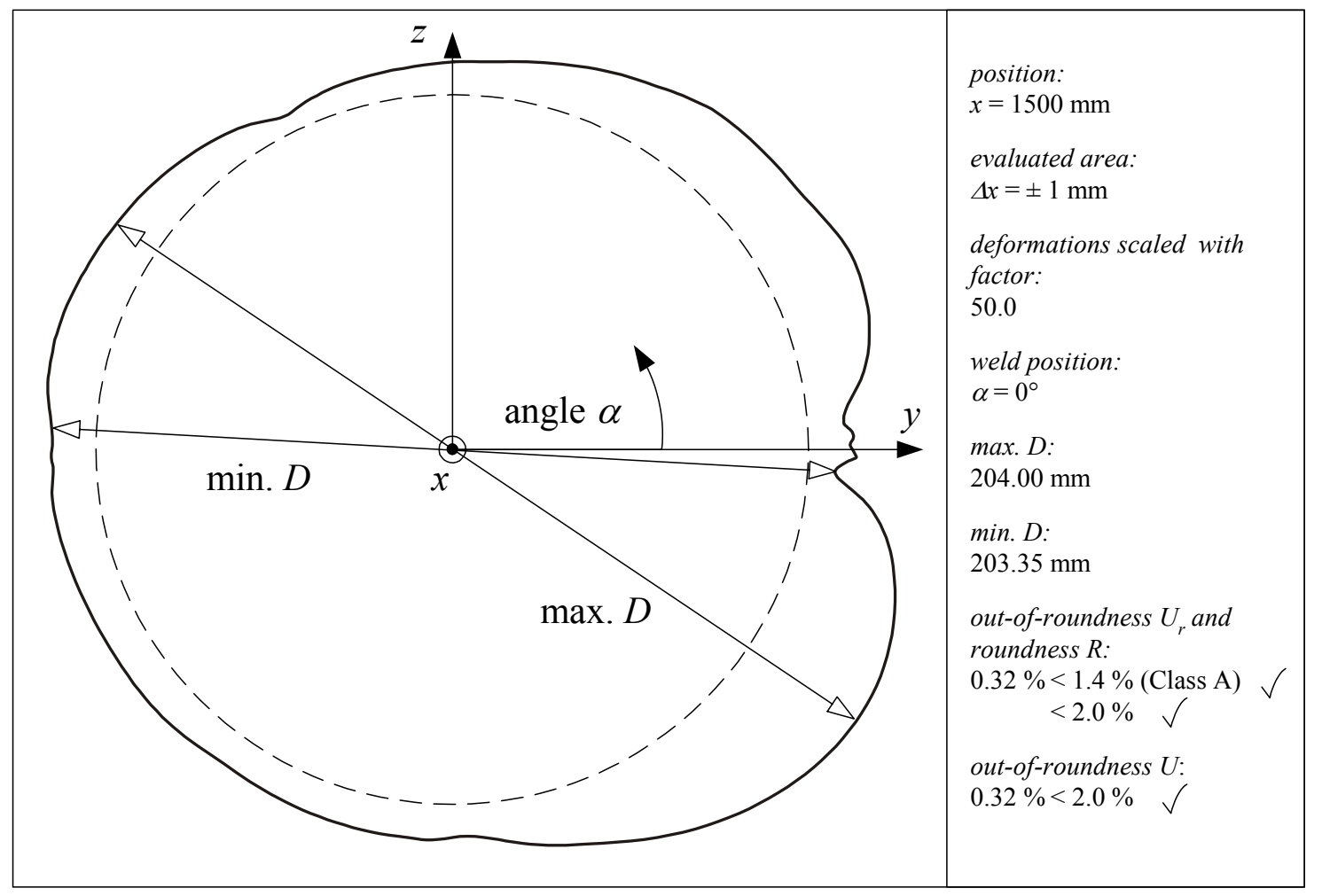

Fig. B.19: Fabrication tolerance - out of roundness at $x=1500 \mathrm{~mm}$ 


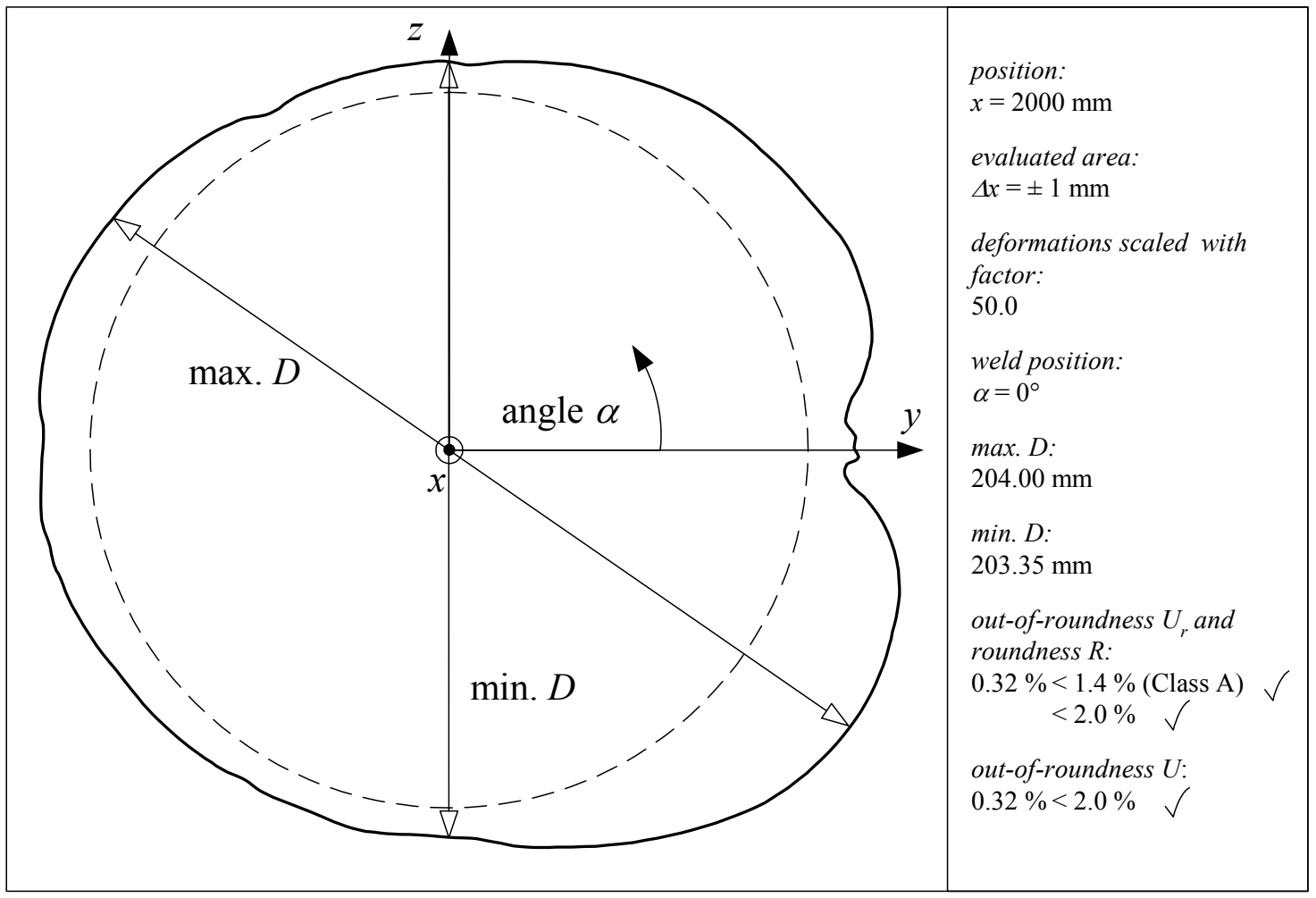

Fig. B.20: Fabrication tolerance - out of roundness at $x=2000 \mathrm{~mm}$

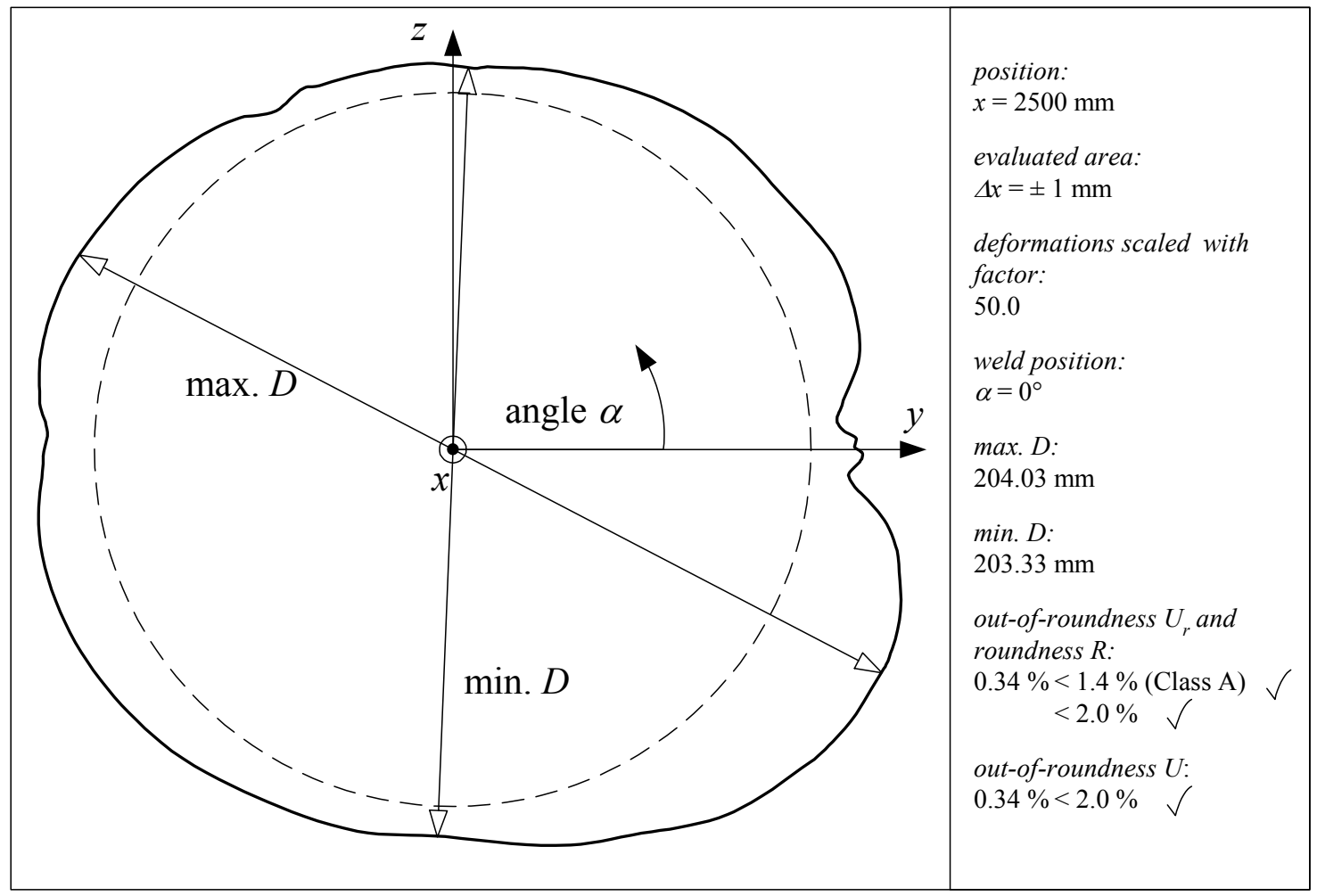

Fig. B.21: Fabrication tolerance - out of roundness at $x=2500 \mathrm{~mm}$ 


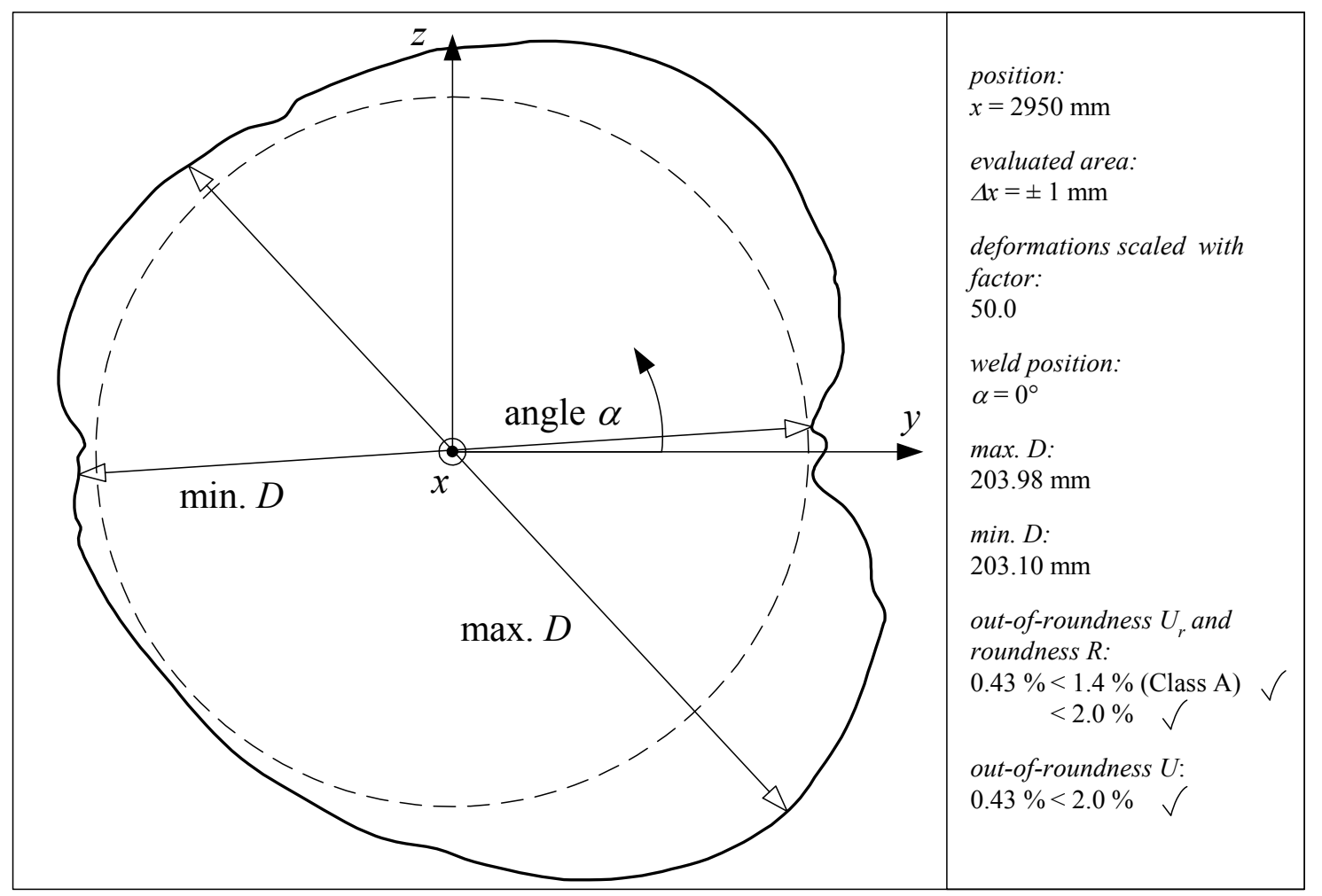

Fig. B.22: Fabrication tolerance - out of roundness at $x=2950 \mathrm{~mm}$

\section{B.2.3 Material properties}

The base material of the cylinder was investigated with seven tensile tests (extraction points shown in Fig. B.23). Four coupons were extracted in longitudinal direction of the tube (W and L 1 to $\mathrm{L} 3$ ) and three coupons were extracted in circumferential direction (C 1 to $\mathrm{C} 3$ ). The extracted circumferential strips were straightened carefully in several steps using a rolling machine. Afterwards the strips were elongated in a testing machine by around $1 \%$ in order to equalise local plastic deformations introduced due to the flattening process. Subsequently the tensile coupons C 1 to C 3 were manufactured from the strips.

The results of the tensile tests are depicted as stress-strain curves in Fig. B.24. The initial measuring length $L_{0}$ for $\mathrm{W}$ and $\mathrm{L} 1$ to $\mathrm{L} 3$ was chosen with $80 \mathrm{~mm}$. The test coupons were small non-proportional coupons according to DIN EN 10002. For the circumferential coupons a larger initial measuring length was picked in order to obtain reliable values for the Young's modulus $\left(L_{0}=160 \mathrm{~mm}\right)$.

In Fig. B. 24 it can be seen that the stress-strain curves for the tensile tests L 1 to L 3 hardly differ, and up to a stress level of nearly $250 \mathrm{~N} / \mathrm{mm}^{2}$ the coupons exhibit a linear material behaviour. Due to the strain hardening in the heat affected zone of the weld the tensile test $\mathrm{W}$ results in a higher tensile strength and a lower ultimate strain. Similar to the case of longitudinal coupons the circumferential coupons exhibit comparable results 
(Fig. B.25). Tab. B.4 summarizes the yield strength and the ultimate strength for the investigated tensile tests.

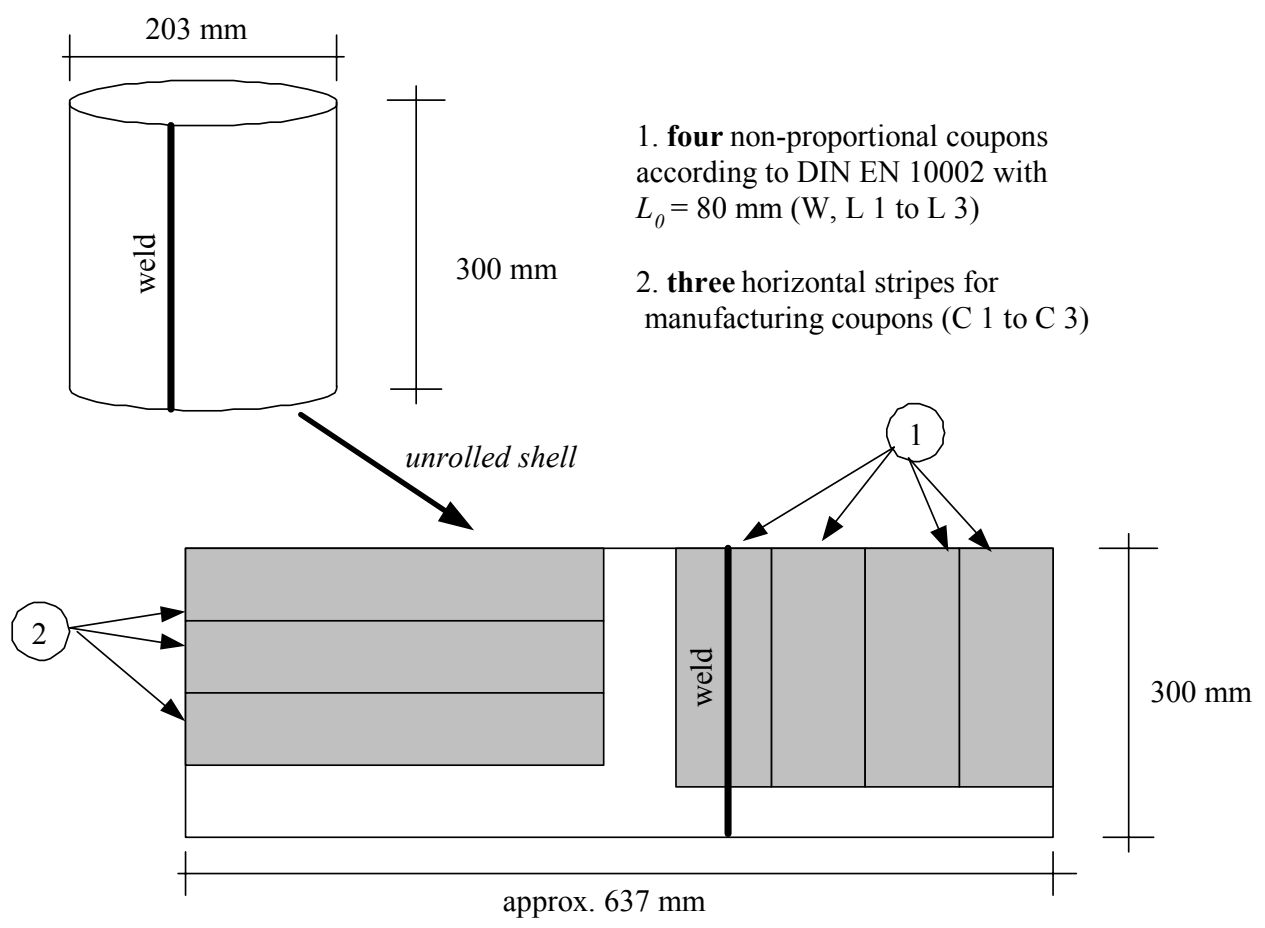

Fig. B.23: Extraction points of the coupons

Tab. B.4: Material properties from tensile tests

\begin{tabular}{|c|c|c|}
\hline coupon & yield strength $R_{e H}\left[\mathrm{~N} / \mathrm{mm}^{2}\right]$ & ultimate strength $R_{m}\left[\mathrm{~N} / \mathrm{mm}^{2}\right]$ \\
\hline L 1 & 312 & 387 \\
\hline L 2 & 304 & 384 \\
\hline L 3 & 301 & 382 \\
\hline W & 383 & 435 \\
\hline C 1 & 314 & 380 \\
\hline C 2 & $R_{p 0,2}=289$ & 375 \\
\hline C 3 & 313 & 378 \\
\hline
\end{tabular}

Tab. B.5: Evaluation of the Young's modulus for the test tube

\begin{tabular}{|c|c|c|}
\hline \multicolumn{2}{|c|}{ Young's modulus $E$} \\
\hline coupon & Young's moduli $\left[\mathrm{N} / \mathrm{mm}^{2}\right]$ & average Young's modulus $E\left[\mathrm{~N} / \mathrm{mm}^{2}\right]$ \\
\hline L 1 & 190000 & \\
\hline L 2 & 178000 & \\
\hline L 3 & 186000 & \\
\hline W & 204500 & \\
\hline C 1 & 189100 & \\
\hline C 2 & 191500 & 190000 \\
\hline C 3 & 192000 & \\
\hline
\end{tabular}




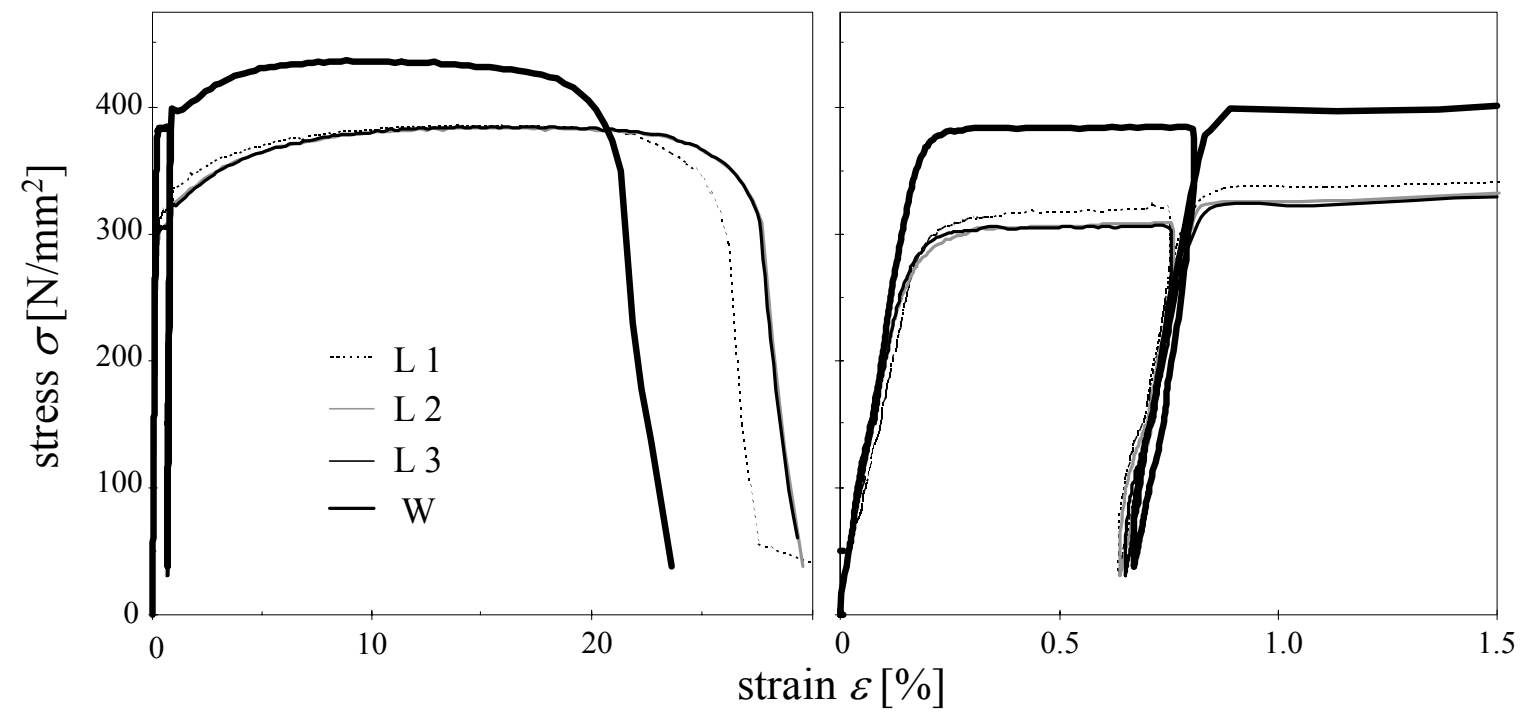

Fig. B.24: Stress-strain curves for the tensile tests for the base material-longitudinal coupons

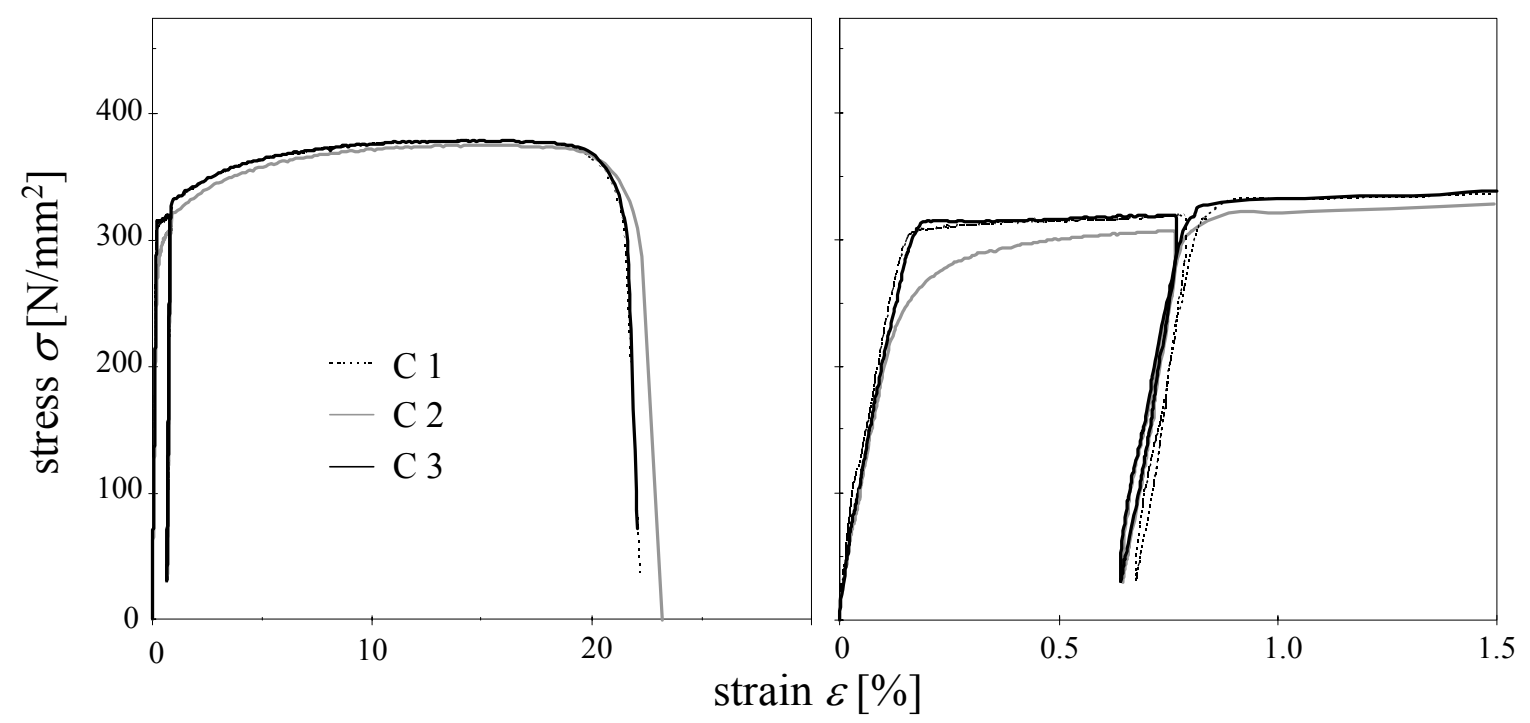

Fig. B.25: Stress-strain curves for the tensile tests for the base material-circumferential coupons

The difference in the transition between the elastic and the plastic region of coupon $\mathrm{C} 2$ in comparison to C 1 and C 3 arises because the latter two strips were cold-formed more intensively. This phenomena can be seen in Fig. B.26 in which a representative longitudinal stress-strain curve is compared to the cases $\mathrm{C} 2$ and $\mathrm{C} 3$. The curve for $\mathrm{C} 2$ is in a similar way non-linear as the curve for $\mathrm{L} 2$, while the coupon $\mathrm{C} 3$ exhibits a linear elastic-perfectly plastic behaviour. The Young's moduli for the circumferential tests do not differ much because a very large initial measuring length was chosen. The stress-strain curves for the circumferential and the longitudinal coupons agree well as seen in Fig. B.26.

The tensile tests for the longitudinal and for the circumferential coupons reveal close agreement concerning the Young's modulus. The results confirm the isotropy in the longitudinal and circumferential direction: 


$$
E \equiv E_{L}=E_{\|} \approx E_{C}=E_{\perp}
$$

For the use in numerical calculations and the interpretation of experimental results the Young's modulus is evaluated statistically in Tab. B.5.

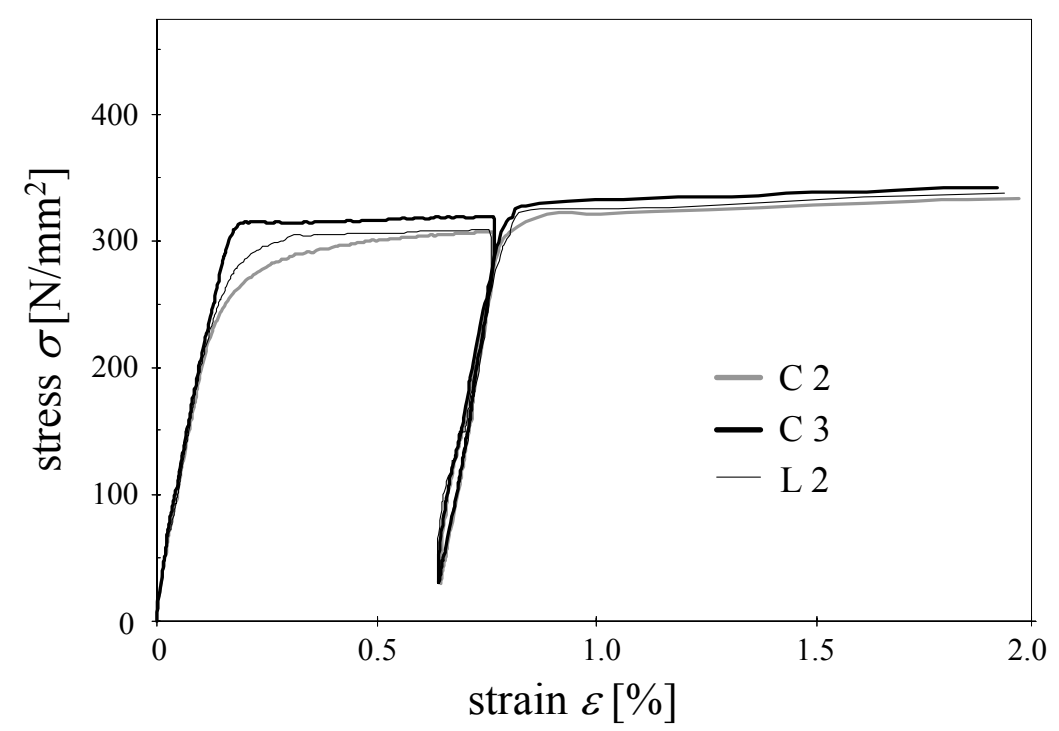

Fig. B.26: Comparison of stress-strain curves for the tensile tests for the base material-two circumferential coupons and one longitudinal coupon 


\section{Annex C: Reduced scale tests}

\section{C.1 General}

For better understanding of the load transfer mechanisms and the soil-structure interaction reduced scale experiments where performed within this project. Additionally, in-situ tests on constructions sites were executed in order to generate experimental results reflecting the tubular piles both installation and loading conditions. A scale reduction from real structures of about 10 was necessary to keep the costs of the experimental program reasonable, while allowing the tests to be conducted under a range of typical soil characters and densities. The set of loading tests was performed at the laboratories of the University of Louvain (UCL) with whom close co-operation was maintained throughout the project. The tests were performed under the supervision of the UCL, and planned closely together with the Universität Karlsruhe. Comments are given on the results from the in-situ tests but are not investigated further at this stage as the laboratory tests are validated with follow-up numerical analyses.

The reduced-scale tests form a basis for interpreting results from numerical analyses and serve as verification for different models. In the following the reduced-scale tests are briefly introduced with information on tests set-up and execution. More information can be found in Charue and Holeyman (2007) and Charue et al. (2007).

\section{C.2 Test and measuring program for the laboratory tests}

The tests performed at the laboratories at the UCL are made in order to provide an experimental basis to consolidate the numerical models and to establish a basis for the proposed design methods. As the experimental test set-up was mostly planned and detailed by the UCL it is referred to Charue and Holeyman (2007) where more details on the planning and the design using preliminary analyses can be found. The general set-up of the tests is a steel tube which is vertically embedded in soil which is confined by a cylindrical test bin and subjected to different elemental loading conditions.

The test program of the laboratory tests consisted of different experiments using 2 tubes. In a first set of tests a (dummy) tube (dt) was used. The second series used a more heavily equipped test tube. In this work it is only dealt with the first test series. As the tests were very time and cost consuming and therefore only a small number of tests were possible it was indispensable to assure the test set-up worked smoothly. Although the applied loads were quite small the sequence of the tests was rather important as the previous test could have an influence on the following test. Thus, the choice for the order of the experiments was made as summarized roughly in Tab. C.1. 
Before the experiments the geometry of the test tube was investigated very accurately by scanning the tube digitally and interpreting the results. The main focus was set on the deviations of the actual geometry from the nominal geometry. The findings were compared with the tolerances given in codes (DIN 1626). Additionally, a segment was cut off before the experiments from the test tube for detailed material property testing. Details on these investigations can be found in Annex B.

Tab. C.1: Test identifiers and sequence

\begin{tabular}{|c|l|c|c|}
\hline order & test identifier & filled/unfilled & tube \\
\hline 1 & dt-uf-T & \multirow{3}{*}{ unfilled } & \multirow{2}{*}{ “dummy" tube } \\
\hline 2 & dt-uf-L & & \\
\hline 3 & dt-uf-A & & \\
\hline
\end{tabular}

Before installation the tubes were instrumented with strain gauges. The test tube was instrumented with 8 simple gauges at 7 different levels (Fig. C.1).

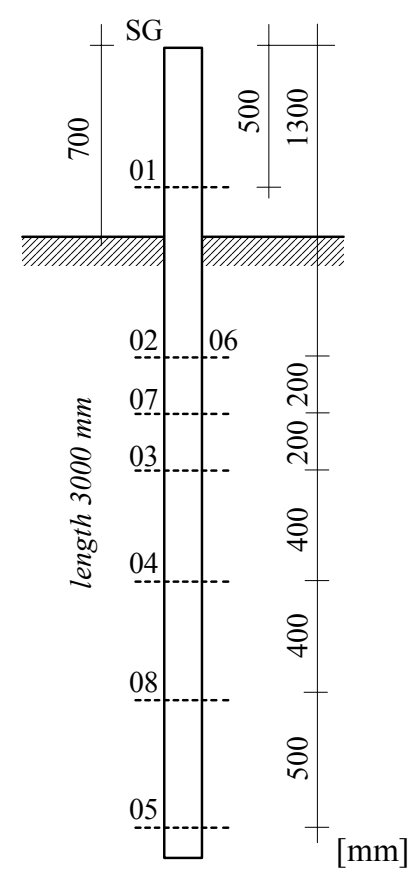

Fig. C.1: Strain gauges (SG) positioning and description for the instrumented layers

The measurement of all strain gauges was performed continuously during the loading of the piles. Moreover, the load and the pile displacements were measured with the monitored hydraulic system of the jacks. The acquisition system connected to the transducers coming from the pile was directly connected to a computer and was built custom made by the UCL. 


\section{C.3 Elements for the laboratory tests}

\section{C.3.1 Tubes}

In the progress of planning the experiments, tubes made of non-alloyed and of stainless steel were taken into consideration. In a first set of studies it was chosen to use tubes made of non-alloyed steel with the main reason of maintaining an elastic response of the structure for at least small loads. Further details on the mechanical measurment of the imperfections and material property tests are given in Annex B.

The chosen tube type made of non-alloyed constructional steel is fabricated by rolling steel plates and welding them together with a single longitudinal weld (Fig. C.2). More details concerning its manufacturing are given in Annex B. The test tube had a length of $3300 \mathrm{~mm}$, an outer diameter of $203 \mathrm{~mm}$, and a wall thickness of $2 \mathrm{~mm}$.
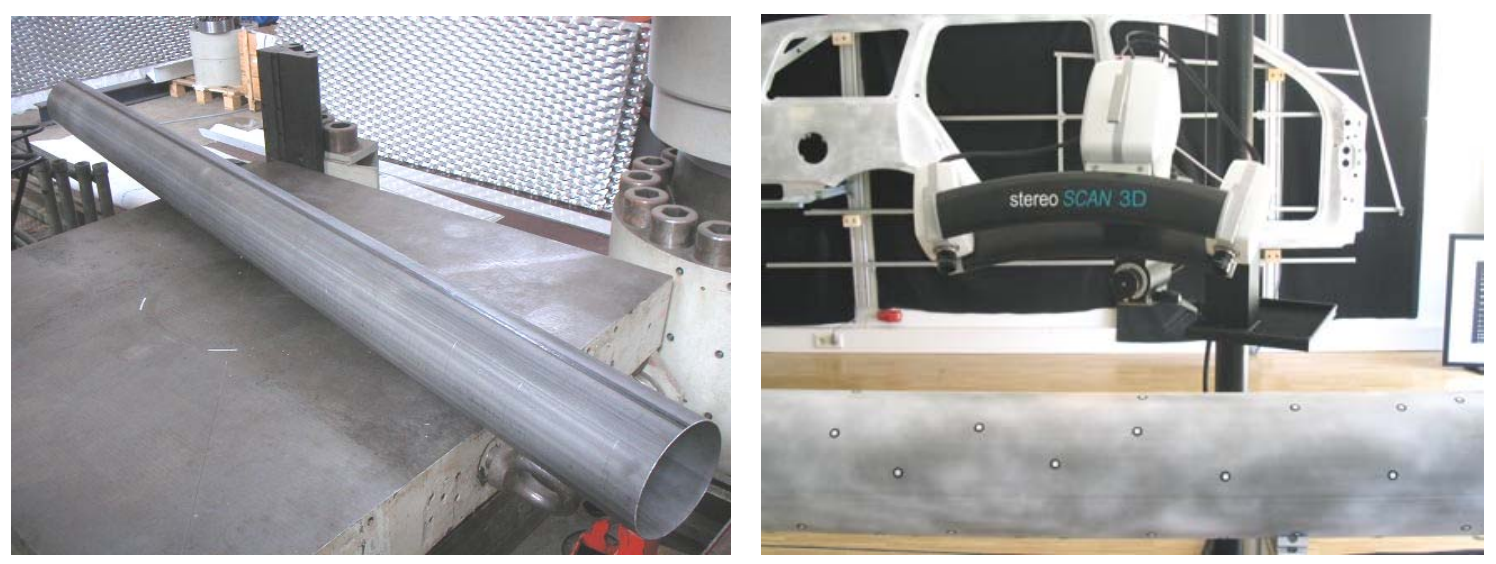

Fig. C.2: Test tube and digital scan

First, a $30 \mathrm{~cm}$ segment was cut off for detailed material property testing, and the remaining $3 \mathrm{~m}$ were scanned digitally in order to record the geometry with all imperfections are precisely as possible (Fig. C.2). The measurement was performed at the company Breuckmann GmbH using newest 3D-metrology techniques with a high resolution digitisation system. The method applied uses a MPT projection unit for obtaining more than 1 million measuring points. The measuring was executed by aligning 120 one-by-one views by pass-point-matching to one set of coordinates. The data from the measuring was evaluated and depicted regarding the deviations from the nominal geometrical values. Fig. C.3 shows an example of the interpreted results from the measurement. Additionally, the wall thickness of the test tube was measured at numerous points by ultrasonic measuring devices. The largest deviations in thickness were $-0.02 \mathrm{~mm}$ and $+0.08 \mathrm{~mm}$ from the nominal wall thickness of $2 \mathrm{~mm}$. More information regarding the applied measurements and the results from the thickness measurement and the digital scanning of the tube can be found in Annex B. 


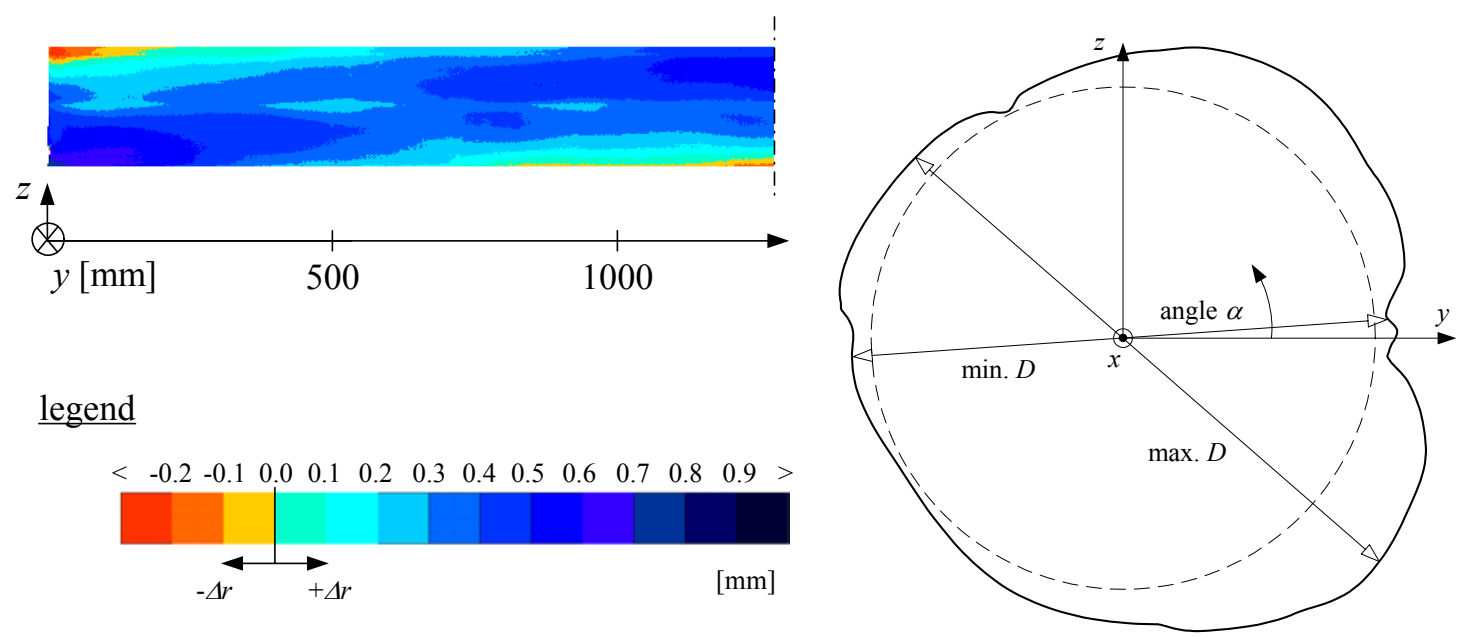

Fig. C.3: Examples for evaluating the data (radial deviations relative to the best-fit-cylinder; Fabrication tolerance - out of roundness)

The tubes were supposed to be made of non-alloyed constructional steel S235 according to the order and the delivery receipt. The material properties were evaluated from several tensile tests in longitudinal and circumferential direction according to DIN EN 100002-1:2001. The results were compared with the data given in the inspection document 3.1.B following DIN EN 10204:2004. Details on the material property tests are given in Annex B. The longitudinal and circumferential tensile tests showed close agreement regarding the Young's modulus. The results confirm the isotropy in the longitudinal and circumferential direction:

$$
E \equiv E_{L}=E_{\|} \approx E_{C}=E_{\perp}
$$

An average Young's modulus of $1900^{\prime} 000 \mathrm{~N} / \mathrm{mm}^{2}$ was evaluated.

\section{C.3.2 Soil}

The type of soil used in the experiments is the so-called "Brusselian Sand" which was acquired from a local quarry near the UCL with a grain size curve according to Fig. C.4. It was chosen to use the "Brusselian Sand" for the tests as it has been studied explicitly in several scientific researches (e.g. Vanden Berghe, 2001). Identification tests (granulometry, water content, soil density) for the sand were performed in laboratories of the UCL. Before applying the loads a cone penetration test (CPT) was performed in order to check on the soil resistance as a function of the depth below the surface. Details can be found in Charue and Holeyman (2007) and Charue et al., 2007.

The installation of the sand was of considerable importance as it controls the relative density of the sand and therefore also the stiffness of the soil. The installation of the sand was performed by using the time-consuming and complex method of pluviating the sand into the bin while taking significant factors such as raining height of the soil or time schedule into account. The installation process was worked out at the UCL (Charue and Holeyman, 2007, and Charue et al., 2007). 


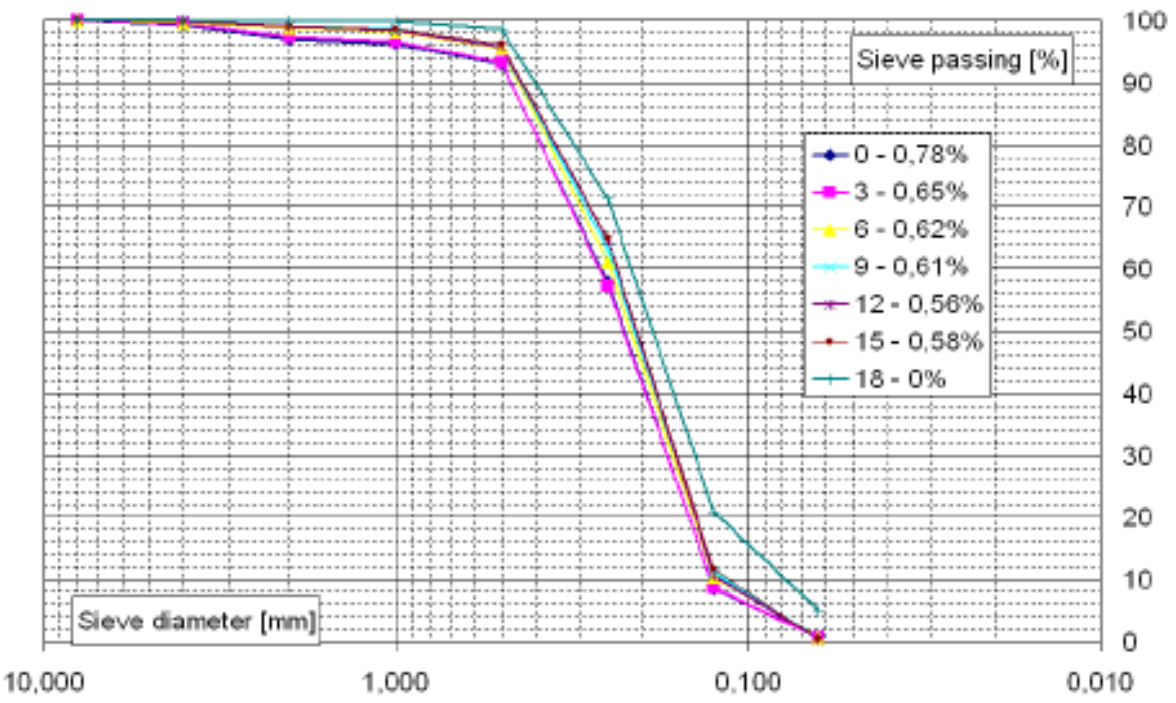

Fig. C.4: Grain size curve for different water contents of "Brusselian Sand" from Charue and Holeyman (2007)

\section{C.4 Set-up and execution of laboratory tests}

\section{C.4.1 General experimental set-up}

The general set-up of the tests is a steel tube which is vertically embedded in soil and subjected to different elemental loading conditions.
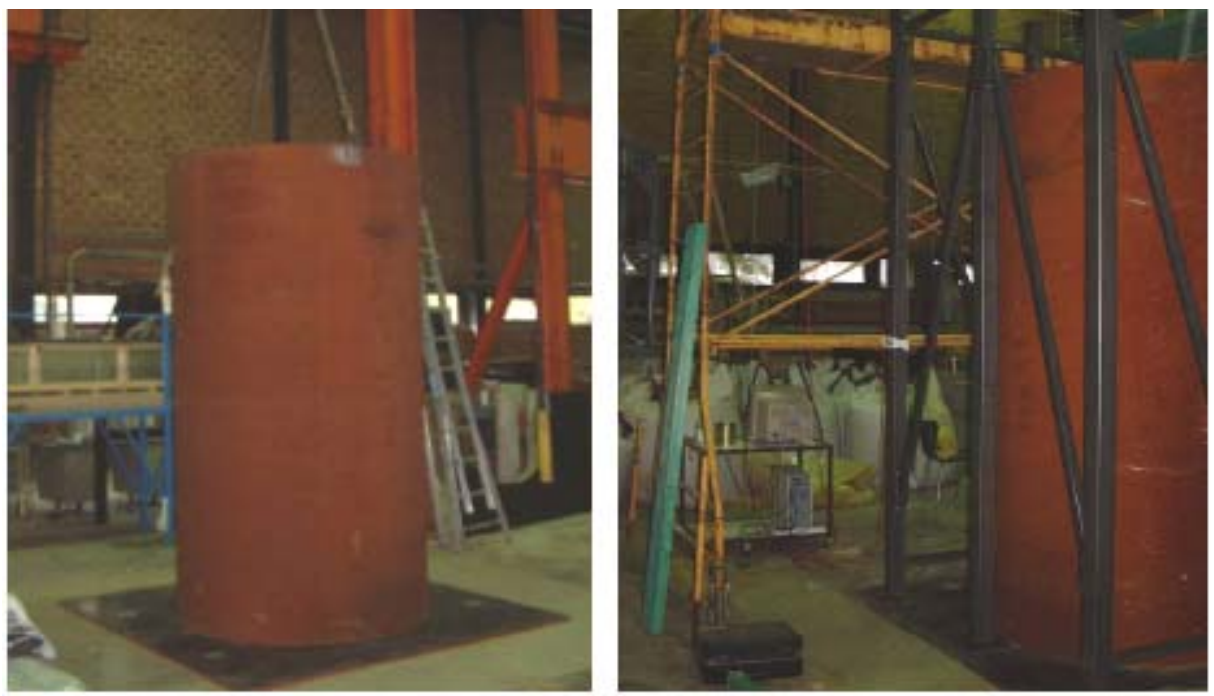

Fig. C.5: Outer confinement for the sand from Charue and Holeyman (2007)

The test bin in which the tube is installed in and which acts as confinement is a cylindrical steel structure of $3 \mathrm{~m}$ height, $1.7 \mathrm{~m}$ of diameter, and $16 \mathrm{~mm}$ of thickness (Fig. C.5). 

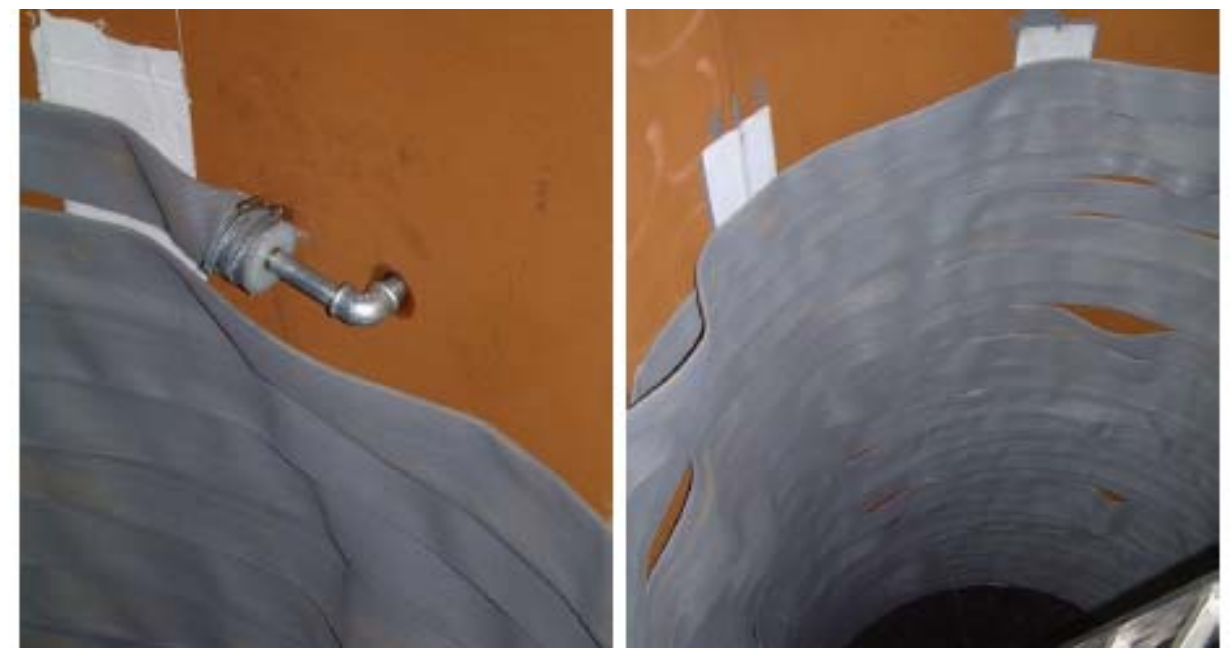

Fig. C.6: Lateral confinement by fire hose from Charue and Holeyman (2007)

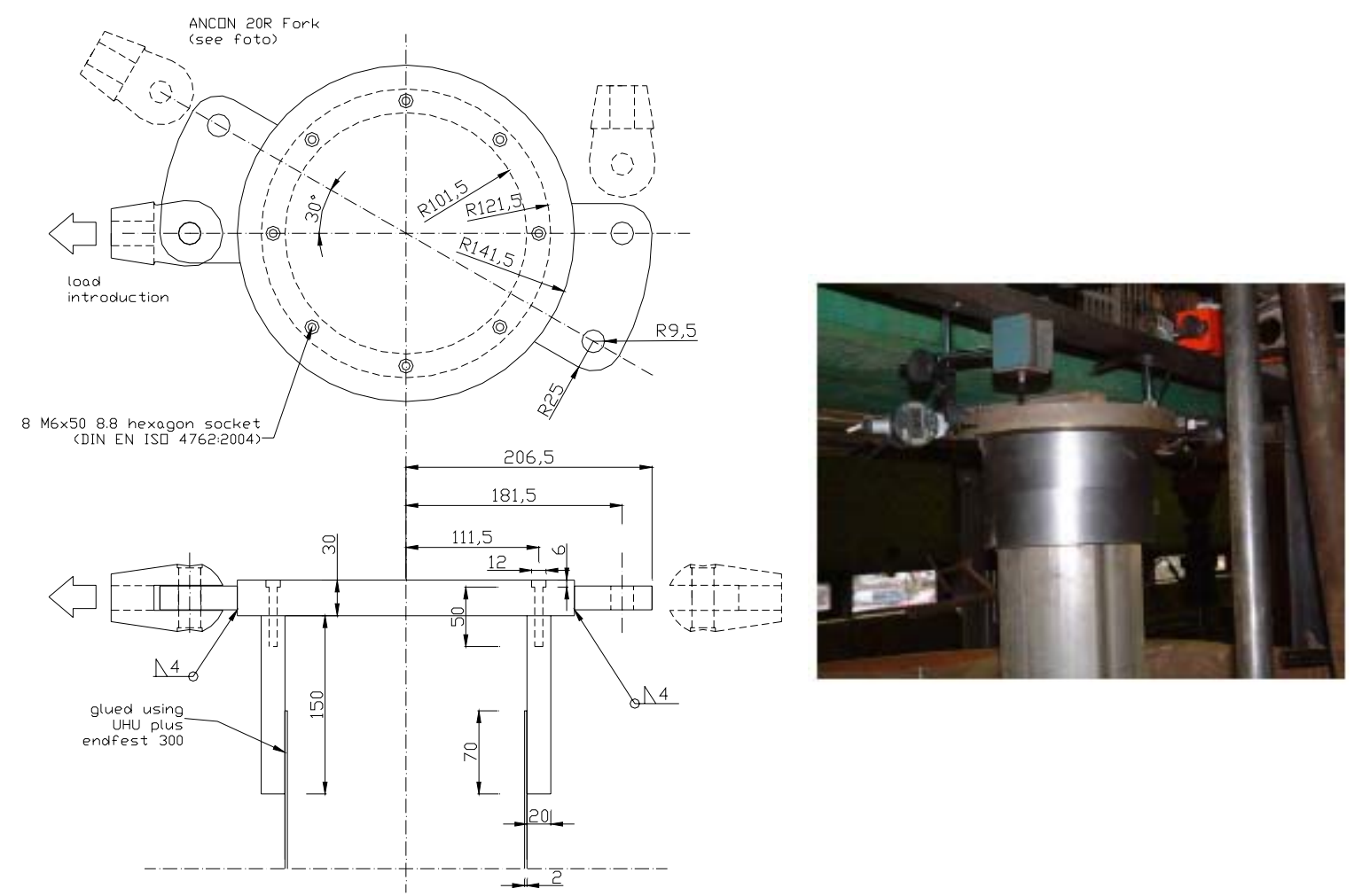

Fig. C.7: First draft of the load introduction device (LID) and final LID

As soil is a material whose behaviour is strongly influenced by its initial and disturbed conditions, the dimensions of the cylinder are important, and for the test set-up they encompass the influence of the radial supports as studied in preliminary tests. The reaction of the soil and therefore the stiffness is highly dependent on the confinement of the soil. Thus, it was chosen to include water filled fire hoses between soil and test bin, which allowed to control the pre-stressing of the soil and as a consequence the stiffness of the soil (Fig. C.6). In this step the top needs to be loaded simultaneously in order to prevent an uplift of the soil and the tube and to establish a uniform stress state. 
The installation of the sand needs to be executed well-controlled by pluviation as the relative density of the sand depends on several parameters (raining height, flow, homogeneity of the raining, shutter porosity, diameter of the shutter, characterisation of the sand, etc.). Intensive studies including numerous raining experiments were performed in order to defined all relevant parameters. More details are e.g. given in Charue and Holeyman (2007).

For the load introduction a pile cap is developed which allows to introduce all loads to the tube with stress distribution as smooth as possible (Fig. C.7). Thus, the load introdution device (LID) is glued to the tube with a fast-setting epoxy resine adhesive with hardener HW 2951 (Araldit ${ }^{\circledR}$ AW 2101).

Since the soil's characteristics change due to the application of lateral confinement by the water hoses, electrical CPT (Cone Penetrometer Test) are performed in the test bin between the subsequent tests.

\section{C.4.2 Axial loading}

The loading is performed by an automatically controlled system (hydraulic system of the laboratory allowing the control of the load, the rate of loading, and the duration of the applied load). The load is applied by a hydraulic jack supported by a stiff loading frame constructed around the test bin.

The aim of the axial load tests is to obtain a relationship between vertical displacement and the evolving opposing force, i.e. a load-displacement relationship for the entire pile. With this information a set of vertical springs can be positioned in numerical analyses representing the meridional shaft friction. A more detailed interpretation shall provide information on the shaft friction at different depths integrated over specific lengths (segments). With this information different spring stiffnesses can be applied in a numerical study. Further, the meridional stress distributions were looked at for a discussion on the uniformity of the stress distribution at different depths.

\section{C.4.3 Torsional loading}

The force is applied to the tube by means of dead weights at two sides diametrically opposed. The plates are connected to the pile cap by means of wires and pulleys. The wires are attached to the pile cap such as both loading directions are parallel and opposite to each other. The loading is performed by putting weights at both ends is simultaneously in order to apply a pure torque on the test tube.

The aim is to obtain a relationship between rotation and the evolving opposing force, i.e. a load-rotation relationship for the entire pile. With this information a set of horizontal springs could be positioned in numerical analyses representing the circumferential shaft friction. Calculating with a lever equivalent to the shell's radius, a number of circumferential springs can be applied. A more detailed interpretation shall provide information on the shaft friction at different depths integrated over specific 
lengths (segments). Then, different spring stiffnesses can be applied in a numerical study.

\section{C.4.4 Lateral loading}

The loading is performed similar to he latter case except that it is performed on one side at a time only. Additionally, the wire is attached centrically to the LID. Two diametrical wires are connected allowing a complete cycling lateral loading.

Firstly, results regarding the deformed shape and the stress and bending moment distribution are valuable since these can be used to verify the predicted results from the preliminary numerical study. In the next step the results needed again to compare the follow-up back-calculations. Secondly, the results shall be used to interpret the uniformity of the stress distribution. Here, it is important to have the stress distributions over the circumference at different cross-sections and over the lengths at different positions. Thirdly, the results shall be used for a back interpretation of the proposed soil models. In this context $p-y$ relationships have to be derived for comparison.

\section{C.5 Testing}

Numerous different tests were performed with the test tube in order to obtain more information on the acquiring system. The interpreted results form an important basis for the follow-up and back-calculations. All different loading types were tested. Further, the radial confinement was varied. The summary of all experiments can be found in Charue and Holeyman (2007). Some results are given at the corresponding sections. 


\section{Annex D: Element tests for „Brusselian Sand“}

\section{D.1 General}

For comparison and for the validation of the material data and the hypoplastic approach the results from experimental and numerical investigations by Vanden Berghe (2001) are compared with element tests performed using the hypoplasticity model implemented into ABAQUS by Fellin and Ostermann (2002). In this context the results of two monotonic drained and undrained triaxial tests are compared.

The hypoplastic parameters were derived by Vanden Berghe (2001) according to the calibration by Bauer (1996) and Herle (2000) with the following tests:

- 2 triaxial compression tests

- 1 triaxial extension test

- 1 oedometric compression

- (1 isotropic oedometric compression)

Mostly, the isotropic oedometric compression is used for deriving the parameter $\beta$. Vanden Berghe (2001) instead uses the oedometric compression. The evaluation of the experiments lead to the following parameters for hypoplasticity which are used for the numerical analyses with ABAQUS:

Tab. D.1: Parameters for hypoplasticity according Vanden Berghe (2001) for „Brusselian Sand“

\begin{tabular}{|c|c|c|c|c|c|c|c|}
\hline$\varphi_{c}\left[^{\circ}\right]$ & $h_{s}[\mathrm{kPa}]$ & $n$ & $e_{d 0}$ & $e_{c 0}$ & $e_{i 0}$ & $\alpha$ & $\beta$ \\
\hline 33 & $2.0 \cdot 10^{6}$ & 0.35 & 0.52 & 0.88 & 1.21 & 0.3 & 1.1 \\
\hline
\end{tabular}

\section{D.2 Drained triaxial compression}

For the triaxial compression a cylindrical specimen is deformed vertically while subjected to a constant external side pressure $\sigma_{2}=\sigma_{3}$ (Fig. D.1). For the drained case the pore water can pass off freely.

The drained triaxial compression test no. 4 according to the indication of Vanden Berghe (2001) is performed under the following conditions:

- geometry: height $h=200 \mathrm{~mm}$ and radius $r=50 \mathrm{~mm}$

- $\quad$ initial void ratio in the test and for ABAQUS $e_{0, V 4}=0.674$

- $\quad$ initial void ratio in simulation by Vanden Berghe $e_{0, \text { sim }}=0.674$

- $\quad$ initial side pressue $\sigma_{2, V 4}=400 \mathrm{kPa}$ 
— test no. 4 by Vanden Berghe (2001)

- - test no. 4 - ABAQUS

$\circ$ o simulation by Vanden Berghe (2001)
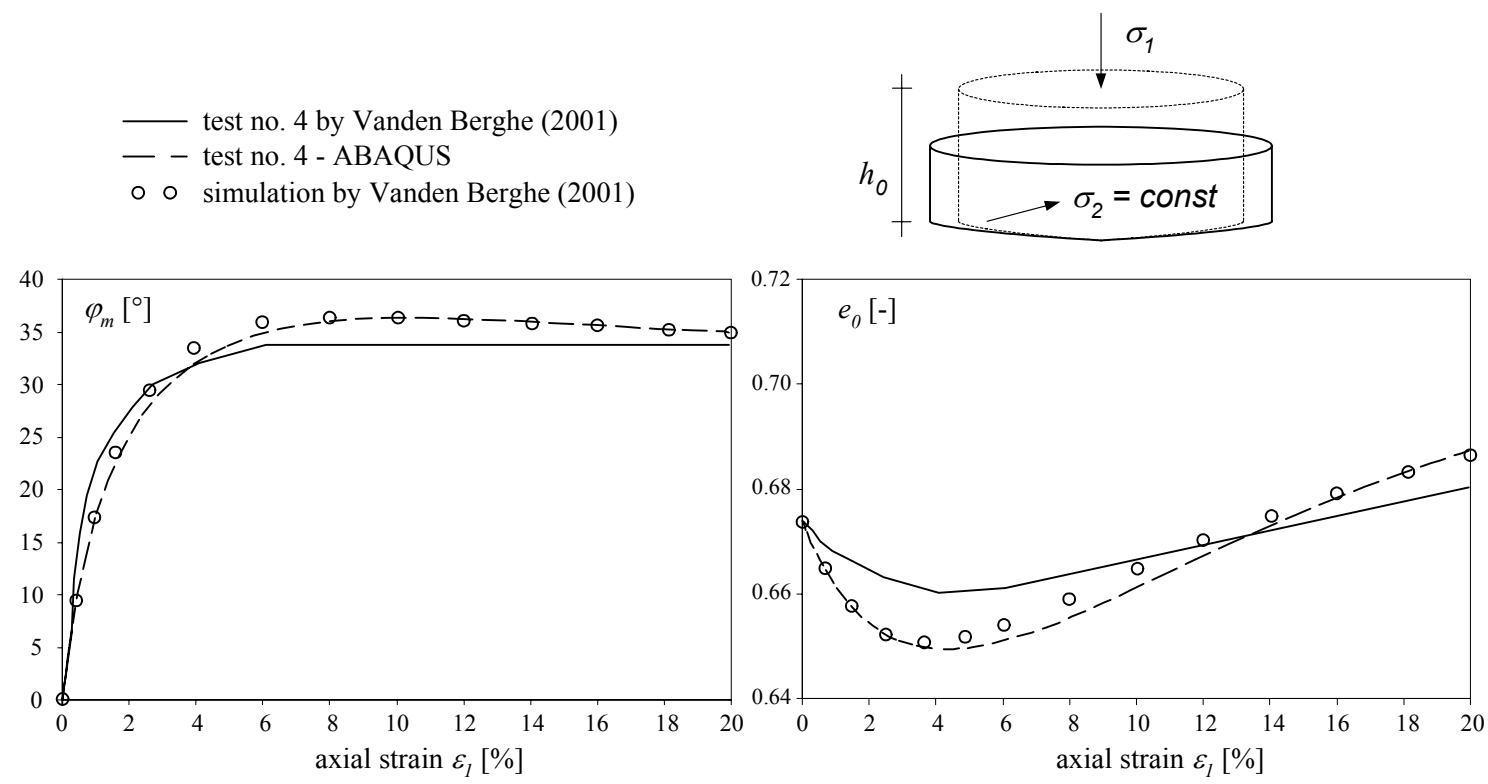

Fig. D.1: Comparison of the numerical results and the findings of test no. 4 by Vanden Berghe (2001) with „Brusselian Sand“ for drained triaxial compression

\section{D.3 Undrained triaxial compression}

For the triaxial compression a cylindrical specimen is deformed vertically while subjected to a constant external side pressure $\sigma_{2}=\sigma_{3}$ (Fig. D.2). For the undrained case the pore water cannot dissipate and as consequence the volume does not change. Using the rate of volume strain a ratio of the vertical and the horizontal strains which are needed for the numerical analyses can be derived as $u_{1} / u_{2}=-2 h / r$.

The undrained triaxial compression tests no. 8 and 9 according to the indication of Vanden Berghe (2001) are performed under the following conditions:

- geometry: height $h=200 \mathrm{~mm}$ and radius $r=50 \mathrm{~mm}$

- initial void ratio in the test and for ABAQUS $e_{0, V 8}=0.686$ and $e_{0, V 9}=0.718$

- initial void ratio in simulation by Vanden Berghe $e_{0, \text { sim }}=0.700$

- $\quad$ initial side pressure $\sigma_{2, V 8}=\sigma_{2, V 9}=200 \mathrm{kPa}$ 


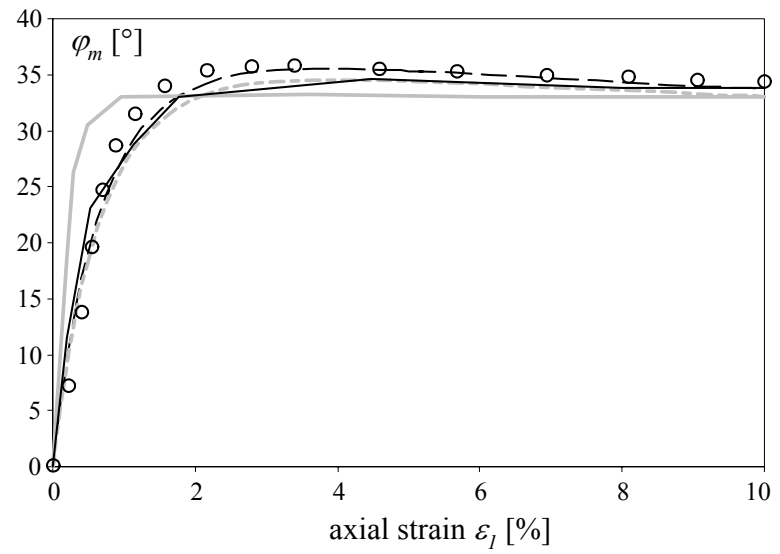

— test Nr. 8 by Vanden Berghe (2001)

test Nr. 9 by Vanden Berghe (2001)

- - test Nr. 8 - ABAQUS

---- test Nr. 9 - ABAQUS

$\circ \circ$ simulation by Vanden Berghe (2001)
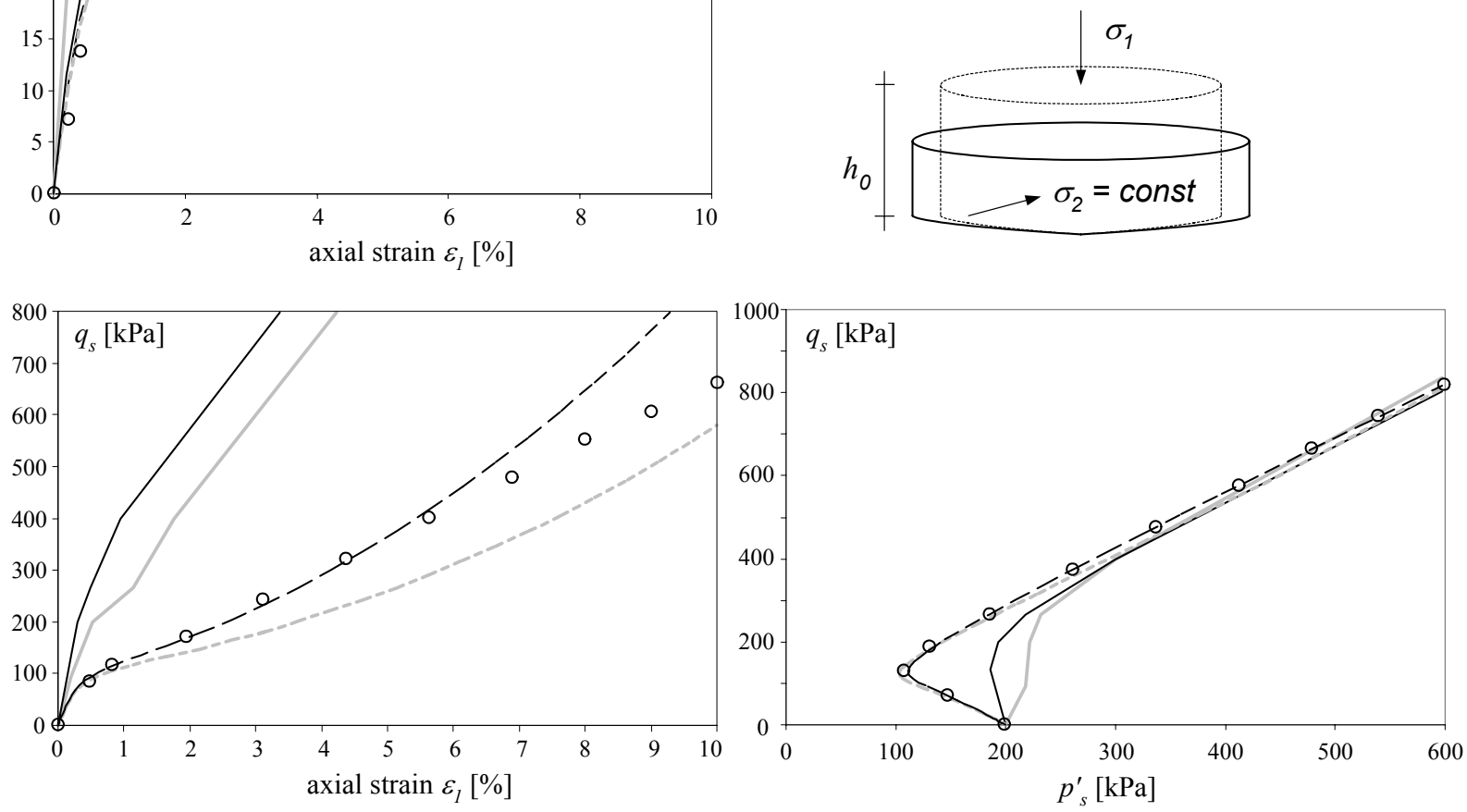

Fig. D.2: Comparison of the numerical results and the findings of test no. 8 and 9 by Vanden Berghe (2001) with „Brusselian Sand" for undrained triaxial compression

\section{D.4 Oedometric compression}

For the oedometric compression a cylindrical specimen is deformed vertically while radial displacements are constrained (Fig. D.3).

The oedometric compression is performed under the following conditions:

- $\quad$ geometry: height $h=20 \mathrm{~mm}$ and radius $r=31.5 \mathrm{~mm}$

- initial void ratio in the test and for ABAQUS $e_{0, V}=0.760$

- $\quad$ initial void ratio in simulation by Vanden Berghe $e_{0, s i m}=0,760$

- $\quad$ initial pressure $\sigma_{1}=1 \mathrm{kPa}$ and $\sigma_{2}=K_{0} \cdot 1 \mathrm{kPa}$ with $K_{0}=0.4$ 

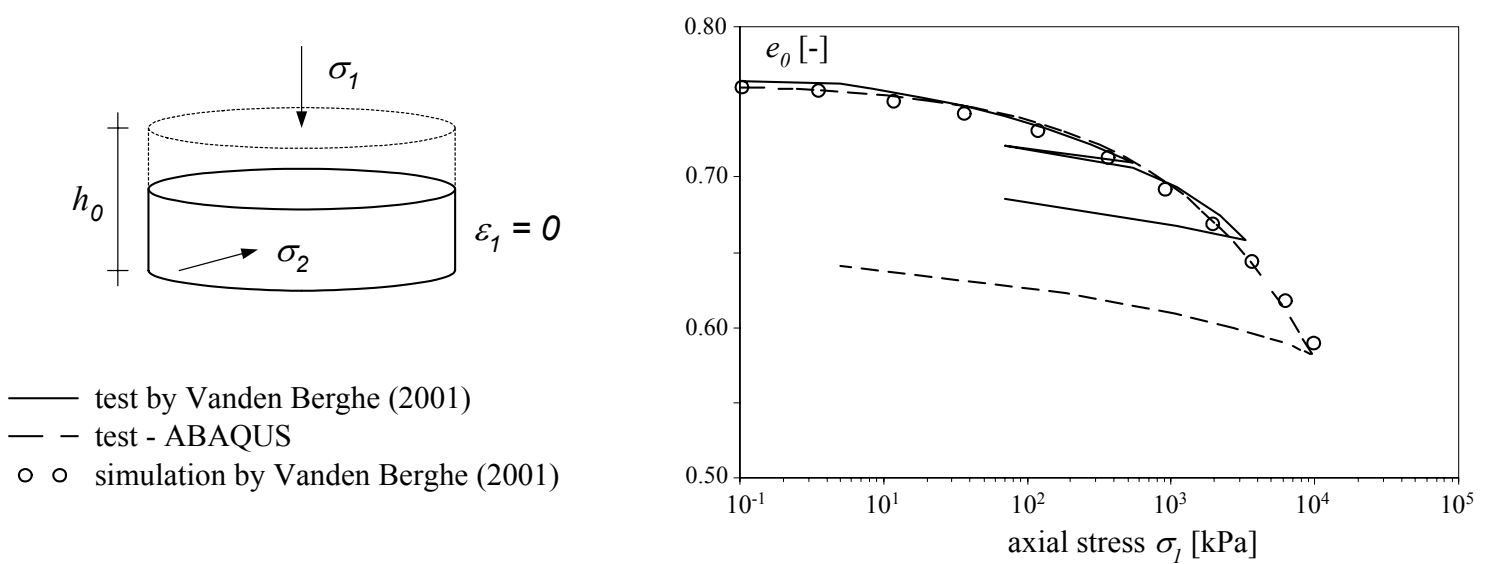

Fig. D.3: Comparison of the numerical results and the findings of test by Vanden Berghe (2001) with „Brusselian Sand“ for oedometric compression 


\section{Annex E: Specific modelling conditions for analyses}

\section{E.1 General}

In order to provide a more detailed insight to the specific modelling conditions used in numerical analyses, the following tables give an overview with respect to geometry, loading, material laws, etc. for different studies. The tables are referred to in the corresponding figure captions.

\section{E.2 Tables}

Tab. E.1: Summary of parameters for Fig. 9.19

\begin{tabular}{|c|c|c|c|c|}
\hline \multicolumn{5}{|c|}{$\begin{array}{l}\text { axially loaded cylindrical shell - linear bifurcation/eigenvalue analyses with soil - } \\
\sigma_{c l}=0.605 \cdot E \cdot t / r\end{array}$} \\
\hline data & \multicolumn{2}{|c|}{ parameter } & value & comments \\
\hline & \multirow{9}{*}{$\widetilde{\widetilde{\sigma}}$} & $K$ & 0.7 & \multirow{2}{*}{ loose sand $-n_{h}=2.2 \mathrm{MN} / \mathrm{m}^{3}$} \\
\hline & & $\delta$ & $20^{\circ}$ & \\
\hline & & $\gamma$ & $15.25 \mathrm{kN} / \mathrm{m}^{3}$ & - \\
\hline \multirow{3}{*}{ (a) } & & $k_{n}$ & $1.2 \mathrm{MN} / \mathrm{m}^{4} \cdot 1 / 2 \mathrm{l}$ & \multirow{6}{*}{$\begin{array}{l}\text { linear springs for tension and } \\
\text { compression; uniformly distributed vs. } z \\
\text { and circumference }\end{array}$} \\
\hline & & $k_{t, c}$ & $1.5 \mathrm{MN} / \mathrm{m}^{4} \cdot 1 / 2 \mathrm{l}$ & \\
\hline & & $k_{t, m}$ & 0 & \\
\hline \multirow{3}{*}{ (b) } & & $k_{n}$ & $1.2 \mathrm{MN} / \mathrm{m}^{4} \cdot 1 / 2 \mathrm{l}$ & \\
\hline & & $k_{t, c}$ & $40 \cdot(1 / 2 l)^{0.45}\left[\mathrm{MN} / \mathrm{m}^{4}\right]$ & \\
\hline & & $k_{t, m}$ & 0 & \\
\hline & \multirow{3}{*}{ 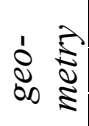 } & $l$ & $0 \mathrm{~m} \div 19 \mathrm{~m}$ & \multirow{3}{*}{$\begin{array}{l}\text { 2D shell elements; boundary conditions: } \\
\text { BC1f/ BC1f from EN 1993-1-6 }\end{array}$} \\
\hline & & $r / t$ & 50 & \\
\hline & & & $900 \mathrm{~mm}$ & \\
\hline
\end{tabular}

Tab. E.2: Summary of parameters for Fig. 9.20

\begin{tabular}{|c|c|c|c|c|}
\hline \multicolumn{5}{|c|}{$\begin{array}{l}\text { axially loaded cylindrical shell - linear bifurcation/eigenvalue analyses with soil - } \\
\sigma_{c l}=0.605 \cdot E \cdot t / r\end{array}$} \\
\hline data & \multicolumn{2}{|c|}{ parameter } & value & comments \\
\hline & \multirow{6}{*}{$\widetilde{\widetilde{\Xi}}$} & $K$ & 0.7 & \multirow{2}{*}{ loose sand $-n_{h}=2.2 \mathrm{MN} / \mathrm{m}^{3}$} \\
\hline & & $\delta$ & $20^{\circ}$ & \\
\hline & & $\gamma$ & $15.25 \mathrm{kN} / \mathrm{m}^{3}$ & - \\
\hline & & $k_{n}$ & $2.2 \mathrm{MN} / \mathrm{m}^{3} \cdot 1 / 2 \mathrm{l} / \mathrm{D}$ & \multirow{3}{*}{$\begin{array}{l}\text { linear springs for tension and } \\
\text { compression; uniformly distributed vs. } z \\
\text { and circumference }\end{array}$} \\
\hline & & $k_{t, c}$ & $1.5 \mathrm{MN} / \mathrm{m}^{4} \cdot 1 / 2 \mathrm{l}$ & \\
\hline & & $k_{t, m}$ & 0 & \\
\hline & \multirow{3}{*}{$\begin{array}{l}1 \\
\dot{\infty} \\
\infty \\
\infty\end{array}$} & $l$ & $0 \mathrm{~m} \div 25 \mathrm{~m}$ & \multirow{3}{*}{$\begin{array}{l}\text { 2D shell elements; boundary conditions: } \\
\text { BC1f/ BC1f from EN 1993-1-6 }\end{array}$} \\
\hline & & $r / t$ & 40,50 & \\
\hline & & $r$ & $450 \mathrm{~mm}, 900 \mathrm{~mm}, 1200 \mathrm{~mm}$ & \\
\hline
\end{tabular}


Tab. E.3: Summary of parameters for Fig. 9.21

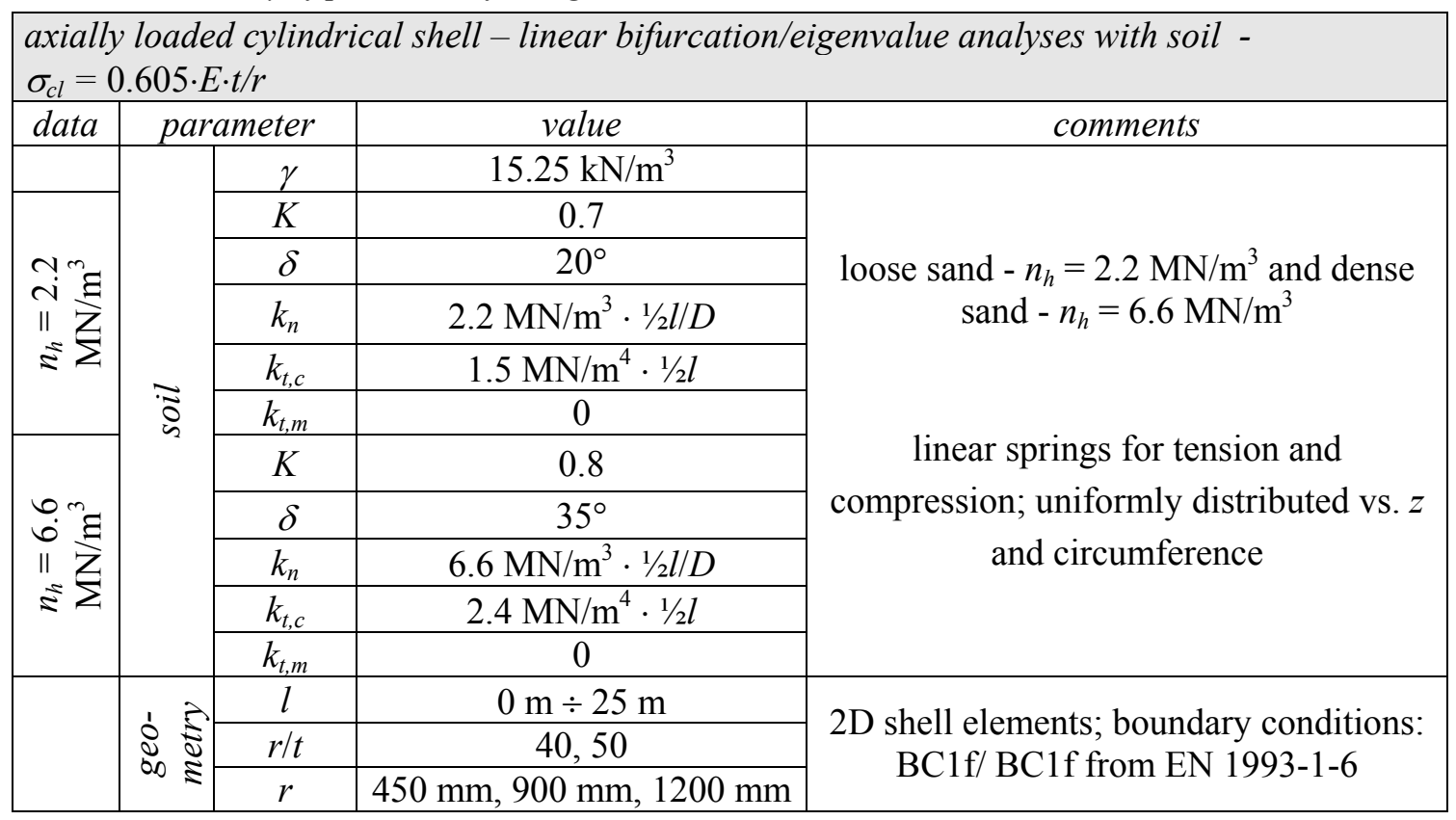

Tab. E.4: Summary of parameters for Fig. 9.22

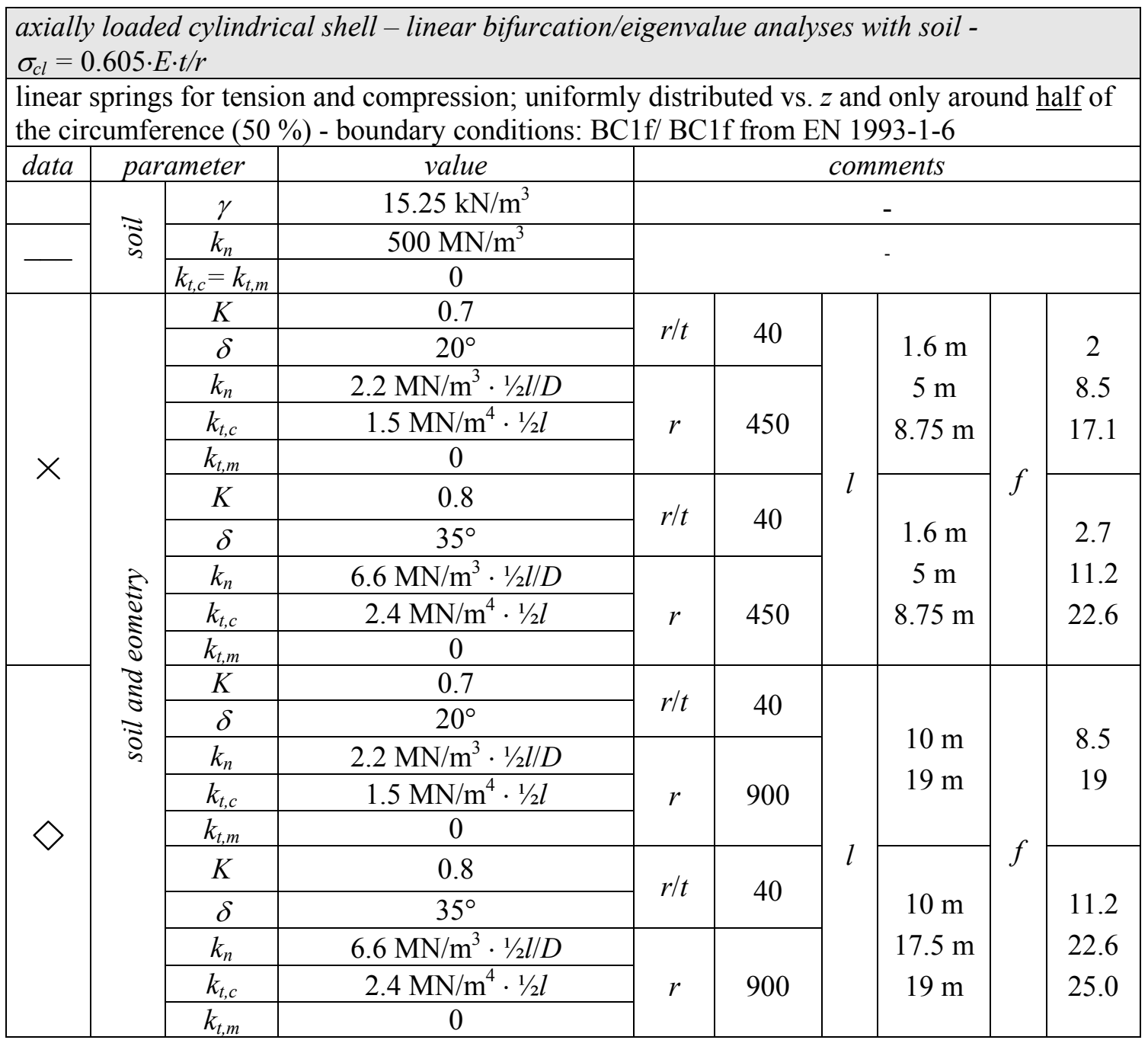


Tab. E.5: Summary of parameters for Fig. 9.23

\begin{tabular}{|c|c|c|c|c|}
\hline \multicolumn{5}{|c|}{$\begin{array}{l}\text { cylindrical shell subjected to e } \\
\text { soil }-\sigma_{c l}=0.92 \cdot E \cdot C_{\theta} / \omega \cdot t / r\end{array}$} \\
\hline data & \multicolumn{2}{|c|}{ parameter } & value & comments \\
\hline & \multirow{6}{*}{$\overrightarrow{\widetilde{\sigma}}$} & $K$ & 0.7 & \multirow{2}{*}{ loose sand $-n_{h}=2.2 \mathrm{MN} / \mathrm{m}^{3}$} \\
\hline & & $\delta$ & $20^{\circ}$ & \\
\hline & & $\gamma$ & $15.25 \mathrm{kN} / \mathrm{m}^{3}$ & - \\
\hline & & $k_{n}$ & $2.2 \mathrm{MN} / \mathrm{m}^{3} \cdot 1 / 2 \mathrm{l} / D$ & \multirow{3}{*}{$\begin{array}{l}\text { linear springs for tension and } \\
\text { compression; uniformly distributed vs. } z \\
\text { and circumference }\end{array}$} \\
\hline & & $k_{t, c}$ & $1.5 \mathrm{MN} / \mathrm{m}^{4} \cdot 1 / 2 \mathrm{l}$ & \\
\hline & & $k_{t, m}$ & $1.5 \mathrm{MN} / \mathrm{m}^{4} \cdot 1 / 2 \mathrm{l}$ & \\
\hline & \multirow{3}{*}{\begin{tabular}{ll|}
1 & $\lambda$ \\
0 & $\vdots$ \\
0 & $\vdots$
\end{tabular}} & $l$ & $0 \mathrm{~m} \div 25 \mathrm{~m}$ & \multirow{3}{*}{$\begin{array}{l}\text { 2D shell elements; boundary conditions: } \\
\text { BC1r/BC3 from EN 1993-1-6 }\left(C_{\theta}=0.6\right)\end{array}$} \\
\hline & & $r / t$ & 40,50 & \\
\hline & & $r$ & $450 \mathrm{~mm}, 900 \mathrm{~mm}, 1200 \mathrm{~mm}$ & \\
\hline
\end{tabular}

Tab. E.6: Summary of parameters for Fig. 9.24

\begin{tabular}{|c|c|c|c|c|}
\hline \multicolumn{5}{|c|}{$\begin{array}{l}\text { cylindrical shell subjected to external pressure-linear bifurcation/eigenvalue analyses with } \\
\text { soil }-\sigma_{c l}=0.92 \cdot E \cdot C_{\theta} / \omega \cdot t / r\end{array}$} \\
\hline data & \multicolumn{2}{|c|}{ parameter } & value & comments \\
\hline \multirow{6}{*}{ 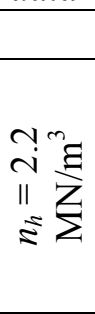 } & \multirow{11}{*}{$\approx$} & $\gamma$ & $15.25 \mathrm{kN} / \mathrm{m}^{3}$ & \multirow{6}{*}{$\begin{array}{l}\text { loose sand }-n_{h}=2.2 \mathrm{MN} / \mathrm{m}^{3} \text { and dense } \\
\text { sand }-n_{h}=6.6 \mathrm{MN} / \mathrm{m}^{3}\end{array}$} \\
\hline & & $K$ & 0.7 & \\
\hline & & $\delta$ & $20^{\circ}$ & \\
\hline & & $k_{n}$ & $2.2 \mathrm{MN} / \mathrm{m}^{3} \cdot 1 / 2 \mathrm{l} / D$ & \\
\hline & & $k_{t, c}$ & $1.5 \mathrm{MN} / \mathrm{m}^{4} \cdot 1 / 2 \mathrm{l}$ & \\
\hline & & $k_{t, m}$ & $1.5 \mathrm{MN} / \mathrm{m}^{4} \cdot 1 / 2 \mathrm{l}$ & \\
\hline \multirow{10}{*}{$\begin{array}{l}\sigma^{m} \Xi \\
\| Z \\
\equiv\end{array}$} & & $K$ & 0.8 & \multirow{5}{*}{$\begin{array}{l}\text { linear springs for tension and } \\
\text { compression; uniformly distributed vs. } z \\
\text { and circumference }\end{array}$} \\
\hline & & $\delta$ & $35^{\circ}$ & \\
\hline & & $k_{n}$ & $6.6 \mathrm{MN} / \mathrm{m}^{3} \cdot 1 / 2 \mathrm{l} / \mathrm{D}$ & \\
\hline & & $k_{t, c}$ & $2.4 \mathrm{MN} / \mathrm{m}^{4} \cdot 1 / 2 \mathrm{l}$ & \\
\hline & & $k_{t, m}$ & $2.4 \mathrm{MN} / \mathrm{m}^{4} \cdot 1 / 2 l$ & \\
\hline & \multirow{5}{*}{ 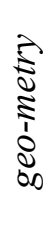 } & $l$ & $0 \mathrm{~m} \div 25 \mathrm{~m}$ & \multirow{5}{*}{$\begin{array}{l}\text { 2D shell elements; boundary conditions: } \\
\text { BC1r/BC3 from EN 1993-1-6 }\left(C_{\theta}=0.6\right)\end{array}$} \\
\hline & & $r / t$ & 40,50 & \\
\hline & & $r$ & $450 \mathrm{~mm}, 900 \mathrm{~mm}, 1200 \mathrm{~mm}$ & \\
\hline & & $r / t$ & 40,50 & \\
\hline & & $r$ & $450 \mathrm{~mm}, 900 \mathrm{~mm}, 1200 \mathrm{~mm}$ & \\
\hline
\end{tabular}


Tab. E. 7: Summary of parameters for Fig. 9.25

cylindrical shell subjected to external pressure - linear bifurcation/eigenvalue analyses with soil $-\sigma_{c l}=0.92 \cdot E \cdot C_{\theta} / \omega \cdot t / r$

linear springs for tension and compression; uniformly distributed vs. $z$ and only around half of the circumference $(50 \%)$

boundary conditions: BC1f/BC1f from EN 1993-1-6

\begin{tabular}{|c|c|c|c|c|c|c|c|c|c|}
\hline data & \multicolumn{2}{|c|}{ parameter } & value & \multirow{2}{*}{\multicolumn{6}{|c|}{$\frac{\text { comments }}{-}$}} \\
\hline & & $\gamma$ & $15.25 \mathrm{kN} / \mathrm{m}^{3}$ & & & & & & \\
\hline \multirow{10}{*}{$x$} & \multirow{20}{*}{ 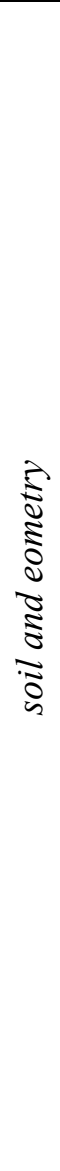 } & $K$ & 0.7 & relt & & \multirow{10}{*}{$l$} & \multirow{5}{*}{$\begin{array}{c}1.6 \mathrm{~m} \\
5 \mathrm{~m} \\
8.75 \mathrm{~m} \\
9.5 \mathrm{~m} \\
13 \mathrm{~m} \\
15.5 \mathrm{~m}\end{array}$} & \multirow{10}{*}{$f$} & 2 \\
\hline & & $\delta$ & $20^{\circ}$ & 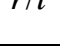 & 40 & & & & 8.5 \\
\hline & & $k_{n}$ & $2.2 \mathrm{MN} / \mathrm{m}^{3} \cdot 1 / 2 \mathrm{l} / D$ & \multirow{3}{*}{$r$} & \multirow{3}{*}{450} & & & & 17.1 \\
\hline & & $k_{t, c}$ & $1.5 \mathrm{MN} / \mathrm{m}^{4} \cdot 1 / 2 \mathrm{l}$ & & & & & & 19 \\
\hline & & $k_{t, m}$ & $1.5 \mathrm{MN} / \mathrm{m}^{4} \cdot 1 / 2 \mathrm{l}$ & & & & & & 35.1 \\
\hline & & $K$ & 0.8 & \multirow{2}{*}{$r / t$} & \multirow{2}{*}{40} & & \multirow{5}{*}{$\begin{array}{c}1.6 \mathrm{~m} \\
5 \mathrm{~m} \\
8.75 \mathrm{~m} \\
9.5 \mathrm{~m} \\
13 \mathrm{~m}\end{array}$} & & \multirow{5}{*}{$\begin{array}{c}2.7 \\
11.2 \\
22.6 \\
25 \\
37.1\end{array}$} \\
\hline & & $\delta$ & $35^{\circ}$ & & & & & & \\
\hline & & $k_{n}$ & $6.6 \mathrm{MN} / \mathrm{m}^{3} \cdot 1 / 2 \mathrm{l} / D$ & \multirow{3}{*}{$r$} & \multirow{3}{*}{450} & & & & \\
\hline & & $k_{t c}$ & $2.4 \mathrm{MN} / \mathrm{m}^{4} \cdot 1 / 2 \mathrm{l}$ & & & & & & \\
\hline & & $k_{t, m}$ & $2.4 \mathrm{MN} / \mathrm{m}^{4} \cdot 1 / 2 \mathrm{l}$ & & & & & & \\
\hline \multirow{10}{*}{$\diamond$} & & $K$ & 0.7 & \multirow{2}{*}{$r / t$} & \multirow{2}{*}{40} & \multirow{10}{*}{$l$} & \multirow{5}{*}{$\begin{array}{c}3.2 \mathrm{~m} \\
10 \mathrm{~m} \\
17.5 \mathrm{~m} \\
19 \mathrm{~m}\end{array}$} & \multirow{10}{*}{$f$} & 21 \\
\hline & & $\delta$ & $20^{\circ}$ & & & & & & 85 \\
\hline & & $k_{n}$ & $2.2 \mathrm{MN} / \mathrm{m}^{3} \cdot 1 / 2 \mathrm{l} / \mathrm{D}$ & \multirow{3}{*}{$r$} & \multirow{3}{*}{900} & & & & 170 \\
\hline & & $k_{t, c}$ & $1.5 \mathrm{MN} / \mathrm{m}^{4} \cdot 1 / 2 \mathrm{l}$ & & & & & & 17.2 \\
\hline & & $k_{t, m}$ & $1.5 \mathrm{MN} / \mathrm{m}^{4} \cdot 1 / 2 \mathrm{l}$ & & & & & & 19 \\
\hline & & $K$ & 0.8 & \multirow{2}{*}{$r / t$} & \multirow{2}{*}{40} & & \multirow{5}{*}{$\begin{array}{c}1.6 \mathrm{~m} \\
10 \mathrm{~m} \\
17.5 \mathrm{~m} \\
19 \mathrm{~m}\end{array}$} & & \multirow{5}{*}{$\begin{array}{c}2.7 \\
11.2 \\
22.6 \\
25.0\end{array}$} \\
\hline & & $\delta$ & $35^{\circ}$ & & & & & & \\
\hline & & $k_{n}$ & $6.6 \mathrm{MN} / \mathrm{m}^{3} \cdot 1 / 2 \mathrm{l} / D$ & \multirow{3}{*}{$r$} & \multirow{3}{*}{900} & & & & \\
\hline & & $k_{t, c}$ & $2.4 \mathrm{MN} / \mathrm{m}^{4} \cdot 1 / 2 \mathrm{l}$ & & & & & & \\
\hline & & $k_{t, m}$ & $2.4 \mathrm{MN} / \mathrm{m}^{4} \cdot 1 / 2 \mathrm{l}$ & & & & & & \\
\hline
\end{tabular}

Tab. E.8: Summary of parameters for Fig. 11.5

\begin{tabular}{|c|c|c|c|}
\hline \multicolumn{4}{|c|}{$\begin{array}{l}\text { cylindrical shell subjected } \\
\sigma_{c l}=0.92 \cdot E \cdot C_{\theta} / \omega \cdot t / r\end{array}$} \\
\hline data & parameter & value & comments \\
\hline & $p_{\text {crit } 0.5}$ & \multicolumn{2}{|c|}{$\begin{array}{l}\text { buckling pressure for "half-pressure", i.e. with external pressure applied } \\
\text { to only } 50 \% \text { of the circumference }\end{array}$} \\
\hline & $p_{\text {crit } 1.0}$ & \multicolumn{2}{|c|}{$\begin{array}{l}\text { buckling pressure for "full-pressure", i.e. with external pressure applied } \\
\text { to } 100 \% \text { of the circumference }\end{array}$} \\
\hline & $l$ & $1.5 \mathrm{~m} \div 15 \mathrm{~m}$ & \multirow{3}{*}{$\begin{array}{l}\text { 2D shell elements; boundary conditions: } \\
\text { BC1r/BC3 from EN 1993-1-6 }\left(C_{\theta}=0.6\right)\end{array}$} \\
\hline & $r / t$ & $40,50,60,75$ & \\
\hline & $r$ & $900 \mathrm{~mm}$ & \\
\hline
\end{tabular}


Tab. E.9: Summary of parameters for Fig. 11.6

\begin{tabular}{|c|c|c|c|}
\hline \multicolumn{4}{|c|}{$\begin{array}{l}\text { cylindrical shell subjected to external pressure - linear bifurcation/eigenvalue analyses - } \\
\sigma_{c l}=0.92 \cdot E \cdot C_{\theta} / \omega \cdot t / r\end{array}$} \\
\hline data & parameter & value & comments \\
\hline & $p_{\text {crit } 0.5}$ & \multicolumn{2}{|c|}{$\begin{array}{l}\text { buckling pressure for "half-pressure", i.e. with external pressure applied } \\
\text { to only } 50 \% \text { of the circumference }\end{array}$} \\
\hline & $p_{\text {crit } 1.0}$ & \multicolumn{2}{|c|}{$\begin{array}{l}\text { buckling pressure for "full-pressure", i.e. with external pressure applied } \\
\text { to } 100 \% \text { of the circumference }\end{array}$} \\
\hline & $l$ & $2.0 \mathrm{~m} \div 30 \mathrm{~m}$ & \multirow{3}{*}{$\begin{array}{l}\text { 2D shell elements; boundary conditions: } \\
\text { BC1f/ BC1f from EN 1993-1-6 }\left(C_{\theta}=1.5\right)\end{array}$} \\
\hline & $r / t$ & $40,50,60,75$ & \\
\hline & $r$ & $900 \mathrm{~mm}$ & \\
\hline
\end{tabular}

Tab. E.10: Summary of parameters for Fig. 11.8

\begin{tabular}{|c|c|c|c|}
\hline \multicolumn{4}{|c|}{$\begin{array}{l}\text { cylindrical shell subjected to external pressure - linear bifurcation/eigenvalue analyses - } \\
\sigma_{c l}=0.92 \cdot E \cdot C_{\theta} / \omega \cdot t / r\end{array}$} \\
\hline data & parameter & value & comments \\
\hline \multirow{2}{*}{ 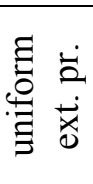 } & $p_{\text {crit } 0.5}$ & \multicolumn{2}{|c|}{$\begin{array}{l}\text { buckling pressure for "half-pressure", i.e. with uniform external } \\
\text { pressure applied to only } 50 \% \text { of the circumference }\end{array}$} \\
\hline & $p_{\text {crit } 1.0}$ & \multicolumn{2}{|c|}{$\begin{array}{l}\text { buckling pressure for "full-pressure", i.e. with uniform external pressure } \\
\text { applied to } 100 \% \text { of the circumference }\end{array}$} \\
\hline \multirow{5}{*}{ 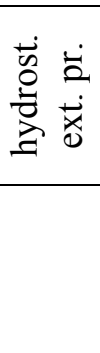 } & $p_{\text {crit } 0.5}$ & \multicolumn{2}{|c|}{$\begin{array}{l}\text { buckling pressure for "half-pressure", i.e. with linear/hydrostatic } \\
\text { external pressure applied to only } 50 \% \text { of the circumference }\end{array}$} \\
\hline & $p_{\text {crit } 1.0}$ & \multicolumn{2}{|c|}{$\begin{array}{l}\text { buckling pressure for "full-pressure", i.e. with linear/hydrostatic } \\
\text { external pressure applied to } 100 \% \text { of the circumference }\end{array}$} \\
\hline & $l$ & $1.8 \mathrm{~m} \div 14.4 \mathrm{~m}$ & \multirow{3}{*}{$\begin{array}{l}\text { 2D shell elements; boundary conditions: } \\
\text { BC1r/BC3 from EN 1993-1-6 }\left(C_{\theta}=0.6\right)\end{array}$} \\
\hline & $r / t$ & 50 & \\
\hline & $r$ & $900 \mathrm{~mm}$ & \\
\hline
\end{tabular}

